\title{
EMPIRICAL DETERMINATION OF THE NEUTRINO \\ FLUX AT THE NOvA EXPERIMENT
}

\author{
A THESIS \\ Submitted to the \\ FACULTY OF SCIENCE \\ PANJAB UNIVERSITY, CHANDIGARH \\ For the degree of
}

DOCTOR OF PHILOSOPHY

2018

KULDEEP KAUR

DEPARTMENT OF PHYSICS

CENTRE OF ADVANCED STUDY IN PHYSICS

PANJAB UNIVERSITY

CHANDIGARH, INDIA 



\section{This thesis is dedicated to my father, S. Gian Singh Maan.}





\section{AKNOWLEDGEMENTS}

I want to begin with thanking God for His abundant blessings in my entire life endeavor.

Thank you, to my supervisor Prof. Vipin Bhatnagar, Department of Physics, Panjab University, Chandigarh, for his constant support, guidance, and motivation throughout my research work. I am very much thankful to him for his precious time and scientific suggestions.

Thank you, to my supervisor Prof. Sanjib R. Mishra, Department of Physics and Astronomy, University of South Carolina, USA, for teaching me many of the finer points of experimentation, statistics and for sharing your intuition in Physics with me. Thank you, for trust and encouragement. This thesis would not have been possible without the countless hours of discussion and support.

Thank you, to my M. Sc teacher Prof. Balbir S. Sandhu and B. Sc teacher Prof. Balwinder Kumar.

Thank you, to my local Fermilab supervisor Dr. John W Cooper, who helped me in every part of this work at Fermilab and special thanks to Dr. Peter Shanahan for all help.

Thank you, to the Chairperson (Prof. Navdeep Goyal), Department of Physics, Dean

of Science (Prof. Devinder Mehta) and Vice-Chancellor (Prof. Arun Kumar Grover), Panjab University, Chandigarh for providing me all support for my research work.

Thank you, to the entire NOvA collaboration, without whose efforts the experimental data would not have existed to write this thesis.

Thank you, to the NOvA Beam Group and especially to Dr. Alexander Radovic, Dr. Raphael Scroeter, Dr. Robert Hatcher, Dr. Giulia Brunetti, Dr. Linda Cremonesi and Dr. Gareth Kafka. They were always available to answer my questions and to help me every time I needed. 
Thank you, to the NOvA Near Detector Group and especially to Prof. Mathew Muether and Dr. Jon Paley for all help and guidance.

Thank you, to the Fermilab Neutrino Division for the support given to the IIFC program.

Thank you, to the Department of Science and Technology for the support given to the IIFC program.

Thank you, to Dr. Xuebing Bu and Dr. H. Duyang for all guidance and teachings.

Thank you, to Dr. Gavin Davies, for proofreading this thesis, motivation, software help and all support. Thank you, to Dr. Fernanda Psihas for all support and motivation.

Thank you, to my cheerful friends Sarabjit Ma'am, Bishu, Ramneek, Harpreet, Pardeep, Kiran, Sukhi and Genius for always having trust in me that I can do it.

Thank you, to Etta Jonson and Stephanie Schuler. They made my life easier. But also to the Fermilab Visa Office, especially to Kappatolia Sherman, who helped to extend my J1 visa.

Thank you, to my father, who this thesis is dedicated. He allowed me to became the woman that I am now; I owe everything to him.

Thank you, to my mother for all love and always make a preference for me Thank you, to my brother, and sister in law for all support and care.

Last but not least, I would like to thank the person who has stood by my side through this thesis and support me in all ways. Thank you Gurinder for all patience, trust and love, this journey would be unimaginable without you.

Date:

Chandigarh Kuldeep Kaur 


\section{Abstract}

An accurate prediction of the neutrino flux is needed for precision oscillation, and is critical for the cross-section measurements. NOvA, a second-generation long-baseline neutrino oscillation experiment at Fermilab, is designed to measure the (anti) electron neutrino appearance and the (anti) muon neutrino disappearance. NOvA uses two functionally identical detectors separated by 810 kilometers at locations 14 milliradians off-axis from the NuMI muon neutrino beam at Fermilab. At these locations the beam energy peaks at $2 \mathrm{GeV}$. This baseline is the longest in the world for an accelerator-based neutrino oscillation experiment, which enhances the sensitivity to the neutrino mass ordering.

This thesis presents a novel method to empirically constrain the neutrino flux at NOvA Detector using the NuMI accelerator facility at the Fermi National Accelerator Laboratory. The precisely constrained flux will be used to conduct precision measurements of neutrino oscillation and cross-section in the NOvA experiment. 



\section{Contents}

List of Figures

List of Tables

1 Introduction 1

1.1 The Neutrino: An Introduction ................. 1

1.1.1 A Brief History of the Neutrino . . . . . . . . . . . . 1

1.1.2 Neutrinos in the Standard Model . . . . . . . . . . . . . . 6 6

1.1.3 Neutrino Oscillation ................... 7

1.1 .4 Matter Effect .................... 11

1.1.5 Neutrino Mass Hierarchy . . . . . . . . . . . . . . . . 12

1.1.6 Evidence of Neutrino Oscillation . . . . . . . . . . . . . 13

1.2 Neutrino Oscillations in NOvA ................. 14

1.3 Importance of Precise Flux Determination in Neutrino Physics . . . . . . . 16

1.4 Outline of the Thesis . . . . . . . . . . . . . . . . . 17

$2 \quad$ NuMI Beam line $\quad 19$

2.1 Introduction . . . . . . . . . . . . . . . . . 19

2.2 NuMI Beam . . . . . . . . . . . . . . . . . . . . . 20

2.2.1 Primary Proton Beam . . . . . . . . . . . . . 22 


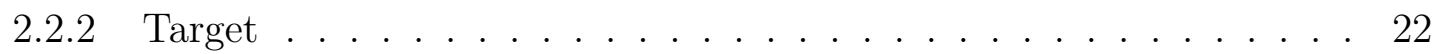

2.2.3 Focusing of Secondary $\pi$ 's and $K$ 's from the Target . . . . . . . . . 23

2.2.4 Decay pipe, Hadron absorber and Muon shield . . . . . . . . . . . 26

2.3 On-Axis versus Off-Axis Beam . . . . . . . . . . . . . [26

2.4 Neutrino Spectra at the NOvA Detectors . . . . . . . . . . . . . 28

2.4.1 Forward Horn Current Mode: . . . . . . . . . . . . . . . . . . 28

2.4.2 Reverse Horn Current Mode: . . . . . . . . . . . . . . . . . 28

2.5 Simulation of NuMI Beam . . . . . . . . . . . . . . . . 30

3 NOvA Detectors

3.1 The NOvA Detector Design . . . . . . . . . . . . . . 33

3.1.1 PVC Modules . . . . . . . . . . . . . . . . . 34

3.1.2 Liquid Scintillator and Wavelength Shifting Fiber . . . . . . . . 34

3.1.3 Readout Electronics . . . . . . . . . . . . . . . 36

3.2 Far Detector . . . . . . . . . . . . . . . . . . . . . . 39

3.3 Near Detector . . . . . . . . . . . . . . . . . 40

3.4 Contributions to the Installation of NOvA ND . . . . . . . . . . 43

3.4.1 Fiber Testing of NOvA ND Modules . . . . . . . . . . . 43

3.4.2 Leak Test of NOvA ND Modules . . . . . . . . . . . . . 48

4 Near Detector Calibration, Event Reconstruction, and Simulation 51

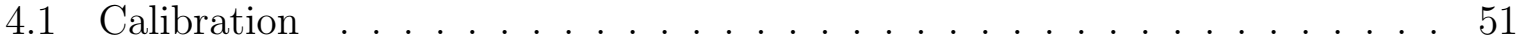

4.1.1 Attenuation Correction ............... 51

4.1.2 Absolute Energy Correction . . . . . . . . . . . . 52

4.2 Event Reconstruction . . . . . . . . . . . . . . . . . . 53

4.2.1 Interaction Separation with DBSCAN . . . . . . . . . 55

4.2.2 Finding Feature Guidelines with a Multi-Hough Transform . . . . . 58 
4.2.3 Vertex Identification with Elastic Arms . . . . . . . . . . . . . 59

4.2.4 Kalman Track . . . . . . . . . . . . . . . . . 59

4.2.5 ReMId ...................... . . . . . . . . . . .

4.2.6 $\nu_{\mu}$ Charged Current Energy Estimators . . . . . . . . . . . 61

4.2.7 Likelihood Based Identifier (LID) . . . . . . . . . . . 62

4.3 Detector Simulation . . . . . . . . . . . . . . . . . 62

5 Prediction of Neutrino Flux 65

5.1 Neutrino Parents ...................... 65

5.1.1 NOvA $\nu$ from Primary and Secondary Interactions . . . . . . . 66

5.1.2 NOvA $\nu$ from Primary and Secondary Nuclear Targets . . . . . . 67

5.2 Sources of the Beam-Transport Uncertainties . . . . . . . . . . . . . 67

5.2.1 Horn Current Miscalibration Uncertainties . . . . . . . . . . . . 70

5.2.2 Horn Position Uncertainties . . . . . . . . . . . . . . . 73

5.2.2.1 Horn 1 Position Unceratinties . . . . . . . . . . . . . 73

5.2.2.2 Horn 2 Position Unceratinties . . . . . . . . . . . . . . 73

5.2.3 Magnetic Field Distribution in the Horns Conductor . . . . . . . . 78

5.2.4 Beam Position on the Target . . . . . . . . . . . . . . . 80

5.2 .5 Beam Spot Size . . . . . . . . . . . . . . 85

5.2.6 Target Position ..................... 88

5.2.7 FLUKA Versions Comparison ............... 92

5.2 .8 G4NuMI vs FLUGG Comparison . . . . . . . . . . . . . 92

5.2.9 Horn Geometry and Water Layer . . . . . . . . . . . . . . . . . 94

5.2.10 Conclusion . . . . . . . . . . . . . . . . . . 96

5.3 Beam Hadron Production Uncertainties . . . . . . . . . . . . . . . . . . . 97

5.3 .1 Event Weights . . . . . . . . . . . . . . 999 
5.3.2 Weights for $\pi^{+}$Production . . . . . . . . . . . . . . 100

5.3.3 Weights for $K^{+}$Production . . . . . . . . . . . . . . 102

5.4 Results . . . . . . . . . . . . . . . . . . . . 108

5.4.1 Combined Constraint . . . . . . . . . . . . . . . 110

6 Constraining $K^{+}$Meson and Contribution to $\nu_{e}$ Flux $\quad 113$

6.1 Introduction and Motivation .................. 113

6.2 Data and Monte Carlo Samples . . . . . . . . . . . . . . . 116

6.3 Event Selection. . . . . . . . . . . . . . . . . . 117

6.3.1 Preselection . . . . . . . . . . . . . . . . 117

6.3.2 Fiducial Volume. . . . . . . . . . . . . . . . . . . 119

6.3.3 Contained Events vs Uncontained Events . . . . . . . . . . . . . . 119

6.3.4 ReMId (Neutral current rejection) . . . . . . . . . . . . . 119

6.3.5 MC Selection Based on Truth . . . . . . . . . . . . . 119

6.4 Method. . . . . . . . . . . . . . . . 121

6.4.1 Inclusive Charged Current Events without E Ead Cut. . . . . . . . . 121

6.4.2 Inclusive Charged Current Events with $\mathrm{E}_{\text {had }}$ Cut . . . . . . . . 123

6.5 Central Value of the $K^{+}$Normalization. . . . . . . . . . . . 125

6.5.1 Fitting the $\nu_{\mu}$ - CC Spectrum .............. 126

6.6 Systematic Uncertainties . . . . . . . . . . . . . . . . 129

6.6.1 Without GENIE Tune . . . . . . . . . . . . . . 129

6.6.2 Hadro-Production: FLUKA vs GEANT4( $\pi /$ K Shape) . . . . . . . 130

6.6.3 Hadron Shower Containment. . . . . . . . . . . . . . . . . . . 130

6.6.4 Variation of Muon Energy. . . . . . . . . . . . . . . 131

6.6.5 Effect of External Hadron Production Data. . . . . . . . . . . . . 132

6.6.6 Cross-section Uncertainty. . . . . . . . . . . . . . . 133 
6.6.7 Genie Re-weight Cross Check. . . . . . . . . . . . . . . . . . 134

6.6.8 Intensity Effect and Time Dependent Modeling Effects . . . . . . . 134

6.6 .9 Detector Modeling . . . . . . . . . . . . . . . . . . . . . 134

6.7 Summary of Systematics. . . . . . . . . . . . . . . . . . . . . 134

6.8 Combined Contribution to $\nu_{e}$ Flux Prediction . . . . . . . . . . . 135

6.9 Conclusion . . . . . . . . . . . . . . . . . . . . . . . . . . . . 138

7 Absolute $\nu$ Flux: $\nu$-e NC Scattering 139

7.1 Introduction . . . . . . . . . . . . . . . . . . . . 139

7.2 Data Sample . . . . . . . . . . . . . . . . . . . . . . . . . 140

7.3 Event Reconstruction . . . . . . . . . . . . . . . . . . . . . . . . . . . 142

7.4 Event Identification and Selection . . . . . . . . . . . . . . . . . . 142

7.5 Data/MC Comparison . . . . . . . . . . . . . . . . . . . 146

7.6 Data Analysis . . . . . . . . . . . . . . . . . . . . . . . . . 154

7.6.1 Background Correction . . . . . . . . . . . . . . . . . 158

$7.6 .2 \quad \nu$-e Signal Counting . . . . . . . . . . . . . . . . . . . 159

7.7 Systematic Uncertainty Study . . . . . . . . . . . . . . . . . . . . . 160

7.7.1 Signal Efficiency Systematic . . . . . . . . . . . . . . . . . 161

7.7.2 Single Particle Requirement . . . . . . . . . . . . . . . . . . 162

7.7.3 Run Condition Noise Systematics . . . . . . . . . . . . . . . . . 163

7.7.4 MC Signal in the Sideband Region . . . . . . . . . . . . . . . 164

7.7.5 Background Normalization . . . . . . . . . . . . . . . 165

7.7.6 Energy Scale in Background . . . . . . . . . . . . . . . 167

7.7.7 Detector Modeling . . . . . . . . . . . . . . . . . . 167

7.7.8 POT Counting, Detector Mass and Beam Intensity . . . . . . . 167 
7.8 Flux Constraint . . . . . . . . . . . . . . . . . . . . . . . . . . 168

7.9 Result . . . . . . . . . . . . . . . . . . 170

8 Summary and Results 171

A Supplementary Material for Chapter 3

B Supplementary material for Beam Transport Systematics 184

B.1 Summary Tables . . . . . . . . . . . . . . . . . . . . . . . . 184

C Supplementary Material for Hadron Production Uncertainty 191

C.1 Cross Sections from NA49 _. . . . . . . . . . . . . . . . . . . 191

C.2 Extended $K$ Coverage Using $K / \pi$ Ratio . . . . . . . . . . . . . 191

C.3 $\pi^{ \pm}$Yields from MIPP . . . . . . . . . . . . . . . . . . . . . . 193

C.4 Weights for $\pi$ Production . . . . . . . . . . . . . . . . . . 194

C.5 Weights for $K^{-}$Production . . . . . . . . . . . . . 195

C.6 Results Using NA49 Pion data . . . . . . . . . . . . . . . . . . . 196

D Supplementary Material for Chapter 6 Part 1

E Supplementary Material for Chapter 6 Part 2

F $\quad$ Supplementary Material for Chapter 6 Part 3

G Supplementary Material for Chapter 7

G.1 Data/MC Comparison . . . . . . . . . . . . . . . . . . . . . 231

G.2 One Loop Electroweak Radiative Corrections to Neutrino-Electron Scattering232

G.3 Particle Likelihoods for $\nu$ - e Elastic Scattering Identification . . . . . . 235

G.4 NC Diffractive $(\mathrm{DFR}) \pi^{0} \ldots \ldots \ldots \ldots \ldots \ldots$ 
List of Publications

Bibliography 



\section{List of Figures}

1.1 Cartoon of the $\mathrm{Wu}$ experiment: if parity was preserved, then the emission of electrons would change if you reversed the orientation of the experiment. Instead, she found that the electrons always shot out the same way relative to the cobalt atoms? spin, showing that the weak force violates the law of

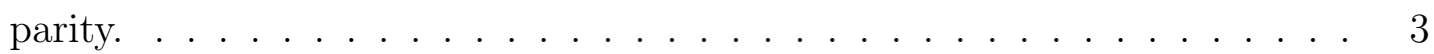

1.2 Charge current (CC) and neutral current (NC) interactions of neutrinos. . 5

1.3 Neutrino-nucleon interaction with kinematic variables. . . . . . . . . . 6

1.4 A conceptual presentation of 2-flavor $\nu$-osicllation. Left: The distribution of the 'oscillated' and the 'un-oscillated' spectra as a function of the neutrino energy $\left(E_{\nu}\right)$. Right: Shows the ratio of oscillated to the un-oscillated spectra as a function of $E_{\nu} \ldots \ldots \ldots \ldots$. . . . . . . . . . . . . . .

1.5 Diagram showing the two possible mass orderings with each state showing the approximate measured flavor combination. . . . . . . . . . . . 13

1.6 The oscillation probabilities $\mathrm{P}\left(\nu_{\mu} \rightarrow \nu_{e}\right)$ and $\mathrm{P}\left(\overline{\nu_{\mu}} \rightarrow \overline{\nu_{e}}\right)$ for the NOvA experiment illustrating the dependence on the remaining unknowns; $\sin ^{2} \theta_{23}$, $\delta_{C P}$, and choice of neutrino mass hierarchy. . . . . . . . . . 15

1.7 Left: NOvA Sensitivity to the mass-hierarchy. Right: NOvA Sensitivity to the CP-violating phase. . . . . . . . . . . . . . . 15

1.8 Measurements of per nucleon $\nu_{\mu}$-CC and $\bar{\nu}_{\mu}$-CC inclusive scattering cross sections divided by neutrino energy as a function of neutrino energy. . . . 17 
2.1 Fermilab's accelerator complex accelerates protons to high energies before sending them out to various experiments. . . . . . . . . . . . . . 20

2.2 Schematic of the NuMI Beam: Shown are the primary proton-C collision, the $\pi^{+}, \pi^{-}, K^{+}, K^{-}$, and $K_{L}^{0}$ mesons that are the primary progenitor of neutrinos, the focusing beam elements, and secondary/tertiary sources

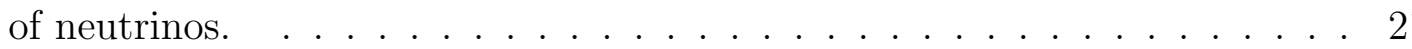

2.3 Daily protons (POT) delivered/recorded all-time by NuMI to NOvA. Highlights the 2016 and 2017 NOvA datasets as well as neutrino/anti-neutrino run periods. 2016 Analysis: 6 Feb 2014 - 1 May 2016: 6.05×10² POT-

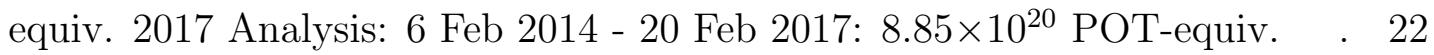

2.4 Relative location of the downstream end of the ME target material with respect to Horn 1 . The target material ends $20 \mathrm{~cm}$ upstream of the start of the idealized Horn 1 which is marked as MCZERO . . . . . . . . . . 24

2.5 Perspective sketch of the ME target core. The primary proton beam runs through the target from left to right. There are 48 segments in the target core, plus two additional Budal monitors (colored red) at the upstream end

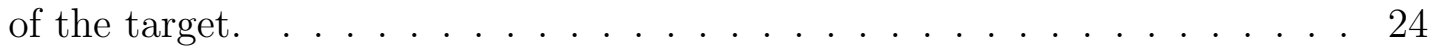

2.6 Detail of the magnetic horns system of the NuMI beam line. The NuMI Beam Line uses a two Horns for focusing. . . . . . . . . . . . . . . . . 25

2.7 Representation of NOvA Near Detector (ND) and Far Detector FD at offaxis position. $\ldots \ldots \ldots \ldots \ldots \ldots \ldots \ldots \ldots \ldots \ldots \ldots \ldots \ldots \ldots \ldots$

2.8 The kinematics and neutrino spectrum in the off-axis beam. The NOvA off-axis spectrum peak at $2 \mathrm{GeV}(14.6 \mathrm{mrad}) . \ldots \ldots \ldots$

2.9 Neutrino CC Spectra in the Forward Horn Current configuration at the NOvA FD and ND. . . . . . . . . . . . . . . . . . 29

2.10 Neutrino CC Spectra in the Reverse Horn Current configuration at the NOvA FD and ND. . . . . . . . . . . . . . . . . . . . . . 29 
3.1 Left: Map of the central United States showing Fermilab, the NuMI beamline, and the NOvA Far Detector site at Ash River, Minnesota. The total distance spanned by the green line is $810 \mathrm{~km}$. Right: Schematic of the NOvA Detectors: The FD and ND are functionally identical. . . . . . . . 34

3.2 Left: Shown is a single-cell, the element of the detector. Right: Fiber routing to the optical connector for the first 16-cells. There are two fiber ends per cell routed to the optical connector. Bottom: Shows the layout of a plane. PVC module assembly showing the end-plate at the bottom and the fiber manifold at the top. Both vertical and horizontal modules have the same configuration.

3.3 A PVC module consists of 32 cells. Each cell is read out by a pixel of an APD. Each APD has 32 pixels. Each APD has a corresponding FEB, which digitizes the signal from the APD. A DCM reads 64 FEBs and sends the information to the buffer node farm. . . . . . . . . . . . . . . 37

3.4 The quantum efficiency comparison between APD (Magenta) and PMT (bialkali photocathode) (Blue). Also shown WLS fiber emission spectra measured at lengths of $0.5,1,2,4,8,16 \mathrm{~m}$, respectively illustrating the shift of the average detected wavelength as fiber length increases. . . . . . 38

3.5 Upper: Partially completed NOvA far detector. Bottom: Top view of NOvA FD, fully instrumented FD Picture. . . . . . . . . . . . 41

3.6 Upper: NOvA ND schematics picture. Botton: Fully instrumented ND Picture. ......................... . . . . . . . . . .

3.7 Expanded assembly of the Fiber Loop Transmission (FLT) QA device. . . 44

3.8 Top: Light router of the Fiber Loop Transmission (FLT) QA device and picture of FTL device. Bottom: The assumed relationship between LED light and ADC output of FLT. 
3.9 ADC output of all Channel 1, 2, 3 and 4 of the Fiber Loop Transmission device while covered with black tape for 100 times called pedestal data. As Fiber Loop Transmission device was covered with black tape so there should be no output, but as shown in the above distribution each channel has small ADC out. Which is used as noise for calibration of respective channel of the Fiber Loop Transmission device. . . . . . . . . . . . . . . 46

3.10 Left: Total raw ADC out of Fiber Loop transmission device for all channels of all modules of NOvA ND. Right: Total calibrated ADC out of Fiber Loop transmission device for all channels using pedestal correction factor for respective channel of all modules of NOvA ND. . . . . . . . . . . . 46

3.11 Left: Total raw ADC out of Fiber Loop transmission device for channel 1, 2, 3 and 4 of all modules of NOvA ND. Right: Total calibrated ADC out of Fiber Loop transmission device for channel 1, 2, 3 and 4 using pedestal correction factor for respective channel of all modules of NOvA ND. . . . 47

3.12 Top Left: Differential pressure for six modules for 60 mins. Top Right: Absolute pressure for six modules for 60 mins. Bottom: Leak test same as upper Left and Right just with the replacement of channel 1 with channel 3 only to make sure there is the leak in the same module as explained in

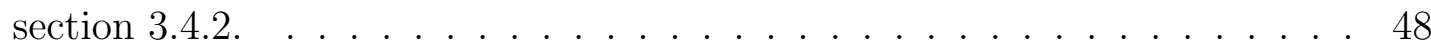

4.1 Left: The final attenuation calibration fit for a channel in the physical ND. Right: The final attenuation calibration fit for a channel in the physical FD. This fit, drawn as a blue line, is considered good. The horizontal axis is distance along the cell depth, measured in centimeters $(\mathrm{cm})$ away from the central depth. The vertical axis is average photo-electrons per path length in $\mathrm{cm}$. Dashed vertical lines indicate the ends of the cell. . . . . . . 53 
4.2 Plot used to create absolute energy calibration for the physical far detector. Each entry in the histogram is a tri-cell hit on a cosmic ray muon which stopped inside the detector. The horizontal axis is the distance of the hit from the end of the track in $\mathrm{cm}$. The vertical axis is the attenuationcorrected energy per path length in $\mathrm{cm}$. The black fit points show the mean of the fit to the distribution for each horizontal bin. Values between 100 and $200 \mathrm{~cm}$ from the end of the track are considered the MIP region and used for the absolute energy calibration. . . . . . . . . . . . . 54

4.3 Example event topologies from data files. Top: Selected $\nu_{\mu}$ ND event. Middle: Selected $\nu_{e}$ ND event. Bottom: Selected $\pi^{0}$ ND event. . . . . . . 54

4.4 Near Detector data event display for full trigger window $550 \mu$ sec. . . . . 56

4.5 Near Detector data event display for NuMI beam window $10 \mu$ sec. . . . . 56

4.6 Simulation: Locations of neutrino interactions that produce activity in the Near Detector. The color scale represents the increasing density of interactions in the detector and the surrounding cavern. . . . . . . . . 63

5.1 Left: Number of $\nu_{\mu}$ as a function of true $\nu$ energy at NOvA Near Detector. Right: Number of $\nu_{e}$ as a function of true $\nu$ energy at NOvA Near Detector. 66

5.2 Left: Number of $\nu_{\mu}$ as a function of true $\nu$ energy at NOvA Near Detector. Right: Number of $\nu_{e}$ as a function of true $\nu$ energy at NOvA Near Detector. 66

5.3 No. of interactions from proton to $\nu_{\mu}$ and $\nu_{e}$ parent meson. . . . . . . 67

5.4 Diagram of Fermi National Laboratory's NuMI beamline. . . . . . . . . . . 70

5.5 Top: FHC neutrino flux at NOA ND (top left) and FD (top right) for all neutrino parents, black is for nominal and red is for the $+2 \mathrm{kA}$ flux, respectively. Bottom: FHC neutrino fluxes ratio of the $+2 \mathrm{kA}$ flux with respect to the nominal flux at the ND (bottom left) and FD (bottom right). 71 
5.6 Top: FHC neutrino flux at NOA ND (top left) and FD (top right) for all neutrino parents, black is for nominal and red is for the $-2 \mathrm{kA}$ flux, respectively. Bottom: FHC neutrino fluxes ratio of the $-2 \mathrm{kA}$ flux with respect to the nominal flux at the ND (bottom left) and FD (bottom right). 71

5.7 Top left: (ND) and top right (FD) is ratio of $\nu$ flux with variants, $\pm 1 \mathrm{kA}$ shift, to nominal $\nu$ flux $(200 \mathrm{kA})$ at NOvA, blue $(+1 \mathrm{kA})$ and red $(-1 \mathrm{kA})$ respectively. Bottom left: FD/ND flux, black is nominal (200 kA), blue is $+1 \mathrm{kA}$ shift $(201 \mathrm{kA})$ and red is $-1 \mathrm{kA}$ shift $(199 \mathrm{kA}) \ldots \ldots$. . . . . . . . 72

5.8 Top: Double-Ratio $\frac{\Phi_{F D / N D}(\text { variant })}{\Phi_{F D / N D}(\text { Std })}$ for $+1 \mathrm{kA}$ shift (blue) and $-1 \mathrm{kA}$ shift (red). Bottom: 1-Double-Ratio to show the effective band representation. . 72

5.9 Top: FHC neutrino flux at NOvA ND (top left) and FD (top right) for all neutrino parents, black is for nominal flux and red is for the Horn1 flux with $+2 \mathrm{~mm}$ shift both in $\mathrm{X}$ and $\mathrm{Y}$. Bottom: FHC neutrino fluxes ratio of the Horn1 flux with $+2 \mathrm{~mm}$ shift both in $\mathrm{X}$ and $\mathrm{Y}$ to the nominal flux at the ND (bottom left) and FD (bottom right). . . . . . . . . . . 74

5.10 Top: FHC neutrino flux at NOvA ND (top left) and FD (top right) for all neutrino parents, black is for nominal flux and red is for the Horn1 flux with $-2 \mathrm{~mm}$ shift both in $\mathrm{X}$ and $\mathrm{Y}$. Bottom: FHC neutrino fluxes ratio of the Horn1 flux with $-2 \mathrm{~mm}$ shift both in $\mathrm{X}$ and $\mathrm{Y}$ to the nominal flux at the ND (bottom left) and FD (bottom right). . . . . . . . . . . . 74

5.11 Top left (ND) and top right (FD) ratio of $\nu$ flux with variants, $\pm 2 \mathrm{~mm}$ shift both in $\mathrm{X}$ and $\mathrm{Y}$ position of Horn 1 to the nominal $\nu$ flux at NOvA, blue (for $+2 \mathrm{~mm}$ ) and red (for $-2 \mathrm{~mm}$ ). Bottom left: FD/ND flux, black is nominal, blue is $+2 \mathrm{~mm}$ shift and red is $-2 \mathrm{~mm}$ shift. . . . . . . . 75 
5.12 Top: Double-Ratio $\frac{\Phi_{F D / N D}(\text { variant })}{\Phi_{F D / N D}(\text { Std })}$ for a $+2 \mathrm{~mm}$ shift (blue) and $-2 \mathrm{~mm}$ shift (red) in the Horn1 X \& Y position. Bottom: 1-Double-Ratio to show the effective band representation. . . . . . . . . . . . . . . 75

5.13 Top: FHC neutrino flux at NOvA ND (top left) and FD (top right) for all neutrino parents, black is for nominal flux and red is for the Horn2 flux with $+2 \mathrm{~mm}$ shift both in $\mathrm{X}$ and Y. Bottom: FHC neutrino fluxes ratio of the Horn2 flux with $+2 \mathrm{~mm}$ shift both in $\mathrm{X}$ and $\mathrm{Y}$ to the nominal flux at the ND (bottom left) and FD (bottom right). . . . . . . . . . . . 76

5.14 Top: FHC neutrino flux at NOvA ND (top left) and FD (top right) for all neutrino parents, black is for nominal flux and red is for the Horn2 flux with $-2 \mathrm{~mm}$ shift both in $\mathrm{X}$ and Y. Bottom: FHC neutrino fluxes ratio of the Horn2 flux with $-2 \mathrm{~mm}$ shift both in $\mathrm{X}$ and $\mathrm{Y}$ to the nominal flux at the ND (bottom left) and FD (bottom right). . . . . . . . . . . 76

5.15 Top left (ND) and top right (FD) ratio of $\nu$ flux with variants, $\pm 2 \mathrm{~mm}$ shift both in $\mathrm{X}$ and $\mathrm{Y}$ position of Horn 2 to the nominal $\nu$ flux at NOvA, blue (for $+2 \mathrm{~mm}$ ) and red (for $-2 \mathrm{~mm}$ ). Bottom left: FD/ND flux, black is nominal, blue is $+2 \mathrm{~mm}$ shift and red is $-2 \mathrm{~mm}$ shift.

5.16 Top: Double-Ratio $\frac{\Phi_{F D / N D} \text { (variant) }}{\Phi_{F D / N D}(\text { Std })}$ for a $+2 \mathrm{~mm}$ shift (blue) and $-2 \mathrm{~mm}$ shift (red) in the Horn1 X \& Y position. Bottom: 1-Double-Ratio to show the effective band representation. . . . . . . . . . . . . . 77

5.17 Top left (ND) and top right (FD) $\nu$ flux at NOvA for all neutrino parents, black is for nominal (linear magnetic field), and red is for the exponential magnetic field. Bottom left(ND) and bottom right (FD) $\nu$ flux ratio of exponential magnetic field to nominal at NOvA. . . . . . . . . . . . 78 
5.18 Top: Double-Ratio $\frac{\Phi_{F D / N D}(\text { variant })}{\Phi_{F D / N D}(\text { Std })}$ for linear (blue) and exponential (red) magnetic field. Bottom: 1-Double-Ratio to show the effective band representation. .......................... . . . . . . . .

5.19 Top: Double-Ratio $\frac{\Phi_{F D / N D}(\text { variant) }}{\Phi_{F D / N D}(\text { Std })}$ for linear (blue) and exponential (red) magnetic field. Bottom: 1-Double-Ratio to show the effective band representation. ......................... 79

5.20 NuMI Proton Beam hits the Medium Energy target, the uncertainties in the proton beam position on target affects the final flux. . . . . . . . . 80

5.21 Top left (ND) and top right (FD) $\nu$ flux at NOvA for all neutrino parents, black is for nominal and red is for the $+0.5 \mathrm{~mm}$ shift of the beam position along the $\mathrm{X}$ direction. Bottom left (ND) and bottom right (FD) $\nu$ fluxes ratio of $+0.5 \mathrm{~mm}$ shift of the beam position along the $\mathrm{X}$ direction to nominal at NOvA. . . . . . . . . . . . . . . . . . 81

5.22 Top left (ND) and top right (FD) $\nu$ flux at NOvA for all neutrino parents, black is for nominal and red is for the $-0.5 \mathrm{~mm}$ shift of the beam position along the $\mathrm{X}$ direction. Bottom left (ND) and bottom right (FD) $\nu$ fluxes ratio of $-0.5 \mathrm{~mm}$ shift of the beam position along the $\mathrm{X}$ direction to nominal at NOvA. . . . . . . . . . . . . . . . 81

5.23 Top left (ND) and top right (FD) $\nu$ flux at NOvA for all neutrino parents, black is for nominal and red is for the $+0.5 \mathrm{~mm}$ shift of the beam position along the $\mathrm{Y}$ direction. Bottom left (ND) and bottom right (FD) $\nu$ fluxes ratio of $+0.5 \mathrm{~mm}$ shift of the beam position along the $\mathrm{Y}$ direction to nominal at NOvA. . . . . . . . . . . . . . . . . 82 
5.24 Top left (ND) and top right (FD) $\nu$ flux at NOvA for all neutrino parents, black is for nominal and red is for the $-0.5 \mathrm{~mm}$ shift of the beam position along the $\mathrm{Y}$ direction. Bottom left (ND) and bottom right (FD) $\nu$ fluxes ratio of $-0.5 \mathrm{~mm}$ shift of the beam position along the $\mathrm{Y}$ direction to nominal at NOvA.

5.25 Top left (ND) and top right (FD), ratio of $\nu$ fluxes with variants, $\pm 0.5 \mathrm{~mm}$ shift of the beam position along the $\mathrm{X}$ direction, blue $(+0.5 \mathrm{~mm})$ and red $(-0.5 \mathrm{~mm})$, to nominal $\nu$ flux at NOvA. Bottom left: FD/ND flux, black is nominal, blue is $+0.5 \mathrm{~mm}$ shift and red is $-0.5 \mathrm{~mm}$ shift of the beam position along the $\mathrm{X}$ direction. . . . . . . . . . . . . . . . .

5.26 Top: Double-Ratio $\frac{\Phi_{F D / N D}(\text { variant })}{\Phi_{F D / N D}(\text { Std })}$ for $+0.5 \mathrm{~mm}$ shift (blue) and $-0.5 \mathrm{~mm}$ shift (red) in beam position on target along the $\mathrm{X}$ direction. Bottom: 1Double-Ratio to show the effective band representation. . . . . . . . . . . 83

5.27 Top left (ND) and top right (FD), ratio of $\nu$ fluxes with variants, $\pm 0.5 \mathrm{~mm}$ shift of the beam position along the $\mathrm{Y}$ direction, blue $(+0.5 \mathrm{~mm})$ and red $(-0.5 \mathrm{~mm})$, to nominal $\nu$ flux at NOvA. Bottom left: FD/ND flux, black is nominal, blue is $+0.5 \mathrm{~mm}$ shift and red is $-0.5 \mathrm{~mm}$ shift of the beam position along the $\mathrm{Y}$ direction. . . . . . . . . . . . . . . 84

5.28 Top: Double-Ratio $\frac{\Phi_{F D / N D}(\text { variant })}{\Phi_{F D / N D}(\text { Std })}$ for $+0.5 \mathrm{~mm}$ shift (blue) and $-0.5 \mathrm{~mm}$ shift (red) in beam position on target along the $\mathrm{Y}$ direction. Bottom: 1Double-Ratio to show the effective band representation. . . . . . . . . . . 84

5.29 Top left (ND) and top right (FD), $\nu$ flux at NOvA for all neutrino parents, black is for nominal and red is for the $-0.2 \mathrm{~mm}$ shift of the beam spot size in both X \& Y directions. Bottom left (ND) and bottom right (FD), $\nu$ fluxes ratio of $-0.2 \mathrm{~mm}$ shift of the beam spot size in both $\mathrm{X} \& \mathrm{Y}$ directions to nominal at NOvA. . . . . . . . . . . . . . . . 85 
5.30 Top left (ND) and top right (FD), $\nu$ flux at NOvA for all neutrino parents, black is for nominal and red is for the $+0.4 \mathrm{~mm}$ shift of the beam spot size in both $\mathrm{X} \& \mathrm{Y}$ directions. Bottom left (ND) and bottom right (FD), $\nu$ fluxes ratio of $+0.4 \mathrm{~mm}$ shift of the beam spot size in both $\mathrm{X} \& \mathrm{Y}$ directions to nominal at NOvA. ..................... 86

5.31 Top left (ND) and top right (FD), ratio of $\nu$ flux with variants, blue $(-0.2 \mathrm{~mm})$, to nominal $\nu$ flux at NOvA. Bottom left: FD/ND flux, black is nominal, blue is $-0.2 \mathrm{~mm}$ shift of the beam spot size in both $\mathrm{x} \& \mathrm{Y}$ directions.

5.32 Top left (ND) and top right (FD), ratio of $\nu$ flux with variants, blue $(+0.4 \mathrm{~mm})$, to nominal $\nu$ flux at NOvA. Bottom left: FD/ND flux, black is nominal, blue is $+0.4 \mathrm{~mm}$ shift of the beam spot size in both $\mathrm{x} \& \mathrm{Y}$ directions.

5.33 Top: Double-Ratio $\frac{\Phi_{F D / N D}(\text { variant })}{\Phi_{F D / N D}(\text { Std })}$ for $-0.2 \mathrm{~mm}$ shift (blue) in the beam spot size in both $\mathrm{X} \& \mathrm{Y}$ directions. Bottom: 1-Double-Ratio to show the effective band representation. . . . . . . . . . . . . . . . 87

5.34 Top: Double-Ratio $\frac{\Phi_{F D / N D}(\text { variant })}{\Phi_{F D / N D}(\text { Std })}$ for $+0.4 \mathrm{~mm}$ shift (blue) in the beam spot size in both $\mathrm{X} \& \mathrm{Y}$ directions. Bottom: 1-Double-Ratio to show the effective band representation. . . . . . . . . . . . . . . . 88

5.35 Top left (ND) and top right (FD), $\nu$ flux at NOvA for all neutrino parents, black is for nominal and red is for the $+7 \mathrm{~mm}$ shift of the target position along the $\mathrm{Z}$ direction. Bottom left (ND) and bottom right (FD), $\nu$ fluxes ratio of $+7 \mathrm{~mm}$ shift of the target position along the $\mathrm{Z}$ direction to nominal at $\mathrm{NOvA}$ 
5.36 Top left (ND) and top right (FD), $\nu$ flux at NOvA for all neutrino parents, black is for nominal and red is for the $-7 \mathrm{~mm}$ shift of the target position along the $\mathrm{Z}$ direction. Bottom left (ND) and bottom right (FD), $\nu$ fluxes ratio of $-7 \mathrm{~mm}$ shift of the target position along the $\mathrm{Z}$ direction to nominal at $\mathrm{NOvA}^{2} \ldots \ldots \ldots \ldots \ldots \ldots$

5.37 Top left (ND) and top right (FD), ratio of $\nu$ flux with variants, blue is $+7 \mathrm{~mm}$ and red is $-7 \mathrm{~mm}$, to nominal $\nu$ flux at NOvA. Bottom left: FD/ND flux, black is nominal, blue is $+7 \mathrm{~mm}$ and red is $-7 \mathrm{~mm}$. . . . . . 90

5.38 Top: Double-Ratio $\frac{\Phi_{F D / N D}(\text { variant })}{\Phi_{F D / N D}(\text { Std })}$ for the $\pm 7 \mathrm{~mm}$ shift (red) of the target position along the $\mathrm{Z}$ direction. Bottom: 1-Double-Ratio to show the effective band representation. . . . . . . . . . . . . . . . . 90

5.39 Top left (ND) and right (FD): $\nu$ flux at NOvA for all neutrino parents, black is for nominal and blue is for the new version of FLUKA (fluka2011.2c.3). Bottom left (ND) and top right (FD) $\nu$ fluxes ratio of the new version of FLUKA (fluka2011.2c.3) to nominal at NOvA. . . . . . . . . . . . . 91

5.40 Top left (ND) and top right (FD), ratio of $\nu$ flux with variant (fluka2011.2c.3) at NOvA. Bottom left: FD/ND flux, flux with variant

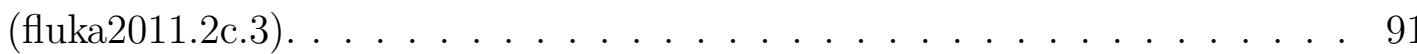

5.41 Top: Double-Ratio $\frac{\Phi_{F D / N D}(\text { variant })}{\Phi_{F D / N D}(S t d)}$ for fluka2011.2c.3. Bottom: 1-DoubleRatio to show the effective band representation. . . . . . . . . . . . 92

5.42 Top left (ND) and top right (FD), $\nu$ flux at NOvA for all neutrino parents, black is for nominal and blue is for the G4NuMI(FTFP_BERT). Bottom left (ND) and bottom right (FD): $\nu$ fluxes ratio of the G4NuMI(FTFP_BERT) to nominal at NOvA. . . . . . . . . . . . . . . . . . . 993 
5.43 Top left (ND) and top right (FD), ratio of $\nu$ flux with variant(G4NuMI(FTFP_BERT)) at NOvA. Bottom left: FD/ND flux, flux with variant(G4NuMI(FTFP_BERT)) . . . . . . . 93

5.44 Top: Double-Ratio $\frac{\Phi_{F D / N D}(\text { variant })}{\Phi_{F D / N D}(\text { Std })}$ for G4NuMI(FTFP_BERT). Bottom: 1Double-Ratio to show the effective band representation. . . . . . . . . . . 94

5.45 Top left (ND) and top right (FD), $\nu$ flux at NOvA for all neutrino parents, black is for nominal and blue is for the New Horn Geometry and Water cooling layer on horn inner conductor. Bottom left (ND) and bottom right (FD), $\nu$ fluxes ratio of theNew Horn Geometry and Water cooling layer on horn inner conductor to nominal at NOvA. . . . . . . . . . . . . . . 95

5.46 Top left (ND) and top right (FD), ratio of $\nu$ flux with variant(New Horn Geometry and Water cooling layer on horn inner conductor) at NOvA. Bottom left: FD/ND flux, flux with variant(New Horn Geometry and Water cooling layer on horn inner conductor) . . . . . . . . . . . . . . 95

5.47 Top: Double-Ratio $\frac{\Phi_{F D / N D}(\text { variant })}{\Phi_{F D / N D}(\text { Std })}$ for New Horn Geometry and Water cooling layer on horn inner conductor v6. Bottom: 1-Double-Ratio to show the effective band representation. . . . . . . . . . . . . . . . . 96

5.48 The distribution for $\pi \rightarrow \nu_{\mu}$ flux as a function of $p_{T}$ and $p_{Z}$ of $\pi$ ancestor. 98

5.49 The distribution for $K \rightarrow \nu_{\mu}$ flux as a function of $p_{T}$ and $p_{Z}$ of $K$ ancestor. 98

5.50 The distribution for $\pi \rightarrow \mu \rightarrow \nu_{e}$ flux as a function of $p_{T}$ and $p_{Z}$ of $\pi$

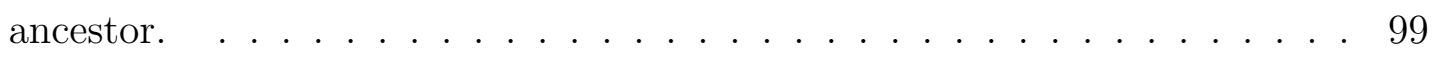

5.51 The distribution for $K \rightarrow \nu_{e}$ flux as a function of $p_{T}$ and $p_{Z}$ of $K$ ancestor. 99

5.52 Left: Demonstration of the external data usage for the phase space coverage of $\pi \rightarrow \nu_{\mu}$ and $\pi \rightarrow \mu \rightarrow \nu_{e}$ flux. Right: Demonstration of the external data usage for the phase space coverage of $K \rightarrow \nu_{e}$ and $K \rightarrow \nu_{\mu}$ flux. . . . 100 
5.53 The $\pi$ yield distributions from data and FLUKA prediction for $\pi^{+}$production of MIPP. . . . . . . . . . . . . . . . . . . . . . . 104

5.54 Invariant cross section and corresponding ratio between data and FLUKA prediction for $\pi^{+}$production of NA49. . . . . . . . . . . . . . 105

5.55 Left: The ratio for pion yield as a function of $p_{Z}$ and $p_{T}$ of pion between G4NuMI and FLUKA using low energy target configuration. Right: $\pi \rightarrow$ $\mu \rightarrow \nu_{e}$ flux from G4NuMI and weighted FLUKA predictions using median energy target configuration. . . . . . . . . . . . . . . . 106

5.56 Invariant cross section and corresponding ratio between data and FLUKA prediction for $K^{+}$production in the extended phase space using the $K^{+} / \pi^{+}$ ratio and $\pi^{+}$production cross section. . . . . . . . . . . . . . . 106

5.57 Invariant cross section and corresponding ratio between data and FLUKA prediction for $K^{+}$production. . . . . . . . . . . . . . . . . 107

5.58 Flux weight as a function of $\nu_{e}$ energy. Uncertainty for the flux weight as a function of $\nu_{e}$ energy. $\ldots \ldots \ldots$. . . . . . . . . . . . . . . 109

5.59 Flux weight as a function of $\nu_{\mu}$ energy. Uncertainty for the flux weight as a function of $\nu_{\mu}$ energy. . . . . . . . . . . . . . . . . . . 109

5.60 The combined uncertainties from different systematics parameters of beam line using different methods at NOvA ND. . . . . . . . . . . . . . 109

5.61 Hadron productiob flux weights for $\nu_{e}$ and $\nu_{\mu}$ at FD. . . . . . . . . 110

5.62 The combined uncertainties from different systematics parameters of beam line using different methods at NOvA FD. . . . . . . . . . . . . . . 110

6.1 Using two body decay formula, Left: Neutrino energy vs pion energy at NOvA axis and on-axis w.r.t the beamline. Right: Neutrino energy vs kaon energy at NOvA axis and on-axis w.r.t the beamline. . . . . . . . . . . . 114 
6.2 Reconstructed Neutrino Energy(TrkCCE) distribution, uncontained: Total muon neutrinoCC MC events (red line), muon neutrinoCC from $\pi^{+}$(purple line), muon neutrinoCC from $K^{+}$(blue line) and muon neutrinoCC from other parents(sea green). . . . . . . . . . . . . . . . . . 115

6.3 Left: The longitudinal pz vs transverse momentum pion distribution at the the target, which are ancestor of contained muon neutrinoCC events at ND. Right: The longitudinal pz vs transverse momentum kaon distribution at the the target, which are ancestor of contained muon neutrinoCC events at ND. . . . . . . . . . . . . . . . . . . . . . 116

6.4 Left: The longitudinal pz vs transverse momentum pion distribution at the the target, which are ancestor of uncontained muon neutrinoCC events at ND. Right: The longitudinal pz vs transverse momentum kaon distribution at the the target, which are ancestor of uncontained muon neutrinoCC events at ND. . . . . . . . . . . . . . . . . . . 116

6.5 The contained vs uncontained selection. . . . . . . . . . . . . 118

6.6 ReMID distribution Left:: Contained sample Right:: uncontained sample. . 120

6.7 Reconstructed Neutrino Energy(TrkCCE) distribution Left: Contained and Right: uncontained: Data rawsignal events[Data- $\left.\mathrm{MC}_{b k g}\right]$ (black solid dot), total muon neutrinoCC MC rawsignal events(red line), muon neutrinoCC from $\pi^{+}$(purple line), muon neutrinoCC from $K^{+}$(blue line) and muon neutrinoCC from other parents(sea green) . . . . . . . . . . . . 123

6.8 Reconstructed Neutrino Energy $(\operatorname{TrkCCE})$ distribution Left: $\mathrm{E}_{\text {Had }}>0.5 \mathrm{GeV}$, contained and Right: $\mathrm{E}_{H a d}>0.5 \mathrm{GeV}$, uncontained : Data rawsignal events[Data-MC $\mathrm{M}_{b k g}$ (black solid dot), total muon neutrinoCC MC rawsignal events(red line), muon neutrinoCC from $\pi^{+}$(purple line), muon neutrinoCC from $K^{+}$(blue line) and muon neutrinoCC from other parents(sea green). . . . . . . . . . . 124 
6.9 Reconstructed Neutrino Energy(TrkCCE) distribution Left: $E_{\text {had }}>1 \mathrm{GeV}$, contained and Right: $\mathrm{E}_{\text {had }}>1 \mathrm{GeV}$, uncontained : Data rawsignal events[Data- $\left.\mathrm{MC}_{b k g}\right]$ (black solid dot), total muon neutrinoCC MC rawsignal events(red line), muon neutrinoCC from $\pi^{+}$(purple line), muon neutrinoCC from $K^{+}$(blue line) and muon neutrinoCC from other parents(sea green). . 124

6.10 Left: $\mathrm{E}_{\mu}$ distribution of Data(solid black dot) and $\mathrm{MC}$ (red line) for contained Sample. Right: $E_{\text {had }}$ distribution of Data(solid black dot) and MC(red line) for contained Sample. . . . . . . . . . . . . . . . 125

6.11 Left: $\mathrm{E}_{\mu}$ distribution of Data(solid black dot) and $\mathrm{MC}($ red line) for uncontained Sample. Right: $E_{\text {had }}$ distribution of Data(solid black dot) and MC(red line) for uncontained Sample. . . . . . . . . . . . . . 125

6.12 Reconstructed Neutrino Energy(TrkCCE) distribution for contained sample: Left: Data events (black solid dot), total MC events (red line), $\nu_{\mu}$ $\mathrm{MC}$ events (blue line), rock events (sea green line), $\overline{\nu_{\mu}}$ events(brown line) and background from others sources (pink line). Right: Data raw signal events[Data-MC $\left.\mathrm{MC}_{b k}\right]$ (black solid dot), total $\nu_{\mu}-\mathrm{CC}$ MC raw signal events(red line), $\nu_{\mu}-\mathrm{CC}$ from $\pi^{+}$( purple line), $\nu_{\mu}$ - CC from $K^{+}$(blue line) and $\nu_{\mu}-$ CC from other parents (sea green) . . . . . . . . . . . 126

6.13 Reconstructed Neutrino Energy (TrkCCE) distribution for uncontained sample: Left: Data events (black solid dot), total MC events (red line), $\nu_{\mu} \mathrm{MC}$ events (blue line), rock events (sea green line), $\overline{\nu_{\mu}}$ events(brown line) and background from others sources (pink line). Right: Data raw signal events[Data- $\left.\mathrm{MC}_{b k g}\right]$ (black solid dot), total $\nu_{\mu}-\mathrm{CC} \mathrm{MC}$ raw signal events(red line), $\nu_{\mu}-$ CC from $\pi^{+}$( purple line), $\nu_{\mu}$ - CC from $K^{+}$(blue line) and $\nu_{\mu}-$ CC from other parents (sea green). . . . . . . . . 127

$6.14 \mathrm{~N}_{\pi}$ and $\mathrm{N}_{K} \chi^{2}$ fit with $1 \sigma$ on central value for $(0.5-10) \mathrm{GeV} . . . . . .128$ 
6.15 The $\nu_{\mu}$ cross-section measurements, especially highlight the NOMAD measurement. . . . . . . . . . . . . . . . . . . . . . 133

6.16 electron neutrino at ND w.r.t their parents information. . . . . . . . 136

6.17 The weights for the electron neutrino flux at ND: Left: Flux weight as a function of electron neutrino true energy with uncertainty using MIPP+NA49 external data. Right: Flux weight as a function of electron neutrino true energy with uncertainty using MIPP+NA49+NOvA(ND muon neutrinoCC) external data. . . . . . . . . . . . . . . 137

6.18 The electron neutrino flux at ND with NA49+ MIPP weights only and with $\mathrm{NA} 49+\mathrm{MIPP}+\mathrm{ND}($ data) weights. . . . . . . . . . . . . . 138

7.2 Top (Left and Right): Distributions of Minimum X and Maximum X of total MC background and MC $\nu$-e signal before applying cuts on Minimum $\mathrm{X}$ and Maximum X. MC are normalized to data POT in each case. The Minimum Xand Maximum $\mathrm{X}$ is defined as the minimum and maximum $\mathrm{X}$ positions of hits on tracks. Bottom (Left and Right): FOM distribution for Minimum Xand Maximum X. . . . . . . . . . . . . . . . . . . . . 147

7.3 Top (Left and Right): Distributions of Minimum Y and Maximum Y of total MC background and MC $\nu$-e signal before applying cuts on Minimum $\mathrm{Y}$ and Maximum Y. MC are normalized to data POT in each case. The Minimum $\mathrm{Y}$ and Maximum $\mathrm{Y}$ is defined as the minimum and maximum Y positions of hits on tracks. Bottom (Left and Right): FOM distribution for Minimum Yand Maximum Y. . . . . . . . . . . . . . . . . . . . . . . 148

7.4 Top (Left and Right): Distributions of Minimum Z and Maximum Z of total MC background and MC $\nu$-e signal before applying cuts on Minimum $\mathrm{Z}$ and Maximum Z. MC are normalized to data POT in each case. The Minimum $\mathrm{Z}$ and Maximum $\mathrm{Z}$ is defined as the minimum and maximum $\mathrm{Z}$ positions of hits on tracks. Bottom (Left and Right): FOM distribution for Minimum Z and Maximum Z. . . . . . . . . . . . . . . . . . . . 149 
7.5 Left: Distributions of $\frac{E_{\text {shower }}}{E_{\text {tot }}}$ of total MC background and MC $\nu$-e signal before applying cuts on $\frac{E_{\text {shower }}}{E_{\text {tot }}}$. MC are normalized to data POT in each case. Right: FoM distribution for $\frac{E_{\text {shower }}}{E_{\text {tot }}} \ldots \ldots \ldots \ldots \ldots \ldots \ldots$

7.6 Left: Distributions of shower vertex energy of total MC background and $\mathrm{MC} \nu$-e signal before applying cuts on the shower vertex energy. MC are normalized to data POT in each case. Right: FoM distribution for shower vertex energy. . . . . . . . . . . . . . . . . . . . 150

7.7 Left: Distributions of the distance between the start point of the shower from the event vertex of total MC background and $\mathrm{MC} \nu$-e signal before applying cuts on the distance between the start point of the shower from the event vertex. MC are normalized to data POT in each case. Right: FoM distribution for distance between the start point of the shower from the event vertex. . . . . . . . . . . . . . . . . . . . 150

7.8 Distributions of EM shower energy of total MC background and MC $\nu$-e signal before applying cuts on $\mathrm{E}_{\text {shower }}$ MC are normalized to data POT in each case. . . . . . . . . . . . . . . . . . . . . 150

7.11 Left: Distributions of $e / \pi^{0}$ PID of total MC background and MC $\nu$-e signal before applying cuts on $e / \pi^{0} \mathrm{PID}$. MC are normalized to data POT in each case. Right: FoM distribution for $e / \pi^{0}$ PID. . . . . . . . . . . . . . 151

7.12 Left: Distributions of $\nu$-e PID of total MC background and MC $\nu$-e signal before applying cuts on $\nu$-e PID. MC are normalized to data POT in each case. Right: FoM distribution for $\nu$-e PID. . . . . . . . . . . . . . . 152

7.13 Left: Distributions of $\mathrm{E} \theta^{2}$ of total MC background and $\mathrm{MC} \nu$-e signal before applying cuts on $\mathrm{E} \theta^{2}$. $\mathrm{MC}$ are normalized to data POT in each case. Right: FoM distribution for $\mathrm{E} \theta^{2} \ldots \ldots \ldots \ldots \ldots \ldots \ldots$ 
7.14 Top: Near Detector data events all hits recorded in a $10 \mu$ sec beam spill at ND. Bottom: Near Detector $\nu$-e data candidate as single forwarding electron after apply selection using PID and kinematic information, giving us confidence in our selection for the signal as single forward EM shower. . 153

7.15 Data/MC of dE/dx in first four planes and Data/MC for $\nu$-e PID inputs. . 155

7.16 Data/MC distribution for (left) $\nu$-e PID and (right) $e / \pi^{0}$ PID. . . . . . 156

$7.17 \mathrm{Up}$ (Left): Data/MC of $\mathrm{E} \theta^{2}$ before background $\mathrm{MC}$ corrections. Up (Right): Data/MC distributions of electron energy in the $E_{e} \theta_{e}^{2}$ signal region before background MC correction. Bottom (Left): Data/MC distributions of electron energy in the $E_{e} \theta_{e}^{2}$ side-band region, before background $\mathrm{MC}$ correction. . . . . . . . . . . . . . . . . . . . 156

7.18 Right (Signal region $0<E_{e} \theta_{e}^{2}<0.005$ ) and Left (Side-band $0.005<E_{e} \theta_{e}^{2}<$ 0.04): Plots to show that background distribution in the side-band region (left side) has kinematics coverage of the background in the signal region. Each kinematic variable distribution in the signal region has its side-band region distribution in parallel, e.g., E, $\mathrm{Q}^{2}$, Bjorken $\mathrm{x}$ and Bjorken y distribution in the signal region right-hand side, to compare these variable with their side-band distributions on the left-hand side. All the kinematic variable distributions, i.e., energy, $\mathrm{Q}^{2}$, Bjorken $\mathrm{x}$ and Bjorken $\mathrm{y}$ in the side-band region overlap the distribution in the signal region. . . . . . . . . 157

7.19 Left: Data/MC $E_{e} \theta_{e}^{2}$ distribution after background correction. Right: Data/MC of electron energy after background correction. . . . . . . . . . 159

7.20 (left) Energy and (right) $\cos (\theta)$ for rock MR Brem in data and MC, compared with $\nu$-e signal events, , area normalized. Rock MR Brem data and $\mathrm{MC}$ are weighted by the ratio of $\nu$-e scattering $\mathrm{MC}$ over $\mathrm{MR}$ Brem $\mathrm{MC}$ in each bin to correct the shape difference between the MR Brem sample and the $\nu$-e scattering signal. . . . . . . . . . . . . . . . . . . . . 162 
7.21 Top: $\nu$-e and $\pi^{0}$ PID for rock MR Brem in data and MC. Bottom: $\mathrm{E} \theta^{2}$ for rock MR Brem in data and MC, compared with $\nu$-e signal events, area normalized. MR Brem data and MC are weighted by the ratio of $\nu$-e scattering MC over MR Brem MC in each bin to correct the shape difference between the MR Brem sample and the $\nu$-e scattering signal. . . . . . . . 163

7.22 $E_{e} \theta_{e}^{2}$ distribution before the background normalization with the definitions of the standard sideband $\left(0.005<E_{e} \theta_{e}^{2}<0.04\right)$, the sideband Region A $\left(0.005<E_{e} \theta_{e}^{2}<0.02\right)$ and the sideband Region B $\left(0.02<E_{e} \theta_{e}^{2}<0.04\right) \ldots 165$

$7.23 \nu$-e signal comparison between standard and stagger geometry . . . . . . 168

A.1 Experimental data from 100 pedestals of Fiber Loop Transmission (FTL) corresponding to channels $5,6,7$, and $8 \ldots \ldots \ldots \ldots \ldots \ldots$

A.7 Experimental data from 100 pedestals of FLT corresponding to channels $29,30,31$ and $32 \ldots \ldots \ldots \ldots \ldots \ldots \ldots \ldots$

A.3 Experimental data from 100 pedestals of FLT corresponding to channels $13,14,15$, and $16 \ldots \ldots \ldots \ldots \ldots \ldots \ldots \ldots \ldots$

A.4 Experimental data from 100 pedestals of FLT corresponding to channels $17,18,19$, and $20 . \ldots \ldots \ldots \ldots \ldots \ldots \ldots \ldots \ldots \ldots \ldots \ldots$

A.5 Experimental data from 100 pedestals of FLT corresponding to channels

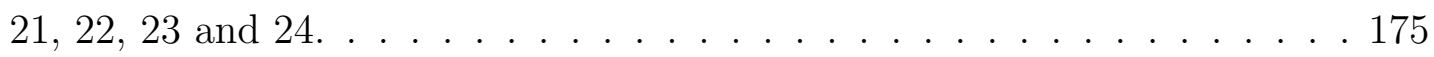

A.6 Experimental data from 100 pedestals of FLT corresponding to channels

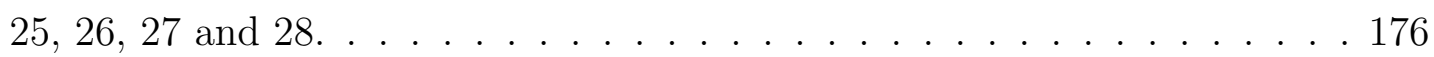

A.8 Raw ADC vs Calibrated ADC response for channel 5, 6, 7 and $8 . \ldots \ldots 177$

A.9 Raw ADC vs Calibrated ADC response for channel 9, 10, 11 and 12 . . . 178

A.10 Raw ADC vs Calibrated ADC response for channel 13, 14, 15 and 16. . . 179 A.11 Raw ADC vs Calibrated ADC response for channel 17, 18, 19 and 20. . . 180 A.12 Raw ADC vs Calibrated ADC response for channel 21, 22, 23 and 24. . . 181 
A.13 Raw ADC vs Calibrated ADC response for channel 25, 26, 27 and 28. . . 182

A.14 Raw ADC vs Calibrated ADC response for channel 29, 30, 31 and $32 . \quad$. 183

C.1 Invariant cross section as a function of $p_{T}$ for different $x_{F}$ of $\pi^{+}$for NA49 from data and FLUKA simulation. . . . . . . . . . . . . . . . . 192

C.2 Invariant cross section as a function of $p_{T}$ for different $x_{F}$ of $\pi^{+}$for NA49 from data and FLUKA simulation. . . . . . . . . . . . . . . . . 193

C.3 Invariant cross section as a function of $p_{T}$ for different $x_{F}$ of $\pi^{-}$for NA49 from data and FLUKA simulation. . . . . . . . . . . . . . . . . . . 194

C.4 Invariant cross section as a function of $p_{T}$ for different $x_{F}$ of $\pi^{-}$for NA49 from data and FLUKA simulation. . . . . . . . . . . . . . . . . 195

C.5 Invariant cross section as a function of $p_{T}$ for different $x_{F}$ of $K^{+}$for NA49 from data and FLUKA simulation. . . . . . . . . . . . . . . . 196

C.6 Invariant cross section as a function of $p_{T}$ for different $x_{F}$ of $K^{-}$for NA49 from data and FLUKA simulation. . . . . . . . . . . . . . . . . 197

C.7 $K / \pi$ ratio from MIPP. . . . . . . . . . . . . . . . . . . . . . . . . . . . 198

C.8 $\pi^{ \pm}$yields from MIPP data. . . . . . . . . . . . . . . . . . . . . . . . . . 198

C.9 Invariant cross section and corresponding ratio between data and FLUKA prediction for $\pi^{+}$production of MIPP. . . . . . . . . . . . . . . . . . . 199

C.10 Invariant cross section and corresponding ratio between data and FLUKA prediction for $\pi^{+}$production of MIPP. . . . . . . . . . . . . . . . . . . 200

C.11 Invariant cross section and corresponding ratio between data and FLUKA prediction for $\pi^{-}$production of MIPP. . . . . . . . . . . . . . . . . 201

C.17 Flux weight as a function of $\nu_{e}$ and $\nu_{\mu}$ energy, where the event weights from NA49 pion results are used. Uncertainty for the flux weight as a function of $\nu_{e}$ and $\nu_{\mu}$ energy, where the event weights from NA49 pion results are used. 
C.13 Invariant cross section and corresponding ratio between data and FLUKA prediction for $\pi^{-}$production of MIPP. . . . . . . . . . . . . . . . 203

C.14 Invariant cross section and corresponding ratio between data and FLUKA prediction for $\pi^{-}$production of NA49. . . . . . . . . . . . . . . 204

C.15 Invariant cross section and corresponding ratio between data and FLUKA prediction for $K^{-}$production. . . . . . . . . . . . . . . . 205

C.16 Invariant cross section and corresponding ratio between data and FLUKA prediction for $K^{-}$production in the extended phase space using the $K^{-} / \pi^{-}$ ratio and $\pi^{-}$production cross section. . . . . . . . . . . . . . 206

C.18 Invariant cross section and corresponding ratio between data and FLUKA prediction for $\pi^{+}$production of NA49. . . . . . . . . . . . . 207

C.19 Invariant cross section and corresponding ratio between data and FLUKA prediction for $\pi^{+}$production of NA49. . . . . . . . . . . . . . 208

C.20 Invariant cross section and corresponding ratio between data and FLUKA prediction for $\pi^{-}$production of NA49. . . . . . . . . . . . . . 209

C.21 Invariant cross section and corresponding ratio between data and FLUKA prediction for $\pi^{-}$production of NA49. . . . . . . . . . . . . . . . . 210

D.1 Left: $\mathrm{E}_{\mu}$ distribution of Data-MC for contained sample without GENIE Tune. Right: $\mathrm{E}_{\text {had }}$ distribution of Data-MC for contained sample without GENIE Tune. . . . . . . . . . . . . . . . . . . . . . . 211

D.2 Reconstructed Neutrino Energy distribution for contained sample without GENIE Tune, Left: Data events and MC with separate background. Right: Raw data signal and MC. . . . . . . . . . . . . . . . . . . 212

D.3 Left: $\mathrm{E}_{\mu}$ distribution of Data-MC for uncontained sample with out GENIE Tune. Right: $\mathrm{E}_{\text {had }}$ distribution of Data-MC for uncontained sample without GENIE Tune. . . . . . . . . . . . . . . . . . . . . . . . . 213 
D.4 Reconstructed Neutrino Energy(TrkCCE) distribution for uncontained sample with out GENIE Tune, Left: Data events and MC with separate background. Right: Raw data signal and MC . . . . . . . . . . . 213

D.5 Reconstructed Neutrino Energy(TrkCCE) distribution rewieghted with FTFP/Fluka weight, Left: Contained and Right: UnContained. . . . . . . 213

D.6 Left: $\mathrm{E}_{\mu}$ distribution of Data-MC for contained sample. Right: $\mathrm{E}_{\text {had }}$ distribution of Data-MC for contained sample for tight fiducial cut. . . . . . 215

D.7 Reconstructed Neutrino Energy(TrkCCE) distribution for contained sample tight fiducial cut, Left: Data events and MC with separate background. Right: Raw data signal and MC. . . . . . . . . . . . . . 215

D.8 Left: $\mathrm{E}_{\mu}$ distribution of Data-MC for uncontained sample. Right: $\mathrm{E}_{\text {had }}$ distribution of Data-MC for uncontained sample for tight fiducial cut. . . . 216

D.9 Reconstructed Neutrino Energy(TrkCCE) distribution for uncontained sample for tight fiducial cut, Left: Data events and MC with separate background. Right: Raw data signal and MC. . . . . . . . . . . . . 217

E.1 Left: $\mathrm{E}_{\mu}$ distribution of Data-MC for contained sample. Right: $\mathrm{E}_{\text {had }}$ distribution of Data-MC for contained sample for $+2 \%$ shift in muon energy. . 218

E.2 Reconstructed Neutrino Energy (TrkCCE) distribution for contained sample $+2 \%$ shift in muon energy, Left: Data events and MC with separate background. Right: Raw data signal and MC . . . . . . . . . . . 219

E.3 Left: $\mathrm{E}_{\mu}$ distribution of Data-MC for contained sample. Right: $\mathrm{E}_{\text {had }}$ distribution of Data-MC for uncontained sample for $+2 \%$ shift in muon energy.220

E.4 Reconstructed Neutrino Energy(TrkCCE) distribution for uncontained sample $+2 \%$ shift in muon energy, Left: Data events and MC with separate background. Right: Raw data signal and MC . . . . . . . . . . 220 
E.5 Left: $\mathrm{E}_{\mu}$ distribution of Data-MC for contained sample. Right: $\mathrm{E}_{\text {had }}$ distribution of Data-MC for contained sample for $-2 \%$ shift in muon energy. . 220

E.6 Reconstructed Neutrino Energy(TrkCCE) distribution for contained sample $-2 \%$ shift in muon energy, Left: Data events and MC with separate background. Right: Raw data signal and MC. . . . . . . . . . . . . 221

E.7 Left: $\mathrm{E}_{\mu}$ distribution of Data-MC for contained sample. Right: $\mathrm{E}_{h a d}$ distribution of Data-MC for contained sample for $-2 \%$ shift in muon energy. . 222

E.8 Reconstructed Neutrino Energy(TrkCCE) distribution for uncontained sample $-2 \%$ shift in muon energy, Left: Data events and MC with separate background. Right: Raw data signal and MC . . . . . . . . . 2223

F.1 Reconstructed Neutrino Energy(TrkCCE) distribution for contained sample MIPP+NA49 error band plus only, Left: Data events and MC with separate background. Right: Raw data signal and MC. . . . . . . . . . 2224

F.2 Reconstructed Neutrino Energy(TrkCCE) distribution for contained sample MIPP+NA49 Error Band Minus Only, Left: Data events and MC with separate background. Right: Raw data signal and MC. . . . . . . . . . 225

F.3 Reconstructed Neutrino Energy(TrkCCE) distribution for contained sample MIPP+NA49 weight for pion only, Left: Data events and MC with separate background. Right: Raw data signal and MC. . . . . . . . . . 226

F.4 Reconstructed Neutrino Energy(TrkCCE) distribution for uncontained sample MIPP+NA49 weight for pion only, Left: Data events and MC with separate background. Right: Raw data signal and MC. . . . . . . . . . . 227

F.5 An example: fReweightMaCCRES, Contained. . . . . . . . . . . . . . . 228

F.6 An example: fReweightMaCCRES, UnContained. . . . . . . . . . . . . 228

G.1 Data/MC of $\mathrm{E} \theta^{2}$ before background $\mathrm{MC}$ corrections, with separate $\nu_{\mu}$ and NC background distributions. . . . . . . . . . . . . . . 2231 
G.2 Distribution of $\sigma_{\nu_{\mu}}$ (left) and $\sigma_{\nu_{e}}$ (right) for corrected, nominal and ratio

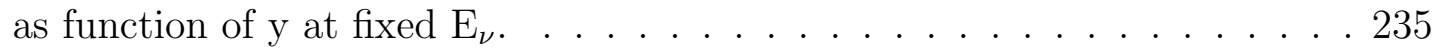

G.3 Distribution of $\sigma \bar{\nu}_{\mu}$ (left) and $\sigma \bar{\nu}_{e}$ (right) for corrected, nominal and ratio as function of $\mathrm{y}$ at fixed $\mathrm{E}_{\nu} \ldots \ldots \ldots \ldots \ldots$. . . . . . . . . . . . . . . .

G.4 Distribution of $\frac{\sigma_{\nu_{\mu}}(\text { RadCorr })}{\sigma_{\nu_{\mu}}(\text { nom })}$ in $\mathrm{y}$ and $\mathrm{E}_{\nu}$ space. . . . . . . . . . . . . 236

G.5 DFR true energy and momentum distributions. . . . . . . . . . . . . . 238

G.6 DFR reconstructed shower energy, E $\theta^{2}$, annLPID and $e / \pi^{0} \mathrm{PID}$ distributions before the cut on PIDs and kinematics $\left(\mathrm{E} \theta^{2}\right)$ distribution i.e distributions with preselection+fiducial $+\mathrm{E}<5 \mathrm{GeV} \ldots \ldots . . . . . . .239$

G.7 DFR reconstructed shower energy, $\operatorname{Cos}(\theta), \mathrm{E} \theta^{2}$, annLPID and $e / \pi^{0} \mathrm{PID}$ distributions after the cut on PIDs and knematics $\left(\mathrm{E} \theta^{2}\right)$ distributions. . . . 239 


\section{List of Tables}

3.1 Table for Far Detector and Near Detector components. . . . . . . . . . . . 39

5.1 Main decay modes and their branching ratios of charged pion, charged kaons, neutral kaons and muons to neutrinos. . . . . . . . . . . 65

5.2 Column(2,3) (RHC) and column(4,5) (FHC): Production of $\pi$ in the NuMI beam line component compared to primary target Carbon for neutrinos at NOvA ND using G4NuMI with GEANT4.9.2p03. . . . . . . . . . . 68

5.3 Tabulated beam transport systematics. . . . . . . . . . . . . . . . . 97

5.4 External data results from various experiments. . . . . . . . . . . . . . 100

6.1 Fractional composition of electron neutrinos from $\mu^{+}, K^{+}$and other parents. . . . . . . . . . . . . . . . . . . . . 114

6.2 Event-selection: Contained. . . . . . . . . . . . . . . . . . . 120

6.3 Event-selection: uncontained. . . . . . . . . . . . . . . . 120

6.4 Iterative Method for final $K^{+}$Norm Calculation. . . . . . . . . . . . . . 122

6.5 $\nu_{\mu}-\mathrm{CC}$ events counts from contained and uncontained samples with GENIE Tune (Nominal). . . . . . . . . . . . . . . . . . . . . . . . . . . . . 128

6.6 The Iteration table for $N_{K}$ with GENIE Tune (Nominal). . . . . . . . . . 129

6.7 Systematics Summary Table. . . . . . . . . . . . . . . 135 
7.1 Cut flow for MC $\nu$ - e signal and background events. The $\varepsilon^{N-1}$ is the efficiency of each cut with respect to the previous ones. $\varepsilon$ is the cumulative efficiency of each cut, defined as the ratio of the number of remaining events, after applying that cut and all previous cuts, to the initial number of events. . . . . . . . . . . . . . . . . . 146

7.2 Event yields in the side band region $\left(0.005<E_{e} \theta_{e}^{2}<0.04\right)$ for Data and MC.158

7.3 Event yields in the signal region $\left(E_{e} \theta_{e}^{2}<0.005\right)$ with and with out background corrections. $\frac{N_{\nu-e}^{\text {Data }}}{N_{\nu-e}^{M C}}$ result is the one with background corrections. . 160

7.4 Summary of the systematic uncertainties for $\frac{N_{\nu e}^{\text {Data }}}{N_{\nu-e}^{M C}} \ldots \ldots . . . . . . .161$

7.5 Summary of the systematic uncertainties in the signal efficiency estimated by rock muon induced EM Shower samples. . . . . . . . . . . . . . . . 162

7.6 Summary of the systematic uncertainties in the selection efficiency of the single particle requirement. The three single-particle requirement cuts on $E_{\text {shower }} / E_{\text {tot }}$, vertex energy and the gap are relax individually. The relaxed cut values are chosen to have a $\sim 90 \%$ selection efficiency for each criterion. The resultant variation in $N_{\nu-e}^{\text {Data }} / N_{\nu-e}^{M C}$ after changing each cut is assigned as the uncertainty. The quadratic sum of the three uncertainties is assigned as the systematic error in the single particle requirement. . . . . . . . . . 164

7.7 Table of iteration for background normalization from sideband region. . . . 164

7.8 Table for flux systematics uncertainties from beam transport and hadroproduction model. . . . . . . . . . . . . . . . . . . . . . . 170

B.1 Number of $\nu_{\mu}$ at NOvA ND separated by parent types in the 0-10 GeV range.185

B.2 Number of $\nu_{\mu}$ at NOvA ND separated by parent types in the 1-3 GeV range.185

B.3 The variation $\delta(\%) \nu_{e}$ and Energy (Mean and RMS) at NO $\nu \mathrm{A} F D$ in the $0-10 \mathrm{GeV}$ range. . . . . . . . . . . . . . . . . . . 186 


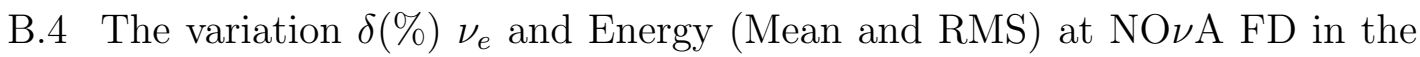
$1-3 \mathrm{GeV}$ range. . . . . . . . . . . . . . . . . . 186

B.5 Number of $\nu_{\mu}$ at NOvA FD separated by parent types in the 0-10 GeV range.186

B.6 Number of $\nu_{\mu}$ at NOvA FD separated by parent types in the 1-3 GeV range.187

B.7 The variation $\delta(\%) \nu_{e}$ and Energy (Mean and RMS) at NO $\nu$ A FD in the $0-10 \mathrm{GeV}$ range. . . . . . . . . . . . . . . . . . . . . . . 187

B.8 The variation $\delta(\%) \nu_{e}$ and Energy (Mean and RMS) at NO $\nu \mathrm{A}$ FD in the $1-3 \mathrm{GeV}$ range. . . . . . . . . . . . . . . . . . . . . 187

B.9 Number of $\nu_{e}$ at NOvA ND separated by parent types in the 0-10 GeV range.188 B.10 Number of $\nu_{e}$ at NOvA ND separated by parent types in the $1-3 \mathrm{GeV}$ range.188

B.11 The variation $\delta(\%) \nu_{e}$ and Energy (Mean and RMS) at NO $\nu$ A FD in the $0-10 \mathrm{GeV}$ range. . . . . . . . . . . . . . . . . . . 189

B.12 The variation $\delta(\%) \nu_{e}$ and Energy (Mean and RMS) at NO $\nu$ A FD in the $1-3 \mathrm{GeV}$ range. . . . . . . . . . . . . . . . . . . . 189

B.13 Number of $\nu_{e}$ at NOvA FD separated by parent types in the 0-10 GeV range.189. B.14 Number of $\nu_{e}$ at NOvA FD separated by parent types in the 1-3 GeV range.190 B.15 The variation $\delta(\%) \nu_{e}$ and Energy (Mean and RMS) at NO $\nu$ A FD in the $0-10 \mathrm{GeV}$ range. . . . . . . . . . . . . . . . . . . . 190

B.16 The variation $\delta(\%) \nu_{e}$ and Energy (Mean and RMS) at NO $\nu$ A FD in the $1-3 \mathrm{GeV}$ range. . . . . . . . . . . . . . . . . . . 190

D.1 $\nu_{\mu}-$ CC event counts for Contained without GENIE Tune. . . . . . . . . . 212

D.2 $\nu_{\mu}-\mathrm{CC}$ event counts for Uncontained without GENIE Tune. . . . . . . . 214

D.3 $K^{+}$Norm without GENIE Tune . . . . . . . . . . . . . . . . . . . 214

D.4 $K^{+}$Norm rewieghted with FTFP/Fluka weight. . . . . . . . . . . . . . 214 
D.5 $\nu_{\mu}-\mathrm{CC}$ event counts for Contained for $\mathrm{E}_{h a d}$ Containment, tight fiducial volume. . . . . . . . . . . . . . . . . . . . . . 216

D.6 $\nu_{\mu}$ - CC event counts for Uncontained for $\mathrm{E}_{\text {had }}$ Containment, tight fiducial volume. . . . . . . . . . . . . . . . . . . . . . . . . 217

D.7 $K^{+}$Norm for $\mathrm{E}_{\text {had }}$ Containment, tight fiducial volume. . . . . . . . . 217

E.1 $\nu_{\mu}-\mathrm{CC}$ event counts for Contained for $\mathrm{E}_{\mu}$ Shift $+2 \%$ in MC. . . . . . 219

E.2 $\nu_{\mu}$ - CC event counts for Uncontained for $\mathrm{E}_{\mu}$ Shift $+2 \%$ in MC. . . . . 221

E.3 $K^{+}$Norm for for $\mathrm{E}_{\mu}$ Shift $+2 \%$ in MC. . . . . . . . . . . . . 221

E.4 $\nu_{\mu}-\mathrm{CC}$ event counts for Contained for $\mathrm{E}_{\mu}$ Shift $-2 \%$ in MC . . . . . 222

E.5 $\nu_{\mu}-\mathrm{CC}$ event counts for Uncontained for $\mathrm{E}_{\mu}$ Shift $-2 \%$ in MC. . . . . . 223

E.6 $K^{+}$Norm for for $\mathrm{E}_{\mu}$ Shift $-2 \%$ in MC. . . . . . . . . . . . 223

F.1 $\nu_{\mu}-\mathrm{CC}$ event counts for Contained for MIPP+NA49 error band plus. . . 225

F.2 $K^{+}$Norm for MIPP+NA49 error band plus only to contained. . . . . . 225

F.3 $\nu_{\mu}$ - CC event counts for Contained for MIPP+NA49 error band minus. . 226

F.4 $K^{+}$Norm for MIPP+NA49 error band minus only to contained. . . . . . . 226

F.5 $\nu_{\mu}-$ CC event counts for Contained for MIPP+NA49 weight for pion only. 227

F.6 $\nu_{\mu}-\mathrm{CC}$ event counts for Uncontained for MIPP+NA49 weight for pion only.227

F.7 $K^{+}$Norm for MIPP+NA49 weight for pion only. . . . . . . . . . 228

F.8 Effect of GENIE parameters $\pm \sigma$, UnContained Sample $\mathrm{K}^{+}$Norm. . . . . 229

F.9 Effect of GENIE parameters $\pm \sigma$, Contained Sample $\pi^{+} 1-3(\mathrm{GeV})$ Norm. . 230

G.1 Summary of the effect of GENIE knobs variation on this analysis, for GENIE knobs. . . . . . . . . . . . . . . . . . . . . . . . . . 232 
G.2 Electroweak couplings in GENIE and in our oneloop calculation of $\nu \mathrm{e}^{-}$ elastic scattering . . . . . . . . . . . . . . . . . . . . 233 



\section{Chapter 1}

\section{Introduction}

\subsection{The Neutrino: An Introduction}

Of all the fundamental particles that compose our universe, the neutrino $(\nu)$ is the most enigmatic. Although its existence was predicted in 1930 when quantum mechanics was still in the crucible of formulation, the neutrino was not discovered until 1956. The neutrino is a fermion; it has no electric charge; it is almost massless; it only participates in the weak-interaction and even more weakly in gravitational interaction yet still more than most elementary particles and might hold answers to some of the most profound questions in particle physics. The fundamental discoveries in the past decade and a half have been dominated by neutrinos, the Higgs discovery being, perhaps, the exception. A brief history of the neutrinos and a synopsis of experimental and theoretical understanding is presented in this section.

\subsubsection{A Brief History of the Neutrino}

The historical account of the neutrino is within the currently held theory of particle physics, known as the Standard Model (SM). Remarkably, this history mirrors the intellectual development of particle physics. 
- $\nu$-Hypothesis (1930): Wolfgang Pauli postulated the existence of neutrino, as massless, chargeless, spin-1/2 particles, to remedy the apparent energy violation in $\beta$-decay [1, 2].

- Fermi Theory of Weak Interaction (1934): The interaction is governing $\beta$-decay, the weak-interaction, and was formulated into a theory by Enrico Fermi [3]. It was Fermi's formulation which cast the 3-body decay of the neutron $(n), n \rightarrow p+e+\nu$ in proper framework, where $p$ is proton and $e$ is electron. Within Fermi's theory, the neutrinos found a theoretical home, but their discovery remained elusive. From the endpoint spectrum of the electron $\left(e^{-}\right)$from neutron-decay, it was evident that neutrinos are almost massless. The current best limit on the neutrino mass is $<2 \mathrm{eV}$ [4].

- Electron Anti-neutrino ( $\left.\bar{\nu}_{e}\right)$ Discovery by Cowan and Reines (1950): The first direct evidence of the neutrinos occurred in South Carolina. Using the neutrinos produced in a nuclear reactor at Savannah River, Cowan and Reines demonstrated the existence of electron anti-neutrino undergoing inverse beta-decay $\bar{\nu}+p \rightarrow n+e^{+}$ [5, 6]. After considering several methods, including a nuclear explosion, they settled on using the enormous flux of electron anti-neutrinos from a nuclear reactor at the Savannah River Nuclear Plant and 10 ton of equipment, including 1400 liters of liquid scintillators. This experiment was the first reactor-neutrino experiment. In June 1956, Reines and Cowan sent a telegram informing Pauli of the discovery. Reines (Cowan passed away) was awarded the Nobel prize 40 years later.

- Discovery of Parity Violation (1956): The next breakthrough came with the prediction of parity violation in weak interaction by Lee and Yang [7]. The prediction was verified by the subsequent discovery of the parity violation in $\beta$-decay by $\mathrm{Wu}$ et al. [8] by measuring the direction of the emitted electrons from magnetically aligned Cobalt-60 decays. The relation between the experiment and the Fermi-theory is pictured in Figure 1.1 . 


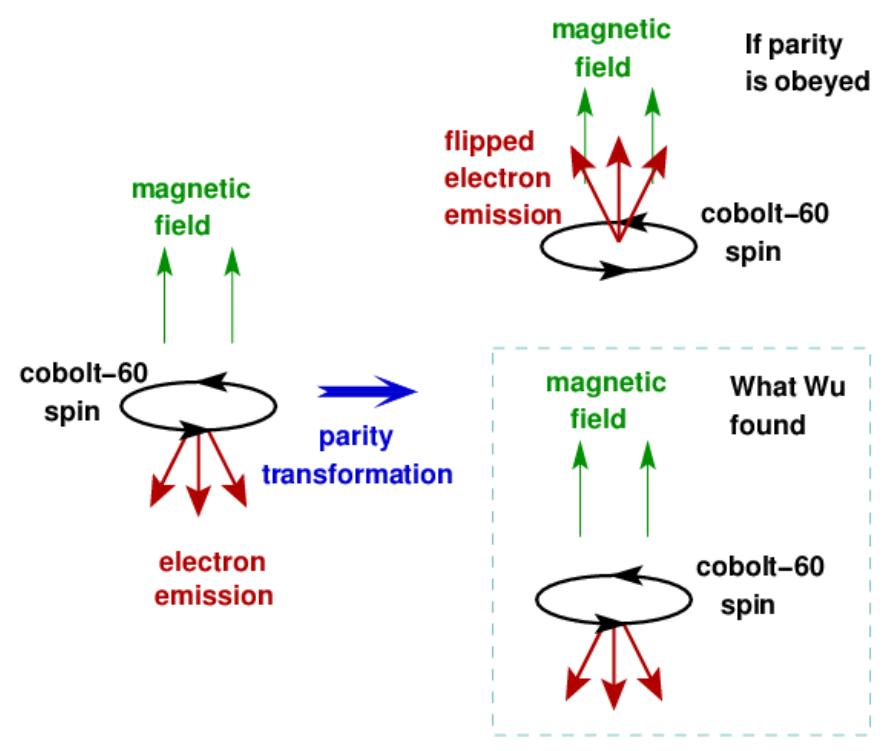

Figure 1.1: Cartoon of the $\mathrm{Wu}$ experiment: if parity was preserved, then the emission of electrons would change if you reversed the orientation of the experiment. Instead, she found that the electrons always shot out the same way relative to the cobalt atoms? spin, showing that the weak force violates the law of parity.

- 2-Component Neutrino Theory (1957): The discovery of parity-violation in the weak interaction impelled a 2-component theory of neutrinos [9] postulating that the neutrino is left-handed, and the antineutrino is right-handed. In this theory, the neutrino with momentum $\mathrm{p}$ has only one spin state, which is always parallel to $\mathrm{p}$. The mass of the neutrino must be zero, and its wave function needs only two components instead of four.

- Discovery of the Muon Neutrino $\left(\nu_{\mu}\right)$ - A 2nd Neutrino (1962): Although the 1950's saw impressive advances in our understanding of neutrinos and the weak interaction, the study of the weak interaction was limited to the low-energy regime, such as in nuclear decays. There was an imperative to study weak interaction at higher energies. An additional puzzle, the lack of $\mu \rightarrow e \gamma$ observation prompted the idea that lepton-number is conserved. If the electron and muon were distinct leptons, there must be neutrinos corresponding to each lepton. The idea of 'Generation of Matter' was taking 
shape, having been first postulated by H. Bhabha in the 1940's. Both topics, weak interactions at higher energy and a second neutrino, was powerfully addressed by the brand-new Alternating Gradient Synchrotron (AGS) and neon-filled detector called a spark chamber at Brookhaven laboratory which discovered the muon neutrino [10].

- Electro-weak Unification (1961): Before the discovery of the muon neutrino, the list of fundamental particles consisted of three hadrons - proton, neutron, and hyperon $(\Lambda)$ and three leptons - electron, muon, and electron neutrino. The muon neutrino spoiled the pseudo-symmetry. The quest for a unified theory of electromagnetism and weak interaction was first proposed by J. Schwinger, and his student, S. Glashow [11]. Their efforts, and efforts of their contemporaries, including A. Salam \& S. Weinberg, culminated in the Electro-Weak (EW) theory [12]. The Electro-Weak theory provided an elegant framework for the known fundamental particles. There are three generations of fundamental particles; each generation has a doublet of charged and neutral leptons and a doublet of quarks. The mass of the $3^{\text {rd }}$ generation is much larger than that of the $2^{\text {nd }}$ generation, which, in turn, is much larger than that of the $1^{\text {st }}$ generation. Much of the universe is composed of the $1^{\text {st }}$ generation particles, electron, electron neutrino, up- and down-quark $(u, d)$ that are subsequently composed of protons and neutrons. Apart from the mass-difference, the three generations are identical in their interactions, a deeply held principle of physics known as the universality principle [13].

- Discovery of the Neutral Current (NC) interactions (1973): Prior to the Electro-Weak theory, the weak interaction was thought to be mediated by the $W$ boson in charge current (CC) interactions such as in $\nu_{\mu}+n \rightarrow \mu^{-}+p$ or neutron-decay. The Electro-Weak theory is based on a non-abelian gauge symmetry [14], $S U_{2} \times U_{1}$. Key to this unification is a deep symmetry between the photon $(\gamma)$ and $Z^{0}$ boson, the neutral boson belonging to the $S U_{2}$ group - the 'mixing' between the two bosons is quantified by the Weak Mixing Angle (WMA), a fundamental parameter of Electro-Weak theory. 


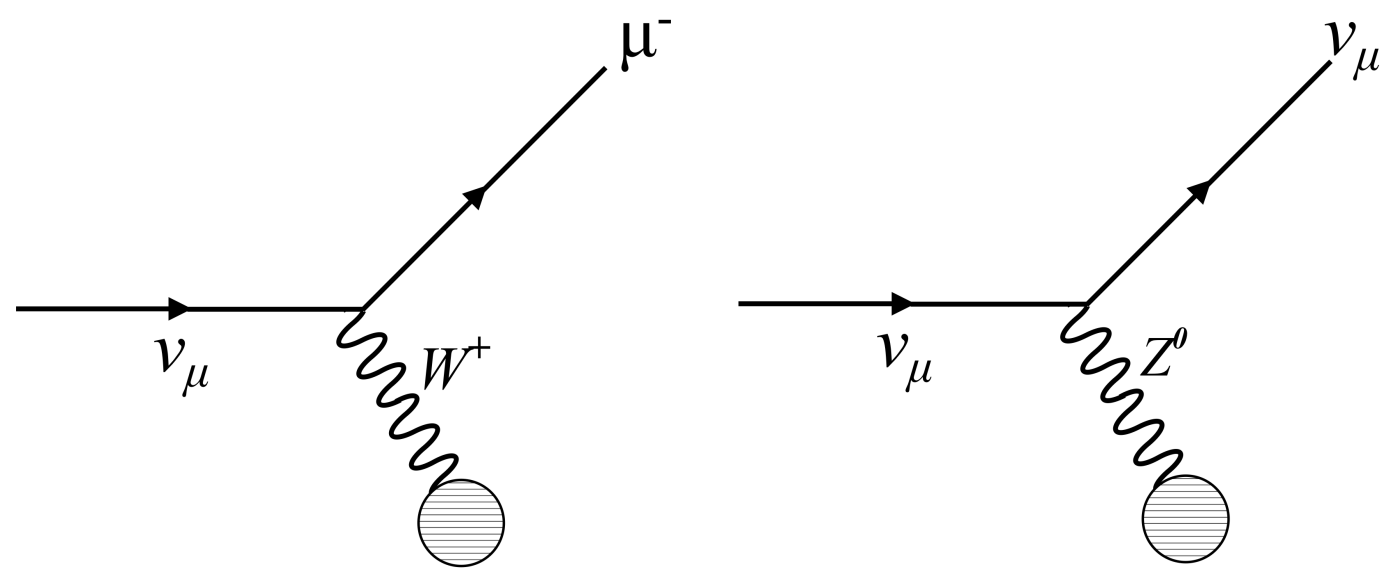

Figure 1.2: Charge current (CC) and neutral current (NC) interactions of neutrinos.

The unification of the two seemingly disparate forces had a set of compelling predictions. One of these was that neutrinos must participate in neutral current (NC) interactions mediated by the $Z^{0}$ boson. Figure 1.2 pictures the two processes. The first evidence of the neutrino-induced NC was provided by the Gargamelle bubble chamber experiment in neutrino electron scattering [15]. Soon after that, a statistically precise measurement of NC was provided by the CalTech-Fermilab experiment [16].

- Three Types of Neutrinos from $Z^{0}$ (1989): The most direct and convincing evidence that there three types of neutrinos - no less or more - came from the precise measurement of the $Z^{0}$ decay-width which directly conveys the $Z^{0} \rightarrow \nu \bar{\nu}$ contribution to the width. The experiments at LEP measured that there are 3 flavor of neutrinos with a $\simeq 1 \%$ precision

- Discovery of tau neutrino $\left(\nu_{\tau}\right)$ - A 3rd Neutrino (2001): The tau neutrinos were detected by the DONUT experiment [17. This experiment collided $800 \mathrm{GeV}$ protons with a block of tungsten. This collision produced $D_{S}$ mesons that subsequently decayed into tau-leptons which then produced tau neutrinos.

The SM theory has emerged as the most precise and thoroughly-tested for rest of fundamental particles. The only departure from the SM picture of the fundamental particles came from neutrinos with more detail on this topic in subsection 1.1 .2 and 1.1.3. 


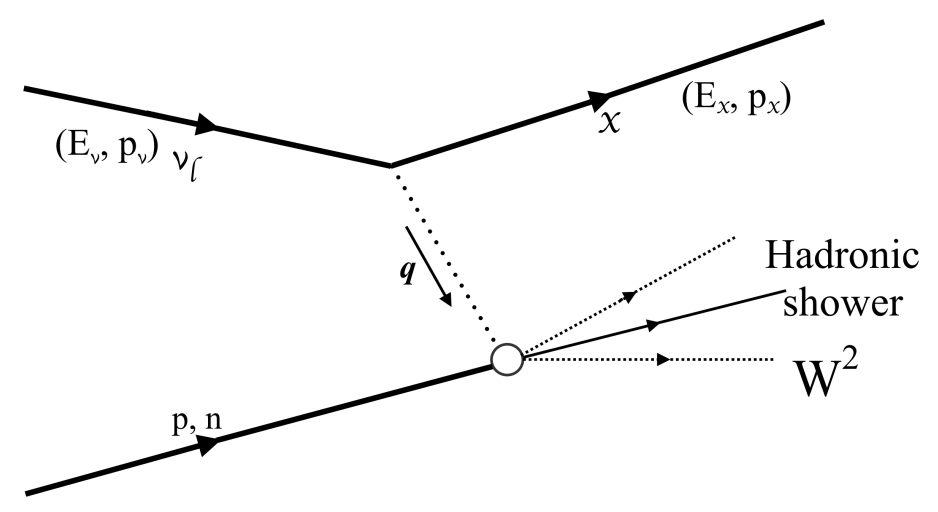

Figure 1.3: Neutrino-nucleon interaction with kinematic variables.

\subsubsection{Neutrinos in the Standard Model}

Two-component neutrino theory tells us that there are no right-handed neutrinos and there are no left-handed antineutrinos. In SM, the (always) left-handed electron neutrino and the left-handed $e^{-}$form a doublet; as do the up and down quark. The second generation comprises left-handed $\left(\nu_{\mu}, \mu^{-}\right)$and $(c, s)$; the third generation comprises $\left(\nu_{\tau}\right.$, $\tau^{-}$) and $(t, b)$. The right-handed $e^{-}, \mu^{-}, \tau^{-}$are singlets. Since the mass term from lagrangian involves $M\left(\bar{\psi}_{L} \psi_{R}+\bar{\psi}_{R} \psi_{L}\right)$ and there are no right-handed neutrino, in the SM neutrinos are massless. Neutrinos participate in CC and NC interaction as shown in Figure 1.2. The NuMI: Neutrinos at the Main Injector $\left(\nu_{\mu}\right)$, Off-Axis: narrow band beam $(2 \mathrm{GeV}), \boldsymbol{\nu}_{\boldsymbol{e}}$ Appearance (NOvA) experiment more details in Chapter 3 , as longest baseline experiment right now (accelerator-based experiment), involves neutrino scattering off nuclear targets. Figure 1.3 shows the schematic of neutrino scattering off a proton/neutron. Following the figure, the interaction is characterized by the kinematic variables such as:

- Hadronic energy: $\nu=E_{\nu}-E_{l}$;

- Four-momentum transfer: $Q^{2}=-q^{2}=2 E_{\nu} E_{l}\left(1-\cos \theta_{l}\right)-m_{l}^{2}$;

- Scaling variable: Fraction momentum of the quark: $x=Q^{2} / 2 M \nu$; 
- Scaling variable: Fraction energy to the hadronic system: $y=\nu / E_{\nu}$;

- Invariant hadronic mass: $W^{2}=M^{2}+2 M E_{\nu} y(1-x)$.

In the SM the neutrino is massless; there is no mixing among the three active flavors of neutrinos. However, the first experimental hints of the neutrino oscillations phenomenon came in 1968 [18]. Ray Davis set up an experiment in the Homestake mine in South Dakota to measure the electron neutrino flux from the Sun as direct confirmation of the theoretical models of nuclear fusion and fission in the Sun. The experiment was located deep underground to reduce cosmic-ray backgrounds and counted the number of ${ }^{37} \mathrm{Ar}$ atoms produced in a 390,000-liter container of tetrachloroethylene through the process $\nu_{e}+{ }^{37} \mathrm{Cl} \rightarrow e^{-}+{ }^{37} \mathrm{Ar}$. The result was a rate of $\nu_{e}$ interactions of about one-third of the theoretical predictions. While the neutrino rate discrepancy was initially attributed to errors in either the measurement or the theoretical prediction, this "solar neutrino problem" persisted when a similar measurement was made in 1989 by the Kamiokande-II experiment in a water-Cherenkov detector [19]. The Super-Kamiokande experiment (SK) in 1998 [20] and the SNO experiment provided conclusive evidence in 2001 [21], signaling that neutrinos are massive and the different flavors mix heralded a new era in the field of particle physics. Ray Davis and Masatoshi Koshiba were awarded the 2002 Nobel Prize for their pioneering contributions to the detection of cosmic neutrinos. The brief calculation for the neutrino oscillation is in the next subsection 1.1.3.

\subsubsection{Neutrino Oscillation}

Let us consider a neutrino with flavor $\alpha\left(\nu_{\alpha}\right)$ and momentum $p$, produced in a CC weak interaction from a charged lepton $l_{\alpha}$. In the standard theory of neutrino oscillations [22, 23], the neutrino flavor state is described by:

$$
\left|\nu_{\alpha}\right\rangle=\sum_{k=1}^{3} U_{\alpha k}^{*}\left|\nu_{k}\right\rangle
$$


The unitary matrix $U$ from Equation 1.1 is the neutrino mixing matrix and $\nu_{k}$ is neutrino mass eigenstate. Next, if we consider the time evolution of a neutrino flavor, $\nu_{\mu}$, for example, Equation 1.2, produced in a pion-decay at $t=0$, after time $t$ the different mass states composing $\nu_{\mu}$ will go out of phase. Thus, at time $t$ there will be a non-zero probability of detecting a neutrino of a flavor $\left(\nu_{e}\right.$ or $\left.\nu_{\tau}\right)$ different from the initial flavor $\left(\nu_{\mu}\right)$. Since neutrino velocity is approximately that of light, $c$, the elapsed time is $t=L / c$, where $L$ is the flight distance where the new flavor is detected. This, in essence, is the idea of neutrino oscillation.

$$
\left|\nu_{\alpha}(t)\right\rangle=\sum_{k=1}^{3} e^{-i E_{k} t} U_{\alpha k}^{*}\left|\nu_{k}\right\rangle
$$

where the $e^{-i E_{k} t}$ is the time-evolution operator for the free particle Hamiltonian in natural units.

For a relativistic free particle, $E_{k}^{2}=p^{2}+m_{k}^{2}$ where we assume $p_{k}=p_{l}=p$, and since neutrino masses are very small with respect to their energies, $E_{k} \approx|p|+\frac{m_{k}^{2}}{2|p|}$. The probability of oscillation from flavor $\alpha$ to flavor $\beta$ is given by

$$
\begin{aligned}
P_{\alpha \rightarrow \beta}(t) & =\left|\left\langle\nu_{\beta}(t)\left|\nu_{\alpha}(t=0)\right| \nu_{\beta}(t) \mid \nu_{\alpha}(t=0)\right\rangle\right|^{2} \\
& =\left|\sum_{k} U_{\alpha k}^{*} U_{\beta k} e^{-i \frac{m_{k}^{2}}{2|p|} t}\right|^{2}
\end{aligned}
$$

Under the assumption that the neutrinos are ultra-relativistic, we can use $t \approx L$ to write $t /|p|$ as $L / E$, where $L$ and $E$ are the distance traveled by the neutrino and the neutrino energy, respectively. $L / E$ is typically expressed in units of $\mathrm{km} / \mathrm{GeV}$.

Taking advantage of the unitarity matrix $U$, Equation 1.3 can be expanded into Equation 1.4. where $\Delta m_{i j}^{2} \equiv m_{i}^{2}-m_{j}^{2}$. The Equation 1.4 for the survival probability $\left(\nu_{\beta}=\nu_{\alpha}\right)$ reduces to Equation 1.5. 


$$
\begin{array}{r}
P_{\alpha \rightarrow \beta}=\delta_{\alpha \beta}-4 \sum_{i j} \operatorname{Re}\left[U_{\alpha i}^{*} U_{\alpha j} U_{\beta i} U_{\beta j}^{*}\right] \sin ^{2}\left(\frac{\Delta m_{i j}^{2}}{4 E} L\right) \\
+2 \sum_{i j} \operatorname{Im}\left[U_{\alpha i}^{*} U_{\alpha j} U_{\beta i} U_{\beta j}^{*}\right] \sin ^{2}\left(\frac{\Delta m_{i j}^{2}}{2 E} L\right) \\
P_{\nu_{\alpha} \rightarrow \nu_{\alpha}}(L, E)=1-4 \sum_{k j}\left|U_{\alpha k}\right|^{2}\left|U_{\alpha j}\right|^{2} \sin ^{2}\left(\frac{\Delta m_{i j}^{2}}{4 E} L\right)
\end{array}
$$

In many experimental cases, one need not consider the full effect of three neutrino flavor mixing, but instead can consider the approximation of two neutrino mixing. If neutrinos had only two flavor states and two mass states, the mixing matrix could be written as in Equation 1.6 and for NOvA experiment the survival probability is calculated by Equation 1.7 .

$$
\begin{gathered}
U=\left(\begin{array}{cc}
\cos \theta & \sin \theta \\
-\sin \theta & \cos \theta
\end{array}\right) \\
P\left(\nu_{\mu} \rightarrow \nu_{\mu}\right)=1-\sin ^{2}\left(2 \theta_{23}\right) \sin ^{2}\left(\frac{1.267 \Delta m_{32}^{2} L}{E}\right)
\end{gathered}
$$

The formula presents the probability that a $\nu_{\mu}$ will remain $\nu_{\mu}$, called the 'disappearance' probability. The two-flavor oscillation involves two parameters: the massdifference $\left(\Delta m^{2}\right)$ and the mixing-strength $(\theta)$ between two mass-eigenstates. In Figure 1.4 the left panel contrasts the 'un-oscillated' and the 'oscillated' spectra as a function of the neutrino energy $\left(E_{\nu}\right)$. The right panel shows the ratio of oscillated to the un-oscillated spectra as a function of $E_{\nu}$. In all oscillation measurements, the final result is invariably encapsulated by the graph on the right or some representation of it. The dip at $2 \mathrm{GeV}$ measures the $\sin ^{2}\left(2 \theta_{23}\right)$ and its width will give $\Delta m_{32}^{2}$. 

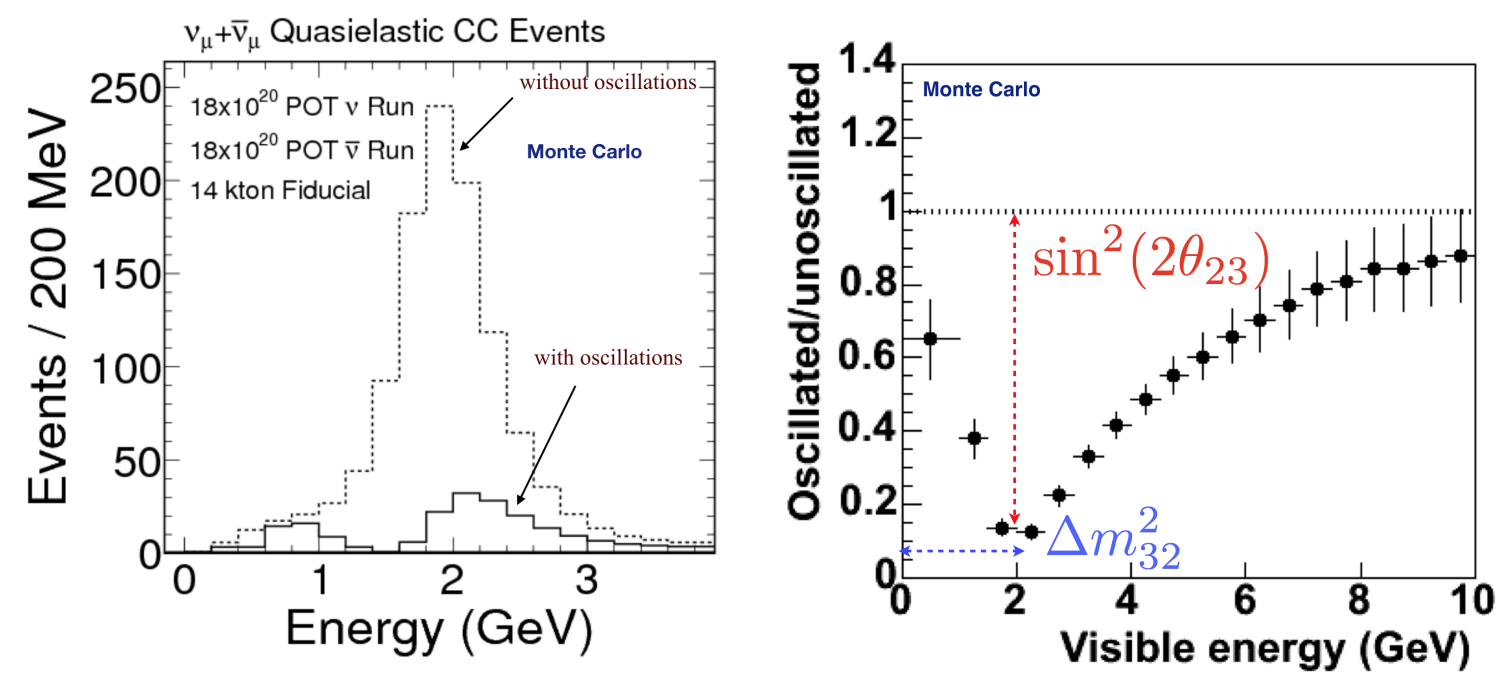

Figure 1.4: A conceptual presentation of 2-flavor $\nu$-osicllation. Left: The distribution of the 'oscillated' and the 'un-oscillated' spectra as a function of the neutrino energy $\left(E_{\nu}\right)$. Right: Shows the ratio of oscillated to the un-oscillated spectra as a function of $E_{\nu}$.

Because there are three flavors of neutrinos, the oscillation formulation must be extended from 2- to 3-flavor oscillation. The 3-flavor oscillation is more complicated than the corresponding 2-flavor. The unitary mixing-matrix, $U$, is a $3 \times 3$ matrix with complex elements. Maki, Nakagawa, and Sakata generalized the Pontecorvo formula to the 3-flavor (or more than 3) picture. The mixing matrix $U$ is referred to as PontecorvoMaki-Nakagawa-Sakata, PMNS, matrix. The PMNS matrix $U$ is shown in Equation 1.8, where $\operatorname{sij}=\sin \theta_{i j}$ and $\operatorname{cij}=\cos \theta_{i j}$.

$$
U=\left(\begin{array}{ccc}
c_{12} c_{13} & s_{12} c_{13} & s_{13} \mathrm{e}^{-\mathrm{i} \delta_{\mathrm{CP}}} \\
-s_{12} c_{23}-c_{12} s_{13} s_{23} \mathrm{e}^{\mathrm{i} \delta_{\mathrm{CP}}} & c_{12} c_{23}-s_{12} s_{13} s_{23} \mathrm{e}^{\mathrm{i} \delta_{\mathrm{CP}}} & c_{13} s_{23} \\
s_{12} s_{23}-c_{12} s_{13} c_{23} \mathrm{e}^{\mathrm{i} \delta_{\mathrm{CP}}} & -c_{12} s_{23}-s_{12} s_{13} c_{23} \mathrm{e}^{\mathrm{i} \delta_{\mathrm{CP}}} & c_{13} c_{23}
\end{array}\right)
$$

The angle $\theta_{12}$ is known as the solar mixing angle for historical reasons. This angle is predominantly measured with solar $(\mathrm{L} \sim 108 \mathrm{~km})$ and reactor neutrinos $(\mathrm{E} \sim 1 \mathrm{MeV})$ where the $\mathrm{L} / \mathrm{E}$ ratio is large. The associated mass splitting is small with $\Delta m_{21}^{2} \approx 8 \times$ $10^{-5} \mathrm{eV}^{2}$. Similarly, $\theta_{23}$ is known as the atmospheric mixing angle and is probed with atmospheric and accelerator neutrinos where the baseline is relatively short $(\mathrm{L} \leq 104$ 
$\mathrm{km})$ and the energies are high $(\mathrm{E} \sim 1 \mathrm{GeV})$ such that $\mathrm{L} / \mathrm{E}$ is comparatively small. The atmospheric sector is associated with a large mass splitting $\Delta m_{32}^{2} \approx \Delta m_{31}^{2} \approx 3 \times 10^{-3} \mathrm{eV}^{2}$. For $\delta$ to possibly be non-zero, all three mixing angles must be non-zero. It should be noted that the PMNS matrix also has two Majorana phases $\alpha_{1}$ and $\alpha_{2}$ that are factored into a fourth sub-matrix, but these phases have no impact on the oscillation results and have been thus suppressed in Equation 1.8. In experimental measurements, there is one more effect that has to be taken into account due to the neutrino interactions with the matter through which neutrinos must traverse before being detected. The matter effect is explained in subsection 1.1.4.

\subsubsection{Matter Effect}

As neutrinos traverse through terrestrial matter, they undergo interactions off the protons, neutrons, and electrons composing the earth crust and mantle. The neutrinos of all flavor undergo neutral current interactions; the NC interaction potential for neutrinos is, $V_{N C} \simeq-G_{F} n_{n}$, where $n_{n}$ is the baryon number density. For anti-neutrino, the sign of the potential is "positive". The $V_{N C}$ does not affect the oscillation measurement. Since electron neutrinos and electron anti-neutrinos can undergo charge current interactions, the resulting potential does affect the oscillation measurement. Equation 1.9 gives the effective potential, Where $n_{e}$ is the number density of electron (the plus sign is for neutrino and the minus for antineutrino) and $G_{F}$ is Fermi's constant. The matter effect alters the oscillation parameters $\theta$ and $\Delta m^{2}$ as shown in Equation 1.10 .

$$
V_{e}= \pm \sqrt{2} G_{F} n_{e}
$$




$$
\begin{aligned}
& P_{\mu e}=s_{23}^{2} \frac{\sin ^{2} 2 \theta_{13}}{C_{13}^{2}} \sin ^{2} C_{13} \Delta-2 \alpha s_{12}^{2} s_{23}^{2} \frac{\sin ^{2} 2 \theta_{13}}{C_{13}^{2}} \sin C_{13} \Delta \\
& \times\left[\Delta \frac{\cos C_{13} \Delta}{C_{13}}\left(1-A \cos 2 \theta_{13}\right)-A \frac{\sin C_{13} \Delta}{C_{13}} \frac{\cos 2 \theta_{13}-A}{C_{13}}\right] \\
& +\alpha s_{13} \sin 2 \theta_{12} \sin 2 \theta_{23} \frac{\sin C_{13} \Delta}{A C_{13}^{2}}\left\{\operatorname { c o s } \delta \left[C_{13} \sin (1+A) \Delta\right.\right. \\
& \left.\left.-\left(1-A \cos 2 \theta_{13}\right) \sin C_{13} \Delta\right]-C_{13} \sin \delta\left[\cos C_{13} \Delta-\cos (1+A) \Delta\right]\right\} \\
& +c_{23}^{2} \frac{\sin ^{2} 2 \theta_{12}}{C_{12}^{2}} \sin ^{2} \alpha C_{12} \Delta-s_{13} \frac{\sin 2 \theta_{12}}{C_{12}} \sin 2 \theta_{23} \frac{(1-\alpha) \sin \alpha C_{12} \Delta}{1+A-\alpha+A \alpha c_{12}^{2}} \\
& \left\{\sin \delta\left[\cos \alpha C_{12} \Delta-\cos (A+\alpha-2) \Delta\right]+\cos \delta[\sin (A+\alpha-2) \Delta\right. \\
& \left.\left.-\sin \alpha C_{12} \Delta\left(\frac{\cos 2 \theta_{12}-\frac{A}{\alpha}}{C_{12}}-\frac{\alpha A C_{12}}{2(1-\alpha)} \frac{\sin ^{2} 2 \theta_{12}}{C_{12}^{2}}\right)\right]\right\} \\
& -2 \alpha s_{13} \sin 2 \theta_{12} \sin 2 \theta_{23} \cos (\Delta+\delta) \frac{\sin A \Delta}{A} \frac{(A-1) \Delta}{(A-1)}
\end{aligned}
$$

\subsubsection{Neutrino Mass Hierarchy}

Since neutrino oscillation implies a non-zero neutrino mass, and from solar neutrino experiment, we know that $m_{2}>m_{1}$ [24]. The sign of the large atmospheric mass splitting has not been measured. An outstanding question regarding the neutrino mass hierarchy arises: Whether the neutrino mass hierarchy is normal, that is $m_{3}>m_{1}$ like those of other fermions or the neutrino mass hierarchy is inverted, that is $m_{3}<m_{1}$ unlike those of other fermions?, as shown in Figure 1.5 . 


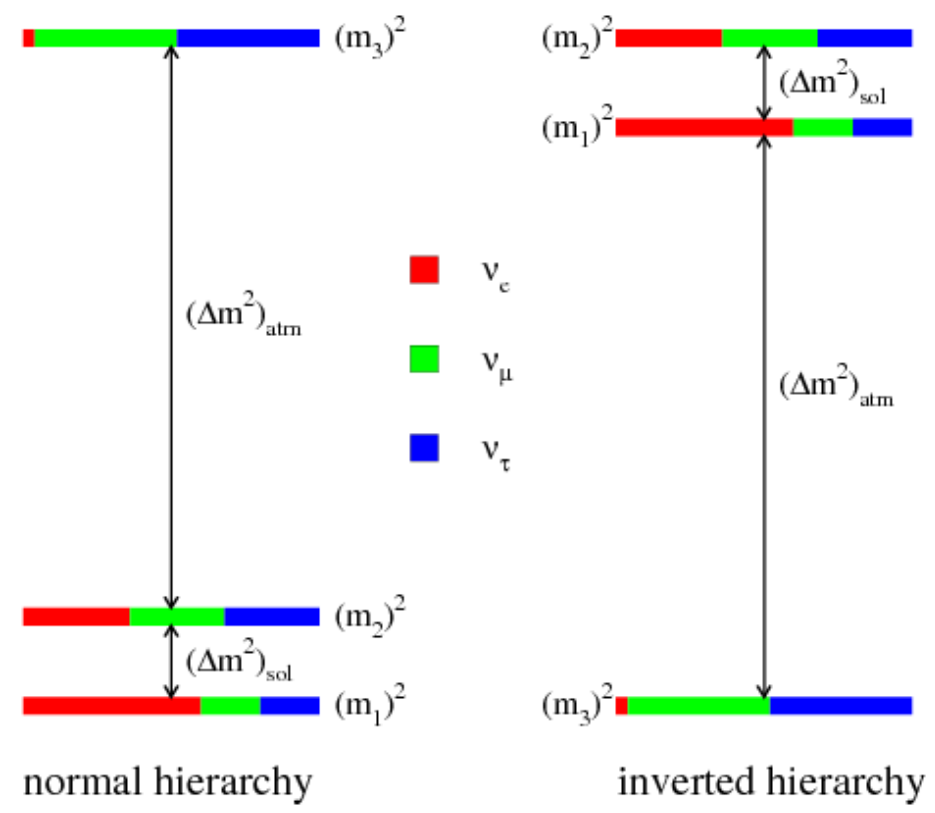

Figure 1.5: Diagram showing the two possible mass orderings with each state showing the approximate measured flavor combination.

\subsubsection{Evidence of Neutrino Oscillation}

A set of experiments have firmly established the existence of neutrino oscillation. The data confirm $\nu_{\mu} \rightarrow \nu_{\tau}$ using atmospheric and accelerator neutrinos, and $\nu_{e} \rightarrow \nu_{\mu}$ using solar and reactor neutrinos.

Atmospheric Neutrino: The first definitive evidence of the oscillation came from the Super-Kamiokande experiment using the atmospheric neutrinos [25]. Data unequivocally showed the $2 \rightarrow 3$ oscillation. The results were verified by other experiments using the atmospheric neutrinos, the precise determination of $\Delta m_{23}^{2}$ was provided by the MINOS experiment at Fermilab [26].

Solar Neutrino: A host of solar neutrino experiments, provided the evidence for the $1 \rightarrow 2$ oscillations. The experiments include the classic Homestake experiment by R. Davis et al., Super-Kamiokande experiment, SAGE and Gallex experiments. The experiments that established the solar neutrino oscillation were the SNO experiment at Sudbury [21] and the Kamland experiment [27]. 


\subsection{Neutrino Oscillations in NOvA}

The two outstanding unknowns are the CP-violating phase $\delta_{C P}$ and the mass hierarchy (subsection 1.1.5). Furthermore, there is a compelling need to measure $\theta_{23}$ with sufficient precision as to resolve the degeneracy. Degeneracy means for example, $\theta_{23}$ tells us if the third mass eigenstate mixes equally with muon and tau neutrino or if it prefers one of the two. An inexorable goal of the field is to measure the parameters with increasing precision permitting one to conduct incisive tests of unitarity, much like the quark sector in the last decade.

The plan for NOvA is is to run until approximately 2024, to collect data for both in the neutrino-mode (Forward Horn Current, FHC) and in the antineutrino-mode (Reverse Horn Current, RHC) in the $700 \mathrm{~kW}$ beam see the Chapter 2. The principal oscillation-physics goals of the NOvA experiment are:

1 $\rightarrow 3$ Sensitivity: NOvA simultaneously measures the $\nu_{\mu} \rightarrow \nu_{e}$ and $\bar{\nu}_{\mu} \rightarrow \bar{\nu}_{e}$. The $1 \rightarrow 3$ sensitivity is pictured in Figure 1.6. The Figure shows the two probabilities for different values of $\delta$ in Normal-Hierarchy $(\mathrm{NH})$ and Inverted-Hierarchy $(\mathrm{IH})$.

$2 \rightarrow 3$ Sensitivity: Using the muon neutrino and muon anti-neutrino disappearance, NOvA will conduct very precise measurements of the $2 \rightarrow 3$ oscillation parameters.

Mass-Hierarchy Sensitivity: NOvA should have a capability to discover the $\nu$ masshierarchy, as shown in Left Figure 1.7.

CP-Vilation Sensitivity: NOvA has limited sensitivity to the $\delta_{C P}$. Right Figure 1.7 presents the anticipated sensitivity to this parameter. 


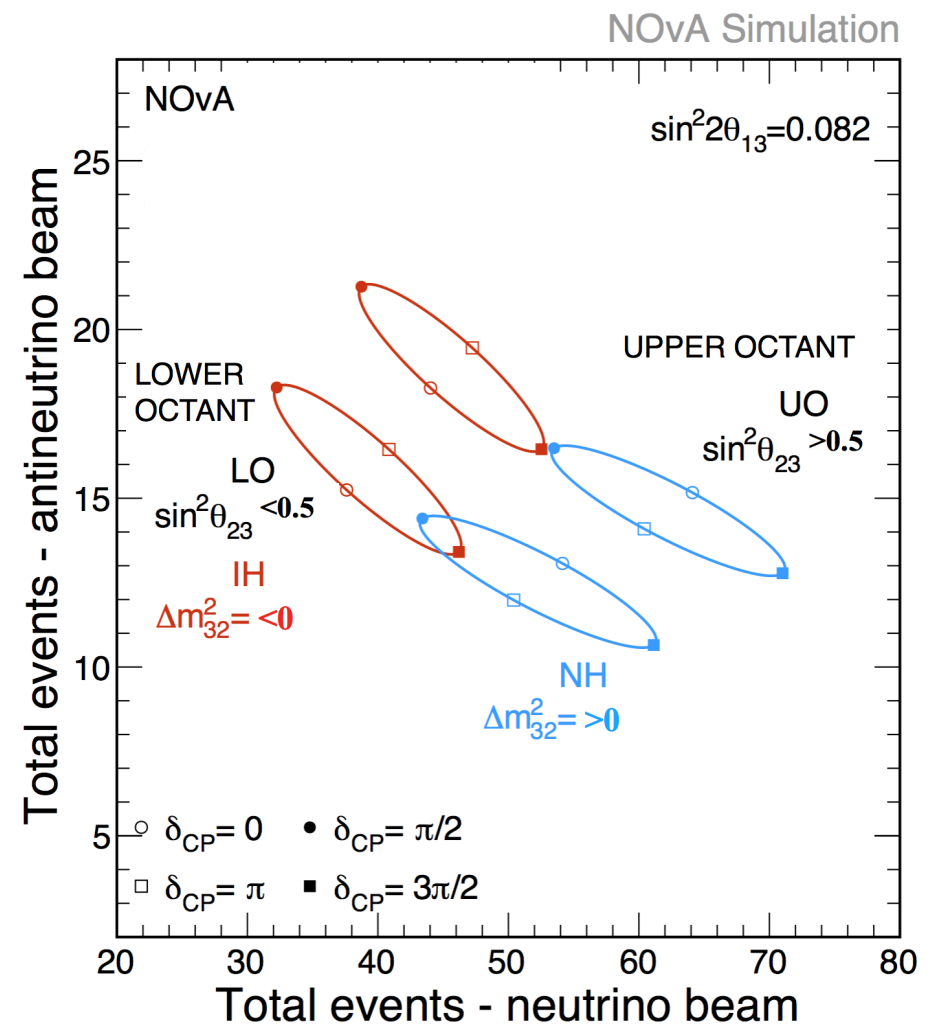

Figure 1.6: The oscillation probabilities $\mathrm{P}\left(\nu_{\mu} \rightarrow \nu_{e}\right)$ and $\mathrm{P}\left(\overline{\nu_{\mu}} \rightarrow \overline{\nu_{e}}\right)$ for the NOvA experiment illustrating the dependence on the remaining unknowns; $\sin ^{2} \theta_{23}, \delta_{C P}$, and choice of neutrino mass hierarchy.
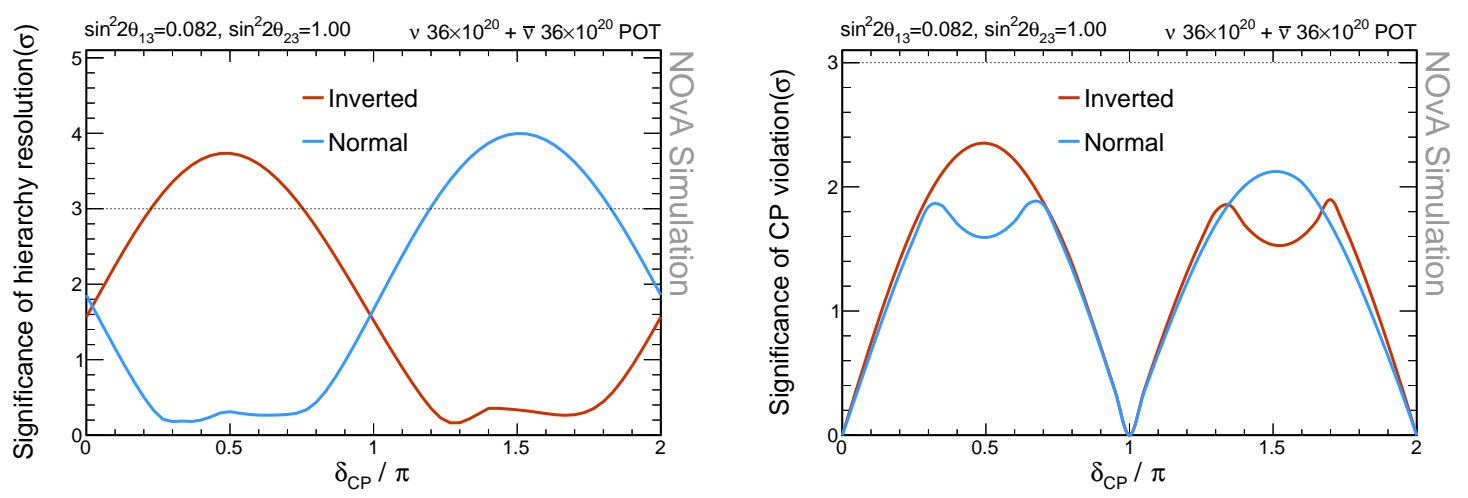

Figure 1.7: Left: NOvA Sensitivity to the mass-hierarchy. Right: NOvA Sensitivity to the CP-violating phase. 


\subsection{Importance of Precise Flux Determination in}

\section{Neutrino Physics}

The challenges in the precision determination of the neutrino oscillation phenomena, both $2 \rightarrow 3$ and $1 \rightarrow 3$ oscillations, is best visualized in Figure 1.4. Un-oscillated Flux: One needs to know with very high precision the un-oscillated flux. At the simplest level, this is best measured by a detector near the source of the neutrino - the Near Detector (ND). The Figure 1.4 shows that in a disappearance measurements the deficit in the oscillated flux at the Far Detector (FD) with respect to the un-oscillated flux, or in an appearance measurement the excess in the oscillated flux at the FD with respect to the un-oscillated flux directly yields the mixing-angle, $\theta$. The spectra that we measure at our experiments are a convolution of the following:

- The neutrinos flux we generated at NuMI beam see in Chapter 2 .

- The neutrino interaction cross-sections (and this comes from our inability to just see neutrinos, but we can only see neutrinos when they interact with something else) .

- Detector/analysis effects.

- and of course oscillation parameters.

NOvA having a ND and FD that are basically identical has a great advantage and it means we can directly measure what we produce at Fermilab, but it is impossible to disentangle the flux from the cross-section. However, the ND measures the spectra of neutrino interaction, and not the flux. To unfold the flux from the ND spectra requires knowledge of neutrino cross-section. However the neutrino and anti-neutrino cross-sections are poorly known in $0.5 \leq E_{\nu} \leq 5 \mathrm{GeV}$ region, a region most sensitive to the oscillation studies with $\Delta \mathrm{m}^{2} \simeq 2 \times 10^{-3} \mathrm{eV}^{2}$. Figure 1.8 [28] presents a compilation of the inclusive $\nu_{\mu}$-CC and $\bar{\nu}_{\mu}$-CC interactions. It is evident that the $\nu_{\mu}$-CC cross-section in $0.5 \leq E_{\nu} \leq 5$ region is not known to better than $20 \%$ uncertainty; the $\bar{\nu}_{\mu}$-CC cross-section is even more poorly known as there is less data available from experiments. 


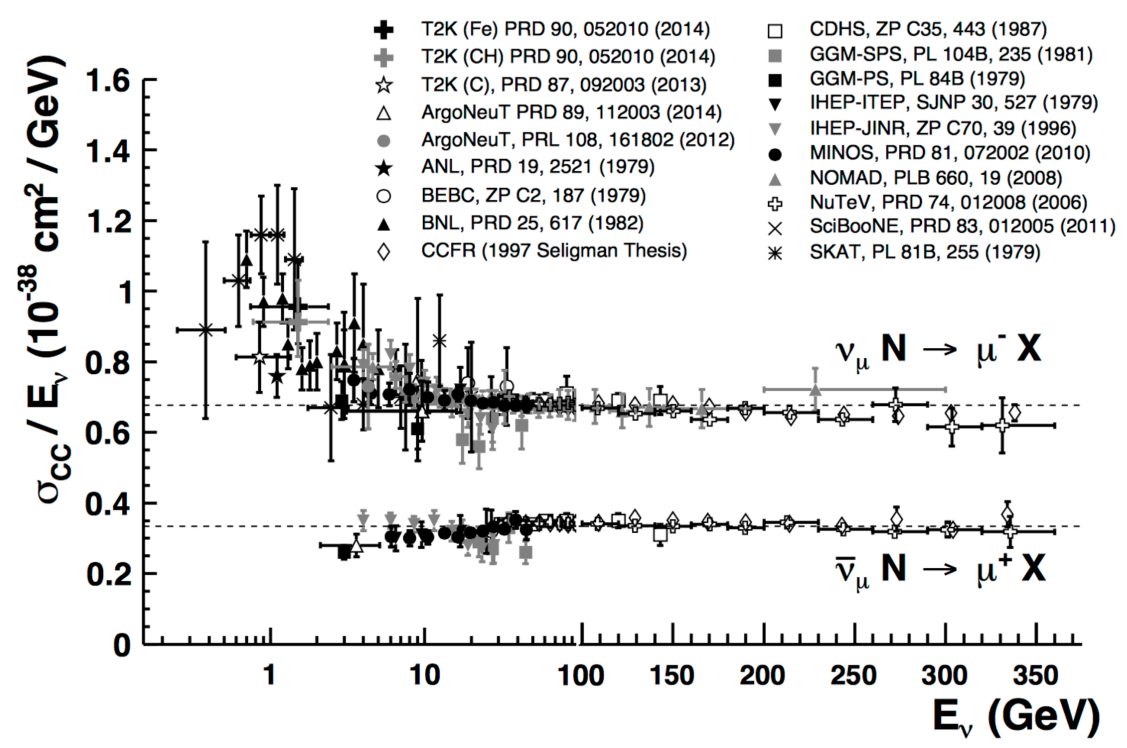

Figure 1.8: Measurements of per nucleon $\nu_{\mu}$-CC and $\bar{\nu}_{\mu}$-CC inclusive scattering cross sections divided by neutrino energy as a function of neutrino energy.

\subsection{Outline of the Thesis}

NOvA generates a beam peaked at $2 \mathrm{GeV}$ of muon neutrinos with an accelerator at Fermilab. The beam passes through two functionally identical liquid scintillator tracking detectors, one located at Fermilab, the other 810 kilometers away in Ash River, Minnesota (MN). The design of the NuMI (Neutrino at the Main Injector), and beam simulation for NOvA is described in Chapter 2. The design of the experiment and a significant personal contribution was made to the ND installation is described in Chapter 3 . The brief explanation for NOvA simulation and reconstruction is described in Chapter 4 . The beam systematic uncertainties from a variety of effects combination of data-driven techniques and simulation studies is described in Chapter 5, the challenges regarding neutrino flux systematics. The methodology and results for Constraining $K^{+}$Meson and Contribution to $\nu_{e}$ are described in Chapter 6. Absolute $\nu$ Flux: $\nu$-Electron Neutral Current Scattering methodology and results in Chapter 7 . The Chapter 8 is Summary and Results for this thesis. 



\section{Chapter 2}

\section{NuMI Beam line}

\section{$2.1 \quad$ Introduction}

This chapter introduces the Neutrinos at the Main Injector (NuMI) beamline. The Fermilab accelerator complex as shown in Figure 2.1 is composed of four accelerators that work in tandem [29]: the linear accelerator (linac), booster, recycler, and main injector. These accelerators produce two primary proton beams, a low energy $(8 \mathrm{GeV})$ proton beam from the Booster and high energy $(120 \mathrm{GeV})$ beam from the Main Injector (MI). These protons hit the target, and produce the secondary beam of pions and kaons which freely decay in decay pipe into muons, and neutrinos that serve a variety of experiments. The $8 \mathrm{GeV}$ beam of protons from the Booster serves as an input to the Main Injector which accelerates this beam to $120 \mathrm{GeV}$. These highly energetic protons are made to strike on a graphite target to generate mesons that subsequently decay to muon neutrinos, resulting in the most intense neutrino beam in the world. Three experiments currently gather data from the NuMI beam line.

The MINOS experiment [30] is a first generation long-baseline neutrino experiment designed to observe the phenomena of neutrino oscillations. MINOS uses two detectors, one located at Fermilab, near to the source of the neutrinos, and the other located $724 \mathrm{~km}$ away, in northern Minnesota, at the Soudan Underground Mine. 


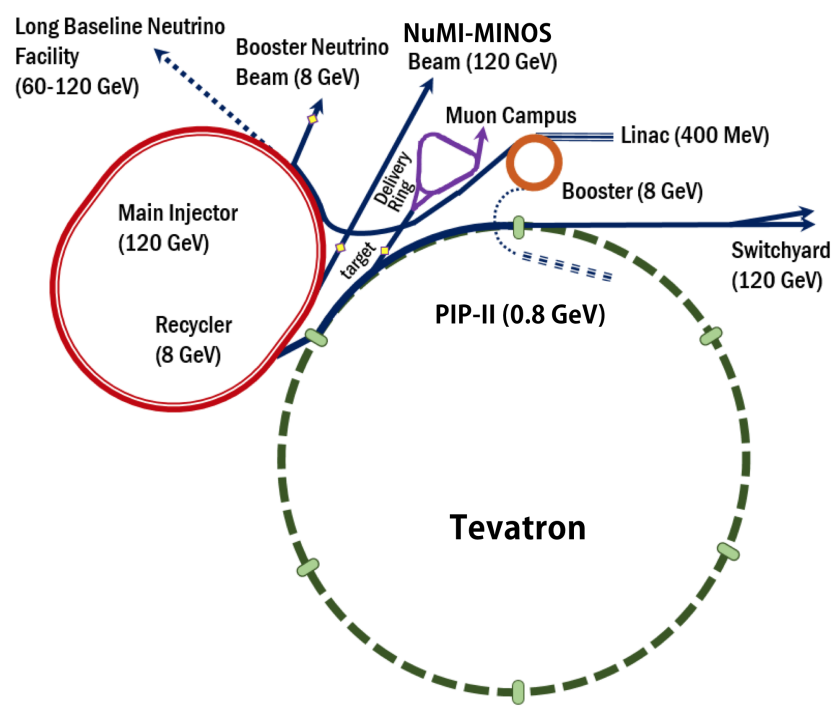

Figure 2.1: Fermilab's accelerator complex accelerates protons to high energies before sending them out to various experiments.

The MINER $\nu \mathbf{A}$ [31] experiment is a neutrino scattering experiment that seeks to measure low energy neutrino interactions both in support of neutrino oscillation experiments and also to study the strong dynamics of the nucleons and nucleus that affect these interactions.

The NOvA experiment, the subject of this thesis, and detailed in Chapter 3, uses the NuMI beam to directly observe and measure the transformation of muon neutrinos into electron neutrinos by measuring the $\nu_{e}$.

\subsection{NuMI Beam}

NuMI (Neutrinos at the Main Injector) as shown in Figure 2.2 has a nominal operating power of $700 \mathrm{~kW}$. In this Figure, daily protons (POT) delivered/recorded all-time by NuMI to NOvA are shown. The original design intensity of NuMI is $700 \mathrm{~kW}$, which was reached in 2017 (See Figure 2.3). NuMI is currently the most intense neutrino source ever built. The Figure 2.3 also highlights the 2016 and 2017 datasets for NOvA experiment, which is used in this thesis only for Forward Horn Current configuration i.e. only the 
neutrino mode data.

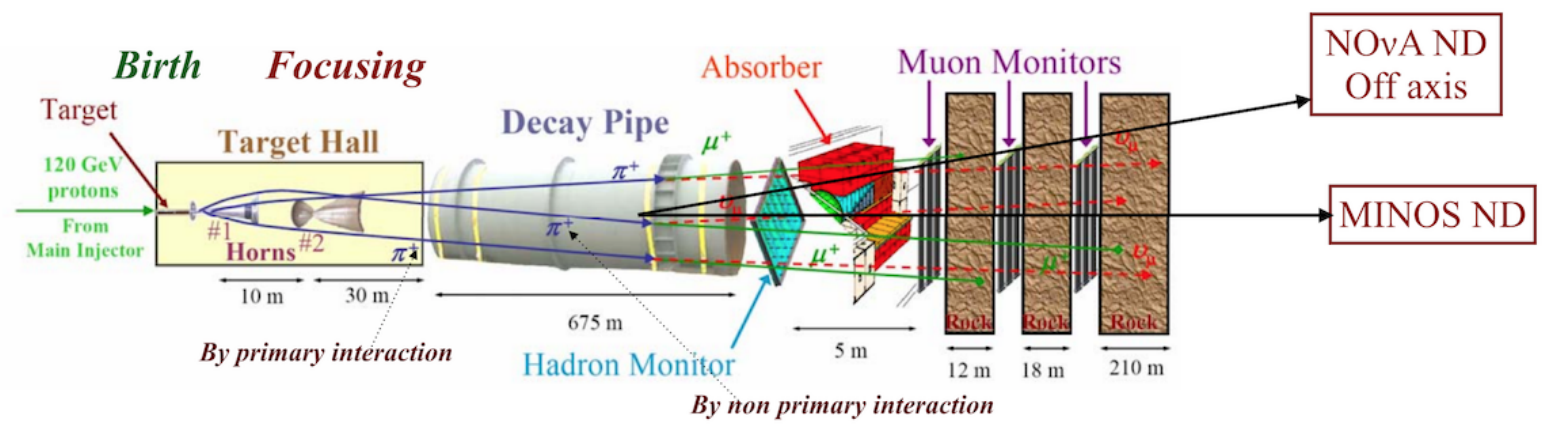

Figure 2.2: Schematic of the NuMI Beam: Shown are the primary proton-C collision, the $\pi^{+}, \pi^{-}, K^{+}, K^{-}$, and $K_{L}^{0}$ mesons that are the primary progenitor of neutrinos, the focusing beam elements, and secondary/tertiary sources of neutrinos.

The NuMI beam itself is structured into packets or clusters of $4.5 \times 10^{12}$ POT called bunches. By limiting the length and frequency of the proton bunches, the NuMI beam can produce a pulsed stream of neutrinos also called spills, which are $10 \mu$ s pulses separated by $1.3 \mathrm{sec}$ intervals and containing 12 bunches each. The purpose of this segmented beam is to localize the neutrino signal in time, which is used to minimize cosmogenic backgrounds. The basic layout of the beamline starts with $120 \mathrm{GeV}$ protons produced by the Main Injector accelerator, which is directed downward at a $158 \mathrm{mrad}$ angle to the NuMI Target Hall. After striking the target, hadron production occurs (pions and kaons). Two parabolic $3 \mathrm{~m}$ long magnetic horns focus these hadrons, pulsed at 200 $\mathrm{kA}$ toward the decay pipe which is $2 \mathrm{~m}$ in diameter, $675 \mathrm{~m}$ in length and inside the decay, pipe mesons are freely allowed to decay into neutrinos and muons. At the end of decay pipe, if there is any left over proton and non-decayed secondary mesons after the decay pipe, there is a Hadron Absorber Hall. After the Hadron Absorber Hall, there is 240 m of Earth shield, to filter the muons resulting from pion and kaon decays, which also separates the Absorber Hall from the Near Detector Hall. Each component of beamline is described in the following subsections, and each of the component has a systematic effect on final neutrino flux, which is the central part of Chapter 5 . 


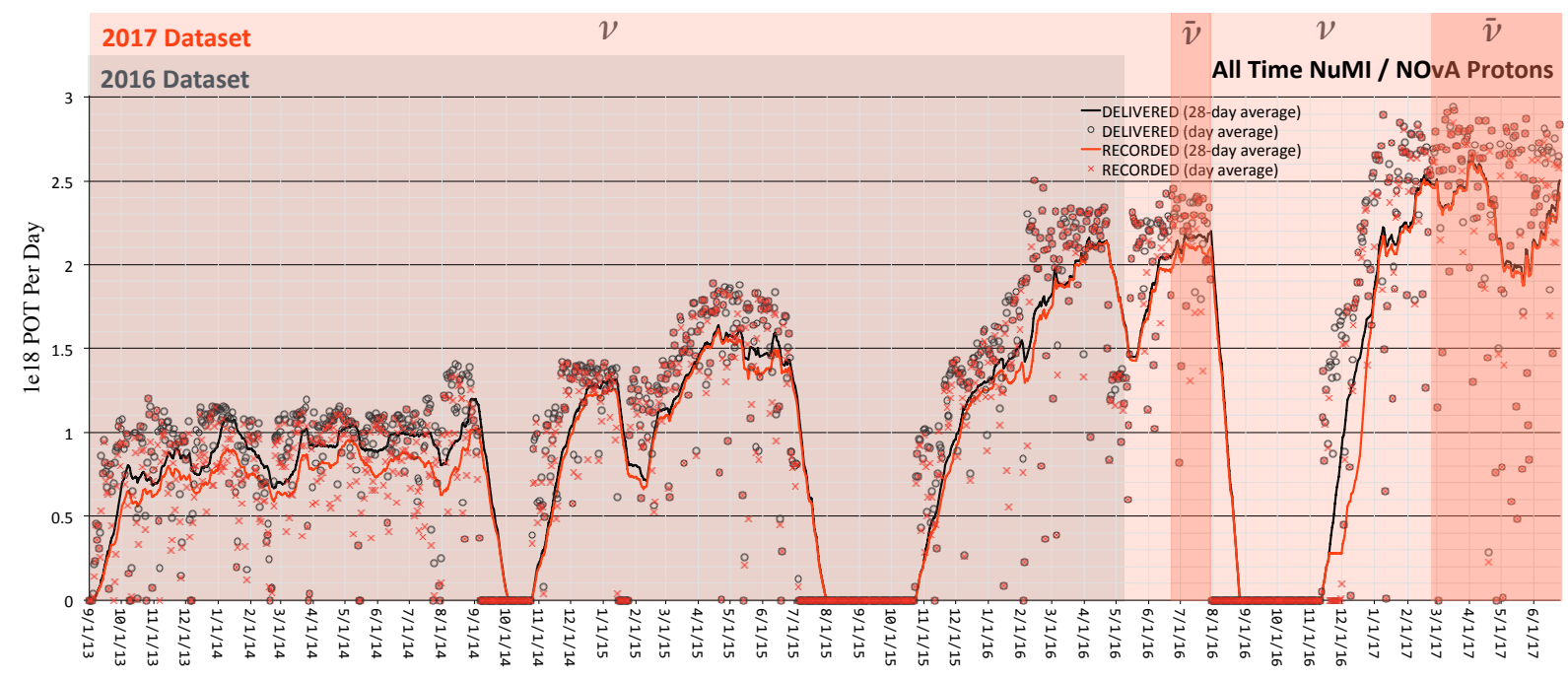

Figure 2.3: Daily protons (POT) delivered/recorded all-time by NuMI to NOvA. Highlights the 2016 and 2017 NOvA datasets as well as neutrino/anti-neutrino run periods. 2016 Analysis: 6 Feb 2014 - 1 May 2016: $6.05 \times 10^{20}$ POT-equiv. 2017 Analysis: 6 Feb 2014 - 20 Feb 2017: $8.85 \times 10^{20}$ POT-equiv.

\subsubsection{Primary Proton Beam}

To select proton energy and intensity one must consider two points according to the basic physics. First is higher energy protons beam will give high energy mesons, which will decay into more energetic neutrinos. Second, the multiplicities of pions and kaons are approximately proportional to the number of protons striking the target (see subsection 2.2.2) multiplied by the proton energy.

\subsubsection{Target}

For the protons beam from MI, the target is the next crucial part in the beamline for the final neutrino beam. NuMI beam uses a graphite target. The target position is shown in the Figure 2.4. The protons interact with nucleons in the graphite and produce mesons, i.e., pions and kaons. The target material is made of the ZXF-5Q graphite grade of Poco Graphite, Inc. which has a density of $1.78 \mathrm{~g} / \mathrm{cm}^{3}$ [32]. The NOvA target consists of a total of 50 graphite segments as shown in Figure 2.5. There are 48 graphite segments (fins) 24 
$\mathrm{mm}$ long in the beam direction equally spaced with gaps of $0.5 \mathrm{~mm}$ between fins. The fins are $7.4 \mathrm{~mm}$ wide by $63 \mathrm{~mm}$ high and are cooled by water circulating in the baseplate at the bottom of the fins. The beam is centered $3.7 \mathrm{~mm}$ from each horizontal edge of the 7.4 $\mathrm{mm}$ wide fins and $3.7 \mathrm{~mm}$ below the top of the $63 \mathrm{~mm}$ high fins. There are two additional Budal Monitors which are identical graphite fins electrically isolated from the rest of the target. One is vertical like the other fins to measure the horizontal position of the beam, and one is mounted horizontally to measure the vertical position of the beam. Each of these fins are electrically isolated from the rest of the target. The total target seen by the beam is 50 fins or $120 \mathrm{~cm}$ of graphite, that corresponds to 2 interaction lengths. There are two uncoupled water cooling systems: the cooling system of graphite target segments and a second system to remove the energy deposited in the target casing. The latter will provide removal of the heat, deposited in the target casing by secondary particles, as well as the heat emitted from target segments tips. The cooling water runs along the target through a helical groove machined at the outer surface of the casing, which is made of heavy-wall pipe. The thin cylindrical jacket covers the target casing with inlet and outlet pipes. The jacket is welded to the target casing at both ends. The inside of the target casing is anodized to obtain the coefficient of emissivity. When the protons hit the target, secondary particles are produced. To enhance the neutrino beam, these secondaries are focused by the focusing system described in the next subsection 2.2 .3 .

\subsubsection{Focusing of Secondary $\pi$ 's and $K$ 's from the Target}

Secondary particles (hadrons) are produced in the graphite target due to proton-nucleus interactions, and need to focus towards the neutrino detectors. A perfect focusing system would collect all secondary particles of the required charge and focus them into a precisely parallel, microscopically thin pencil beam. This ideal gathering system is unfortunately not realistically attainable. The most efficient focusing system which matches NuMI requirements consists of a set of magnetic horns as shown in Figure 2.6. Each horn consists of two cylindrically symmetric current sheets; a thin-walled, cone-shaped, aluminum inner 


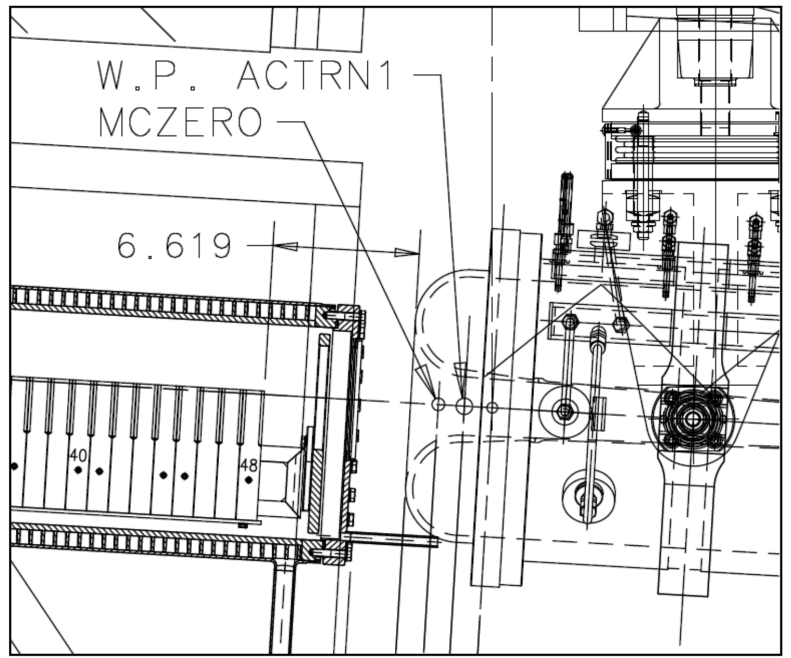

Figure 2.4: Relative location of the downstream end of the ME target material with respect to Horn 1. The target material ends $20 \mathrm{~cm}$ upstream of the start of the idealized Horn 1 which is marked as MCZERO.

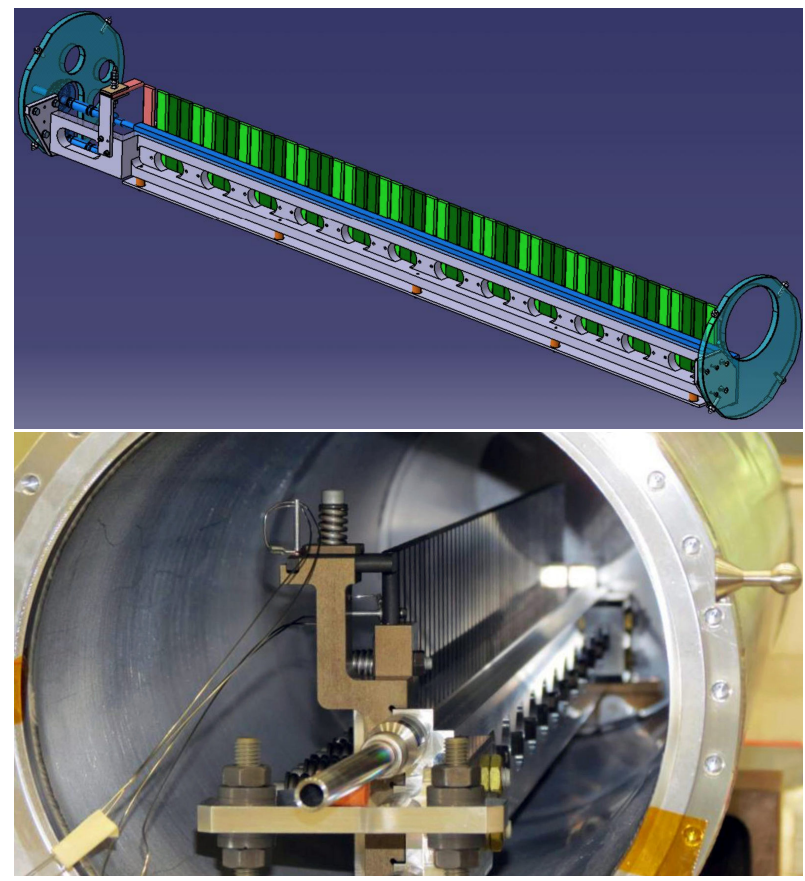

Figure 2.5: Perspective sketch of the ME target core. The primary proton beam runs through the target from left to right. There are 48 segments in the target core, plus two additional Budal monitors (colored red) at the upstream end of the target. 

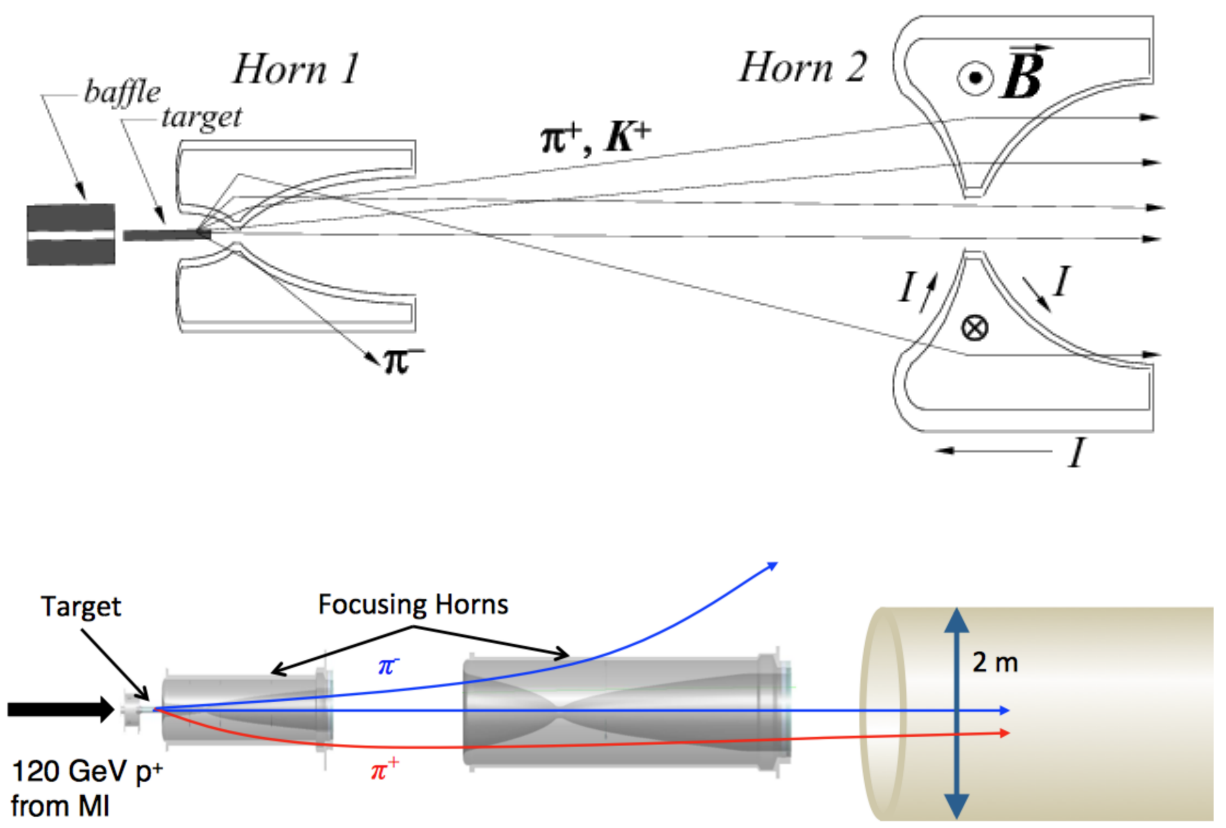

Figure 2.6: Detail of the magnetic horns system of the NuMI beam line. The NuMI Beam Line uses a two Horns for focusing.

conductor and a thin-walled cylindrical outer conductor with a strong current (roughly 200 $\mathrm{kA}$ ) moving out along the inner element and back along the outer wall. The magnetic field is contained between the inner and outer conductors. The shape of the inner conductor has been studied at various laboratories around the world, and usually, it is either a simple or modified parabola. These magnetic horns can focus efficiently even if the particles do not all start from the same point and have been efficient over a $1.5 \mathrm{~m}$ longitudinal distance upstream of the horn and allows a more extended production target (as in case of NuMI the target is $120 \mathrm{~cm}$ long) which increased percentage of interacting protons. A customized electrical system able to deliver $200 \mathrm{kA}$ over a $1 \mathrm{~ms}$ pulse has been designed to energize the horns. The different focusing modes and their respective flux at NOvA detectors are described in section 2.4. NuMI effective focusing system focus the secondary particles into decay pipe, more explanation see subsection 2.2.4. 


\subsubsection{Decay pipe, Hadron absorber and Muon shield}

The particles emanating from the target hall area enter the $675 \mathrm{~m}$ long steel pipe. The NuMI decay pipe is $2 \mathrm{~m}$ in diameter within a larger excavated tunnel. The decay pipe starts $46 \mathrm{~m}$ downstream of the NuMI target and is filled with He because creating a perfect vacuum in the decay pipe is more expensive. The pipe is embedded into concrete shielding, so the particles moving in the outward direction are stopped either in the walls of the pipe or the surrounding shielding. Since the energy deposition of off-angle particles heats the decay pipe and the surrounding concrete, the temperature of the shield is maintained by water cooling system. The decay length of a pion sets the length of the decay pipe. NOvA neutrinos come from pions that have energy $\approx 5 \mathrm{GeV}$, and the decay length of such pion is $336 \mathrm{~m}$. However, the same NuMI beamline is also used for other experiments, like MINOS and MINERvA. Their neutrinos come from pions having energy $\approx 5$ to $10 \mathrm{GeV}$, and for a $10 \mathrm{GeV}$ pion, the decay length is about $560 \mathrm{~m}$. The hadron absorber hall stops the residual protons and leftover mesons, but the neutrinos and some of the muons pass through it. The absorber is a box $5.5 \mathrm{~m}$ wide $\times 5.6 \mathrm{~m}$ tall $\times 8.5 \mathrm{~m}$ long. It consists of 4.75 m long, water cooled, aluminum core. The core is surrounded by layers of steel blocks and a layer of concrete shielding. The threshold in momentum for muons is $3-4 \mathrm{GeV}$. Muons with momentum less than this are absorbed. The muons which are remaining in the NuMI beam after the absorber hall are ranged out in the so-called muon shield, which is $240 \mathrm{~m}$ of solid dolomite rock between the absorber and the MINOS ND and NOvA ND hall. Without a muon shield, the Near Detector electronics would be overloaded.

\subsection{On-Axis versus Off-Axis Beam}

Off-Axis Beam: Pions and kaons decay isotropically in their centers of mass resulting in a relatively broad neutrino beam energy spectrum. For small angles, the flux and energy of neutrinos produced from the pion decay in flight and intercepted by a detector 


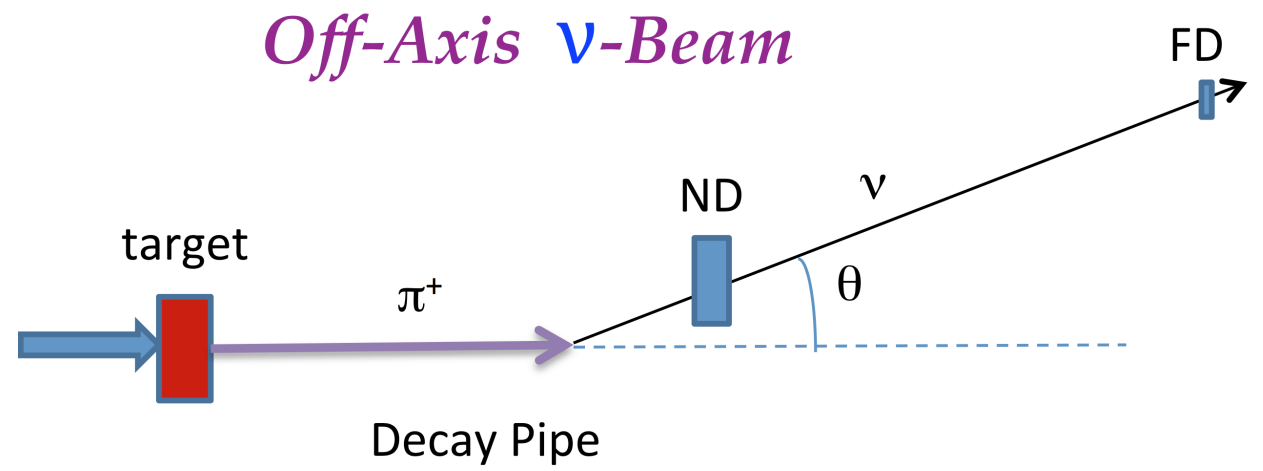

Figure 2.7: Representation of NOvA Near Detector (ND) and Far Detector FD at off-axis position.
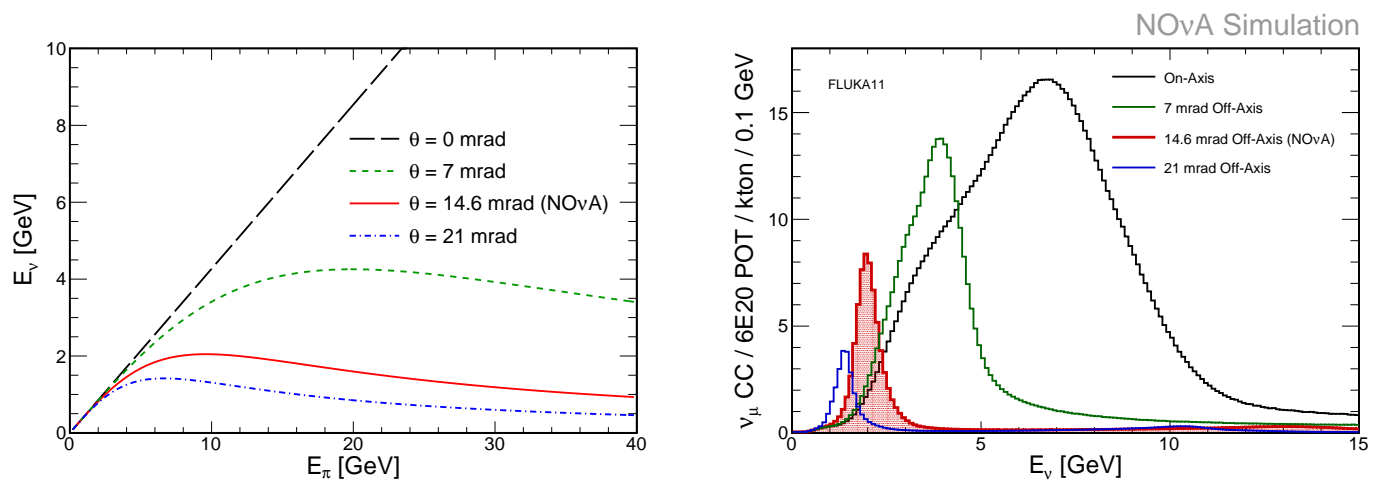

Figure 2.8: The kinematics and neutrino spectrum in the off-axis beam. The NOvA off-axis spectrum peak at $2 \mathrm{GeV}(14.6 \mathrm{mrad})$.

of area $\mathrm{A}$ and located at distance $\mathrm{z}$ are given in the lab frame by Equations 2.1 and 2.2. Figures 2.7 and 2.8 picture the conceptual idea and the spectrum in an off-axis beam.

$$
\begin{gathered}
F=\frac{2 \gamma}{1+\gamma^{2} \theta^{2}} \frac{A}{4 \pi z^{2}} \\
E_{\nu}=\left[\frac{0.43 E_{\pi}}{1+\gamma_{2} \theta_{2}}\right]
\end{gathered}
$$

Where $\theta$ is the angle between the pion direction and the neutrino direction, $E_{\pi}$ the energy of the parent pion, $m_{\pi}$ the mass of the pion and $\gamma=E_{\pi} / m_{\pi}$. The expressions for the neutrinos from the corresponding charged $K$ decays are identical except that 0.43 
is replaced by 0.96 resulting in a more energetic and broader distribution for identical meson energies (see example of plots in Chapter 6). The neutrino flux peaks in the forward direction for all meson energies, which is the reason that, in general, neutrino detectors are placed on axis. As the neutrino direction deviates from the meson direction, however, the relationship between the pion energy and neutrino energy flattens as shown in Figure 2.8 for $\theta=14$ mrad most pion decays result in neutrino with $\mathrm{E}=2 \mathrm{GeV}$, with some smearing around that value.

\subsection{Neutrino Spectra at the NOvA Detectors}

Since the principal goal of NOvA is the measurement of the difference in the probability of $\nu_{\mu} \rightarrow \nu_{e}$ and $\bar{\nu}_{\mu} \rightarrow \bar{\nu}_{e}$ oscillations, it is essential to separately create neutrino and antineutrino beams. The NOvA experiment runs in two modes:

\subsubsection{Forward Horn Current Mode:}

Focus-positive or Forward-Horn-Current (FHC) mode is the mode in which positive particles are focused and negative particles are defocused. As a result, most of the neutrinos are $\nu_{\mu}$ whereas the $\bar{\nu}_{\mu}$ are at a few percent level. Figure 2.9 shows the expected $\nu_{\mu}, \bar{\nu}_{\mu}$, $\nu_{e}$, and $\bar{\nu}_{e}$ charge current event spectra at the Far and Near Detectors in the FHC mode.

\subsubsection{Reverse Horn Current Mode:}

The Focus-Negative, or Reverse Horn Current (RHC) mode does the opposite, however, the contamination $\left(\nu_{\mu}\right)$ is larger than that in the FHC mode. Figure 2.10 shows the expected $\nu_{\mu}, \bar{\nu}_{\mu}, \nu_{e}$, and $\bar{\nu}_{e}$ charge current event spectra at the Far and Near Detectors in the RHC mode. 

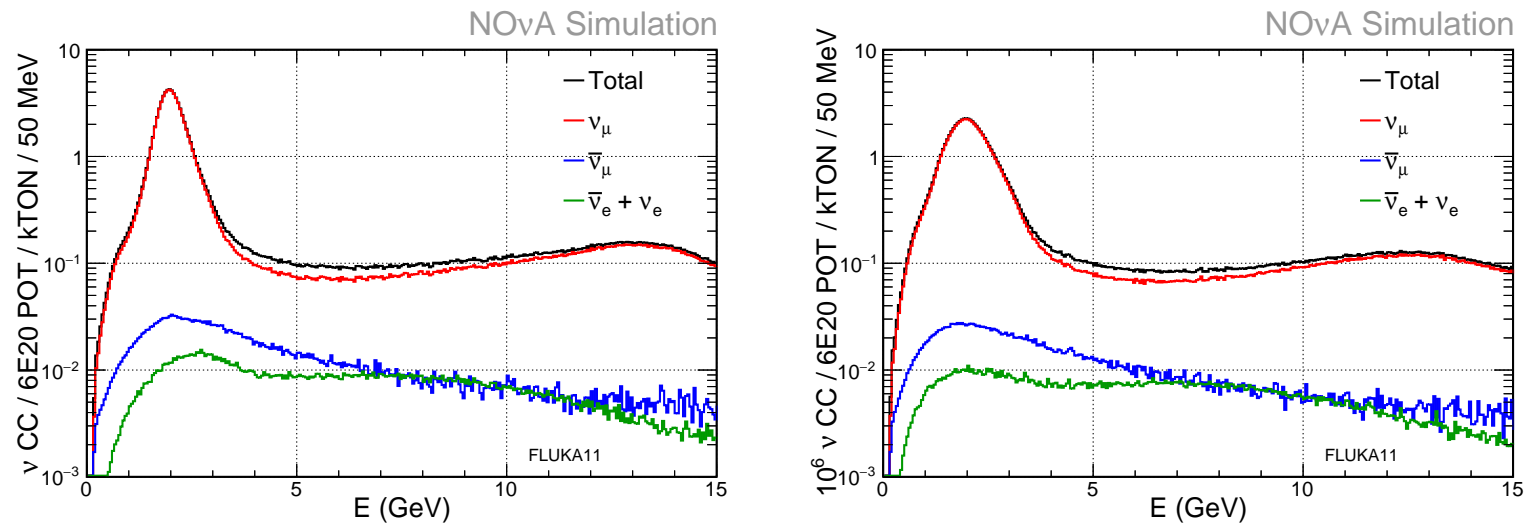

\begin{tabular}{|c|c|c|}
\hline Event Rates & / 6e20 POT & / kton \\
\hline Far Detector & $(1-3) \mathrm{GeV}$ & $(0-120) \mathrm{GeV}$ \\
\hline Total & 68.0 & 109.1 \\
$\nu_{\mu}$ & 66.5 & 102.6 \\
$\overline{\nu_{\mu}}$ & 1.1 & 4.1 \\
$\nu_{e}+\overline{\nu_{e}}$ & 0.4 & 2.4 \\
\hline$(1,3) \mathrm{GeV}:\left(\nu_{e}+\overline{\nu_{e}}\right) / \nu_{\mu}$ & $=$ & $0.6 \%$ \\
\hline$(0,120) \mathrm{GeV}:\left(\nu_{e}+\overline{\nu_{e}}\right) / \nu_{\mu}$ & $=$ & $2.3 \%$ \\
\hline
\end{tabular}

\begin{tabular}{|c|c|c|}
\hline Event Rates & / 6e20 POT & / kton \\
\hline Near Detector & $(1-3) \mathrm{GeV}$ & $(0-120) \mathrm{GeV}$ \\
\hline Total & 55.1 & 95.6 \\
$\nu_{\mu}$ & 53.7 & 89.9 \\
$\overline{\nu_{\mu}}$ & 1 & 3.7 \\
$\nu_{e}+\overline{\nu_{e}}$ & 0.4 & 2.1 \\
\hline$(1-3) \mathrm{GeV}:\left(\nu_{e}+\overline{\nu_{e}}\right) / \nu_{\mu}$ & $=$ & $0.7 \%$ \\
\hline$(0-120) \mathrm{GeV}:\left(\nu_{e}+\overline{\nu_{e}}\right) / \nu_{\mu}$ & $=$ & $2.3 \%$ \\
\hline
\end{tabular}

Figure 2.9: Neutrino CC Spectra in the Forward Horn Current configuration at the NOvA FD and ND.
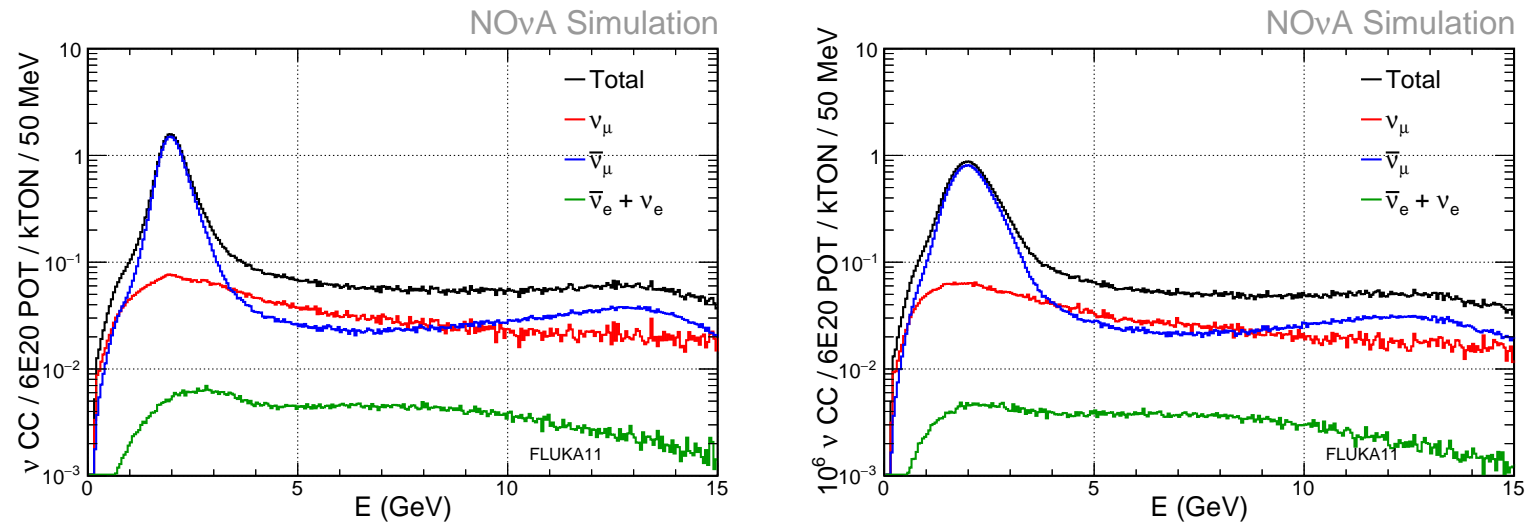

\begin{tabular}{|c|c|c|}
\hline Event Rates & / 6e20 POT & / kton \\
\hline Far Detector & $(1-3) \mathrm{GeV}$ & $(0-120) \mathrm{GeV}$ \\
\hline Total & 26.7 & 48.8 \\
$\nu_{\mu}$ & 2.7 & 14.1 \\
$\overline{\nu_{\mu}}$ & 23.8 & 33.4 \\
$\nu_{e}+\overline{\nu_{e}}$ & 0.2 & 1.3 \\
\hline$[1,3] \mathrm{GeV}:\left(\nu_{e}+\bar{\nu}_{e}\right) / \nu_{\mu}$ & $=$ & $0.8 \%$ \\
\hline$[0,120] \mathrm{GeV}:\left(\nu_{e}+\overline{\nu_{e}}\right) / \nu_{\mu}$ & $=$ & $3.9 \%$ \\
\hline
\end{tabular}

\begin{tabular}{|c|c|c|}
\hline Event Rates & / 6e20 POT & / kton \\
\hline Near Detector & $(1-3) \mathrm{GeV}$ & $(0-120) \mathrm{GeV}$ \\
\hline Total & 21.7 & 42.5 \\
$\nu_{\mu}$ & 2.4 & 12.5 \\
$\overline{\nu_{\mu}}$ & 19.2 & 28.9 \\
$\nu_{e}+\overline{\nu_{e}}$ & 0.2 & 1.1 \\
\hline$(1-3) \mathrm{GeV}:\left(\nu_{e}+\overline{\nu_{e}}\right) / \nu_{\mu}$ & $=$ & $1 \%$ \\
\hline$(0-120) \mathrm{GeV}:\left(\nu_{e}+\overline{\nu_{e}}\right) / \nu_{\mu}$ & $=$ & $3.8 \%$ \\
\hline
\end{tabular}

Figure 2.10: Neutrino CC Spectra in the Reverse Horn Current configuration at the NOvA FD and ND. 


\subsection{Simulation of NuMI Beam}

The official simulation software used by NOvA to simulate the entire beamline is a combination of Fluka [33, 34] and Geant4 [35]. This combination is called Flugg [36], a tool that adds on to Fluka an interface to Geant4 geometry. A simple model of the incoming proton beam is used as a source of primary particles. The primary output of this code is an n-tuple representing the decays of secondaries that give rise to neutrinos. The Flugg simulation code falls into two broad categories: the simulation of the physical processes, made by Fluka (Fortran) and the geometry description, written using Geant4 $(\mathrm{C}++)$. As an interplay of these two parts, Fluka performs particle interactions track particle properties and write output files, and whenever Fluka goes to make a query about the geometry, the Flugg code passes this query to Geant4, 337. FLUKA [38] is a general purpose tool for calculations of particle transport and interactions with matter, covering an extended range of applications spanning from proton and electron accelerator shielding to target design, calorimetry, activation, dosimetry, detector design, Accelerator Driven Systems, cosmic rays, neutrino physics, radiotherapy etc.

- For momentum from threshold to $5 \mathrm{GeV} / \mathrm{c}$ : The FLUKA hadron nucleus inelastic interactions are described in terms of resonance production and decay up to a few $\mathrm{GeV}$.

- The PEANUT model is used (Glauber-Gribov cascade and high energy collisions, Generalized intra-nuclear cascade, Pre-equilibrium emission and Evaporation/fragmentation/Fission and Final de-excitation).

- At higher energies, a model based on Dual Parton Model takes over. DPM is a particular quark/parton string model and provides reliable results up to $\sim \mathrm{TeV}$.

Besides Flugg, a second simulator is used by the NOvA experiment, based on Geant4 only is G4NuMI. The G4NuMI code builds up a representation of the NuMI 
beamline geometry and interfaces it with the Geant4 physics tools. The Physics lists used for this thesis study are FTFP_BERT and QGSP_BERT (FTF: FriGof string model, QGS: Quark Gluon String model, P: G4Precompound model used for de-excitation and BERT: Bertini-style cascade $(<\sim 10 \mathrm{GeV}))$.

- FTFP_BERT: contains all standard EM processes, uses BerGni-style cascade for hadrons $<5 \mathrm{GeV}$ and uses FTF (FriGof) model for high energies $(>4 \mathrm{GeV}$ ).

- QGSP_BERt: contains all standard EM processes, uses BerGni-style cascade up to 9.5 GeV, QGS model for high energies (> $20 \mathrm{GeV}$ ) and LEP (Low Energy Parameterized models) in between.

G4NuMI and Flugg make use of the same geometry. This software is often used to make cross-checks with the Flugg results. Although the studies shown in this thesis come from the Flugg simulator, G4NuMI is used to make comparisons with Flugg.

The NOvA experiment for which NuMI beam configuration is shifted from LE (Low energy mode) to ME (Medium energy mode) is described in Chapter 3, NOvA is designed as a finely segmented twin (near and far) detector experiment offering superb event identification capability. 



\section{Chapter 3}

\section{NOvA Detectors}

\subsection{The NOvA Detector Design}

As Fermilab's flagship experiment, NOvA studies neutrinos produced by the NuMI (described in Chapter 2) neutrino beam. The experiment measures oscillations in the electron (anti)neutrino appearance and the muon (anti)neutrino disappearance channels. It consists of two functionally identical detectors placed $14 \mathrm{mrad}$ off the NuMI beam axis: the $0.3 \mathrm{kt}$ Near Detector $(\mathrm{ND})$, close to the neutrino source underground at Fermilab $(\sim 0.8$ $\mathrm{km})$, and the 14kt Far Detector (FD) in Ash River, Minnesota $(\sim 810 \mathrm{~km})$ as shown in left Figure 3.1. The NOvA detectors are functionally identical in order to cancel systematic uncertainties in the analysis. It schematic is pictured in right Figure 3.1. The NOvA detectors are $65 \%$ active low-Z tracking calorimeters [39] designed to detect electrons, as from $\nu_{e}$-induced CC interactions. Both detectors consist of extruded cells as shown in Figure 3.2 of PVC plastic filled with liquid scintillator to form a three dimensional tracking calorimeter. The general detector design will be described first in the following subsections and then sections will explain the specifics for the Near and Far Detectors. 

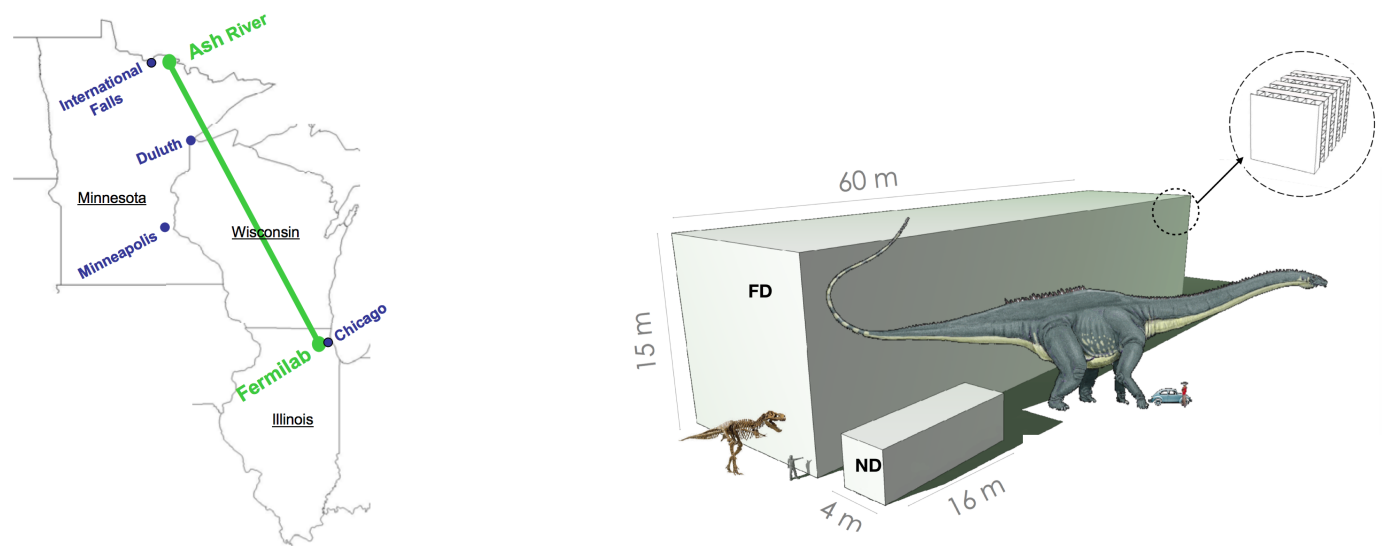

Figure 3.1: Left: Map of the central United States showing Fermilab, the NuMI beam-line, and the NOvA Far Detector site at Ash River, Minnesota. The total distance spanned by the green line is $810 \mathrm{~km}$. Right: Schematic of the NOvA Detectors: The FD and ND are functionally identical.

\subsubsection{PVC Modules}

The fundamental building block for the NOvA Far and Near Detectors is the PVC cell shown in the left Figure 3.2. A PVC module consists of a 32-cell PVC extrusion assembly; pairs of 16-cell PVC extrusions are glued together side-by-side to form a 32-cell module as shown in the right Figure 3.2. One end of the module is capped with a reflective plastic seal. The 32-cells each contain a looped wavelength-shifting fiber, described in section 3.1 .2 , that is routed through the fiber manifold and terminates in an optical connector mounted on the fiber manifold. The cells are coated with titanium dioxide $\left(\mathrm{TiO}_{2}\right)$ which is $90 \%$ reflective for 430 -nanometer wavelength light. The PVC cells provide the structural support for the detector. The 32-cell modules make the horizontal and vertical plane for the detectors as shown in the circle of the right Figure 3.1.

\subsubsection{Liquid Scintillator and Wavelength Shifting Fiber}

The cells are filled with a liquid scintillator that is by mass $94.63 \%$ mineral oil, $5.23 \%$ pseudocumene (scintillator), 0.14\% PPO (waveshifter), 0.0016\% bis-MSB (waveshifter), 0.001\% Stadis-425 (anti-static), and 0.001\% Vitamin E (anti-oxidant) [40]. This solution 


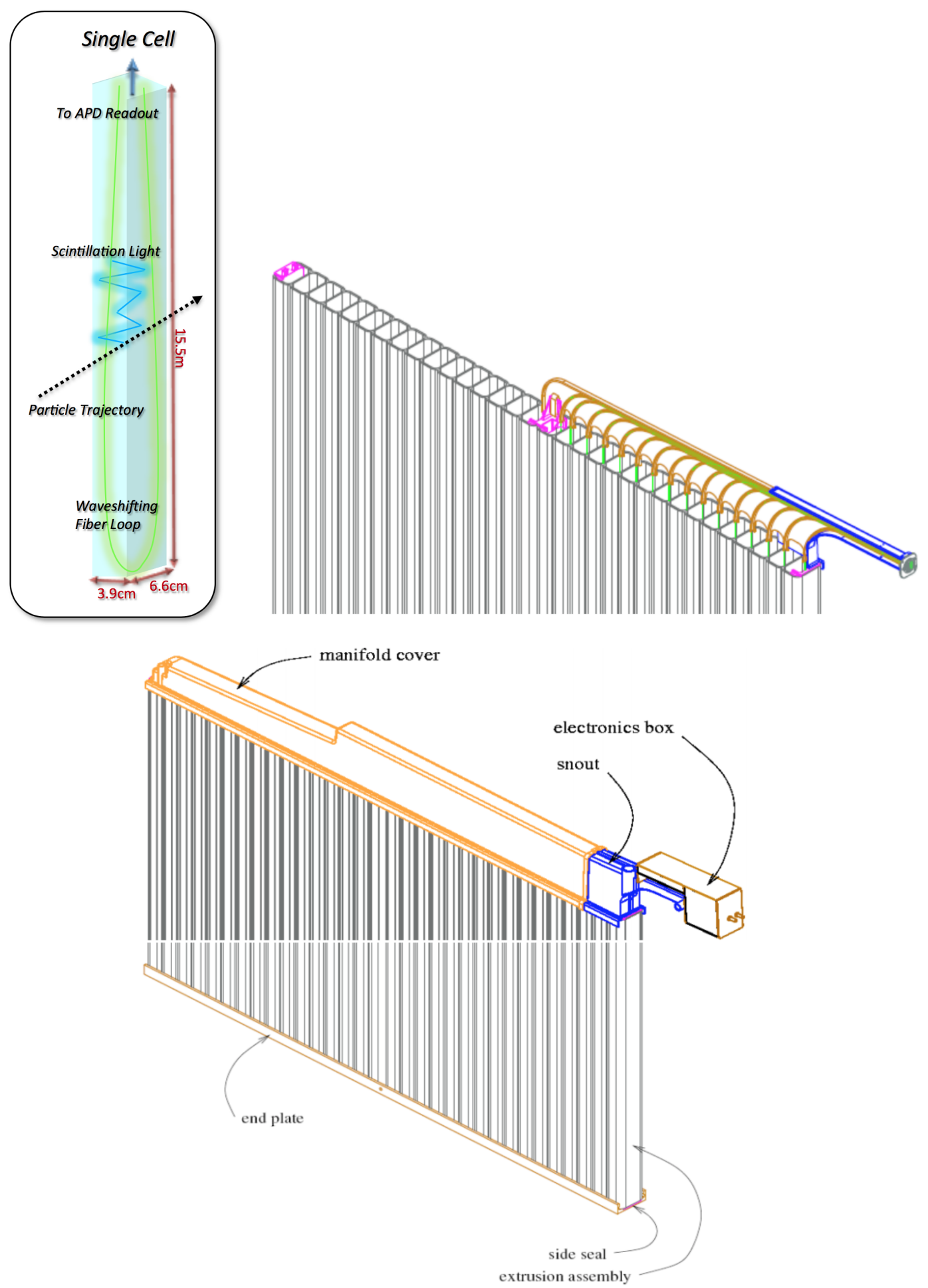

Figure 3.2: Left: Shown is a single-cell, the element of the detector. Right: Fiber routing to the optical connector for the first 16-cells. There are two fiber ends per cell routed to the optical connector. Bottom: Shows the layout of a plane. PVC module assembly showing the end-plate at the bottom and the fiber manifold at the top. Both vertical and horizontal modules have the same configuration. 
produces scintillation light in the near ultraviolet and shifts it to the visible region of 380-450 nm. Within each cell, a 0.7-millimeter double-clad Kuraray wavelength-shifting (WLS, did service work for ND modules fiber testing see section 3.4) fiber is looped down the entire cell length in a $U$ shape. Both ends of the fiber are read out by a single photodetector which improves collection efficiency. The fiber absorbs light in the violetblue range and emits in the blue-green (450-600 nm) range. NOvA is using a loop of a $0.7 \mathrm{~mm}$ diameter fiber inside of each PVC extrusion cell as shown in Figure 3.2. Plastic wavelength shifting (WLS) fibers provide an efficient method for collecting light generated in the long liquid scintillator filled cells of the detector. The violet light (425nm) emitted by the scintillator is absorbed by a fluorescent dye in the WLS fiber. The blue-green (450$650 \mathrm{~nm}$ ) light emitted by the dye is partially trapped within the fiber by total internal reflection. Once trapped much of the short wavelength light $(520 \mathrm{~nm})$ is attenuated while traveling through a full length of WLS fiber, however, the longer wavelengths, are only weakly attenuated. This light, coupled with the high quantum efficiency of avalanche photodiodes at long wavelengths yields a strong signal for minimum ionizing particles traversing anywhere along the length of a cell.

\subsubsection{Readout Electronics}

Let's start from a diagram in the Figure 3.3. Each end of the wavelength shifting fiber in each cell is connected to a pixel on a 32-pixel Hamamatsu avalanche photodiode (APD). The APD was chosen for its high quantum efficiency of 85\%, as shown in Figure 3.4 . High quantum efficiency is desired in order to make long cells, 15.6 meters in the far detector, and still see a minimum signal of 20 photoelectrons as specified in the technical design criteria. A comparison of APDs to photomultiplier tubes (PMTs) for the NOvA light spectrum is shown in Figure 3.4. The APDs are operated at $-15^{\circ} \mathrm{C}$ to reduce thermal noise. The voltage is determined individually for each channel for a gain of 150 for FD, 100 for ND, and is approximately 425 volts. The noise thresholds are set around 10 
photoelectrons. A thin and transparent paralene coating is put on the surface of each APD to isolate from humidity.

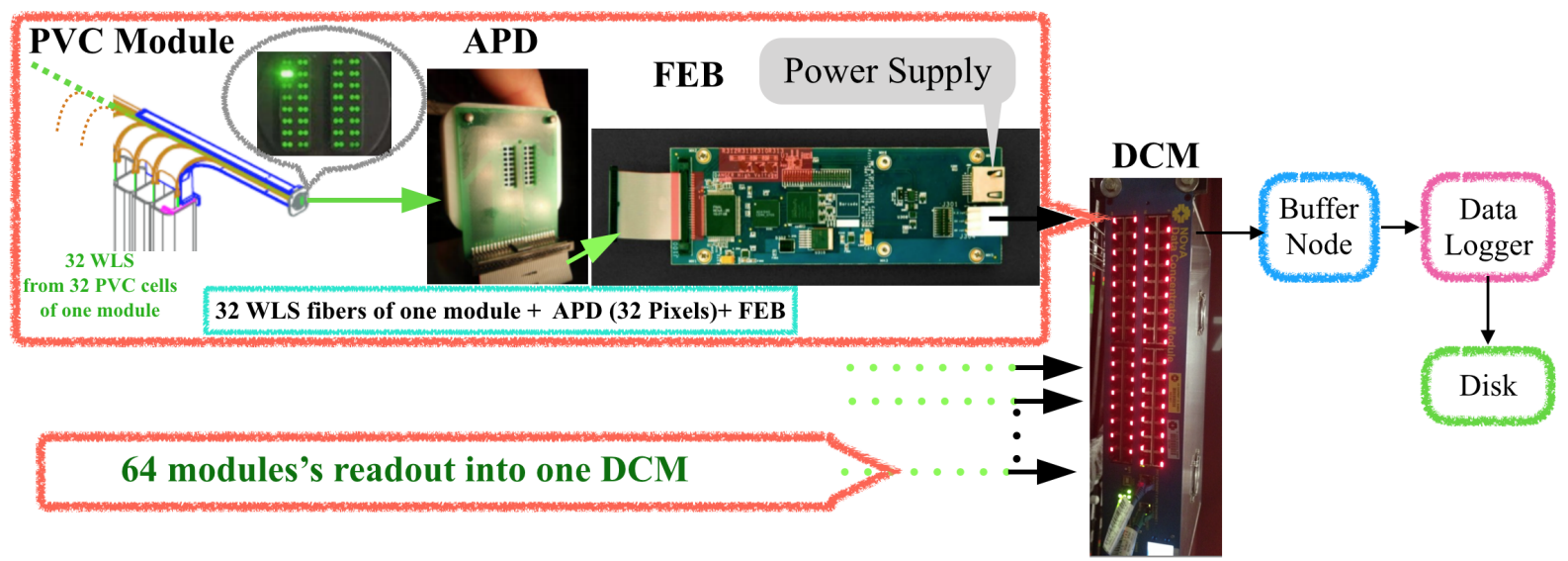

Figure 3.3: A PVC module consists of 32 cells. Each cell is read out by a pixel of an APD. Each APD has 32 pixels. Each APD has a corresponding FEB, which digitizes the signal from the APD. A DCM reads 64 FEBs and sends the information to the buffer node farm.

A system of tubes flow dry nitrogen gas through the APD housings to keep ice from forming on the surface. The APD reads these relatively small levels of photons and amplifies the signal by a factor of one hundred into a level capable of being read by sensitive, low-noise electronics. The amplified signal is read by a Front End Board (FEB). There is one FEB for each APD. The FEB reads the analog signal from the APD and converts it into digital hits above the threshold. The hit information from the FEB is collected by a Data Concentrator Module (DCM), one DCM reads 64 FEBs. The system encompassing the FEBs, DCMs, and buffer nodes is called the data acquisition (DAQ) system. There are many DCMs on each detector; they send all the collected hit information for a five millisecond time window to a single buffer node. The buffer node then executes triggering decision code that decides if any portion of the data should be written out to disk. NOvA employs a round-robin system with its buffer nodes. There are many buffer nodes, arranged in a ring. The first set of 5-millisecond data is sent to the first buffer node in the ring. The next set of 5-millisecond data is sent to the second buffer node and so forth until all the buffer nodes have received a set of 5-millisecond data. Then the first buffer node gets its second set of 5-millisecond data. This second set overwrites 


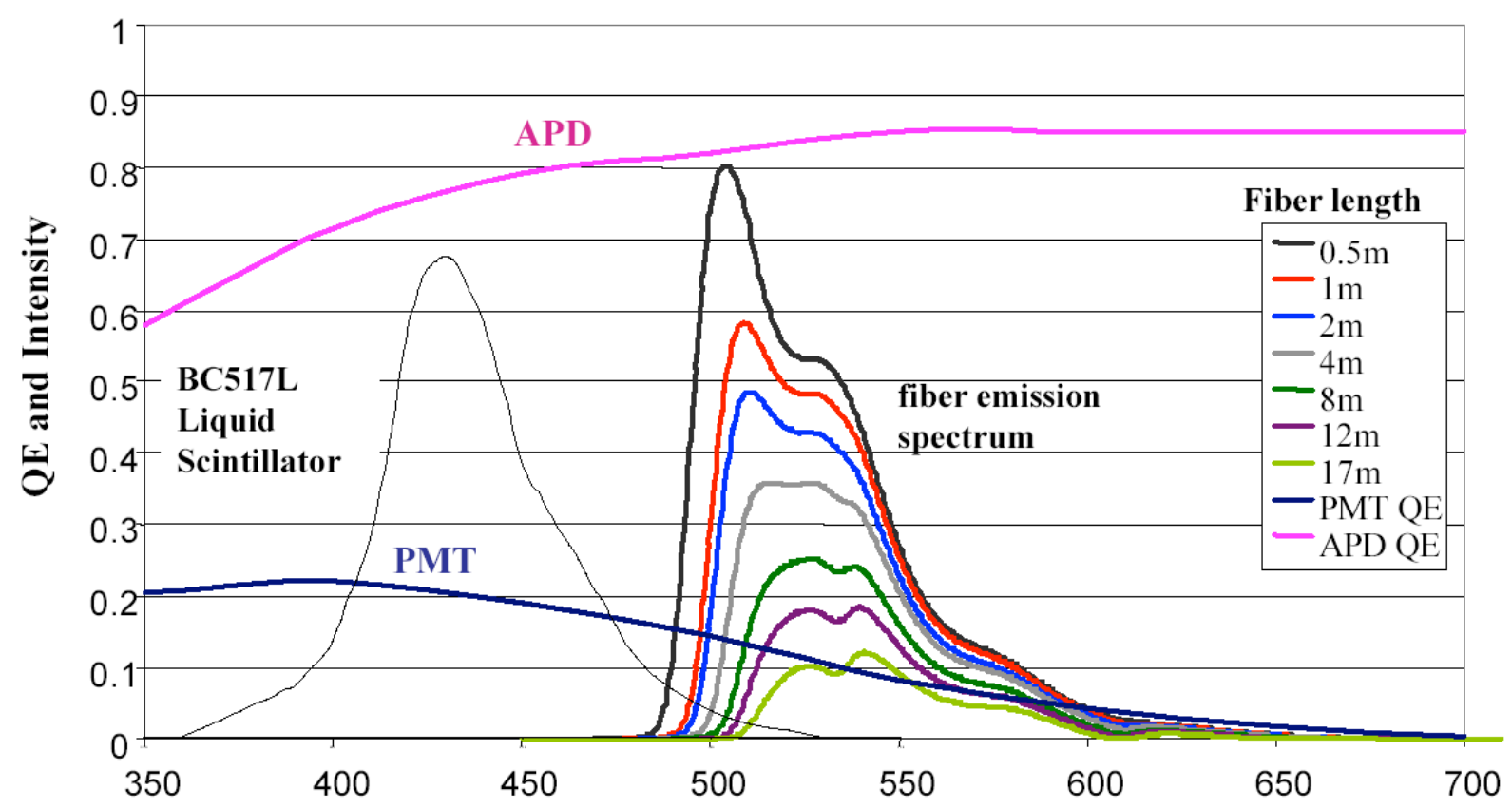

Figure 3.4: The quantum efficiency comparison between APD (Magenta) and PMT (bialkali photocathode) (Blue). Also shown WLS fiber emission spectra measured at lengths of $0.5,1,2,4,8,16 \mathrm{~m}$, respectively illustrating the shift of the average detected wavelength as fiber length increases.

the first; therefore, the triggering decision code has a limited amount of time to decide if hits should be saved to disk before the hits are lost. Currently, the software has about three seconds to decide, though this number will likely increase in the future. Without the round-robin system, this time would be much shorter. The three seconds is enough processing time for reasonably complex data-driven trigger algorithms to complete. For the oscillation data, a data-driven algorithm is not necessary, since an accelerator event drives trigger decisions. Runs and sub runs organize triggered readout windows. During a run, manually-set detector configurations do not change. A run ends when it has 64 sub runs, when the total run duration is 24 hours, or when the detector stops taking data. A sub run ends when it has a duration of 1 hour, the file size reaches 1 GB, or the detector stops taking data. For the near detector, sub run is 3 hours, and sub run 10-15 mins. 


\begin{tabular}{|l|l|l|}
\hline Components & Far Detector & Near Detector \\
\hline \hline Cells per Module & 32 & 32 \\
\hline Modules per Plane & 12 & $3(3,2)$ \\
\hline Cells per Plane & 384 & $96(96,64)$ \\
\hline Planes per Block & 32 & $32(\mathrm{n} / \mathrm{a})$ \\
\hline Number of Blocks & 28 & $6(2)$ \\
\hline Number of Cells & 344,064 & $18,432(1,760)$ \\
\hline Number of Planes & 896 & $192(22+10$ steel $)$ \\
\hline Cell Depth [cm] & 5.64 & 5.64 \\
\hline Cell Width [cm] & 3.6 & 3.6 \\
\hline Cell Length [cm] & 1550 & $399(399,274)$ \\
\hline Detector X Dimension Extents $[\mathrm{cm}]$ & -780 to 780 & -200 to 200 \\
\hline Detector Y Dimension Extents [cm] & -780 to 780 & -200 to $200(-200$ to 70$)$ \\
\hline Detector Z Dimension Extents $[\mathrm{cm}]$ & 0 to 5,962 & 0 to $1,280(1,280$ to 1,560$)$ \\
\hline Detector Mass [ton] & 14,363 & 293 \\
\hline Liquid Scintillator [gal] & $2,674,000$ & 41,140 \\
\hline Wavelength-shifting Fiber $[\mathrm{km}]$ & 11,116 & 188 \\
\hline
\end{tabular}

Table 3.1: Table for Far Detector and Near Detector components.

\subsection{Far Detector}

The NOvA far detector is located near Ash River in northern Minnesota, $810 \mathrm{~km}$ away from the near detector. The far detector is situated in a detector hall such that $1 / 6$ of the detector is above the surface of the Earth and the bottom 5/6 of the detector is below the surface of the Earth. The detector hall roof is constructed with concrete and a 12inch barite overburden layer. The total overburden protects against cosmic rays with the minimum shielding depth of $3 \mathrm{~m}$, nine radiation lengths. The layout of the detector hall 
and a picture of the detector are shown in Figure 3.5. The FD is measured the oscillated flux for neutrino oscillation study of NOvA Chapter 1 .

\subsection{Near Detector}

The NOvA near detector is located in a cavern at Fermilab $1 \mathrm{~km}$ downstream of the NuMI beam and $100 \mathrm{~m}$ underground. The layout of the cavern and a picture of the detector are shown in Figure 3.6. The ND is used to measure the composition of the un-oscillated beam Chapter 2. In parallel to help in oscillation measurements, the NOvA near detector provides an excellent opportunity for the measurement of various neutrino interactions. Due to the underground position of ND, it gets cosmic shielding from cosmic rays. However, there is a background contribution to the ND beam events coming from the rock muons. On the other hand, the data from the rock muons also help to optimize the PIDs for the ND see Chapter 7. The tabulated information for the FD and ND component is in Table 3.1 . 


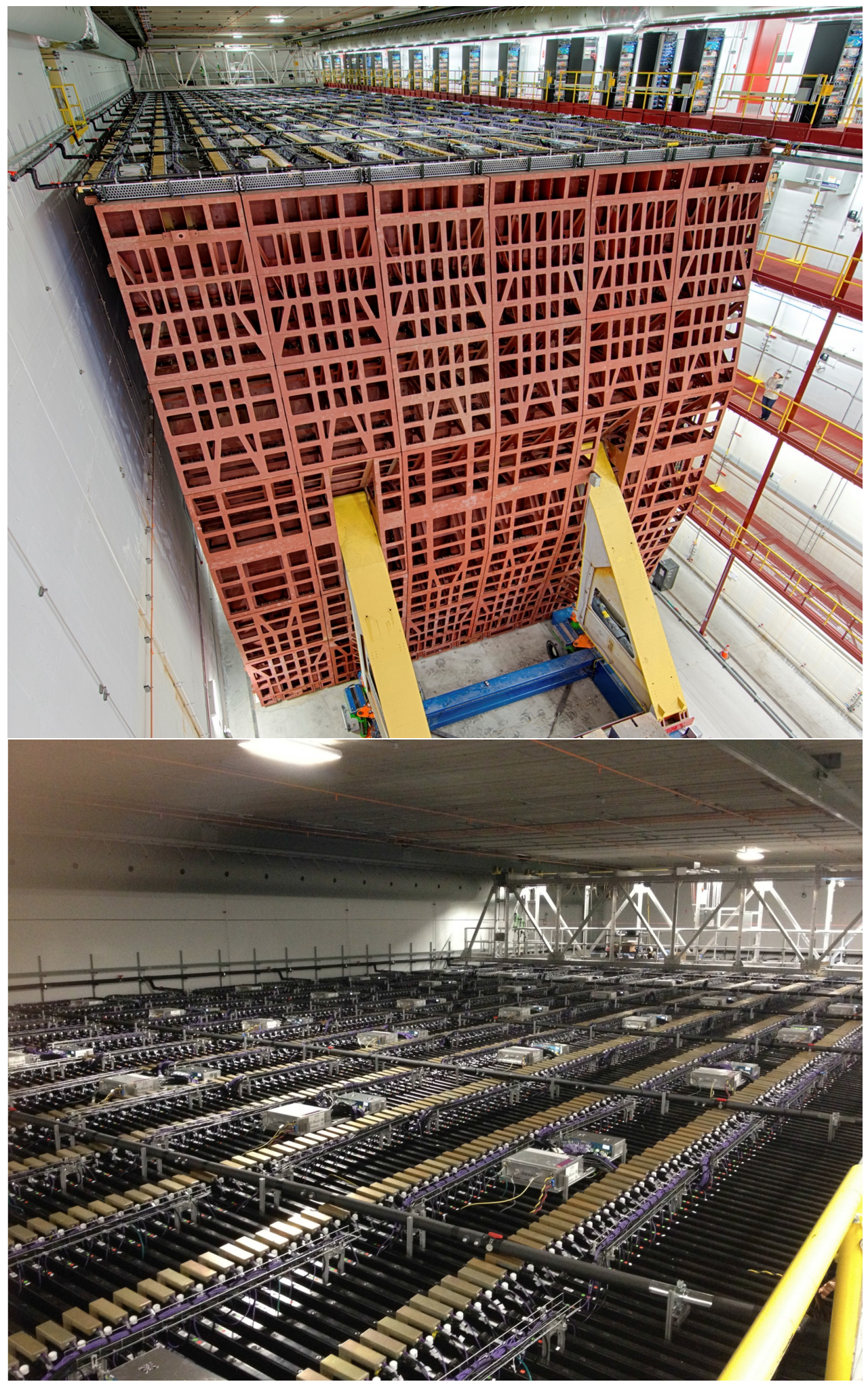

Figure 3.5: Upper: Partially completed NOvA far detector. Bottom: Top view of NOvA FD, fully instrumented FD Picture. 

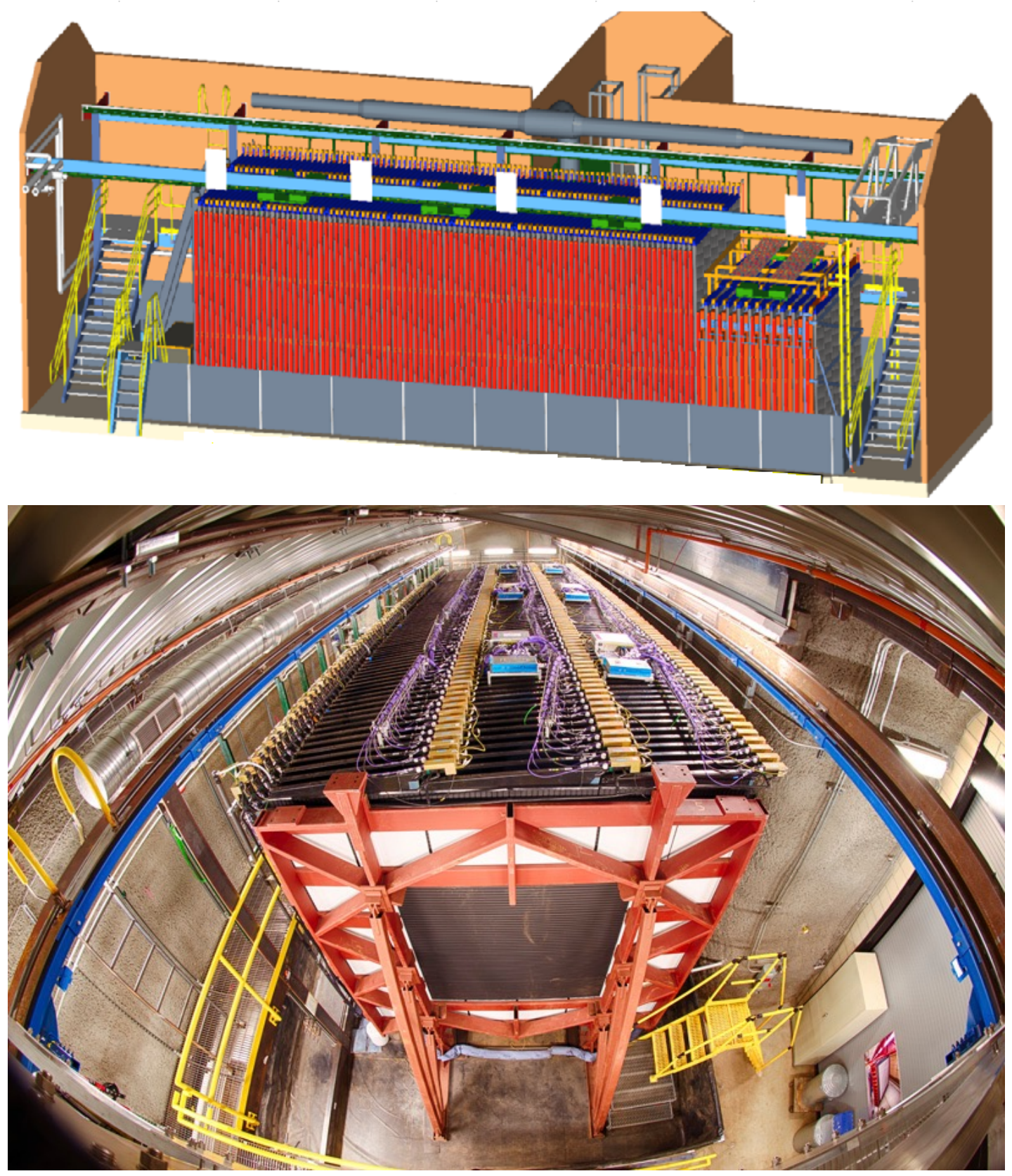

Figure 3.6: Upper: NOvA ND schematics picture. Botton: Fully instrumented ND Picture. 


\subsection{Contributions to the Installation of NOvA ND}

During this thesis work got the opportunity to work on the installation of the NOvA Near Detector (ND) as part of the installation team. The ND consists of PVC cells filled with liquid scintillator. Each cell contains a loop of bare Kuraray Y11 wavelength-shifting (WLS) fiber optic cable to collect the scintillation light, both ends of which lead to an avalanche photodiode for readout. Installation of the NOvA Near Detector involved many different tasks (both major and minor) which were accomplished as described below.

- All the fibers connecting the ND modules were tested to ensure the integrity of the WLS fibers meet the quality, no breakage, and design specifications.

- Optical connectors for all the NOvA ND modules were inspected for any malfunction.

- A significant work went into the module leak determination tests for ensuring that the detector modules were free of leaks before these were filled with the scintillating mineral oil.

- APDs were checked for the required noise sensitivity threshold levels for them to pass the quality assurance test.

\subsubsection{Fiber Testing of NOvA ND Modules}

The Fiber Loop Transmission (FLT) [41] QA device pumps red light into one end of each of 32 fiber loops in a NOvA module and measures the light received from the other end. The FLT is modular with a light router, an array of red light-emitting diodes (LED) and phototransistors (PT), and the control board is shown in the expanded assembly drawing demonstrated in Figure 3.7. The light sources are 32 red LEDs while the light receptors are 32 Phototransistors. A clear fiber light router, as shown during its construction in Figure 3.8, has 32 fiber pairs glued into a faceplate and then polished. The pattern of fiber pairs matches the end pattern of 32 WLS fiber loops in a NOvA module. The design 
of the fibers in the back plate of the router matches that of the LED and PT array. The severe bending of the light pipe fibers results in a wide range of intrinsic transmission.

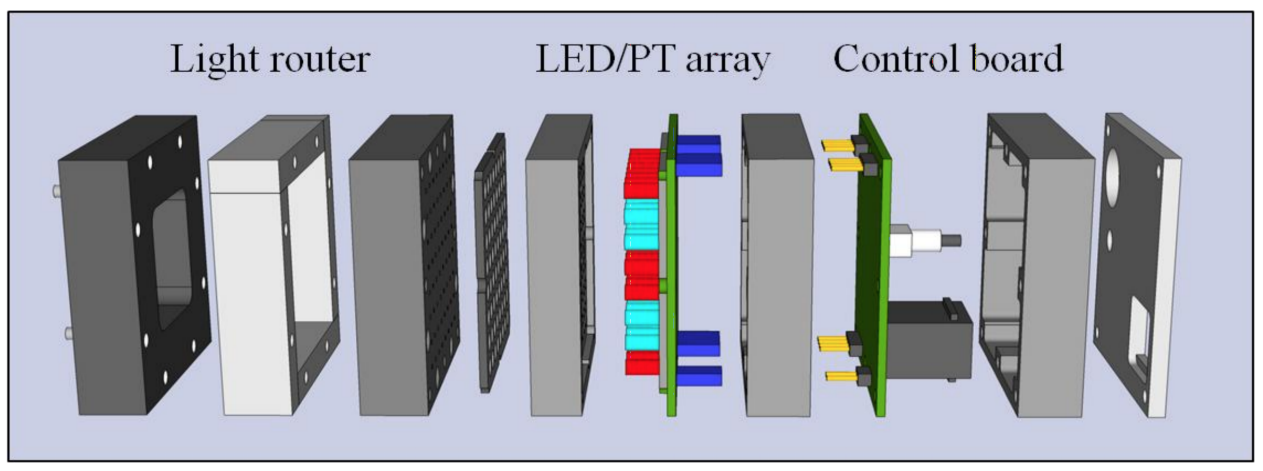

Figure 3.7: Expanded assembly of the Fiber Loop Transmission (FLT) QA device.

One member of each fiber pair is routed to its LED, and the other member is routed to its phototransistor. The transistor outputs are connected in parallel and feed the total current to a single ADC (analog-digital converter). Thus the current signal readout by the ADC is the sum of the currents produced by all 32 phototransistors. The phototransistors produce a dark current measurement of just a few counts on the 10bit ADC. A signal seen above the dark current is due to light from a single red LED piped through the corresponding WLS fiber loop of the NOvA module and received by the corresponding phototransistor in the FLT. The LED and phototransistor arrays are isolated from each other by a black foam gasket. We assumed a linear correlation between LED light and ADC value as shown in Figure 3.8. To check current noise value of the FLT device, did 100 pedestal data (means cover the FLT device with the black tape and do the experiment), e.g., as shown in Figure 3.9 pedestal data for all channels can be found in the appendix A. Total raw ADC comparison with Calibrated ADC is shown in Fugure 3.10. After subtracting the pedestal correction factor, we obtained raw ADC output, which we calibrated using mean value for that channel from all modules: $\frac{N_{A D C}-\text { PedestalConts }}{\text { Mean }} \times 100$ as shown in the Figure 3.11. If a fiber was not installed correctly in the cell of the module, the raw ADC out was approximately zero and module was rejected for installation. 

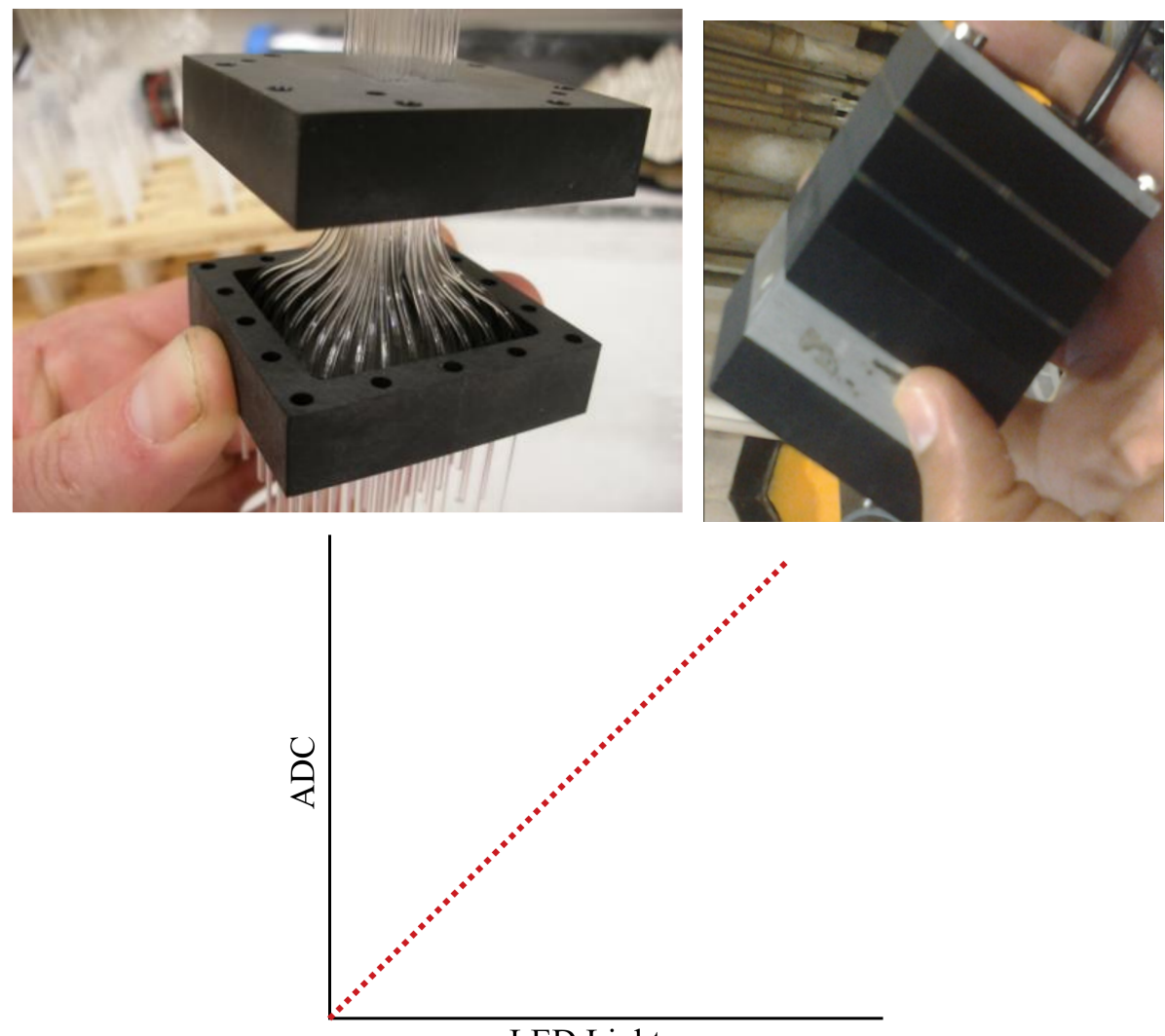

LED Light

Figure 3.8: Top: Light router of the Fiber Loop Transmission (FLT) QA device and picture of FTL device. Bottom: The assumed relationship between LED light and ADC output of FLT. 

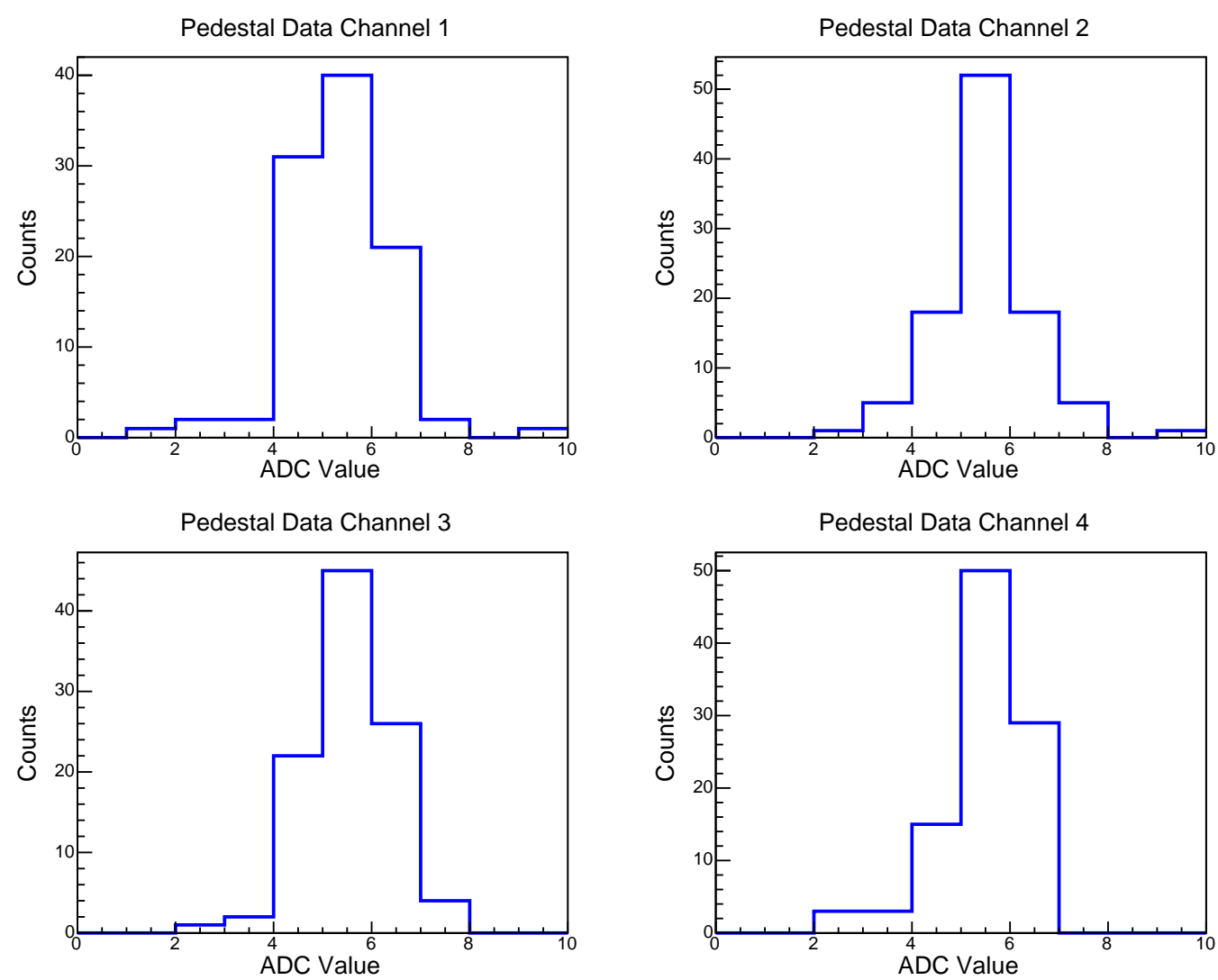

Figure 3.9: ADC output of all Channel 1,2, 3 and 4 of the Fiber Loop Transmission device while covered with black tape for 100 times called pedestal data. As Fiber Loop Transmission device was covered with black tape so there should be no output, but as shown in the above distribution each channel has small ADC out. Which is used as noise for calibration of respective channel of the Fiber Loop Transmission device.
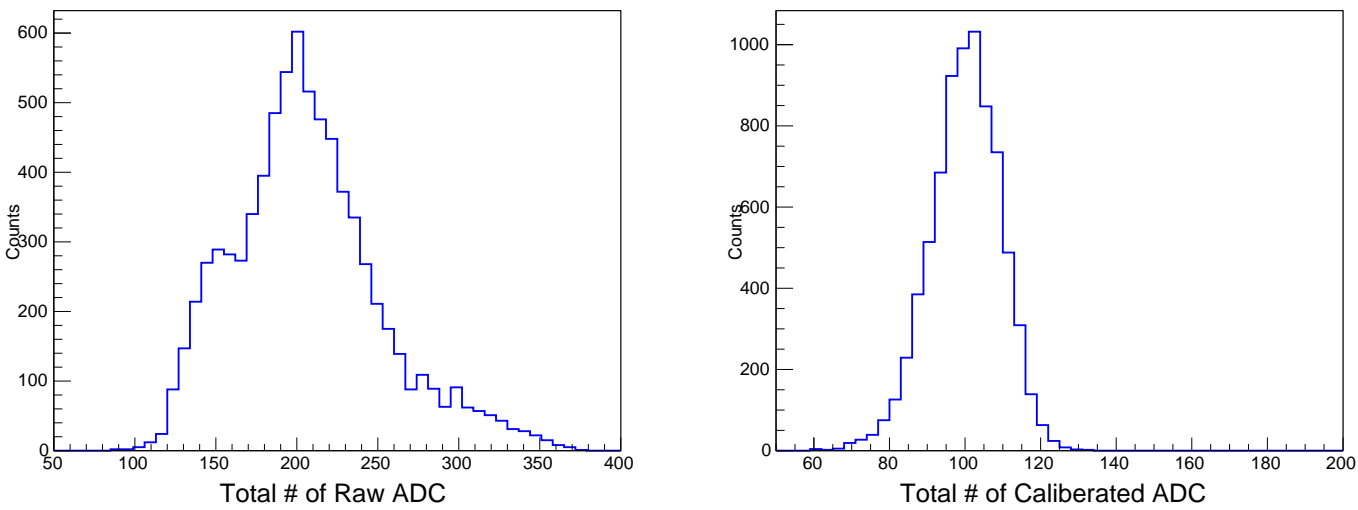

Figure 3.10: Left: Total raw ADC out of Fiber Loop transmission device for all channels of all modules of NOvA ND. Right: Total calibrated ADC out of Fiber Loop transmission device for all channels using pedestal correction factor for respective channel of all modules of NOvA ND. 

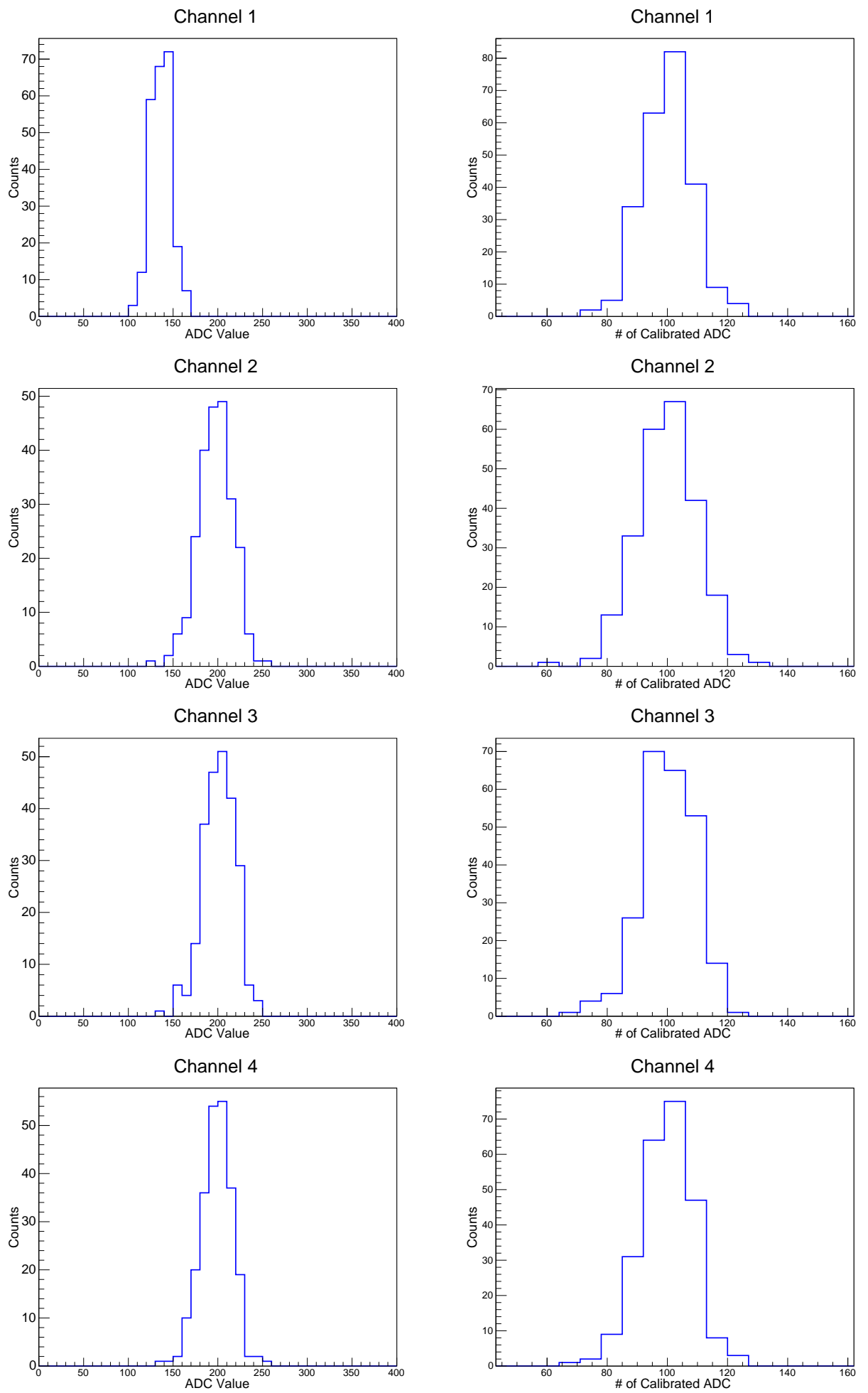

Figure 3.11: Left: Total raw ADC out of Fiber Loop transmission device for channel 1, 2, 3 and 4 of all modules of NOvA ND. Right: Total calibrated ADC out of Fiber Loop transmission device for channel 1,2, 3 and 4 using pedestal correction factor for respective channel of all modules of NOvA ND. 

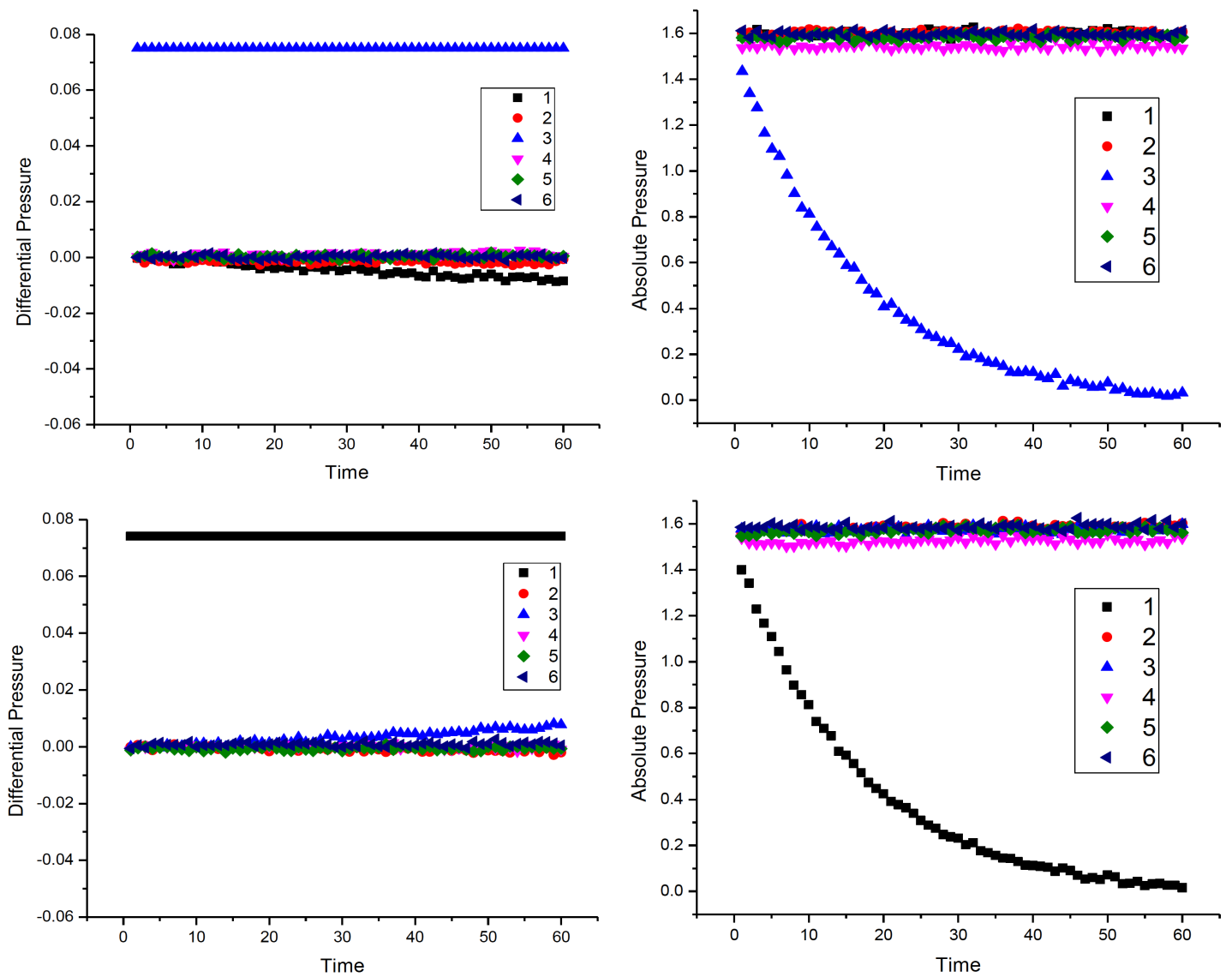

Figure 3.12: Top Left: Differential pressure for six modules for 60 mins. Top Right: Absolute pressure for six modules for 60 mins. Bottom: Leak test same as upper Left and Right just with the replacement of channel 1 with channel 3 only to make sure there is the leak in the same module as explained in section 3.4 .2 .

\subsubsection{Leak Test of NOvA ND Modules}

The purpose of the leak test was to confirm that the liquid scintillator would not leak out of the modules once filled. The module volume is pressurized and the pressure is recorded over the course of one hour. If there was a leak in the module, pressure would decrease as shown in Figure 3.12, Otherwise, it would remain constant. In Figure 3.12 channel 3 showed leakage in module number 3 . Need to double check that it is the leakage in the module 3 or a problem of leak testing system. We replaced channel 3 with channel 1 and repeated the test for 60 mins. It showed decrease in pressure for channel 1, which 
confirmed that there was leakage in the same module. 



\section{Chapter 4}

\section{Near Detector Calibration, Event Reconstruction, and Simulation}

\subsection{Calibration}

Calibration is necessary to understand how to translate a hit in a specific cell at a specific depth into an energy deposition. Calibration has sequential stages that allow this translation. The energy calibration of the NOvA detectors is divided into two phases: a relative calibration that corrects for attenuation and aging of the detector [42], and an absolute calibration that converts an energy deposition recorded by the APD into physical units of $\mathrm{GeV}$ [43].

\subsubsection{Attenuation Correction}

Attenuation calibration corrects cell-to-cell differences. A collection of hits is chosen and hits are required to be on well-reconstructed muon cosmic rays. If possible, the tri-cell criterion is also required; that is, a hit is only used if the cells above it and below it in the plane also have hits. This criterion allows for the more precise calculation of the path length and thus $\mathrm{dE} / \mathrm{dx}$. In cases where there isn't enough statistics to make the tri-cell 
criterion (such as having bad channels next to a good channel), one can instead require that the cells in the planes before and after the hit also have a hit. In the worst cases, such as corner cells, there is no requirement, and instead, an average path length over all the directions that the track can have are used. Given a collection of hits in a cell with known path length, one can make populations that have similar path lengths. Next, a 2D plot of the distance down the length of the cell vs. $(\mathrm{ADC} / \mathrm{cm})$ is constructed. The profile of this plot is taken and fit to determine the attenuation correction for this cell. For most of the length of the fiber, the shape of the attenuation correction is approximated to be that of two exponentials. Near the top and bottom of the cells, the data shows a strong roll-off effect due to the end structure of the cells. Some channels show strong deviations from the double exponential fit. In some cases, this was due to extremely noisy channels located nearby in space, allowing fake cosmic tracks to be produced. However, most of the time noisy behavior is not evident and the source of the discrepancy is unknown. An interpolation fit is applied on top of the double exponential fit. If the interpolation fit is more than $15 \%$ different from the original fit, the channel is marked as uncalibrated. Figure 4.1 displays the attenuation fit for a typically good channel in the near detector (left) and for a typically good channel in the far detector (right). Aging effects are corrected for by monitoring the mean response in a cell over time and correcting back to the calibration period.

\subsubsection{Absolute Energy Correction}

After cell-to-cell differences are removed, one needs to set an absolute scale for the energy. It allows one to translate a hit's charge corrected into reconstruction energy deposition $\mathrm{GeV}$. The absolute energy scale is set with a population of cosmic muon tracks that stop within the detector is used. Since muon energy loss in the detector is well understood, one can start at the end of the track (where the muon energy is zero) and work backward with the Bethe-Bloch formula to determine the $\frac{d E}{d x}$ along the track. The MIP region of 

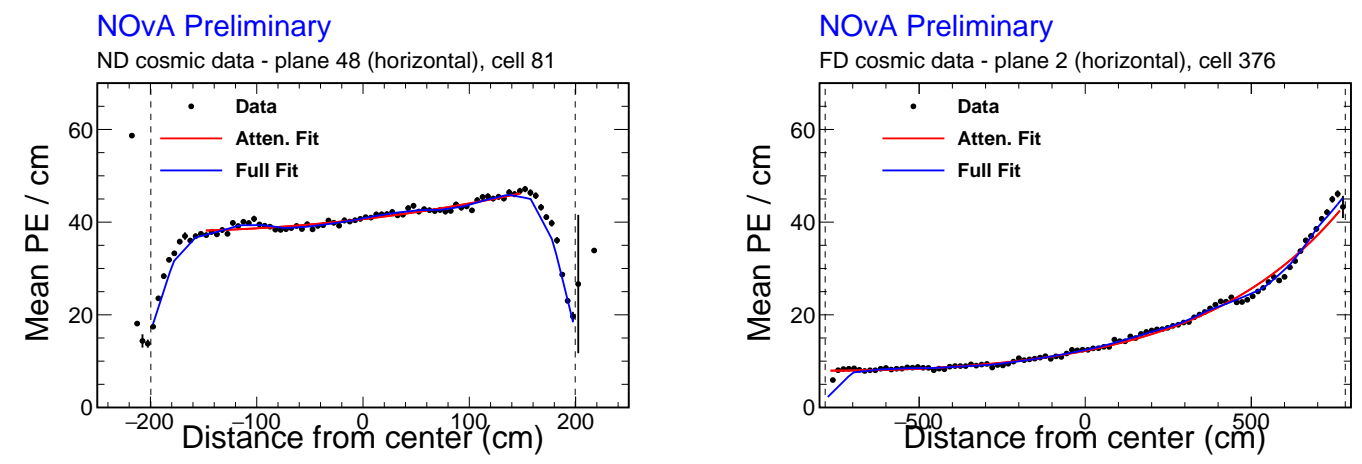

Figure 4.1: Left: The final attenuation calibration fit for a channel in the physical ND. Right: The final attenuation calibration fit for a channel in the physical FD. This fit, drawn as a blue line, is considered good. The horizontal axis is distance along the cell depth, measured in centimeters $(\mathrm{cm})$ away from the central depth. The vertical axis is average photo-electrons per path length in $\mathrm{cm}$. Dashed vertical lines indicate the ends of the cell.

the track, where $\frac{d E}{d x}$ is approximately constant, is found. Figure 4.2 displays the MIP region for stopping muon tracks in the far detector. A scale factor is then determined that translates the energy units given by the attenuation calibration into GeV.

\subsection{Event Reconstruction}

NOvA event reconstruction in data and Monte Carlo (MC) is a multiple step process. The common event topologies are shown in Figure 4.3. The figure shows one detector view for each event, and the cell hits are colored by the charge deposited and depth of the shade relates to the amount of charge deposited. The top panel is a $\nu_{\mu} \mathrm{CC}$ event having a track-like muon, a minimum-ionizing particle (MIP). The middle panel shows a $\nu_{e} \mathrm{CC}$ event having an electron in the output. The electron has a distinct shower shape with the energy deposition per plane rising and falling, providing separation of electron and muon events relatively straightforward. The neutral current (NC) interaction shown in the bottom panel with a single $\pi^{0}$ serves as background in most of the NOvA analyses for active neutrinos $\left(\nu_{\mu}, \nu_{e}\right)$, and it is the most challenging background for studies. However, $\mathrm{NC}$ acts as the signal for sterile search in NOvA. The decay mode of $\pi^{0}$ into two photons 


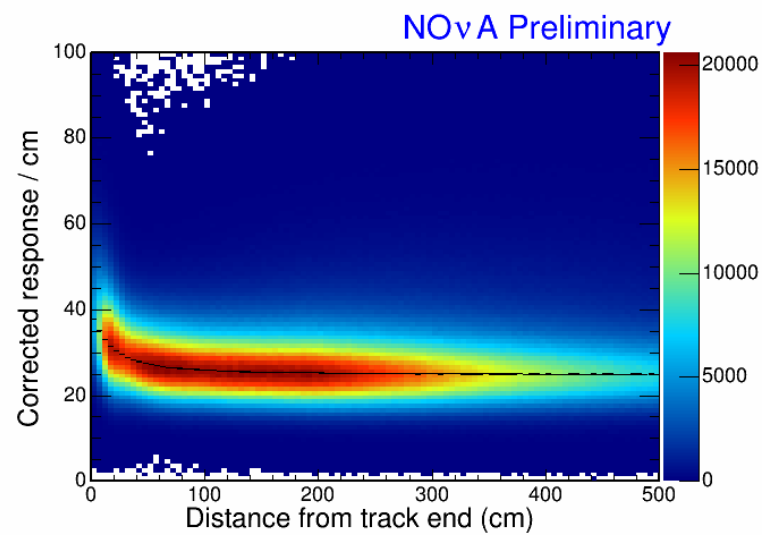

Figure 4.2: Plot used to create absolute energy calibration for the physical far detector. Each entry in the histogram is a tri-cell hit on a cosmic ray muon which stopped inside the detector. The horizontal axis is the distance of the hit from the end of the track in $\mathrm{cm}$. The vertical axis is the attenuation-corrected energy per path length in $\mathrm{cm}$. The black fit points show the mean of the fit to the distribution for each horizontal bin. Values between 100 and $200 \mathrm{~cm}$ from the end of the track are considered the MIP region and used for the absolute energy calibration.

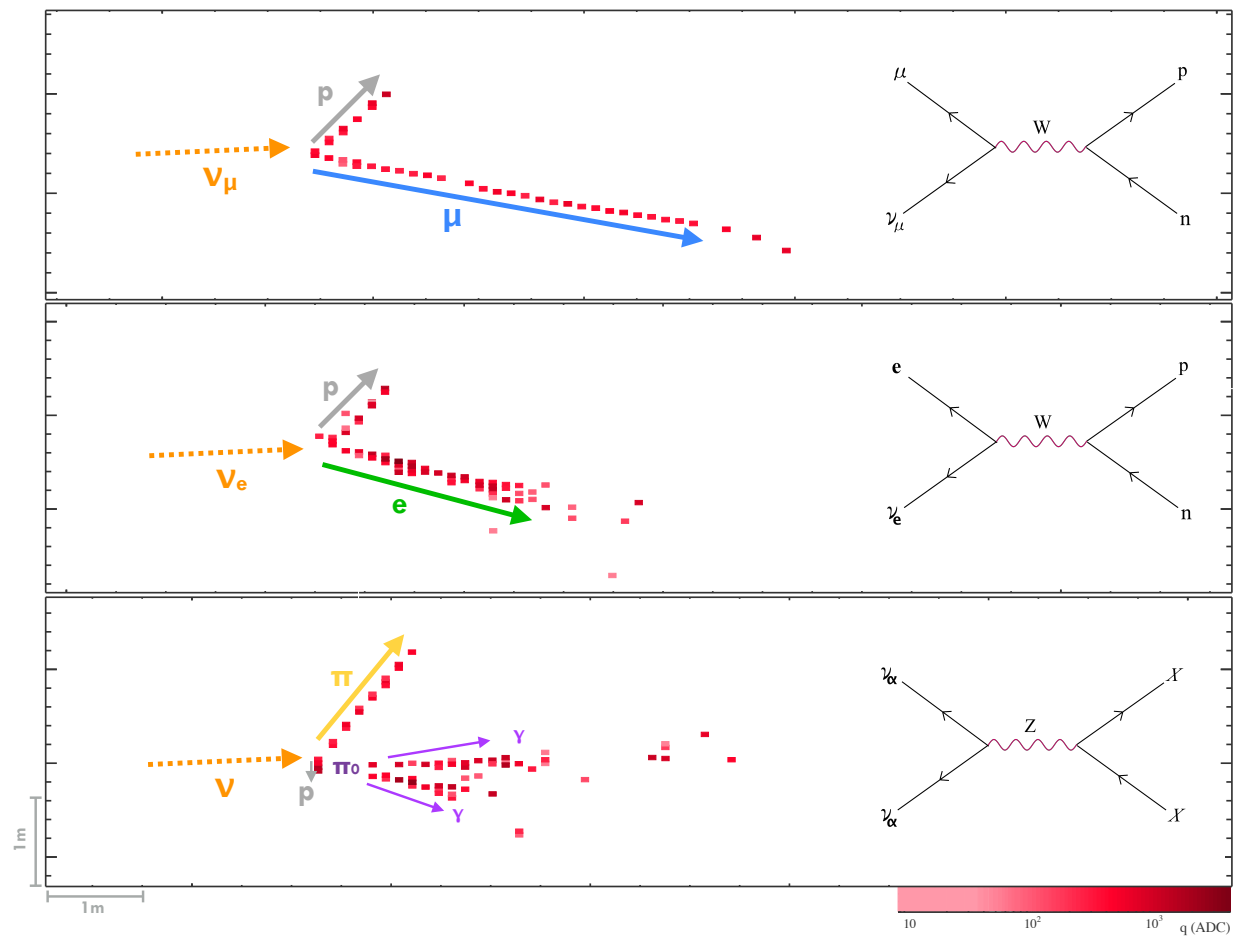

Figure 4.3: Example event topologies from data files. Top: Selected $\nu_{\mu}$ ND event. Middle: Selected $\nu_{e}$ ND event. Bottom: Selected $\pi^{0}$ ND event. 
has $98.8 \%$ [44] Branching ratio. As these photons also produce electromagnetic showers, it is hard to distinguish between them and electron. The photons, before converting into an $\mathrm{e}^{-} / \mathrm{e}^{+}$pair, travel some distance, which in the NOvA detectors is a photon conversion distance of $53 \mathrm{~cm}$ (9 plane widths). The photons from $\pi^{0}$ can be identified by their gap between the vertex and the start of the showers, which is why NOvA was designed with low-Z materials to yield a longer conversion distance. The gap between the vertex and the start of the showers is used to distinguish between a photon and electron electromagnetic shower. Additionally, the very start of a photon shower has a $\mathrm{dE} / \mathrm{dx}$ profile of approximately 2 MIP, but for an electron, there is only one shower and one MIP activity. The following subsection will give a brief explanation of each important reconstruction step that used in this thesis.

\subsubsection{Interaction Separation with DBSCAN}

The recorded trigger window for data in NOvA is of length $550 \mu \mathrm{sec}$ as shown in Figure 4.4. The window records multiple unrelated events inside the detector from different interactions. The real physics interaction from beam neutrinos occurs for a short period of $\sim 10 \mu \mathrm{sec}$ as shown in Figure 4.5. The first step in event reconstruction is to arrange all of the calibrated hits (these hits have $\mathrm{x}, \mathrm{y}, \mathrm{z}$ and charge information with time) from a 550 $\mu$ sec readout window into clusters. The reconstruction subsequently locates the hits in the clusters that have a probability of occuring from the same particle and creates individual particle tracks. These reconstructed 3D tracks are used to identify particle types and estimate the total event energy. The clusters of hits that are roughly contiguous in time and space are called slices. Each slice would correlate to one physics interaction (neutrino interaction or cosmic ray). First, we locate a global event vertex for each cluster of hits and from this vertex identify the tracks both long (created by the primary lepton) and short (due to a recoiling proton). This identification is crucial for increased reconstructed event energy resolution. 


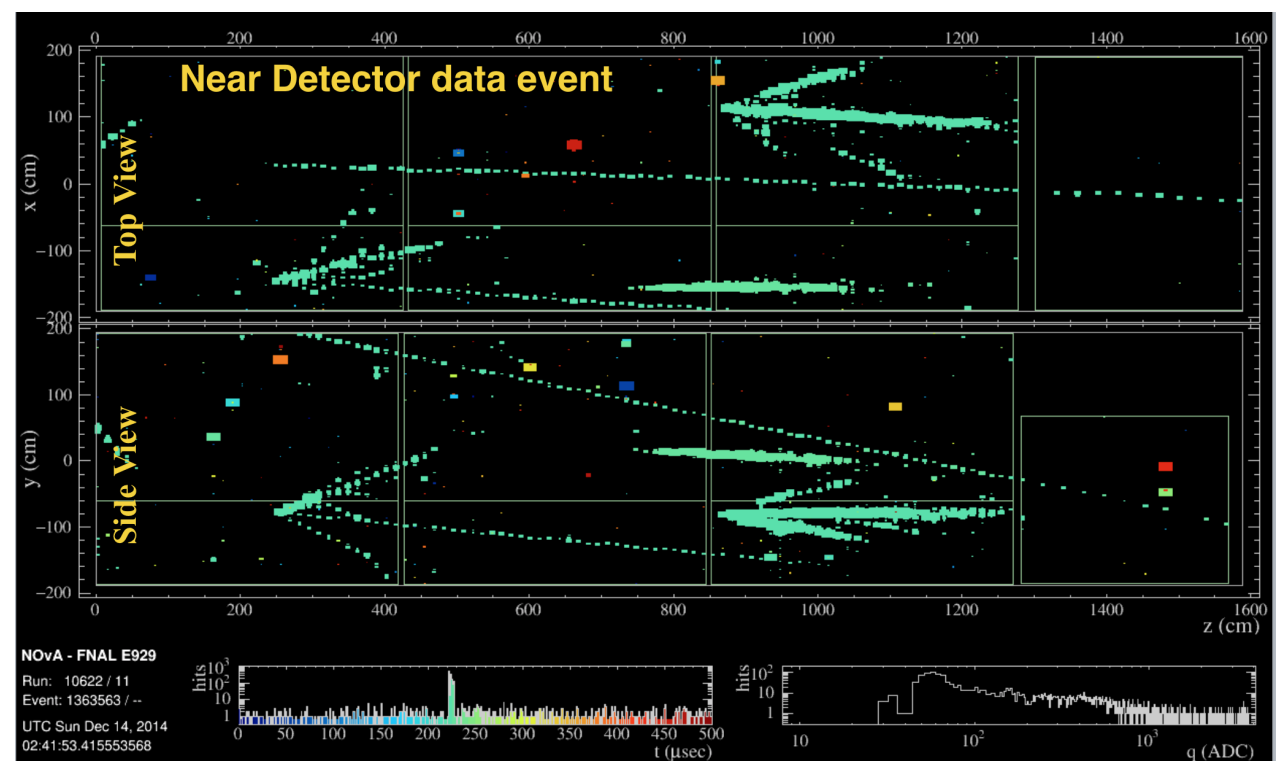

Figure 4.4: Near Detector data event display for full trigger window $550 \mu$ sec.

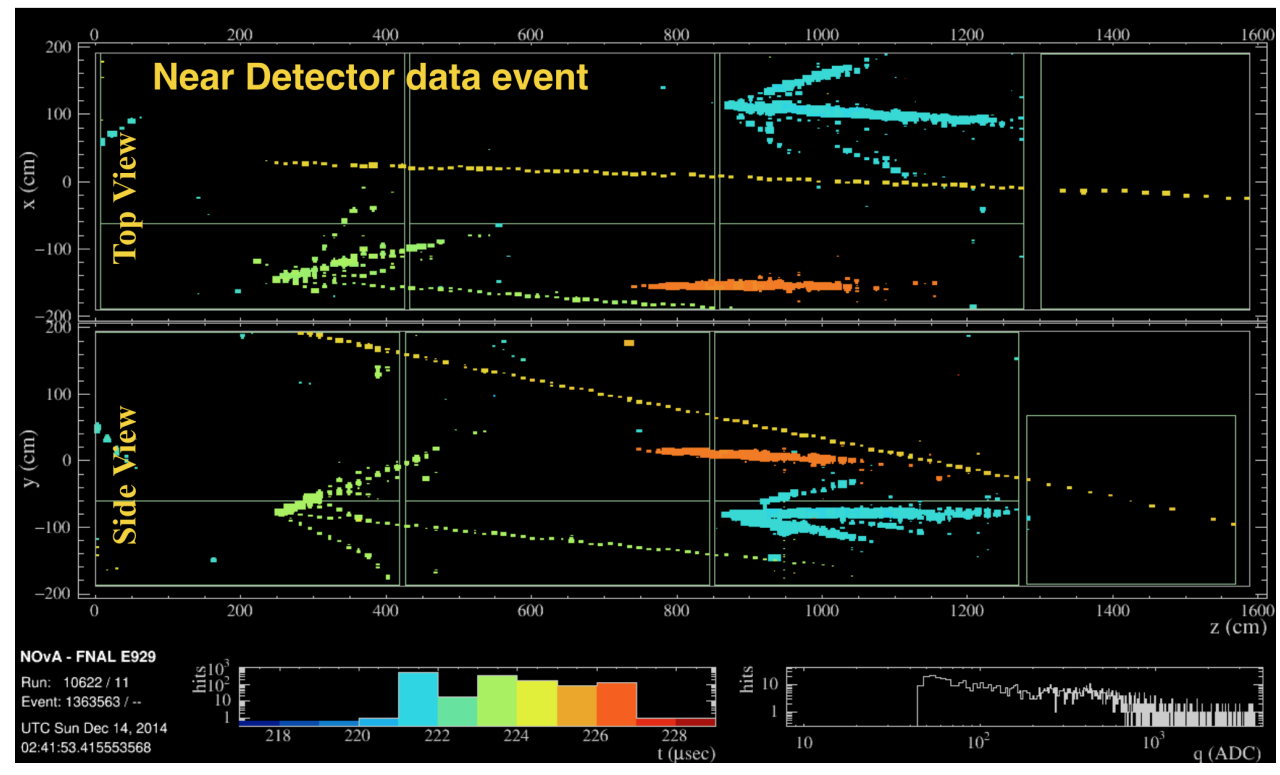

Figure 4.5: Near Detector data event display for NuMI beam window $10 \mu$ sec. 
The information from a single triggered channel is called a "hit" and collection of hits from an arbitrary readout window is called "data event," currently $550 \mu$ sec. The hit contains information regarding the plane and cell numbers for that channel, the total ADC, and hit time. These hits are categorized into two slice types: signal and noise. For separation of the signal hits from the noise hits and distinction of a cluster of signal hits that originate from different sources, NOvA uses an expanding density-based clustering algorithm (DBSCAN), described in [45], which makes use of the available spatial and temporal information. DBSCAN clusters are separable from each other by regions in which the density of points in some parameter space drops below some critical density. This algorithm has success even when the clusters have irregular shapes and when the density of background noise hits is relatively constant within the parameter space. For each hit, the algorithm calculates the local density by counting the number of hits that are within a certain "distance" of that hit. The "distance" can be computed with any score function that is appropriate for the specific clustering application. The DBSCAN algorithm makes a cluster, which is compromised of two types of hits: core hits and border hits. The core hits within the critical distance must have at least the minimum number of neighboring hits (neighbors). The border hits are included in a cluster only if they are a neighbor of a core point but have less than the minimum number of neighbors. The algorithm expands the cluster around the core points to make clusters, by looping over all points. Once it finds a core point, it begins a cluster and adds that point and its neighbors to the cluster and checks all other points in its neighborhood until it terminates at a border point. Once this loop completes, it returns to the original list of points and repeats the process for the next unassigned core point and so on. After this process, if there are still any hits not assigned to a cluster, they are labeled as noise. The final non-noise slice object requirement is to have a minimum of three hits in each view which is very rare for coincident noise. 


\subsubsection{Finding Feature Guidelines with a Multi-Hough Trans-}

\section{form}

After slicing has been applied the next reconstruction stage is to run a Hough transform algorithm on each slice to find the prominent lines. The Hough transform technique is a relatively widely used algorithm that utilizes a voting procedure. The Hough transformation is designed to identify significant lines or features in a $2 \mathrm{D}$ image composed of pixels or points for pattern recognition problems. In NOvA, the algorithm is modified to improve the robustness against noise using a pair of points [46]. This algorithm is applied to each detector view separately by taking pairs of hits in the event. It calculates the line that passes through a pair of hits with the perpendicular distance from the origin to the line $\rho$, and $\theta$, the angle between $\rho$ and the x-axis in polar coordinates. It makes parameter space map (called a Hough map) and fills it with a Gaussian smeared vote. The Gaussian voting is:

$$
\text { vote }=e^{\frac{-\left(\rho-\rho_{o}\right)^{2}}{2 \sigma_{\rho}^{2}}} e^{\frac{-\left(\theta-\theta_{o}\right)^{2}}{2 \sigma_{\theta}^{2}}}
$$

where $\sigma_{\rho}=3 / \sqrt{12}$ and $\sigma_{\theta}=3 / \mathrm{d} \sqrt{6}$ (where $\mathrm{d}$ is the distance between the two hits in the detector).

The region of interaction inside the detector makes a peak in the Hough map that can use the Hough lines that characterize the event. Peaks in the Hough space map are identified as the coordinates of a line. For the separation of the signal from the noise, a threshold for peak identification is set as the average height of all $\rho, \theta$ bins in the Hough space. To reduce the tendency to make spurious lines an iterative procedure is used. At first the highest peak in the Hough map is found and then a line is formed from the weighted average $\rho$ and $\theta$ of a $7 \times 7$ grid of bins surrounding the peak. Cell hits that fall within 6 centimeters (one cell depth) of the line are removed except for the most 
upstream and downstream hit, which could be shared with other tracks coming from a common vertex. After hit removal, the Hough map is recalculated, and a new peak is found. This process repeats until no new lines remain or the maximum number of lines (currently 10) is reached. The primary performance criterion for the algorithm is for the dominant Hough lines to pass close to and form intersections near the primary interaction point of the slice.

\subsubsection{Vertex Identification with Elastic Arms}

The output from the Multi-Hough algorithm is used as input for the Elastic Arms algorithm to use as a seed for finding the global event vertex. This algorithm is based on a method of the same name listed in [47]. The basic template for a NOvA event is a vertex with one or more particle tracks emanating outwards from that vertex. In the Elastic Arms method, once a vertex has been identified, each particle track is approximated by an "arm" (a vector pointing away from the vertex) whose direction can be adjusted to fit the event. For the application of this method to NOvA data, the number of arms is taken to be the largest number of good Hough lines found for the event in either the $\mathrm{XZ}$ or $\mathrm{YZ}$ views.

\subsubsection{Kalman Track}

KalmanTrack is a Kalman-filter based algorithm which takes in a cluster of hits from Slicer and groups the cell hits from either the vertical or horizontal cells into 2D tracks [48]. KalmanTrackMerge takes the $2 \mathrm{D}$ tracks from each view and attempts to merge them into 3D tracks. The information from the 3D tracks and unmatched $2 \mathrm{D}$ tracks are written out to the file. This information includes the position of the most upstream (lowest z) part of the track, called the start position, the position of the most downstream (highest $\mathrm{z}$ ) part of the track, called the end position, the all the hits associated with each track, and 
the trajectory points determined to exist along the path of the track. A track is defined as a mostly continuous string of cells that would result from the energy deposited by a single, non-showering particle. This tracker uses linear, piecewise segments to describe a trajectory and assumes the scatter is consistent with a muon.

\subsubsection{ReMId}

ReMId (Reconstructed Muon Identification) is an algorithm that attempts to identify a muon track. A $\nu_{\mu}$ charged current interaction is identified if it has a 3D muon track, as determined by ReMId. ReMId uses a k-Nearest Neighbors (kNN) algorithm to make its determination. More information about $\mathrm{kNN}$ algorithms is in [49]. The input variables are: log-likelihoods that use the energy deposited per unit length $\mathrm{dE} / \mathrm{dx}$ profile of the track, log-likelihoods based on the scattering observed on the track, the track length, and the fraction of planes used to created the $\mathrm{dE} / \mathrm{dx}$ profile log-likelihood. To use the $\mathrm{dE} / \mathrm{dx}$ profile information of a track, ReMId determines the log-likelihood ( $\mathrm{LL}^{E}$ ) that a particle of type j created the track, where:

$$
L L_{j}^{E}=\frac{1}{N_{\text {plane }}} \Sigma_{i} P_{i}^{j}
$$

For $\mathrm{LL}^{E}$, the $\mathrm{dE} / \mathrm{dx}$ profile of the track is measured at plane $\mathrm{i}$ and the probability, $\mathrm{P}_{i}^{j}$, of a particle of type $\mathrm{j}$ to have the measured $(\mathrm{dE} / \mathrm{dx})$ profile at this distance from the end of the track is calculated from a histogram created using simulated events under the assumption of particle type $\mathrm{j}$. The number of planes for the $\mathrm{dE} / \mathrm{dx}$ profile is measured on the track is $\mathrm{N}_{\text {plane }}$ and is used to normalize the LL. The difference in the values of the LL under the pion and the muon assumptions is taken to form the final ( $\mathrm{dE} / \mathrm{dx}$ ) profile LL variable input into the ReMId $\mathrm{kNN}$. 
To use the scattering information of a track, the LL ${ }^{\text {scat }}$ is defined as:

$$
L L_{j}^{\text {scat }}=\frac{1}{N_{\text {scat }}} \Sigma_{i} P_{i}^{j}
$$

where $\mathrm{P}_{i}^{j}$ is the probability that a particle of type $\mathrm{j}$ has the measured scatter at a distance of $\mathrm{i}$ from the end of the track, as determined by a histogram created using simulated events under the assumption of particle type $\mathrm{j}$. $\mathrm{N}_{\text {plane }}$ is used to normalize the LL. The measured scatter $s$ is defined as:

$$
s=\frac{\theta^{2}}{d}
$$

where $\theta$ is the measured scattering angle for that trajectory point and $d$ is the distance from the last measured scatter. The $\mathrm{kNN}$ returns a value between 0 and 1: 1 being muon-like and 0 being background type. The analysis uses a cut of 0.7 to determine if the track is deemed muonic.

\subsection{6 $\nu_{\mu}$ Charged Current Energy Estimators}

The numu-CC energy estimator, called NumuEnergy on NOvA, attempts to measure muon, hadronic, and neutrino energies for each slice using various assumptions. For an energy object to be returned, the slice must have contained at least one 3D Kalman track. This is neutrino energy that is tuned for the entire charged current population. This is the neutrino energy used in the first and second analyses. It is a sum of reconstructed muon energy and reconstructed hadronic energy. The muon energy is determined from the track length of the 3D Kalman track with the highest ReMId value. This track length is converted into reconstructed muon energy using a spline fit. The visible hadronic energy is defined as the sum of energy on all slice hits not on the muon track as well as the energy on the track in the vertex region that exceeds minimum ionizing particle values. 
This visible energy is then fit with a spline fit to the true neutrino energy minus the reconstructed muon energy. For more details see [50].

\subsubsection{Likelihood Based Identifier (LID)}

The basic idea of the Likelihood-based identifier (LID) is to use the shower-energy profile to separate electrons from muons, $\pi^{0}$ 's, and other hadrons. Different particles have very different energy-depositions in the detector. For example, the electron deposits energy through ionization in the first few planes then starts a shower; the photon is a shower that follows a gap in the first few planes, and the muon registers as a long minimum ionizing particle (MIP) track. This makes it possible to identify particles by comparison of shower/track shapes with different particle hypotheses. LID compares the longitudinal and transverse energy deposition in the primary shower to template histograms for various simulated particles. The likelihood differences among different particle hypotheses and other topological variables are used as input to an artificial neural network to construct the primary classifier. The energy range of events selected with this primary method is further restricted to 1.5 to $2.7 \mathrm{GeV}$ to remove additional backgrounds from cosmic radiation. For further details see Ref. [51]. We have used modified version of this PID for our $\nu-e$ elastic scattering experiment as described in Chapter 7 .

\subsection{Detector Simulation}

To simulate the propagation of particles through the NOvA detector, we use Geant4 as shown in Figure 4.6. The MC in this thesis is with physics list QGSP BERT HP. This configuration uses the quark-gluon string (QGS) model [52] for high energy interactions of protons, neutrons, pions, kaons, and nuclei. Here, high energy means greater than 20 $\mathrm{GeV}$. The Bertini cascade (BERT) model [53, 54, 55] is used to simulate primary protons, neutrons, pions and kaons with energies below $10 \mathrm{GeV}$. The low energy parametrized 


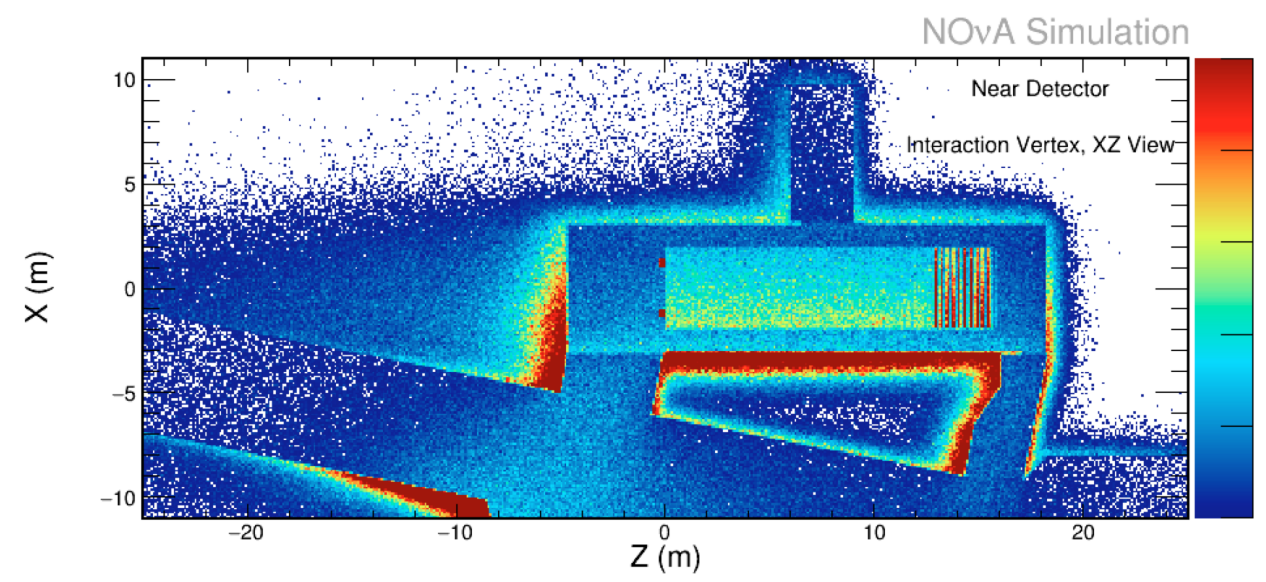

Figure 4.6: Simulation: Locations of neutrino interactions that produce activity in the Near Detector. The color scale represents the increasing density of interactions in the detector and the surrounding cavern.

(LEP) Model is used for intermediate energies and low energy particles not modeled by BERT. Using BERT instead of LEP for all lower energy hadronic modeling results in better agreement to data. In general, it creates more secondary protons and neutrons than LEP. To simulate the de-excitation of nuclei, the G4Precompound (P) model is used. To simulate proton and neutron inelastic interactions, the Axen-Wellisch Parametrization of cross sections is used. To simulate pion inelastic interactions, a table of Barashenkov cross sections is used. A data-driven high precision neutron (NeutronHP) simulation is used to model the interactions of neutrons with energies less than $20 \mathrm{MeV}$ down to thermal energies. 



\section{Chapter 5}

\section{Prediction of Neutrino Flux}

\subsection{Neutrino Parents}

The main decay modes that produce neutrinos are presented in Table 5.1. Charged pions and charged kaons create predominantly muon neutrinos and a small component of electron neutrinos. This is shown in Figure 5.1 and Figure 5.2. Muons can also be generated in these decays and contribute to the electron neutrino flux, but these are highly suppressed because they are mostly absorbed before they decay.

\begin{tabular}{|c||c|c|}
\hline Decay & Chanel & Branching ratio (\%) \\
\hline 1 & $\pi^{ \pm} \rightarrow \mu^{ \pm}+\nu_{\mu}\left(\bar{\nu}_{\mu}\right)$ & 99.9877 \\
2 & $\pi^{ \pm} \rightarrow e^{ \pm}+\nu_{e}\left(\bar{\nu}_{e}\right)$ & 0.0123 \\
\hline 3 & $K^{ \pm} \rightarrow \mu^{ \pm}+\nu_{\mu}\left(\bar{\nu}_{\mu}\right)$ & 63.55 \\
4 & $K^{ \pm} \rightarrow \pi^{0}+e^{ \pm}+\nu_{e}\left(\bar{\nu}_{e}\right)$ & 5.07 \\
5 & $K^{ \pm} \rightarrow \pi^{0}+\mu^{ \pm}+\nu_{\mu}\left(\bar{\nu}_{\mu}\right)$ & 3.353 \\
\hline 6 & $K_{L}^{0} \rightarrow \pi^{ \pm}+e^{\mp}+\nu_{e}$ & 40.55 \\
7 & $K_{L}^{0} \rightarrow \pi^{ \pm}+\mu^{\mp}+\nu_{\mu}$ & 27.04 \\
\hline 8 & $\mu^{ \pm} \rightarrow e^{ \pm}+\nu_{e}\left(\bar{\nu}_{e}\right)+\bar{\nu}_{\mu}\left(\nu_{\mu}\right)$ & 100.0 \\
\hline
\end{tabular}

Table 5.1: Main decay modes and their branching ratios of charged pion, charged kaons, neutral kaons and muons to neutrinos.

Accurate $\nu_{\mu}, \bar{\nu}_{\mu}, \nu_{e}$, and $\bar{\nu}_{e}$ flux prediction is needed for the precision oscilla- 
tion studies; it is crucial for the cross-section measurements in the Near Detector (ND). The largest source of error in the flux modeling comes from the poorly known production cross-section of the $\pi^{+}, K^{+}, \pi^{-}, K^{-}$and $K^{0}$ mesons in proton-target collision. Additional errors are incurred from the simulation of the beam-transport and re-interaction of hadrons during the transport.
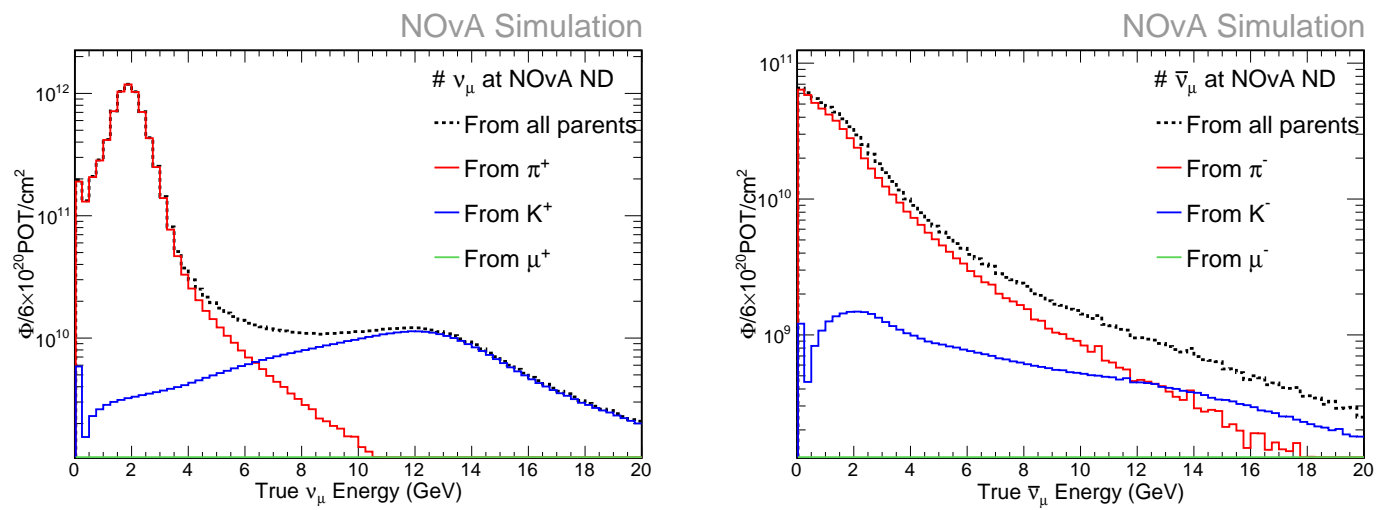

Figure 5.1: Left: Number of $\nu_{\mu}$ as a function of true $\nu$ energy at NOvA Near Detector. Right: Number of $\nu_{e}$ as a function of true $\nu$ energy at NOvA Near Detector.
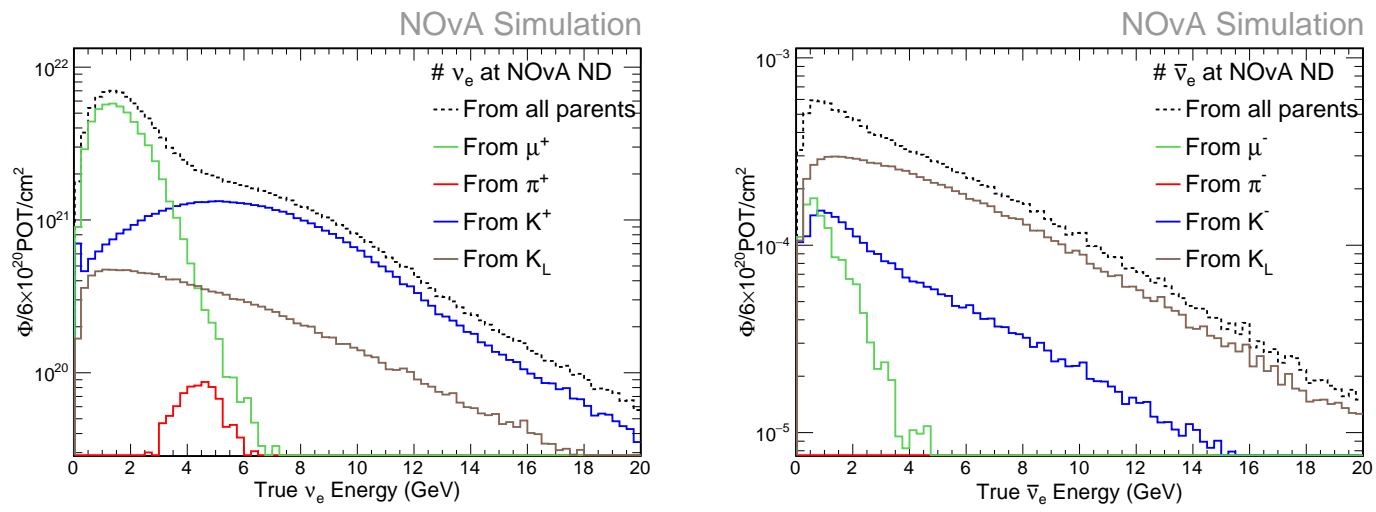

Figure 5.2: Left: Number of $\nu_{\mu}$ as a function of true $\nu$ energy at NOvA Near Detector. Right: Number of $\nu_{e}$ as a function of true $\nu$ energy at NOvA Near Detector.

\subsubsection{NOvA $\nu$ from Primary and Secondary Interactions}

Since the NuMI target is a thick target, as mentioned in Chapter 2, the NOvA neutrino flux is not only come from primary meson produced at target but also by secondary 
meson as shown in Figure $5.3, \approx 55 \% \nu$ flux for NOvA is from the direct proton interaction (secondary mesons, named as 1 interaction), which means there is $\approx 45 \%$ meson production that is not covered by the data results from thin target experiments.
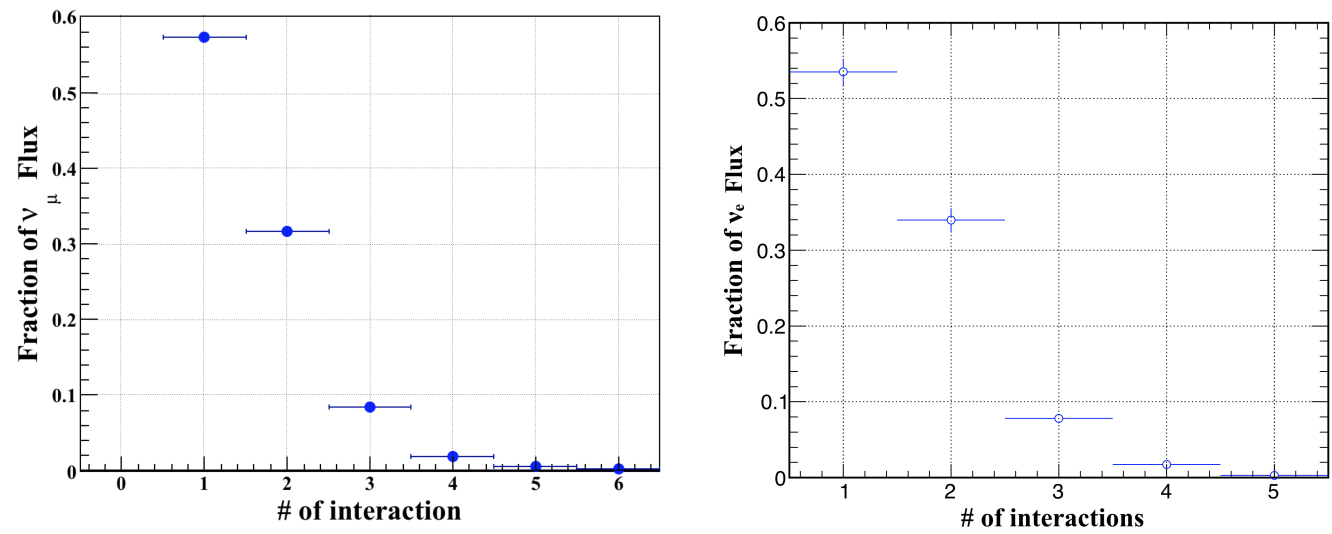

Figure 5.3: No. of interactions from proton to $\nu_{\mu}$ and $\nu_{e}$ parent meson.

\subsubsection{NOvA $\nu$ from Primary and Secondary Nuclear Targets}

Although the principal source of neutrinos are the mesons produced in the $120 \mathrm{GeV}$ primary proton collision with the graphite NuMI-target, about 10-20\% of (anti)neutrinos are produced in interactions in the nuclear elements that reside in the beam. Downstream of primary graphite target $\mathrm{C}$, protons encounter different A's(nuclei) i.e He, Al, Fe, N, H, $\mathrm{O}$, Si etc as shown in Table 5.2. Most of the contamination is because of production in secondary nuclear elements.

\subsection{Sources of the Beam-Transport Uncertainties}

This study includes variations in parameters associated with the beam transport (see Figure 5.4), simulating neutrino flux at the NOvA detectors, and presenting the variations in the flux at ND, FD, and Far to Near Ratio (F/N). The NOvA beam simulation is based 


\begin{tabular}{|c||c|c|c|c|}
\hline$\nu$ mode & RHC & RHC & FHC & FHC \\
\hline Element & $\% \pi^{-}$ & $\% \pi^{+}$ & $\% \pi^{+}$ & $\% \pi^{-}$ \\
\hline Carbon & 83.7 & 35.9 & 85.2 & 31.0 \\
Iron & 5.12 & 30.9 & 4.96 & 37.0 \\
Aluminium & 4.21 & 12 & 4.08 & 10.3 \\
Nitrogen & 2.94 & 11.4 & 2.11 & 6.25 \\
Helium & 2.27 & 6.93 & 2.33 & 12.4 \\
Oxygen & 1.65 & 2.68 & 1.14 & 2.87 \\
Beryllium & 0.12 & 0.11 & 0.17 & 0.002 \\
Hydrogen & 0.01 & 0.05 & 0.03 & 0.00 \\
Chromium & 0.01 & 0.02 & 0.002 & 0.003 \\
Silicon & 0.004 & 0.01 & 0.002 & 0.004 \\
\hline
\end{tabular}

Table 5.2: Column(2,3) (RHC) and column(4,5) (FHC): Production of $\pi$ in the NuMI beam line component compared to primary target Carbon for neutrinos at NOvA ND using G4NuMI with GEANT4.9.2p03.

on Flugg 2009-3d and Fluka (2011.2b.6) part of the Numi-X "Black Bird" release [56]. In the studies presented here, the "nominal" conditions are:

- $975 \times 500 \mathrm{k}=4.875 \times 10^{8}$ total POTs

- Forward Horn Current (FHC)

- Horn Current 200 kA (nominal)

- Linear magnetic field distribution in the horn

- Beam spot size $1.1 \mathrm{~mm}$ in both $\mathrm{X}$ and $\mathrm{Y}$

- PEANUT generator turned on for all energies

The FluxReader framework [57] is used to generate neutrino fluxes at both the ND \& FD, where the flux is defined as $\Phi=\# \nu / \mathrm{cm}^{2}$ for all POTs. In this chapter we present the subset of beam transport uncertainties that are relevant to the NOvA 2015 [58, 59, 60], 2016 [61, 62] and 2017 63] oscillation analysis. The effect of each beam transport parameter on different types of neutrino flavor at the ND and the FD in the tabulated form are shown in Appendix B.1, [64, 65]. Also, variations of the neutrino energy scale, 
$0 \leq E_{\nu} \leq 10 \mathrm{GeV}$ and $1 \leq E_{\nu} \leq 3 \mathrm{GeV}$ are included.

\section{Beam Transports are:}

- Horn Current shifted by $\pm 1 k A$ w.r.t nominal

- Horn1 position shifted by $\pm 2 \mathrm{~mm}$ both in $\mathrm{X}$ and $\mathrm{Y}$ w.r.t nominal

- Horn2 position shifted by $\pm 2 \mathrm{~mm}$ both in $\mathrm{X}$ and $\mathrm{Y}$ w.r.t nominal

- Magnetic field distribution changed to an exponential magnetic field distribution (0.77 cm skin depth) in the horn skin

- Beam position on the target shifted by $\pm 0.5 \mathrm{~mm}$ in $\mathrm{X} \& \mathrm{Y}$ separately

- Beam spot size shifted by $-0.2 \mathrm{~mm}$ and $+0.4 \mathrm{~mm}$ both in $\mathrm{X}$ and $\mathrm{Y}$ w.r.t nominal $1.1 \mathrm{~mm}$ in $\mathrm{X}$ and $\mathrm{Y}$

- Target position shifted by $\pm 7 \mathrm{~mm}$ shift w.r.t nominal

- FLUKA versions comparison

- G4NuMI vs FLUGG comparison

- Improved horn description and addition of a water cooling layer on the horn inner conductor 66

For a given variation (Up/Down) of a parameter (Current, Alignment, etc.), we show the following:

- Variant Flux, $\Phi_{\text {Variant }}$, (shift from the nominal value of a given parameter) and Nominal (Standard) Flux, $\Phi_{\text {Std }}$, at both ND and FD

- $\frac{\Phi_{\text {Variant }}}{\Phi_{\text {Std }}}$ at ND and FD

- $\Phi(F D / N D)$ for both variant and standard

- The double ratio $=\frac{\Phi_{F D / N D}(\text { variant })}{\Phi_{F D / N D}(\text { Std })}$ and DiffDouble-Ratio $=1-\frac{\Phi_{F D / N D}(\text { variant })}{\Phi_{F D / N D}(\text { Std })}$ 


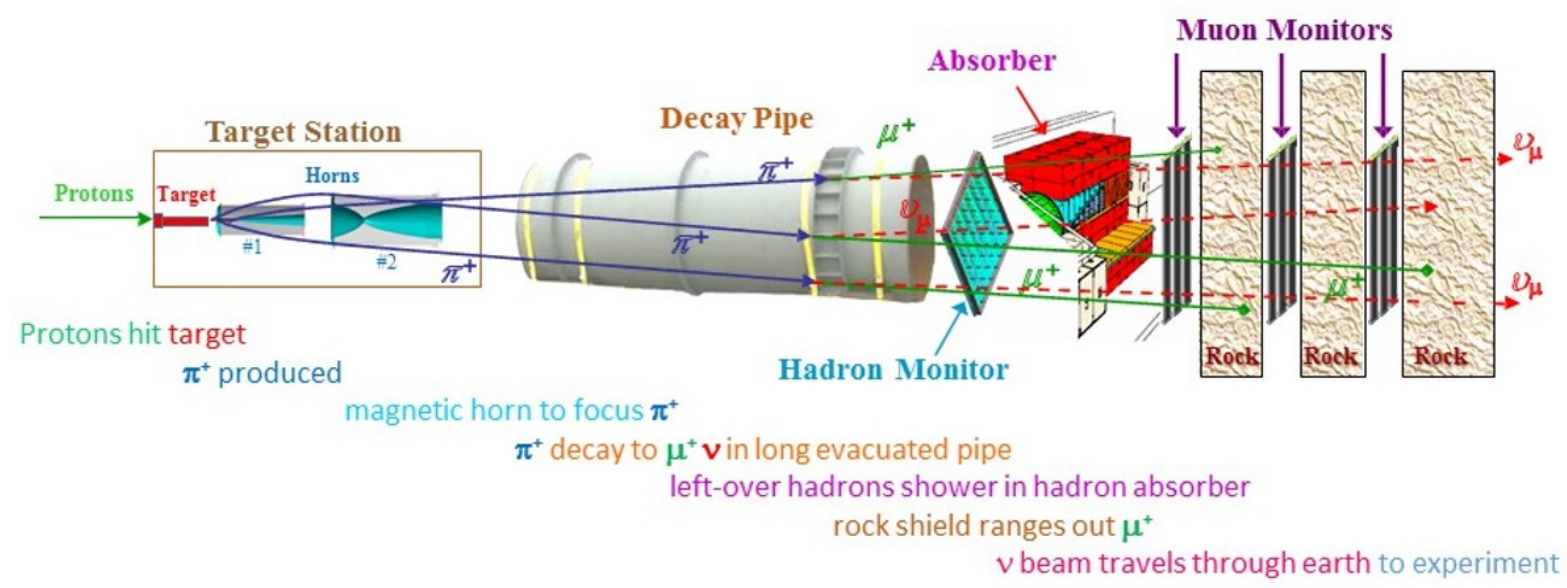

Figure 5.4: Diagram of Fermi National Laboratory's NuMI beamline.

\subsubsection{Horn Current Miscalibration Uncertainties}

In this subsection we will show the effect of the shift in absolute value of the horn current which affects the focusing of all particles [67]. The uncertainty in the distribution of the current in the horn conductors will be discussed in subsection 5.2.3. We consider a horn current scale shift of $\pm 1 \mathrm{kA}$ with respect to the nominal current of $200 \mathrm{kA}$. We have used beamline monitor data to finalize the shift in the horns current value [68]. Additionally, we repeated this exercise for a wide variety of horn current values with respect to the nominal current, e.g. $\pm 10 \%$ and $\pm 5 \%$. We calculated the uncertainties for horn current miscalibration as a difference with the nominal value see Appendix B.1. The key point we checked are variation in number of $\nu$ at both detectors and change in energy scale for all $\nu$ flavors at ND and FD. The flux at FD \& ND changes by $\pm 3 \%$ in the $0-10 \mathrm{GeV}$ range for a $\pm 1 \mathrm{kA}$ variation as shown in Figures 5.5, 5.6 and 5.7. The FD/ND flux changes by $\pm 1 \%$ in the $0-10 \mathrm{GeV}$ range for a $\pm 1 \mathrm{kA}$ variation, as shown in Figure 5.8. The uncertainty due to horn current miscalibration is small for oscillation study of NOvA. 

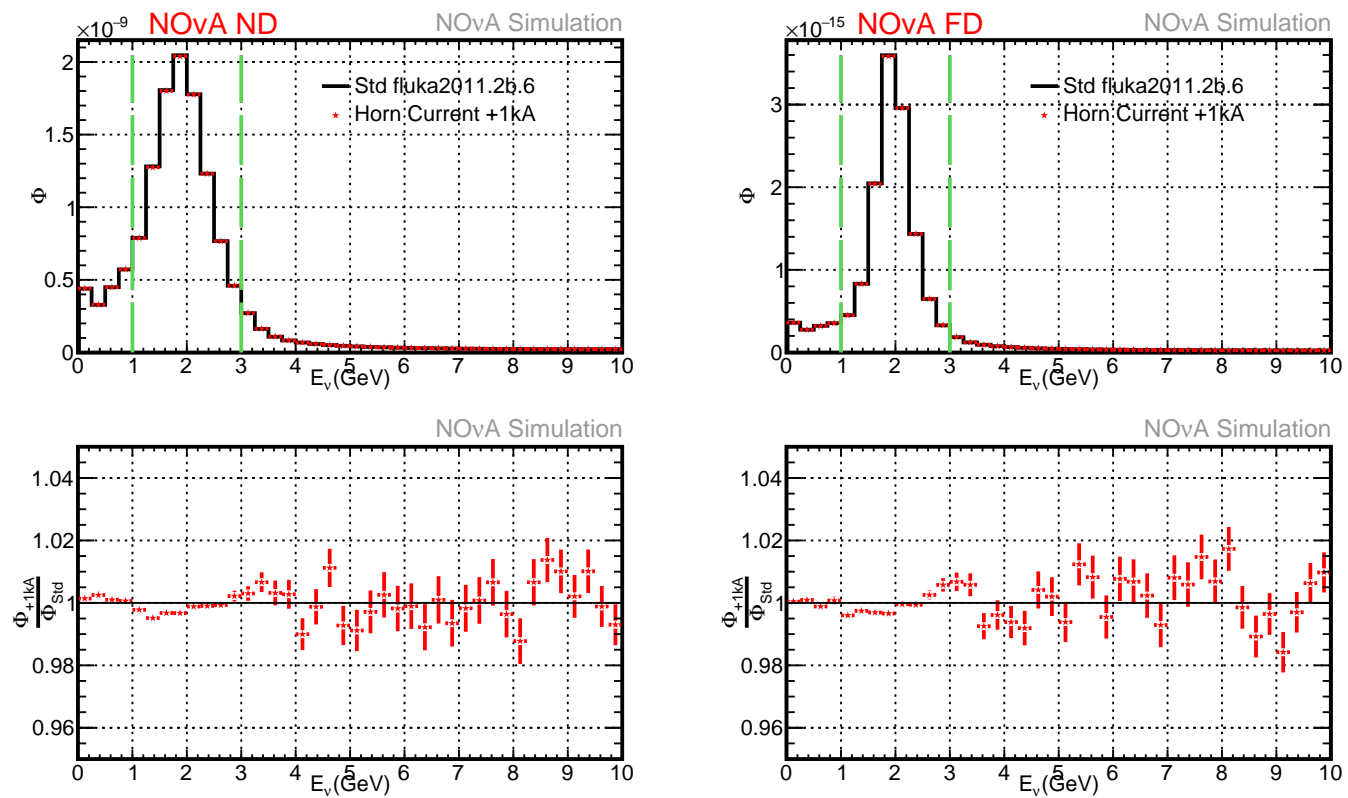

Figure 5.5: Top: FHC neutrino flux at NOA ND (top left) and FD (top right) for all neutrino parents, black is for nominal and red is for the $+2 \mathrm{kA}$ flux, respectively. Bottom: FHC neutrino fluxes ratio of the $+2 \mathrm{kA}$ flux with respect to the nominal flux at the ND (bottom left) and FD (bottom right).
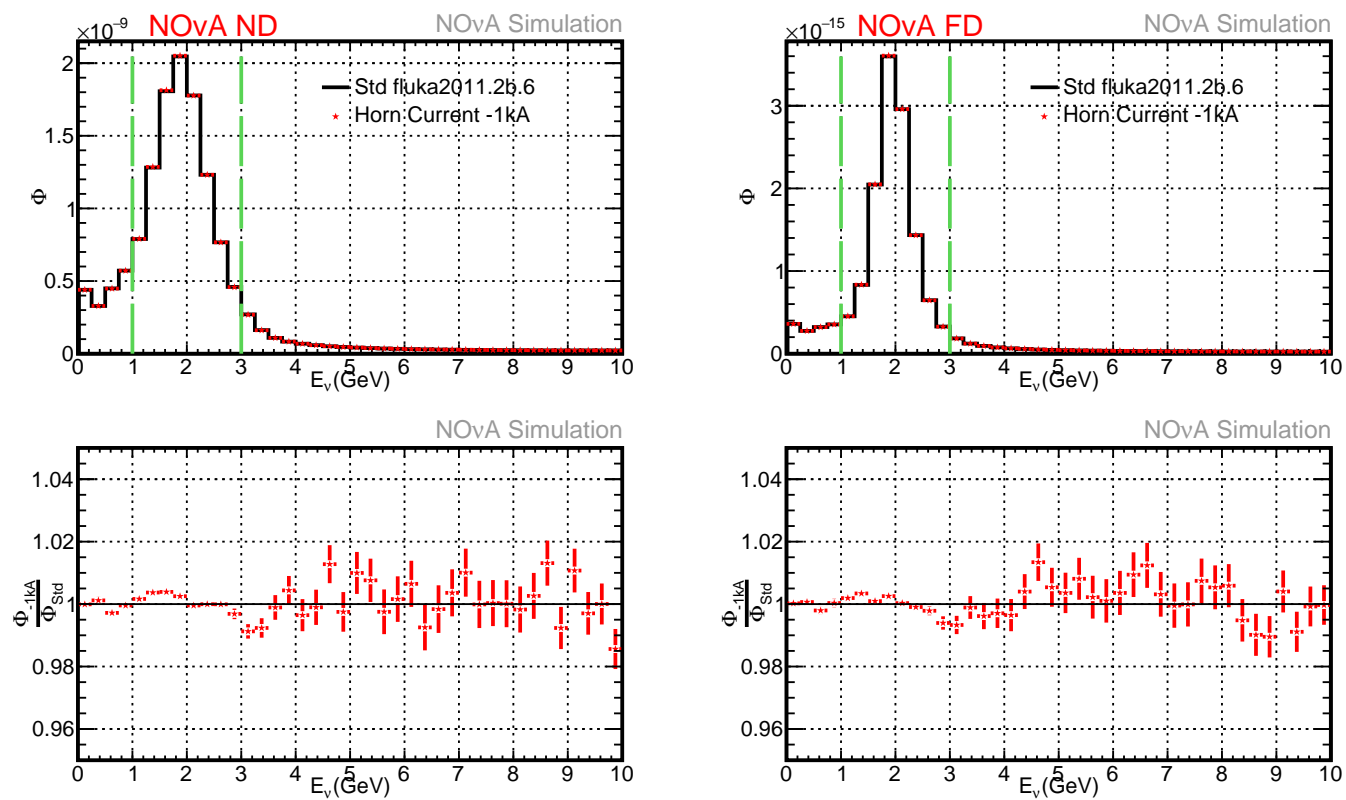

Figure 5.6: Top: FHC neutrino flux at NOA ND (top left) and FD (top right) for all neutrino parents, black is for nominal and red is for the $-2 \mathrm{kA}$ flux, respectively. Bottom: FHC neutrino fluxes ratio of the $-2 \mathrm{kA}$ flux with respect to the nominal flux at the ND (bottom left) and FD (bottom right). 

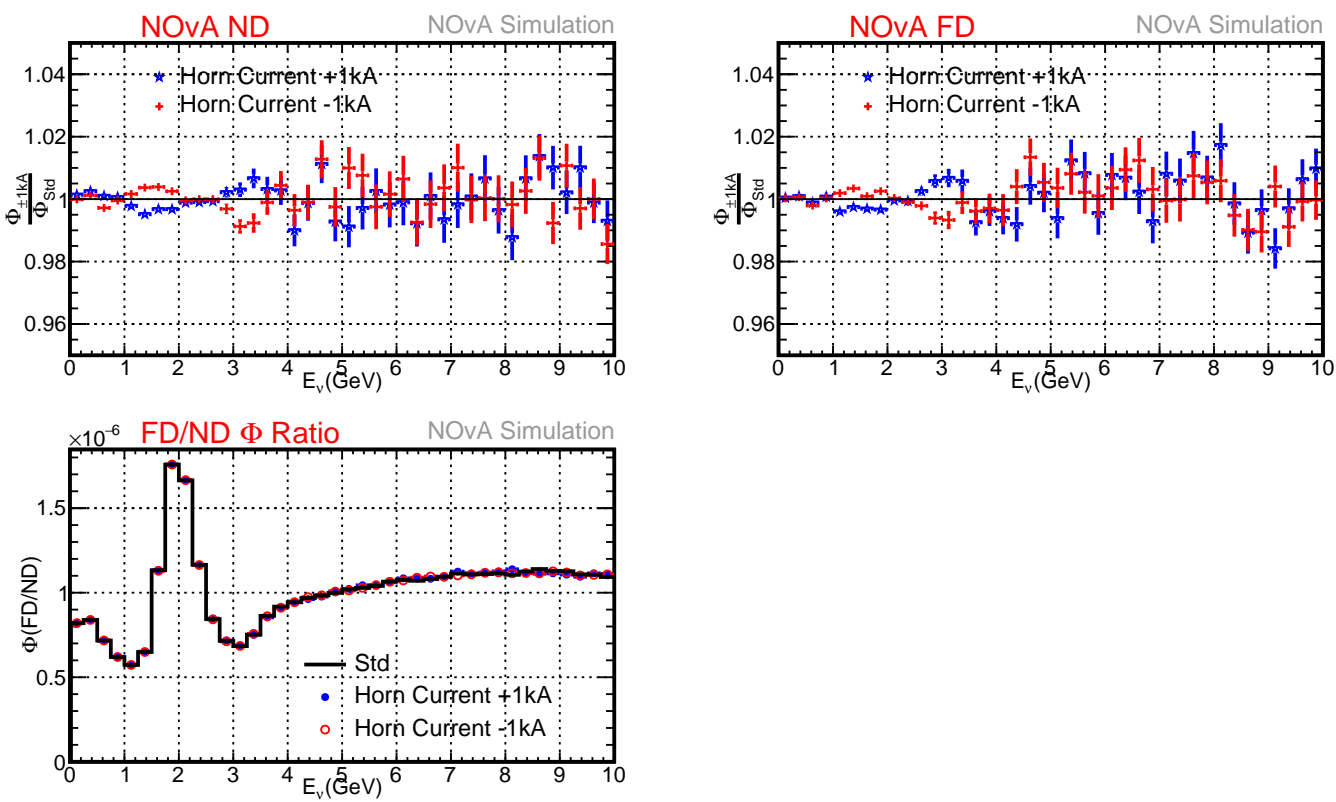

Figure 5.7: Top left: (ND) and top right (FD) is ratio of $\nu$ flux with variants, $\pm 1 \mathrm{kA}$ shift, to nominal $\nu$ flux $(200 \mathrm{kA})$ at NOvA, blue $(+1 \mathrm{kA})$ and red $(-1 \mathrm{kA})$ respectively. Bottom left: FD/ND flux, black is nominal (200 kA), blue is $+1 \mathrm{kA}$ shift $(201 \mathrm{kA})$ and red is $-1 \mathrm{kA}$ shift $(199 \mathrm{kA})$.
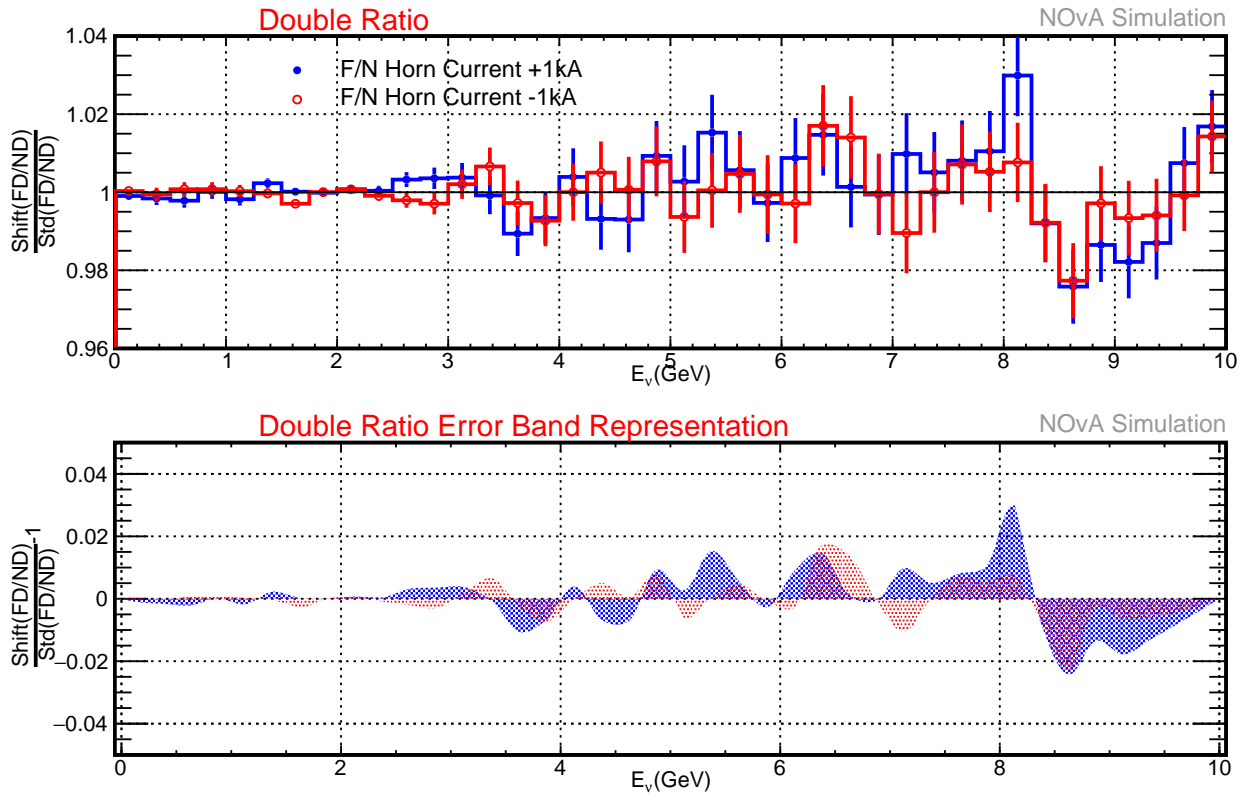

Figure 5.8: Top: Double-Ratio $\frac{\Phi_{F D / N D}(\text { variant })}{\Phi_{F D / N D}(\text { Std })}$ for $+1 \mathrm{kA}$ shift (blue) and $-1 \mathrm{kA}$ shift (red). Bottom: 1-Double-Ratio to show the effective band representation. 


\subsubsection{Horn Position Uncertainties}

Misalignment of the beamline elements causes changes to the nominal neutrino spectrum. It is estimated that the position of the horns was measured with an error of $3 \mathrm{~mm}$. Mismodelling of the horn position affects the focusing of secondary particles, which in turn affects the geometric ray tracing of pions and kaons through the horns and ultimately affects the final neutrino spectrum at the near and far detectors.

\subsubsection{Horn 1 Position Unceratinties}

Misalignment of the beamline elements causes changes to the nominal neutrino spectrum. Misalignment of Horn 1 position in the beam line as shown in Figure 5.4 affects the focusing of secondary particles, which in turn affects the geometric ray tracing of pions and kaons through the horns [67]) and ultimately affects the final neutrino spectrum at ND \& FD. Flux at FD \& ND changes by $\pm 2 \%$ in the $0-10 \mathrm{GeV}$ range for a $\pm 2 \mathrm{~mm}$ variation in $\mathrm{X}$ and $\mathrm{Y}$ as shown in Figures 5.9, 5.10 and 5.11. The FD/ND flux changes by less than $1 \%$ in the $0-10 \mathrm{GeV}$ range for a $\pm 2 \mathrm{~mm}$ variation in $\mathrm{X}$ and $\mathrm{Y}$, as shown in Figure 5.12 .

\subsubsection{Horn 2 Position Unceratinties}

Misalignment of Horn 2 position in the beam line as shown in Figure 5.4 affects the focusing of secondary particles, which in turn affects the geometric ray tracing of pions and kaons through the horns [67]) and ultimately affects the final neutrino spectrum at ND \& FD. Flux at FD \& ND changes by $\pm 2 \%$ in the $0-10 \mathrm{GeV}$ range for a $\pm 2 \mathrm{~mm}$ variation in $\mathrm{X}$ and $\mathrm{Y}$ as shown in Figures 5.13, 5.14 and 5.15. The FD/ND flux changes by less than $1 \%$ in the $0-10 \mathrm{GeV}$ range for a $\pm 2 \mathrm{~mm}$ variation in $\mathrm{X}$ and $\mathrm{Y}$, as shown in Figure 5.16 . 

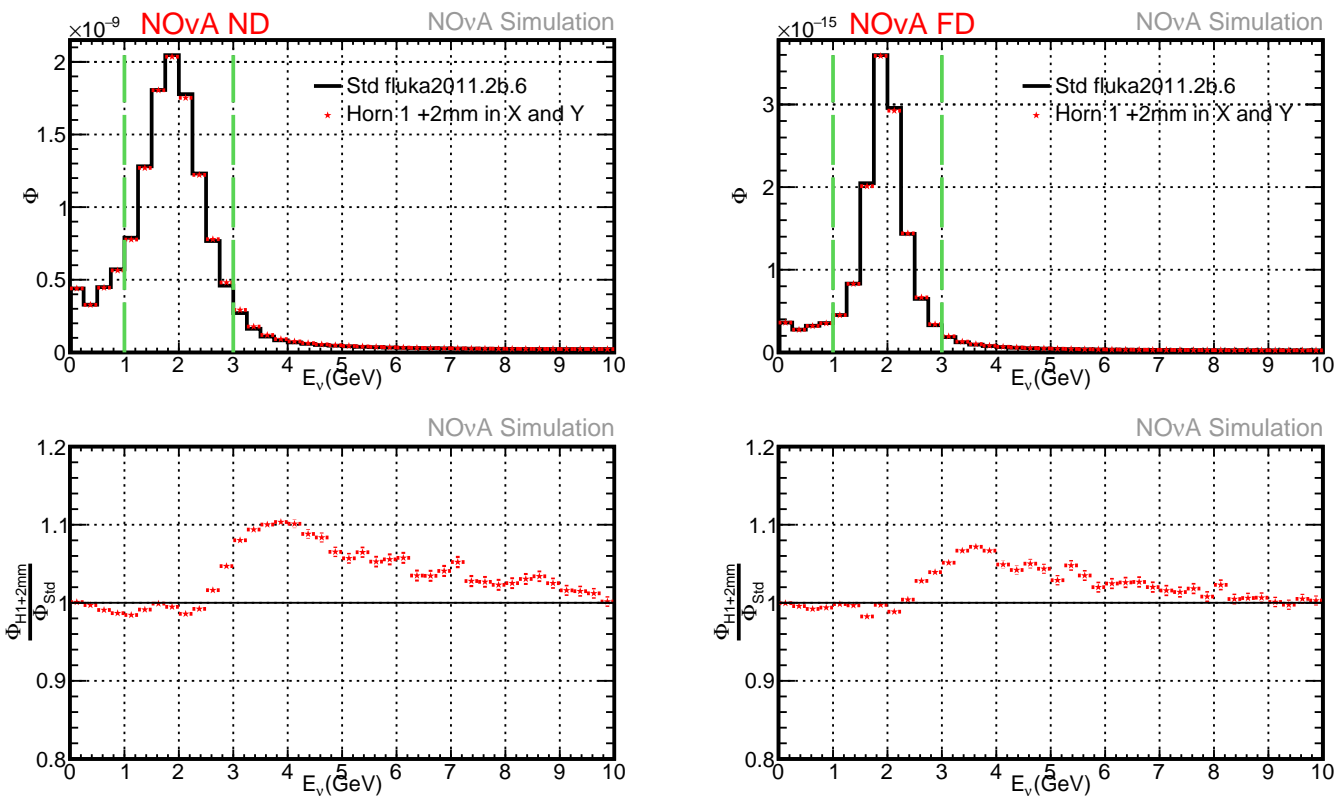

Figure 5.9: Top: FHC neutrino flux at NOvA ND (top left) and FD (top right) for all neutrino parents, black is for nominal flux and red is for the Horn1 flux with $+2 \mathrm{~mm}$ shift both in X and Y. Bottom: FHC neutrino fluxes ratio of the Horn1 flux with $+2 \mathrm{~mm}$ shift both in $\mathrm{X}$ and $\mathrm{Y}$ to the nominal flux at the ND (bottom left) and FD (bottom right).
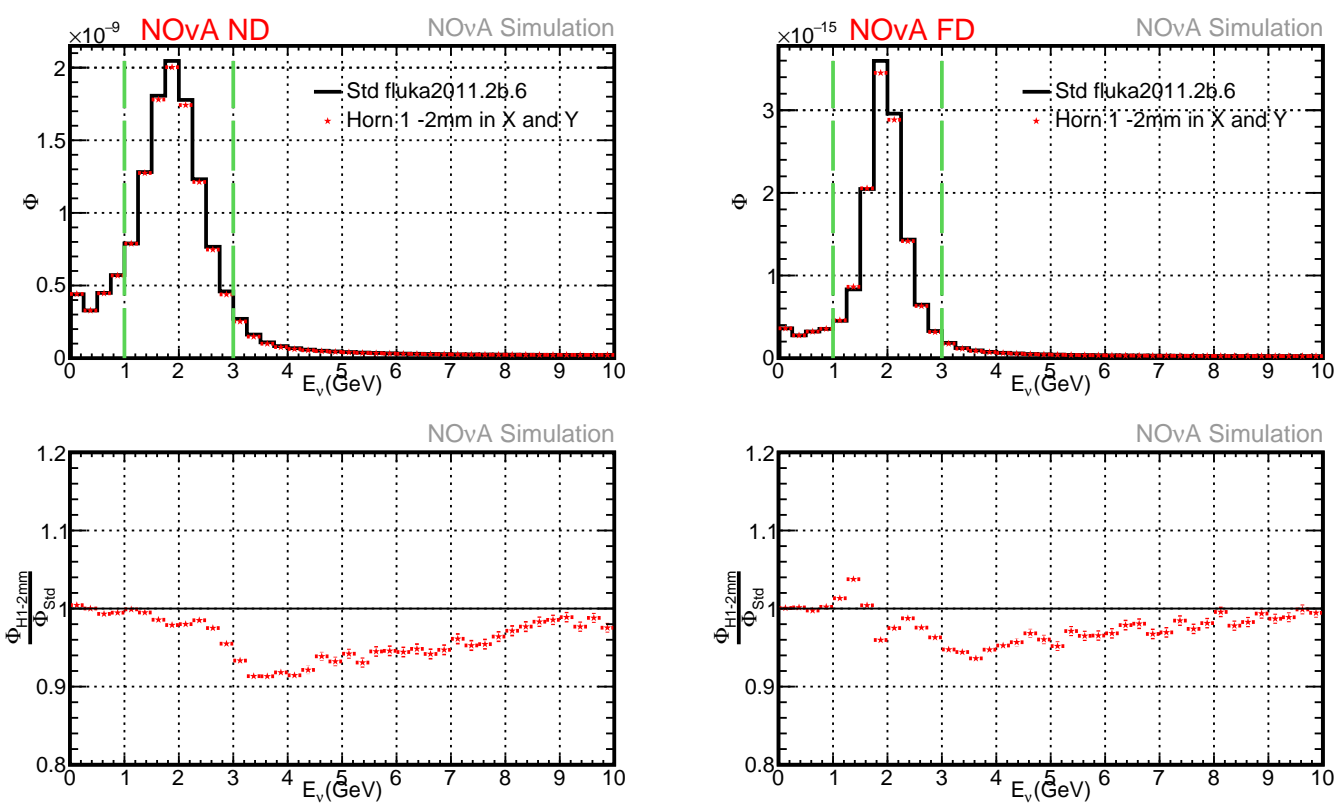

Figure 5.10: Top: FHC neutrino flux at NOvA ND (top left) and FD (top right) for all neutrino parents, black is for nominal flux and red is for the Horn1 flux with -2mm shift both in X and Y. Bottom: FHC neutrino fluxes ratio of the Horn1 flux with $-2 \mathrm{~mm}$ shift both in $\mathrm{X}$ and $\mathrm{Y}$ to the nominal flux at the ND (bottom left) and FD (bottom right). 

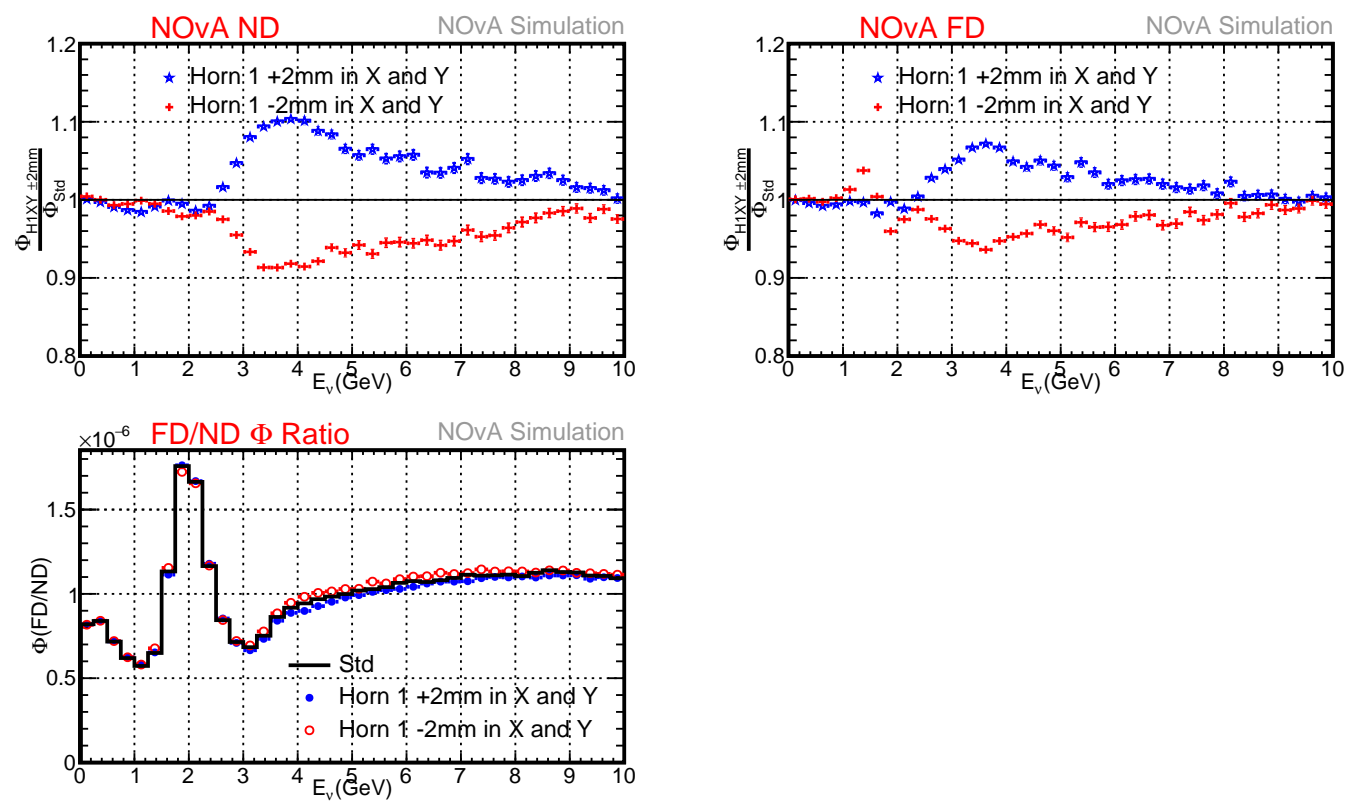

Figure 5.11: Top left (ND) and top right (FD) ratio of $\nu$ flux with variants, $\pm 2 \mathrm{~mm}$ shift both in $\mathrm{X}$ and $\mathrm{Y}$ position of Horn 1 to the nominal $\nu$ flux at NOvA, blue (for $+2 \mathrm{~mm}$ ) and red (for $-2 \mathrm{~mm}$ ). Bottom left: FD/ND flux, black is nominal, blue is $+2 \mathrm{~mm}$ shift and red is $-2 \mathrm{~mm}$ shift.
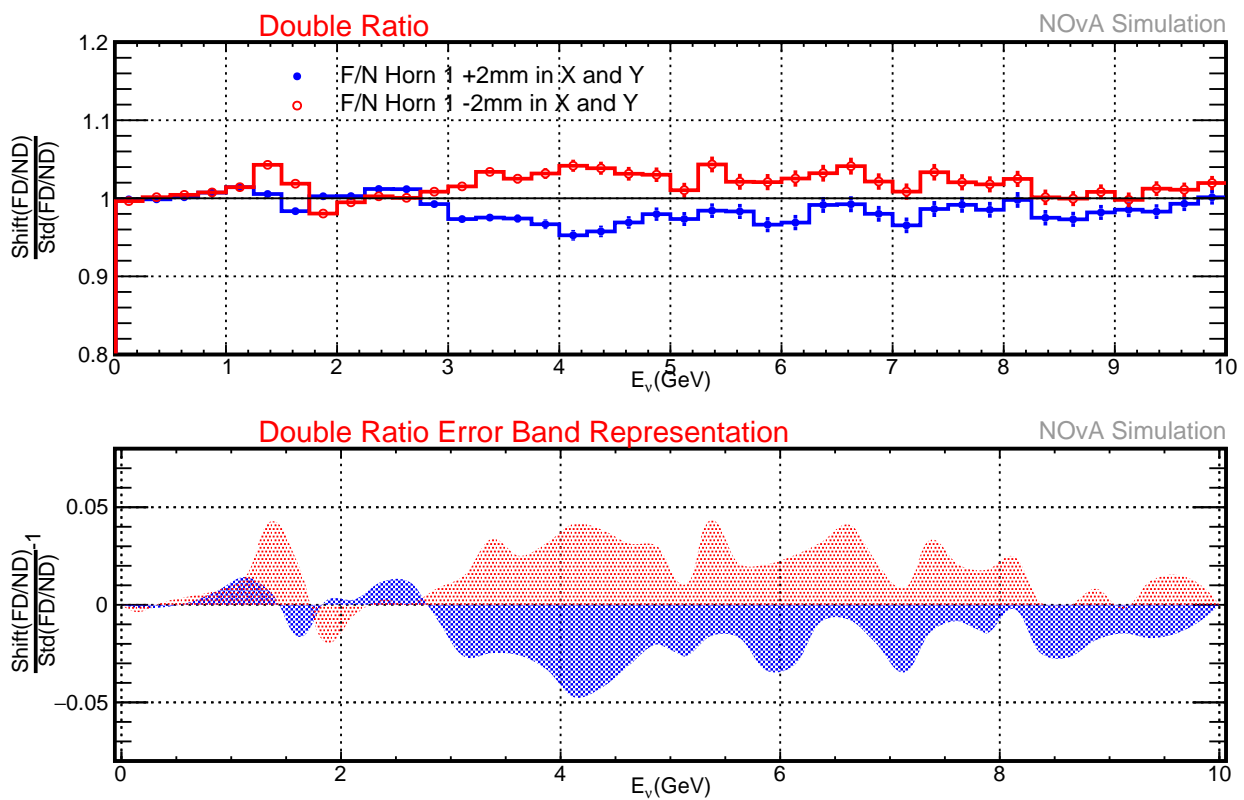

Figure 5.12: Top: Double-Ratio $\frac{\Phi_{F D / N D}(\text { variant) }}{\Phi_{F D / N D}(\text { Std })}$ for a $+2 \mathrm{~mm}$ shift (blue) and $-2 \mathrm{~mm}$ shift (red) in the Horn1 X \& Y position. Bottom: 1-Double-Ratio to show the effective band representation. 

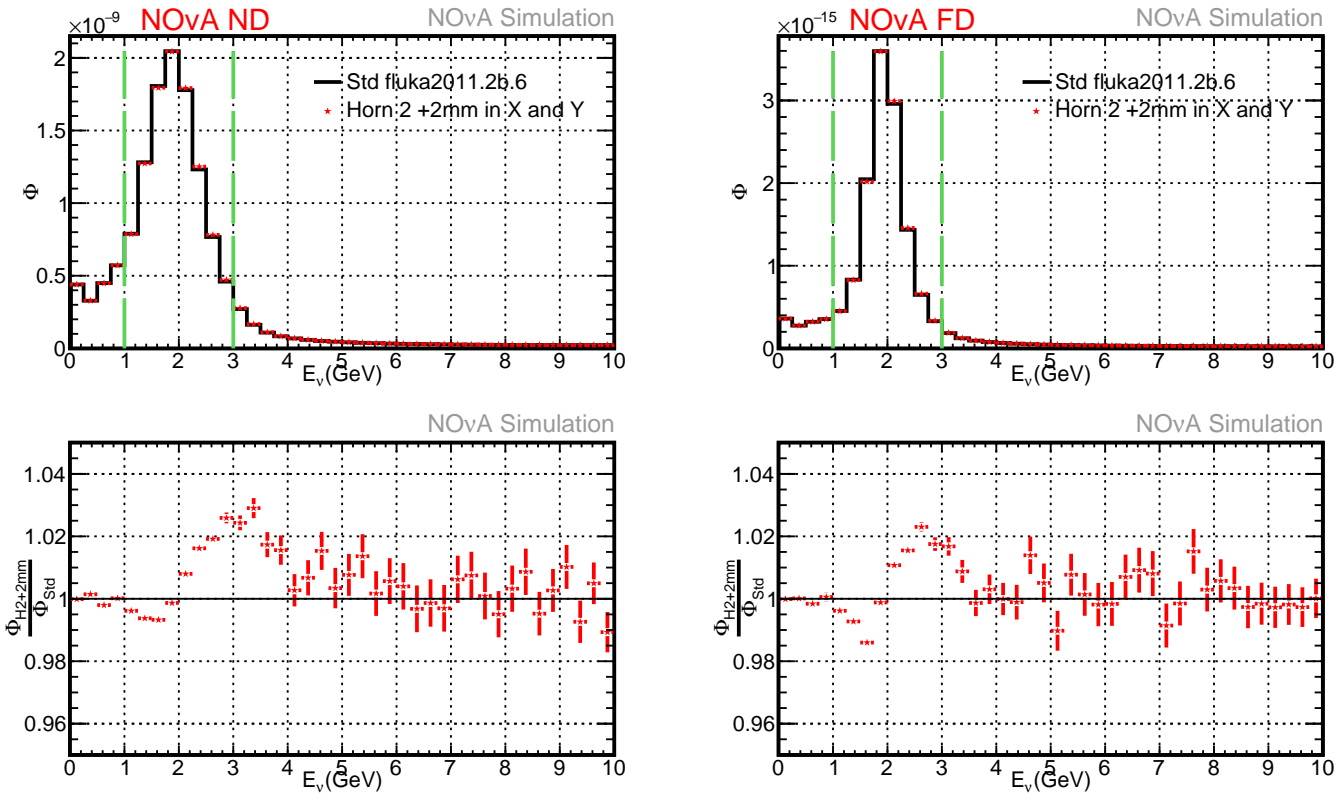

Figure 5.13: Top: FHC neutrino flux at NOvA ND (top left) and FD (top right) for all neutrino parents, black is for nominal flux and red is for the Horn2 flux with $+2 \mathrm{~mm}$ shift both in X and Y. Bottom: FHC neutrino fluxes ratio of the Horn2 flux with $+2 \mathrm{~mm}$ shift both in $\mathrm{X}$ and $\mathrm{Y}$ to the nominal flux at the ND (bottom left) and FD (bottom right).
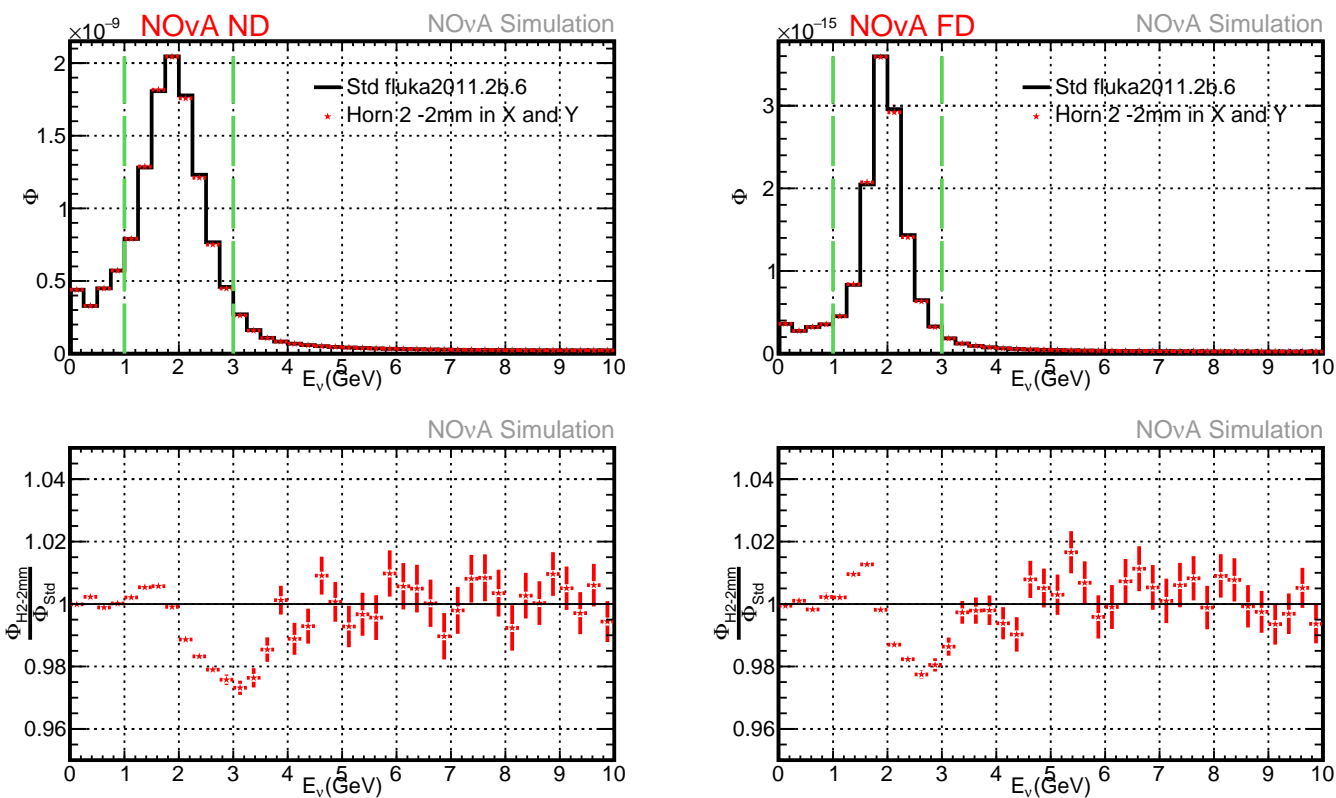

Figure 5.14: Top: FHC neutrino flux at NOvA ND (top left) and FD (top right) for all neutrino parents, black is for nominal flux and red is for the Horn2 flux with -2mm shift both in X and Y. Bottom: FHC neutrino fluxes ratio of the Horn2 flux with $-2 \mathrm{~mm}$ shift both in X and $\mathrm{Y}$ to the nominal flux at the ND (bottom left) and FD (bottom right). 

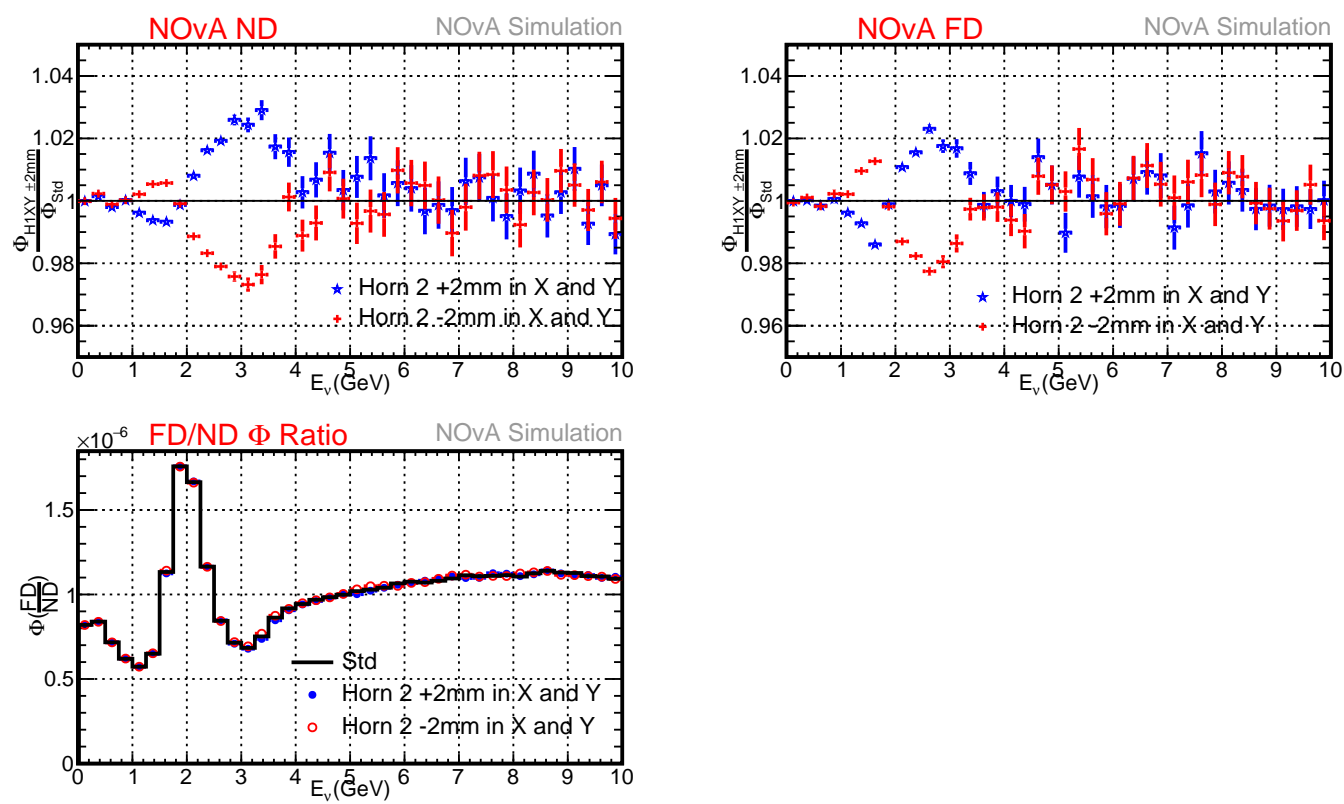

Figure 5.15: Top left (ND) and top right (FD) ratio of $\nu$ flux with variants, $\pm 2 \mathrm{~mm}$ shift both in $\mathrm{X}$ and $\mathrm{Y}$ position of Horn 2 to the nominal $\nu$ flux at NOvA, blue (for $+2 \mathrm{~mm}$ ) and red (for $-2 \mathrm{~mm}$ ). Bottom left: FD/ND flux, black is nominal, blue is $+2 \mathrm{~mm}$ shift and red is $-2 \mathrm{~mm}$ shift.
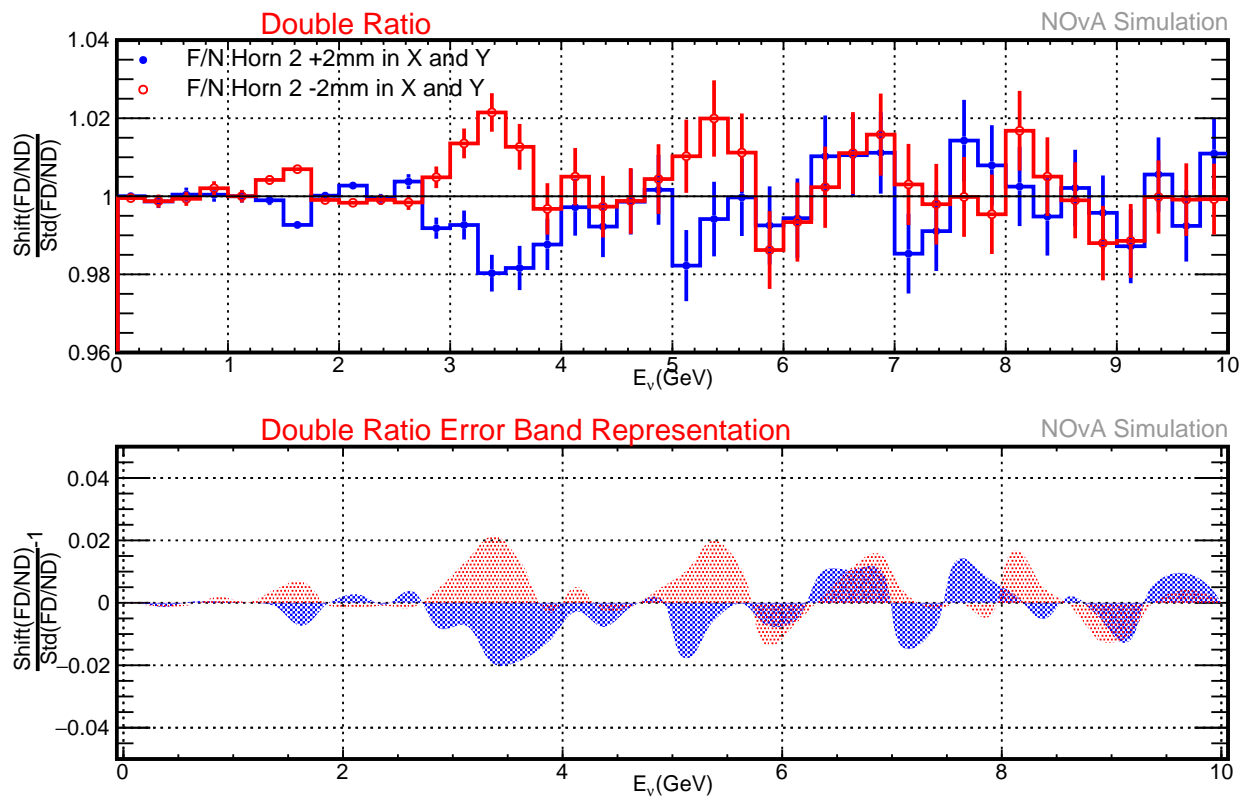

Figure 5.16: Top: Double-Ratio $\frac{\Phi_{F D / N D}(\text { variant })}{\Phi_{F D / N D}(\text { Std })}$ for a $+2 \mathrm{~mm}$ shift (blue) and $-2 \mathrm{~mm}$ shift (red) in the Horn1 X \& Y position. Bottom: 1-Double-Ratio to show the effective band representation. 


\subsubsection{Magnetic Field Distribution in the Horns Conductor}

Another uncertainty comes from the modeling of the magnetic field distribution inside the material (aluminum) of the horn. The default model in the beam simulation uses a magnetic field that decreases linearly [56]. We consider an alternative model which makes the simplistic assumption that the magnetic field decreases exponentially with the skin depth $(0.77 \mathrm{~cm}$ skin depth). We assume that the difference in the neutrino flux induced by the change of magnetic field model conservatively brackets the systematic error on the magnetic field distribution inside the horn material. Flux at FD \& ND changes by $\pm 4 \%$ in the $0-10 \mathrm{GeV}$ range as shown in Figures 5.17 and 5.18. The FD/ND flux changes smaal in the $1-3 \mathrm{GeV}$ range, as shown in Figure 5.19 .
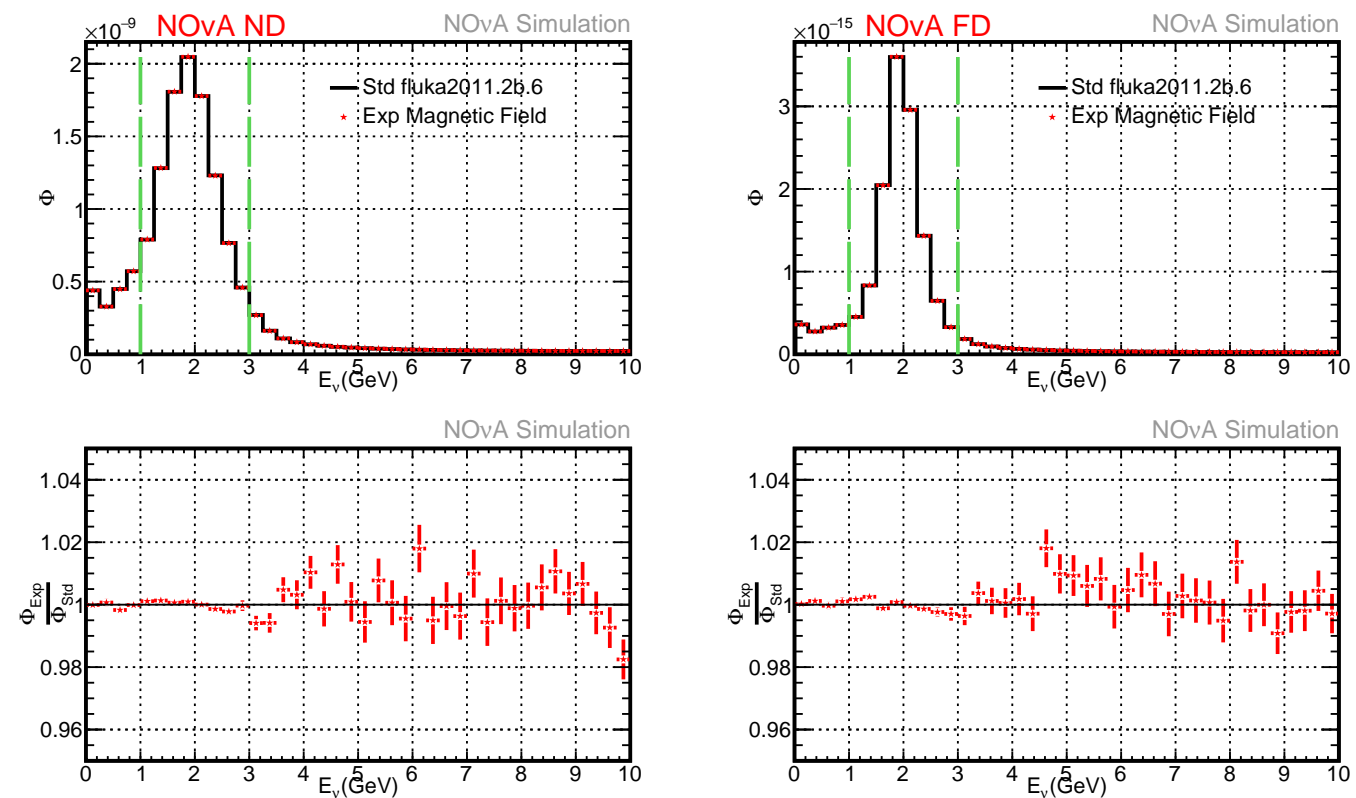

Figure 5.17: Top left (ND) and top right (FD) $\nu$ flux at NOvA for all neutrino parents, black is for nominal (linear magnetic field), and red is for the exponential magnetic field. Bottom left(ND) and bottom right (FD) $\nu$ flux ratio of exponential magnetic field to nominal at NOvA. 

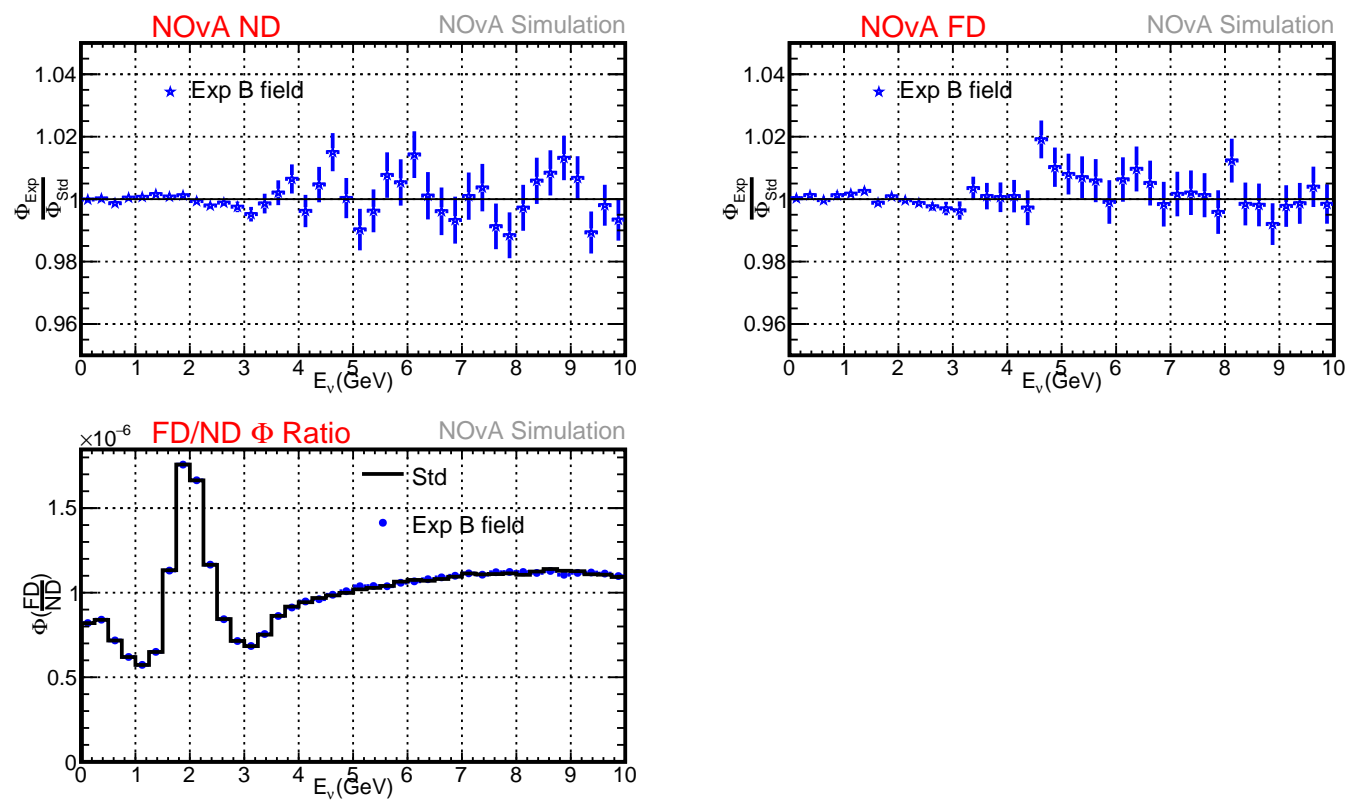

Figure 5.18: Top: Double-Ratio $\frac{\Phi_{F D / N D}(\text { variant) }}{\Phi_{F D / N D}(\text { Std })}$ for linear (blue) and exponential (red) magnetic field. Bottom: 1-Double-Ratio to show the effective band representation.
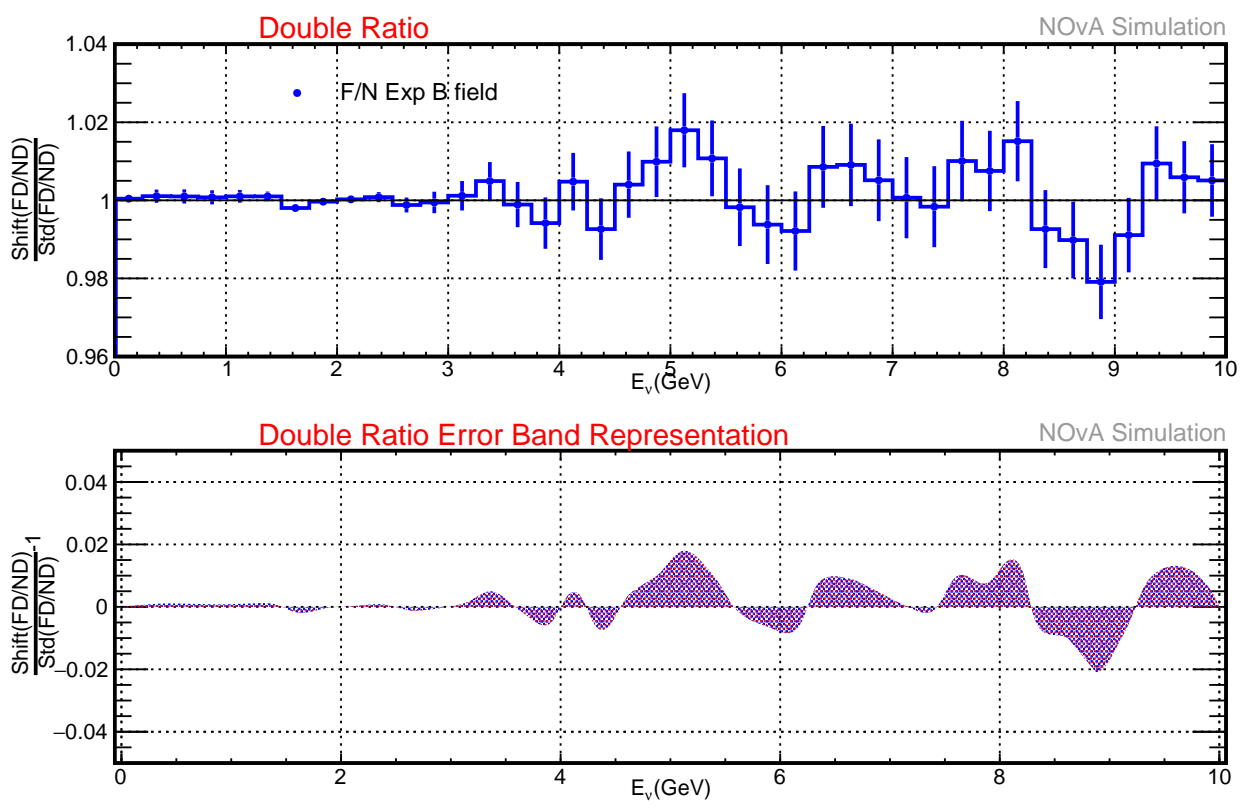

Figure 5.19: Top: Double-Ratio $\frac{\Phi_{F D / N D}(\text { variant) }}{\Phi_{F D / N D}(\text { Std })}$ for linear (blue) and exponential (red) magnetic field. Bottom: 1-Double-Ratio to show the effective band representation. 


\subsubsection{Beam Position on the Target}

Previous scans of the proton beam across the target [67] showed that the uncertainty on the beam position is $\pm 0.5 \mathrm{~mm}$ in both $\mathrm{X} \& \mathrm{Y}$ directions, as shown in Figure 5.20 . The NOvA detectors being off-axis, we expect that uncertainty to have a symmetric effect on the neutrino flux along $\mathrm{X}$ and asymmetric along $\mathrm{Y}$ (MINOS sees a symmetric effect along both $\mathrm{X}$ and $\mathrm{Y}$ as expected for an on-axis experiment [69]). Flux at FD \& ND changes by $\pm 4 \%$ in the $0-10 \mathrm{GeV}$ range for a $\pm 0.5 \mathrm{~mm}$ variation along the $\mathrm{X}$ direction, as shown in Figures 5.21 and 5.22 . Flux at FD \& ND changes by $-1 \%$ in the $0-10 \mathrm{GeV}$ range for a $\pm 0.5 \mathrm{~mm}$ variation along the $\mathrm{Y}$ direction, as shown in Figures 5.23 and 5.24 . The FD/ND flux changes by $\pm 1 \%$ in the 0 - $10 \mathrm{GeV}$ range for a variation of $\pm 0.5 \mathrm{~mm}$ in both $\mathrm{X}$ and $\mathrm{Y}$ directions, as shown in Figures 5.25, 5.26, 5.27 and 5.28.

\section{NOvA-era ME running}

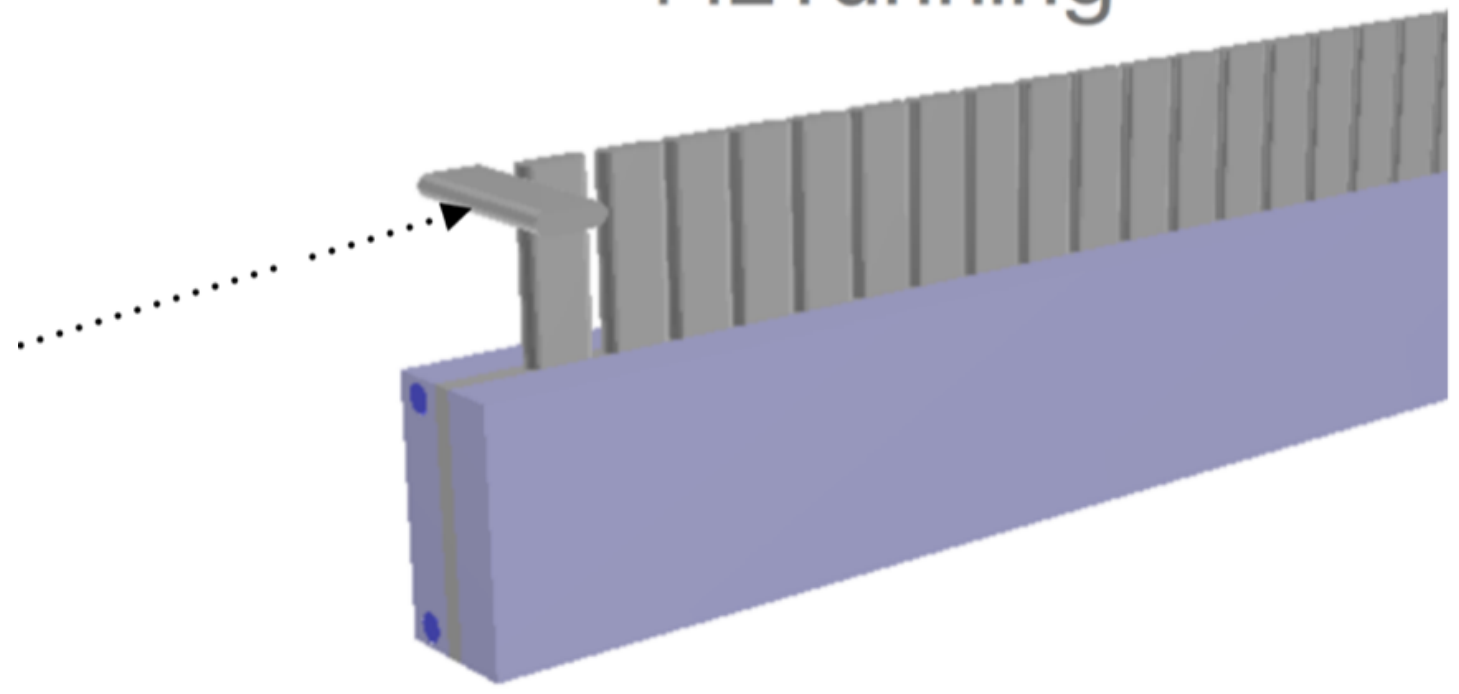

Figure 5.20: NuMI Proton Beam hits the Medium Energy target, the uncertainties in the proton beam position on target affects the final flux. 

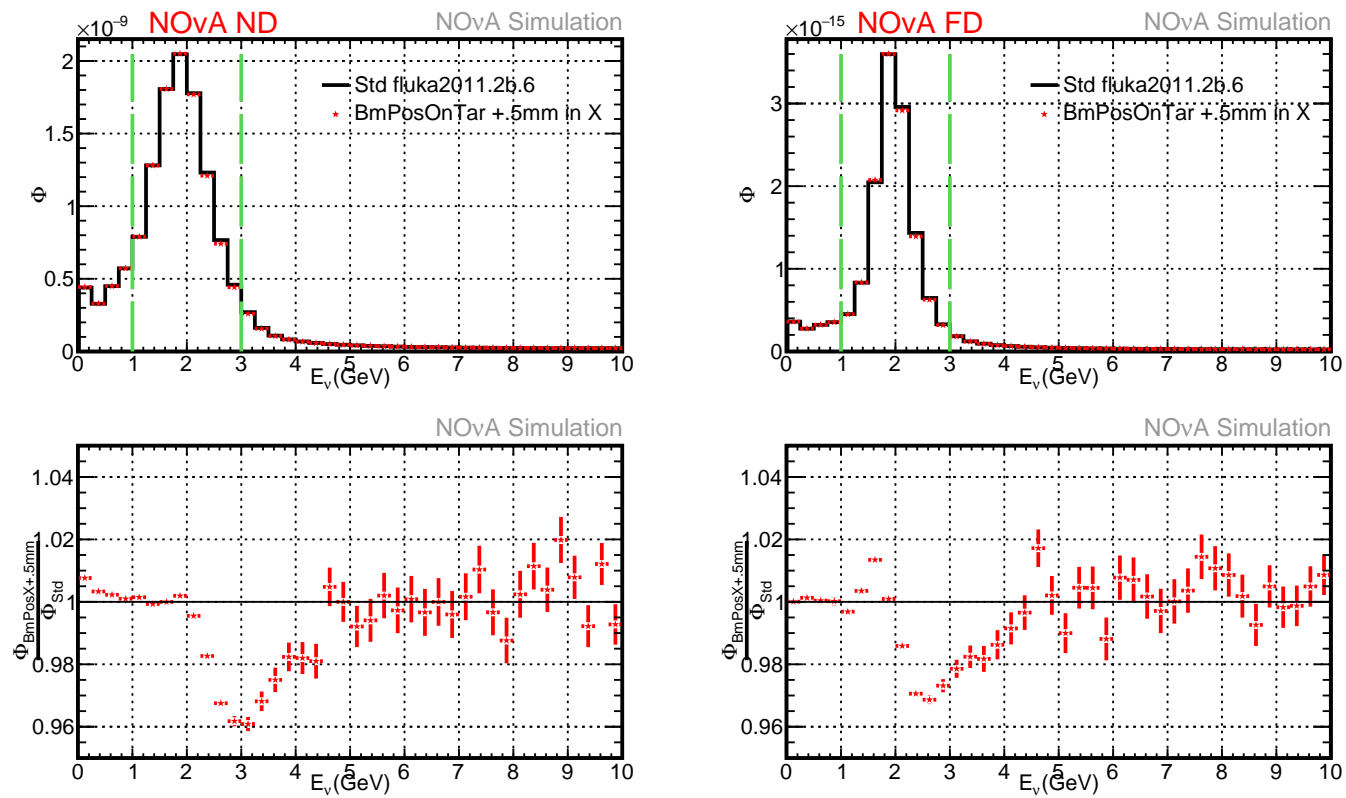

Figure 5.21: Top left (ND) and top right (FD) $\nu$ flux at NOvA for all neutrino parents, black is for nominal and red is for the $+0.5 \mathrm{~mm}$ shift of the beam position along the $\mathrm{X}$ direction. Bottom left (ND) and bottom right (FD) $\nu$ fluxes ratio of $+0.5 \mathrm{~mm}$ shift of the beam position along the $\mathrm{X}$ direction to nominal at NOvA.
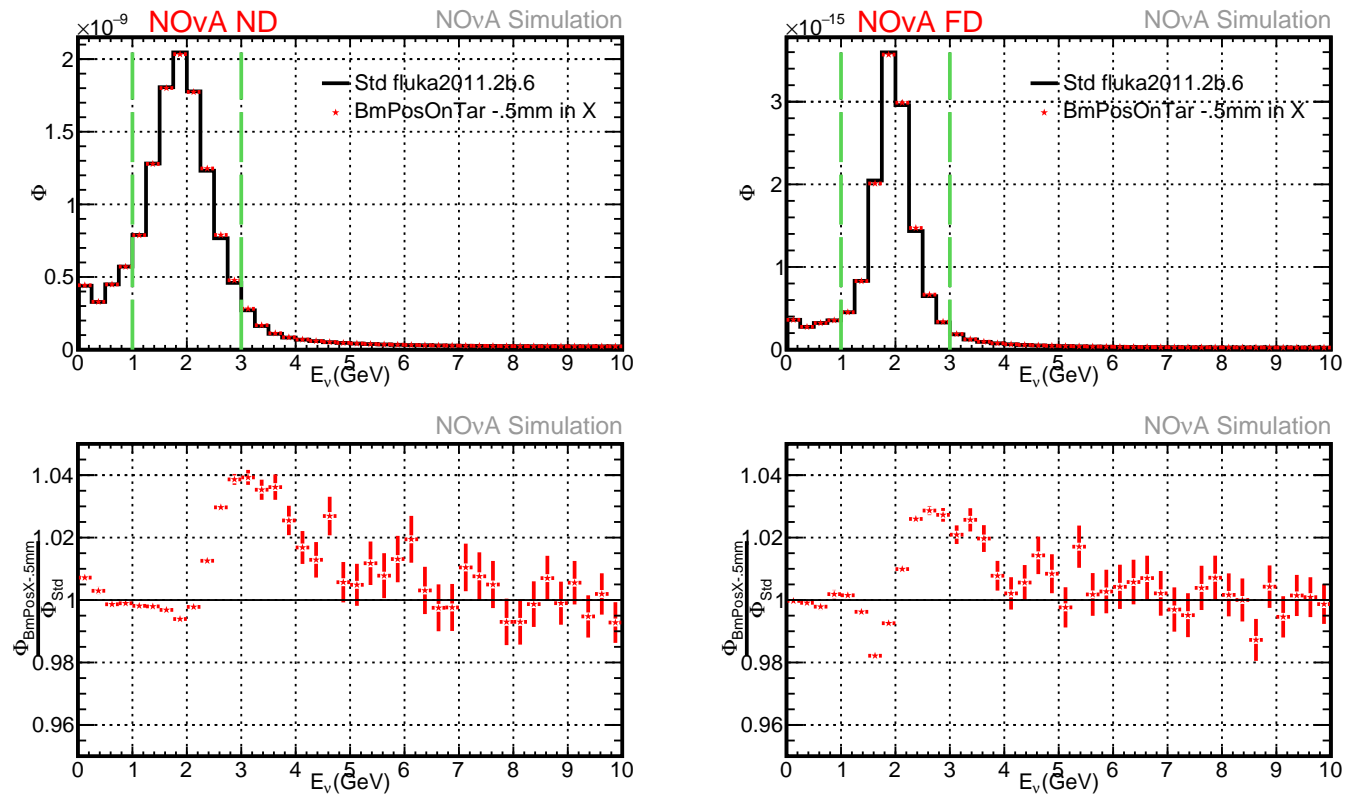

Figure 5.22: Top left (ND) and top right (FD) $\nu$ flux at NOvA for all neutrino parents, black is for nominal and red is for the $-0.5 \mathrm{~mm}$ shift of the beam position along the $\mathrm{X}$ direction. Bottom left (ND) and bottom right (FD) $\nu$ fluxes ratio of $-0.5 \mathrm{~mm}$ shift of the beam position along the $\mathrm{X}$ direction to nominal at NOvA. 

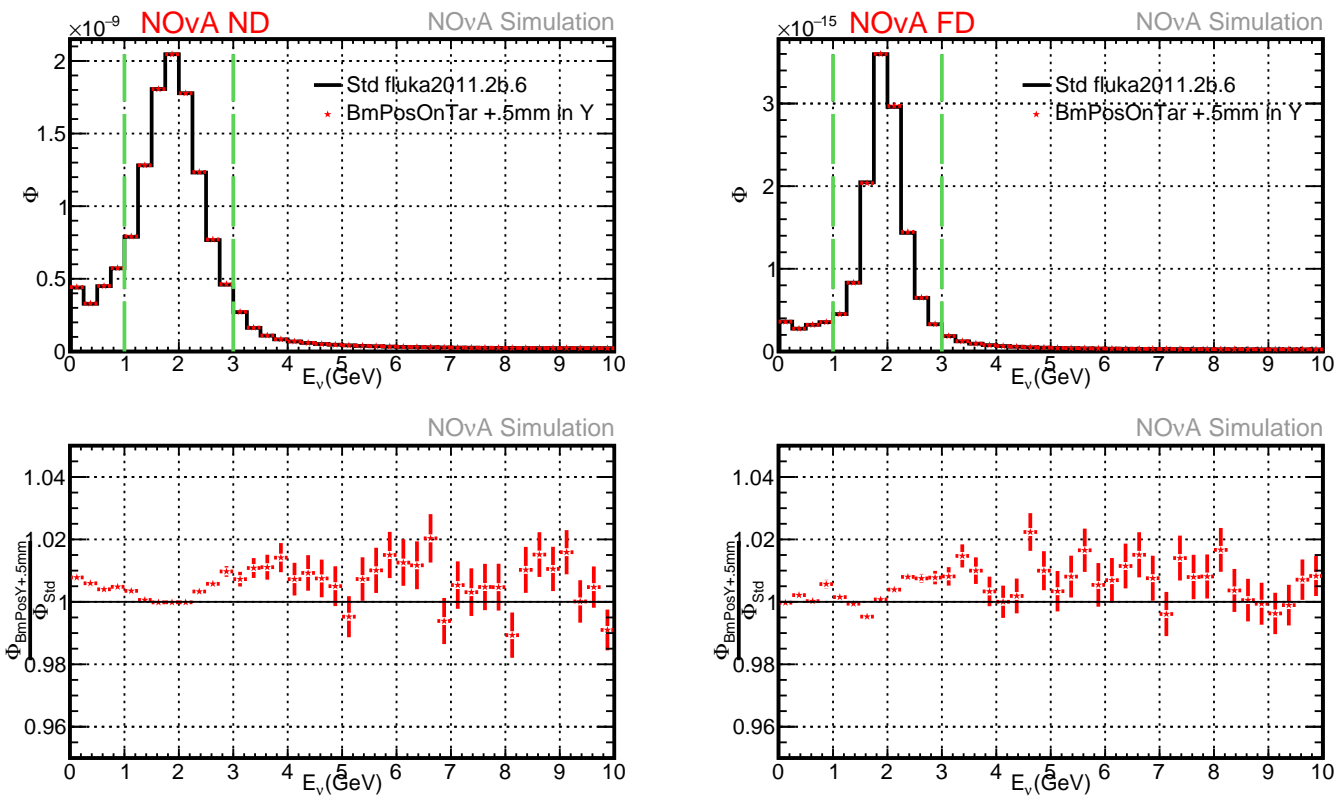

Figure 5.23: Top left (ND) and top right (FD) $\nu$ flux at NOvA for all neutrino parents, black is for nominal and red is for the $+0.5 \mathrm{~mm}$ shift of the beam position along the $\mathrm{Y}$ direction. Bottom left (ND) and bottom right (FD) $\nu$ fluxes ratio of $+0.5 \mathrm{~mm}$ shift of the beam position along the $\mathrm{Y}$ direction to nominal at NOvA.
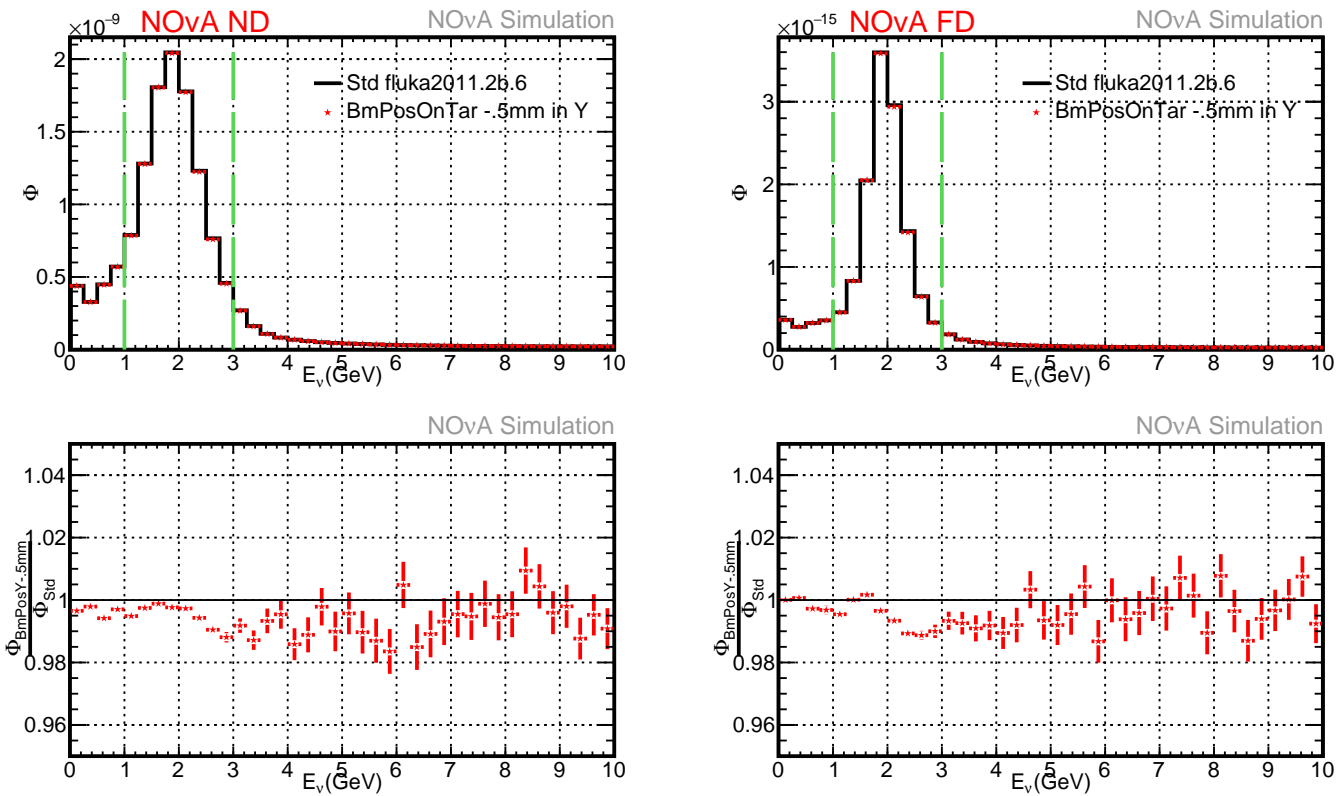

Figure 5.24: Top left (ND) and top right (FD) $\nu$ flux at NOvA for all neutrino parents, black is for nominal and red is for the $-0.5 \mathrm{~mm}$ shift of the beam position along the $\mathrm{Y}$ direction. Bottom left (ND) and bottom right (FD) $\nu$ fluxes ratio of $-0.5 \mathrm{~mm}$ shift of the beam position along the $\mathrm{Y}$ direction to nominal at NOvA. 

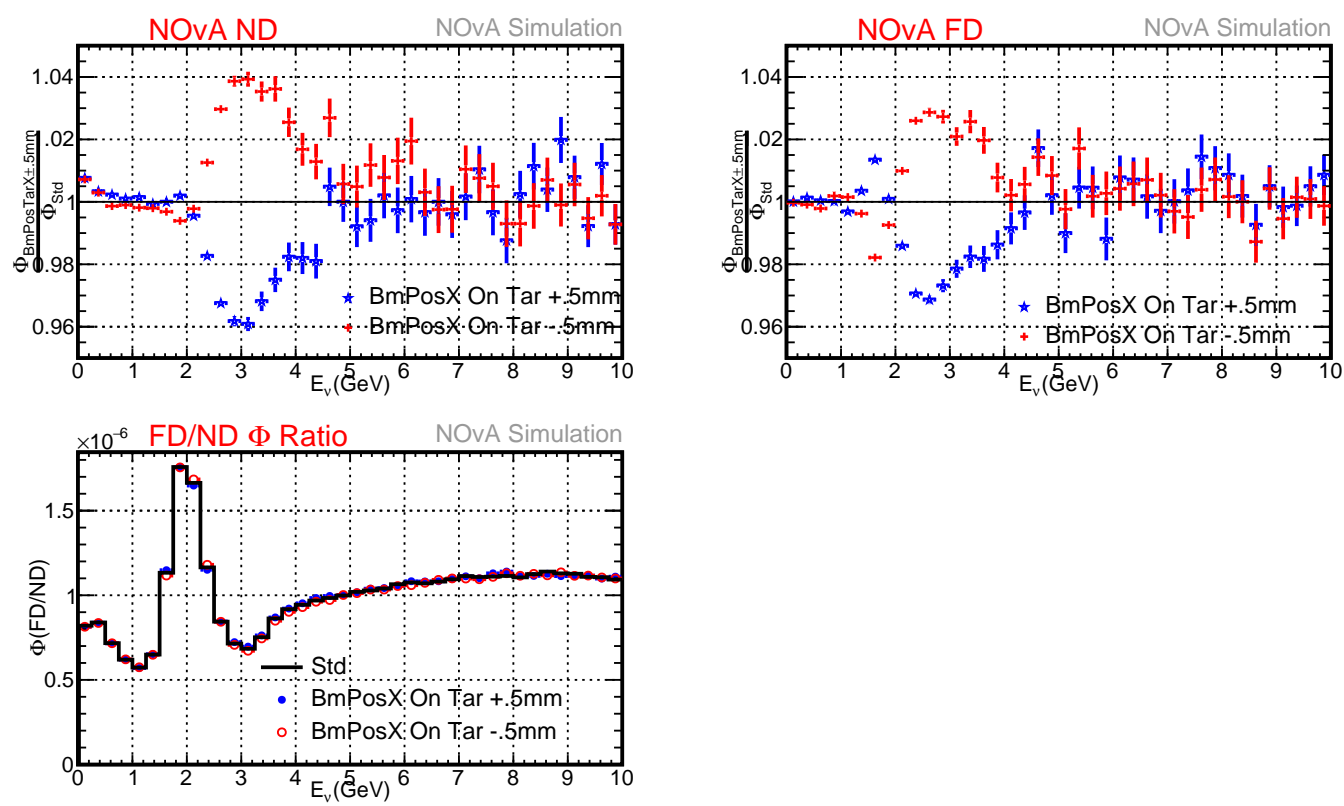

Figure 5.25: Top left (ND) and top right (FD), ratio of $\nu$ fluxes with variants, $\pm 0.5 \mathrm{~mm}$ shift of the beam position along the $\mathrm{X}$ direction, blue $(+0.5 \mathrm{~mm})$ and red $(-0.5 \mathrm{~mm})$, to nominal $\nu$ flux at NOvA. Bottom left: FD/ND flux, black is nominal, blue is $+0.5 \mathrm{~mm}$ shift and red is $-0.5 \mathrm{~mm}$ shift of the beam position along the $\mathrm{X}$ direction.
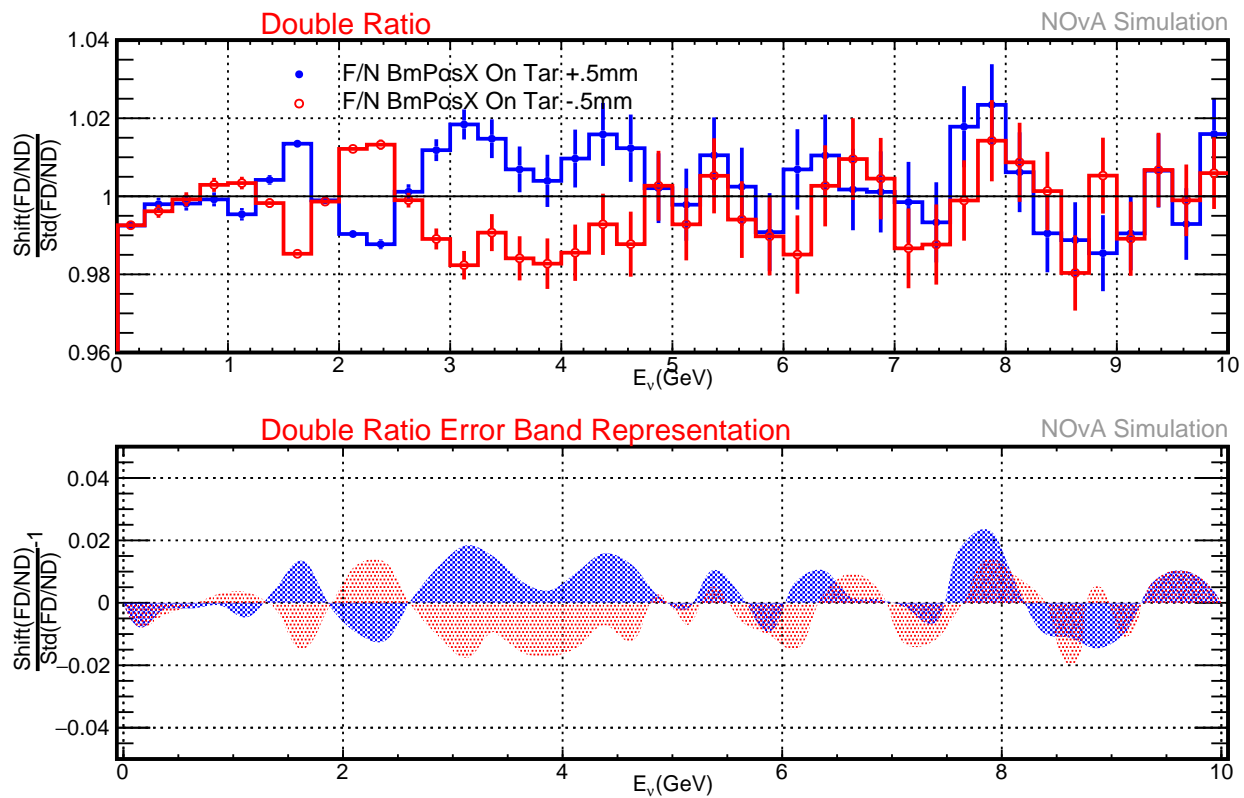

Figure 5.26: Top: Double-Ratio $\frac{\Phi_{F D / N D}(\text { variant })}{\Phi_{F D / N D}(\text { Std })}$ for $+0.5 \mathrm{~mm}$ shift (blue) and $-0.5 \mathrm{~mm}$ shift (red) in beam position on target along the X direction. Bottom: 1-Double-Ratio to show the effective band representation. 

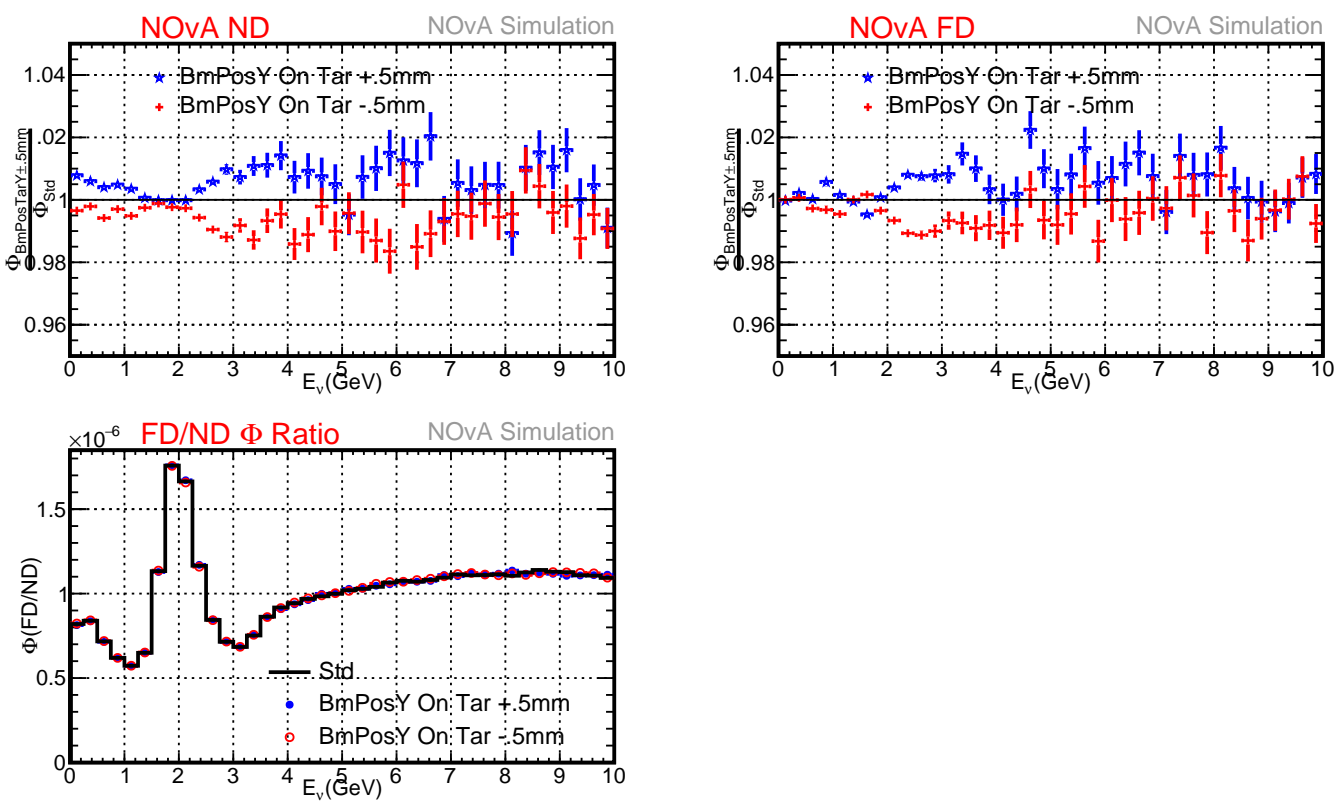

Figure 5.27: Top left (ND) and top right (FD), ratio of $\nu$ fluxes with variants, $\pm 0.5 \mathrm{~mm}$ shift of the beam position along the $\mathrm{Y}$ direction, blue $(+0.5 \mathrm{~mm})$ and red $(-0.5 \mathrm{~mm})$, to nominal $\nu$ flux at NOvA. Bottom left: FD/ND flux, black is nominal, blue is $+0.5 \mathrm{~mm}$ shift and red is $-0.5 \mathrm{~mm}$ shift of the beam position along the $\mathrm{Y}$ direction.
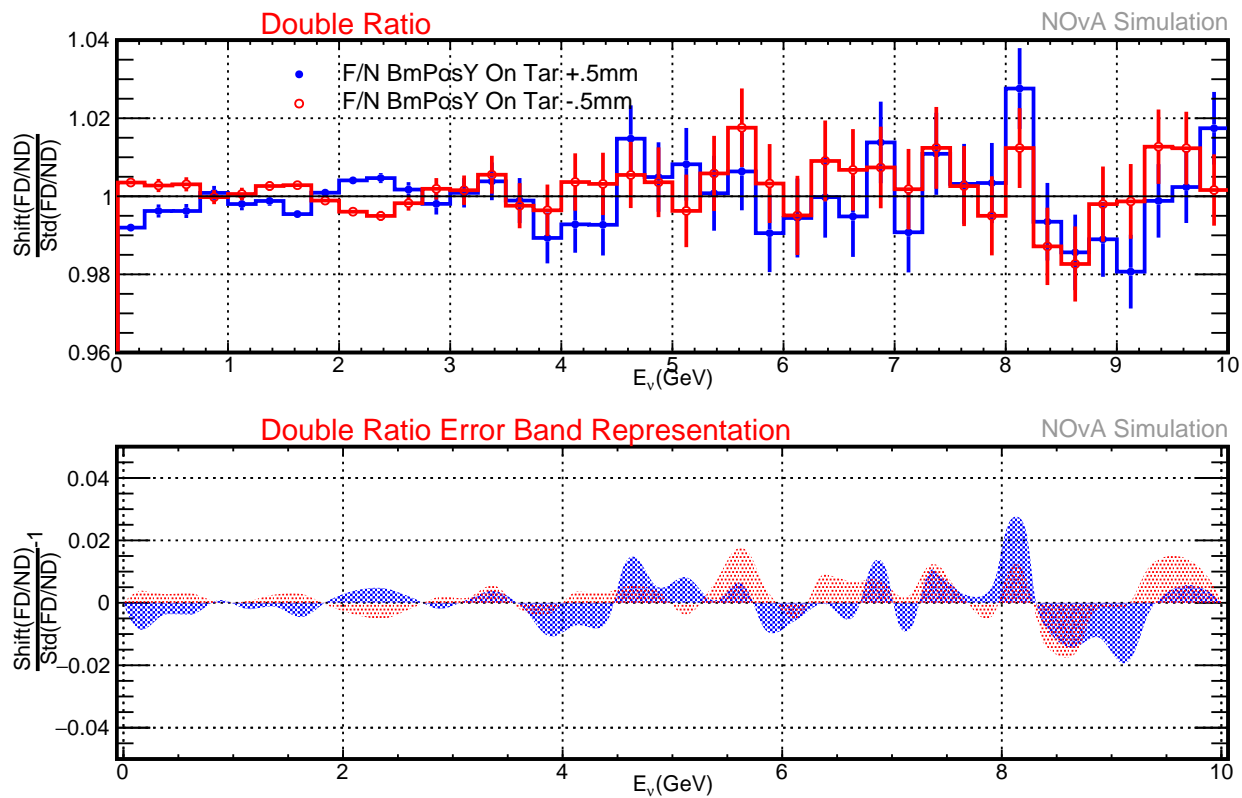

Figure 5.28: Top: Double-Ratio $\frac{\Phi_{F D / N D}(\text { variant) }}{\Phi_{F D / N D}(\text { Std })}$ for $+0.5 \mathrm{~mm}$ shift (blue) and $-0.5 \mathrm{~mm}$ shift (red) in beam position on target along the Y direction. Bottom: 1-Double-Ratio to show the effective band representation. 


\subsubsection{Beam Spot Size}

The nominal beam simulation has beam spot size $1.1 \mathrm{~mm}$. For this study we varied it by $-0.2 \mathrm{~mm}$ both in $\mathrm{X} \& \mathrm{Y}$ i.e. $0.9 \mathrm{~mm}$ both in $\mathrm{X} \& \mathrm{Y}$ and by $+0.4 \mathrm{~mm}$ both in $\mathrm{X} \& \mathrm{Y}$ i.e. $1.4 \mathrm{~mm}$ both in $\mathrm{X} \& \mathrm{Y}$. The value of the shift is obtained by taking the difference between the beam spot size corresponding to two NuMI running modes [68, [70], $0.9 \mathrm{~mm}$ for medium energy configuration. The later $1.5 \mathrm{~mm}$ is the beam spot size expected when running NuMI at $700 \mathrm{~kW}$ [71]. Flux at FD \& ND changes $\leq 1 \%$ for $-0.2 \mathrm{~mm}$ both in $\mathrm{X} \& \mathrm{Y}$ and changes $\leq 3 \%$ for $+0.4 \mathrm{~mm}$ both in $\mathrm{X} \& \mathrm{Y}$ as shown in Figures $5.29-5.30$. The effect is negligible at $2 \mathrm{GeV}$ for $\mathrm{FD} / \mathrm{ND}$ ratio for $-0.2 \mathrm{~mm}$ both in $\mathrm{X} \& \mathrm{Y}$ and for $+0.4 \mathrm{~mm}$ both in $\mathrm{X} \& \mathrm{Y}$ as shown in Figure 5.31-5.34.
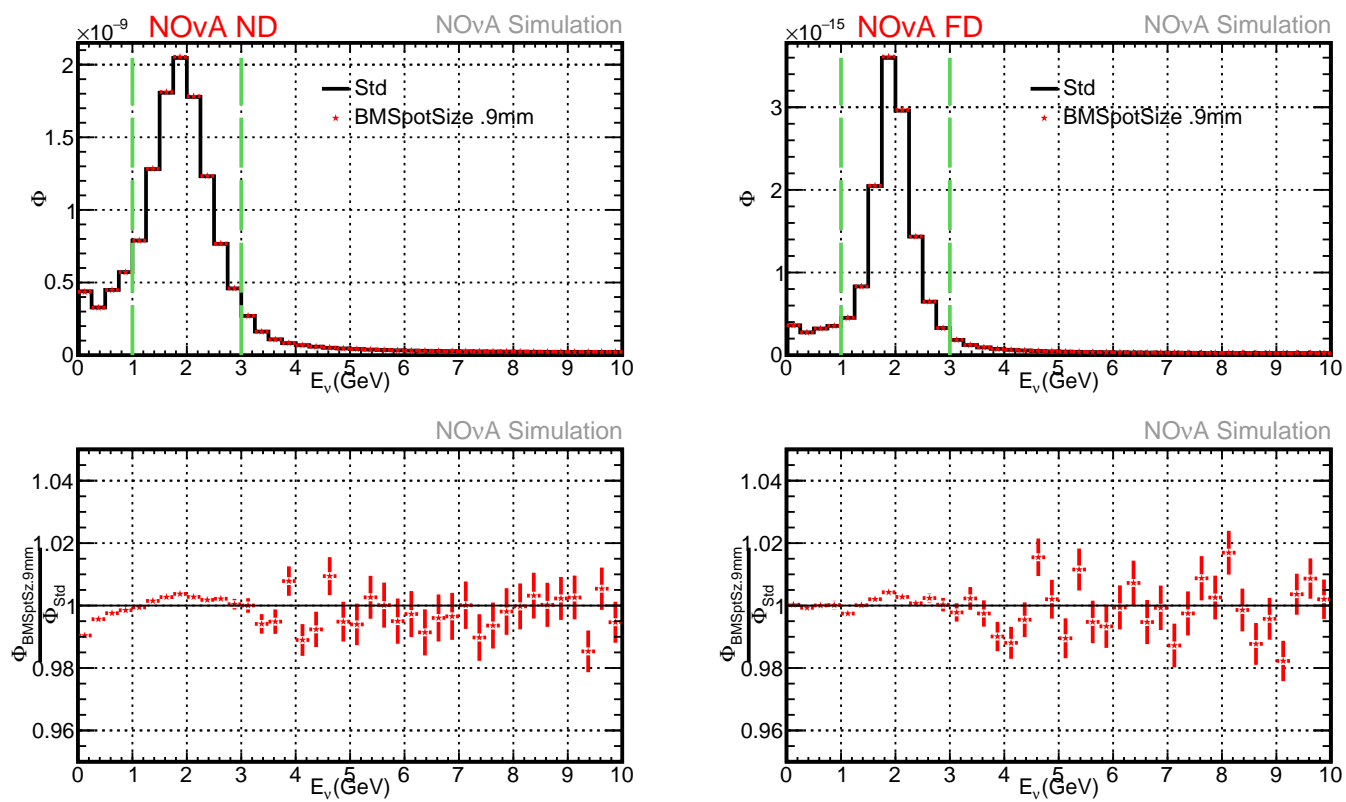

Figure 5.29: Top left (ND) and top right (FD), $\nu$ flux at NOvA for all neutrino parents, black is for nominal and red is for the $-0.2 \mathrm{~mm}$ shift of the beam spot size in both $\mathrm{X} \&$ $\mathrm{Y}$ directions. Bottom left (ND) and bottom right (FD), $\nu$ fluxes ratio of $-0.2 \mathrm{~mm}$ shift of the beam spot size in both X \& Y directions to nominal at NOvA. 

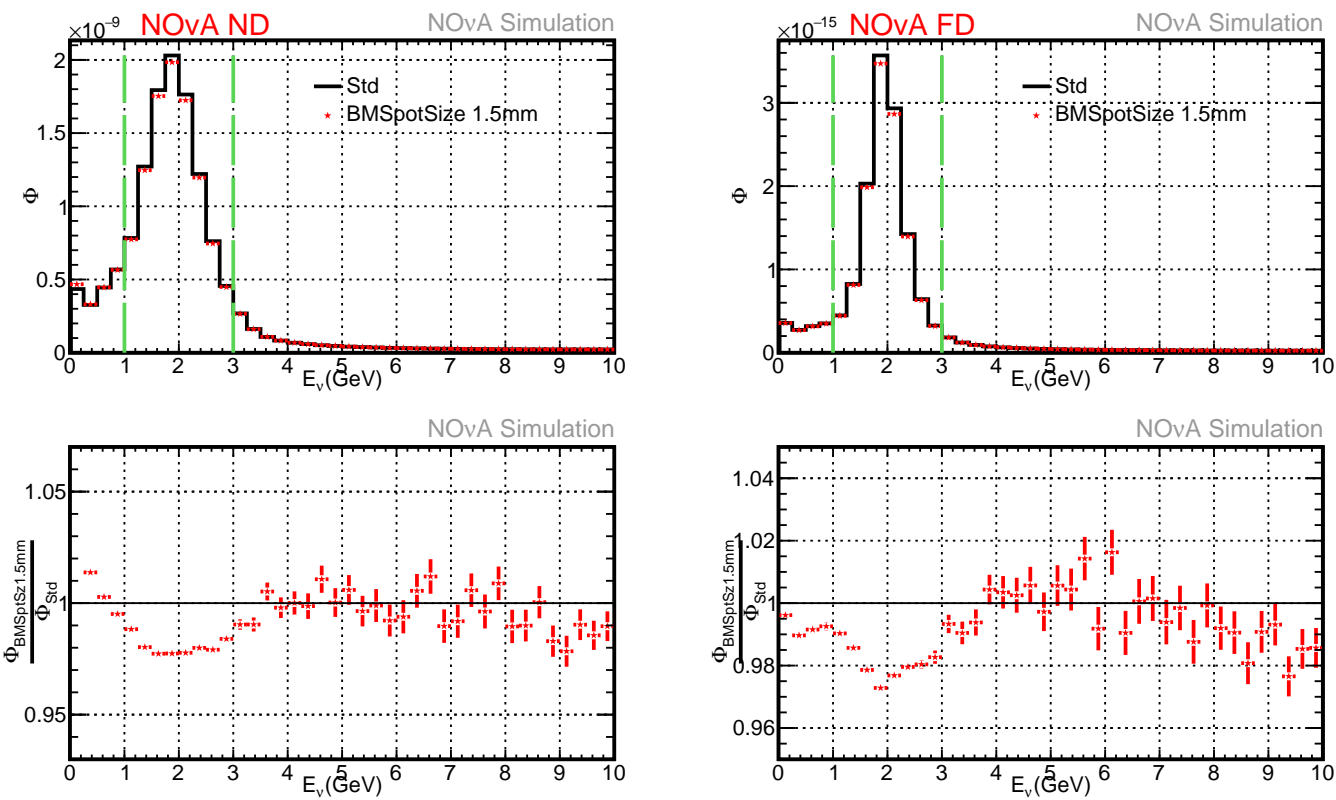

Figure 5.30: Top left (ND) and top right (FD), $\nu$ flux at NOvA for all neutrino parents, black is for nominal and red is for the $+0.4 \mathrm{~mm}$ shift of the beam spot size in both $\mathrm{X} \&$ $\mathrm{Y}$ directions. Bottom left (ND) and bottom right (FD), $\nu$ fluxes ratio of $+0.4 \mathrm{~mm}$ shift of the beam spot size in both $\mathrm{X} \& \mathrm{Y}$ directions to nominal at NOvA.
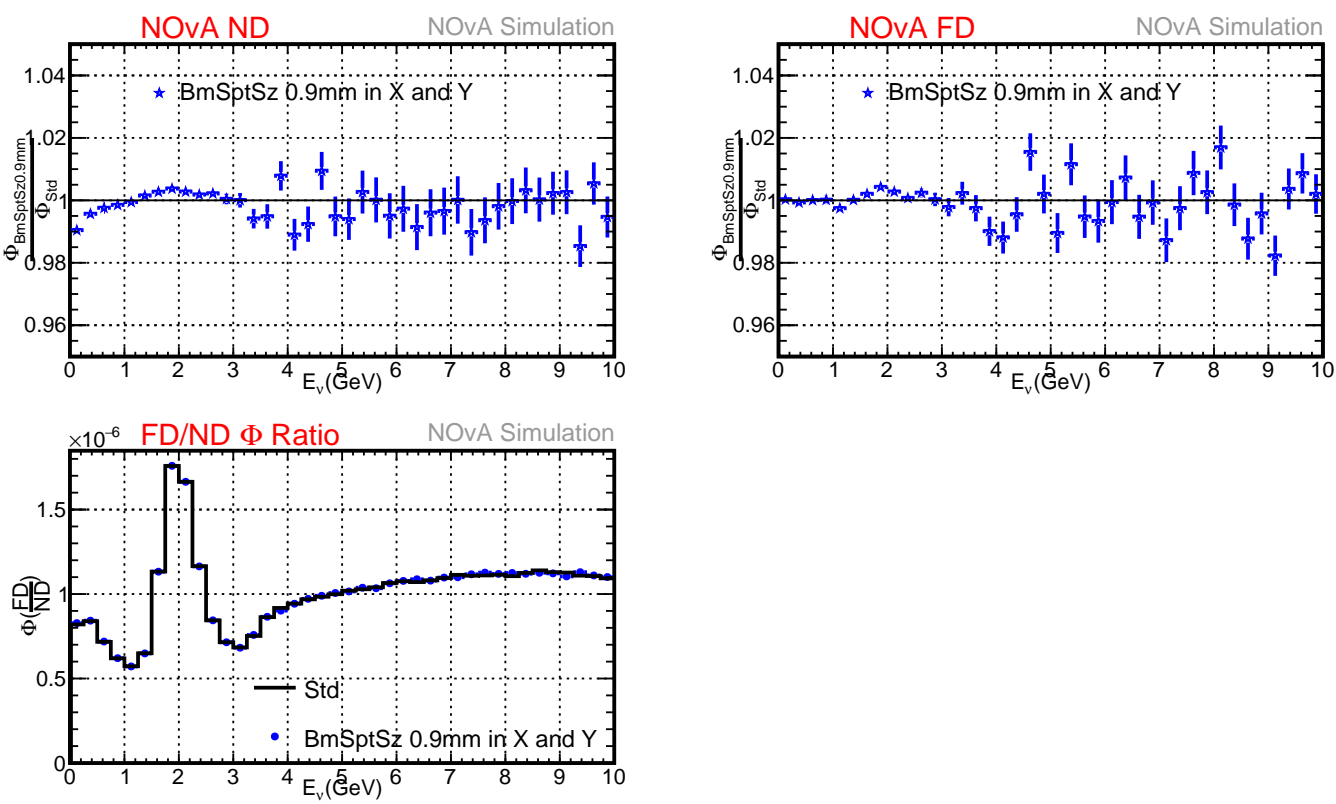

Figure 5.31: Top left (ND) and top right (FD), ratio of $\nu$ flux with variants, blue $(-0.2 \mathrm{~mm})$, to nominal $\nu$ flux at NOvA. Bottom left: FD/ND flux, black is nominal, blue is $-0.2 \mathrm{~mm}$ shift of the beam spot size in both $\mathrm{x} \& \mathrm{Y}$ directions. 

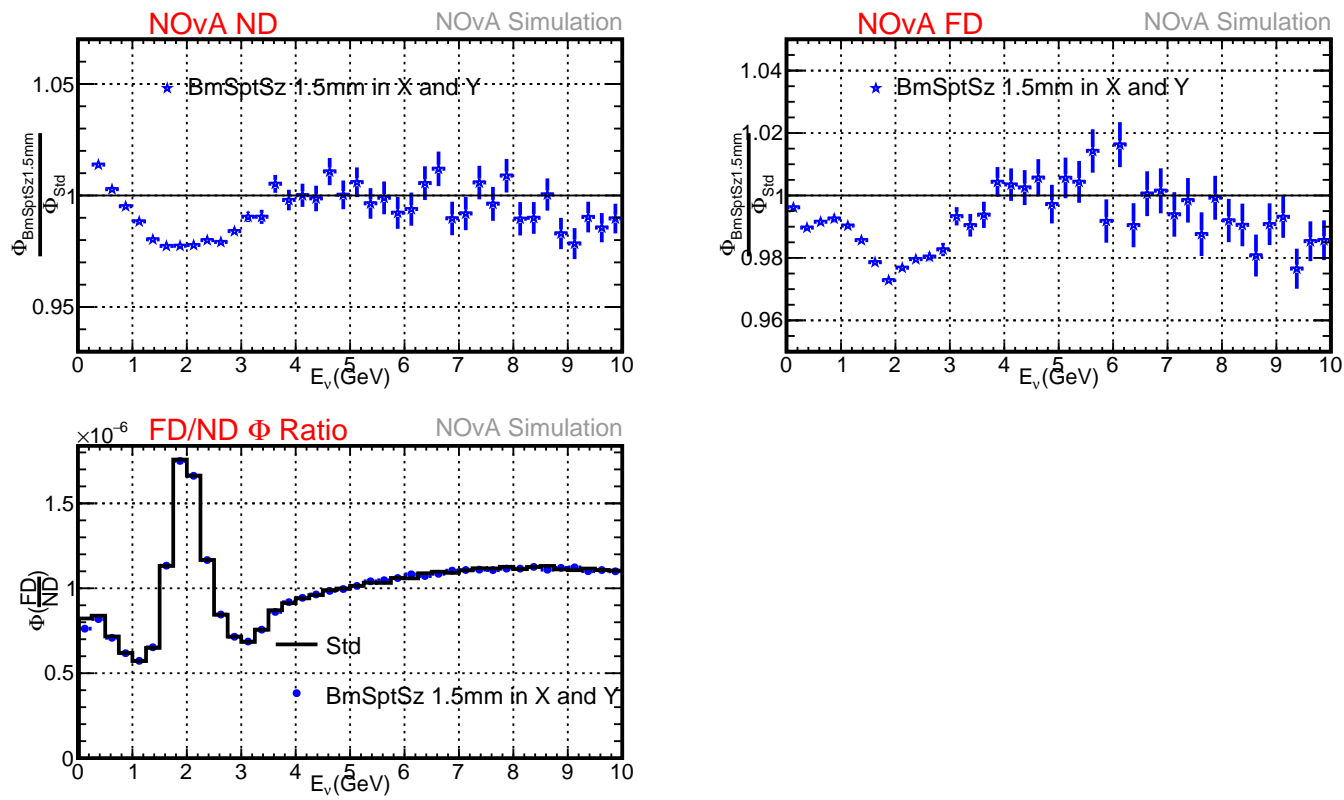

Figure 5.32: Top left (ND) and top right (FD), ratio of $\nu$ flux with variants, blue $(+0.4 \mathrm{~mm})$, to nominal $\nu$ flux at NOvA. Bottom left: FD/ND flux, black is nominal, blue is $+0.4 \mathrm{~mm}$ shift of the beam spot size in both $\mathrm{x} \& \mathrm{Y}$ directions.
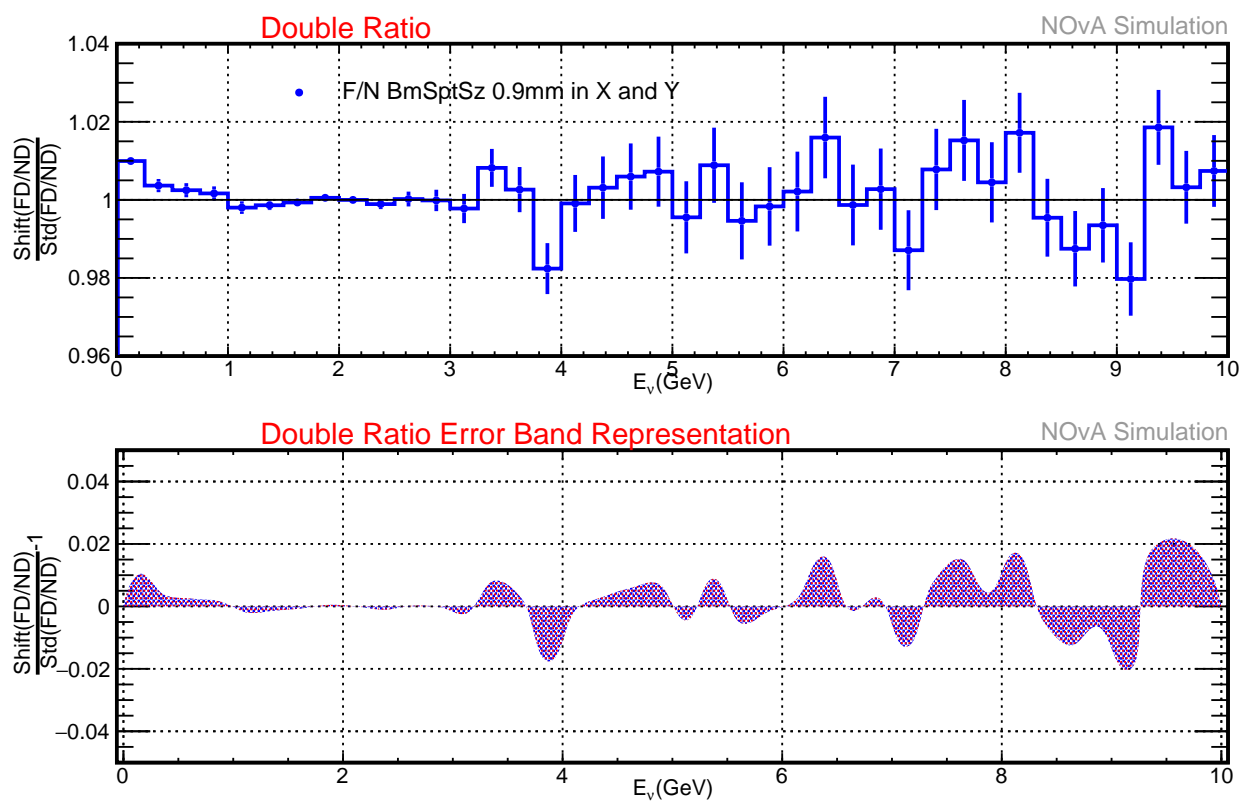

Figure 5.33: Top: Double-Ratio $\frac{\Phi_{F D / N D}(\text { variant) }}{\Phi_{F D / N D}(\text { Std })}$ for $-0.2 \mathrm{~mm}$ shift (blue) in the beam spot size in both X \& Y directions. Bottom: 1-Double-Ratio to show the effective band representation. 

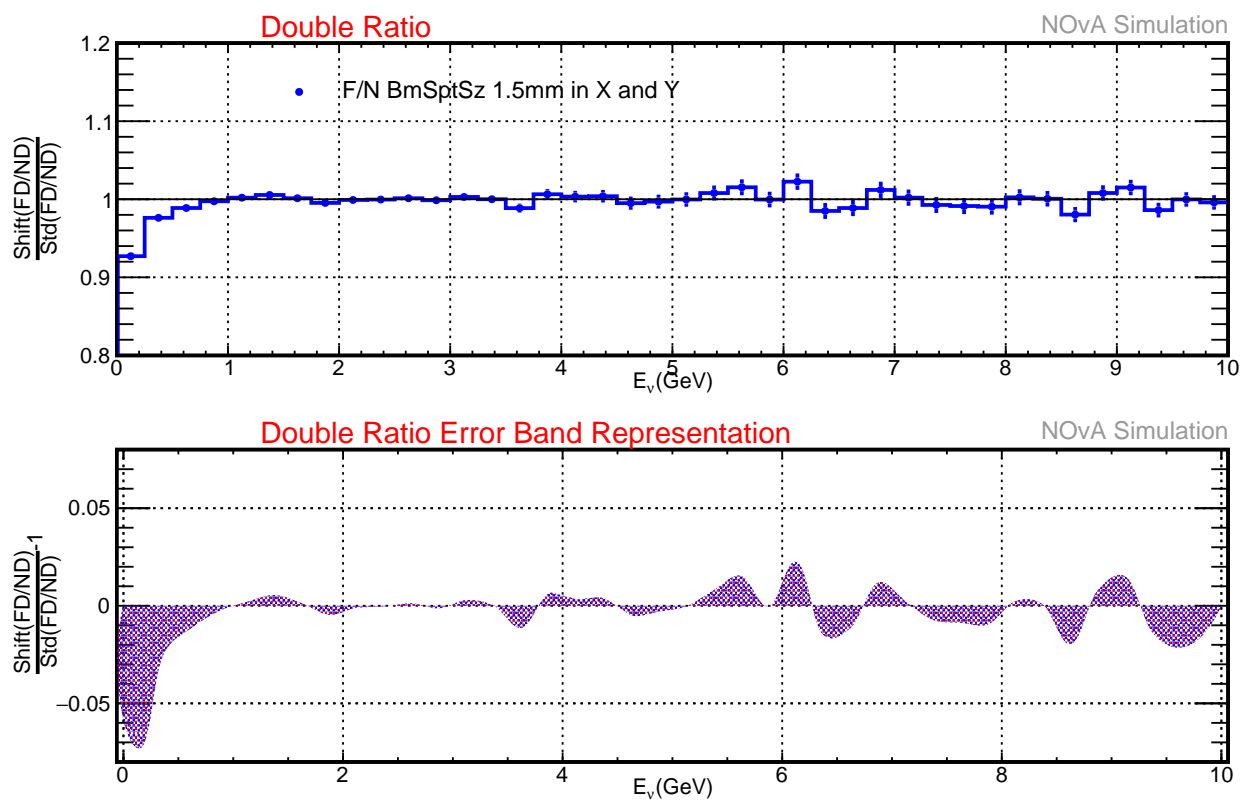

Figure 5.34: Top: Double-Ratio $\frac{\Phi_{F D / N D}(\text { variant })}{\Phi_{F D / N D}(\text { Std })}$ for $+0.4 \mathrm{~mm}$ shift (blue) in the beam spot size in both X \& Y directions. Bottom: 1-Double-Ratio to show the effective band representation.

\subsubsection{Target Position}

In the NuMI beam for neutrino flux, target is most important part. If there is any uncertainty in it's position it will change the intensity of number of pions and kaons focus by the horns. Therefore a shift in the target position with respect to Horn 1 affects the final neutrino spectrum at ND and FD. After consulting with the NuMI technical experts and considering all reasonable effects, we decided to apply a $\pm 7 \mathrm{~mm}$ shift to the target position along the $\mathrm{Z}$ direction. The effect is almost negligible, on the order of $1 \%$, for ND and FD and FD/ND, as shown in Figures 5.35 and 5.36. The effect is negligible at $2 \mathrm{GeV}$ for $\mathrm{FD} / \mathrm{ND}$ ratio for $\pm 7 \mathrm{~mm}$ shift to the target position as shown in Figure 5.37-5.38. 

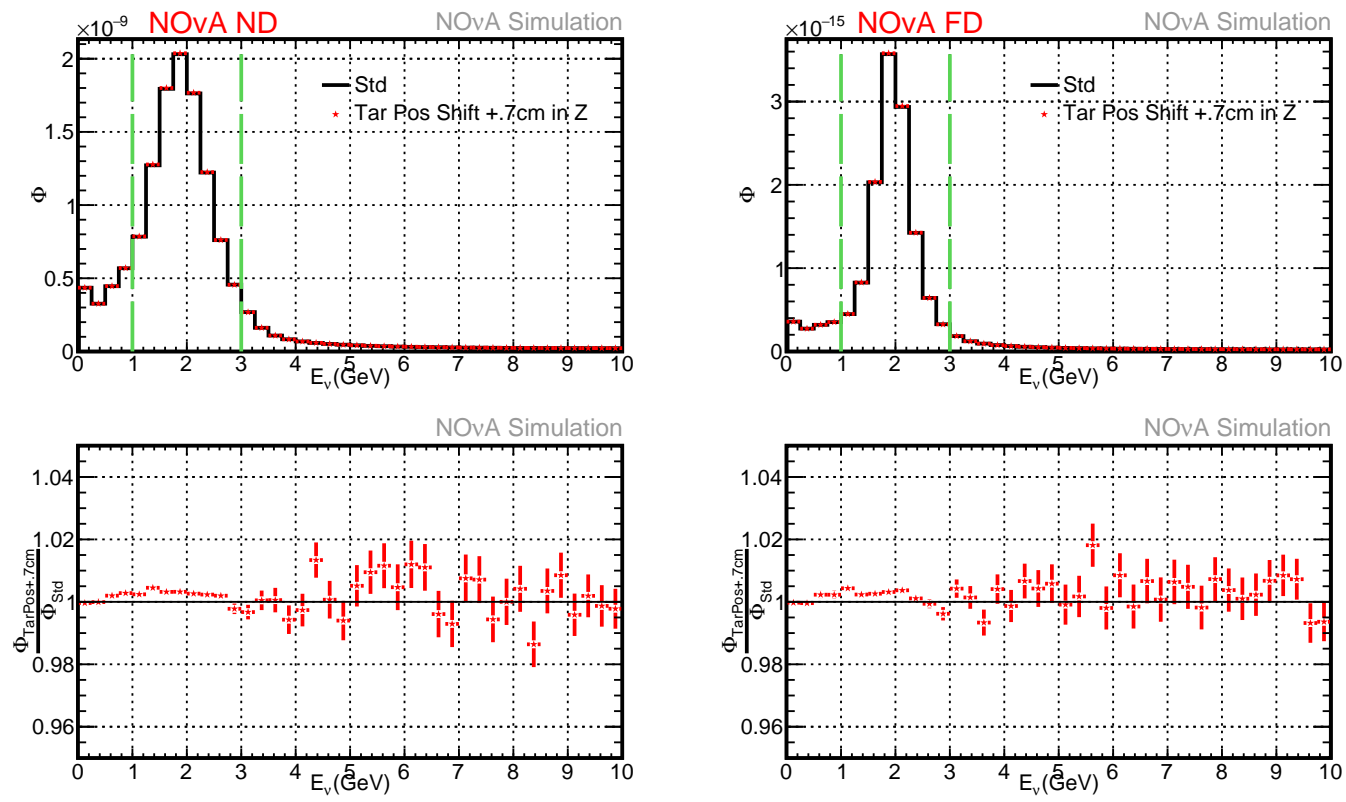

Figure 5.35: Top left (ND) and top right (FD), $\nu$ flux at NOvA for all neutrino parents, black is for nominal and red is for the $+7 \mathrm{~mm}$ shift of the target position along the $\mathrm{Z}$ direction. Bottom left (ND) and bottom right (FD), $\nu$ fluxes ratio of $+7 \mathrm{~mm}$ shift of the target position along the $\mathrm{Z}$ direction to nominal at $\mathrm{NOvA}$.
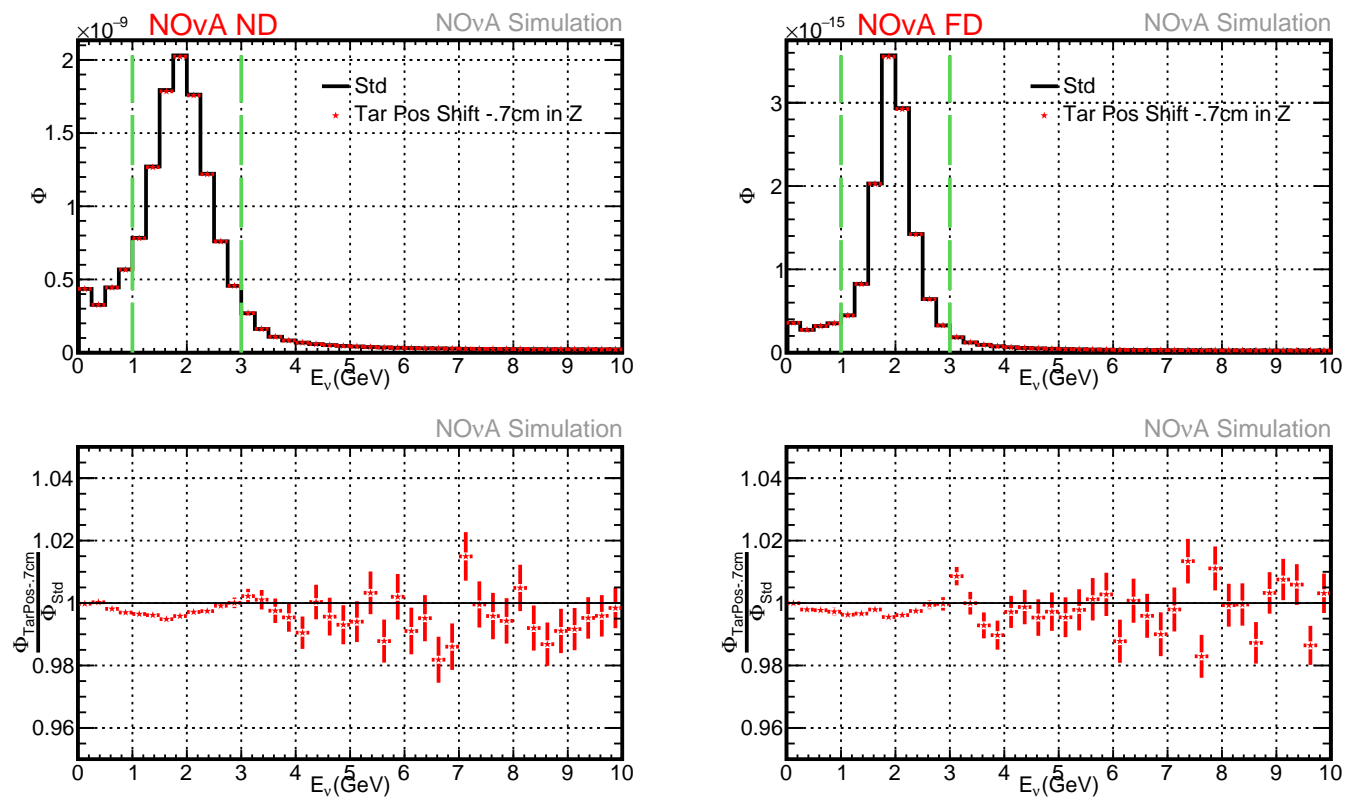

Figure 5.36: Top left (ND) and top right (FD), $\nu$ flux at NOvA for all neutrino parents, black is for nominal and red is for the $-7 \mathrm{~mm}$ shift of the target position along the $\mathrm{Z}$ direction. Bottom left (ND) and bottom right (FD), $\nu$ fluxes ratio of $-7 \mathrm{~mm}$ shift of the target position along the $\mathrm{Z}$ direction to nominal at $\mathrm{NOvA}$. 

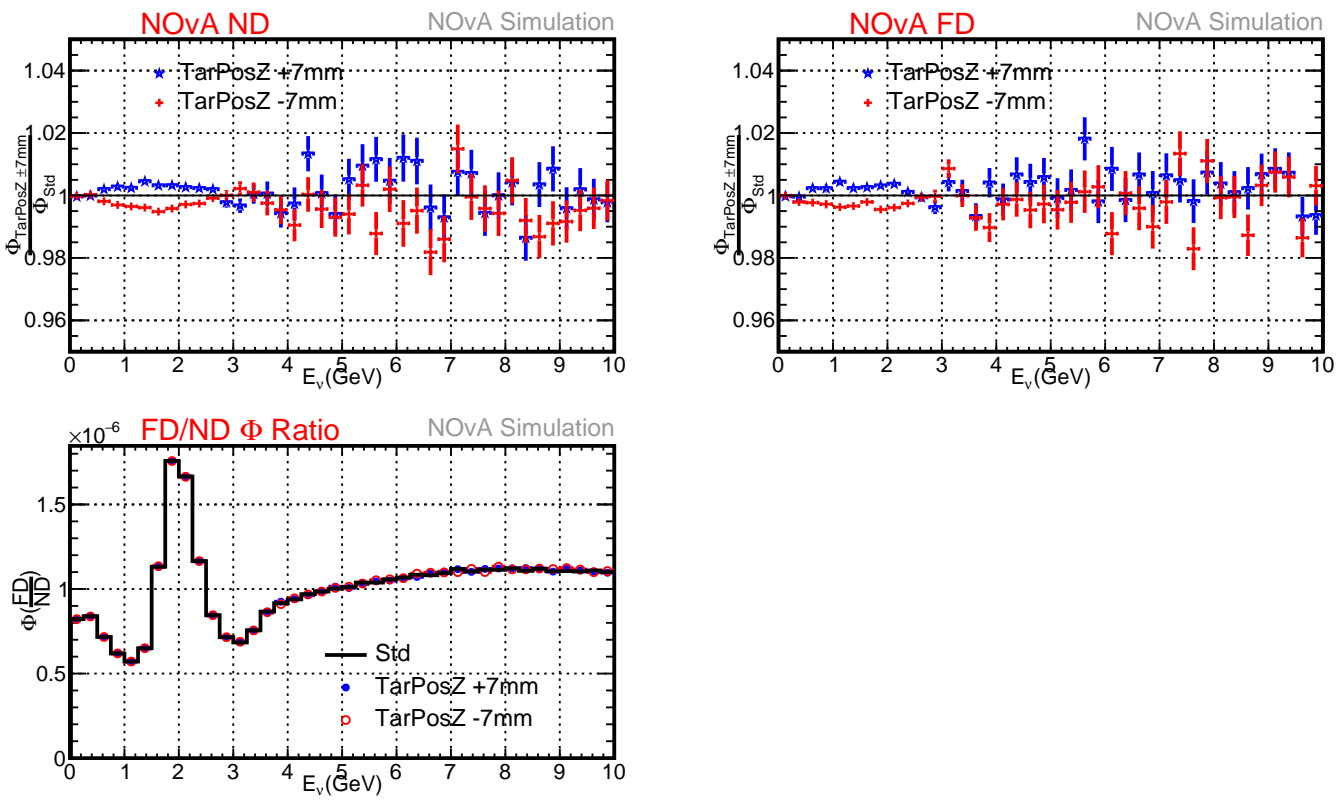

Figure 5.37: Top left (ND) and top right (FD), ratio of $\nu$ flux with variants, blue is $+7 \mathrm{~mm}$ and red is $-7 \mathrm{~mm}$, to nominal $\nu$ flux at NOvA. Bottom left: FD/ND flux, black is nominal, blue is $+7 \mathrm{~mm}$ and red is $-7 \mathrm{~mm}$.
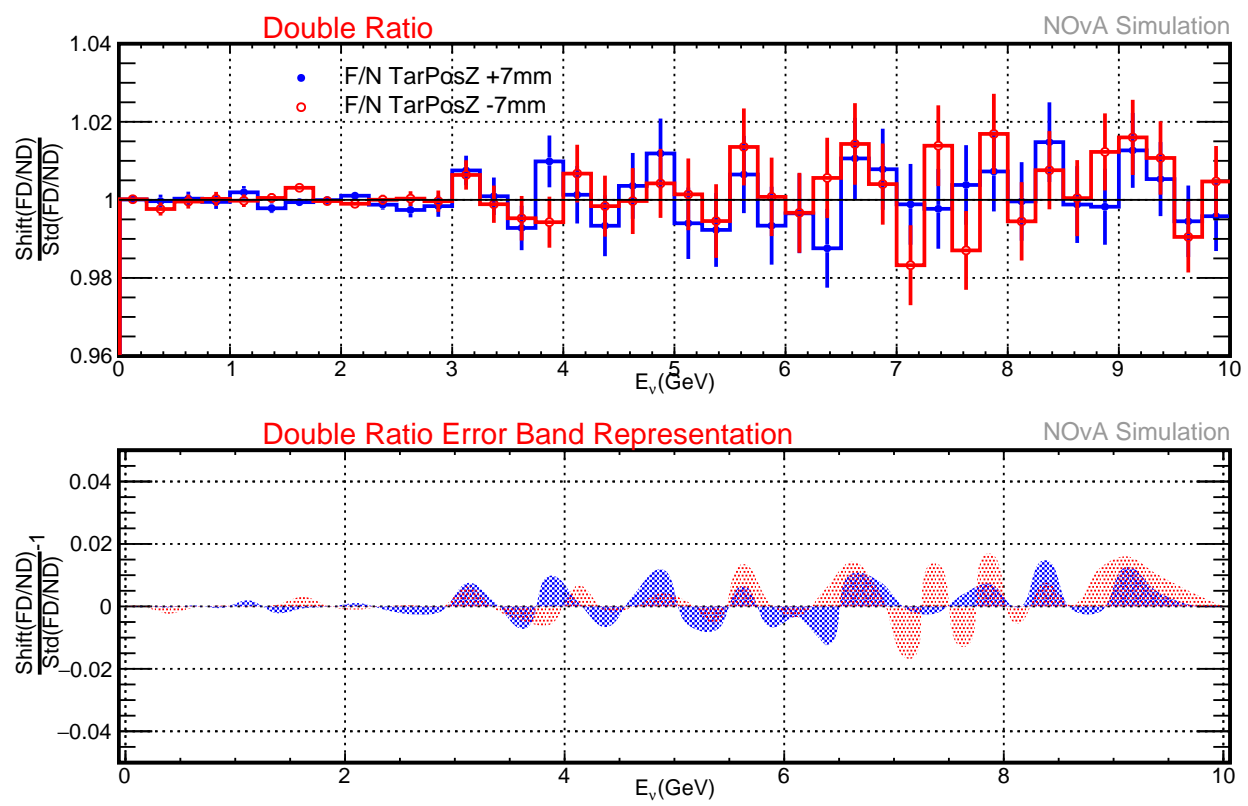

Figure 5.38: Top: Double-Ratio $\frac{\Phi_{F D / N D}(\text { variant) }}{\Phi_{F D / N D}(\text { Std })}$ for the $\pm 7 \mathrm{~mm}$ shift (red) of the target position along the $\mathrm{Z}$ direction. Bottom: 1-Double-Ratio to show the effective band representation. 

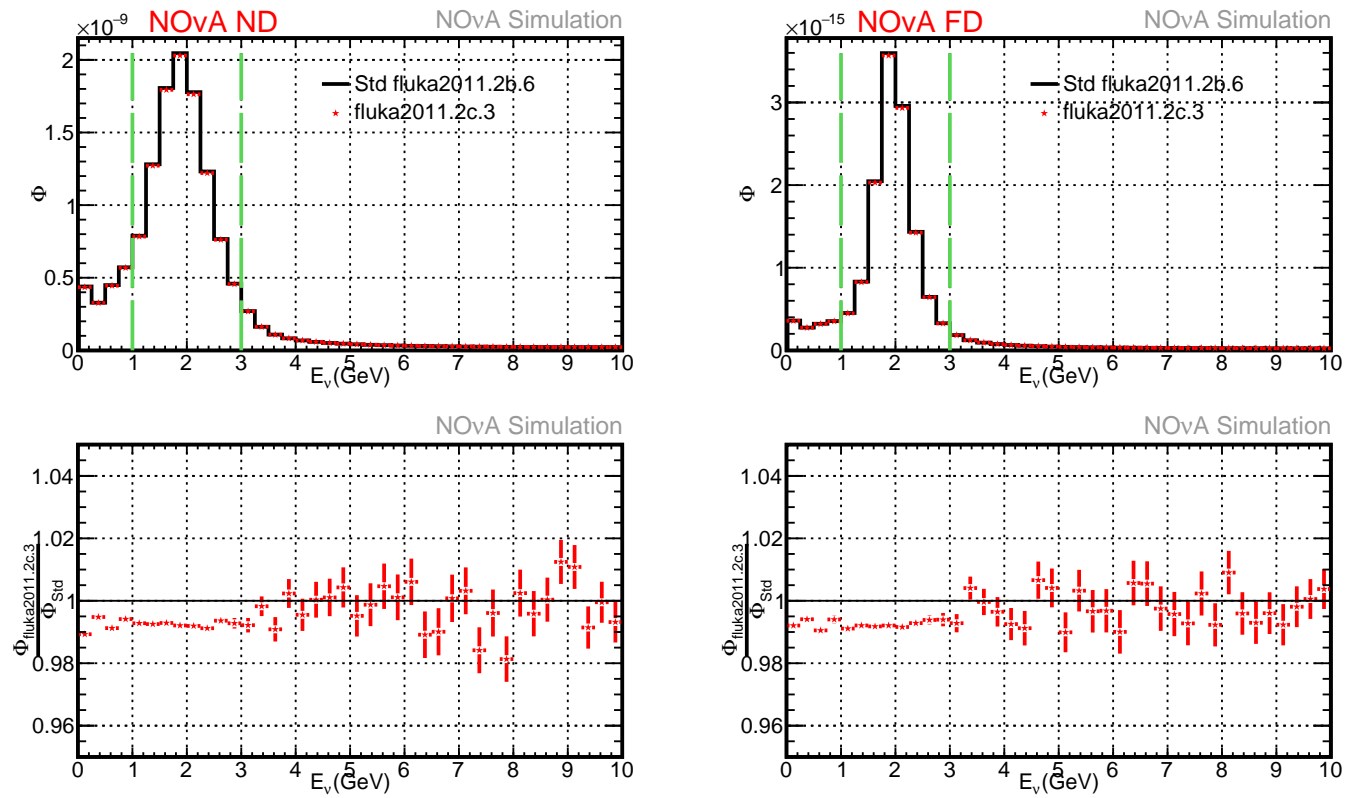

Figure 5.39: Top left (ND) and right (FD): $\nu$ flux at NOvA for all neutrino parents, black is for nominal and blue is for the new version of FLUKA (fluka2011.2c.3). Bottom left (ND) and top right (FD) $\nu$ fluxes ratio of the new version of FLUKA (fluka2011.2c.3) to nominal at NOvA.
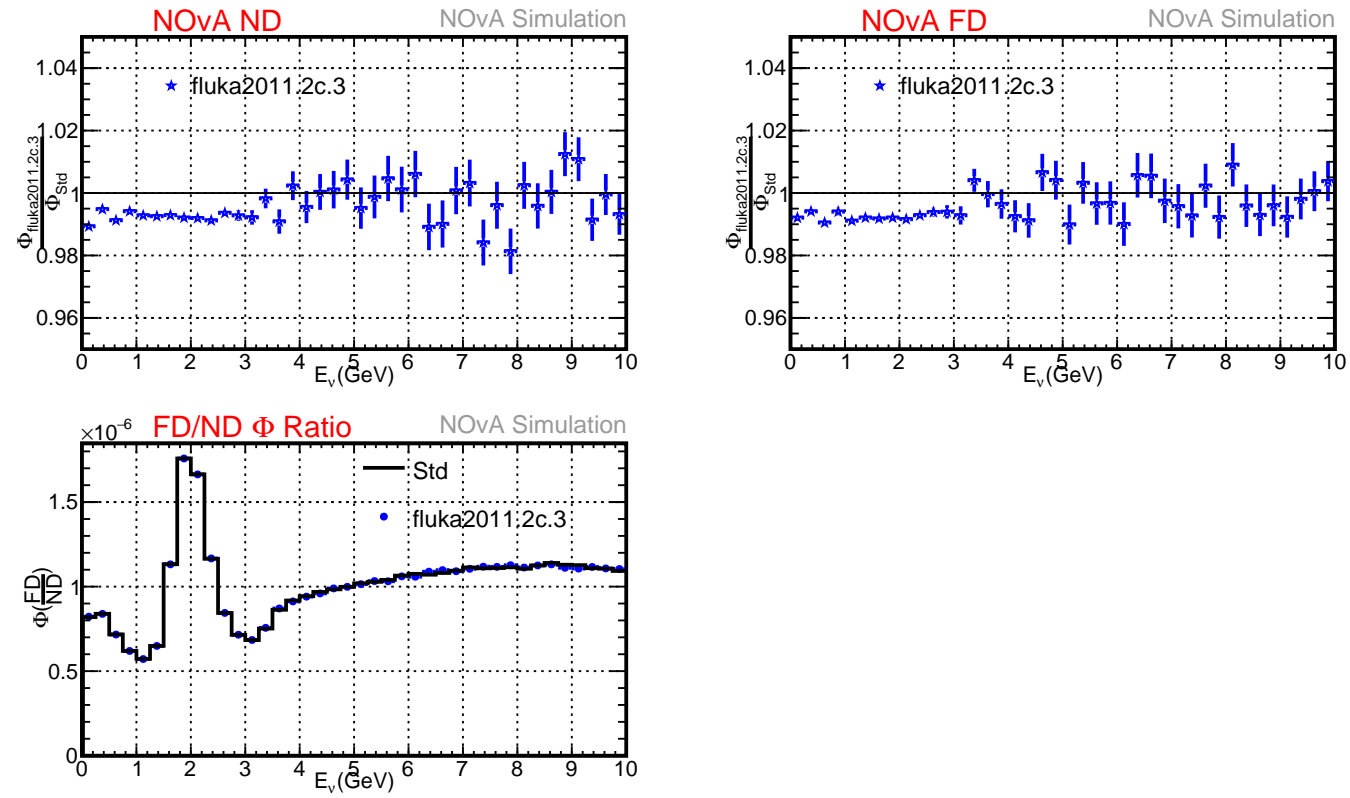

Figure 5.40: Top left (ND) and top right (FD), ratio of $\nu$ flux with variant (fluka2011.2c.3) at NOvA. Bottom left: FD/ND flux, flux with variant (fluka2011.2c.3). 

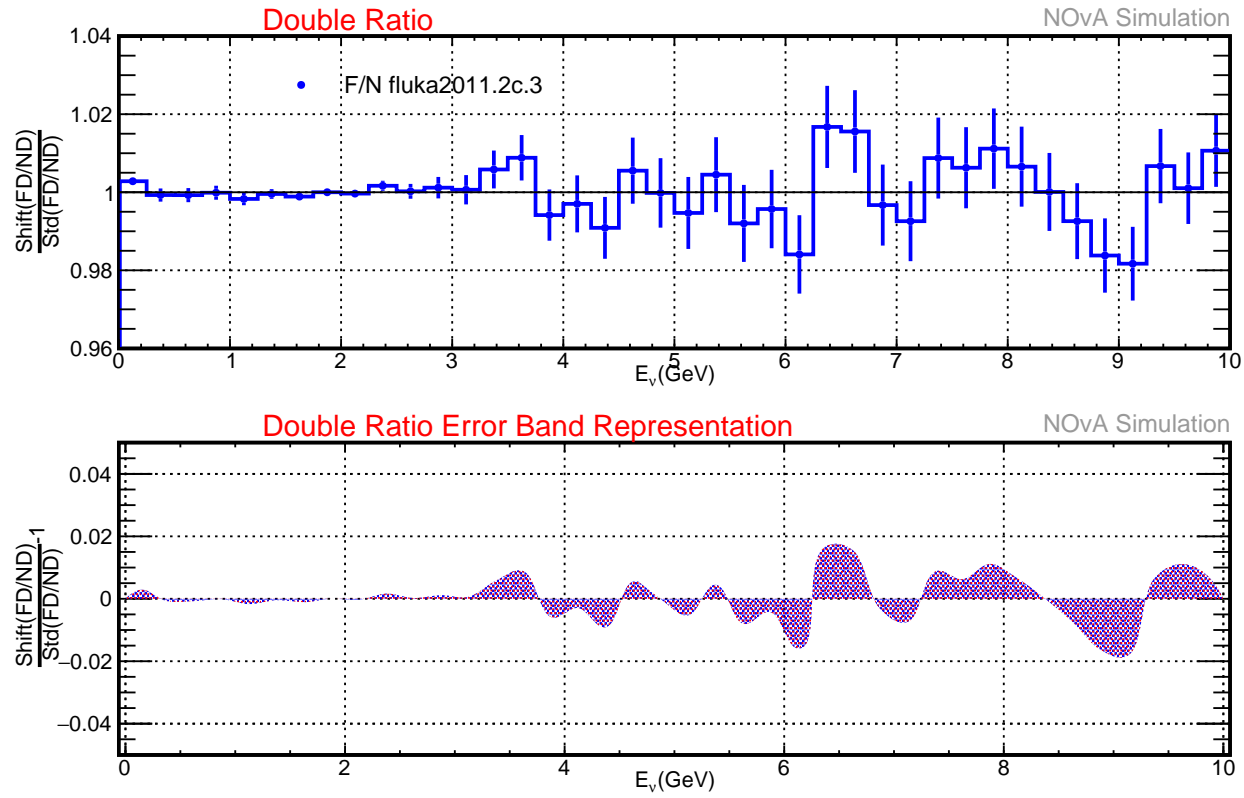

Figure 5.41: Top: Double-Ratio $\frac{\Phi_{F D / N D}(\text { variant })}{\Phi_{F D / N D}(\text { Std })}$ for fluka2011.2c.3. Bottom: 1-DoubleRatio to show the effective band representation.

\subsubsection{FLUKA Versions Comparison}

For the NOvA beam simulation for this study, we had used FLUKA. The FLUKA [72] the event generator has a time bomb for short period of time. To include all the effects from the new version if they have any, need to compare the neutrino flux at NOvA with new and old nominal version of NOvA. The variation is very small and comparison between the latest version of FLUKA with respect to the nominal version for ND and FD and $\mathrm{FD} / \mathrm{ND}$ is shown in Figures $5.39-5.41$.

\subsubsection{G4NuMI vs FLUGG Comparison}

An additional model spread uncertainty is derived from the comparison between G4NuMI(FTFP_BERT) vs FLUGG for ND and FD and FD/ND and is shown in Figures $5.42,5.43$ and 5.44 . 

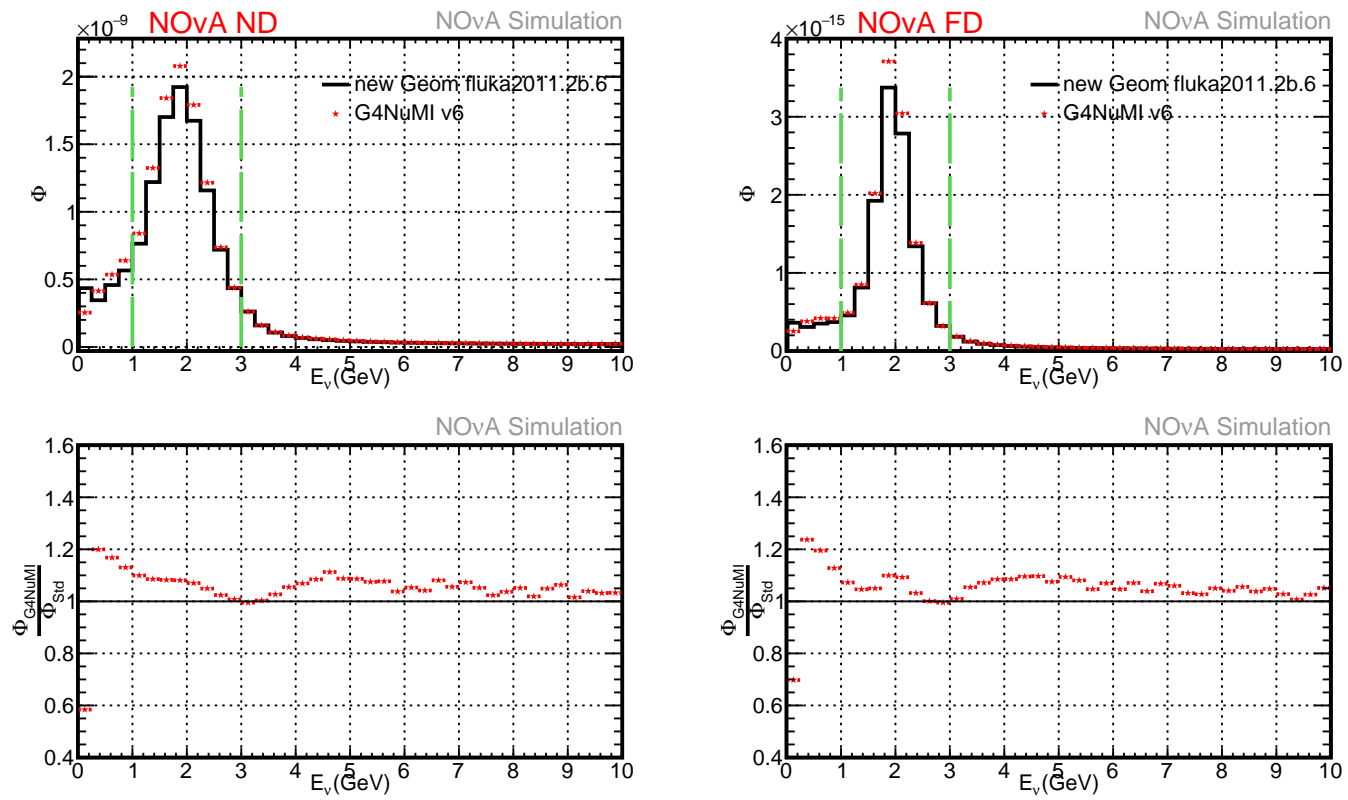

Figure 5.42: Top left (ND) and top right (FD), $\nu$ flux at NOvA for all neutrino parents, black is for nominal and blue is for the G4NuMI(FTFP_BERT). Bottom left (ND) and bottom right (FD): $\nu$ fluxes ratio of the G4NuMI(FTFP_BERT) to nominal at NOvA.
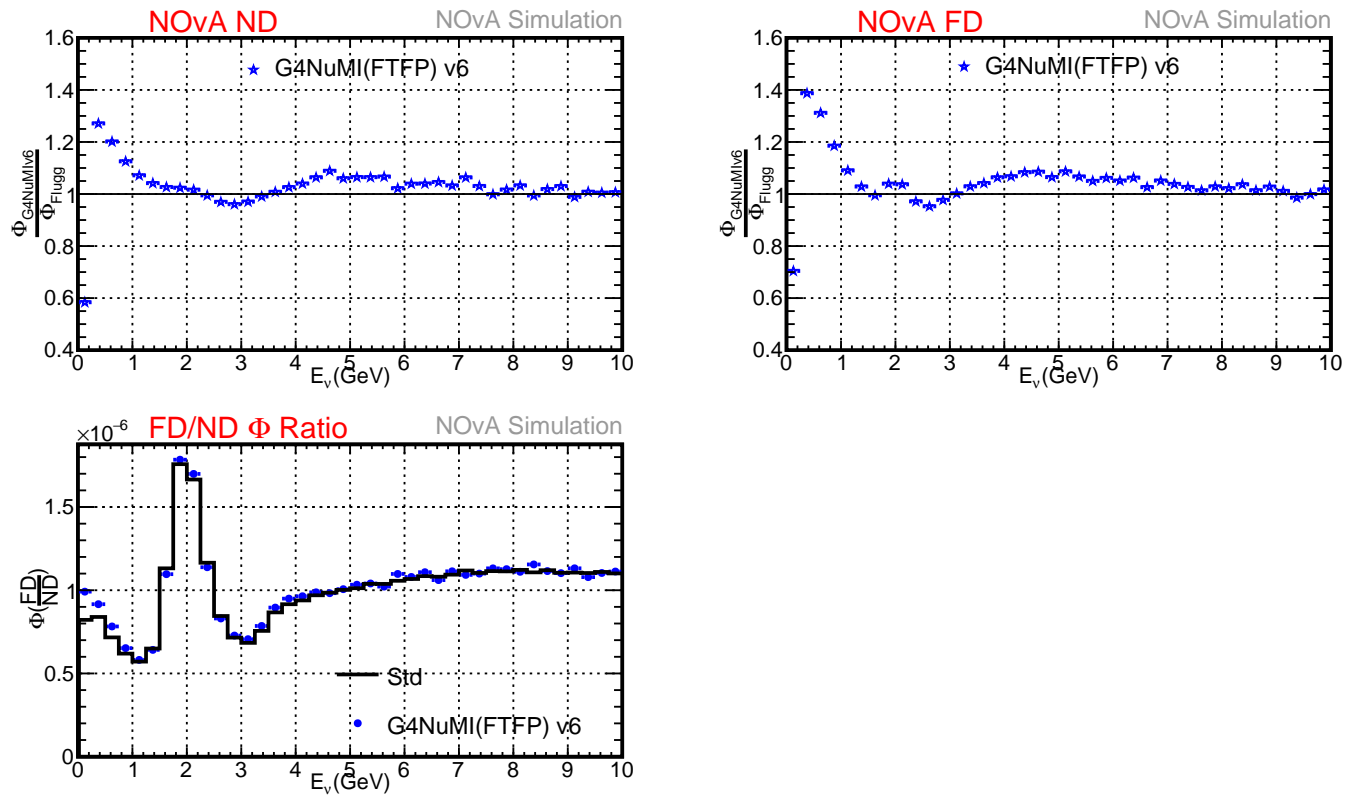

Figure 5.43: Top left (ND) and top right (FD), ratio of $\nu$ flux with variant(G4NuMI(FTFP_BERT)) at NOvA. Bottom left: FD/ND flux, flux with variant(G4NuMI(FTFP_BERT)) 

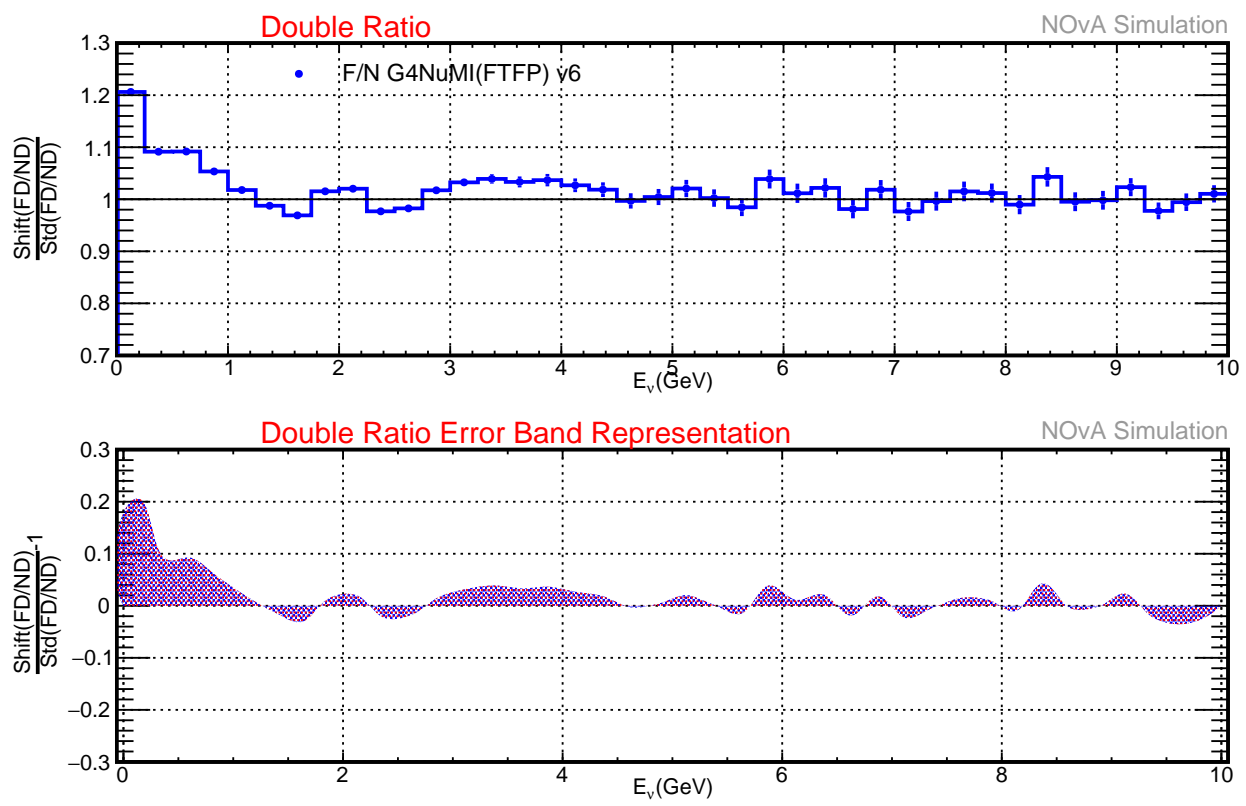

Figure 5.44: Top: Double-Ratio $\frac{\Phi_{F D / N D}(\text { variant })}{\Phi_{F D / N D}(\text { Std })}$ for G4NuMI(FTFP_BERT). Bottom: 1-Double-Ratio to show the effective band representation.

\subsubsection{Horn Geometry and Water Layer}

A more precise description of the horn geometry and the addition of a cooling water layer on the inner horn conductor have been implemented in the FLUGG geometry, which was not in the nominal flux package. The thickness of the water cooling layer on the horns inner conductor was measured to be $1 \pm 0.5 \mathrm{~mm}$. Several studies with the FLUGG simulations showed the effect of water layers of different thicknesses and different geometry descriptions [66]. In this study, the only effect of the new horn geometry and water layer is checked. Due to the new horn geometry and water layer, the effect is $\sim 4 \%$ at ND and FD as shown in Figures 5.45, 5.46, but for the FD/ND ratio the effect is almost negligible at $2 \mathrm{GeV}$ i.e. the NovA oscillation region of interest as shown in Figure 5.47. 

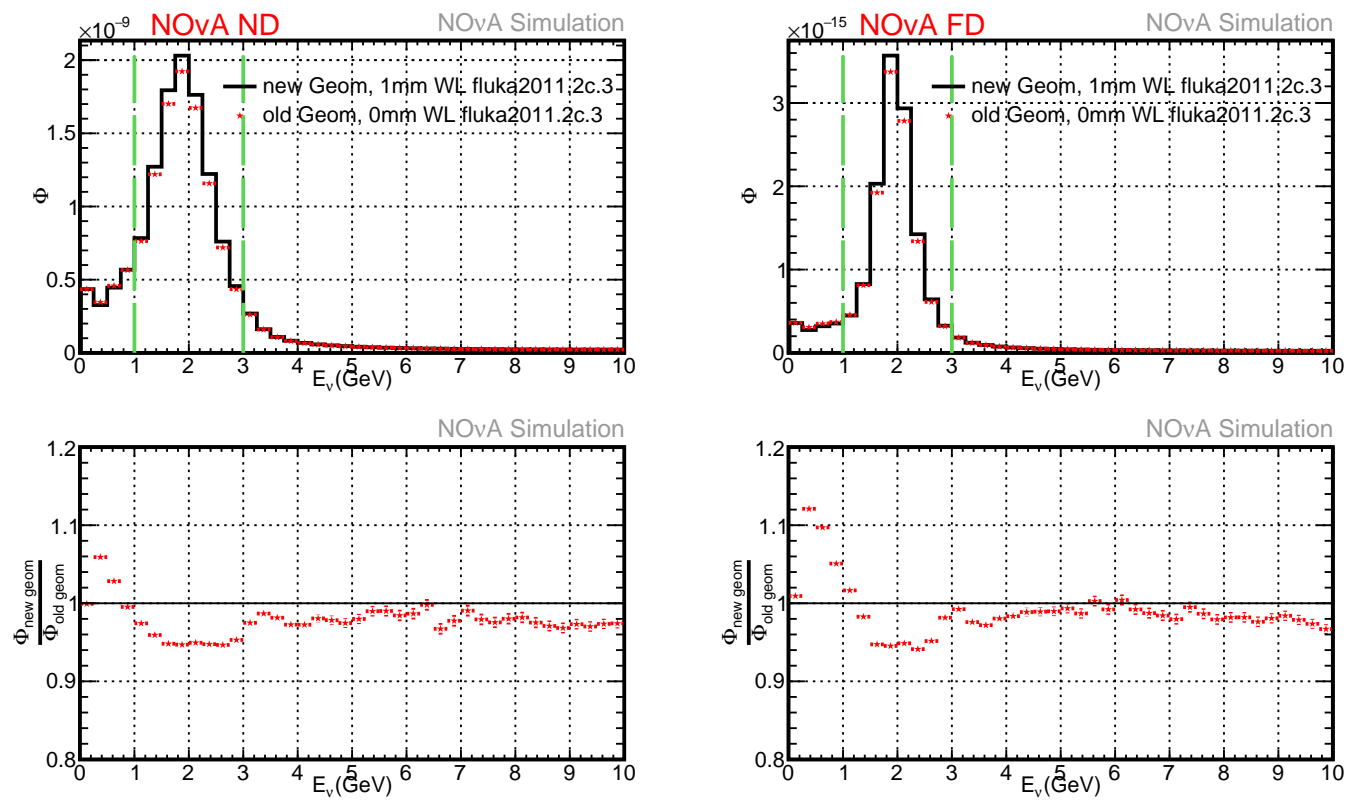

Figure 5.45: Top left (ND) and top right (FD), $\nu$ flux at NOvA for all neutrino parents, black is for nominal and blue is for the New Horn Geometry and Water cooling layer on horn inner conductor. Bottom left (ND) and bottom right (FD), $\nu$ fluxes ratio of theNew Horn Geometry and Water cooling layer on horn inner conductor to nominal at NOvA.
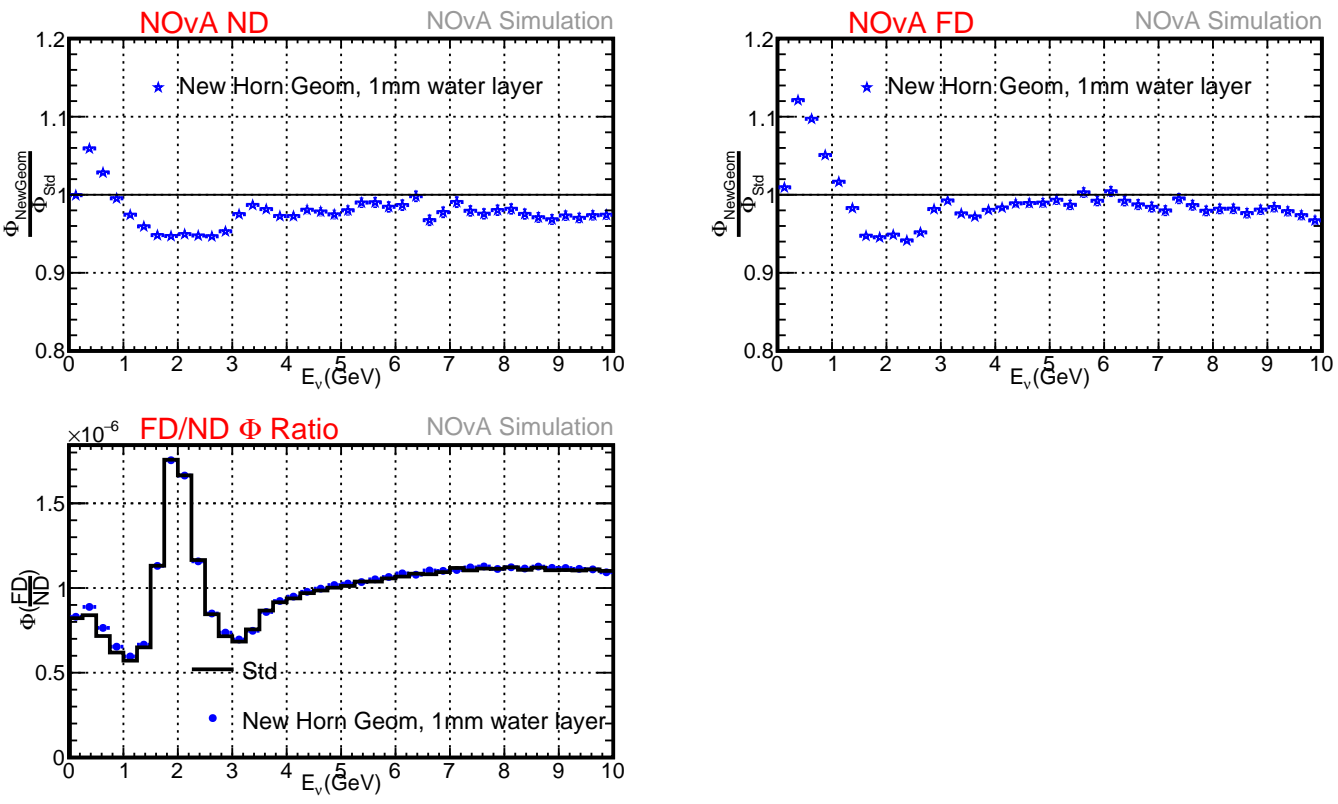

Figure 5.46: Top left (ND) and top right (FD), ratio of $\nu$ flux with variant(New Horn Geometry and Water cooling layer on horn inner conductor) at NOvA. Bottom left: FD/ND flux, flux with variant(New Horn Geometry and Water cooling layer on horn inner conductor) 

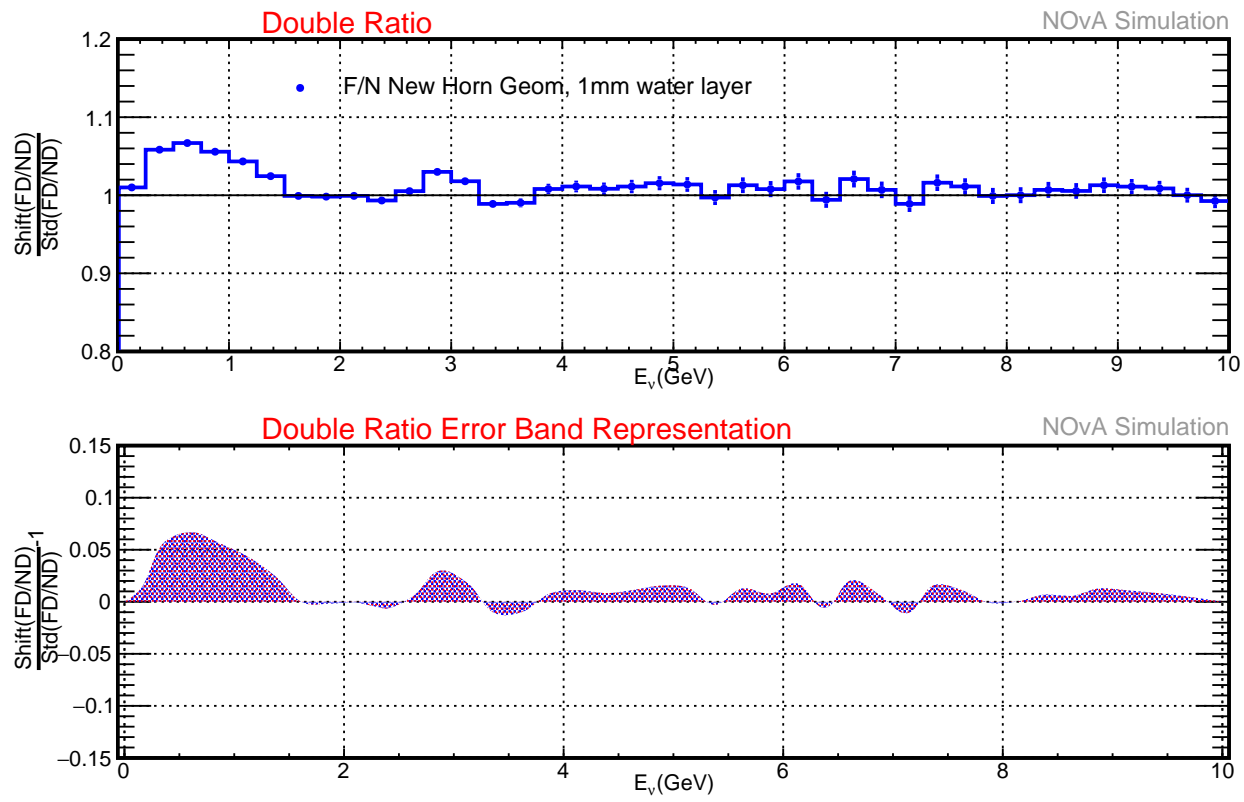

Figure 5.47: Top: Double-Ratio $\frac{\Phi_{F D / N D}(\text { variant })}{\Phi_{F D / N D}(\text { Std })}$ for New Horn Geometry and Water cooling layer on horn inner conductor v6. Bottom: 1-Double-Ratio to show the effective band representation.

\subsubsection{Conclusion}

All shifts affect the energy scale less than $1 \%$ because of the off-axis nature of NOvA. All shifts affect the neutrino flux to the tune of $1-2 \%$, and only the new horn geometry and water layer implementation has the most significant effect on the flux, on the order of $4 \%$ at ND and FD but stays negligible when considering the Far to Near Ratio. As a conservative estimation, each beam systematic shift is summed in quadrature to obtain the final effect of all beam transport related uncertainties. 


\begin{tabular}{|c||c|c|}
\hline Shift & ND $(1-3) \mathrm{GeV} \delta(\%)$ & $\mathrm{FD}(1-3) \mathrm{GeV} \delta(\%)$ \\
\hline \hline Standard(Fluka2011.2b.6) & 0.000 & 0.000 \\
\hline Horn Current +1 kA & 0.219 & 0.168 \\
\hline Horn Current -1KA & -0.164 & -0.093 \\
\hline Beam position on X-axis +0.5 mm & 0.678 & 0.683 \\
\hline Beam position on X-axis -0.5 mm & -0.273 & -0.235 \\
\hline Beam position on Y-axis +0.5 mm & -0.132 & -0.175 \\
\hline Beam position on Y-axis -0.5 mm & 0.362 & 0.450 \\
\hline Horn 1 +2 mm in X and Y & 0.383 & 0.393 \\
\hline Horn 1 -2 mm in X and Y & 1.696 & 1.786 \\
\hline Horn 2 +2 mm in X and Y & -0.370 & -0.299 \\
\hline Horn 2 -2 mm in X and Y & 0.516 & 0.472 \\
\hline Exponential B field & -0.021 & 0.016 \\
\hline Beam Spot Size on the target 0.9 mm mm & -0.299 & -0.285 \\
\hline Beam Spot Size on the target 1.5 mm mm & 2.268 & 2.360 \\
\hline Fluka2011.2c.3 & 0.848 & 0.847 \\
\hline Target Position in Z +7 mm & -0.294 & -0.266 \\
\hline Target Position in Z -7 mm & 0.356 & 0.339 \\
\hline New Horn 1 mm Water Layer & 4.947 & 4.425 \\
\hline G4NuMI(FTFP_BERTv6) & -1.368 & -1.368 \\
\hline
\end{tabular}

Table 5.3: Tabulated beam transport systematics.

\subsection{Beam Hadron Production Uncertainties}

The phase space of the transverse momentum $\left(p_{T}\right)$ and longitudinal momentum $\left(p_{Z}\right)$ for the meson ancestor of NOvA neutrinos are shown in Figures 5.48 and 5.49 for $\nu_{\mu}$ and Figures 5.50 and 5.51 for $\nu_{e}$. As one can see, for the energy region of $1<E_{\nu}<3 \mathrm{GeV}$, the majority of flux is located within $p_{T}<0.5 \mathrm{GeV}$ and $4<p_{Z}<15 \mathrm{GeV}$ region from pions and $2<p_{Z}<15 \mathrm{GeV}$ from Kaons.

As shown in the $\nu_{e}$ inclusive cross section measurement [73], the flux uncertainty is the single dominant uncertainty, which would limit the precision for all kinds of cross section measurements. It is essential to use all available external data to help constrain the flux uncertainty. As summarized in Table 5.3, there are data results for both pion [74] and kaon [75] production from the NA49 experiment using a similar proton energy as NOvA. The kaon and pion production ratio from the MIPP experiment [76] could help 

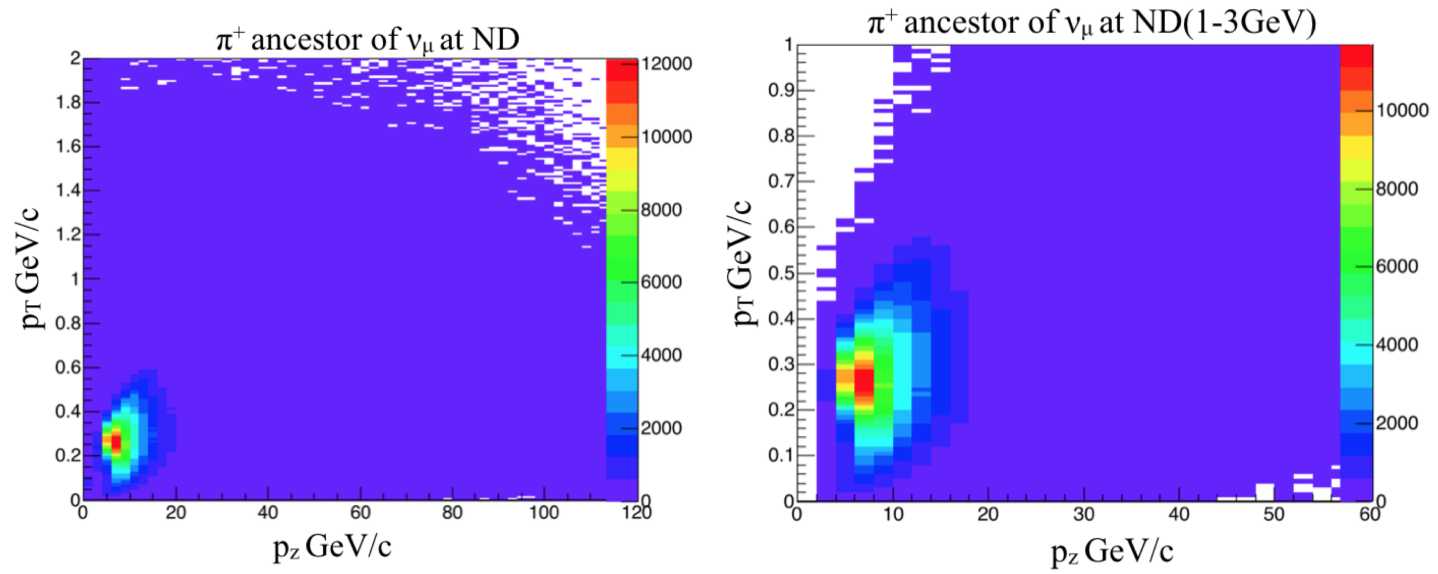

Figure 5.48: The distribution for $\pi \rightarrow \nu_{\mu}$ flux as a function of $p_{T}$ and $p_{Z}$ of $\pi$ ancestor.
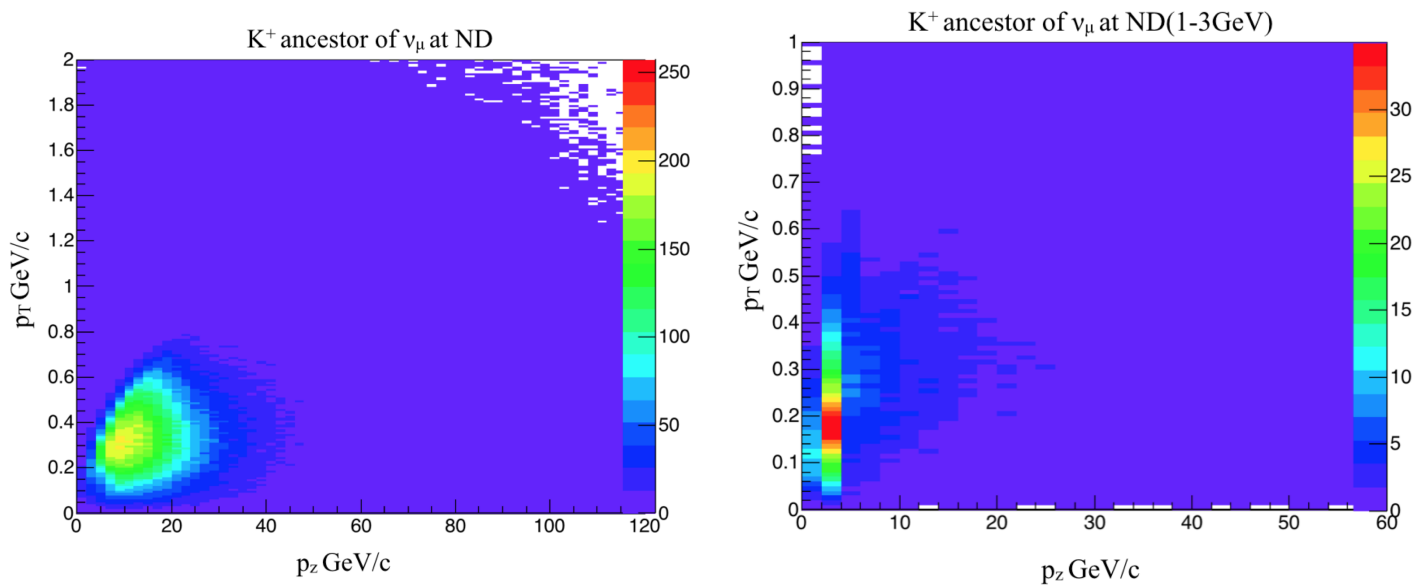

Figure 5.49: The distribution for $K \rightarrow \nu_{\mu}$ flux as a function of $p_{T}$ and $p_{Z}$ of $K$ ancestor.

extend the kaon production coverage. Besides that, there is also a precise measurement for the pion production from MIPP [77].

The available external data as listed in Table 5.3 is divided into two parts, one part is secondary mesons, and the other part is tertiary and other mesons. This chapter, presents the weights for $\pi^{+}$ancestor of $\pi \rightarrow \mu \rightarrow \nu_{e}$ flux and $\pi \rightarrow \nu_{\mu}$ flux in NOvA dominant phase space in Section 5.3.2. The weights for $K$ ancestor of $K \rightarrow \nu_{e}$ flux and $K \rightarrow \nu_{\mu}$ flux are presented in Section 5.3.3. The results for the weights as a function of $\nu$ energy and the corresponding systematic uncertainty is shown in Section 6.5 . 

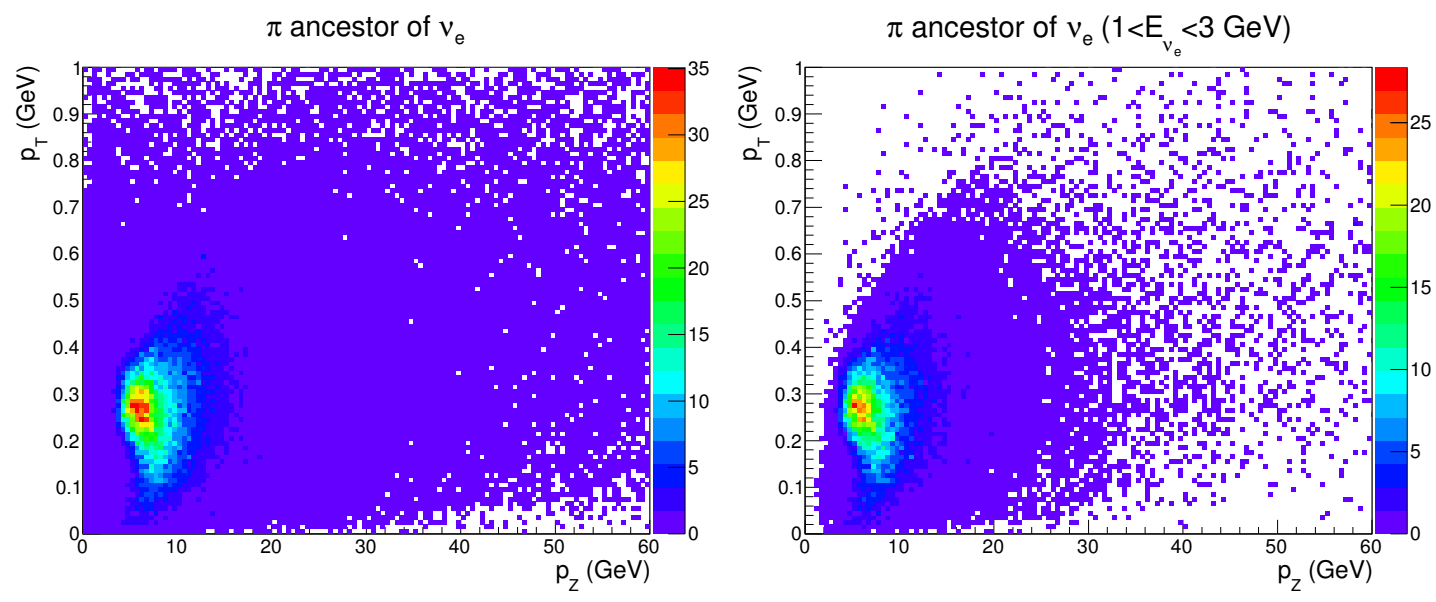

Figure 5.50: The distribution for $\pi \rightarrow \mu \rightarrow \nu_{e}$ flux as a function of $p_{T}$ and $p_{Z}$ of $\pi$ ancestor.
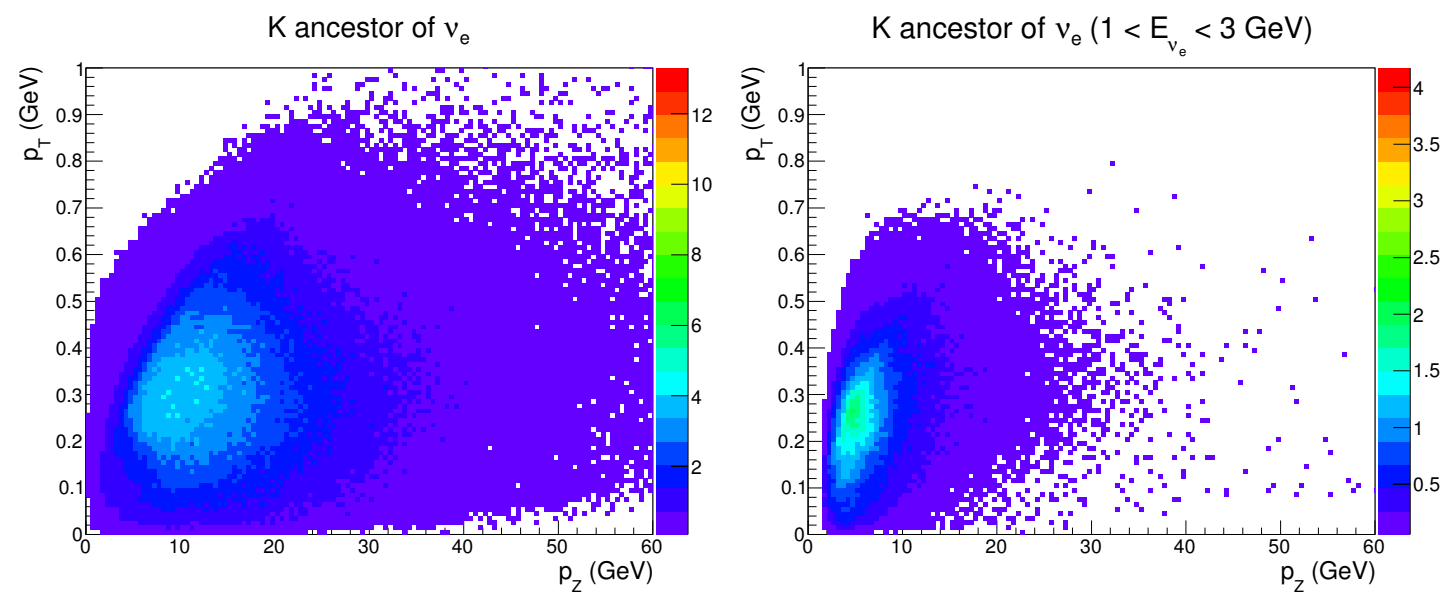

Figure 5.51: The distribution for $K \rightarrow \nu_{e}$ flux as a function of $p_{T}$ and $p_{Z}$ of $K$ ancestor.

\subsubsection{Event Weights}

The event weights are derived based on the ratio between data and FLUKA prediction.

The bin center and the bin size for the FLUKA are the same as the ones used in the experimental data results. The following subsections, present the distributions from both data and FLUKA, and the ratio between them (a.k.a. weights). The invariant crosssection distributions for both $\pi$ and $K$ production of NA49 are presented in Appendix C.1. The $K / \pi$ ratio used in this analysis is shown in Appendix C.2, and data coverage of the original MIPP data is shown in Appendix C.3. 


\begin{tabular}{|c|c|c|c|c|c|}
\hline Experiment & data type & $p_{T}$ range $(\mathrm{GeV})$ & $x_{F}$ range & target & proton energy $(\mathrm{GeV})$ \\
\hline NA49 & pion production & $0-2$ & $0-0.5$ & thin Carbon & 158 \\
\hline NA49 & kaon production & $0-1$ & $0-0.2$ & thin Carbon & 158 \\
\hline MIPP & kaon/pion ratio & $0-2$ & $0.2-0.5$ & thin Carbon & 120 \\
\hline MIPP & pion production & $0-2$ & $0-0.5$ & thick Carbon & 120 \\
\hline
\end{tabular}

Table 5.4: External data results from various experiments.

\subsubsection{Weights for $\pi^{+}$Production}

As demonstrated in Figure 5.52 (left), we use the $\pi$ yield data results from MIPP for all $\pi$ ancestor in following phase space:

- $0.3<p_{Z}<0.5 \mathrm{GeV}$ with $p_{T}<0.4 \mathrm{GeV}$;

- $0.5<p_{Z}<2 \mathrm{GeV}$ with $p_{T}<0.5 \mathrm{GeV}$;

- $4<p_{Z}<6 \mathrm{GeV}$ with $p_{T}<0.4 \mathrm{GeV}$;

- $6<p_{Z}<8 \mathrm{GeV}$ with $p_{T}<0.5 \mathrm{GeV}$;

- $8<p_{Z}<68 \mathrm{GeV}$ with $p_{T}<2 \mathrm{GeV}$.
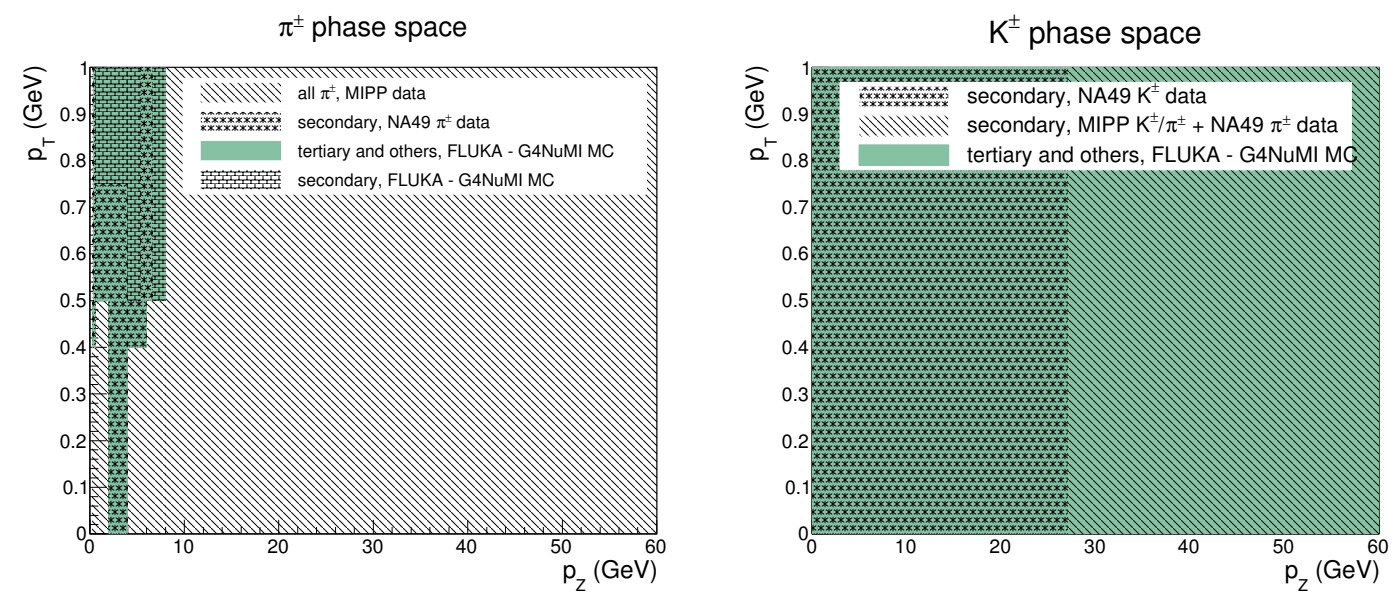

Figure 5.52: Left: Demonstration of the external data usage for the phase space coverage of $\pi \rightarrow \nu_{\mu}$ and $\pi \rightarrow \mu \rightarrow \nu_{e}$ flux. Right: Demonstration of the external data usage for the phase space coverage of $K \rightarrow \nu_{e}$ and $K \rightarrow \nu_{\mu}$ flux.

The $\pi$ yield distributions for data and FLUKA in the NOvA dominant phase space are shown in Figure 5.53. The event weights as a function of $p_{T}$ for each $p_{Z}$ bin 
are shown in the bottom part of these distributions. The data and FLUKA comparisons and corresponding event weights for the rest $\pi^{+}$phase space and the $\pi^{-}$production are shown in Appendix C.4. The target file from FLUKA is simulated with the low-energy configuration for the beamline target.

The invariant cross section of $\pi$ production data results from NA49 are used for the secondary $\pi$ only, where the phase space is not covered by the MIPP $\pi$ yield:

- $-0.005<x_{F}<0.005$ with $p_{T}<2 \mathrm{GeV}$;

- $0.005<x_{F}<0.035$ with $p_{T}<0.75 \mathrm{GeV}$;

- $0.045<x_{F}<0.055$ with $p_{T}<2 \mathrm{GeV}$.

The FLUKA prediction for NA49 is based on a simplified carbon target, more details are in Ref [78]. The invariant cross section for $\pi^{+}$in these regions are shown in Figure 5.54 .

For the the residual phase space that are not covered by either MIPP data or NA49 data, we use the difference between FLUKA and G4NuMI with FTFP_BERT physics list as the systematic uncertainty:

- $0.6<p_{Z}<4.2 \mathrm{GeV}$ with $p_{T}>0.75 \mathrm{GeV}$ for secondary $\pi$;

- $4.2<p_{Z}<5.4 \mathrm{GeV}$ with $p_{T}>0.4 \mathrm{GeV}$ for secondary $\pi$;

- $6.6<p_{Z}<8 \mathrm{GeV}$ with $p_{T}>0.5 \mathrm{GeV}$ for secondary $\pi$;

- $0.3<p_{Z}<0.5 \mathrm{GeV}$ with $p_{T}>0.4 \mathrm{GeV}$ for non-secondary $\pi$;

- $0.5<p_{Z}<2 \mathrm{GeV}$ with $p_{T}>0.5 \mathrm{GeV}$ for non-secondary $\pi$;

- $2<p_{Z}<4 \mathrm{GeV}$ with $p_{T}>0 \mathrm{GeV}$ for non-secondary $\pi$;

- $4<p_{Z}<6 \mathrm{GeV}$ with $p_{T}>0.4 \mathrm{GeV}$ for non-secondary $\pi$;

- $6<p_{Z}<8 \mathrm{GeV}$ with $p_{T}>0.5 \mathrm{GeV}$ for non-secondary $\pi$. 
The event weights are set as 1 whilst assigning the difference between FLUKA and G4NuMI as a corresponding uncertainty. There is $\approx 10-20 \%$ uncertainty for the secondary $\pi$ responsible for the NOvA (NuMI) neutrinos, and $\approx 20 \%$ uncertainty for the non-secondary $\pi$ responsible for the NOvA neutrinos. Conservatively, we take $20 \%$ as the systematic uncertainty for these $\pi \mathrm{s}$ that are not covered by the data results from either MIPP or NA49.

We directly use the pion yield weights between MIPP data and FLUKA prediction using low energy NuMI beam target, and apply these weights to the NOvA flux prediction with FLUKA in the medium energy neutrino beam. By doing so, we assume the pion yield weights between data and FLUKA prediction is consistent between low energy and median energy target configurations. To quantify the potential effect due to different target configurations, we perform a mock data test. We treat the MC prediction from G4NuMI (as explained in Section. 2.5) with the FTFP_BERT physics list as the data. Consequently we derive the pion yield ratio between G4NuMI and FLUKA in low energy mode using the same $p_{T}$ and $p_{Z}$ as the MIPP data results (see Figure 5.55 (left)). Finally we apply these ratios to the FLUKA prediction in median energy mode, and compare the weighted FLUKA with the G4NuMI median energy prediction. The results are shown in Figure 5.55 (right), where we can see the weighted FLUKA prediction is systematically lower than G4NuMI prediction by $\approx 10 \%$. We take this $10 \%$ as an additional systematic uncertainty to apply on the MIPP data and FLUKA weights.

\subsubsection{Weights for $K^{+}$Production}

As demonstrated in Figure 5.52 (right), we use the invariant cross-section data results from NA49 for the secondary $K$. The direct measurement results from NA49 cover the phase space of $p_{Z}<27 \mathrm{GeV}$ with $p_{T}<1 \mathrm{GeV}$. The data and FLUKA comparison with the corresponding weights for $K^{+}$are shown in Figure 5.57. The $K / \pi$ ratio from the MIPP data results together with the $\pi$ production cross section from NA49 data extend 
the $K$ phase space coverage up to $p_{Z}=60 \mathrm{GeV}$. The data and FLUKA comparisons for this extended phase space are shown in Figure 5.56, which covers $27<p_{Z}<60$ $\mathrm{GeV}$ with $p_{T}<2 \mathrm{GeV}$. For the non-secondary $K$ (having interactions $N_{\text {int }}>1$ ), use the difference between FLUKA and G4NuMI with the FTFP_BERT physics list as the systematic uncertainty. The relative difference between FLUKA and G4NuMI is $\approx 10 \%$ in the energy region $0.5<E_{\nu}<2 \mathrm{GeV}$, and $20-30 \%$ for $E_{\nu}>2 \mathrm{GeV}$. The difference in each energy bin is used as the uncertainty, and we conservatively require the uncertainty to be at least 20\%. The data and FLUKA comparisons and the corresponding event weights for the $K^{-}$production are shown in Appendix C.5. The $K^{0}$ accounts for $\approx 30 \%$ of the $K$ flux, which is $\approx 10 \%$ of total flux in the energy region $1<E_{\nu}<3 \mathrm{GeV}$, we use the event weights from $K^{+}$for these $K^{0}$ due to lack of data. 

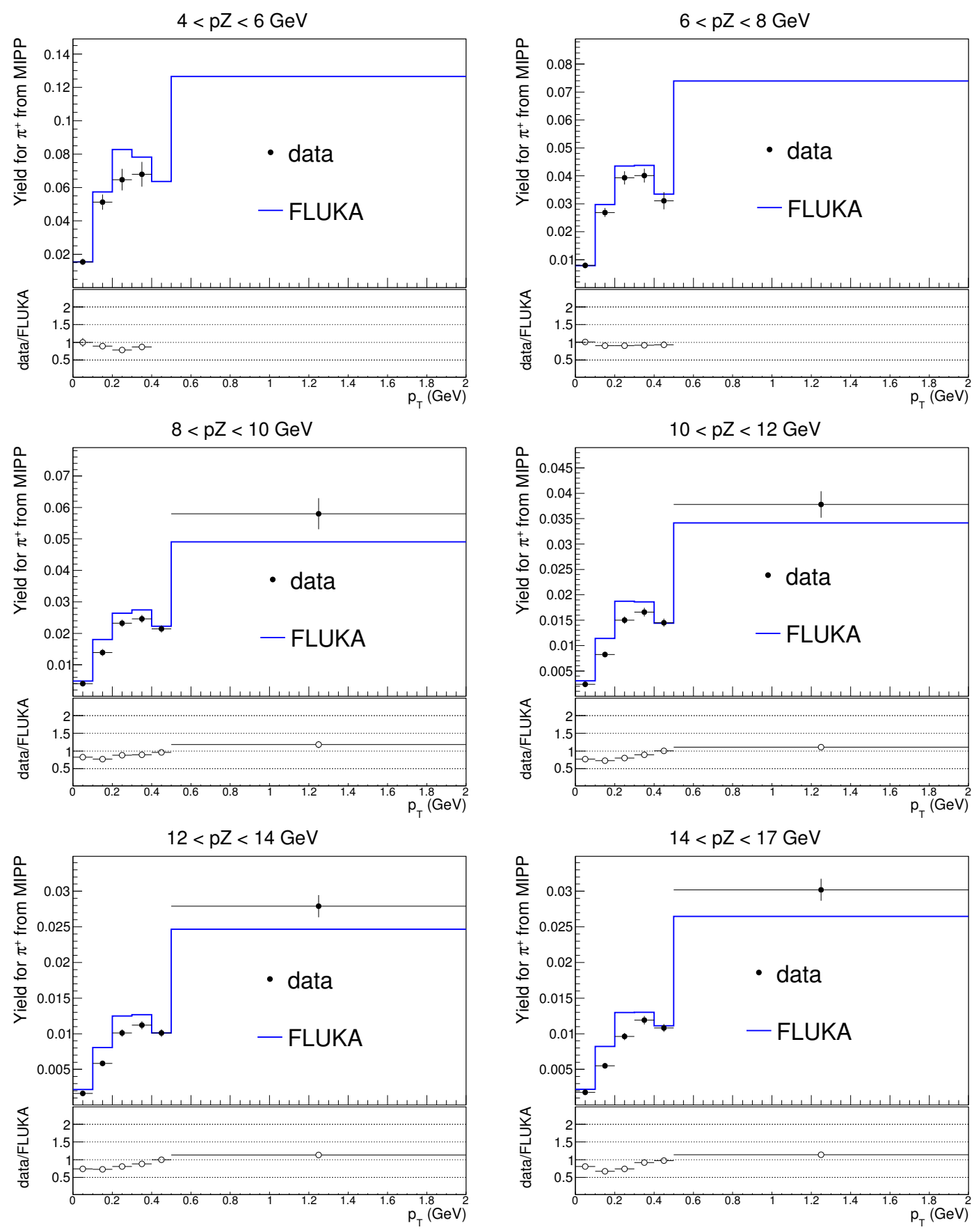

Figure 5.53: The $\pi$ yield distributions from data and FLUKA prediction for $\pi^{+}$production of MIPP. 

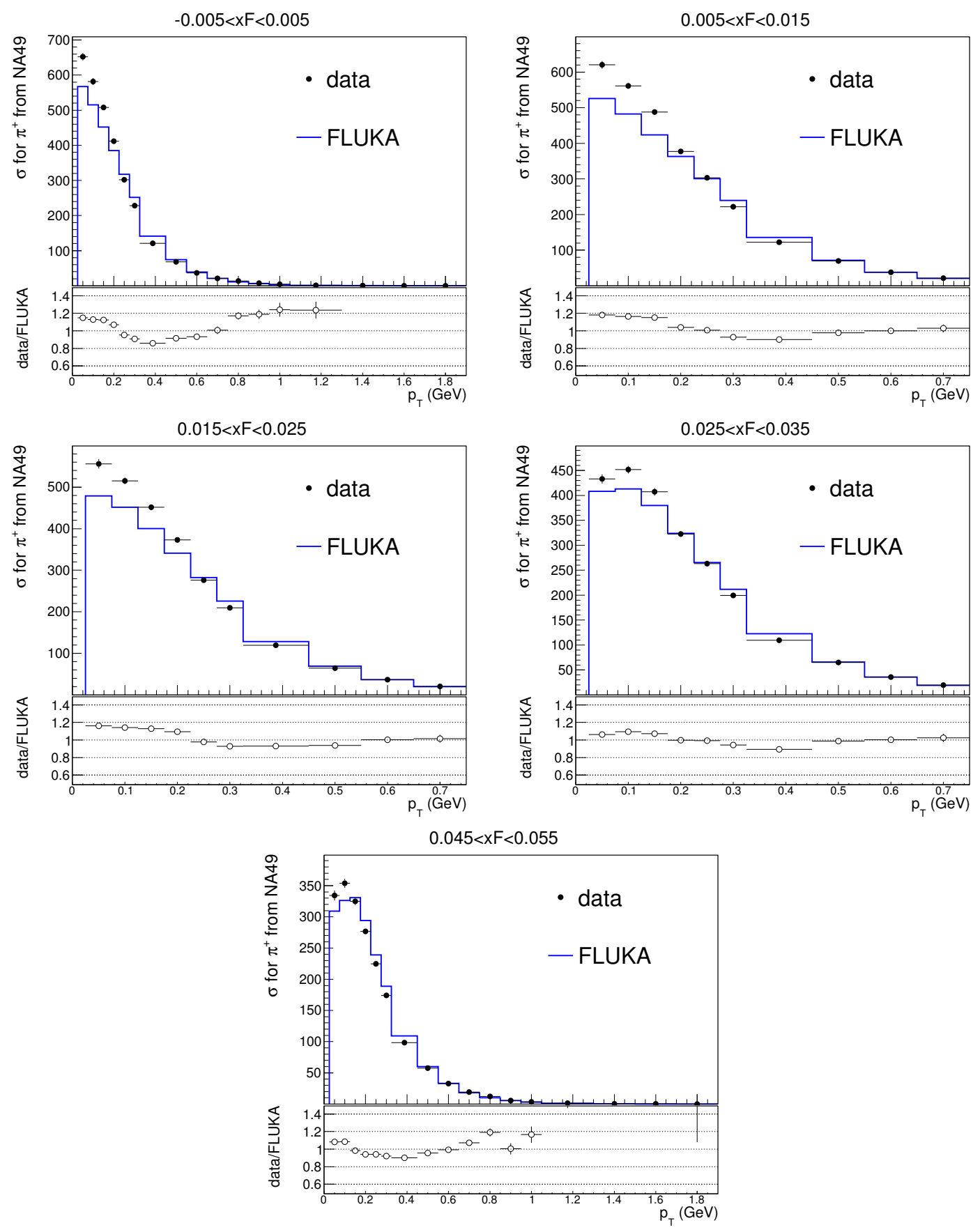

Figure 5.54: Invariant cross section and corresponding ratio between data and FLUKA prediction for $\pi^{+}$production of NA49. 

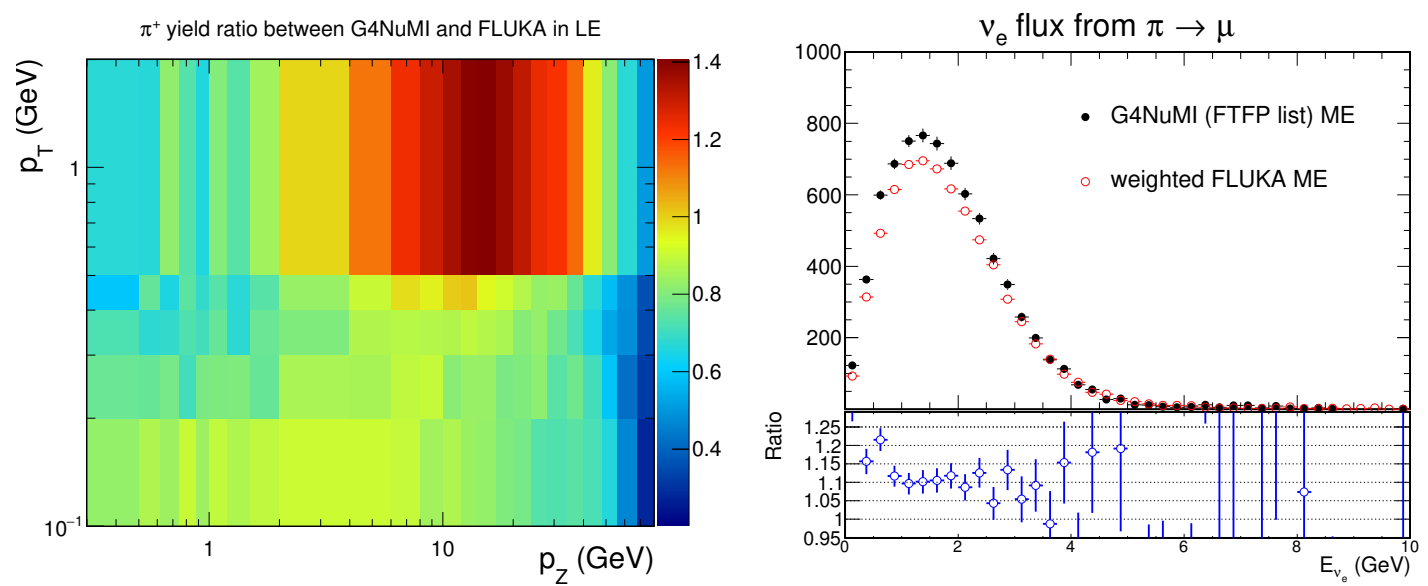

Figure 5.55: Left: The ratio for pion yield as a function of $p_{Z}$ and $p_{T}$ of pion between G4NuMI and FLUKA using low energy target configuration. Right: $\pi \rightarrow \mu \rightarrow \nu_{e}$ flux from G4NuMI and weighted FLUKA predictions using median energy target configuration.
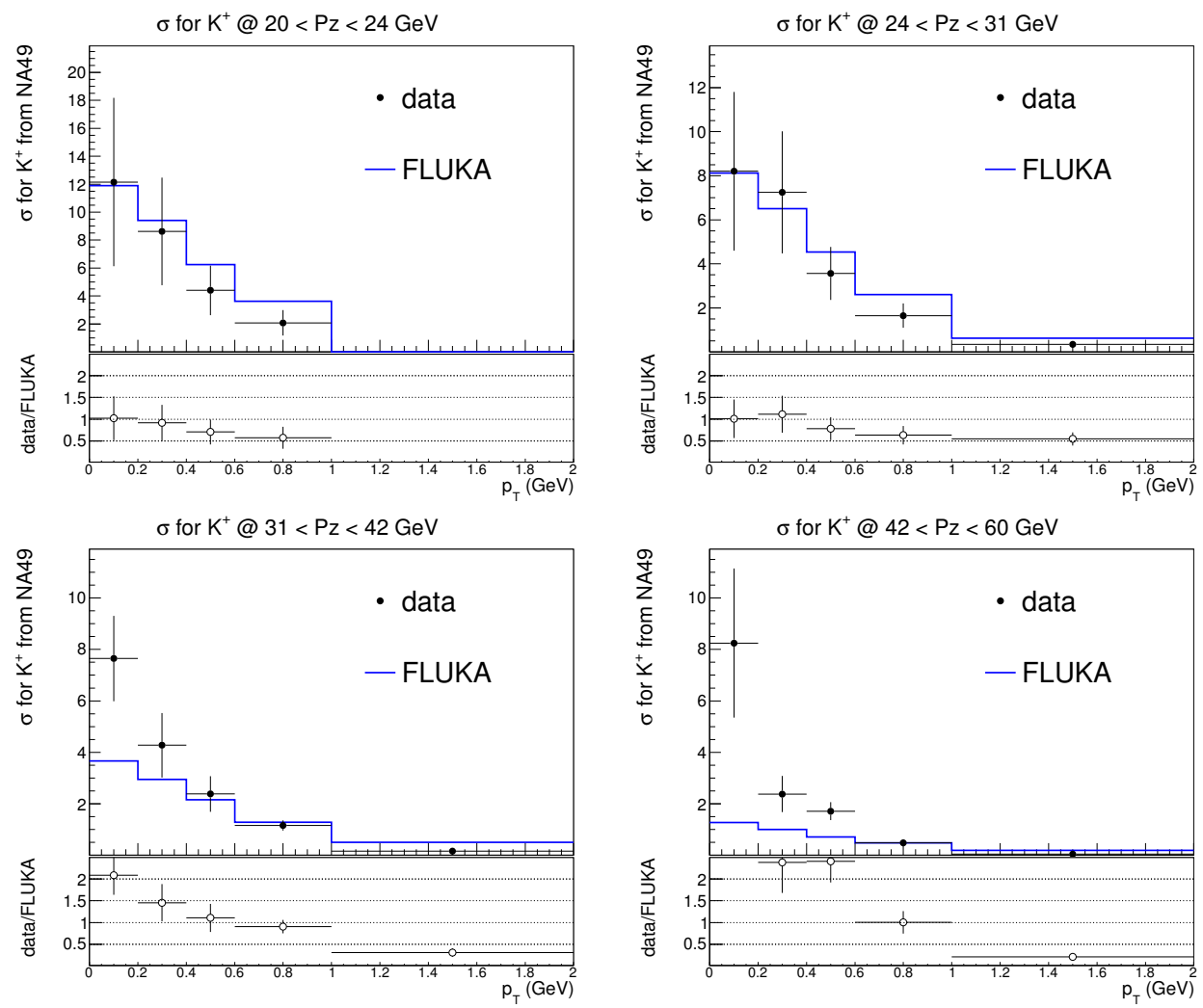

Figure 5.56: Invariant cross section and corresponding ratio between data and FLUKA prediction for $K^{+}$production in the extended phase space using the $K^{+} / \pi^{+}$ratio and $\pi^{+}$production cross section. 

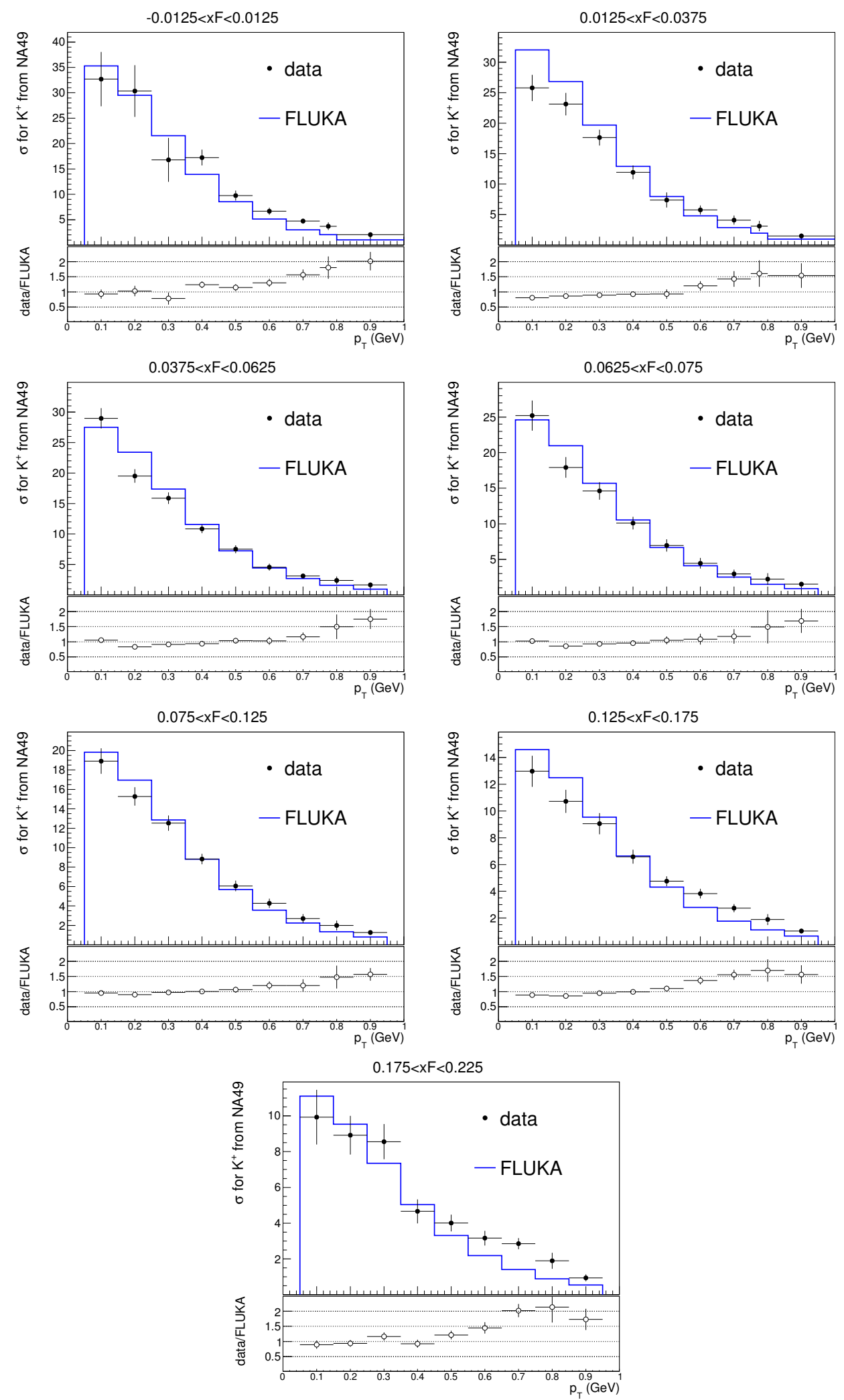

Figure 5.57: Invariant cross section and corresponding ratio between data and FLUKA prediction for $K^{+}$production. 


\subsection{Results}

We have developed an event-by-event weight framework to utilize the weights as described in previous sections, as well as to quantify the final weight on the $\nu$ flux and the corresponding uncertainty. We analyze the same FLUKA $\nu$ (for different flavors separately) events 1000 times, for each time, apply a random Gaussian weight, where the mean is measured from the event weights as described in the previous section (Section 5.3.1) based on the $p_{Z}$ and $p_{T}$ for the meson ancestor. The $\sigma$ is the corresponding uncertainty for the event weights. For the phase space not covered by any data, we treat the mean as 1 . There is no clear correlation matrix for the systematic uncertainties from the data results, thus we simply treat $\sigma$ are fully correlated.

The final flux weight at the ND as a function of $\nu_{e}$ energy is shown in Figure 5.58 (left ). The weight is $\approx 0.9$ at $1 \mathrm{GeV}$ and $\approx 0.95$ at $3 \mathrm{GeV}$. The uncertainty for the flux weight is shown in Figure 5.58 (right), which is $\approx 9 \%$ at $1 \mathrm{GeV}$, and $\approx 11 \%$ at $3 \mathrm{GeV}$. The incrementing shape starting around $3 \mathrm{GeV}$ is due to the $K$ flux component increasing rapidly. Similarly, for $\nu_{\mu}$ at the ND the weight and their respective uncertainties is shown in Figure 5.59 .

We also performed a closure test by using the NA49 pion invariant cross section results instead of the MIPP pion yield (see Appendix C.6). The uncertainty is $\approx 10 \%$, The flux weight has a similar trend as the one using MIPP data. However, as expected the size of the flux weight for this closure test is smaller, since we only apply the NA49 pion results to the secondary pion ancestors, and use a $20 \%$ systematic uncertainty for the non-secondary pion ancestors. 

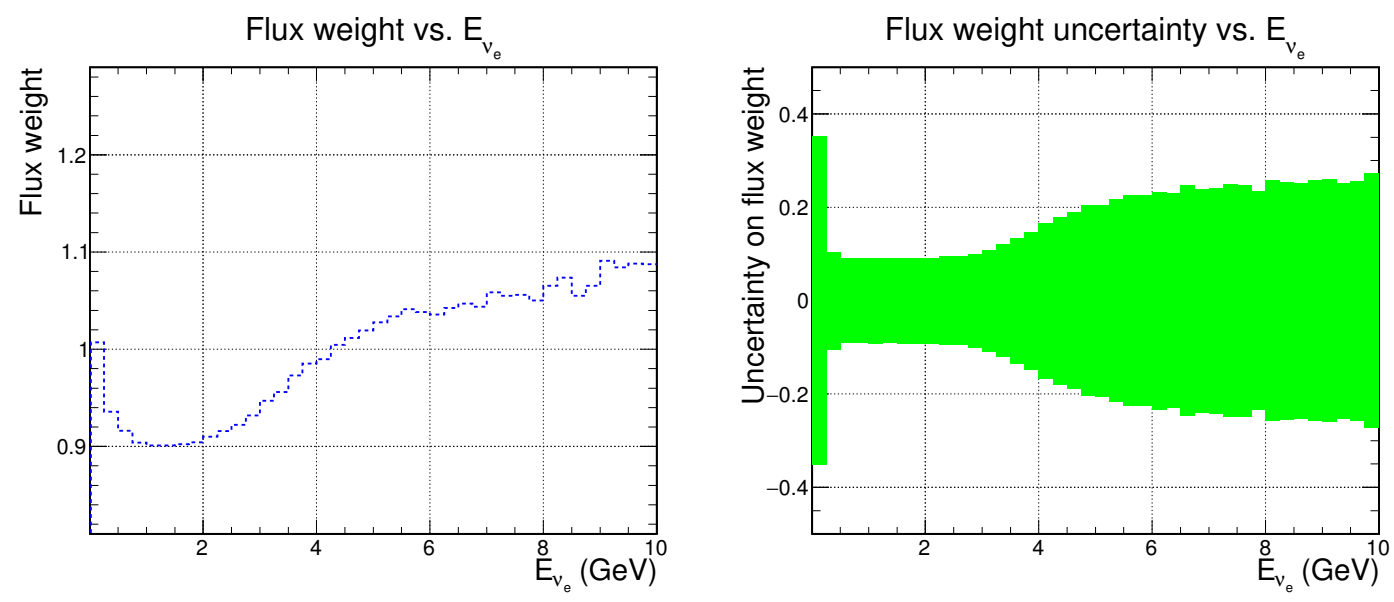

Figure 5.58: Flux weight as a function of $\nu_{e}$ energy. Uncertainty for the flux weight as a function of $\nu_{e}$ energy.
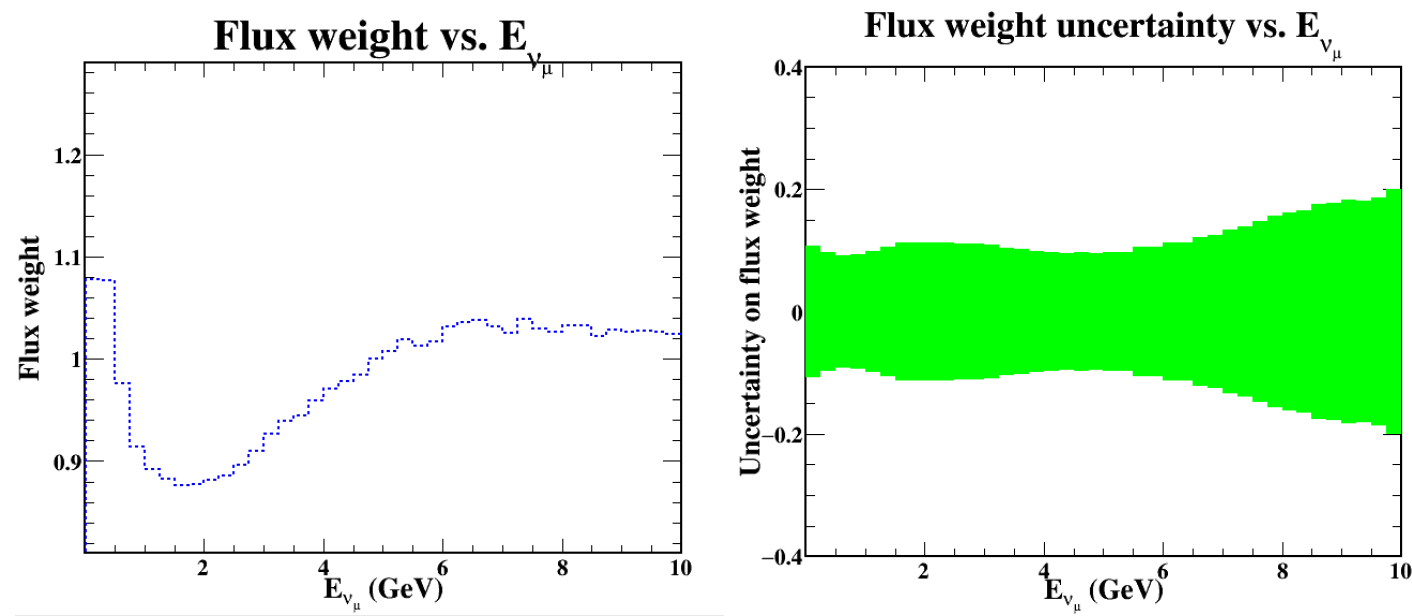

Figure 5.59: Flux weight as a function of $\nu_{\mu}$ energy. Uncertainty for the flux weight as a function of $\nu_{\mu}$ energy.
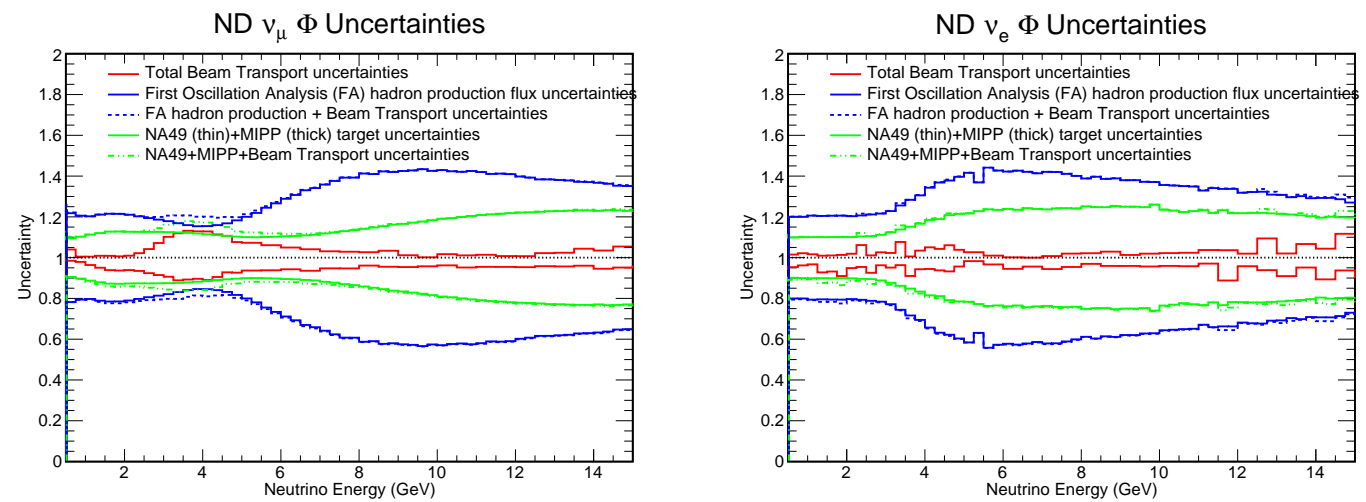

Figure 5.60: The combined uncertainties from different systematics parameters of beam line using different methods at NOvA ND. 

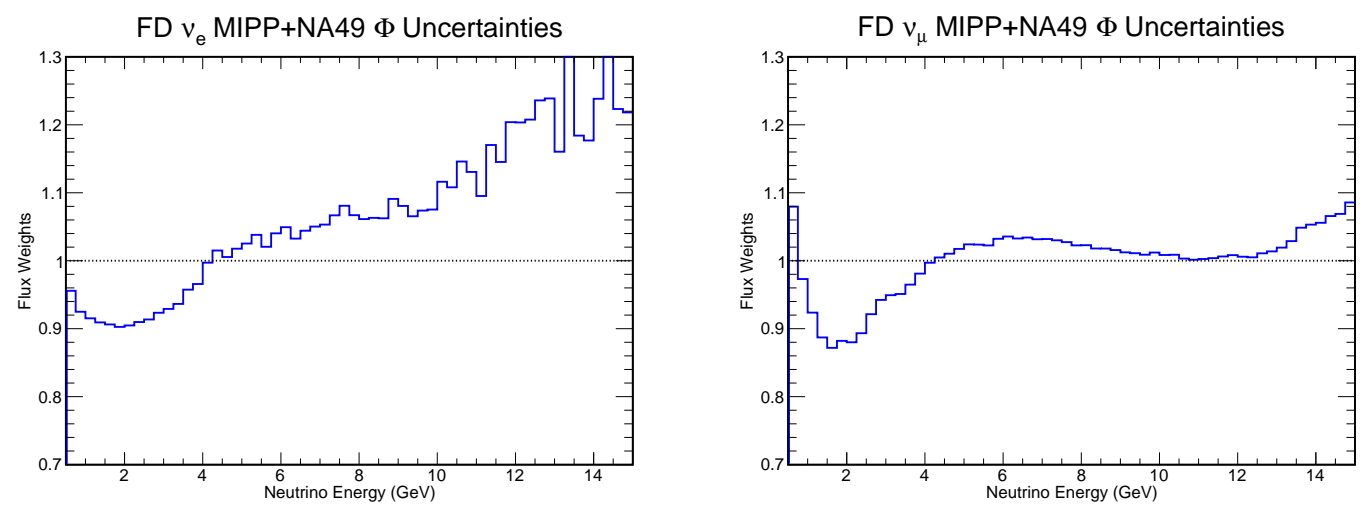

Figure 5.61: Hadron productiob flux weights for $\nu_{e}$ and $\nu_{\mu}$ at FD.
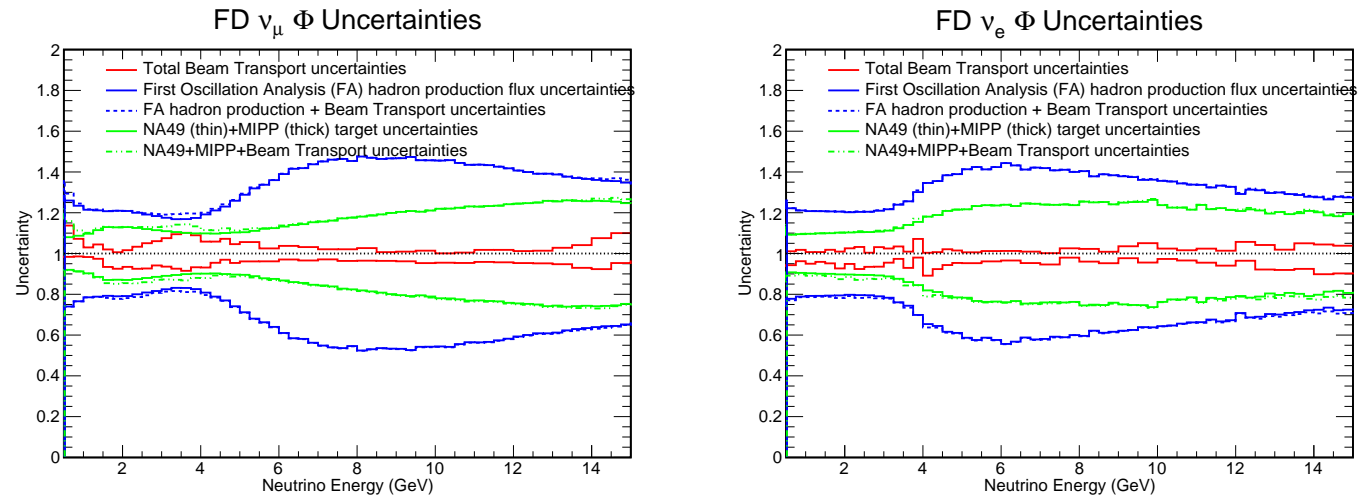

Figure 5.62: The combined uncertainties from different systematics parameters of beam line using different methods at NOvA FD.

\subsubsection{Combined Constraint}

After working on beam-line systematics studies for the main oscillation analyses, I gained significant knowledge of different beam parameters. The highest systematics for flux comes from hadron production cross-section poor knowledge. For the first (2015) NOvA oscillation analyses, I worked on beam transport studies very intimately. Also, for hadron production study there was a model for conservative uncertainties using on NA49 data only. However, based on NA49 data, the variations are larger than that expected by two different models, FTFP_BERT and Fluka. This method had very large hadron production uncertainties around $20 \%$ for ND and $18 \%$ for FD as shown in Figures $5.60-5.62$. For the oscillation study the total flux uncertainties will cancel out using the FD/ND ratio method, but if someone wants to do any measurement in the ND, e.g., for cross-section 
study, these large flux uncertainties are a problem since NuMI beamline is using thick target data but NA49 is only thin target data. Measurements in the ND would need to use thick target data from MIPP experiment. Using MIPP experiment data combine with NA49 as mentioned in the above sections the hadron production uncertainties reduced from $20 \%$ to $9-10 \%$ in oscillation region-of-interest $1-3 \mathrm{GeV}$ as shown in Figures 5.60 5.62 for ND and FD. These figures also show uncertainties from beam transports only for $\mathrm{ND}$ and FD and the combined effect of hadron production uncertainties combined with beam transport uncertainties as a funtion of neutrino energy. Chapter 6, presents the combined effect of MIPP+NA49 data with ND data for $\nu_{e}$ flux constraint only but with large energy region 0-10 GeV. Chapter 7 presents the study for absolute flux constraints using ND data for $\nu$ on electron scattering for 0-120 GeV energy region. 



\section{Chapter 6}

\section{Constraining $K^{+}$Meson and Contribution to $\nu_{e}$ Flux}

\subsection{Introduction and Motivation}

The beam $\nu_{e}$ and $\bar{\nu}_{e}$ fluxes are especially pertinent to the $\nu_{e}$ and $\bar{\nu}_{e}$ appearance measurements that lead to a determination of the neutrino mass hierarchy and the $\delta_{C P}$. In the neutrino mode, the $\nu_{e}$ flux in NOvA is dominated by $\mu^{+}$and $K^{+}$. In the $\mathrm{E}_{\nu_{e}} \leq 3 \mathrm{GeV}$ region, the $\mu^{+}$decay $\left(\mu^{+} \rightarrow e^{+}+\bar{\nu}_{\mu}+\nu_{e}\right)$ as shown in Table 5.1 dominates, where the $\mu^{+}$is almost entirely produced from the $\pi^{+}$decay $\left(\pi^{+} \rightarrow \mu^{+}+\nu_{\mu}\right)$. In the $\mathrm{E}_{\nu_{e}} \geq 3.5 \mathrm{GeV}$ region, the $K^{+}$decay (via $K_{e 3}$ ) dominates as shown in Table 6.1. This is shown in Figure 5.2. As displayed in Figure 6.1, the neutrinos produced on-axis demonstrate a strong dependence on the parent pion (left) and kaon (right) energy. As opposed to the off-axis weak energy dependence of those produced from pion compared to kaon (only include two body decay which covers $63.55 \%$ as shown in Table 5.1).

The $\mu^{+}$component, in principle, can be determined from the $\nu_{\mu}$ - CC data in $0.5 \leq E \nu_{\mu} \leq 3.5 \mathrm{GeV}$. However, in this region error in the $\nu_{\mu}-\mathrm{CC}$ cross-section is poorly known, $15 \%$, or larger. On the other hand, whereas the cross-section for $\nu_{\mu}-\mathrm{CC}$ 


\begin{tabular}{|c||c|c|c|c|}
\hline$\nu_{e} \%$ & $0-10(\mathrm{GeV})$ & $1-3(\mathrm{GeV})$ & $<4 \mathrm{GeV}$ & $>4 \mathrm{GeV}$ \\
\hline$\mu^{+}$ & $50.8 \%$ & $81.3 \%$ & $71.5 \%$ & $11.47 \%$ \\
$K^{+}$ & $36.9 \%$ & $11.4 \%$ & $19.04 \%$ & $67.65 \%$ \\
Other & $12.3 \%$ & $7.3 \%$ & $9.42 \%$ & $21.07 \%$ \\
\hline
\end{tabular}

Table 6.1: Fractional composition of electron neutrinos from $\mu^{+}, K^{+}$and other parents.
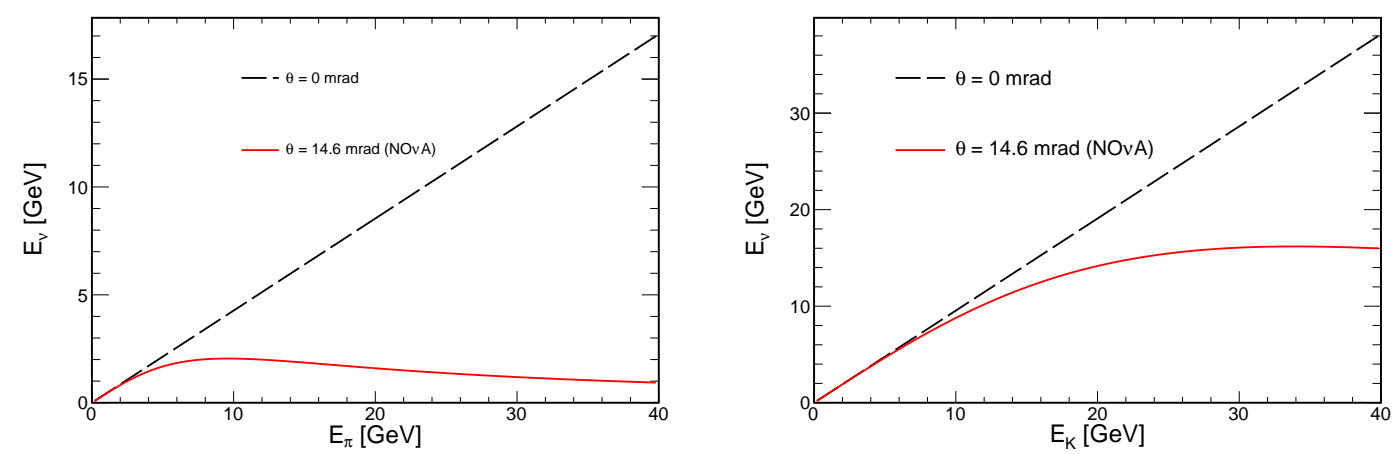

Figure 6.1: Using two body decay formula, Left: Neutrino energy vs pion energy at NOvA axis and on-axis w.r.t the beamline. Right: Neutrino energy vs kaon energy at NOvA axis and on-axis w.r.t the beamline.

in $\mathrm{E}_{\nu_{\mu}} \geq 4 \mathrm{GeV}$ is better known $(\approx 5 \%)$ [79], in an on-axis experiment the $K^{+}$-induced neutrinos are not separated from the $\pi^{+}$-neutrinos. However, in the off-axis beam of NOvA the $\nu_{\mu}$ from $K^{+}$are clearly separated from the $\pi^{+}$in $\mathrm{E}_{\nu_{\mu}} \geq 4.5 \mathrm{GeV}$ using uncontained $\nu_{\mu} \mathrm{CC}$ events in ND, as pictured in Figure 6.2 and their parent $p_{z}$ vs $p_{t}$ distribution of the target $\left(t p_{z}, t_{p_{t}}\right)$ is shown in Figures 6.3 and 6.4 .

This analysis extracts constraints on the $K^{+}$and $\pi^{+}$yields using the contained and uncontained $\nu_{\mu}$ induced charged current (CC) events in the ND. The constrained $K^{+}$yield, in conjunction with the hadro-production $(\mathrm{MIPP}+\mathrm{NA} 49)$ constraints on $\pi^{+}$ allow the $\nu_{e}$ flux prediction with a $11 \%$ precision in $0.5 \leq E_{\nu} \leq 10 \mathrm{GeV}$. In summary, this analysis will be used for:

1. $\nu_{e}$ flux Prediction: The ND data-driven constraint on $K^{+}$allows for a precise $\nu_{e}$-flux prediction for $\mathrm{E}_{\nu_{e}}>3.5 \mathrm{GeV}$.

2. $\frac{F D}{N D}$ flux Prediction: More precise constraint on $\frac{F D}{N D}$ flux for $\nu_{e} \& \nu_{\mu}$ flux. 
3. $\nu_{\mu}$ Physics:

- Provides a cross-check for absolute $\nu_{\mu} \mathrm{CC}$ cross-section measurement.

- For all other analyses, $\sigma(\mathrm{X}) / \sigma\left(\right.$ Inclusive $\left.\nu_{\mu} \mathrm{CC}\right)$, where $\mathrm{X}=$ Neutral Curent (NC), Quasi-Elastic (QE), Resononce (Res), or Coherent pion (CohPi), the constrained $K^{+}$flux translates to a commensurately precise prediction for $\mathrm{E}_{\nu}>$ $3.5 \mathrm{GeV}$.

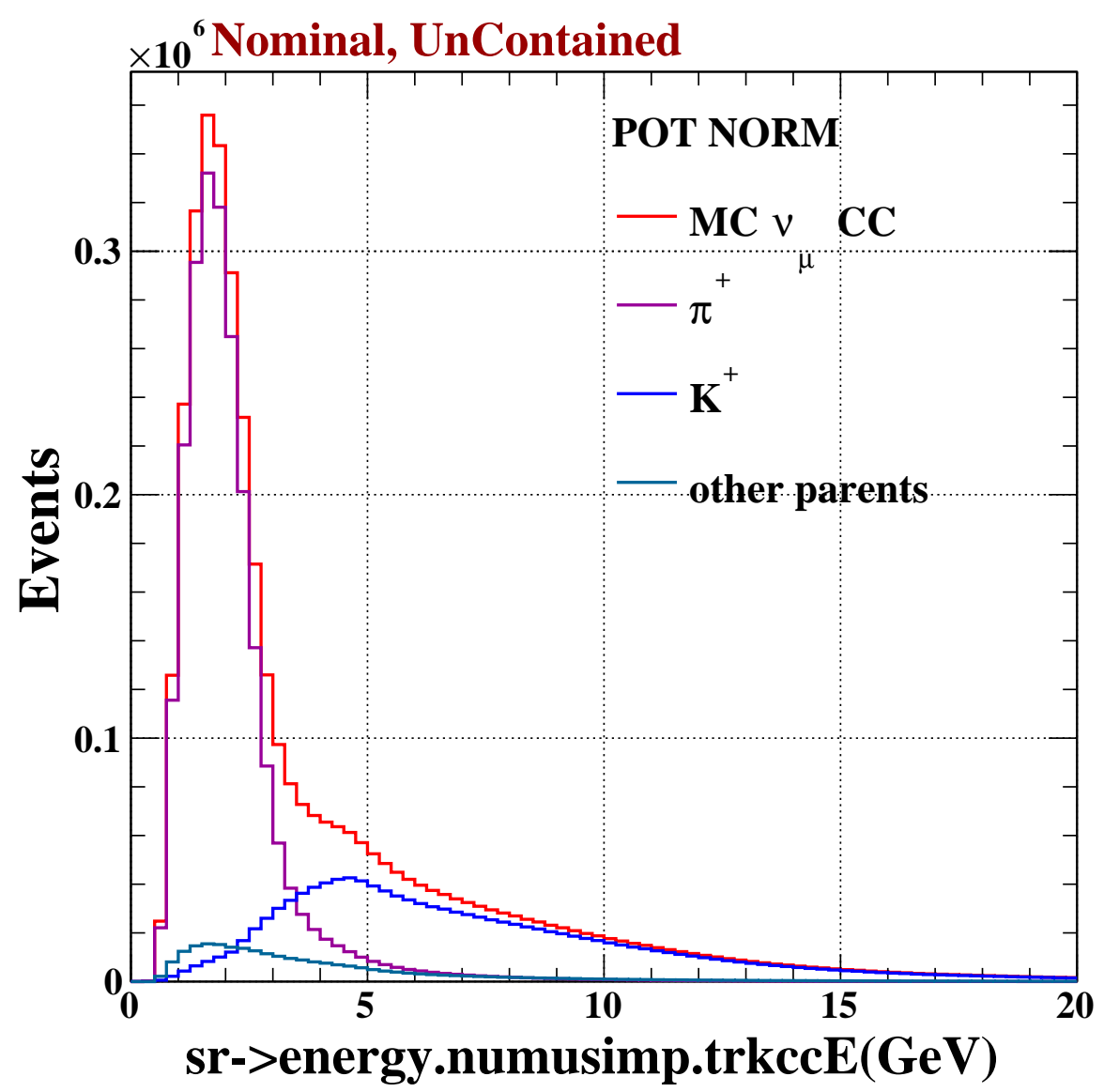

Figure 6.2: Reconstructed Neutrino Energy(TrkCCE) distribution, uncontained: Total muon neutrinoCC MC events (red line), muon neutrinoCC from $\pi^{+}$(purple line), muon neutrinoCC from $K^{+}$(blue line) and muon neutrinoCC from other parents(sea green). 

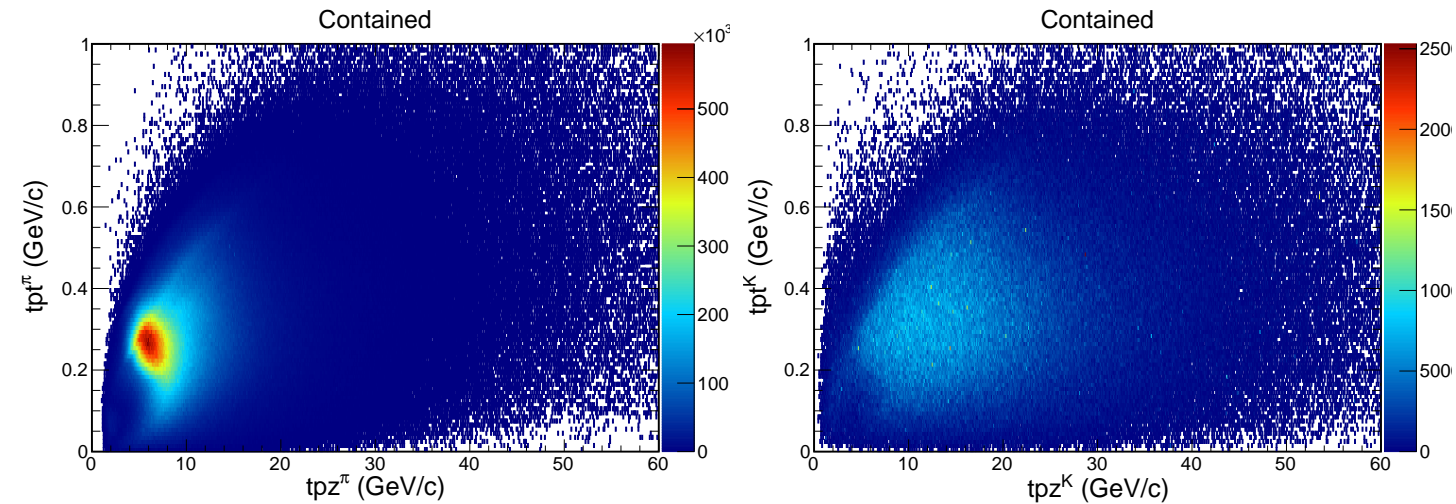

Figure 6.3: Left: The longitudinal pz vs transverse momentum pion distribution at the the target, which are ancestor of contained muon neutrinoCC events at ND. Right: The longitudinal $\mathrm{pz}$ vs transverse momentum kaon distribution at the the target, which are ancestor of contained muon neutrinoCC events at ND.
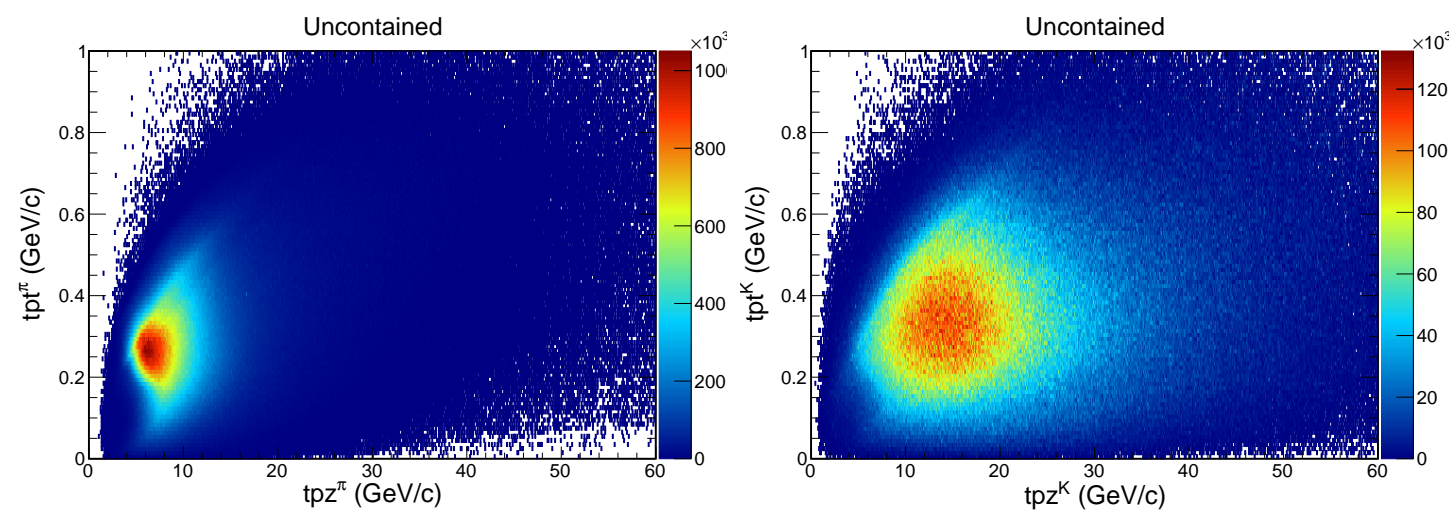

Figure 6.4: Left: The longitudinal pz vs transverse momentum pion distribution at the the target, which are ancestor of uncontained muon neutrinoCC events at ND. Right: The longitudinal pz vs transverse momentum kaon distribution at the the target, which are ancestor of uncontained muon neutrinoCC events at ND.

\subsection{Data and Monte Carlo Samples}

This analysis has been performed using CAF's and the CAFAna framework [80] of the NOvA experiment. The data being analyzed for this analysis are the same as the data used in the oscillation analyses concluded in the Summer of 2016. The data is collected by the NOvA Near Detector between 16 Aug, 2014 - 20 Jan, 2016 (2016, Second Analysis, $\mathrm{SA})$, with $3.7 \times 10^{20}$ protons on target $(\mathrm{POT})$. Neutrino interactions are simulated using the GENIE generator and reconstruction is done as explained in Chapter 4. Nominal 
Flux is based on Flugg see Chapter 2,

\subsection{Event Selection.}

This section details the data quality selection for NOvA data as well as the event selection. The selection includes cuts to ensure data quality, background rejection and signal selection.

\subsubsection{Preselection}

- We ensure that the $\nu_{\mu}$ - CC energy algorithm has returned valid energy for the slice. The $\nu_{\mu}-\mathrm{CC}$ requires that there is a $3 \mathrm{~d}$ muon track in an event, this ensures all slices selected have at least one $3 \mathrm{~d}$ track that has been reconstructed as a muon.

- Events with less than 20 hits are more likely to be due to NC interactions and are often not well reconstructed. These events are removed. Coincidentally this will remove events less than about $400 \mathrm{MeV}$ in energy.

- If events are very vertical, these are very likely to be due to cosmic muons. Though the rate of this is tiny at the Near Detector, to remove all such events in our selection the number of continuous planes is greater than four.

- The cosmic track [81] reconstruction looks for long straight tracks in the detector and will identify Near Detector muon tracks from the beam. This selection is necessary for the Far detector cosmic muon rejection and is included here for consistency between the two detectors. 

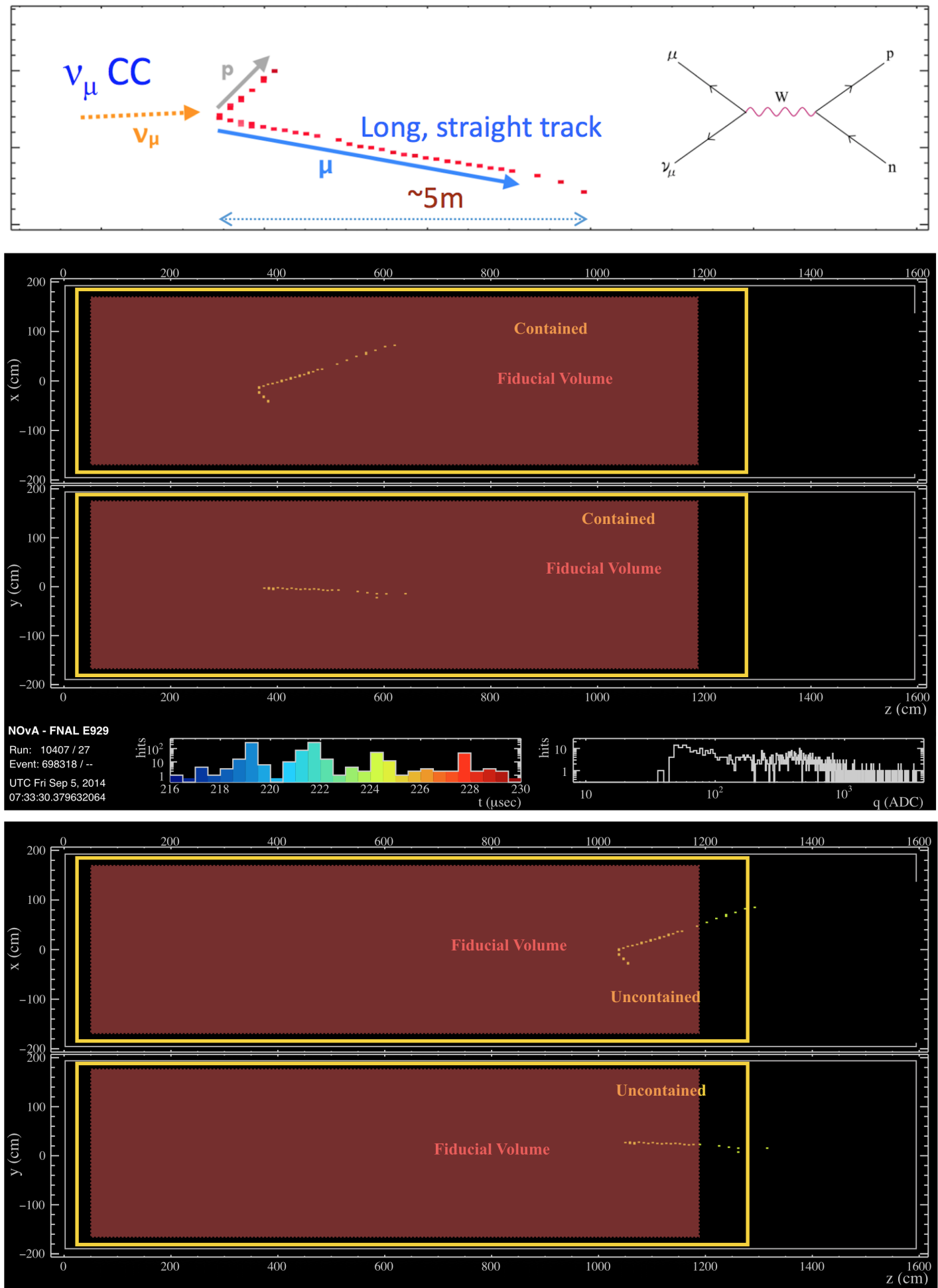

NOVA - FNAL E929

Run: $10407 / 27$

UTC Fri Sep 5, 2014

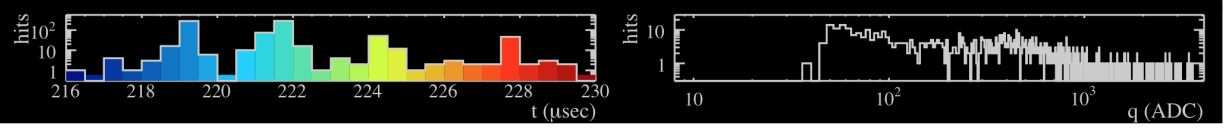

Figure 6.5: The contained vs uncontained selection. 


\subsubsection{Fiducial Volume.}

This is a veto cut for particles entering the detector caused by neutrino interactions in the material surrounding the detector, i.e., rock. We are selecting events that have interactions inside the active region of ND. The fiducial volume (FV) for this study is defined as $-170<\mathrm{x}<170,-170<\mathrm{y}<170$ and $30<\mathrm{z}<1150 \mathrm{~cm}$.

\subsubsection{Contained Events vs Uncontained Events}

This is a muon track containment and uncontainment selection as shown in Figure 6.5. the kalman track (see Subsection 4.2.4) belonging to best track value starts inside the active region $(\mathrm{FV})$. For contained events this track should stop inside the containment region (inside detector) i.e $-185<\mathrm{x}<185,-185<\mathrm{y}<185$ and $19<\mathrm{z}<1275 \mathrm{~cm}$. For uncontained events this track should stop outside the detector i.e $-185<\mathrm{x}<185,-185<$ $\mathrm{y}<185$ and $19<\mathrm{z}<1275 \mathrm{~cm}$.

\subsubsection{ReMId (Neutral current rejection)}

ReMId [49] (also see Section 4.2.5) cut is applied to reject NC events. The standard selection is the removal of all events where the response of this ReMId gives a score < 0.75. The ReMId distributions both for contained and uncontained samples are shown in Figure 6.6, left and right respectively.

\subsubsection{Selection Based on Truth}

- Parent's selection is based on ancestor information exiting the target using pdg code information 211 for $\pi^{+}$and 321 for $K^{+}$.

- Rock background events are informed on truth vertex information [82] if an inter- 

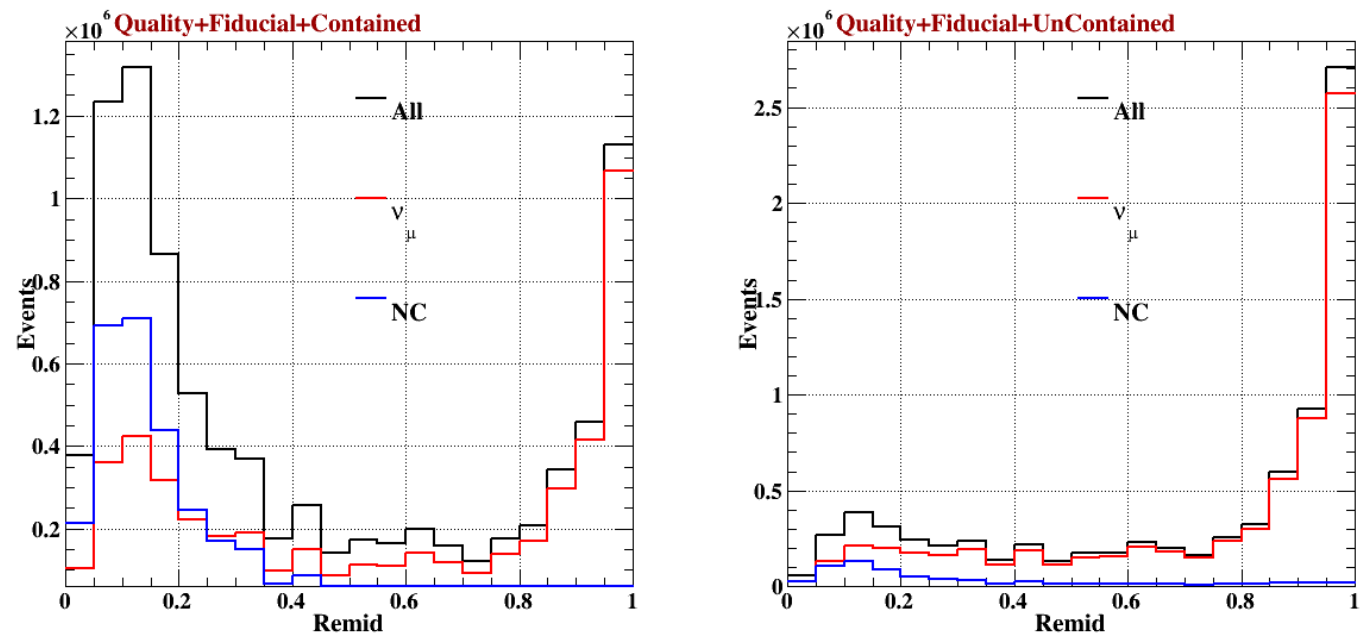

Figure 6.6: ReMID distribution Left:: Contained sample Right:: uncontained sample.

action vertex satisfies any of the following conditions we are going to use that as rock event i.e $-180>\mathrm{x}>180,-180>\mathrm{y}>180$ and $\mathrm{z}<30 \mathrm{~cm}$.

Selection cut table for contained and uncontained events are shown in Table 6.2 and Table 6.3 .

\begin{tabular}{|c||c|c|c|c|c|c|c|}
\hline Cut & Data & MC & muon neutrinoCC & $\overline{\nu_{\mu}}$ & NC & Rock & Others \\
\hline \hline Fiducial & 8669077 & 8461313.6 & 6563296.6 & 263334.8 & 1313492.1 & 193202.1 & 127988.1 \\
Cont & 3262354 & 3248223.5 & 1878460.4 & 41787 & 1066681.4 & 162746.6 & 98548.1 \\
REMID & 1080850 & 1085999.4 & 1006094.5 & 26165.7 & 44073 & 6282.3 & 3383.8 \\
\hline
\end{tabular}

Table 6.2: Event-selection: Contained.

\begin{tabular}{|c||c|c|c|c|c|c|c|}
\hline Cut & Data & MC & muon neutrinoCC & $\overline{\nu_{\mu}}$ & NC & Rock & Others \\
\hline \hline Fiducial & 8669077 & 8461313.6 & 6563296.6 & 263334.8 & 1313492.1 & 193202.1 & 127988.1 \\
UnCont & 5406723 & 5213090.2 & 4684836.3 & 221547.8 & 246810.7 & 30455.5 & 29439.9 \\
REMID & 4087471 & 3934078.3 & 3696085 & 194548.2 & 33072.4 & 7012 & 3360.7 \\
\hline
\end{tabular}

Table 6.3: Event-selection: uncontained. 


\subsection{Method.}

This section outlines the method used to determine the $K^{+}$and $\pi^{+}$yields using the $\nu_{\mu}$ induced charged current (CC) events in the NOvA ND. The CC ND data are divided into two samples: Contained Sample, composed of $\nu_{\mu}$ - CC events where the muon ranges out in the active ND before the muon catcher. These are typically low energy events. uncontained Sample composed of $\nu_{\mu}$ - CC events where the muon traverses through the active ND and enters into the muon-catcher. These are typically higher-energy events. The $K^{+}$constraint is derived from the $\nu_{\mu}$ - CC measured in these two samples: the Contained sample serves as an in-situ constraint on the pion-normalization; next, the uncontained sample in visible energy $>4.5 \mathrm{GeV}$ yields the kaon-normalization. In this analysis, the MC samples, comprising $\nu_{\mu}-\mathrm{CC}, \bar{\nu}_{\mu}-\mathrm{CC}$, neutral current events (NC), rock-events, etc. are all normalized using the data proton-on-target (POT).

\subsubsection{Inclusive Charged Current Events without $\mathrm{E}_{\text {had }}$ Cut.}

The total reconstructed $\nu_{\mu}$ (Energy Estimation 4.2.6) energy distribution of the contained sample is shown in Figure 6.7 (left), shows that the $\nu_{\mu}$ from $\pi^{+}$dominates the distribution. The total reconstructed $\nu_{\mu}$ energy distribution of the uncontained sample is shown in Figure 6.7 (right), where the $\nu_{\mu}$ from $K^{+}$is dominant above $E_{\nu} \geq 4.5 \mathrm{GeV}$ and the $\nu_{\mu}$ from $\pi^{+}$is at a few percent levels. Although there is an evident discrepancy in the shape, the overall yield of the $\pi^{+}$and $K^{+}$are discernible from the $E_{\nu}$ distributions in the two samples. The shape discrepancy between data and MC arises from three principal sources: 1) the differential cross-section of $\pi^{+} / K^{+}$production, $d^{2} \sigma / d x_{F} d P_{T}$; 2) the $\nu_{\mu} \mathrm{CC}$ cross-section modeling; and 3) event reconstruction model. The systematic uncertainty analysis attempts to account for the corresponding effect on the overall normalization of the mesons due to these uncertainties. The method of determining the 
overall mesons' yield is as follows:

1. Obtain the Contained $\nu_{\mu}-\mathrm{CC}$.

2. Subtract the contributions to the $\nu_{\mu}$ - CC from all sources other than $\pi^{+}$such as shown in Figure 6.7. It should be noted that these background events are small $\left(\leq 1 \%\right.$ of the observed $\left.\nu_{\mu}-\mathrm{CC}\right)$

3. Fit the $\pi^{+}$normalization in the $0.75-3 \mathrm{GeV}$ region. In this analysis all meson normalizations are with respect to the standard beam simulation which is used for the hadro-production and for the beam-transport [83]. Since the statistical error in meson normalization is typically $\approx 1 \%$, which is the case for all the variants in this analysis, the dominant concern and, hence, the focus, is on determining the systematic errors from various sources. Typically the $\pi^{+}$yield is close to unity, with respect to the beam prediction, within $\approx 3 \%$.

4. Obtain the $\nu_{\mu}-\mathrm{CC}$ in the Uncontained sample, and subtract the background to get the $K^{+}$contribution.

5. Using the $\pi^{+}$normalization determined in (3), fit the $K^{+}$normalization in $4.5 \leq$ $E_{\nu} \leq 10 \mathrm{GeV}$.

6. Iterate the entire chain between contained (pion) and uncontained region (kaon) until converge. This method is tabulated in Table. 6.4

\begin{tabular}{|c||c|}
\hline 1 & RawDataSignal=Data-(NC+Anti+Rock+Others $)_{M C}$ \\
\hline 2 & $N_{\pi}=\frac{\text { RawDataSignal }-\left(\nu_{\mu} \text { CC from } K^{+} \text {and other parents except } \pi^{+}\right)}{\nu_{\mu} \text { from } \pi^{+}}$ \\
\hline 3 & $N_{K}=\frac{\left.\text { RawDataSignal }-{ }_{\left(\nu_{\mu}\right.} \text { CC from } \pi^{+} \text {and other parents except } K^{+}\right)}{\nu_{\mu} \text { from } K^{+}}$ \\
\hline
\end{tabular}

Table 6.4: Iterative Method for final $K^{+}$Norm Calculation. 

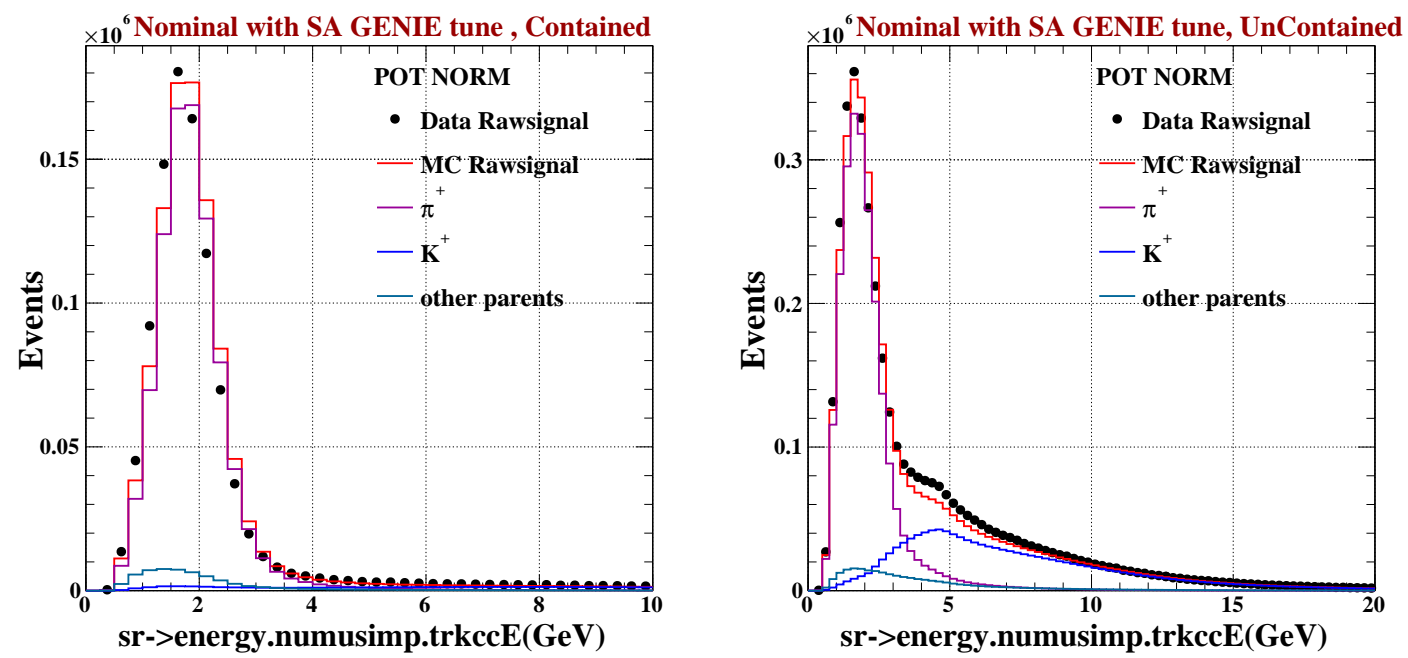

Figure 6.7: Reconstructed Neutrino Energy(TrkCCE) distribution Left: Contained and Right: uncontained: Data rawsignal events[Data- $\left.\mathrm{MC}_{b k g}\right]$ (black solid dot), total muon neutrinoCC MC rawsignal events(red line), muon neutrinoCC from $\pi^{+}$(purple line), muon neutrinoCC from $K^{+}$(blue line) and muon neutrinoCC from other parents(sea green).

\subsubsection{Inclusive Charged Current Events with $\mathrm{E}_{\text {had }}$ Cut}

The $K^{+}$separation from the $\pi^{+}$sample is cleaner using kinematic cuts, for example a cut on the hadron energy $\geq 0.5$ or $1 \mathrm{GeV}$ results in a distinct $K^{+}$- rich region in the $\nu_{\mu}$ - CC spectrum. Figures 6.8 and 6.9 shows the $\nu_{\mu}-\mathrm{CC}$ distribution with $E_{\mathrm{Had}}>0.5$ $\mathrm{GeV}$ and $>1 \mathrm{GeV}$. The $K^{+}$vs $\pi^{+}$separation is more pronounced with the $E_{\mathrm{Had}}$ cut than without the $E_{\mathrm{Had}}$ cut. However, the $E_{\mathrm{Had}}$ distribution is poorly understood as evidenced in Figures 6.10 and 6.11 (for Contained and uncontained sample). Thus, given the rather intractable discrepancy in the $E_{\mathrm{Had}}$ despite the tuned-Genie in the second analysis, this sample cannot be used with appreciable precision to extract the $K^{+}$and $\pi^{+}$normalization. Once we understand the $\mathrm{E}_{\text {had }}$ distribution, this method holds promise as evidenced by the figures. 

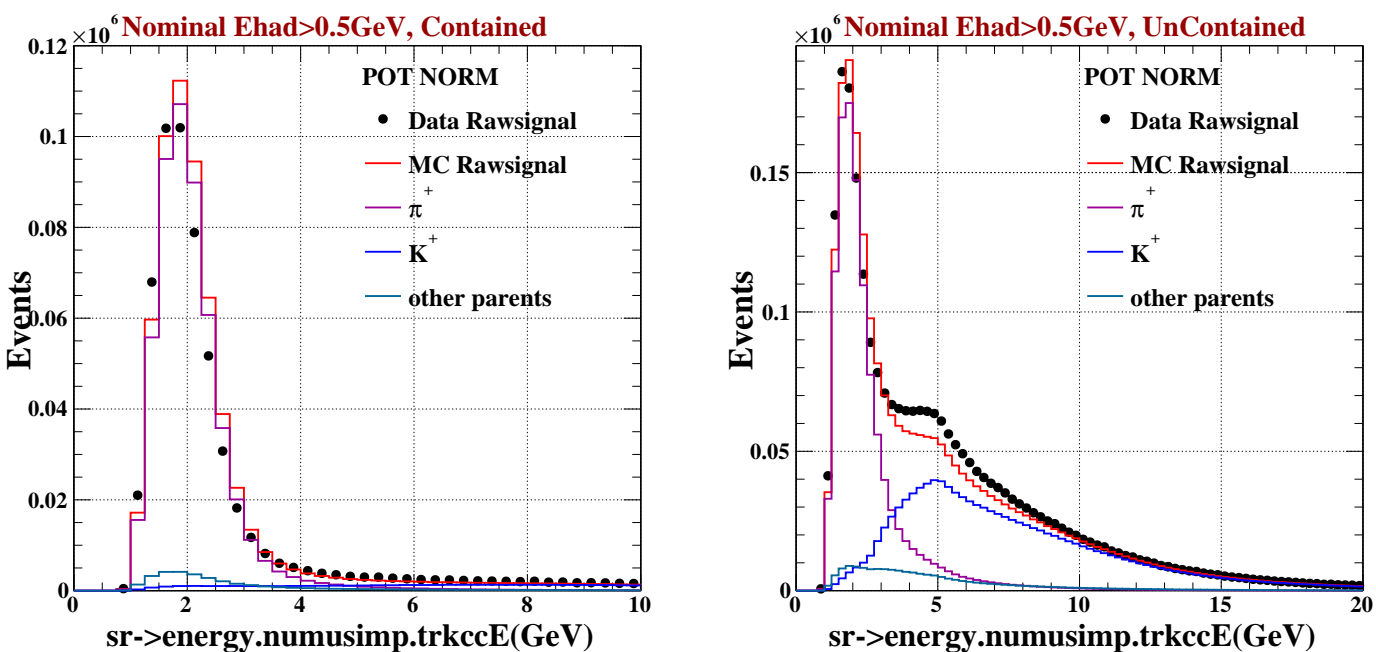

Figure 6.8: Reconstructed Neutrino Energy(TrkCCE) distribution Left: $\mathrm{E}_{H a d}>0.5 \mathrm{GeV}$, contained and Right: $\mathrm{E}_{\text {Had }}>0.5 \mathrm{GeV}$, uncontained : Data rawsignal events[Data$\left.\mathrm{MC}_{b k g}\right]$ (black solid dot), total muon neutrinoCC $\mathrm{MC}$ rawsignal events(red line), muon neutrinoCC from $\pi^{+}$(purple line), muon neutrinoCC from $K^{+}$(blue line) and muon neutrinoCC from other parents(sea green).
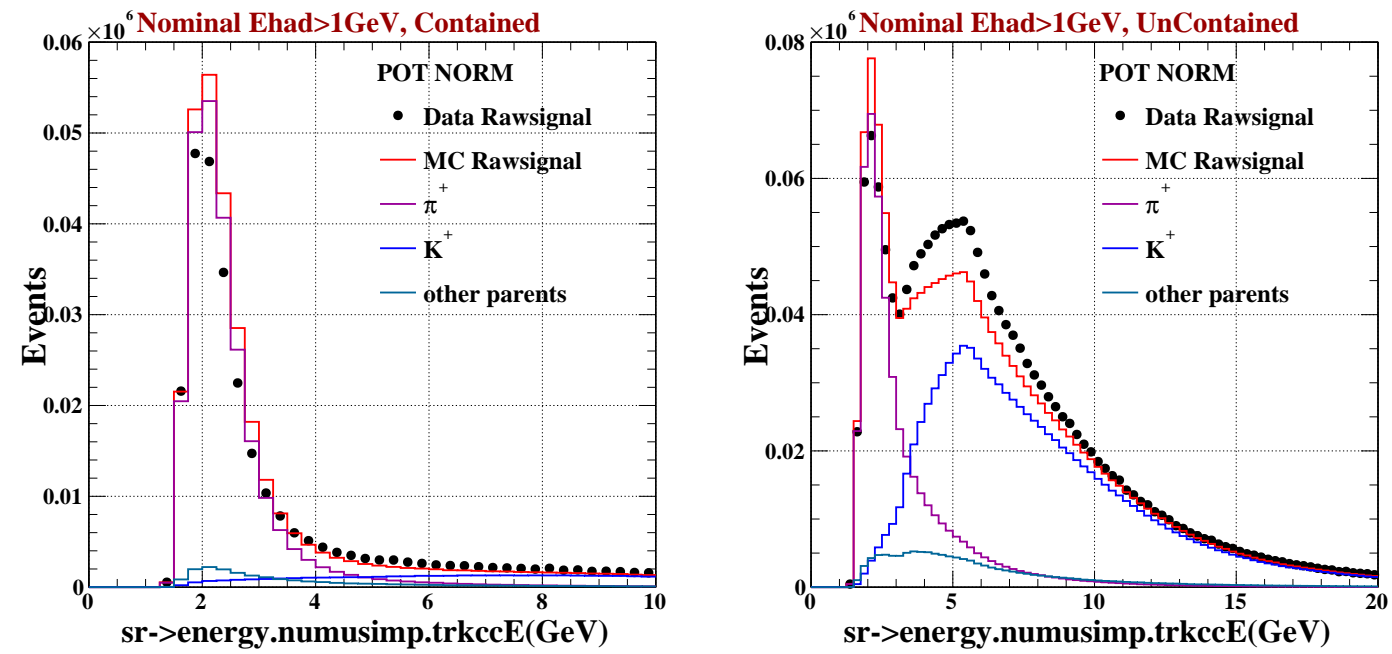

Figure 6.9: Reconstructed Neutrino Energy(TrkCCE) distribution Left: $E_{\text {had }}>1 \mathrm{GeV}$, contained and Right: $\mathrm{E}_{\text {had }}>1 \mathrm{GeV}$, uncontained : Data rawsignal events[Data$\left.\mathrm{MC}_{b k g}\right]$ (black solid dot), total muon neutrinoCC MC rawsignal events(red line), muon neutrinoCC from $\pi^{+}$(purple line), muon neutrinoCC from $K^{+}$(blue line) and muon neutrinoCC from other parents(sea green). 

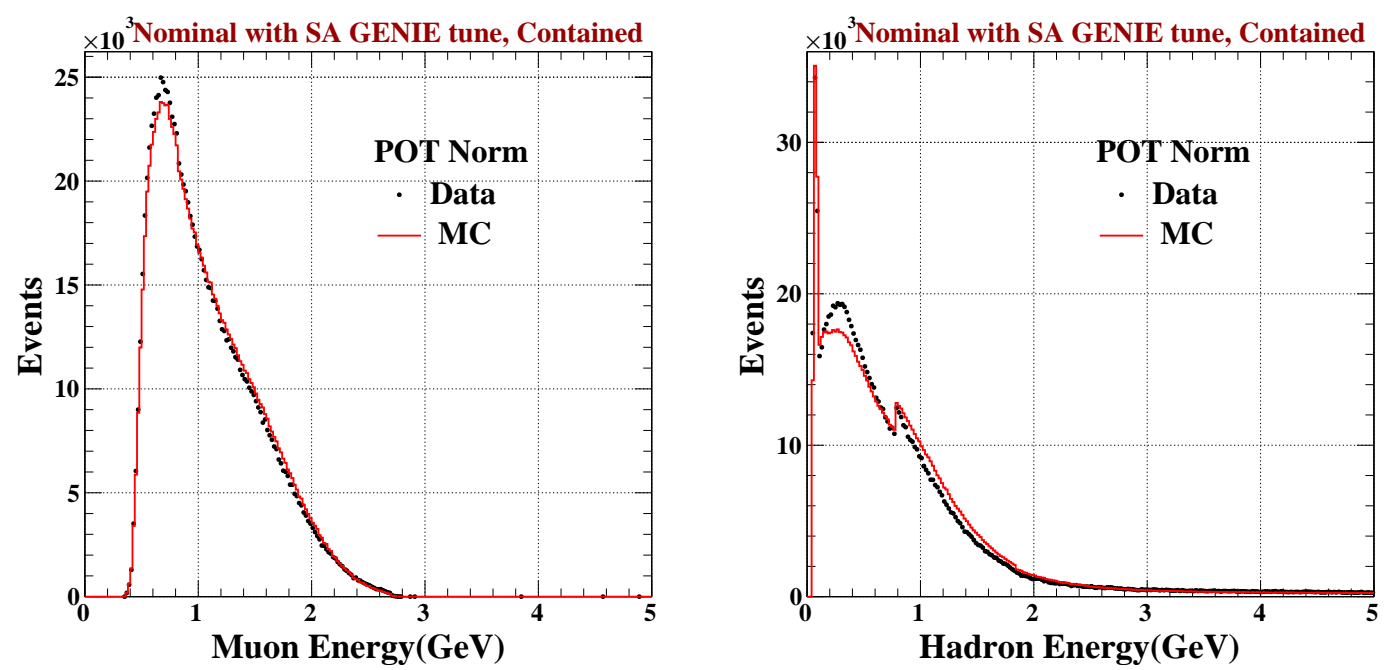

Figure 6.10: Left: $\mathrm{E}_{\mu}$ distribution of Data(solid black dot) and $\mathrm{MC}$ (red line) for contained Sample. Right: $\mathrm{E}_{\text {had }}$ distribution of Data(solid black dot) and MC(red line) for contained Sample.
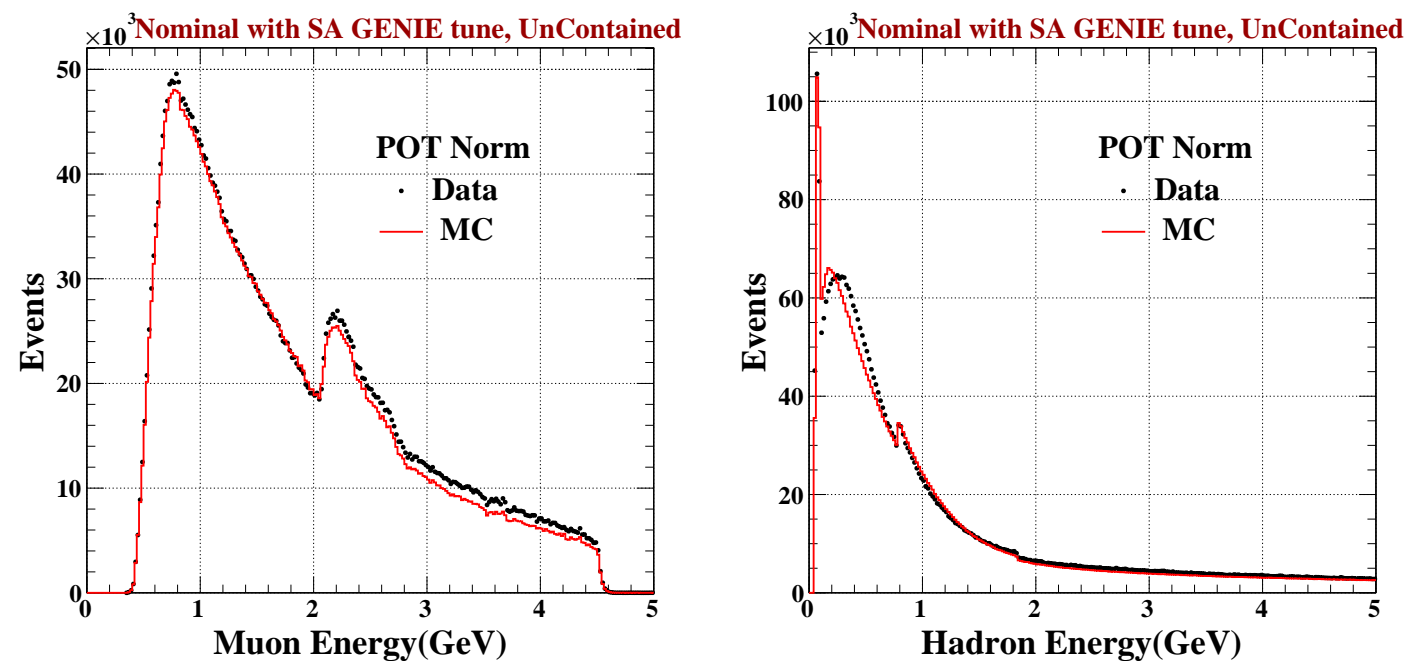

Figure 6.11: Left: $\mathrm{E}_{\mu}$ distribution of Data(solid black dot) and $\mathrm{MC}$ (red line) for uncontained Sample. Right: $\mathrm{E}_{\text {had }}$ distribution of Data(solid black dot) and MC(red line) for uncontained Sample.

\subsection{Central Value of the $K^{+}$Normalization.}

The 2016 second analysis [61, 62] muon neutrino disappearance and electron neutrino appearance, used GENIE [84] weights for our nominal MC. For the central value calculation 
for this analysis we used the same GENIE weights. We have presented Data-MC comparison for $E_{\mu}$ distribution, $E_{h a d}$ distribution as shown in Figure 6.10 \& 6.11, reconstructed energy distributions as shown in Figure 6.12 \& 6.13, and their respective tabulated information is also included both for the contained and the uncontained sample as shown in Table 6.5 (column 2) and 6.5 (column 3). Using method in Subsection 6.4.1 as mentioned above, the central value for $\pi^{+}$is equal to 0.98 and central value for $K^{+}$is equal to 1.17 as shown in Table 6.6.
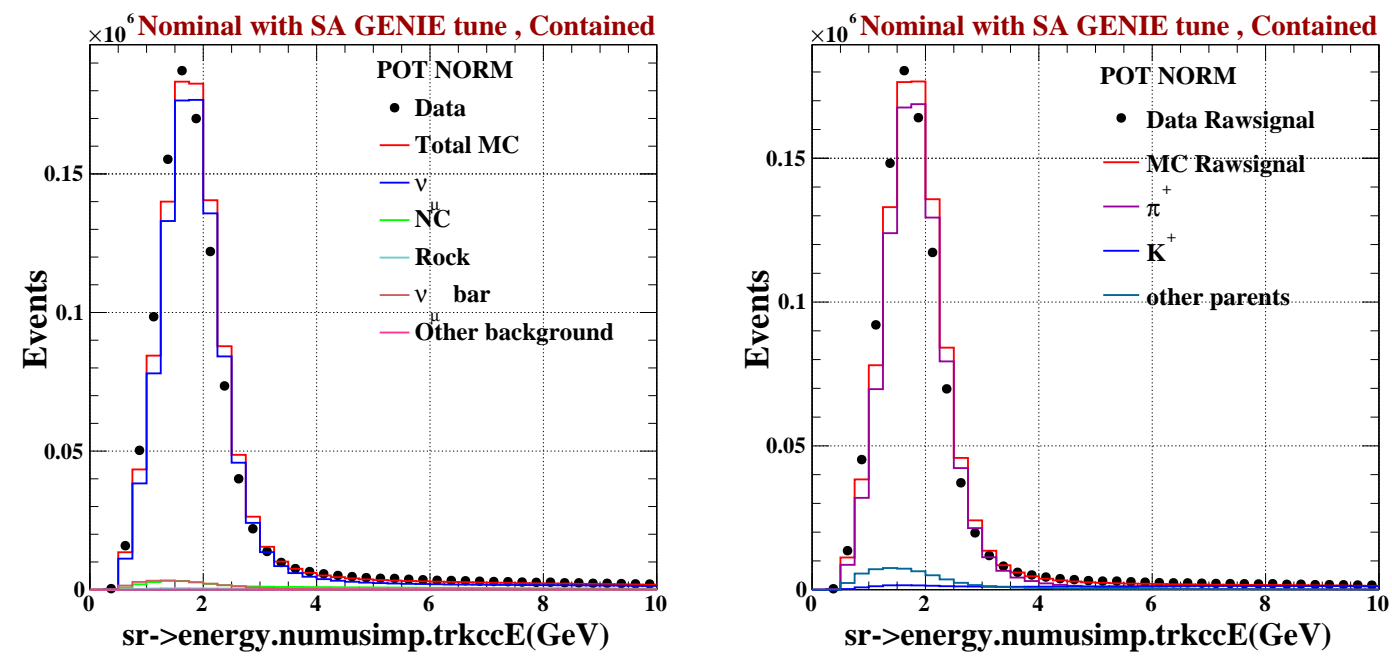

Figure 6.12: Reconstructed Neutrino Energy(TrkCCE) distribution for contained sample: Left: Data events (black solid dot), total MC events (red line), $\nu_{\mu} \mathrm{MC}$ events (blue line), rock events (sea green line), $\overline{\nu_{\mu}}$ events(brown line) and background from others sources (pink line). Right: Data raw signal events[Data-MC $\left.\mathrm{MCkg}_{b}\right]$ (black solid dot), total $\nu_{\mu}-\mathrm{CC}$ MC raw signal events(red line), $\nu_{\mu}$ - CC from $\pi^{+}$( purple line), $\nu_{\mu}-$ CC from $K^{+}$(blue line) and $\nu_{\mu}$ - CC from other parents (sea green).

\subsubsection{Fitting the $\nu_{\mu}$ - CC Spectrum}

As a cross check, we fitted the measured contained and uncontained spectrum for the Pion $\left(N_{\pi}\right)$ and Kaon normalization $\left(N_{K}\right)$. The MC spectrum, with the $N_{\pi}$ and $N_{K}$ floating, is fitted to the measured Data spectrum, and the $\chi^{2}$ is calculated as in Equation 6.1. 

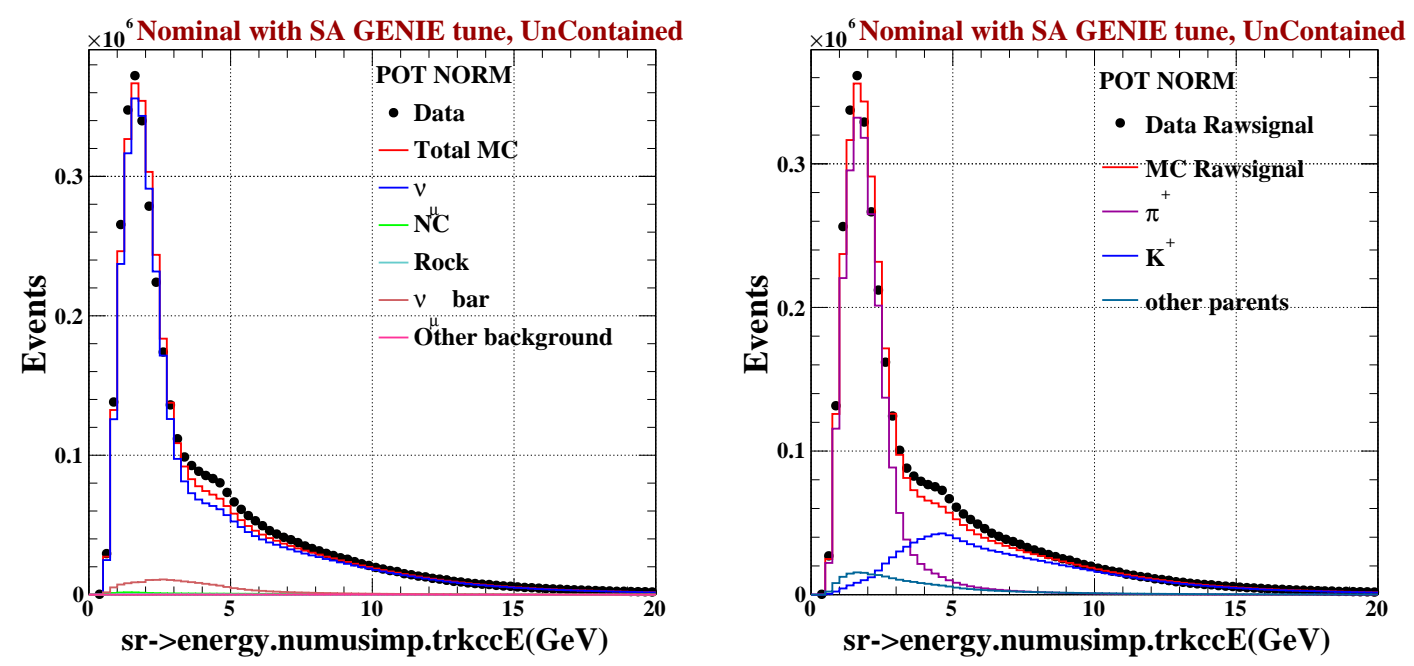

Figure 6.13: Reconstructed Neutrino Energy (TrkCCE) distribution for uncontained sample: Left: Data events (black solid dot), total MC events (red line), $\nu_{\mu} \mathrm{MC}$ events (blue line), rock events (sea green line), $\overline{\nu_{\mu}}$ events(brown line) and background from others sources (pink line). Right: Data raw signal events[Data- $\left.\mathrm{MC}_{b k g}\right]$ (black solid dot), total $\nu_{\mu}$ - CC MC raw signal events(red line), $\nu_{\mu}$ - CC from $\pi^{+}$( purple line), $\nu_{\mu}$ - CC from $K^{+}$(blue line) and $\nu_{\mu}$ - CC from other parents (sea green).

$$
\chi^{2}=\Sigma_{b i n} \frac{(D-M)^{2}}{\left(D_{e r r}\right)^{2}+\left(M_{e r r}\right)^{2}} .
$$

where $\mathrm{D}$ is the data, $\mathrm{M}$ is the $\mathrm{MC}, \mathrm{D}_{\text {err }}$ is the data statistical uncertainty and $\mathrm{M}_{\text {err }}$ is the MC statistical uncertainty, and the $\mathrm{M}$ is described as in Equation 6.2.

$$
M=N_{\pi} \times \pi+N_{K} \times K+\text { other parents. }
$$

Figure 6.14 shows the two dimensional contour of $N_{K}$ versus $N_{\pi}$ in the range 0.5-10 GeV. Since there is a manifest shape discrepancy, especially in the pion-region, the $\chi^{2}$ is quite large, i.e. $\chi^{2} / \mathrm{DOF}$ is much greater than unity. Consequently, the reduced- $\chi^{2}$, $\chi^{2} / \mathrm{DOF}$, is varied by one unit to ascertain the error. Figure 6.14 shows that the central values of $N_{\pi}$ and $N_{K}$ are the same as the previous method; the corresponding statistical error of $N_{\pi}$ is $\approx 1.5 \%$ and that of $N_{K}$ is $\approx 2.5 \%$. These uncertainties, although much smaller than the systematic uncertainties, are included in the uncertainties budget. The two parameter $\chi^{2}$ fit gives $\mathrm{N}_{\pi}=0.987$ and $\mathrm{N}_{K}=1.175$ as show in Figure 6.14 with $1 \sigma$ 


\begin{tabular}{|c||c|c|}
\hline Sample & Contained & Unconatined \\
\hline Variable/Energy range & $.75-3$ & $4.5-10$ \\
\hline Data & 918740 & 923601 \\
MC & 936970.39 & 820953.21 \\
Raw Data Signal (D- M·Bkg) & 874155.92 & 858433.65 \\
Signal MC & 892386.31 & 755785.86 \\
Background MC & 44584.08 & 65167.35 \\
From Pion parents & 834517.72 & 81963.89 \\
From Kaon parents & 11332.82 & 615038.79 \\
From others parents & 46535.78 & 58783.18 \\
\hline Raw & Signal w.r.t parent & type \\
\hline Raw Data Signal from $\pi$ & 816287.32 & 184611.68 \\
Raw Data Signal from $K$ & 45228.77 & 717686.58 \\
Signal MC from $\pi$ & 834517.72 & 81963.89 \\
Signal MC from $K$ & 11332.82 & 615038.79 \\
$N_{\pi}$ & 0.98 & 2.25 \\
$N_{K}$ & 3.99 & 1.17 \\
\hline
\end{tabular}

Table 6.5: $\nu_{\mu}-\mathrm{CC}$ events counts from contained and uncontained samples with GENIE Tune (Nominal).

effect. We also checked this fit using Minuit (ROOT) and the results are approximately same.

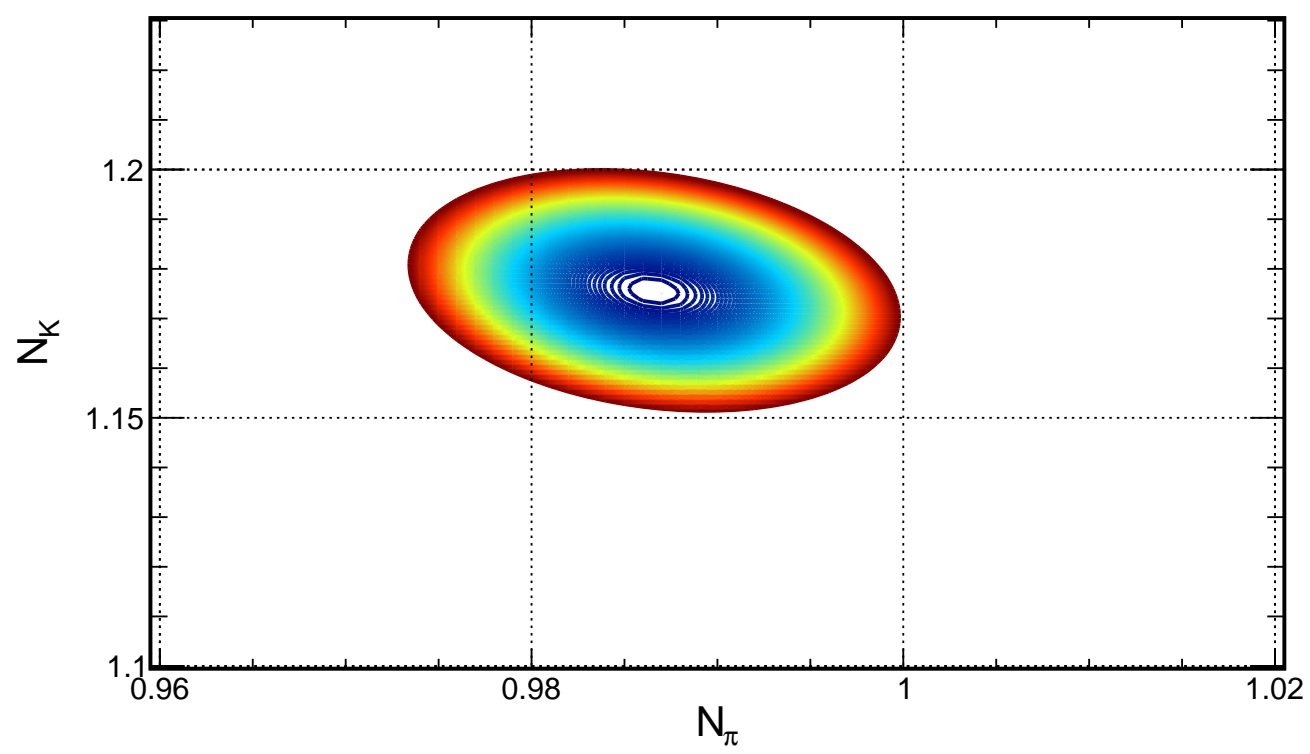

Figure 6.14: $\mathrm{N}_{\pi}$ and $\mathrm{N}_{K} \chi^{2}$ fit with $1 \sigma$ on central value for $(0.5-10) \mathrm{GeV}$. 


\begin{tabular}{|c|}
\hline$N_{\pi}$ for $.75-3 \mathrm{GeV}$ in contained sample $\sim 0.98($ Table 6.4$)$ \\
\hline after normalization of $\pi^{+}($eq.1) for uncontained sample \\
$N_{K}$ is $1.18(4-10 \mathrm{GeV})-N_{\pi} 0.96(0.75-3 \mathrm{GeV})$ \\
$-N_{K} 1.19(4-10 \mathrm{GeV})-N_{\pi} 0.98(0.75-3 \mathrm{GeV})$ \\
$-N_{K} 1.18(4-10 \mathrm{GeV})-N_{\pi} 0.96(0.75-3 \mathrm{GeV})$ \\
$<-$ after iteration \\
\hline \hline$N_{\pi}$ for .75-3 GeV in contained sample $\sim 0.98($ Table 6.4$)$ \\
\hline after normalization of $\pi^{+}($eq.1) for uncontained sample \\
$N_{K}$ is $1.17(4.5-10 \mathrm{GeV})-N_{\pi} 0.98(0.75-3 \mathrm{GeV})$ \\
$-N_{K} 1.17(4.5-10 \mathrm{GeV})-N_{\pi} 0.98(0.75-3 \mathrm{GeV})$ \\
$-N_{K} 1.17(4.5-10 \mathrm{GeV})-N_{\pi} 0.98(0.75-3 \mathrm{GeV})$ \\
$<-\mathrm{after}$ iteration \\
\hline \hline$N_{\pi}$ for .75-3 GeV in contained sample $\sim 0.98(T a b l e[6.4)$ \\
\hline after normalization of $\pi^{+}($eq.1) for uncontained sample \\
$N_{K}$ is $1.16(5-10 \mathrm{GeV})-N_{\pi} 0.98(0.75-3 \mathrm{GeV})$ \\
$<-$ after iteration \\
\hline
\end{tabular}

Table 6.6: The Iteration table for $N_{K}$ with GENIE Tune (Nominal).

\subsection{Systematic Uncertainties}

Relevant systematic sources have been considered for this analysis. For the systematics on the central value, each section has $E_{\mu}$ distribution, $E_{\text {had }}$ distribution, reconstructed energy distributions and their respective tabulated information both for the contained and the uncontained sample.

\subsubsection{Without GENIE Tune}

The second muon neutrino disappearance and electron neutrino appearance analysis on NOvA have used GENIE tune [84] for less discrepancy between data and MC. Data-MC comparison without GENIE tune events gives us a systematic uncertainty for cross-section in this analysis. The value of $\mathrm{N}_{\pi^{+}}$is 1.00 and the $\mathrm{N}_{K^{+}}$is 1.15 without GENIE tune to the MC as shown in Table D.3. We are going to use this difference as a systematics uncertainty i.e. $2.04 \%$ for $\mathrm{N}_{\pi^{+}}$and $1.71 \%$ for $\mathrm{N}_{K^{+}}$on central value from the nominal 
case.

The details of this systematic study are presented in Appendix D. Here we summarize the estimate of the systematic uncertainty on $N_{K}$ estimated using the difference between the case having no MEC events and GENIE tune with nominal. Data/MC comparison for this study are shown in Figures D.1-D.4 both for contained and uncontained samples for reconstructed energy distribution, $\mathrm{E}_{\mu}$ and $E_{\mathrm{Had}}$, Table D.1- D.2 represent the tabulated information.

\subsubsection{Hadro-Production: FLUKA vs GEANT4( $\pi /$ K Shape)}

We need to check uncertainties related to shape discrepancy from hadron production modeling between data and MC. We have used another hadron production model from GEANT4 physics list FTFP_BERT for flux simulation using G4NuMI. For this case the value of $\mathrm{N}_{\pi^{+}}$is 0.97 and the $\mathrm{N}_{K^{+}}$is 1.14. This measurement gives systematics uncertainty for shape discrepancy, which is the difference between these with nominal i.e. $2.04 \%$ for $\mathrm{N}_{\pi^{+}}$and $2.6 \%$ for $\mathrm{N}_{K^{+}}$as shown in Table D.4. The details of this systematic study are presented in Appendix D. Here we summarize the estimate of the systematic uncertainty on $N_{K}$ due to hadron production uncertainty, the estimation from the difference in the neutrino flux from FTFP_BERT versus FLUKA. Table D.4 presents the percentage difference in $N_{\pi}$ and $N_{K}$ and Figure D.5 shows the Data-MC raw signal comparison for contained sample(left) and uncontained sample(right).

\subsubsection{Hadron Shower Containment.}

Our analysis is based on a sample, which is the selection of uncontained muon track for muon neutrino $\mathrm{CC}$ events. For this method we have to check uncertainties for $E_{\text {Had }}$ energy leakage between data and MC. We have tested hadron shower containment very thoroughly as presented in [85]. 
- Tight fiducial volume (FV), i.e interaction vertex should be inside a region defined as $-100<\mathrm{x}<100,-100<\mathrm{y}<100$ and $100<\mathrm{z}<600 \mathrm{~cm}$ as compared to Section 6.3.2. Also muon tracks belong to highest ReMId value inside this region mean tight region for containment start point

- Tracks of selected event which do not belong to highest ReMId value should be contained inside the region defined as $-185<\mathrm{x}<185,-185<\mathrm{y}<185$ and $19<\mathrm{z}$ $<1275 \mathrm{~cm}$.

The $\mathrm{N}_{\pi^{+}}$is 0.95 and $\mathrm{N}_{K^{+}}$is 1.21 and contributed uncertainty on the central value of $\mathrm{N}_{\pi^{+}}$is $3.1 \%$ and $\mathrm{N}_{K^{+}}$is $3.4 \%$ as shown in Table D.7. The details of this systematic study is presented in Appendix D. Here we summarize the estimate of the systematic uncertainty on $N_{K}$ due to hadron shower energy leakage, estimated using the tight fiducial volume verses nominal. Table D.7 presents the percentage difference in $N_{\pi}$ and $N_{K}$ and Figures D.6, D.8 show the Data-MC for $\mathrm{E}_{\mu}$ and $E_{\mathrm{Had}}$. Figures D.7, D.9 and Tables-D.5, D.6 show Data-MC comparison for reconstructed energy for tight fiducial volume.

\subsubsection{Variation of Muon Energy.}

The reconstructed neutrino energy used in this analysis is colloquially referred to on NOvA as TrkCCE. It is a sum of reconstructed muon energy and reconstructed hadronic energy. To include the effect of uncertainty in muon energy estimation, we have included $\pm 2 \%$ shift as a conservative shift on $\mathrm{E}_{\mu}$ with respect to the first and second analysis of NOvA. The details of this systematic study are presented in Appendix E. Shifted muon energy by $+2 \%$ and repeated the analysis. The value of $\mathrm{N}_{\pi^{+}}$is 0.981 and $\mathrm{N}_{K^{+}}$is 1.16 for $+2 \%$ shift in muon energy. Systematics uncertainty for $\mathrm{N}_{\pi^{+}}$is $0.1 \%$ and for $\mathrm{N}_{K^{+}}$is $0.9 \%$ for $+2 \%$ shift in $\mathrm{E}_{\mu}$ as shown in Table E.3, Figures E.2, E.4 and Tables-E.1, E.2 show the Data-MC comparison for reconstructed energy both for contained and uncontained 
sample. Figures E.1, E.3 show Data-MC for $\mathrm{E}_{\mu}$ and $E_{\mathrm{Had}}$ for $+2 \%$ shift in $\mathrm{E}_{\mu}$. We now shifted the muon energy by $-2 \%$ and reanalyzed. The value of $\mathrm{N}_{\pi^{+}}$is 0.981 and $\mathrm{N}_{K^{+}}$ is 1.12 for $+2 \%$ shift in muon energy. Systematic uncertainty for $\mathrm{N}_{\pi^{+}}$is $0.1 \%$ and for $\mathrm{N}_{K^{+}}$is $2.6 \%$ as shown in Table E.6. Figures E.6, E.8 and Tables E.4, E.5 show the Data-MC comparison for reconstructed energy both for the contained and uncontained sample. Figures E.5, E.7 show Data-MC for $\mathrm{E}_{\mu}$ and $E_{\mathrm{Had}}$ for $-2 \%$ shift in $\mathrm{E}_{\mu}$.

\subsubsection{Effect of External Hadron Production Data.}

The default flux is simulated by FLUGG, with significant uncertainty from hadron production. Using external data [86] from MIPP and NA49 experiments there are weights with uncertainties to constraint the $\nu$ flux. As in this analysis, we are using $\pi^{+}$normalization from the contained sample as explained in Section 6.4 to fix the $\pi^{+}$contribution for $\mathrm{N}_{K^{+}}$. First we see the effect of MIPP + NA49 uncertainties before the pion normalization only for the contained sample. Second we observe the effect of MIPP+NA49 weights uncertainties up and down separately only to contain the sample. In the third case, we will see the effect of MIPP+NA49 weights only for pion in the contained sample and uncontained sample. The details of this systematic study are presented in Appendix F. We shifted the contained sample with MIPP+NA49 plus error band and got $\mathrm{N}_{\pi^{+}}$and repeated the procedure with the nominal uncontained sample. The uncertainty for $\mathrm{N}_{K^{+}}$ is $0.9 \%$ as shown in Table F.2. Figure F.1 and Table F.1 show Data-MC for reconstructed energy for the shifted the contained sample with MIPP+NA49 plus error band. We then shifted the contained sample with MIPP+NA49 minus error band and got $\mathrm{N}_{\pi^{+}}$and repeated the procedure with the nominal uncontained sample. The uncertainty for $\mathrm{N}_{K^{+}}$is $1.7 \%$ as shown in Table F.4. Figure F.2 and Table F.3 show Data-MC for reconstructed energy for the shifted the contained sample with MIPP+NA49 minus error band. Finally we shifted the contained and uncontained sample with MIPP+NA49 weights only for $\pi$. The uncertainty for $N_{\pi}$ is $11.7 \%$ and $N_{K}$ is $1.7 \%$ as shown in Table F.7 Figure F.3, F.4 
and Table F.5, F.6 show Data-MC for reconstructed energy for the shifted the contained sample with MIPP+NA49 weights only for $\pi$ both for contained and uncontained samples.

\subsubsection{Cross-section Uncertainty.}

Using the NOMAD [79] data result, the cross-section uncertainty for higher energy $\nu_{\mu}-$ CC events is 5\%, as shown in Figure 6.15.

$$
v_{\mu} \mathrm{N} \rightarrow \mu^{-} X
$$

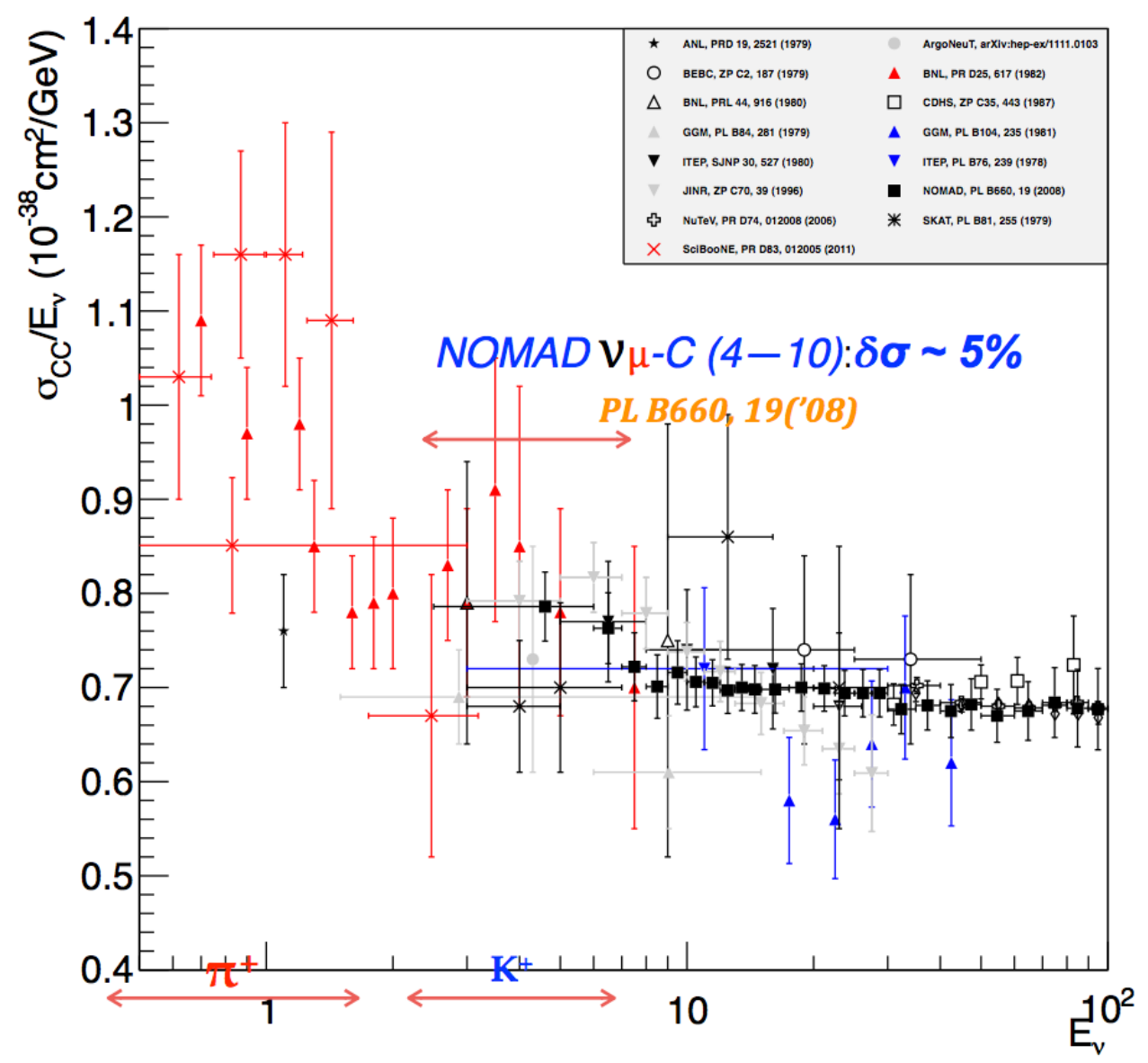

Figure 6.15: The $\nu_{\mu}$ cross-section measurements, especially highlight the NOMAD measurement. 


\subsubsection{Genie Re-weight Cross Check.}

GENIE is the default neutrino event generator used by NOvA. It provides a re-weighting scheme for neutrino interactions. The details of this systematic study is presented in Appendix F. We shifted the GENIE parameter with $1 \sigma$, we have included the effect of relevant parameters for our analysis as shown in Table F.8, F.9 and Figures F.5, F.6 show Data-MC comparison. The total uncertainties for uncontained sample for Kaon is $4.95 \%$ and the total uncertainties for contained sample for pion between $1-3 \mathrm{GeV}$ is $8 \%$.

\subsubsection{Intensity Effect and Time Dependent Modeling Effects}

In $\nu_{\mu}$ oscillation analysis [87] effect of these uncertainties is of the order of $<1 \%$.

\subsubsection{Detector Modeling}

The alternative GEANT physics lists had almost 1-2\% effect on most reconstructed distributions [88.

\subsection{Summary of Systematics.}

The summary of the systematic study is presented in Table 6.7 with regards to the determination of $N_{K}$. To estimate the error due to the hadro-production uncertainties, we employ two methods, FLUKA vs FTFP and the error-band on the pion/kaon production from the MIPP/NA49 data; however, only the error from MIPP/NA49 is used, the former method being used as a cross-check only. Similarly, to estimate the cross-section uncertainty, variations in the GENIE-parameters, as well as the inclusive $\nu_{\mu}$ - CC cross-section results form NOMAD are used; however, only the GENIE numbers are chosen as the representative error. 


\begin{tabular}{|c|c|c|}
\hline Source & $\pi^{+}$ & $K^{+}$ \\
Central & 0.98 & 1.17 \\
Statistical & $1.5 \%$ & $2.0 \%$ \\
\hline \hline Systematics & $\pi^{+}$ & $K^{+}$ \\
& & \\
\hline \hline Experimental & & \\
E $_{\mu}$ Shift +-2\% & $+-.3 \%$ & $+-2.6 \%$ \\
Ehad containment & $3.1 \%$ & $3.4 \%$ \\
Variation in: Energy-Range & $0.10 \%$ & $1.02 \%$ \\
\hline Hadro-Production & & \\
FLUKA .vs FTFP & $2.04 \%$ & $2.6 \%$ \\
MIPP/NA49 Error-Band (+/-) & $-/+10 \%$ & $+/-1.7 \%$ \\
\hline Cross-Section & & \\
Without GENIE tune & $2.04 \%$ & $1.71 \%$ \\
Genie Parameters' variation & $8.0 \%$ & $4.9 \%$ \\
NOMAD muon neutrinoCC Data [79] & $\mathrm{n} / \mathrm{a}$ & $5.0 \%$ \\
\hline \hline Total & & \\
& $13.4 \%$ & $8.0 \%$ \\
\hline
\end{tabular}

Table 6.7: Systematics Summary Table.

\subsection{Combined Contribution to $\nu_{e}$ Flux Prediction}

Need to combine the constraints from:

- Constraints from the external Hadro-Production Experiments, MIPP \& NA49 (see Chapter 5).

- Constraints from the $K^{+}$-Normalization using NOvA ND as we presented in this Chapter.

The dominant uncertainties in the neutrino flux come from poor knowledge of hadron production modeling. We need to constrain the flux using external hadron production data (MIPP, NA49) [86]. NOvA flux is from NuMI target which is a thick carbon target. 


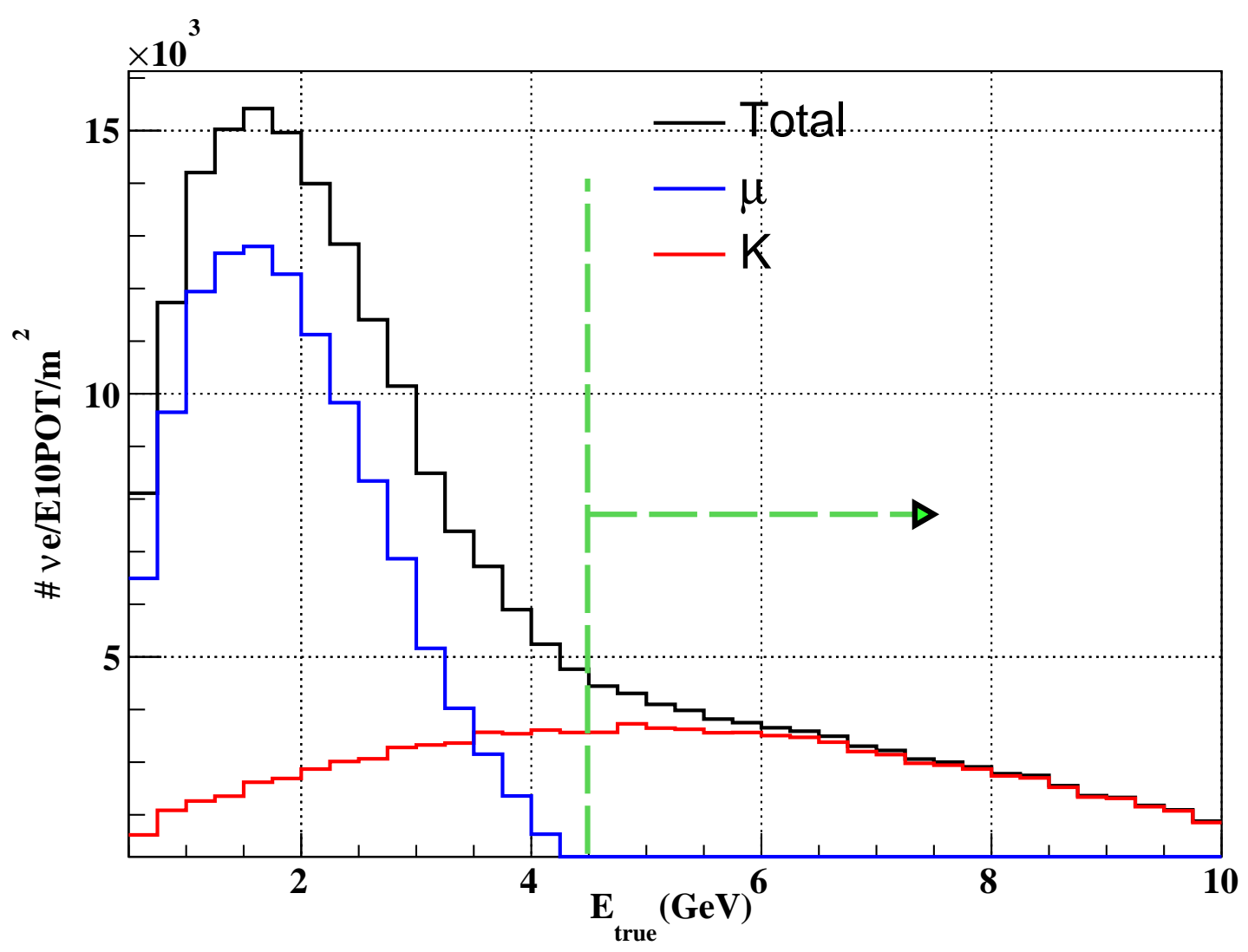

Figure 6.16: electron neutrino at ND w.r.t their parents information.

Unfortunately we don't have enough data from external hadron production experiments for $K$. We only have data from thick target experiments for $\pi$. All other data that we have are for thin target, e.g., NA49 experiment data. We already have weights for electron neutrino flux using external experimental data [86] as shown in Figure 6.17 (top). Due to lack of thick target data for Kaons the uncertainties in the higher energy region are enormous. Fortunately we can use NOvA ND $\nu_{\mu}$ - CC data to constrain Kaon yield above $4.5 \mathrm{GeV}$ where the contribution from kaons dominates as shown in Figure 6.16. The electron neutrino flux weights and certainties as a function of true energy using results from external experiment data as in Section 5.4 and the NOvA ND $\nu_{\mu}$ - CC data weights can be seen in Figure 6.17 (bottom). The electron neutrino flux after applying constraints from external experimental data and the NOvA ND $\nu_{\mu}-\mathrm{CC}$ data weights is shown in Figure 6.18 

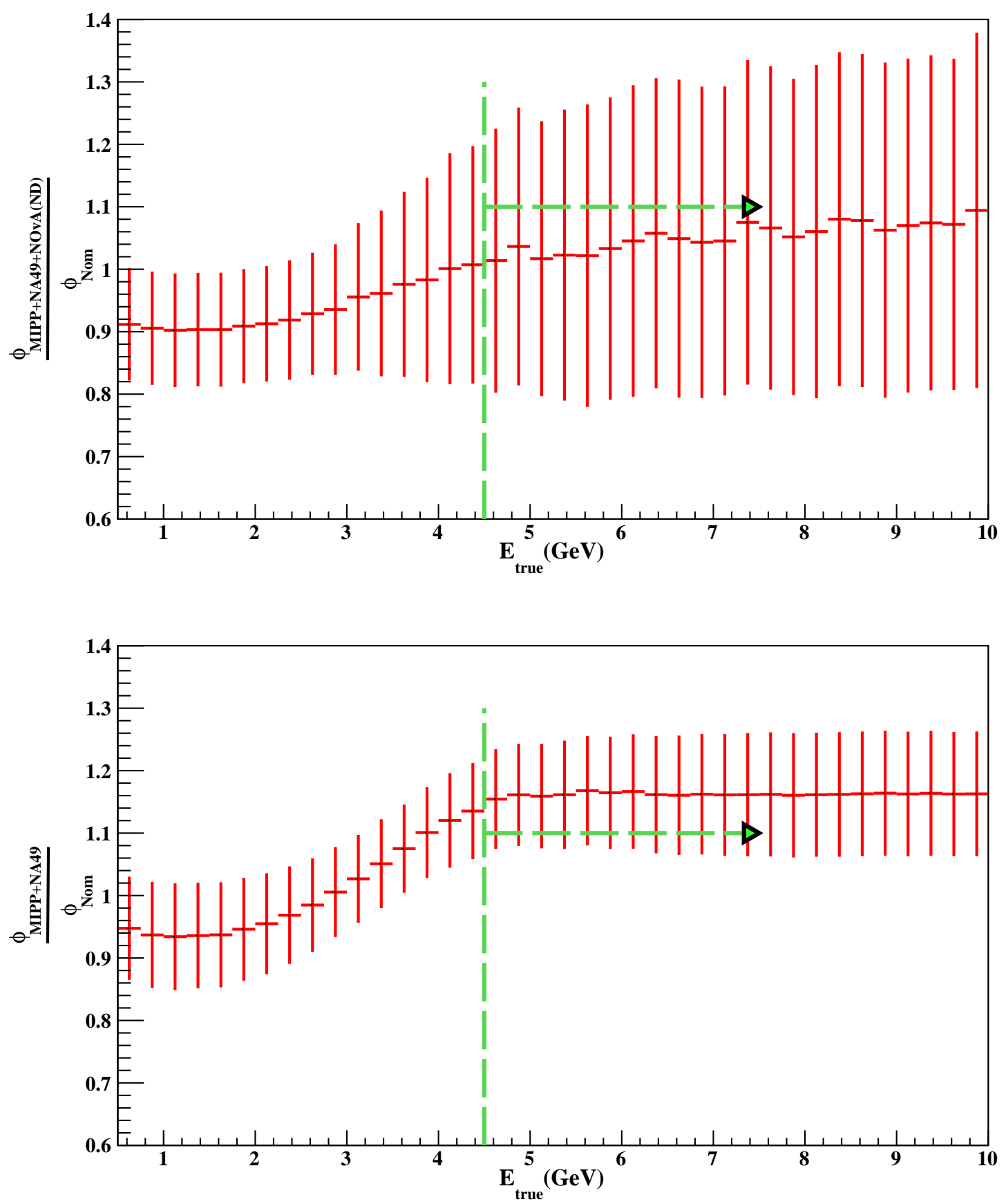

Figure 6.17: The weights for the electron neutrino flux at ND: Left: Flux weight as a function of electron neutrino true energy with uncertainty using MIPP+NA49 external data. Right: Flux weight as a function of electron neutrino true energy with uncertainty using MIPP+NA49+NOvA(ND muon neutrinoCC) external data. 


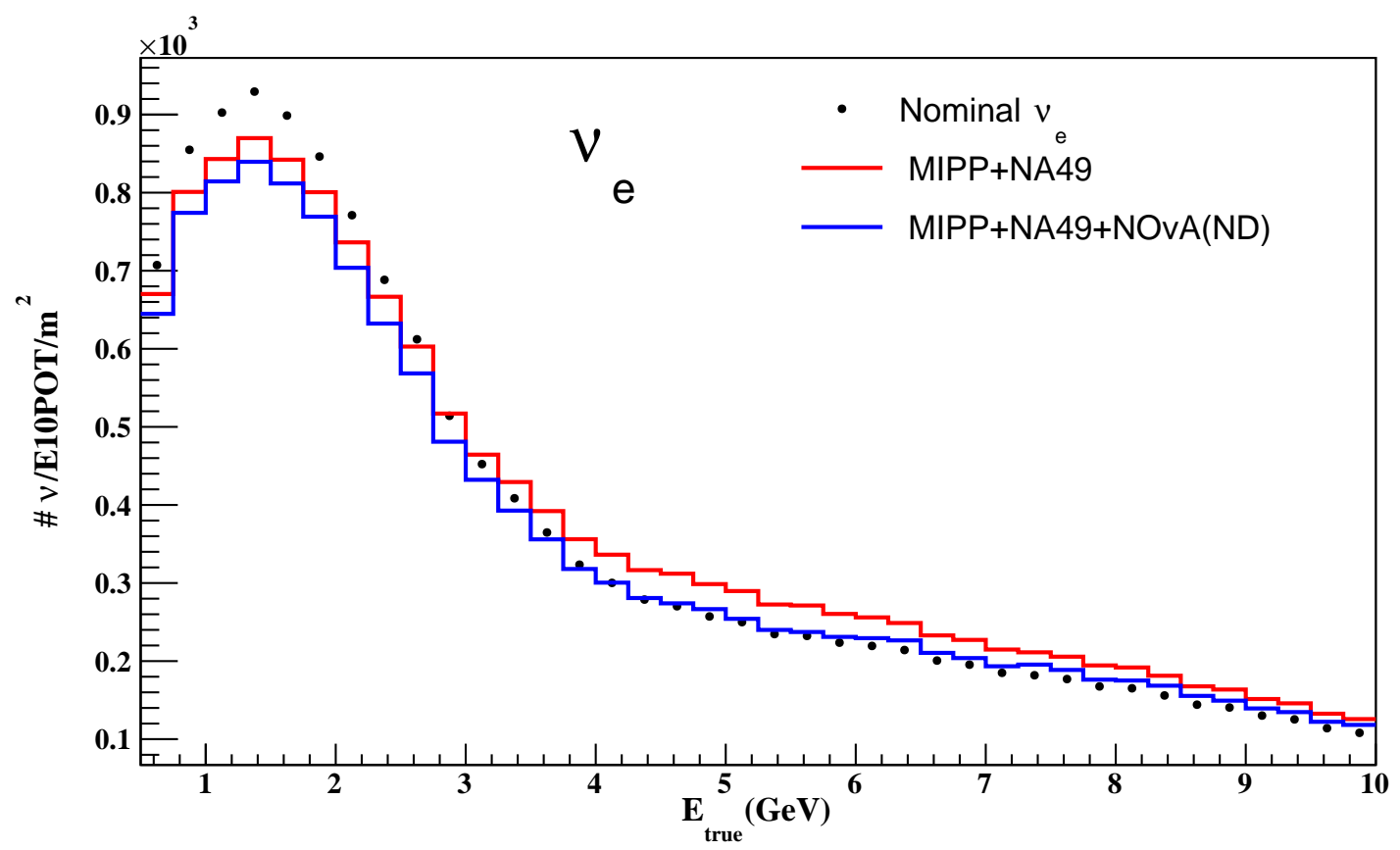

Figure 6.18: The electron neutrino flux at ND with NA49+ MIPP weights only and with $\mathrm{NA} 49+\mathrm{MIPP}+\mathrm{ND}($ data $)$ weights.

\subsection{Conclusion}

In summary, presented an analysis which constrains the $K^{+}$yield in the NOvA neutrino beam using the measured $\nu_{\mu}-\mathrm{CC}$ spectrum at the ND: The off-axis nature of the beam makes $\pi^{+}$versus $K^{+}$separation possible. We determine a $K^{+}$normalization of $1.17 \pm 0.005$ (Stat) \pm 0.10 (Syst) relative to the FLUKA hadro-production model; the corresponding normalization for the $\pi^{+}$yield is $0.98 \pm 0.13($ Stat $\oplus$ Syst). Sources of systematic errors in the meson yields include reconstruction, hadro-production, and cross-section modeling. Using the constrained $K^{+}$, we extract a prediction of the flux in NOvA with a $10 \%$ uncertainty in the region $E_{\nu} \geq 3.5 \mathrm{GeV}$. 


\section{Chapter 7}

\section{Absolute $\nu$ Flux: $\nu$-e NC Scattering}

\subsection{Introduction}

In the weak interaction perturbation theory, $\nu$-e interactions involve only free leptons, whose amplitude is precisely predicted in the Standard Model (SM) [22]. The lowest order Feynman diagrams of the neutrino-electron elastic scattering are shown in Figure 7.1.
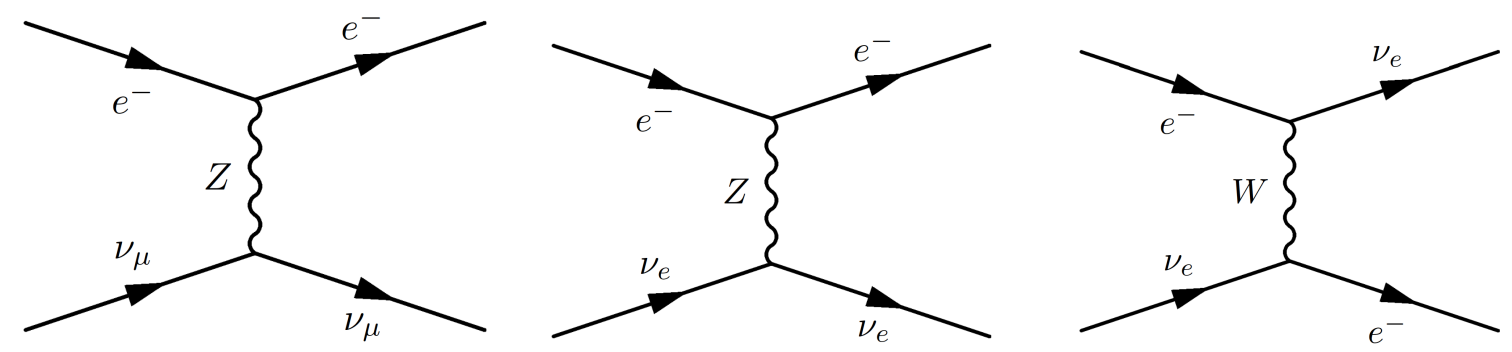

Figure 7.1: Lowest order Feynman diagrams of the $\nu$-e elastic scattering.

The first observation of a handful of $\nu$-e elastic scattering events was done by the Gargamelle bubble chamber in 1973 [89]. This experiment demonstrated the existence of the $\mathrm{Z}$ boson and neutral currents, a milestone towards the establishment of the electroweak theory. Since then, several experiments such as CHARM [90] and BNL E734 [91] have 
furthered the study of the $\nu$-e elastic scattering. Due to the cross-section of $\nu$-e elastic scattering being calculated with SM precisely; it can be used to calibrate the neutrino fluxes at accelerator neutrino experiments, whose beams have large uncertainties caused by inadequate knowledge of hadron productions by proton on the beam target. In this Chapter, we present a measurement of elastic scattering and the measurement of the absolute neutrino flux at the NOvA experiment based on the $\nu$-e elastic scattering. This work will provide a substantial constraint to the flux uncertainty for both near detector (ND) cross-section measurements and far detector (FD) oscillation analyses at NOvA and will demonstrate a flux constraint method for DUNE [92]. $\nu$-e elastic scattering is an elastic two-body collision, and the kinematics are given by:

$$
\cos \theta_{e}=1-\frac{m_{e}(1-y)}{E_{e}}
$$

where $\theta_{e}$ is the angle of the outgoing electron with respect to the neutrino beam, $m_{e}$ is the electron mass, $E_{e}$ is the electron energy in the final state. The parameter $y$ is defined as $y=T_{e} / E_{\nu}$, where $T_{e}$ is the electron kinetic energy and $E_{\nu}$ is the neutrino energy. For neutrino energy at GeV level, Equation 7.1 can be approximated as $E_{e} \theta_{e}^{2}=2 m_{e}(1-y)$ since $0 \leq \mathrm{y} \leq 1, E_{e} \theta e^{2}$ is less than $2 m_{e}$. Therefore, the signal we are looking for is a single, very forward-going electron shower with $E_{e} \theta_{e}^{2}$ peaking around zero.

\subsection{Data Sample}

We perform this analysis on an ND data sample consisting of $8.28 \times 10^{20}$ protons-ontarget (POT) exposure. The data were recorded in the ND between 16 August, 2014 - 10 January, 2017. Monte Carlo (MC) simulation was used to study the signal and background for $\nu$-e scattering, as well as the neutrino flux. For the neutrino beam, we use FLUKA [72] to model hadron production in the NOvA target and use the FLUGG ([93]) interface to 
GEANT4 94] to simulate the focusing and decay of those hadrons in the NuMI beam. In the NOvA detectors, interactions of neutrinos are simulated by the GENIE generator [95] , and detector responses are simulated by GEANT4. The customized detector simulation chain for NOvA detectors is described in Ref [96].

In GENIE(v2.10.4), the cross-section of the $\nu$-e elastic scattering signal is calculated at the tree level. With the limit $m_{e}<<E_{\nu}$ for all active neutrinos and anti-neutrinos, differential cross section of $\nu$-e elastic scattering is given by Equation 7.2 .

$$
\frac{d \sigma\left(\nu+e^{-} \rightarrow \nu+e^{-}\right)}{d y}=\frac{G_{F}^{2} s}{\pi}\left[C_{L L}^{2}+C_{L R}^{2}(1-y)^{2}\right]
$$

where $\mathrm{G}_{F}$ is the Fermi weak coupling constant, $s$ is the square of the total energy in the center-of-mass frame, and $y \equiv \frac{T_{e}}{E_{\nu}}$ where $T_{e}$ is the electron kinetic energy and $E_{\nu}$ is the neutrino energy. The couplings $\mathrm{C}_{L L}$ and $\mathrm{C}_{L R}$ depend on the neutrino flavor and whether the incident particle is a neutrino or anti-neutrino. For $\nu_{\mu}$ and $\nu_{\tau}, \mathrm{C}_{L L}=$ $\frac{1}{2}+\sin ^{2} \theta_{W}$ because the interaction contains interfering contributions from the neutralcurrent interaction that is present for all flavors and from a charged-current interaction that is present only for electron neutrinos. To improve the precision of the simulated $\nu$-e elastic scattering cross-section, we perform radiative corrections to the original GENIE $\nu$-e elastic scattering cross-section by tuning $C_{L L}$ and $C_{L R}$ to one-loop values predicted using global fits to electroweak data to include the additional low-energy terms due to radiative corrections [97] and one-loop electroweak couplings from recent global fits to electroweak data [98], details are shown in the Appendix G.2. This method is the same as what was used by J. Park et. al. in MINERvA's $\nu$-e elastic scattering paper [99]. After radiative corrections, the cross-section of $\nu$-e elastic scattering decreases $3.2 \%$ from the default GENIE value. The size of this signal Monte Carlo simulation (MC) is $6.75 \times 10^{22}$ POT. For the background simulation, we use $2.68 \times 10^{21}$ POT inclusive NOvA official ND MC sample, composed of $\nu_{\mu}$ charged current $\left(\nu_{\mu}\right.$-CC $), \nu_{e}$ charged current $\left(\nu_{e}-\mathrm{CC}\right)$ 
and neutral current (NC) events. Meson exchange current events (MEC) [100] are added and tuned in this simulation. This sample also includes neutrino interactions in the rock surrounding the ND. This analysis has been performed using Common Analysis Files (CAF's) and the CAFAna framework [80].

\subsection{Event Reconstruction}

Standard NOvA reconstruction (see Chapter 4) is used to reconstruct $\nu$-e elastic scattering events. The event reconstruction begins with clustering hits by space-time coincidence to separate beam events from cosmic rays in a trigger window. This procedure can collect together hits from a single neutrino interaction (slice). The slices then serve as the foundation for all later reconstruction stages [101]. Next, a modified Hough transform is applied to identify prominent straight-line features in a slice. Then the lines are tuned in an iterative procedure until they converge to the interaction vertex of that slice. Prongs are then reconstructed based on distances from hits to the lines associated with each of the particles that paths are emanating from the reconstructed vertex [102]-[103]. We define the shower core based on the prong direction provided by the prong cluster, then collect signal hits in a column around this core [104]. Because the electron deposits energy through ionization in the first few planes then starts a shower, we require the radius to be twice the cell width for the first eight planes (see Chapter 3) from the start point of the shower and 20 times the cell width for other planes.

\subsection{Event Identification and Selection}

Standard NOvA data quality and timing cuts are used to select beam neutrino events under the standard beam and detector conditions. As discussed in Section 7.1, the signal we are looking for is a single, very forward-going electron shower with $E_{e} \theta^{2}$ peaking around 
zero.

A preselection is applied to remove apparent $\nu_{\mu} \mathrm{CC}$ interactions produced in the detector. The criteria are tuned from the preselection of the NOvA $\nu_{e}$ appearance analysis with loose cuts:

- $L<800 \mathrm{~cm}$, where $L$ is the length of the longest prong

- $N_{\text {Plane }}<120$, where $N_{\text {Plane }}$ is the number of planes of the longest prong

- $N_{\text {Cell }}<600$, where $N_{\text {Cell }}$ is the total number of cells in a slice

To suppress backgrounds induced by neutrino interactions in the rock (mostly) upstream of the ND, prior to the event identification ( $\nu$-e ID and $\left.e / \pi^{0} \mathrm{ID}\right)$, we restrict the distance of the primary shower from the detector edges. These fiducial cuts are applied on the minimum and maximum $\mathrm{X}, \mathrm{Y}$ and $\mathrm{Z}$ position of hits on prongs in an event:

- Minimum $\mathrm{X}>-170 \mathrm{~cm}$, Maximum $\mathrm{X}<170 \mathrm{~cm}$

- Minimum $\mathrm{Y}>-170 \mathrm{~cm}$, Maximum $\mathrm{Y}<190 \mathrm{~cm}$

- Minimum Z>75 cm, Maximum Z $<1250 \mathrm{~cm}$

Where Min X, Y, Z are the minimum X, Y and Z positions of hits on tracks in an event and Maximum $\mathrm{X}, \mathrm{Y}$ and $\mathrm{Z}$ are the maximum $\mathrm{X}, \mathrm{Y}$ and $\mathrm{Z}$ positions of hits on prongs in an event. The fiducial volume requirement is more strick at the front of the detector where most of the rock events enter the ND volume. Distributions, cuts and Figures of Merit (FOM) (defined later) of Maximum X, Y and Z and Min X, Y and Z are shown in Figure 7.2 , 7.3 and 7.4 .

The $\nu$-e elastic scattering signal is single particle events. However, due to cell noise and imperfection of reconstruction, in a reconstructed single electron event, there could be cell energies that are not associated with the most energetic reconstructed shower, and cell hits caused by the electron could be clustered as more than one showers. In order to reject backgrounds with multiple final state particles while keeping the signal efficiency, 
we require that the primary shower must contain most of the cell energies, and no vertex interaction is found in the detector, and off-shower cell hits and minor reconstructed showers with small energies are allowed. Specifically, to select single-particle events, we require:

- $E_{\text {shower }} / E_{\text {tot }}>0.9$, where $E_{\text {shower }}$ is the energy of the most energetic shower and $E_{t o t}$ is the total energy in the slice, as shown in Figure 7.5.

- vertex energy $=0 \mathrm{GeV}$, where vertex energy is the sum of cell energies above thresholds, excluding the leading shower, within \pm 8 planes of the event vertex as shown in Figure 7.6 .

- Gap $<20 \mathrm{~cm}$, where the gap is the distance between the start point of the primary shower and the event vertex (if a minor, secondary shower is reconstructed, the event vertex is not necessarily the start point of the primary shower), as shown in Figure 7.7 .

Our event classifiers have limited performance for low energy backgrounds, so we require that the energy of the most energetic shower should be greater than $0.5 \mathrm{GeV}$. In the high energy region, the rate of $\nu_{e}$-CC is much higher than the $\nu$-e elastic scattering signal even after the event identification, so the maximum energy of $5.0 \mathrm{GeV}$ is required on the primary shower as shown in Figure 7.8. The distribution and selection optimization of the most energetic shower energy are shown in Figure 7.8. For the event identification, an artificial neural network (ANN [51]) is trained to identify $\nu$-e elastic scattering events ( $\nu$-e ID). The inputs to the ANN are 12 particle likelihood differences between electron and other particle hypotheses $(e-\gamma, e-\mu$, etc.) for the most energetic shower. These likelihoods are calculated by comparing the longitudinal and transverse energy deposition in the primary shower to template histograms generated by simulated $e, \gamma, \mu, \pi^{0}, p, n$ and $\pi^{ \pm}$. The particle likelihoods are defined and determined by the same method used in the LID $\nu_{e}$ selector for NOvA's first $\nu_{e}$ oscillation analyses [104]. More details of the particle likelihood calculation can be found in Appendix G.3. The $\nu$-e ID is required to 
be greater than 0.9 to select the signal events. The distribution and selection optimization of $\nu$-e ID are shown in Figure 7.4. Data/MC of inputs and outputs of $\nu$-e ID are plotted in Section 7.5 .

The dominant background particles after $\nu$-e ID selection are $\pi^{0}$ 's from coherent $(\mathrm{COH})$ interactions. A $\pi^{0}$ can be misidentified as an electron by $\nu$-e ID when only one of the daughter photons is successfully reconstructed, or two daughter photons merge into one shower. The $\mathrm{COH} \pi^{0}$ production occurs with small momentum transfer and no quantum numbers (charge, spin, isospin) exchange, which is almost collinear with the incident neutrino, similar to the emitting direction of the electron in $\nu$-e elastic scattering events. Figure 7.9 (left) shows the true mother particle that matches to the most energetic shower in each event that survives $\nu$-e ID and previous cuts. Figure 7.9 (right) shows types of interaction of backgrounds with $\pi^{0}$ s associated with most energetic showers after $\nu$-e ID and previous selections. To further reject $\pi^{0}$ backgrounds after the $\nu$-e ID selection, a second $\mathrm{ANN}$ event selector, $e / \pi^{0} \mathrm{ID}$, is used. The Figure 7.10 show the effectiveness of $e / \pi^{0}$ ID. The signature to distinguish an electron from $\pi^{0}$ backgrounds is the electron's minimum ionization peaks before the multiple scattering (shower) happens, so $\mathrm{dE} / \mathrm{dx}$ in the first four planes are used to form the input of this $\mathrm{ANN} . e / \pi^{0}$ ID is required to be greater than 0.8 to suppress $\pi^{0}$ backgrounds. The distribution, cut and FOM of $e / \pi^{0}$ ID are shown in Figure 7.4. Data/MC of inputs and outputs of $e / \pi^{0}$ ID are plotted in Section 7.5 .

Finally, for the most energetic shower in the final state (electron candidate), the product of energy and the squared angle with respect to the beam $\left(E_{e} \theta^{2}\right)$ must be less than $0.005 \mathrm{GeV} \times \operatorname{rad}^{2}$, as shown in Figure 7.13. Most of the above selection criteria are chosen to maximize the FOM defined as $\frac{S}{\sqrt{\left(S+B+\delta B^{2}\right)}}$, where $S$ and $B$ are the numbers of signal and background events, and $\delta B$ is the systematic uncertainty in the background. We conservatively assume $\delta B=0.3 B$ according to the $\nu_{e}$ appearance analyses and the $\nu_{e}$-CC cross-section measurement at NOvA. The distributions and selection optimizations 
of the selected variables are shown in Figure $7.2,7.13$, with each distribution plotted with the cuts imposed on the other variables. In distributions such as Maximum Zand $e / \pi^{0} \mathrm{ID}$, there are statistical fluctuations in backgrounds due to limited numbers of events after other selections. To prevent selection bias caused by statistical jumps up and down, loose cuts are applied to those variables, instead of chosen maxima of the FOMs. Table. 7.1 shows the cut flow for the event selection for signal and background. The efficiency $\varepsilon^{N-1}$ indicate the efficiency of each cut concerning the previous ones. The parameter $\varepsilon$ is the cumulative efficiency of each cut, defined as the ratio of the number of remaining events, after application of that cut and all previous cuts, to the initial number of events. Figure 7.14 show event displays for beam neutrino interactions in an ND trigger window with a simulated $\nu$-e elastic scattering signal event before and after the event selection. One can find that the event selection keeps the single electron signal from the $\nu$-e elastic scattering while removing all other neutrino interactions in the same trigger window.

\begin{tabular}{|c|c|c|c|c|c|c|}
\hline Cut & $N_{\text {sig }}$ & $\varepsilon_{\text {sig }}^{N-1}(\%)$ & $\varepsilon_{\text {sig }}(\%)$ & $N_{b k g}$ & $\varepsilon_{b k g}^{N-1}(\%)$ & $\varepsilon_{b k g}(\%)$ \\
\hline No cut & 4184.69 & 100 & 100 & $1.53 \mathrm{e}+08$ & 100 & 100 \\
Preselection & 4184.15 & 99.99 & 99.99 & $1.50 \mathrm{e}+08$ & 98.15 & 98.15 \\
Containment & 1800.33 & 43.03 & 43.02 & $4.83 \mathrm{e}+07$ & 32.26 & 31.66 \\
Signal-particle requirement & 847.07 & 47.05 & 20.24 & 656773 & 1.36 & 0.43 \\
E(0.5-5)GeV & 541.36 & 63.91 & 12.94 & 176923 & 26.94 & 0.12 \\
$\nu$-e PID & 485.47 & 89.68 & 11.60 & 21385.7 & 12.09 & 0.01 \\
e/ $\pi^{0}$ PID & 338.33 & 69.69 & 8.08 & 3326.22 & 15.55 & 0.0021 \\
E $\theta^{2}$ & 308.81 & 91.28 & 7.38 & 44.40 & 1.33 & $2.91 \mathrm{e}-05$ \\
\hline
\end{tabular}

Table 7.1: Cut flow for MC $\nu$ - e signal and background events. The $\varepsilon^{N-1}$ is the efficiency of each cut with respect to the previous ones. $\varepsilon$ is the cumulative efficiency of each cut, defined as the ratio of the number of remaining events, after applying that cut and all previous cuts, to the initial number of events.

\subsection{Data/MC Comparison}

The distributions in Figure 7.15 compare distributions of several input variables to the $\nu$-e ID and $e / \pi^{0}$ ID in data with those from MC, MC backgrounds separated into their 

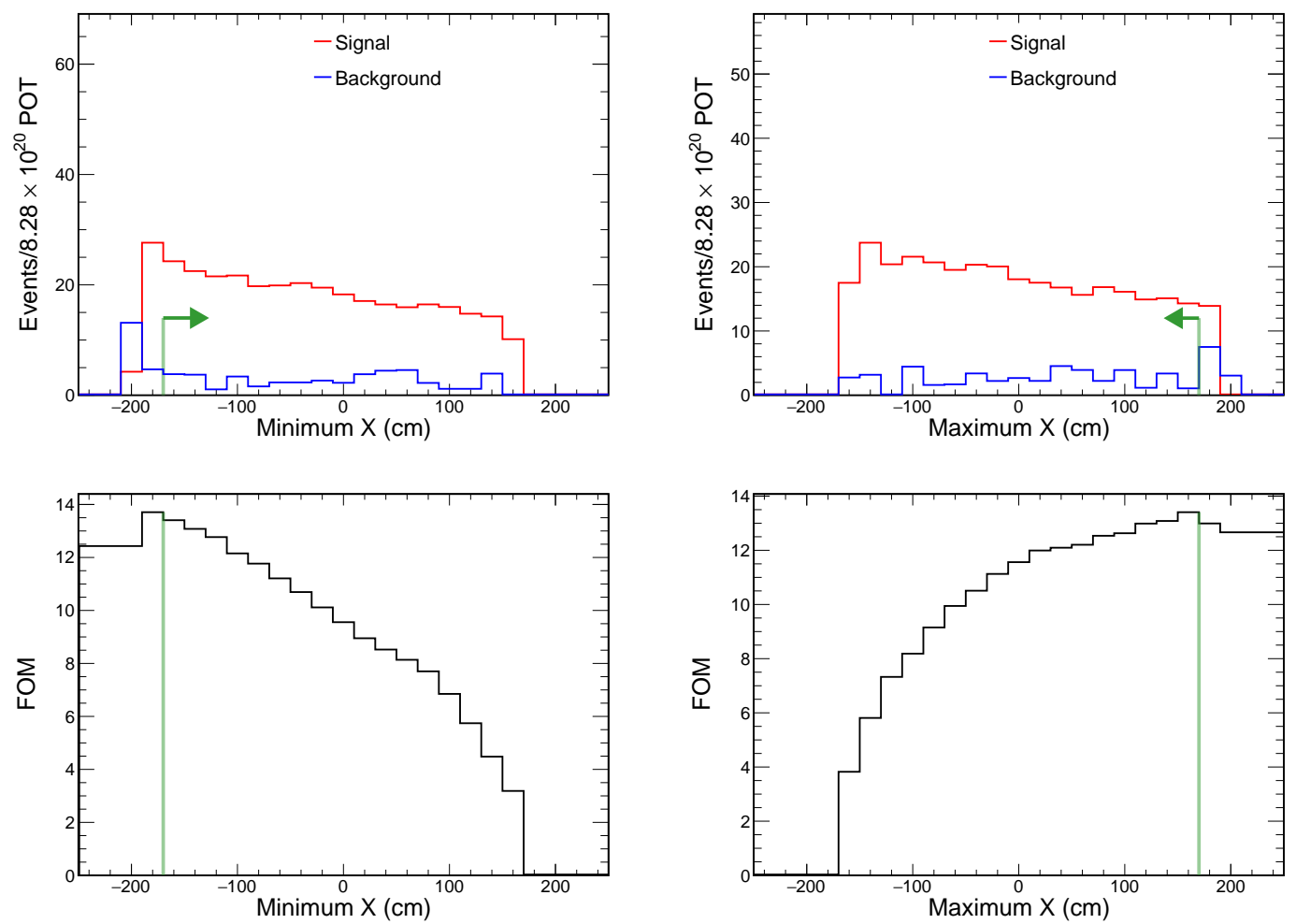

Figure 7.2: Top (Left and Right): Distributions of Minimum X and Maximum X of total MC background and MC $\nu$-e signal before applying cuts on Minimum X and Maximum $\mathrm{X}$. MC are normalized to data POT in each case. The Minimum Xand Maximum X is defined as the minimum and maximum $\mathrm{X}$ positions of hits on tracks. Bottom (Left and Right): FOM distribution for Minimum Xand Maximum X.

respective components. Each distribution is plotted after the event selections cuts have been imposed. The signal and background MC are normalized to the exposure (POT) of the data. 

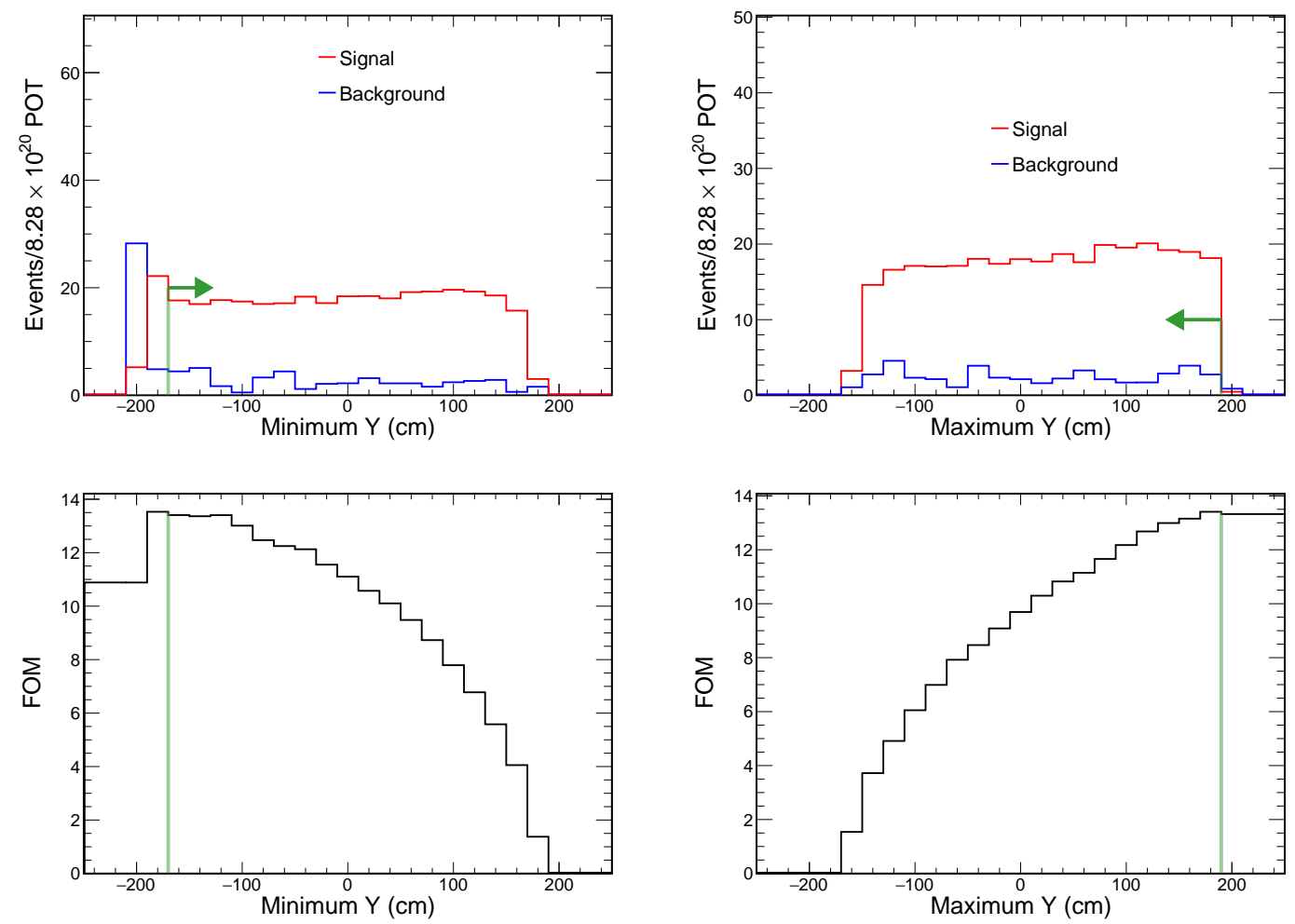

Figure 7.3: Top (Left and Right): Distributions of Minimum Y and Maximum Y of total MC background and MC $\nu$-e signal before applying cuts on Minimum Y and Maximum Y. MC are normalized to data POT in each case. The Minimum Y and Maximum Y is defined as the minimum and maximum Y positions of hits on tracks. Bottom (Left and Right): FOM distribution for Minimum Yand Maximum Y. 

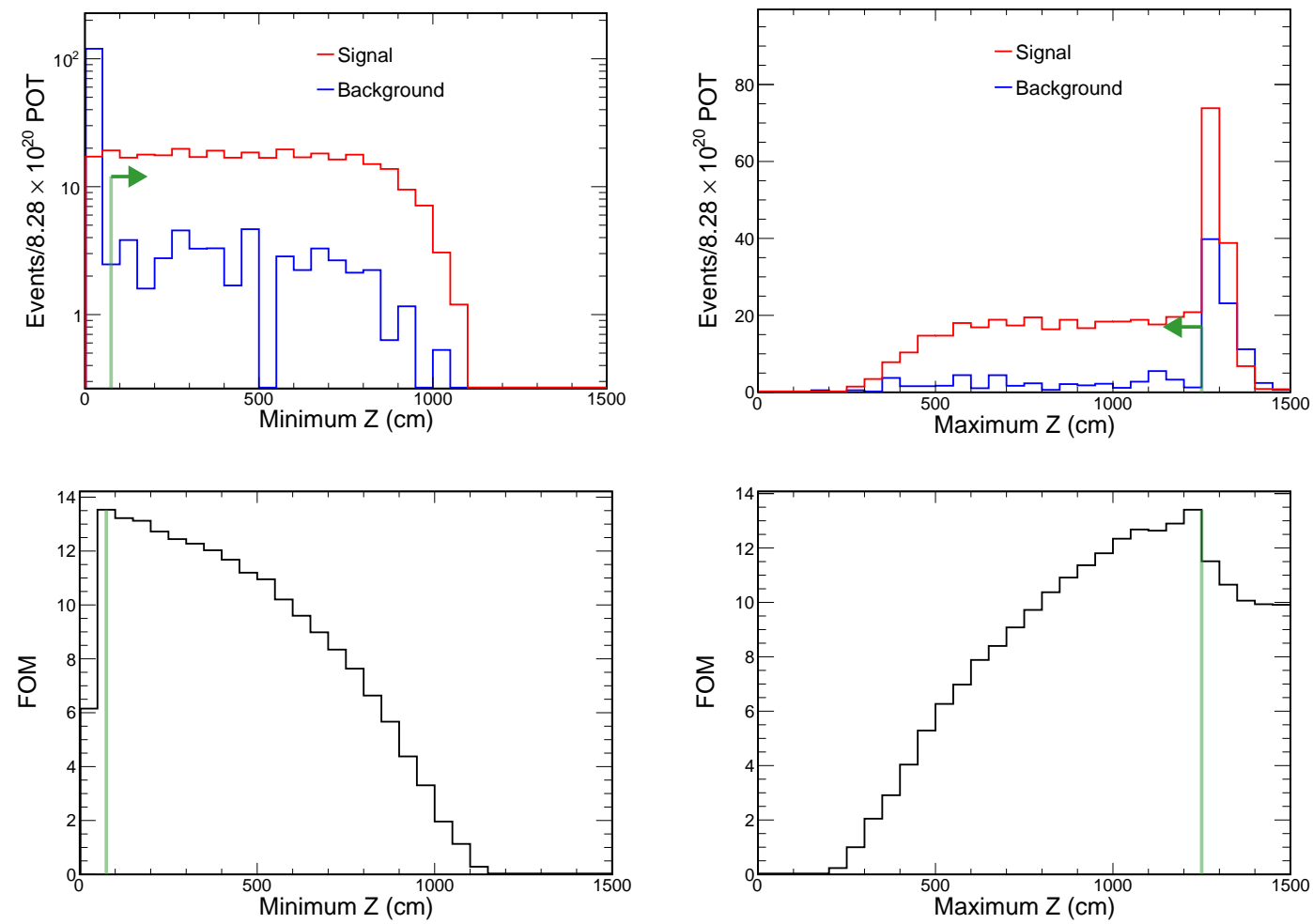

Figure 7.4: Top (Left and Right): Distributions of Minimum Z and Maximum Z of total MC background and MC $\nu$-e signal before applying cuts on Minimum $\mathrm{Z}$ and Maximum Z. MC are normalized to data POT in each case. The Minimum Z and Maximum Z is defined as the minimum and maximum $\mathrm{Z}$ positions of hits on tracks. Bottom (Left and Right): FOM distribution for Minimum $\mathrm{Z}$ and Maximum $\mathrm{Z}$.
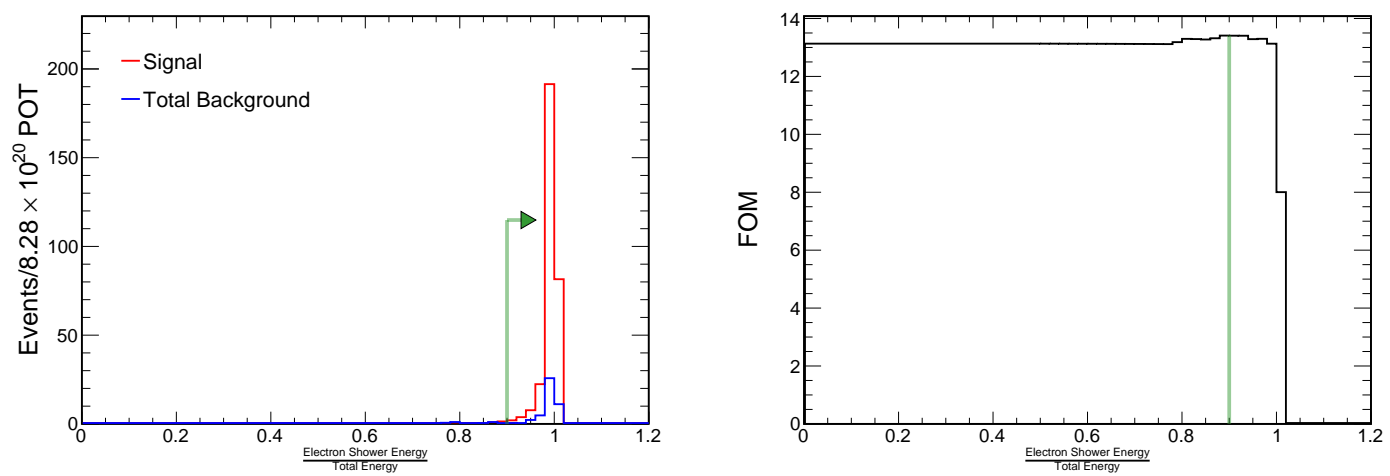

Figure 7.5: Left: Distributions of $\frac{E_{\text {shower }}}{E_{\text {tot }}}$ of total MC background and MC $\nu$-e signal before applying cuts on $\frac{E_{\text {shower }}}{E_{\text {tot }}}$. MC are normalized to data POT in each case. Right: FoM distribution for $\frac{E_{\text {shower }}}{E_{\text {tot }}}$. 

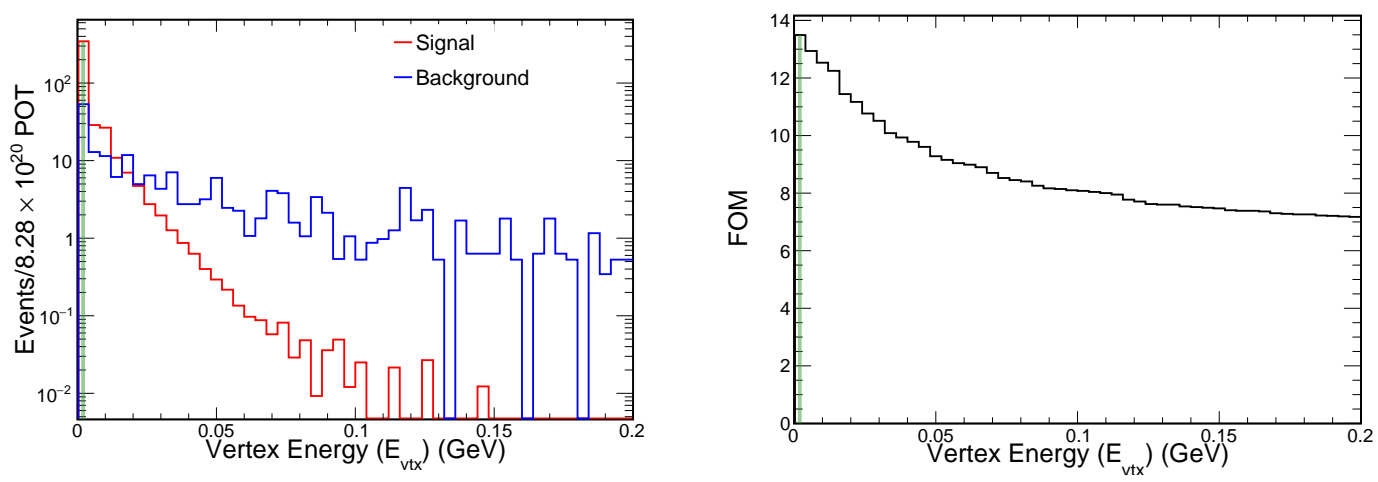

Figure 7.6: Left: Distributions of shower vertex energy of total MC background and MC $\nu$-e signal before applying cuts on the shower vertex energy. MC are normalized to data POT in each case. Right: FoM distribution for shower vertex energy.
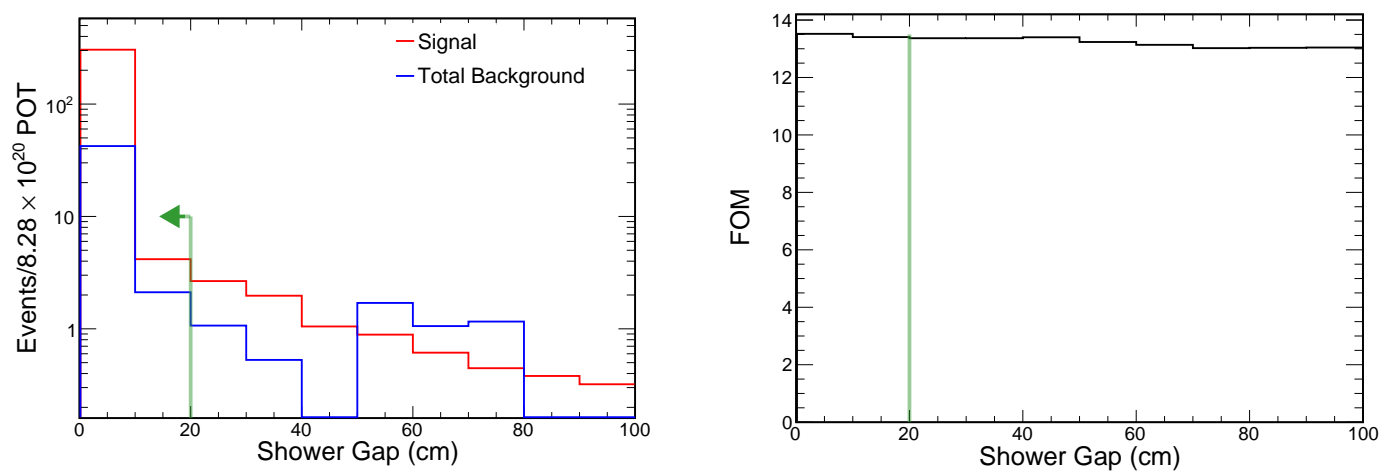

Figure 7.7: Left: Distributions of the distance between the start point of the shower from the event vertex of total MC background and MC $\nu$-e signal before applying cuts on the distance between the start point of the shower from the event vertex. MC are normalized to data POT in each case. Right: FoM distribution for distance between the start point of the shower from the event vertex.

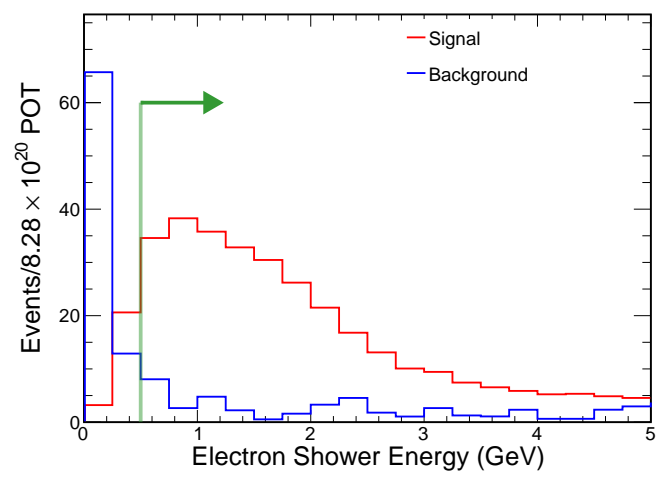

Figure 7.8: Distributions of EM shower energy of total MC background and MC $\nu$-e signal before applying cuts on $\mathrm{E}_{\text {shower }}$. MC are normalized to data POT in each case. 

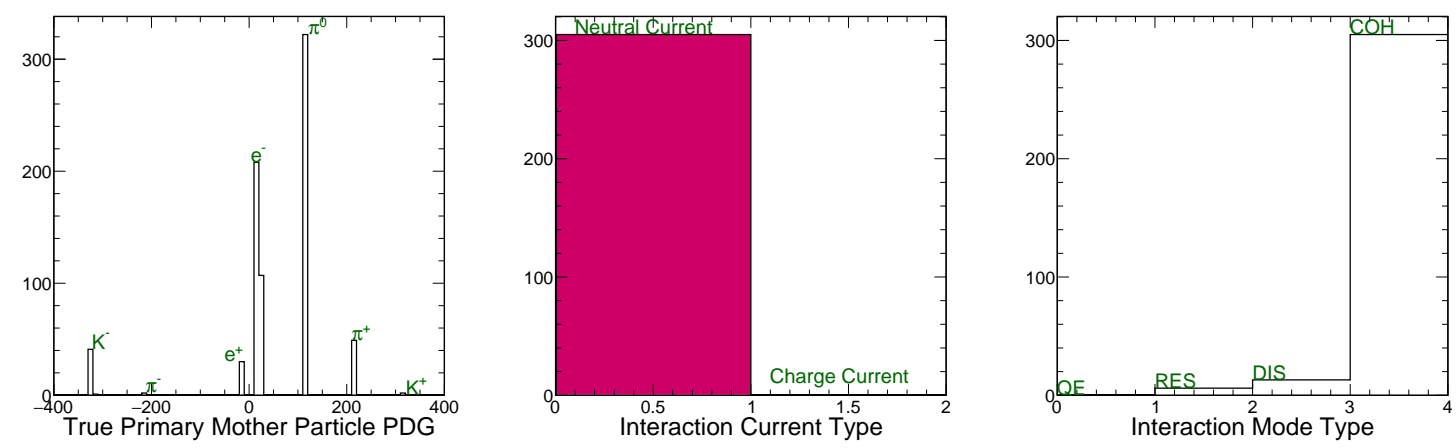

Figure 7.9: Left: True mother particle of EM shower after preselection and $\nu$-e PID > 0.9 and $\mathrm{E} \theta^{2}<0.005\left(\mathrm{GeV} \times \mathrm{rad}^{2}\right)$, Middle: Interaction Current type information after preselection and $\nu$-e PID $>0.9$ and $\mathrm{E} \theta^{2}<0.005\left(\mathrm{GeV} \times \mathrm{rad}^{2}\right)$, Right: Interaction mode information after preselection and $\nu$-e PID $>0.9$ and $\mathrm{E} \theta^{2}<0.005\left(\mathrm{GeV} \times \operatorname{rad}^{2}\right)$.
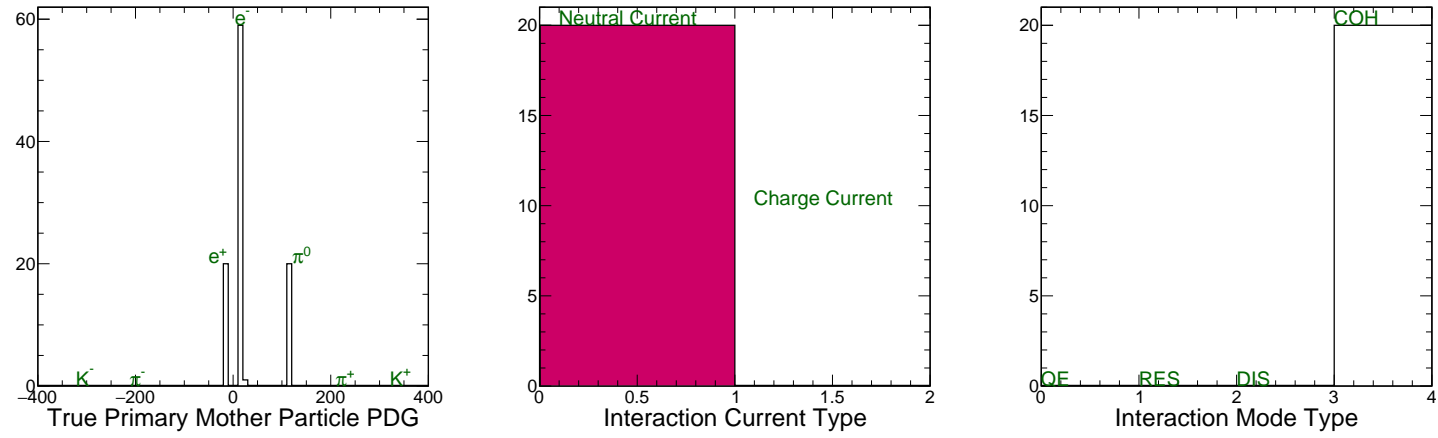

Figure 7.10: Left: True mother particle of EM shower after preselection and $\nu$-e PID > $0.9, \mathrm{E} \theta^{2}<0.005\left(\mathrm{GeV} \times \mathrm{rad}^{2}\right)$ and $\mathrm{e} / p i^{0} \mathrm{PID}>0.8$, Middle: Interaction Current type information after preselection and $\nu$-e PID $>0.9, \mathrm{E} \theta^{2}<0.005\left(\mathrm{GeV} \times \operatorname{rad}^{2}\right)$ and e $/ p i^{0}$ PID $>0.8$, Right: Interaction mode information after preselection and $\nu$-e PID $>0.9$, $\mathrm{E} \theta^{2}<0.005\left(\mathrm{GeV} \times \mathrm{rad}^{2}\right)$ and $\mathrm{e} / p i^{0} \mathrm{PID}>0.8$.
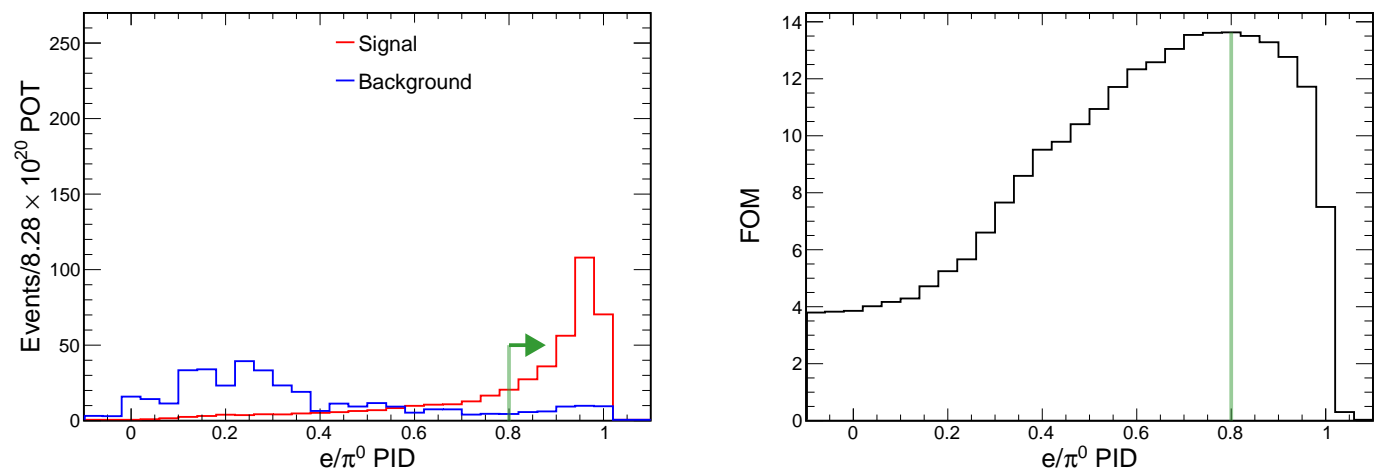

Figure 7.11: Left: Distributions of $e / \pi^{0}$ PID of total MC background and MC $\nu$-e signal before applying cuts on $e / \pi^{0}$ PID. MC are normalized to data POT in each case. Right: FoM distribution for $e / \pi^{0}$ PID. 

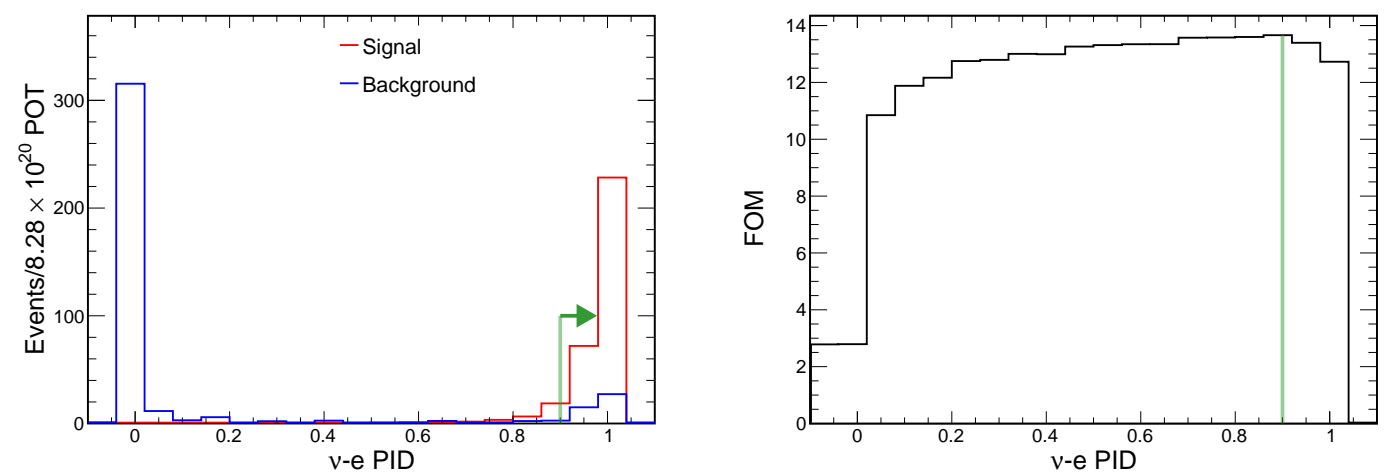

Figure 7.12: Left: Distributions of $\nu$-e PID of total MC background and MC $\nu$-e signal before applying cuts on $\nu$-e PID. MC are normalized to data POT in each case. Right: FoM distribution for $\nu$-e PID.
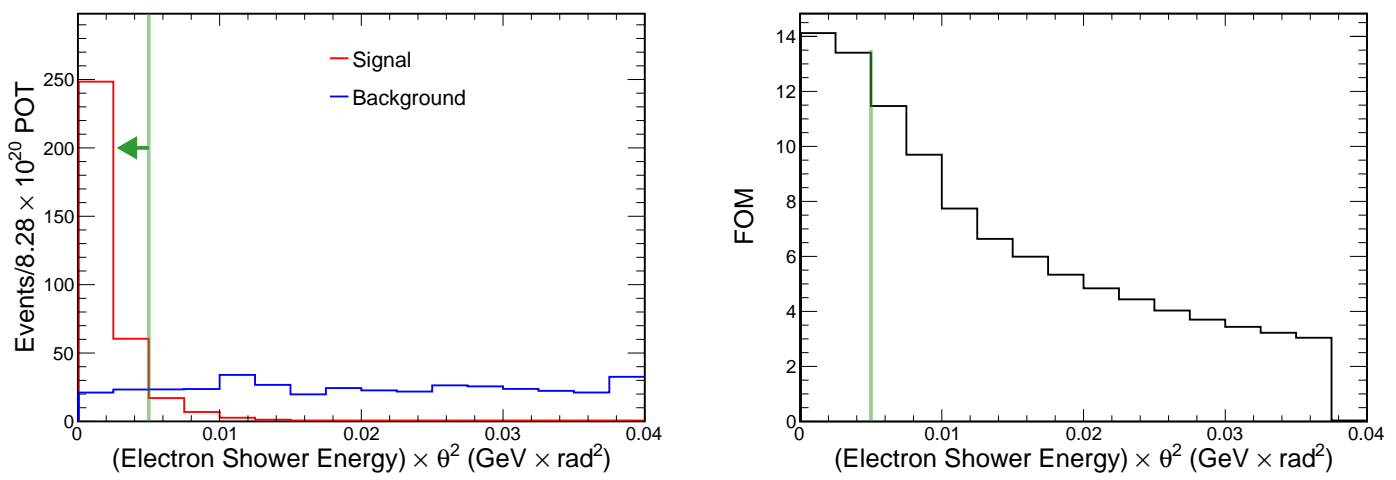

Figure 7.13: Left: Distributions of $\mathrm{E} \theta^{2}$ of total $\mathrm{MC}$ background and $\mathrm{MC} \nu$-e signal before applying cuts on $\mathrm{E} \theta^{2}$. $\mathrm{MC}$ are normalized to data POT in each case. Right: FoM distribution for $\mathrm{E} \theta^{2}$. 

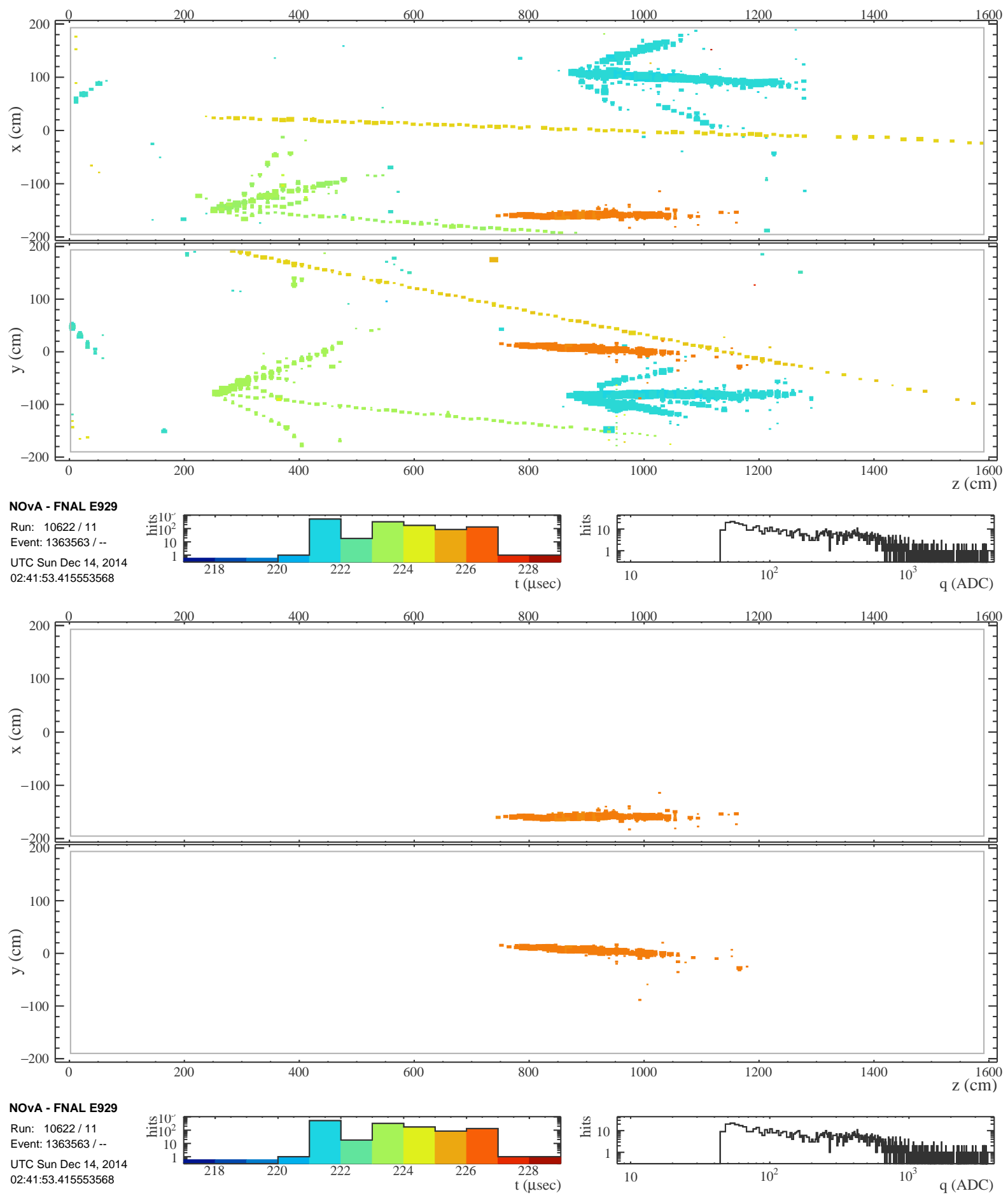

Figure 7.14: Top: Near Detector data events all hits recorded in a $10 \mu$ sec beam spill at ND. Bottom: Near Detector $\nu$-e data candidate as single forwarding electron after apply selection using PID and kinematic information, giving us confidence in our selection for the signal as single forward EM shower. 
Data/MC distributions of longitudinal and transverse log-likelihood differences for $e / \gamma, e / \pi^{0}$ and $e / \mu$ are shown in Figure 7.15 as examples of the inputs of the $\nu$-e ID. Data/MC distributions of the input variables of the $e / \pi^{0} \mathrm{ID}, \mathrm{dE} / \mathrm{dx}$ in first four planes in the most energetic shower, are shown in Figure 7.15. Agreements between the MC simulation and the data are quite good for the inputs of both IDs.

The $\nu$-e ID and $e / \pi^{0}$ ID data and MC distributions are shown in Figure 7.16 (left and right respectively). Each distribution is plotted with the cuts on the other variables imposed (mean each distribution has all other selections applied i.e. N-1 cuts). Agreements between the MC simulation and the data are reasonable within few \%. Figure 7.16 (left) shows somewhat difference between data and MC shapes in the background region $\left(e / \pi^{0} \mathrm{ID}<0.1\right)$, but due to the selection cut is far from that region, it doesn't affect the selection efficiency and the analysis.

The kinematic variable $E_{e} \theta_{e}^{2}$ data/MC distributions for $\nu$-e elastic scattering after all selections are shown in Figure 7.17 (For separate $\nu_{\mu}$ and NC background distribution see G.1). The signal region in the $E_{e} \theta_{e}^{2}$ distribution is $E_{e} \theta_{e}^{2}<0.005$, and the side-band region is defined as $0.005<E_{e} \theta_{e}^{2}<0.04$ (see Section 7.6.1). The electron candidate energy data/MC distributions after all selections in the signal and side-band regions are shown in Figure 7.17 (left and right respectively). The electron candidate energy is the calorimetric energy of the most energetic shower with corrections of dead material and position.

\subsection{Data Analysis}

The nominal MC for this analysis has GENIE weights [105] applied, and kaon/pion components in the MC flux are corrected according to the $\nu_{e}$ oscillation SA study (normalized kaon with 1.17 and pion with 0.98) [106]. 

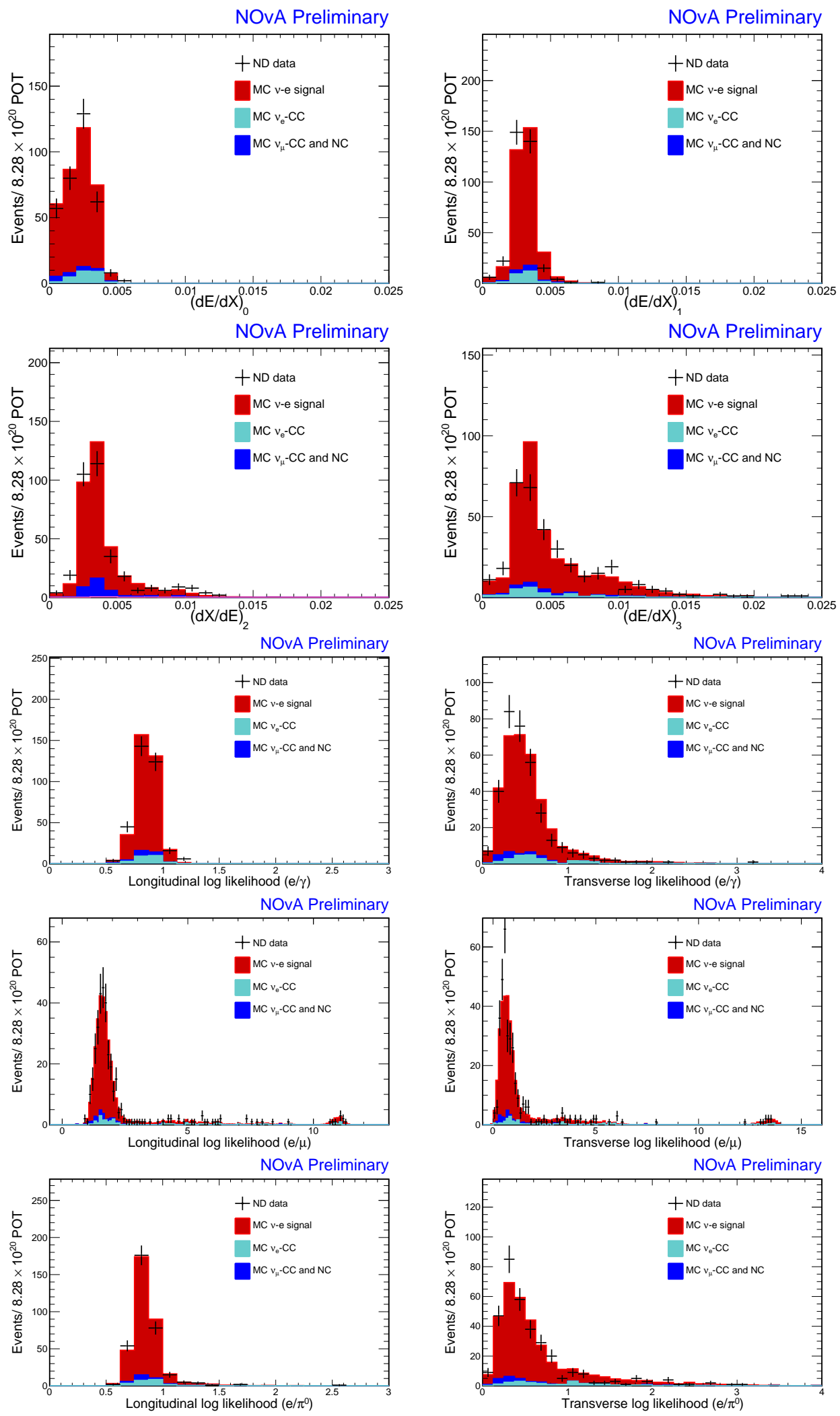

Figure 7.15: Data/MC of $\mathrm{dE} / \mathrm{dx}$ in first four planes and Data/MC for $\nu$-e PID inputs. 

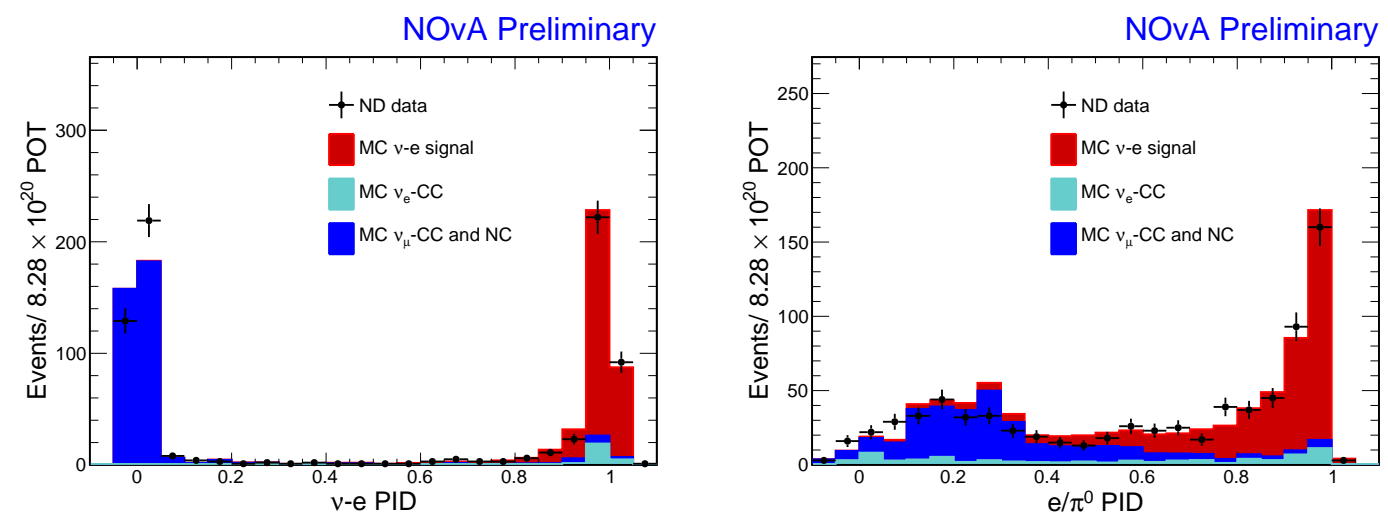

Figure 7.16: Data/MC distribution for (left) $\nu$-e PID and (right) $e / \pi^{0} \mathrm{PID}$.
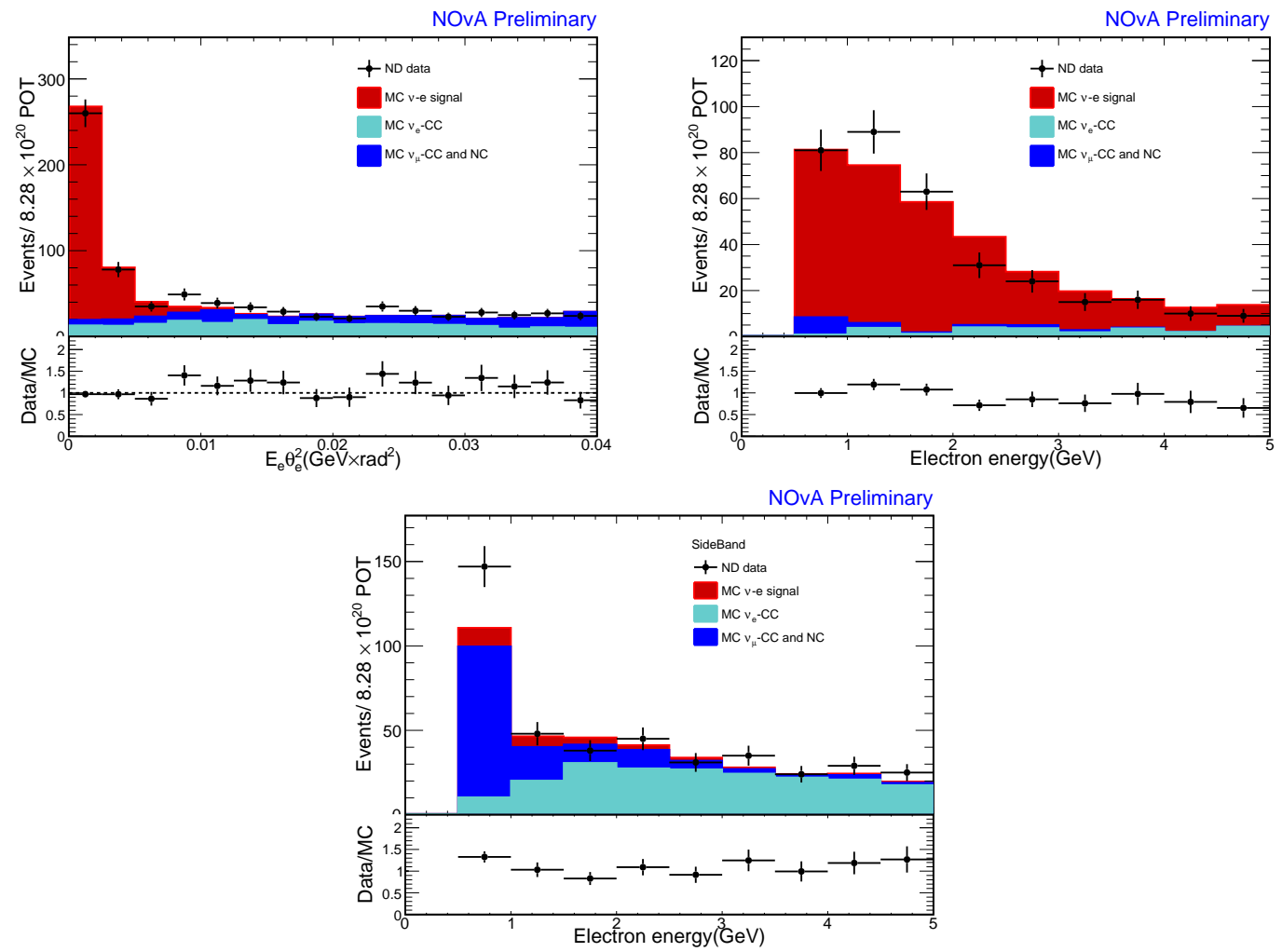

Figure 7.17: Up (Left): Data/MC of $\mathrm{E} \theta^{2}$ before background $\mathrm{MC}$ corrections. Up (Right): Data/MC distributions of electron energy in the $E_{e} \theta_{e}^{2}$ signal region before background MC correction. Bottom (Left): Data/MC distributions of electron energy in the $E_{e} \theta_{e}^{2}$ side-band region, before background $\mathrm{MC}$ correction. 

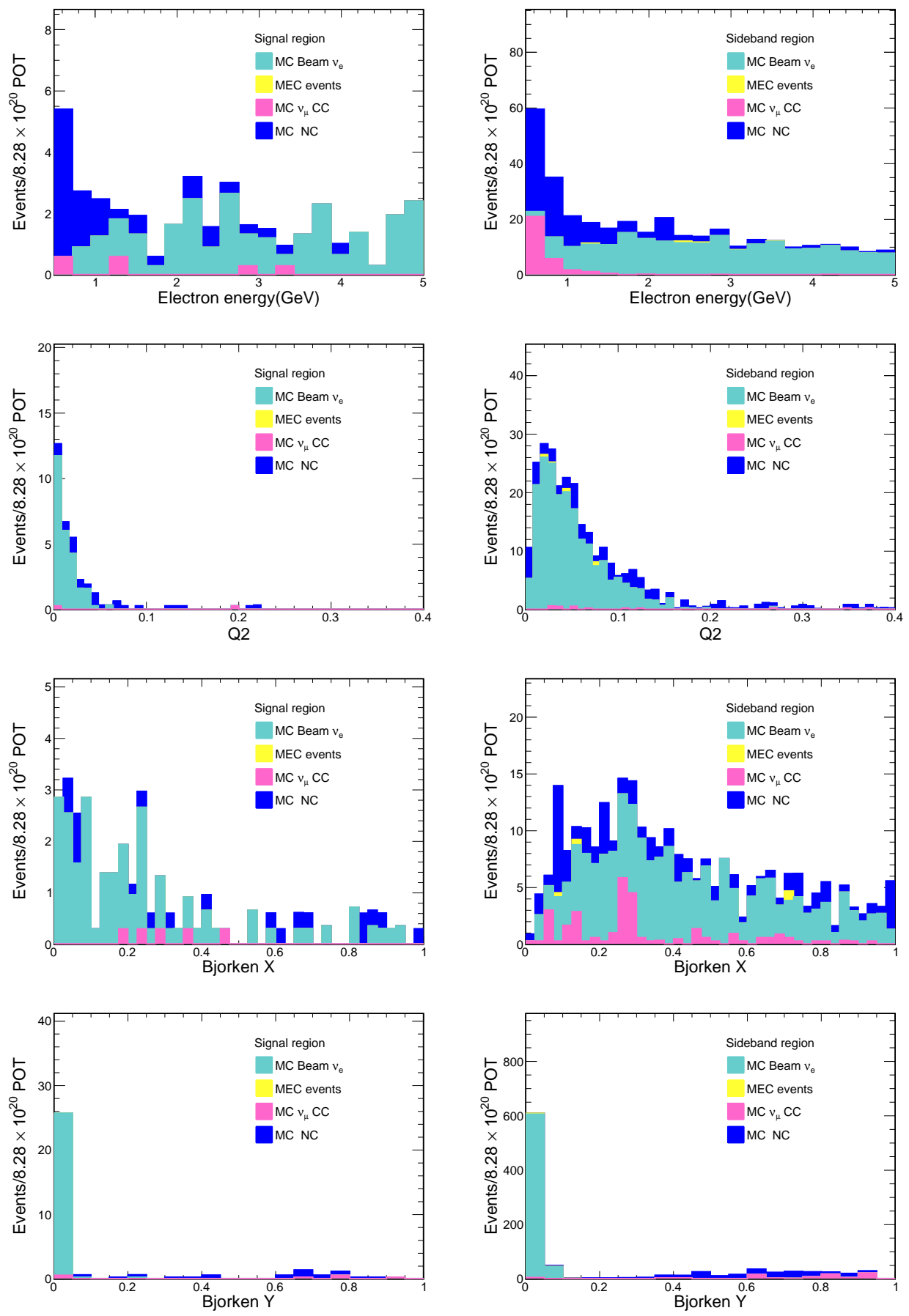

Figure 7.18: Right (Signal region $0<E_{e} \theta_{e}^{2}<0.005$ ) and Left (Side-band $0.005<E_{e} \theta_{e}^{2}<$ 0.04): Plots to show that background distribution in the side-band region (left side) has kinematics coverage of the background in the signal region. Each kinematic variable distribution in the signal region has its side-band region distribution in parallel, e.g., E, $\mathrm{Q}^{2}$, Bjorken $\mathrm{x}$ and Bjorken $\mathrm{y}$ distribution in the signal region right-hand side, to compare these variable with their side-band distributions on the left-hand side. All the kinematic variable distributions, i.e., energy, $\mathrm{Q}^{2}$, Bjorken $\mathrm{x}$ and Bjorken $\mathrm{y}$ in the side-band region overlap the distribution in the signal region. 


\begin{tabular}{|c|c|}
\hline Sideband & $\mathrm{N}(0.5-5) \mathrm{GeV}$ \\
\hline \hline Data & 422 \\
\hline MC & 374.5 \\
\hline Data-MC & 47.5 \\
\hline NC bkg & 110.6 \\
\hline$\nu_{e}$ CC bkg & 200.8 \\
\hline$\nu_{\mu}$ CC bkg & 31.8 \\
\hline MEC bkg & 1.91 \\
\hline Total sig MC & 29.5 \\
\hline Total bkg MC & 345.0 \\
\hline
\end{tabular}

Table 7.2: Event yields in the side band region $\left(0.005<E_{e} \theta_{e}^{2}<0.04\right)$ for Data and MC.

\subsubsection{Background Correction}

The background in the signal region needs to be estimated and subtracted to obtain $\mathrm{N}_{\nu \text {-on-e }}^{\text {Data }}$. This procedure is subject to systematic uncertainties because mis-modeling of both the background and the neutrino flux can bias the signal measurement. The sideband region (SB) $0.005<E_{e} \theta_{e}^{2}<0.04$ is chosen as shown in Section 7.4. So the yield from $\nu$-e signal is small (less than $1 / 10$ of the total number of side-band events, see Table 7.2), and the background event kinematics in the side-band region are similar to the signal region. Electron candidate energy and MC truth of kinematic variables $Q^{2}$, Bjorken $x$ and Bjorken $y$ [107] in backgrounds in signal and side-band regions as shown in Figure 7.18. $Q^{2}$ is the negative four-momentum transfer squared between the incoming neutrino and the outcome hadrons. Bjorken $x$ is the Bjorken scaling variable defined as $Q^{2} / 2 M \nu$, where $M$ is the nucleon mass, and $\nu$ is the neutrino's energy loss in the nucleon rest frame. Bjorken $y$ is the fractional energy loss of the incoming neutrino defined as $\nu / E_{\nu}$, where $E_{\nu}$ is the incoming neutrino energy. One can find that the kinematic variable distributions in the side-band region can well cover those in the signal region. The width of the side-band is seven times of the signal region, large enough to reduce statistical uncertainties when performing background correction. Event yields in the side- 
band region $\left(0.005<E_{e} \theta_{e}^{2}<0.04\right)$ for Data and $\mathrm{MC}$ are listed in the Table 7.2 . If we assume $N C, \nu_{e} \mathrm{CC}$ and $\nu_{\mu} \mathrm{CC}$ MC backgrounds contribute evenly to the Data/MC difference, the background normalization (BN) factor multiplied to each MC background component in signal region is $B N=\frac{\left(\text { Data-MC }-M C_{(\text {Sig) }}\right)^{S B}}{M C_{\text {TotBkg }}^{S B}}=1.138 \pm 0.07$ (stat).
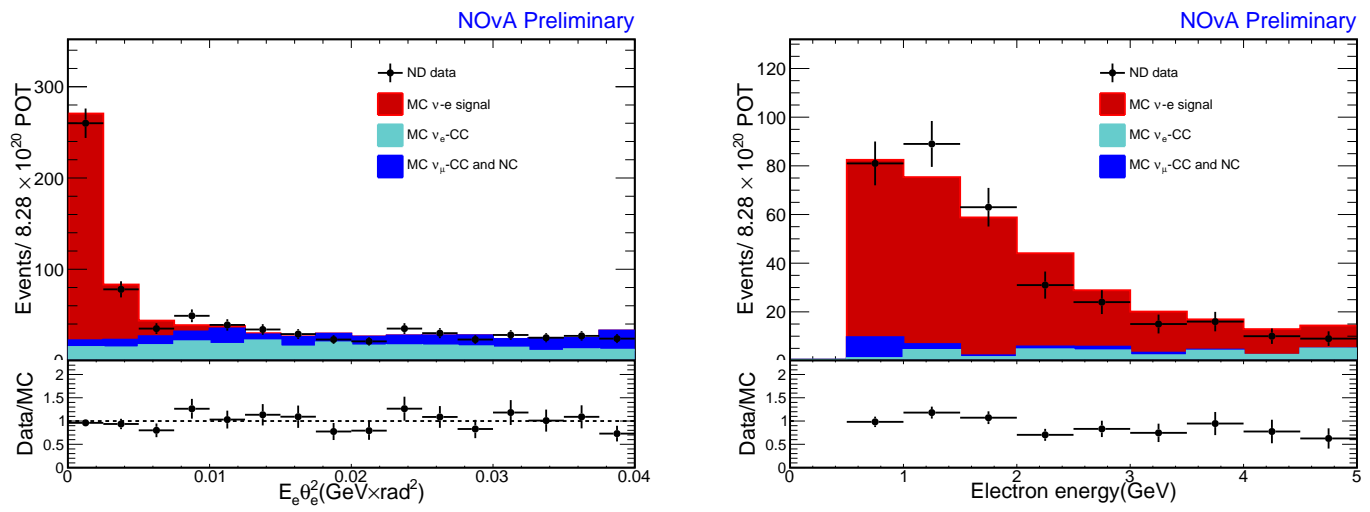

Figure 7.19: Left: Data/MC $E_{e} \theta_{e}^{2}$ distribution after background correction. Right: Data/MC of electron energy after background correction.

\subsection{2 $\quad \nu$-e Signal Counting}

After background correction from side-band region the $E_{e} \theta_{e}^{2}$ distributions for Data/MC are shown in Figure 7.19, all cuts on other variables are applied. After event selection, 338.0 data events are observed in the signal region $E_{e} \theta_{e}^{2}<0.005 \mathrm{GeV} \times \operatorname{rad}^{2}$. The yield of the $\nu$-e elastic scattering signal in data is counted by subtracting corrected MC background prediction from the total selected data events in the signal region. After the background subtraction, we observe $292.9 \pm 19.0$ neutrino-electron elastic scattering events in the data, and Data/MC ratio $\frac{N_{\nu-e}^{\text {Data }}}{N_{\nu-e}^{M C}}$ is measured to be $0.95 \pm 0.06$ (stat), statistical uncertainty from side-band background correction is included. Data and MC event yields in the signal region are listed in Table 7.3 . The selected electron candidate energy distribution in data, signal and background-corrected MC is shown in Figure 7.19. 


\begin{tabular}{|c|c|c|}
\hline Signal & $\mathrm{N}(0.5-5) \mathrm{GeV}$, with bkg correction & $\mathrm{N}(0.5-5) \mathrm{GeV}$, without bkg correction \\
\hline \hline Data & 338.0 & 338.0 \\
\hline MC pred. & 353.9 & 348.4 \\
\hline Bg. MC & 45.1 & 39.6 \\
\hline$\nu_{e}$ CC bkg & 29.3 & 25.7 \\
\hline$\nu_{\mu}$ CC bkg & 2.1 & 1.8 \\
\hline NC bkg (NC COH bkg $)$ & $13.7(7.2)$ & $12.1(6.3)$ \\
\hline$\nu$-e MC & 308.8 & 308.8 \\
\hline$\nu$-e Data (bkg subtracted) & $292.9 \pm 19.0$ & $298.4 \pm 18.7$ \\
\hline \hline$\frac{N_{\nu-e}^{D a t a}}{N_{\nu-e}^{M C}}$ & $0.95 \pm 0.06$ & $0.97 \pm 0.06$ \\
\hline
\end{tabular}

Table 7.3: Event yields in the signal region $\left(E_{e} \theta_{e}^{2}<0.005\right)$ with and with out background corrections. $\frac{N_{\nu-e}^{D a t a}}{N_{\nu-e}^{M C}}$ result is the one with background corrections.

\subsection{Systematic Uncertainty Study}

The total number of $\nu$-e elastic scatters provides a constraint on the integrated neutrino flux per POT:

$$
\Phi(\text { Data })=\Phi(M C) \times \frac{N_{\nu-e}^{\text {Data }}}{N_{\nu-e}^{M C}},
$$

where $\Phi($ Data $)$ is the measured neutrino flux per POT in data, $\Phi(M C)$ is the simulated flux per POT, and $\frac{N_{\nu-e}^{D a t a}}{N_{\nu-e}^{M C}}$ is the ratio of the measured numbers of $\nu$-e scattering events in data and MC. The measured number of $\nu$-e elastic scattering events is the product of the $\nu$-e elastic scattering cross section, the detector mass, the neutrino flux, and the detection efficiency. The cross-section of $\nu$-e elastic scattering is well predicted, but signal efficiency, detector simulation, and POT calculation cause systematic uncertainties in the measurement of $\nu$-e scattering events. Besides, uncertainties in the backgrounds also cause systematic uncertainty on the $\nu$-e counting. These systematic errors are discussed in this section and summarized in Table 7.4. All systematic uncertainties in the table are summed in quadrature to obtain the total systematic uncertainty. The flux related uncertainties that affect the integrated simulated flux $\Phi(M C)$ are discussed in the next Section. Figure 7.14 show event displays for beam neutrino interactions in an ND trigger 
window with a simulated $\nu$-e elastic scattering signal event before and after the event selection. One can find that the event selection keeps the single electron signal from the $\nu$-e elastic scattering while removing all other neutrino interactions in the same trigger window.

\begin{tabular}{|c|c|}
\hline Sources & $\frac{N_{\nu-e}^{\text {Data }}}{N_{\nu-e}^{M C}}$ \\
\hline Signal efficiency & 0.046 \\
\hline Single-particle requirement & 0.042 \\
\hline Background Normalization & 0.013 \\
\hline Detector modeling & 0.009 \\
\hline POT counting, Detector Mass and Beam Intensity & 0.009 \\
\hline Total Systematics & 0.065 \\
\hline
\end{tabular}

Table 7.4: Summary of the systematic uncertainties for $\frac{N_{\nu-e}^{D a t a}}{N_{\nu-e}^{M C}}$

\subsubsection{Signal Efficiency Systematic}

We used rock muon induced Bremsstrahlung (muon-removed brem (MR Brem)) showers in the ND to estimate the systematic uncertainty in the signal efficiency. Rock muons refer to the muons produced in neutrino interactions in the rock surrounding the ND. Original muons in MR Brem samples are removed using the technique described in Ref [108] and [109]. The electron energy and $\cos \left(\theta_{e}\right)$ distributions of the MR Brem shower selected from rock muon Data and MC, compared with $\nu$-e elastic scattering signal events, are shown in Figure 7.20, $\theta_{e}$ is the angle from the shower to the beam direction. The energy and angle distributions in the MR Brem shower sample overlaps with the energy and angle distributions in the $\nu$-e elastic scattering signal.

The efficiency difference for MR Brem Data and MC differences in the $\nu$-e PID, $e / \pi^{0} \mathrm{PID}$, and $E_{e} \theta_{e}^{2}$ selection are assigned as uncertainties for these cuts. The total uncertainty in the signal selection is the square root of the quadratic sum of all these three terms. Because we directly check the Data/MC difference in the $E_{e} \theta_{e}^{2}$ selection, the uncertainties caused by EM shower angular resolution and energy calibration are included 
in this MR Brem shower study. While testing one efficiency, cuts on other two variables are applied. To better mimic selection efficiencies for signal, we re-weight distributions of $\nu$-e $\mathrm{ID}, e / \pi^{0} \mathrm{ID}$ and $E_{e} \theta_{e}^{2}$ in MR Brem MC and Data to match corresponding distributions in the $\nu$-e signal MC sample, as shown in Figure 7.21. The resultant systematic uncertainties are shown in Table 7.5 .
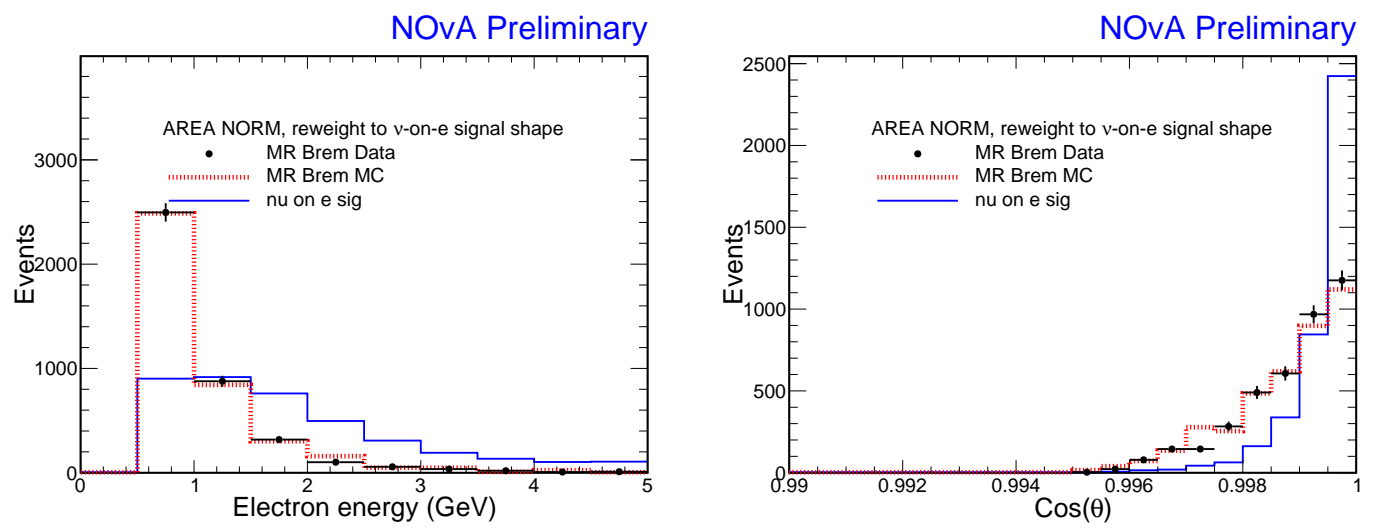

Figure 7.20: (left) Energy and (right) $\cos (\theta)$ for rock MR Brem in data and MC, compared with $\nu$-e signal events, , area normalized. Rock MR Brem data and MC are weighted by the ratio of $\nu$-e scattering $\mathrm{MC}$ over $\mathrm{MR}$ Brem $\mathrm{MC}$ in each bin to correct the shape difference between the MR Brem sample and the $\nu$-e scattering signal.

\begin{tabular}{|c|c|c|c|}
\hline Efficiency & $\mathrm{MC}$ & Data & $($ Data-MC) $/ \mathrm{MC}$ \\
\hline$E_{e} \theta_{e}^{2}$ & 0.906 & 0.901 & 0.006 \\
\hline$e / \pi^{0} \mathrm{PID}$ & 0.733 & 0.700 & 0.045 \\
\hline$\nu$-e PID & 0.941 & 0.934 & 0.007 \\
\hline \hline Total & & & 0.046 \\
\hline
\end{tabular}

Table 7.5: Summary of the systematic uncertainties in the signal efficiency estimated by rock muon induced EM Shower samples.

\subsubsection{Single Particle Requirement}

Due to inaccuracies in the shower clustering and noise simulation for Data and MC cause systematic uncertainties in the selection efficiency of the single particle requirement. We relax the three single-particle requirement cuts on $E_{\text {shower }} / E_{t o t}$, vertex energy and the gap individually. The relaxed cut values are chosen to have a $\sim 90 \%$ selection efficiency 

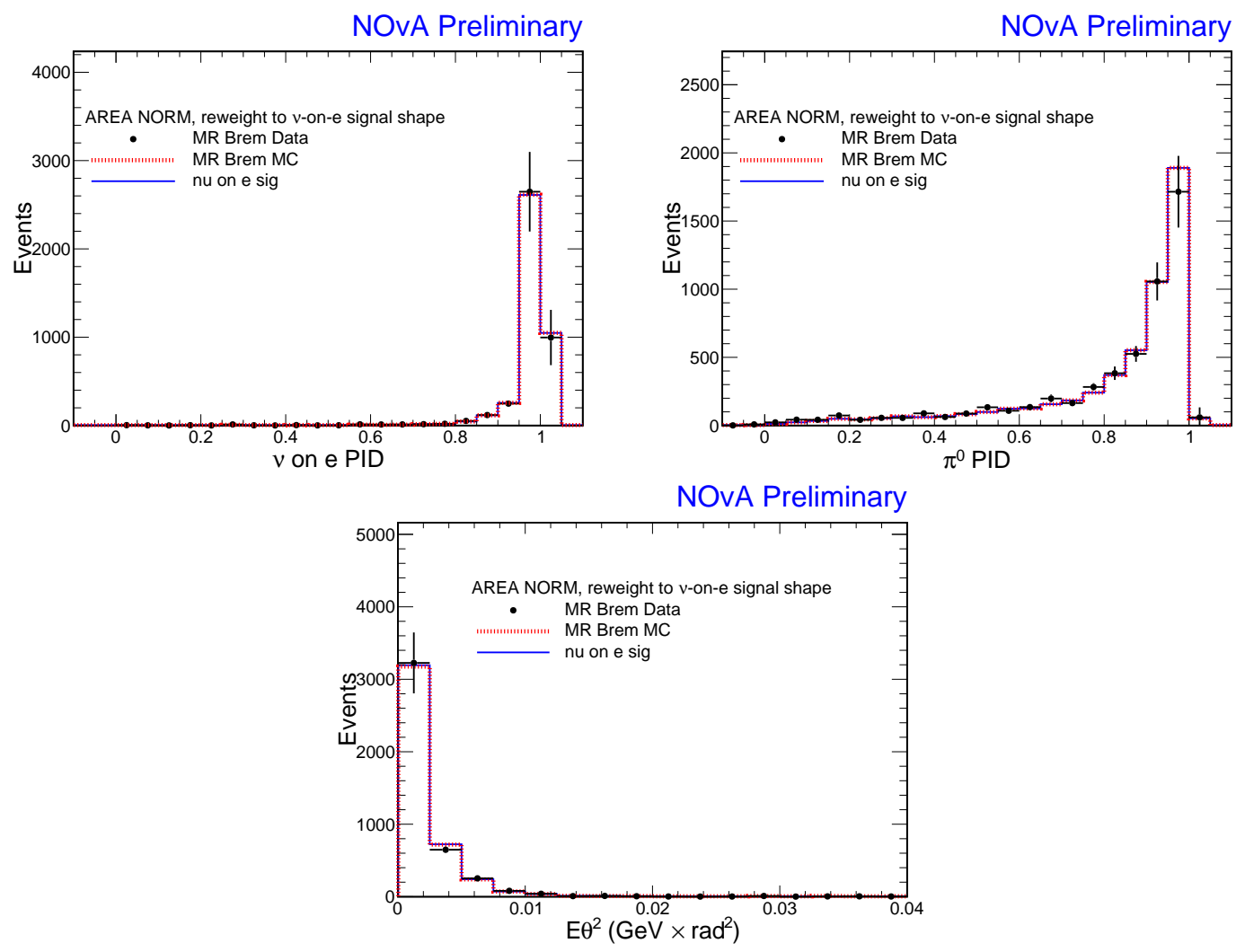

Figure 7.21: Top: $\nu$-e and $\pi^{0}$ PID for rock MR Brem in data and MC. Bottom: $\mathrm{E} \theta^{2}$ for rock MR Brem in data and MC, compared with $\nu$-e signal events, area normalized. MR Brem data and $\mathrm{MC}$ are weighted by the ratio of $\nu$-e scattering $\mathrm{MC}$ over MR Brem MC in each bin to correct the shape difference between the MR Brem sample and the $\nu$-e scattering signal.

for each criterion. The resultant variation in $N_{\nu-e}^{D a t a} / N_{\nu-e}^{M C}$ after changing each cut is assigned as the uncertainty. The quadratic sum of the three uncertainties is assigned as the systematic error in the single particle requirement, as shown in Table 7.6. The MR Brem sample is generated by the muon removal algorithm, which has a different vertex behavior compared with a real neutrino interaction, so we do not use it to validate the efficiency of the single-particle requirement.

\subsubsection{Run Condition Noise Systematics}

According to Ref. [110] these effects are negligible. The effect count only for 2015 because of coarse timing noise effect was there but for rest of the period has fine timing and they 


\begin{tabular}{|c|c|c|}
\hline Selection & $\frac{N_{\nu-e}^{\text {Data }}}{N_{\nu-e}^{M C}}$ & uncertainty $\left(\frac{\text { nom-shift }}{\text { nom }}\right)$ \\
\hline $\begin{array}{c}\text { Standard single particle requirement } \\
\text { Section } 7.4\end{array}$ & 0.948 & 0 \\
\hline \hline Vertex energy $<0.02$ & 0.907 & 0.042 \\
\hline gap $<80$ & 0.951 & 0.003 \\
\hline$E_{\text {shower }}>0.8$ & 0.944 & 0.005 \\
\hline$E_{\text {tot }}$ & & 0.042 \\
\hline Total syst. & & \\
\hline
\end{tabular}

Table 7.6: Summary of the systematic uncertainties in the selection efficiency of the single particle requirement. The three single-particle requirement cuts on $E_{\text {shower }} / E_{t o t}$, vertex energy and the gap are relax individually. The relaxed cut values are chosen to have $\mathrm{a} \sim 90 \%$ selection efficiency for each criterion. The resultant variation in $N_{\nu-e}^{\text {Data }} / N_{\nu-e}^{M C}$ after changing each cut is assigned as the uncertainty. The quadratic sum of the three uncertainties is assigned as the systematic error in the single particle requirement.

did not find much effect from noise. As in the ND we always have fine timing so the impact of this uncertainty on the $\nu$-e signal selection is negligible.

\subsubsection{Signal in the Sideband Region}

The background correction factor is calculated by subtracting the MC signal from Data in the sideband region. To consider the effect of the MC signal in the sideband region, we use the measured $N_{\nu-e}^{\text {Data }} / N_{\nu-e}^{M C}$ from the signal region to correct the MC signal in the sideband region, and re-calculate the background normalization factor $B N$ in Session 7.6.1. The new background normalization factor is then used to correct the MC background yield in the signal region and re-calculate $N_{\nu-e}^{\text {Data }} / N_{\nu-e}^{M C}$. This process is repeated for three iterations, as shown in Table 7.7. No significant change is observed in the $N_{\nu-e}^{D a t a} / N_{\nu-e}^{M C}$ result.

\begin{tabular}{|c|c|c|}
\hline Iteration no. & $N_{\nu-e}^{\text {Data }} / N_{\nu-e}^{M C}$ & BN (background normalization factor) \\
\hline 1 & 1 & 1.138 \\
2 & 0.95 & 1.142 \\
3 & 0.95 & 1.142 \\
\hline
\end{tabular}

Table 7.7: Table of iteration for background normalization from sideband region. 


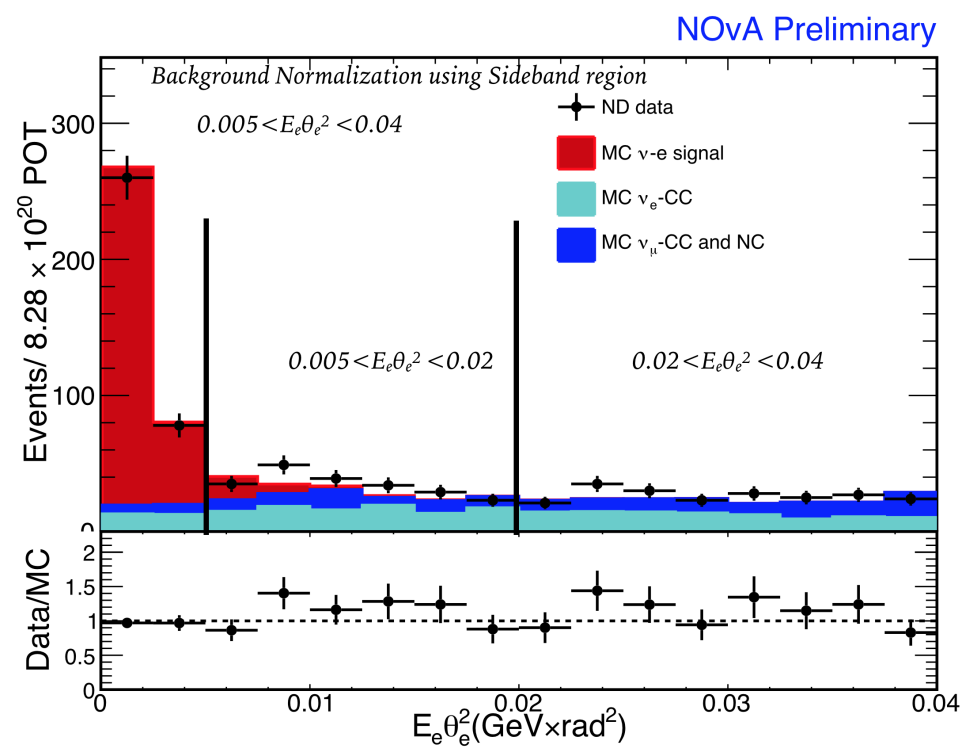

Figure 7.22: $E_{e} \theta_{e}^{2}$ distribution before the background normalization with the definitions of the standard sideband $\left(0.005<E_{e} \theta_{e}^{2}<0.04\right)$, the sideband Region A $\left(0.005<E_{e} \theta_{e}^{2}<\right.$ $0.02)$ and the sideband Region $\mathrm{B}\left(0.02<E_{e} \theta_{e}^{2}<0.04\right)$.

\subsubsection{Background Normalization}

We use GENIE knobs [84] to check the effect of background modeling uncertainties on this analysis. For each knob one $\sigma$, background normalization is re-calculated by sideband data and GENIE re-weighted MC. The uncertainty in $\frac{N_{\nu-e}^{D a t a}}{N_{\nu-e}^{M C}}$ is calculated as:

$$
\text { Uncertainty }=\frac{\left(B N *(b k g)_{s g}\right)_{r w}-\left(\left(B N *(b k g)_{s g}\right)_{s t d}\right.}{\left(B N_{s b} *(b k g)_{s g}\right)_{s t d}},
$$

where $B N$ is the background correction factor $B N=\frac{\left(D a t a-M C_{(\text {Sig) }}\right)^{S B}}{M C_{T o t B k g}^{S B}}$ from sideband $(\mathrm{SB}),(b k g)_{s g}$ is the MC background events in the signal region before background correction. The subscript $r w$ represents re-weighted with respect to $\pm \sigma$ shift in the respective GENIE parameter and the subscript std stands for the nominal condition without re-weight. For the variation of coherent $\pi^{0}$, we use the NOvA measurement described in [111. The variation for each term of the GENIE reweight can be found in Table G.1. 
The overall variation is $9 \%$ for the total MC background, which is 0.013 in $\frac{N_{\nu-e}^{D a t a}}{N_{\nu-e}^{M C}}$.

We don't have NC diffractive (DFR) $\pi^{0}$ events simulated in the official NOvA MC, the details are shown in the Appendix G.4. We simulate a stand-alone DFR sample and put it in the analysis to study the effect of including the DFR background. We end up with 2.54 DFR events in the sideband region and zero in the signal region, which causes a variation of 0.002 in $\frac{N_{\nu-e}^{\text {Data }}}{N_{\nu-e}^{M C}}$. We therefore consider the effect from DFR to be negligible.

In the background correction, we scale the background interaction modes uniformly according to the sideband data for the entire background. To further check the uncertainty, we change only the scale for one background component, i.e., $\mathrm{NC} / \nu_{e} \mathrm{CC}$ component alone, assuming the Data/MC difference in sideband is only from that background component. The resulting $\nu$-e Data/MC is 0.982 for the $\mathrm{NC}$ correction and 0.979 for the $\nu_{e} \mathrm{CC}$ correction. The largest variation in $\frac{N_{\nu-e}^{\text {Data }}}{N_{\nu-e}^{M C}}$ from nominal is 0.002 , which we consider to be negligible.

To check the background normalization uncertainty caused by the different kinematics in the signal and sideband regions, we divide sideband region $\left(0.005<E_{e} \theta_{e}^{2}<0.04\right)$ into two sub-regions $\mathrm{A}$ and $\mathrm{B}$. Region $\mathrm{A}\left(0.005<E_{e} \theta_{e}^{2}<0.02\right)$ is closer to the signal region while the Region $\mathrm{B}\left(0.02<E_{e} \theta_{e}^{2}<0.04\right)$ is further away, as shown in Figure 7.22 . We then use these two sub-regions to calculate background normalization factors individually. Region A gives a background normalization factor of 1.165 and region $\mathrm{B}$ gives us 1.066. Applying the two different scale factors to the background in the signal region makes resultant changes 0.004 and 0.008 in $\frac{N_{\nu-e}^{D a t a}}{N_{\nu-e}^{M C}}$. These variations can be covered easily by the GENIE re-weight study. 


\subsubsection{Energy Scale in Background}

Birks-Chou and energy calibration effects can affect the energy scale of signal and background. For the signal, these effects have been covered by the data-driven rock shower study. For the background, we use $\pi^{0}$ as a control sample to study the systematic uncertainty due to the energy scale. As our selection PIDs are trained to select EM shower, the background passes these PIDs also EM dominant. In the signal region, more than $90 \%$ of background is an electron, $\pi^{0}$ or $\gamma$ shower. We take the Data/MC difference in $\pi^{0}$ nominal mass (1.5\%) from the $\mathrm{COH} \pi^{0}$ analysis note [111] as the energy scale uncertainty of EM shower and shift the shower energy in selected background MC. A maximum variation of 0.002 is observed in $\frac{N_{\nu-e}^{D a t a}}{N_{\nu-e}^{M C}}$, which is neglectable.

\subsubsection{Detector Modeling}

In NOvA simulation, the default ND geometry is the "staggered" geometry, which has the intentional plane to plane stagger based on survey data. To check the effect of the geometry simulation, we compare simulated signal $E_{e} \theta_{e}^{2}$ distributions between the stagger geometry and the ideal geometry, as shown in Figure 7.23. The ideal ND geometry assumes a perfect detector alignment. The difference 0.009 between the two samples is assigned as the detector modeling uncertainty in $\frac{N_{\nu-e}^{D a t a}}{N_{\nu-e}^{M C}}$.

\subsubsection{POT Counting, Detector Mass and Beam Intensity}

According to Ref. [112, the systematic uncertainty on the POT counting is $0.5 \%$. Another $0.7 \%$ uncertainty is found by calculation of the detector mass [113. According to Ref. [114, the uncertainty caused by mis-modeling of beam intensity is $0.47 \%$. Adding these three terms in quadrature, we have a systematic uncertainty 0.009 in $\frac{N_{\nu e}^{D a t a}}{N_{\nu-e}^{M C}}$. 


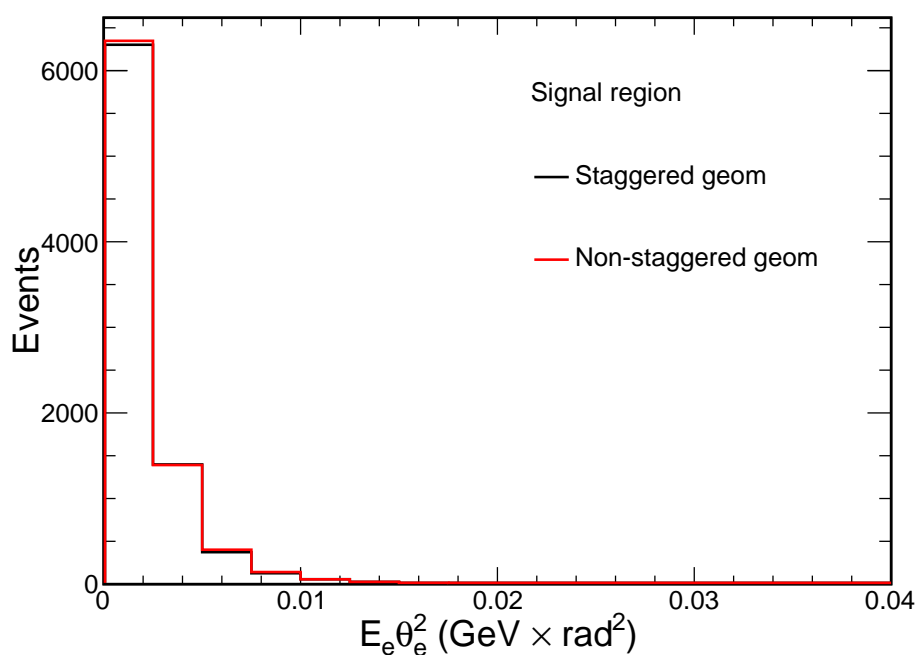

Figure 7.23: $\nu$-e signal comparison between standard and stagger geometry

\subsection{Flux Constraint}

As discussed in the first paragraph of Section 7.7, the flux in data can be calculated as:

$$
\Phi(\text { Data })=\Phi(M C) \times \frac{N_{\nu-e}^{\text {Data }}}{N_{\nu-e}^{M C}}
$$

The integrated flux from $0.5 \mathrm{GeV}$ to $120 \mathrm{GeV}$ in the near detector $\mathrm{MC}$ is $\Phi(M C)$

$=1.32 \times 10^{-8} \nu / \mathrm{cm}^{2} / \mathrm{POT}$. Using $\frac{N_{\nu-e}^{D a t a}}{N_{\nu-e}^{M C}}$, the integrated flux at ND is measured to be:

$$
\Phi(\text { Data })=\Phi(M C) \times \frac{N_{\nu-e}^{\text {Data }}}{N_{\nu-e}^{M C}}=1.32 \times 10^{-8} \times 0.95=1.25 \times 10^{-8} \nu / \mathrm{cm}^{2} / \mathrm{POT}
$$

Based on the propagation of uncertainty, the statistical uncertainty of $\Phi($ measured $)$ is: 
$\sigma_{\text {stat. }}[\Phi($ Data $)]=\Phi(M C) \times \sigma_{\text {stat. }}\left[\frac{N_{\nu-e}^{\text {Data }}}{N_{\nu-e}^{M C}}\right]=1.32 \times 10^{-8} \times 0.06=0.08 \times 10^{-8} \nu / \mathrm{cm}^{2} / \mathrm{POT}$

The goal of this analysis is to use the measured $\nu$-e signal to do a integrated flux measurement. Flux-shape-related uncertainty can potentially affects this analysis by changing the background kinematic shapes and the $\nu$-e scattering cross section. Flux normalization on the other hand should have no effect on the analysis.

Flux shape uncertainty may come from the modeling of hadron production and beam transport. To study the effect, we re-weight the MC flux by external hadron production data MIPP and NA49 with PPFX and compare the measured flux $\left(\Phi \times \frac{\text { Data }}{\text { MC }}\right)$ from the nominal. The beam transport uncertainty is studied by varying beam simulation parameters. Table 7.8 summarizes the final effect from hadron production model and beam transport, and the relative effect on integrated flux measurement is $2.2 \%$.

The relative systematic uncertainty in the flux measurement from the $\frac{N_{\nu-e}^{D a t a}}{N_{\nu-e}^{M C}}$ measurement is

$$
\frac{\sigma_{\text {syst. }}\left(\frac{N_{\nu-e}^{\text {Data }}}{N_{\nu-e}^{M C}}\right)}{\frac{N_{\nu-e}^{D a t a}}{N_{\nu-e}^{M C}}}=0.065 / 0.95=6.8 \%
$$

Combine the systematic uncertainty from flux shape and the systematic uncertainty from the $\frac{N_{\nu-e}^{\text {Data }}}{N_{\nu-e}^{M C}}$ measurement quadratically, the total systematic uncertainty of $\Phi($ Data $)$ is $0.09 \times 10^{-8} \nu / \mathrm{cm}^{2} / \mathrm{POT}$. 


\begin{tabular}{|c|c|c|c|c|}
\hline Sources & Flux without correction $/ \mathrm{POT} / \mathrm{cm}^{2}$ & Data/MC & Corrected Flux $/$ POT/cm & Uncertainty \\
\hline Nominal & 1.32 & 0.95 & 1.254 & 0.0 \\
\hline Hadro-production (PPFX) & 1.23 & 1.04 & 1.279 & 0.025 \\
\hline Beam Transport plus & 1.35 & 0.92 & 1.242 & 0.012 \\
Beam Transport minus & 1.24 & 1.01 & 1.252 & 0.002 \\
\hline \hline Total & & & $0.028(2.2 \%)$ \\
\hline
\end{tabular}

Table 7.8: Table for flux systematics uncertainties from beam transport and hadroproduction model.

\section{$7.9 \quad$ Result}

In summary, observed $292.9 \pm 19.0$ (stat.) neutrino-electron elastic scattering events in the data, and the Data/MC ratio for this process is measured to be

$$
\frac{N_{\nu-e}^{\text {Data }}}{N_{\nu-e}^{M C}}=0.95 \pm 0.06 \text { (stat.) } \pm 0.07 \text { (syst.) }
$$

The integrated flux in Monte Carlo from $0.5 \mathrm{GeV}$ to $120 \mathrm{GeV}$ at NOvA ND is $\Phi(M C)=$ $1.32 \times 10^{-8} \nu / \mathrm{cm}^{2} /$ POT. Using the Data $/ M C$ ratio of neutrino-electron elastic scattering events we observe, the flux is determined to be:

$$
\Phi(\text { Data })=[1.25 \pm 0.08(\text { stat. }) \pm 0.09(\text { syst. })] \times 10^{-8} \nu / \mathrm{cm}^{2} / \mathrm{POT}
$$

The result presented here may differ from the from an upcoming NOvA publication (in progress) due to the addition of decay in flight data/MC sample for systematics study compared to rock muons. 


\section{Chapter 8}

\section{Summary and Results}

An accurate prediction of the neutrino flux is needed for precision oscillation and is critical for the cross-section measurements. NOvA, a second-generation long-baseline neutrino oscillation experiment at Fermilab, is designed to measure the (anti) electron neutrino appearance and the (anti) muon neutrino disappearance. NOvA comprises two finely segmented, liquid scintillator detectorsat $14 \mathrm{mrad}$ off-axis in the NuMI beam. Data from the external hadro-production experiments measurements (MIPP, NA49), and, importantly, from the NOvA Near Detector provide powerful constraints on the (anti) muon neutrino and (anti) electron neutrino fluxes. Additionally, the measurement of the neutrinoelectron elastic scattering provides an in situ constraint on the absolute flux. This thesis presents a novel method to empirically constrain the neutrino flux at NOvA using NuMI accelerator facility at the Fermi National Accelerator Laboratory. The precisely constrained flux will be used to conduct precision measurements of neutrino oscillation and cross-section in the NOvA experiment. The studies related to Near Detector installation are presented. To ensure the best performance of Near Detector modules several tests have been done before installation. All installed Near Detector modules passed fiber and leak test.

The first part of the thesis shows the systematics uncertainties for beam transport 
for NOvA Oscillation study (used in NovA oscillation analyses) and hadron-production flux uncertainties using external experiments data. The weight is $\approx 0.9$ at $1 \mathrm{GeV}$ and $\approx 0.95$ at $3 \mathrm{GeV}$. The uncertainty for the flux weight is is $\approx 9 \%$ at $1 \mathrm{GeV}$, and $\approx 11 \%$ at $3 \mathrm{GeV}$. The incrementing shape starting around $3 \mathrm{GeV}$ is due to the $K$ flux component is increasing rapidly.

The second part shows $K^{+}$normalization using Near Detector muon neutrino charge current data with $3.7 \times 10^{2} 0$ protons-on-target, second analysis (2016) data set. The results from this analysis are used for second oscillation analysis of NOvA. The combined effect on electron neutrino flux using $K^{+}$normalization from Near Detector data and external experiment is also presented. We presented an analysis which constrains the $K^{+}$yield in the NOvA neutrino beam using the measured $\nu_{\mu}$-CC spectrum at the ND: The off-axis nature of the beam makes $\pi^{+}$versus $K^{+}$separation possible. We determine a $K^{+}$normalization of $1.17 \pm 0.005$ (Stat) \pm 0.10 (Syst) relative to the FLUKA hadro-production model; the corresponding normalization for the $\pi^{+}$yield is $0.98 \pm 0.13($ Stat $\oplus$ Syst $)$. Sources of systematic errors in the meson yields include reconstruction, hadro-production, and cross-section modeling. Using the constrained $\mathrm{K}^{+}$, we extract a prediction of the flux in NOvA with a $10 \%$ error in the region $E_{\nu} \geq 3.5 \mathrm{GeV}$.

The final part of the analysis shows first NOvA preliminary constraint on absolute flux using Near Detector neutrino-electron elastic scattering data with 8.28 $\times 10^{20}$ proton-on-target. Observed $292.9 \pm 19.0$ (stat.) neutrino-electron elastic scattering events in the data, and the Data/MC ratio for this process is measured to be $\frac{N_{\nu-e}^{\text {Data }}}{N_{\nu-e}^{M C}}=0.95 \pm 0.06$ (stat.) \pm 0.07 (syst.). The integrated flux in Monte Carlo from 0.5 $\mathrm{GeV}$ to $120 \mathrm{GeV}$ at NOvA ND is $\Phi(M C)=1.32 \times 10^{-8} \nu / \mathrm{cm}^{2} /$ POT. Using the Data $/ M C$ ratio of neutrino-electron elastic scattering events we observe, the flux is determined to be: $\Phi($ Data $)=[1.25 \pm 0.08($ stat. $) \pm 0.09$ (syst. $)] \times 10^{-8} \nu / \mathrm{cm}^{2} / \mathrm{POT}$ 


\section{Appendix A}

\section{Supplementary Material for}

\section{Chapter 3}
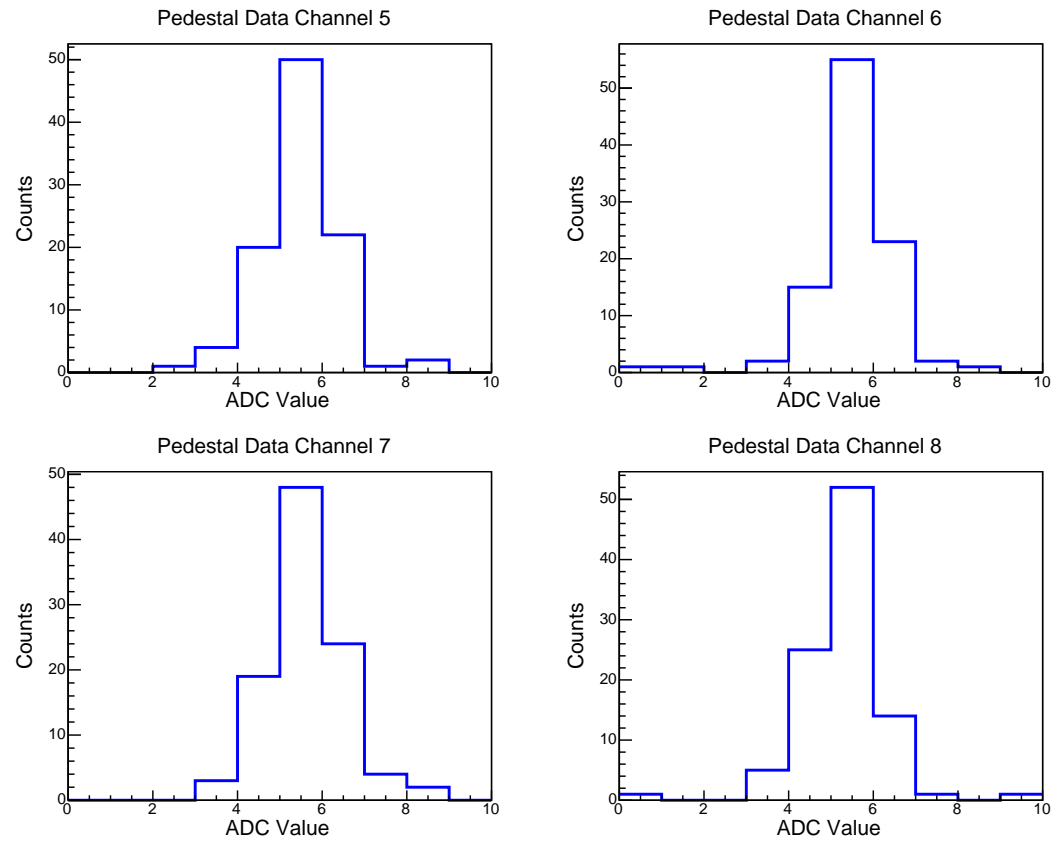

Figure A.1: Experimental data from 100 pedestals of Fiber Loop Transmission (FTL) corresponding to channels $5,6,7$, and 8 . 

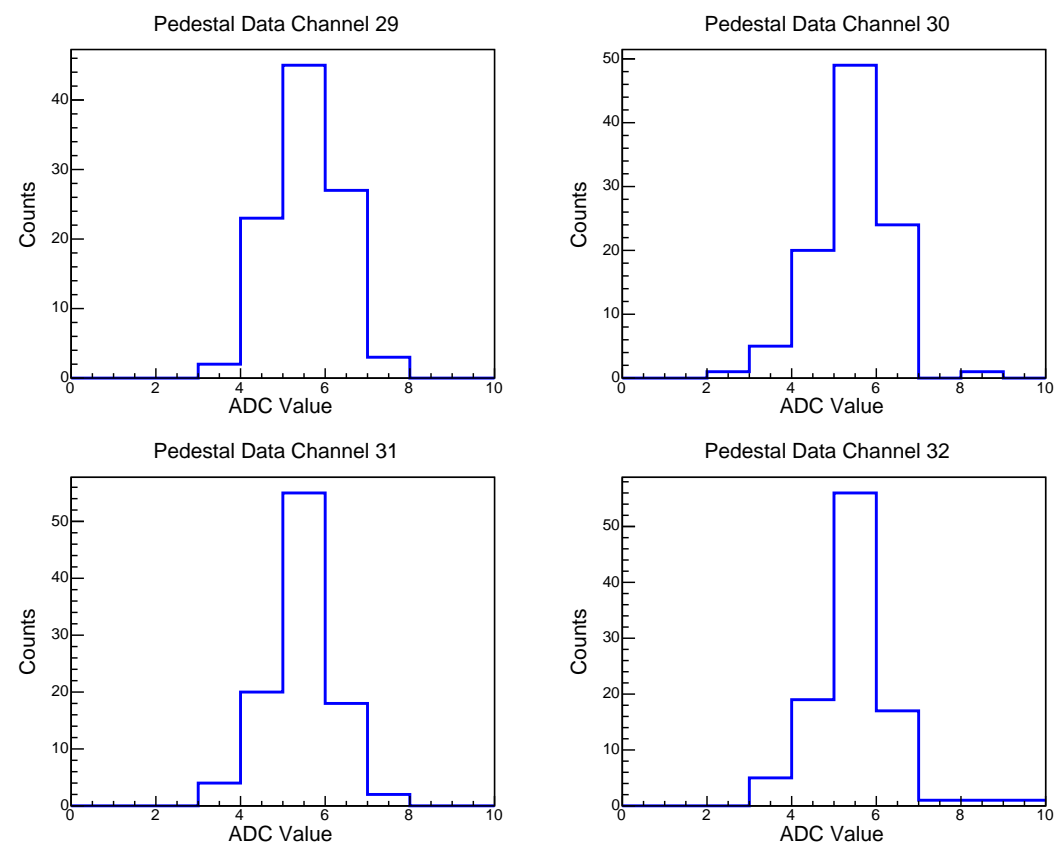

Figure A.7: Experimental data from 100 pedestals of FLT corresponding to channels 29, 30,31 and 32 .
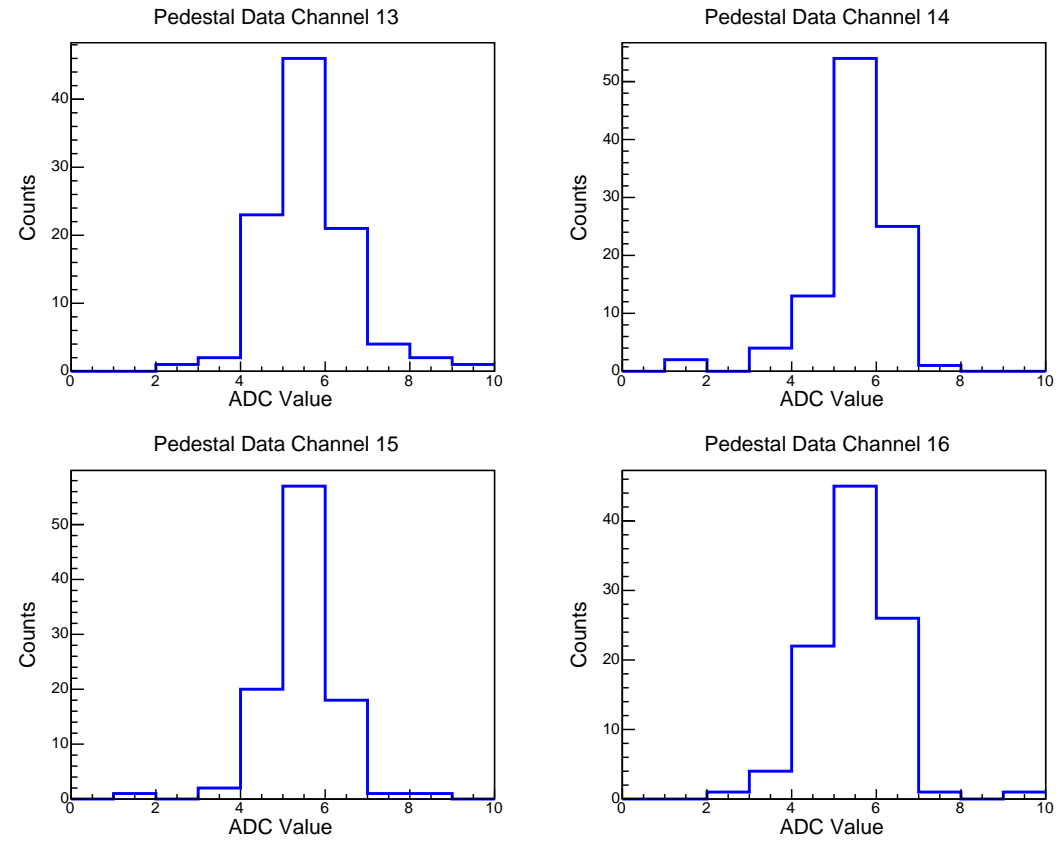

Figure A.3: Experimental data from 100 pedestals of FLT corresponding to channels $13,14,15$, and 16 . 

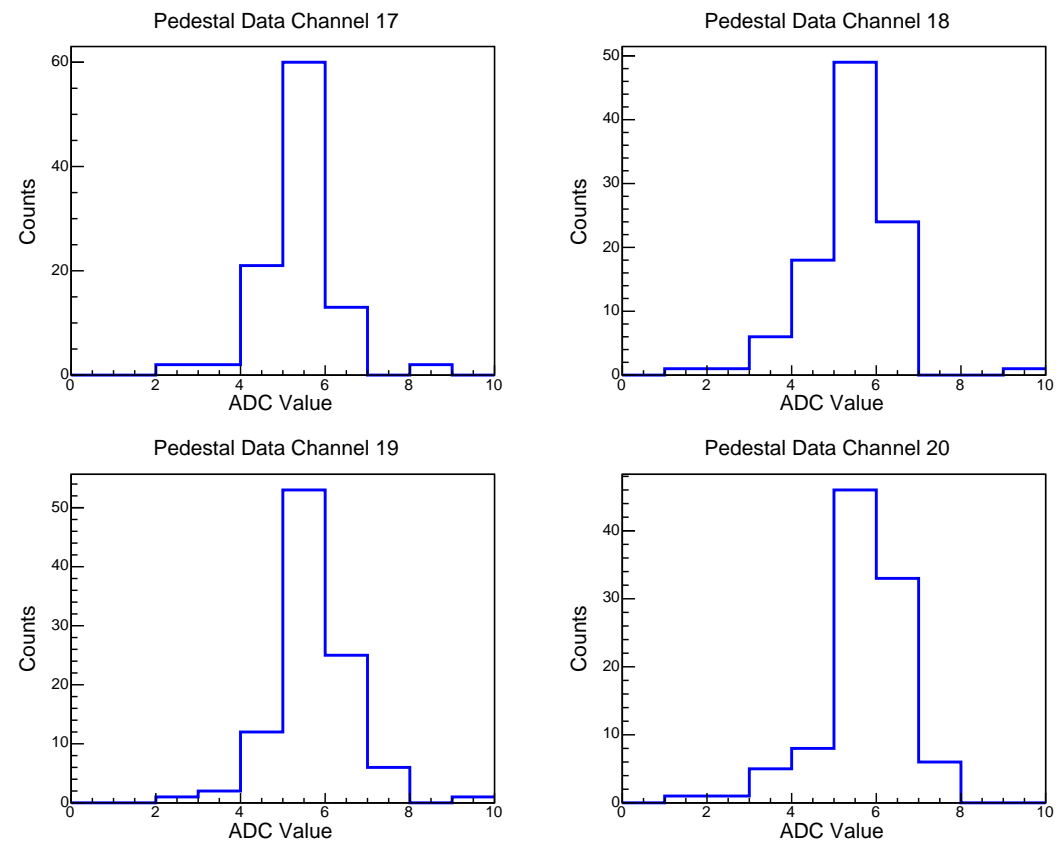

Figure A.4: Experimental data from 100 pedestals of FLT corresponding to channels 17, 18,19 , and 20 .
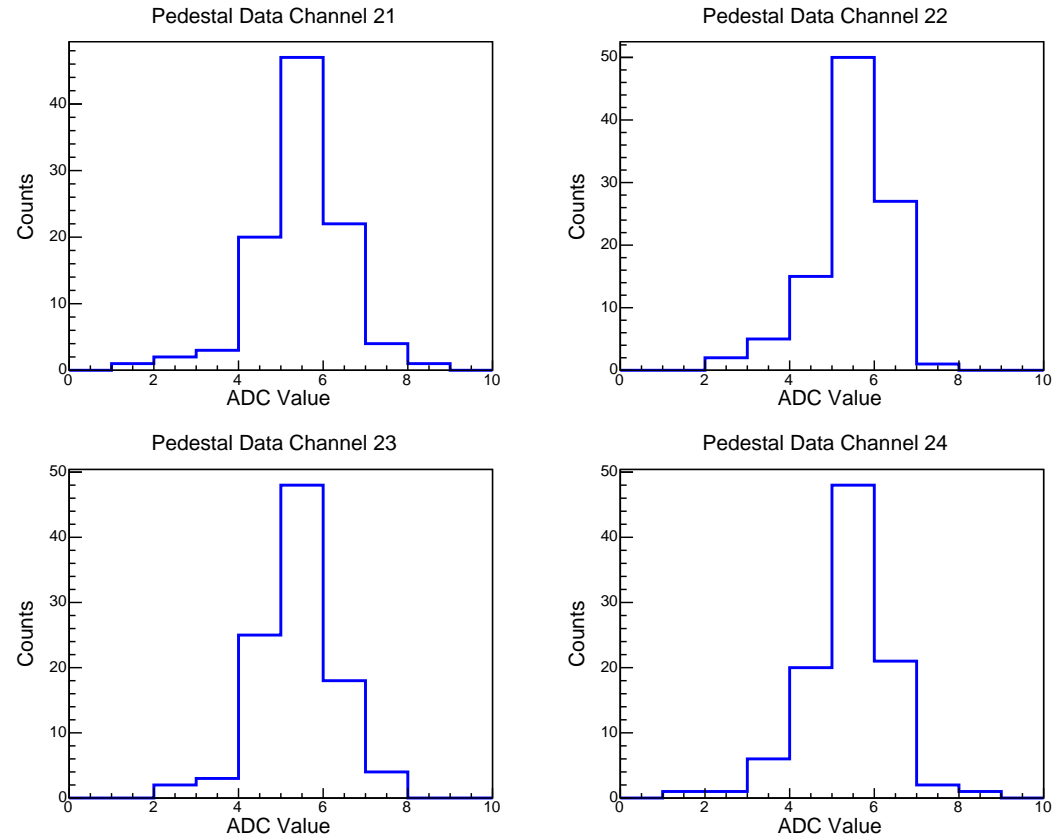

Figure A.5: Experimental data from 100 pedestals of FLT corresponding to channels 21, 22,23 and 24. 

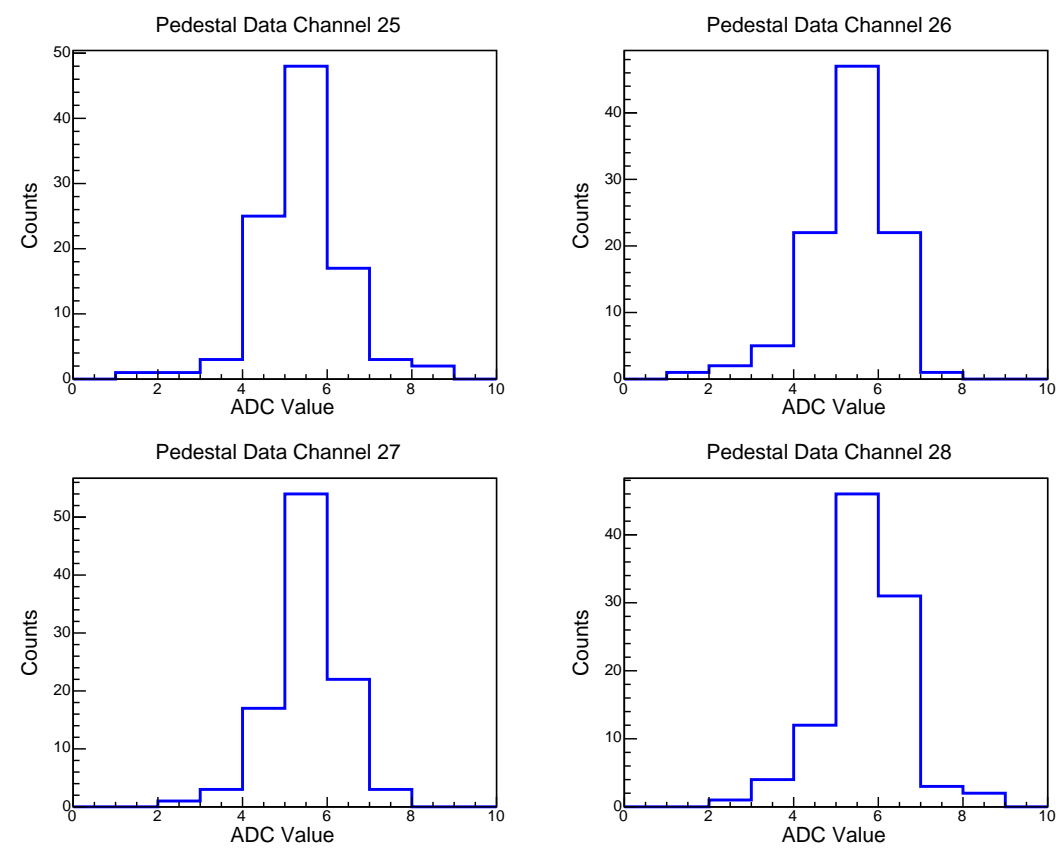

Figure A.6: Experimental data from 100 pedestals of FLT corresponding to channels 25, 26,27 and 28 . 

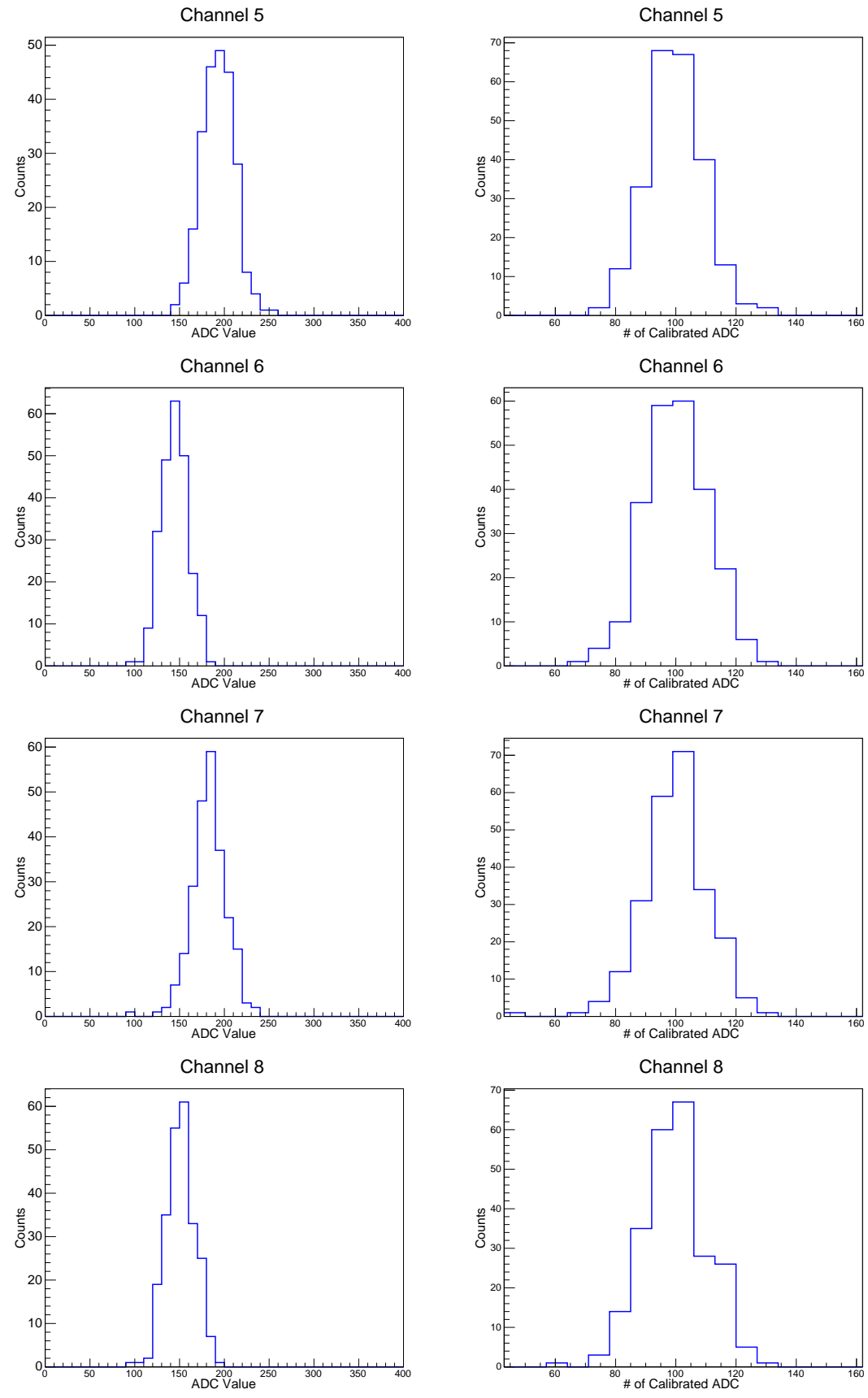

Figure A.8: Raw ADC vs Calibrated ADC response for channel 5, 6, 7 and 8. 

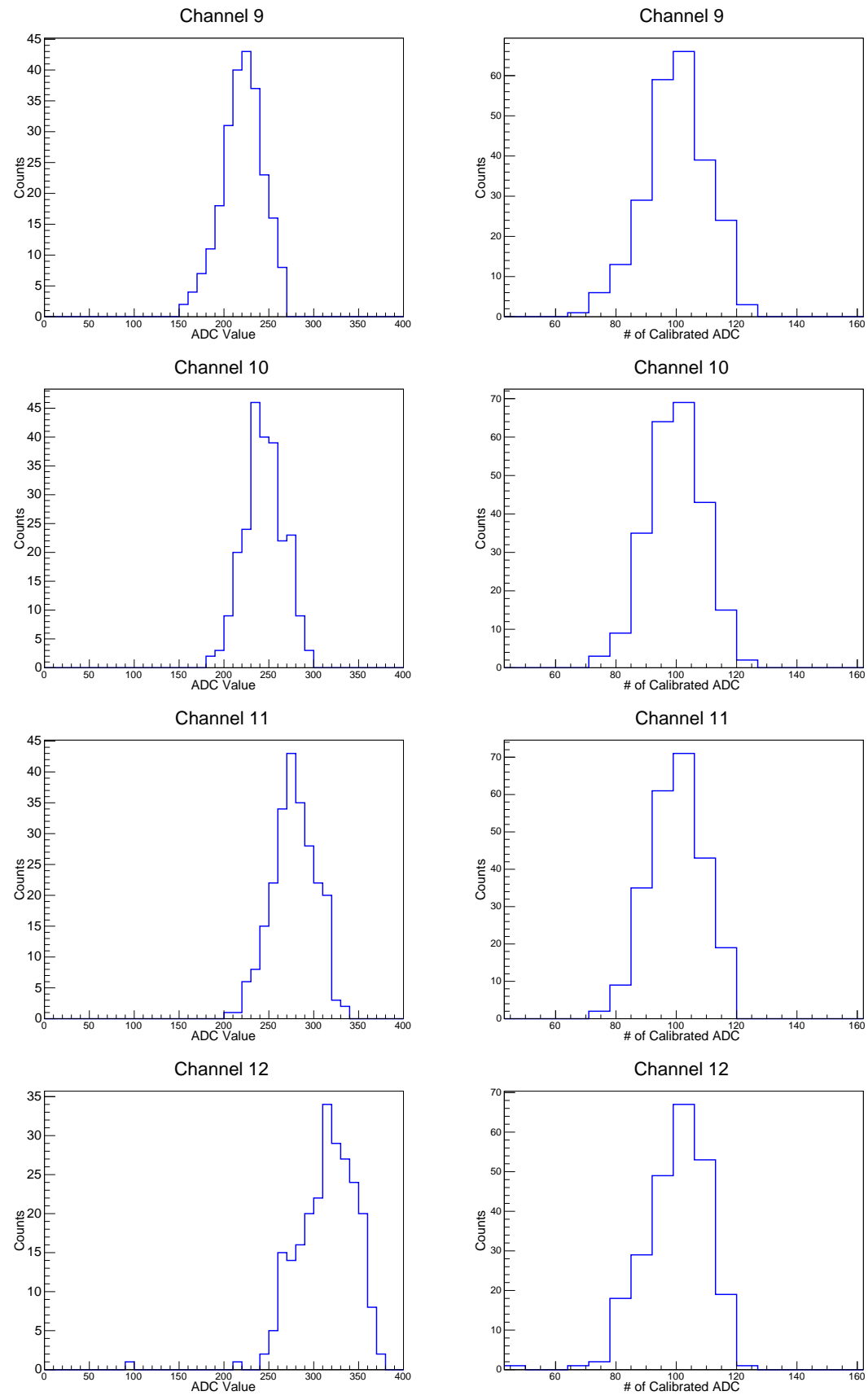

Figure A.9: Raw ADC vs Calibrated ADC response for channel 9, 10, 11 and 12. 

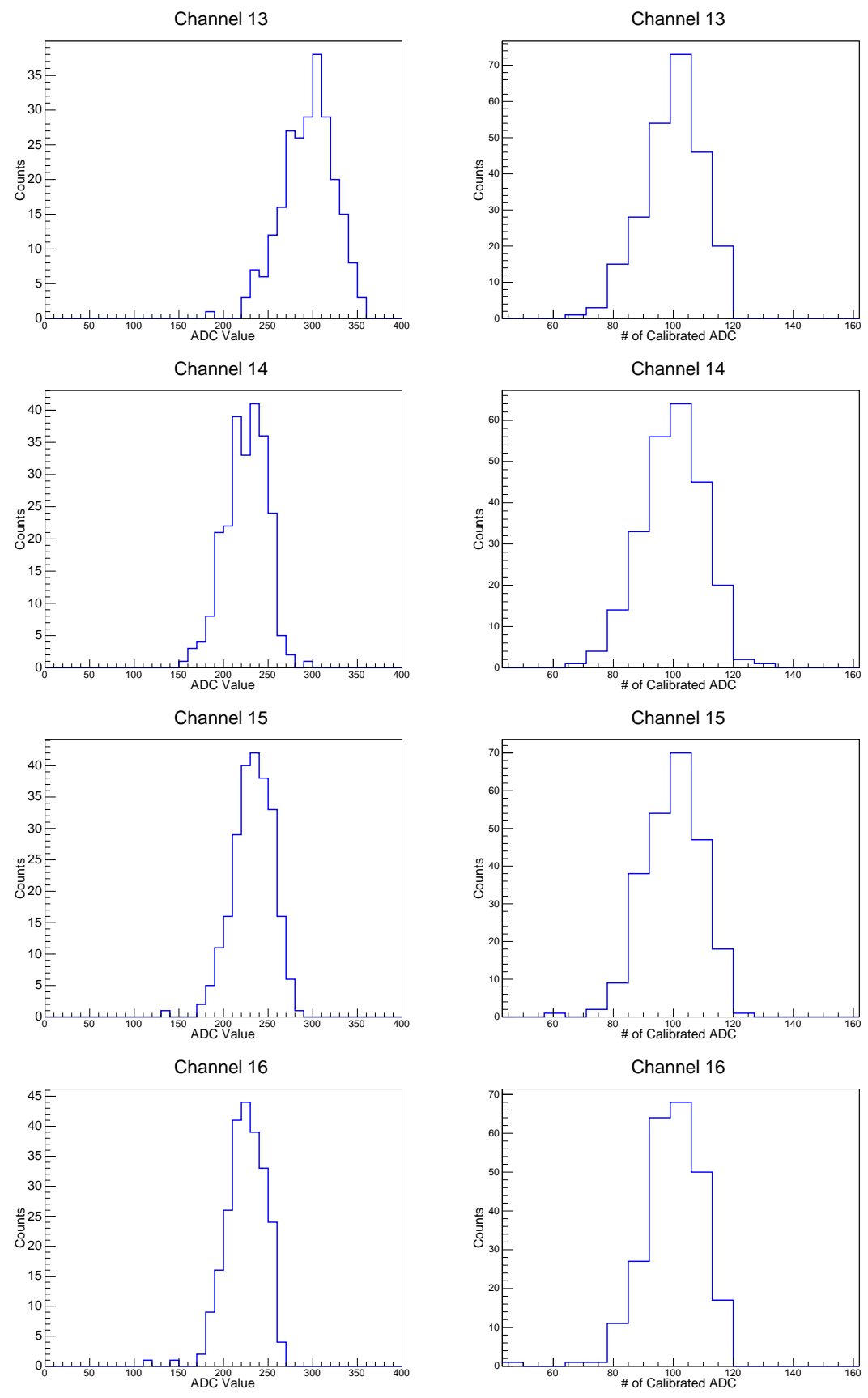

Figure A.10: Raw ADC vs Calibrated ADC response for channel 13, 14, 15 and 16. 

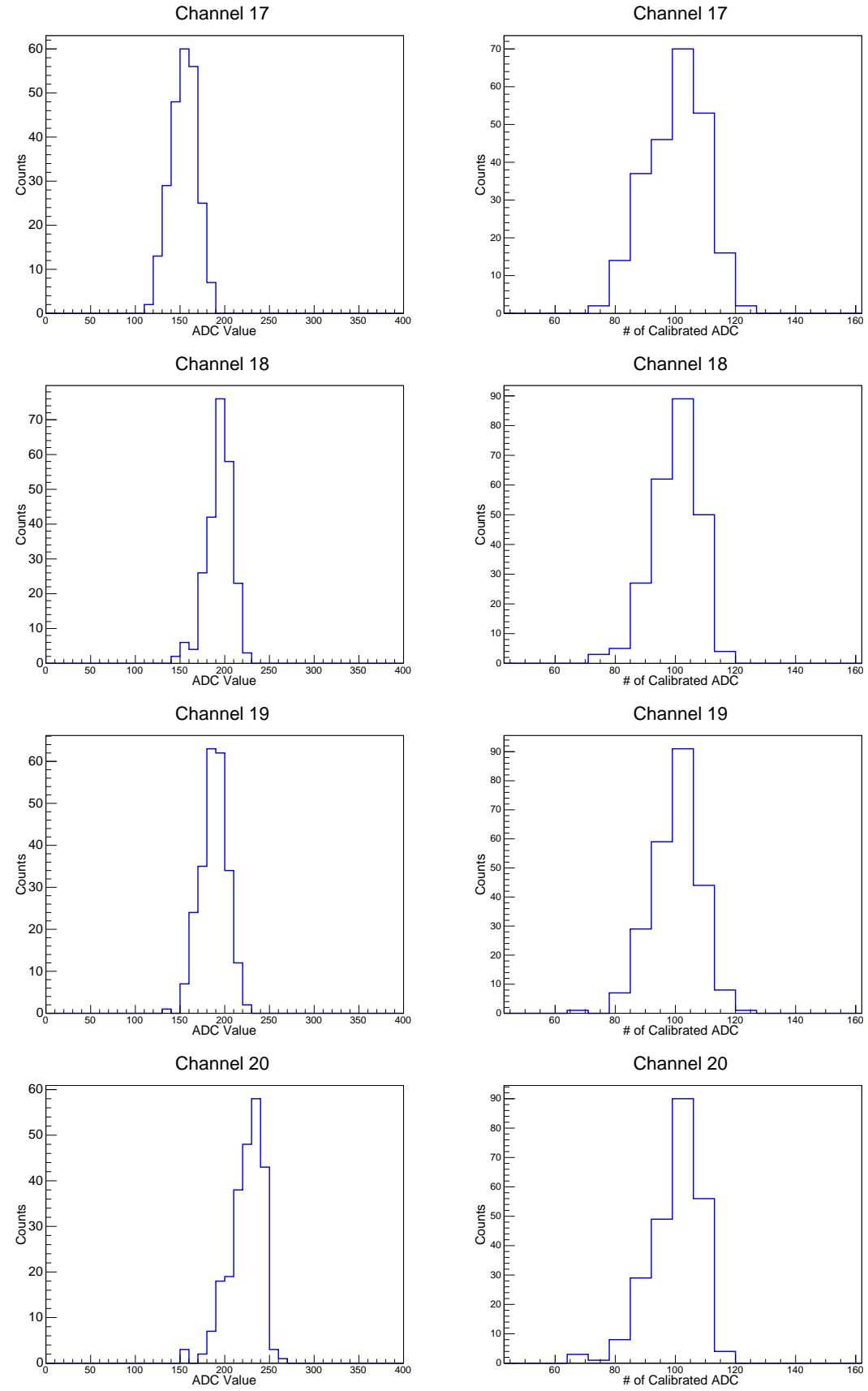

Figure A.11: Raw ADC vs Calibrated ADC response for channel 17, 18,19 and 20. 

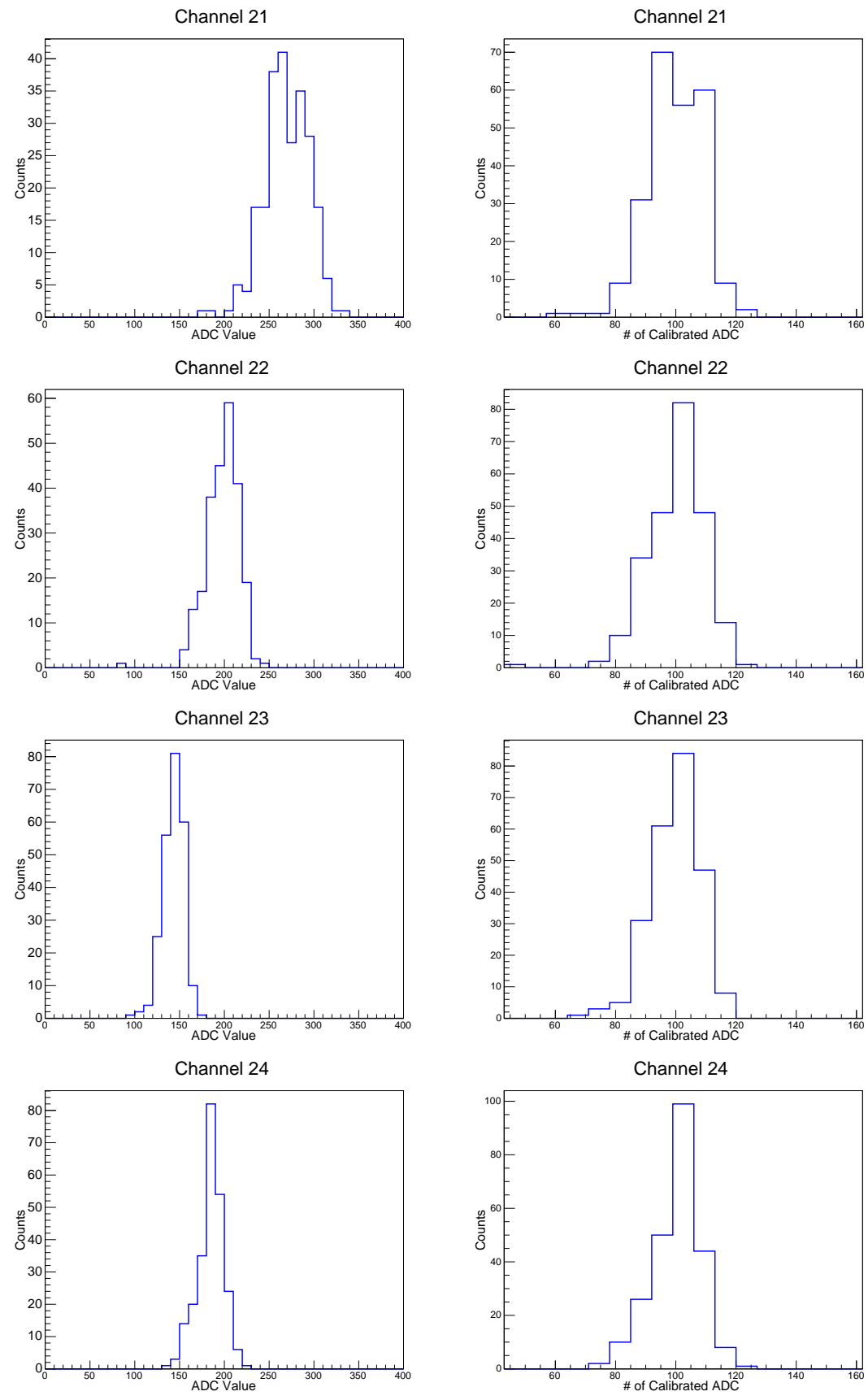

Figure A.12: Raw ADC vs Calibrated ADC response for channel 21, 22, 23 and 24. 

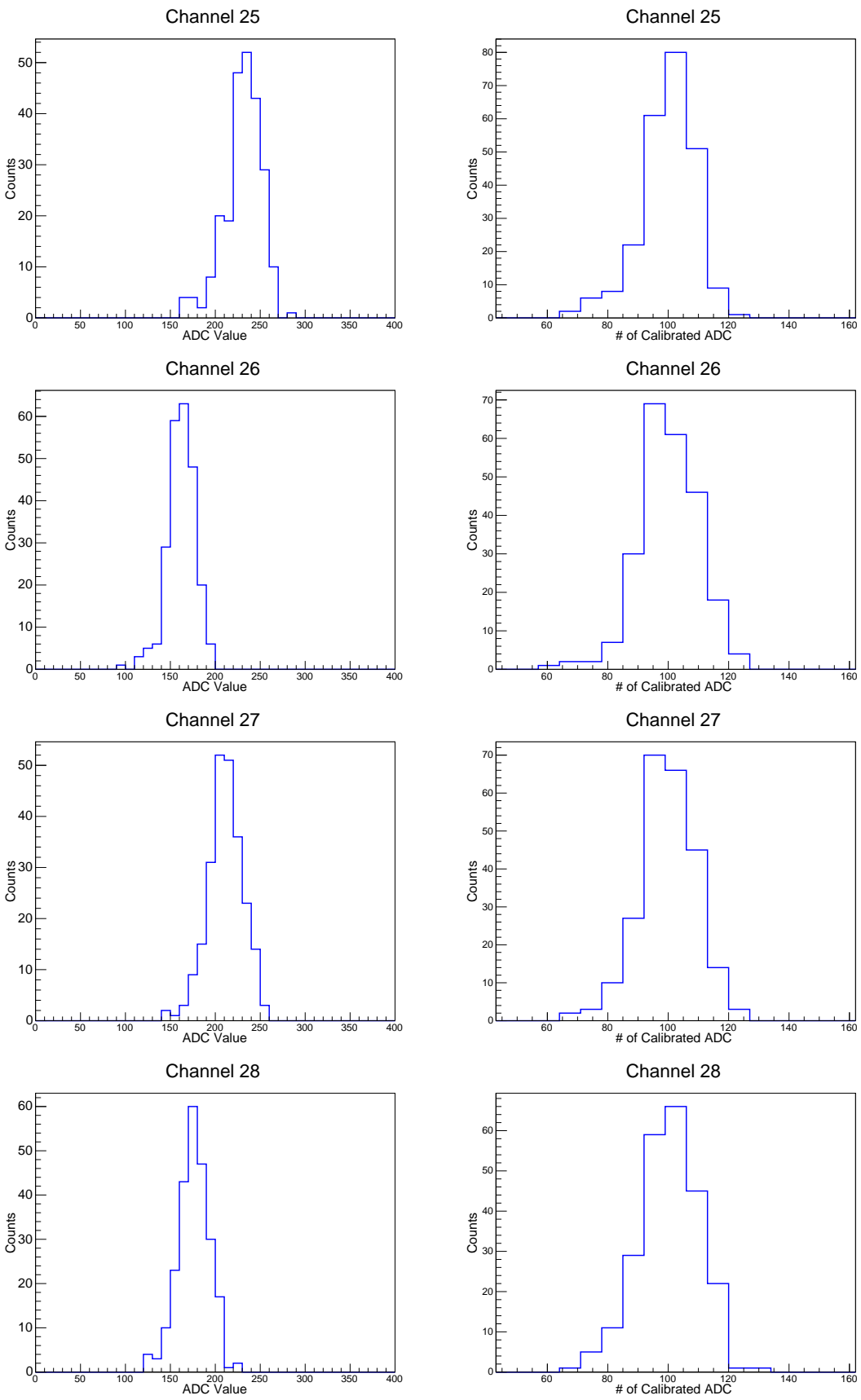

Figure A.13: Raw ADC vs Calibrated ADC response for channel 25, 26, 27 and 28. 

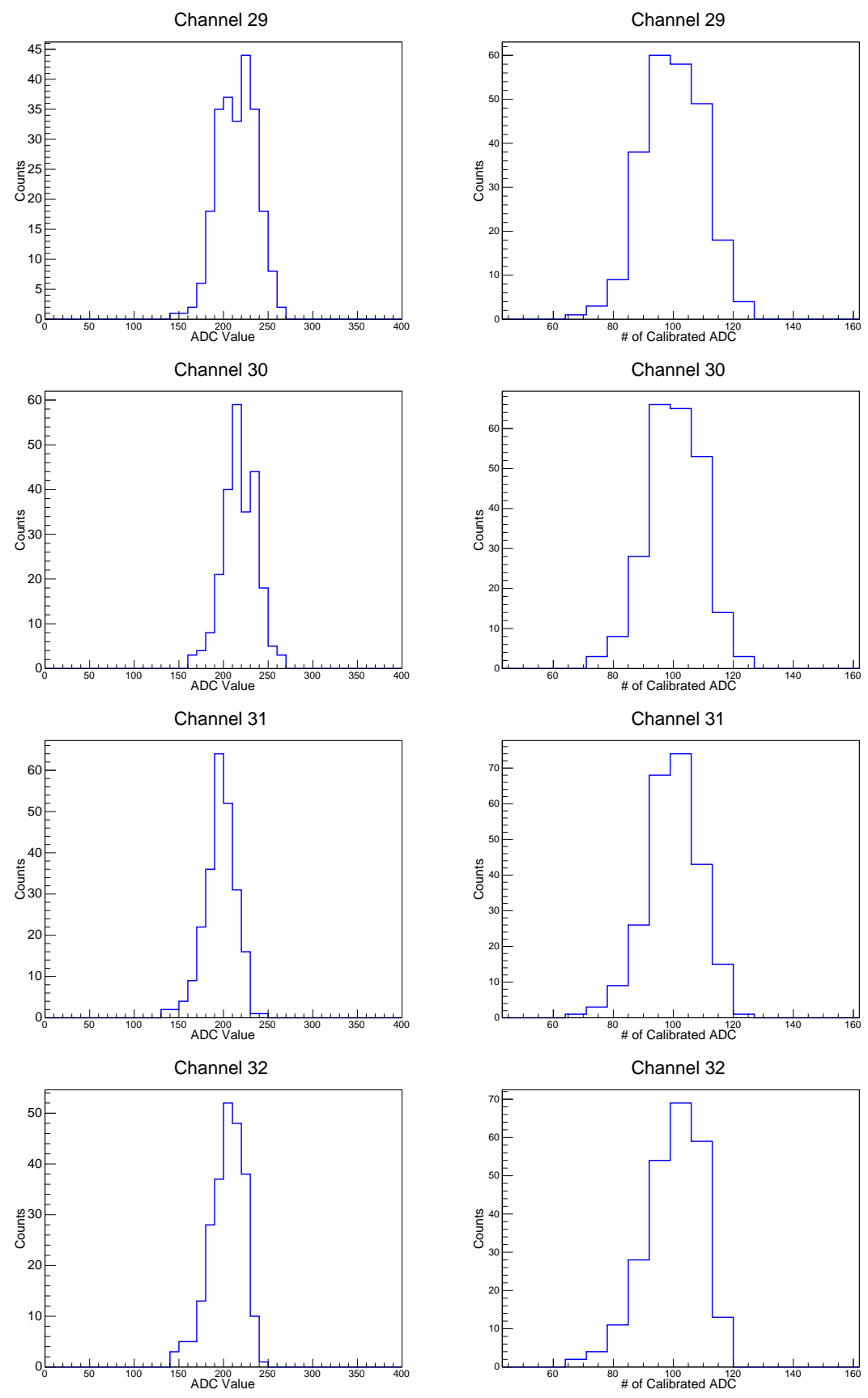

Figure A.14: Raw ADC vs Calibrated ADC response for channel 29, 30, 31 and 32. 


\section{Appendix B}

\section{Supplementary material for Beam Transport Systematics}

\section{B.1 Summary Tables}

This section shows results of the full beam transport systematics studies (full statistics) in tabular form. The following results are shown:

- Number of $\nu_{\mu}$ and $\nu_{e}$ from parents $\left(\pi^{ \pm}, K^{ \pm}\right.$, etc. $)$at the $\mathrm{NO} \nu \mathrm{A}$ detectors for two energy ranges, $0<E_{\nu}<10$ and $1<E_{\nu}<3 \mathrm{GeV}$

- Average $E_{\nu}$ and RMS for the energy ranges $0<E_{\nu}<10$ and $1<E_{\nu}<3 \mathrm{GeV}$. This gives the variation in the overall energy-scale due to the transport uncertainties considered. 


\begin{tabular}{cccccc}
\hline Shift/Parent & $\pi^{+}$ & $K^{+}$ & $\mu^{-}$ & Rest & Total \\
\hline Std(fluka2011.2b.6) & $1.174 \mathrm{E}-08$ & $3.683 \mathrm{E}-10$ & $2.450 \mathrm{E}-12$ & $1.244 \mathrm{E}-11$ & $1.213 \mathrm{E}-08$ \\
$+1 \mathrm{kA}$ & $1.173 \mathrm{E}-08$ & $3.687 \mathrm{E}-10$ & $2.393 \mathrm{E}-12$ & $1.249 \mathrm{E}-11$ & $1.211 \mathrm{E}-08$ \\
$-1 \mathrm{kA}$ & $1.175 \mathrm{E}-08$ & $3.680 \mathrm{E}-10$ & $2.327 \mathrm{E}-12$ & $1.249 \mathrm{E}-11$ & $1.214 \mathrm{E}-08$ \\
BposX+.5mm & $1.166 \mathrm{E}-08$ & $3.684 \mathrm{E}-10$ & $2.297 \mathrm{E}-12$ & $1.250 \mathrm{E}-11$ & $1.204 \mathrm{E}-08$ \\
BposX-.5mm & $1.180 \mathrm{E}-08$ & $3.681 \mathrm{E}-10$ & $2.435 \mathrm{E}-12$ & $1.249 \mathrm{E}-11$ & $1.218 \mathrm{E}-08$ \\
BposY+.5mm & $1.177 \mathrm{E}-08$ & $3.684 \mathrm{E}-10$ & $2.455 \mathrm{E}-12$ & $1.253 \mathrm{E}-11$ & $1.215 \mathrm{E}-08$ \\
BposY-.5mm & $1.169 \mathrm{E}-08$ & $3.681 \mathrm{E}-10$ & $2.381 \mathrm{E}-12$ & $1.238 \mathrm{E}-11$ & $1.208 \mathrm{E}-08$ \\
H1 +2mm X \& Y & $1.178 \mathrm{E}-08$ & $3.662 \mathrm{E}-10$ & $2.380 \mathrm{E}-12$ & $1.244 \mathrm{E}-11$ & $1.216 \mathrm{E}-08$ \\
H1 -2mm X \& Y & $1.150 \mathrm{E}-08$ & $3.693 \mathrm{E}-10$ & $2.317 \mathrm{E}-12$ & $1.246 \mathrm{E}-11$ & $1.188 \mathrm{E}-08$ \\
H2 +2mm in X \& Y & $1.179 \mathrm{E}-08$ & $3.680 \mathrm{E}-10$ & $2.369 \mathrm{E}-12$ & $1.247 \mathrm{E}-11$ & $1.218 \mathrm{E}-08$ \\
H2 -2mm in X \& Y & $1.168 \mathrm{E}-08$ & $3.685 \mathrm{E}-10$ & $2.453 \mathrm{E}-12$ & $1.244 \mathrm{E}-11$ & $1.206 \mathrm{E}-08$ \\
Exp B field & $1.174 \mathrm{E}-08$ & $3.683 \mathrm{E}-10$ & $2.379 \mathrm{E}-12$ & $1.242 \mathrm{E}-11$ & $1.213 \mathrm{E}-08$ \\
BmSptSz .9mm & $1.177 \mathrm{E}-08$ & $3.683 \mathrm{E}-10$ & $2.343 \mathrm{E}-12$ & $1.235 \mathrm{E}-11$ & $1.215 \mathrm{E}-08$ \\
\hline \hline fluka2011.2c.3 & $1.164 \mathrm{E}-08$ & $3.666 \mathrm{E}-10$ & $2.459 \mathrm{E}-12$ & $1.250 \mathrm{E}-11$ & $1.202 \mathrm{E}-08$ \\
BMSptSz 1.5mm & $1.143 \mathrm{E}-08$ & $3.655 \mathrm{E}-10$ & $2.549 \mathrm{E}-12$ & $1.264 \mathrm{E}-11$ & $1.181 \mathrm{E}-08$ \\
TarPosZ +7mm & $1.167 \mathrm{E}-08$ & $3.672 \mathrm{E}-10$ & $2.475 \mathrm{E}-12$ & $1.247 \mathrm{E}-11$ & $1.205 \mathrm{E}-08$ \\
TarPosZ -7mm & $1.160 \mathrm{E}-08$ & $3.661 \mathrm{E}-10$ & $2.400 \mathrm{E}-12$ & $1.247 \mathrm{E}-11$ & $1.198 \mathrm{E}-08$ \\
NewHorn ImmWL & $1.120 \mathrm{E}-08$ & $3.623 \mathrm{E}-10$ & $2.385 \mathrm{E}-12$ & $1.229 \mathrm{E}-11$ & $1.157 \mathrm{E}-08$ \\
G4NuMI(FTFPv6) & $1.181 \mathrm{E}-08$ & $3.501 \mathrm{E}-10$ & $2.898 \mathrm{E}-12$ & $1.224 \mathrm{E}-11$ & $1.218 \mathrm{E}-08$ \\
\hline
\end{tabular}

Table B.1: Number of $\nu_{\mu}$ at NOvA ND separated by parent types in the 0-10 GeV range.

\begin{tabular}{cccccc}
\hline Shift/Parent & $\pi^{+}$ & $K^{+}$ & $\mu^{-}$ & Rest & Total \\
\hline Std(fluka2011.2b.6) & $9.610 \mathrm{E}-09$ & $4.344 \mathrm{E}-11$ & $1.114 \mathrm{E}-12$ & $3.754 \mathrm{E}-12$ & $9.659 \mathrm{E}-09$ \\
$+1 \mathrm{kA}$ & $9.589 \mathrm{E}-09$ & $4.344 \mathrm{E}-11$ & $1.061 \mathrm{E}-12$ & $3.764 \mathrm{E}-12$ & $9.637 \mathrm{E}-09$ \\
$-1 \mathrm{kA}$ & $9.626 \mathrm{E}-09$ & $4.339 \mathrm{E}-11$ & $1.071 \mathrm{E}-12$ & $3.760 \mathrm{E}-12$ & $9.674 \mathrm{E}-09$ \\
BposX+.5mm & $9.545 \mathrm{E}-09$ & $4.354 \mathrm{E}-11$ & $1.023 \mathrm{E}-12$ & $3.774 \mathrm{E}-12$ & $9.593 \mathrm{E}-09$ \\
BposX-.5mm & $9.637 \mathrm{E}-09$ & $4.349 \mathrm{E}-11$ & $1.143 \mathrm{E}-12$ & $3.775 \mathrm{E}-12$ & $9.685 \mathrm{E}-09$ \\
BposY+.5mm & $9.623 \mathrm{E}-09$ & $4.352 \mathrm{E}-11$ & $1.131 \mathrm{E}-12$ & $3.770 \mathrm{E}-12$ & $9.671 \mathrm{E}-09$ \\
BposY-.5mm & $9.575 \mathrm{E}-09$ & $4.332 \mathrm{E}-11$ & $1.099 \mathrm{E}-12$ & $3.728 \mathrm{E}-12$ & $9.624 \mathrm{E}-09$ \\
H1 +2mm X \& Y & $9.573 \mathrm{E}-09$ & $4.338 \mathrm{E}-11$ & $1.089 \mathrm{E}-12$ & $3.751 \mathrm{E}-12$ & $9.622 \mathrm{E}-09$ \\
H1 -2mm X \& Y & $9.446 \mathrm{E}-09$ & $4.350 \mathrm{E}-11$ & $1.074 \mathrm{E}-12$ & $3.746 \mathrm{E}-12$ & $9.495 \mathrm{E}-09$ \\
H2 +2mm in X \& Y & $9.646 \mathrm{E}-09$ & $4.338 \mathrm{E}-11$ & $1.118 \mathrm{E}-12$ & $3.739 \mathrm{E}-12$ & $9.694 \mathrm{E}-09$ \\
H2 -2mm in X \& Y & $9.560 \mathrm{E}-09$ & $4.340 \mathrm{E}-11$ & $1.167 \mathrm{E}-12$ & $3.763 \mathrm{E}-12$ & $9.609 \mathrm{E}-09$ \\
Exp B field & $9.612 \mathrm{E}-09$ & $4.338 \mathrm{E}-11$ & $1.092 \mathrm{E}-12$ & $3.759 \mathrm{E}-12$ & $9.661 \mathrm{E}-09$ \\
BmSptSz .9mm & $9.639 \mathrm{E}-09$ & $4.329 \mathrm{E}-11$ & $1.105 \mathrm{E}-12$ & $3.731 \mathrm{E}-12$ & $9.687 \mathrm{E}-09$ \\
\hline \hline fluka2011.2c.3 & $9.529 \mathrm{E}-09$ & $4.304 \mathrm{E}-11$ & $1.106 \mathrm{E}-12$ & $3.766 \mathrm{E}-12$ & $9.577 \mathrm{E}-09$ \\
BMSptSz 1.5mm & $9.311 \mathrm{E}-09$ & $4.386 \mathrm{E}-11$ & $1.161 \mathrm{E}-12$ & $3.844 \mathrm{E}-12$ & $9.360 \mathrm{E}-09$ \\
TarPosZ +7mm & $9.559 \mathrm{E}-09$ & $4.313 \mathrm{E}-11$ & $1.141 \mathrm{E}-12$ & $3.777 \mathrm{E}-12$ & $9.607 \mathrm{E}-09$ \\
TarPosZ -7mm & $9.497 \mathrm{E}-09$ & $4.303 \mathrm{E}-11$ & $1.084 \mathrm{E}-12$ & $3.754 \mathrm{E}-12$ & $9.545 \mathrm{E}-09$ \\
NewHorn ImmWL & $9.056 \mathrm{E}-09$ & $4.481 \mathrm{E}-11$ & $1.058 \mathrm{E}-12$ & $3.659 \mathrm{E}-12$ & $9.105 \mathrm{E}-09$ \\
G4NuMI(FTFPv6) & $9.662 \mathrm{E}-09$ & $4.298 \mathrm{E}-11$ & $1.198 \mathrm{E}-12$ & $3.781 \mathrm{E}-12$ & $9.710 \mathrm{E}-09$ \\
\hline
\end{tabular}

Table B.2: Number of $\nu_{\mu}$ at NOvA ND separated by parent types in the 1-3 GeV range. 


\begin{tabular}{cccc}
\hline Shift/Parent & $\delta(\%)$ & RMS $(\mathrm{GeV})$ & Mean \\
\hline Std(fluka2011.2b.6) & 0.000 & 1.269 & 2.043 \\
$+1 \mathrm{kA}$ & 0.137 & 1.271 & 2.044 \\
$-1 \mathrm{kA}$ & -0.094 & 1.268 & 2.042 \\
BposX+.5mm & 0.709 & 1.270 & 2.035 \\
BposX-.5mm & -0.446 & 1.270 & 2.050 \\
BposY+.5mm & -0.223 & 1.270 & 2.044 \\
BposY-.5mm & 0.412 & 1.268 & 2.041 \\
H1 +2mm X \& Y & -0.295 & 1.282 & 2.062 \\
H1 -2mm X \& Y & 1.998 & 1.264 & 2.028 \\
H2 +2mm in X \& Y & -0.429 & 1.268 & 2.048 \\
H2 -2mm in X \& Y & 0.519 & 1.271 & 2.038 \\
Exp B field & -0.006 & 1.269 & 2.042 \\
BmSptSz .9mm & -0.204 & 1.267 & 2.043 \\
\hline \hline fluka2011.2c.3 & 0.884 & 1.270 & 2.045 \\
BMSptSz 1.5mm & 1.723 & 1.277 & 2.040 \\
TarPosZ +7mm & -0.258 & 1.270 & 2.044 \\
TarPosZ -7mm & 0.324 & 1.271 & 2.045 \\
NewHorn ImmWL & 3.739 & 1.283 & 2.034 \\
G4NuMI(FTFPv6) & -1.304 & 1.259 & 2.039 \\
\hline
\end{tabular}

Table B.3: The variation $\delta(\%) \nu_{e}$ and Energy (Mean and RMS) at $\mathrm{NO} \nu \mathrm{A} F \mathrm{~F}$ in the $0-10 \mathrm{GeV}$ range.

\begin{tabular}{cccc}
\hline Shift/Parent & $\delta(\%)$ & RMS $(\mathrm{GeV})$ & Mean \\
\hline Std(fluka2011.2b.6) & 0.000 & 0.457 & 1.920 \\
$+1 \mathrm{kA}$ & 0.219 & 0.457 & 1.920 \\
$-1 \mathrm{kA}$ & -0.164 & 0.457 & 1.919 \\
BposX+.5mm & 0.678 & 0.455 & 1.915 \\
BposX-.5mm & -0.273 & 0.459 & 1.924 \\
BposY+.5mm & -0.132 & 0.457 & 1.920 \\
BposY-.5mm & 0.362 & 0.456 & 1.919 \\
H1 +2mm X \& Y & 0.383 & 0.459 & 1.923 \\
H1 -2mm X \&Y & 1.696 & 0.457 & 1.916 \\
H2 +2mm in X \&Y & -0.370 & 0.458 & 1.924 \\
H2 -2mm in X \& Y & 0.516 & 0.456 & 1.915 \\
Exp B field & -0.021 & 0.457 & 1.919 \\
BmSptSz .9mm & -0.299 & 0.457 & 1.920 \\
\hline \hline fluka2011.2c.3 & 0.848 & 0.457 & 1.920 \\
BMSptSz 1.5mm & 2.268 & 0.457 & 1.919 \\
TarPosZ +7mm & -0.294 & 0.457 & 1.919 \\
TarPosZ -7mm & 0.356 & 0.457 & 1.920 \\
NewHorn ImmWL & 4.947 & 0.458 & 1.917 \\
G4NuMI(FTFPv6) & -1.368 & 0.455 & 1.908 \\
\hline
\end{tabular}

Table B.4: The variation $\delta(\%) \nu_{e}$ and Energy (Mean and RMS) at $\mathrm{NO} \nu \mathrm{A}$ FD in the $1-3 \mathrm{GeV}$ range.

\begin{tabular}{cccccc}
\hline Shift/Parent & $\pi^{+}$ & $K^{+}$ & $\mu^{-}$ & Rest & Total \\
\hline Std(fluka2011.2b.6) & $1.320 \mathrm{E}-14$ & $4.292 \mathrm{E}-16$ & $2.090 \mathrm{E}-18$ & $1.405 \mathrm{E}-17$ & $1.364 \mathrm{E}-14$ \\
$+1 \mathrm{kA}$ & $1.318 \mathrm{E}-14$ & $4.295 \mathrm{E}-16$ & $2.050 \mathrm{E}-18$ & $1.410 \mathrm{E}-17$ & $1.363 \mathrm{E}-14$ \\
-1kA & $1.321 \mathrm{E}-14$ & $4.289 \mathrm{E}-16$ & $2.030 \mathrm{E}-18$ & $1.409 \mathrm{E}-17$ & $1.365 \mathrm{E}-14$ \\
BposX+.5mm & $1.310 \mathrm{E}-14$ & $4.287 \mathrm{E}-16$ & $2.030 \mathrm{E}-18$ & $1.409 \mathrm{E}-17$ & $1.355 \mathrm{E}-14$ \\
BposX-.5mm & $1.324 \mathrm{E}-14$ & $4.281 \mathrm{E}-16$ & $2.080 \mathrm{E}-18$ & $1.400 \mathrm{E}-17$ & $1.368 \mathrm{E}-14$ \\
BposY+.5mm & $1.323 \mathrm{E}-14$ & $4.284 \mathrm{E}-16$ & $2.140 \mathrm{E}-18$ & $1.411 \mathrm{E}-17$ & $1.367 \mathrm{E}-14$ \\
BposY-.5mm & $1.314 \mathrm{E}-14$ & $4.295 \mathrm{E}-16$ & $2.070 \mathrm{E}-18$ & $1.401 \mathrm{E}-17$ & $1.358 \mathrm{E}-14$ \\
H1 +2mm X \& Y & $1.319 \mathrm{E}-14$ & $4.261 \mathrm{E}-16$ & $2.060 \mathrm{E}-18$ & $1.405 \mathrm{E}-17$ & $1.363 \mathrm{E}-14$ \\
H1 -2mm X \& Y & $1.295 \mathrm{E}-14$ & $4.295 \mathrm{E}-16$ & $2.040 \mathrm{E}-18$ & $1.405 \mathrm{E}-17$ & $1.340 \mathrm{E}-14$ \\
H2 +2mm in X \& Y & $1.324 \mathrm{E}-14$ & $4.291 \mathrm{E}-16$ & $2.050 \mathrm{E}-18$ & $1.409 \mathrm{E}-17$ & $1.368 \mathrm{E}-14$ \\
H2 -2mm in X \& Y & $1.314 \mathrm{E}-14$ & $4.295 \mathrm{E}-16$ & $2.110 \mathrm{E}-18$ & $1.404 \mathrm{E}-17$ & $1.359 \mathrm{E}-14$ \\
Exp B field & $1.320 \mathrm{E}-14$ & $4.293 \mathrm{E}-16$ & $2.070 \mathrm{E}-18$ & $1.402 \mathrm{E}-17$ & $1.364 \mathrm{E}-14$ \\
BmSptSz .9mm & $1.323 \mathrm{E}-14$ & $4.305 \mathrm{E}-16$ & $2.020 \mathrm{E}-18$ & $1.399 \mathrm{E}-17$ & $1.368 \mathrm{E}-14$ \\
\hline \hline fluka2011.2c.3 & $1.308 \mathrm{E}-14$ & $4.275 \mathrm{E}-16$ & $2.130 \mathrm{E}-18$ & $1.410 \mathrm{E}-17$ & $1.352 \mathrm{E}-14$ \\
BMSptSz 1.5mm & $1.279 \mathrm{E}-14$ & $4.186 \mathrm{E}-16$ & $2.230 \mathrm{E}-18$ & $1.400 \mathrm{E}-17$ & $1.323 \mathrm{E}-14$ \\
TarPosZ +7mm & $1.312 \mathrm{E}-14$ & $4.283 \mathrm{E}-16$ & $2.140 \mathrm{E}-18$ & $1.406 \mathrm{E}-17$ & $1.356 \mathrm{E}-14$ \\
TarPosZ -7mm & $1.304 \mathrm{E}-14$ & $4.267 \mathrm{E}-16$ & $2.100 \mathrm{E}-18$ & $1.405 \mathrm{E}-17$ & $1.348 \mathrm{E}-14$ \\
NewHorn ImmWL & $1.262 \mathrm{E}-14$ & $4.232 \mathrm{E}-16$ & $2.040 \mathrm{E}-18$ & $1.383 \mathrm{E}-17$ & $1.306 \mathrm{E}-14$ \\
G4NuMI(FTFPv6) & $1.341 \mathrm{E}-14$ & $4.120 \mathrm{E}-16$ & $2.530 \mathrm{E}-18$ & $1.393 \mathrm{E}-17$ & $1.384 \mathrm{E}-14$ \\
\hline
\end{tabular}

Table B.5: Number of $\nu_{\mu}$ at NOvA FD separated by parent types in the 0-10 GeV range. 


\begin{tabular}{cccccc}
\hline Shift/Parent & $\pi^{+}$ & $K^{+}$ & $\mu^{-}$ & Rest & Total \\
\hline Std(fluka2011.2b.6) & $1.171 \mathrm{E}-14$ & $4.760 \mathrm{E}-17$ & $1.020 \mathrm{E}-18$ & $4.030 \mathrm{E}-18$ & $1.176 \mathrm{E}-14$ \\
$+1 \mathrm{kA}$ & $1.169 \mathrm{E}-14$ & $4.759 \mathrm{E}-17$ & $9.900 \mathrm{E}-19$ & $4.030 \mathrm{E}-18$ & $1.174 \mathrm{E}-14$ \\
$-1 \mathrm{kA}$ & $1.172 \mathrm{E}-14$ & $4.755 \mathrm{E}-17$ & $9.700 \mathrm{E}-19$ & $4.030 \mathrm{E}-18$ & $1.177 \mathrm{E}-14$ \\
BposX+.5mm & $1.163 \mathrm{E}-14$ & $4.753 \mathrm{E}-17$ & $9.500 \mathrm{E}-19$ & $4.030 \mathrm{E}-18$ & $1.168 \mathrm{E}-14$ \\
BposX-.5mm & $1.174 \mathrm{E}-14$ & $4.748 \mathrm{E}-17$ & $1.010 \mathrm{E}-18$ & $4.020 \mathrm{E}-18$ & $1.179 \mathrm{E}-14$ \\
BposY+.5mm & $1.173 \mathrm{E}-14$ & $4.749 \mathrm{E}-17$ & $1.020 \mathrm{E}-18$ & $4.030 \mathrm{E}-18$ & $1.178 \mathrm{E}-14$ \\
BposY-.5mm & $1.165 \mathrm{E}-14$ & $4.762 \mathrm{E}-17$ & $1.020 \mathrm{E}-18$ & $4.020 \mathrm{E}-18$ & $1.171 \mathrm{E}-14$ \\
H1 +2mm X \& Y & $1.166 \mathrm{E}-14$ & $4.747 \mathrm{E}-17$ & $9.900 \mathrm{E}-19$ & $4.020 \mathrm{E}-18$ & $1.171 \mathrm{E}-14$ \\
H1 -2mm X \& Y & $1.150 \mathrm{E}-14$ & $4.760 \mathrm{E}-17$ & $9.900 \mathrm{E}-19$ & $4.010 \mathrm{E}-18$ & $1.155 \mathrm{E}-14$ \\
H2 +2mm in X \& Y & $1.174 \mathrm{E}-14$ & $4.756 \mathrm{E}-17$ & $9.800 \mathrm{E}-19$ & $4.020 \mathrm{E}-18$ & $1.180 \mathrm{E}-14$ \\
H2 -2mm in X \& Y & $1.165 \mathrm{E}-14$ & $4.760 \mathrm{E}-17$ & $1.010 \mathrm{E}-18$ & $4.030 \mathrm{E}-18$ & $1.170 \mathrm{E}-14$ \\
Exp B field & $1.171 \mathrm{E}-14$ & $4.757 \mathrm{E}-17$ & $1.000 \mathrm{E}-18$ & $4.040 \mathrm{E}-18$ & $1.176 \mathrm{E}-14$ \\
BmSptSz .9mm & $1.174 \mathrm{E}-14$ & $4.769 \mathrm{E}-17$ & $9.700 \mathrm{E}-19$ & $4.030 \mathrm{E}-18$ & $1.179 \mathrm{E}-14$ \\
\hline \hline fluka2011.2c.3 & $1.161 \mathrm{E}-14$ & $4.724 \mathrm{E}-17$ & $1.010 \mathrm{E}-18$ & $4.040 \mathrm{E}-18$ & $1.166 \mathrm{E}-14$ \\
BMSptSz 1.5mm & $1.133 \mathrm{E}-14$ & $4.650 \mathrm{E}-17$ & $1.100 \mathrm{E}-18$ & $4.000 \mathrm{E}-18$ & $1.139 \mathrm{E}-14$ \\
TarPosZ +7mm & $1.164 \mathrm{E}-14$ & $4.733 \mathrm{E}-17$ & $1.030 \mathrm{E}-18$ & $4.050 \mathrm{E}-18$ & $1.169 \mathrm{E}-14$ \\
TarPosZ -7mm & $1.157 \mathrm{E}-14$ & $4.720 \mathrm{E}-17$ & $1.040 \mathrm{E}-18$ & $4.030 \mathrm{E}-18$ & $1.162 \mathrm{E}-14$ \\
NewHorn ImmWL & $1.105 \mathrm{E}-14$ & $4.999 \mathrm{E}-17$ & $9.900 \mathrm{E}-19$ & $3.900 \mathrm{E}-18$ & $1.110 \mathrm{E}-14$ \\
G4NuMI(FTFPv6) & $1.177 \mathrm{E}-14$ & $4.762 \mathrm{E}-17$ & $1.140 \mathrm{E}-18$ & $4.100 \mathrm{E}-18$ & $1.182 \mathrm{E}-14$ \\
\hline
\end{tabular}

Table B.6: Number of $\nu_{\mu}$ at NOvA FD separated by parent types in the 1-3 GeV range.

\begin{tabular}{cccc}
\hline Shift/Parent & $\delta(\%)$ & RMS $(\mathrm{GeV})$ & Mean \\
\hline Std(fluka2011.2b.6) & 0.000 & 1.174 & 2.095 \\
+1kA & 0.117 & 1.176 & 2.096 \\
-1kA & -0.074 & 1.174 & 2.095 \\
BposX+.5mm & 0.676 & 1.176 & 2.090 \\
BposX-.5mm & -0.296 & 1.175 & 2.101 \\
BposY+.5mm & -0.210 & 1.175 & 2.097 \\
BposY-.5mm & 0.432 & 1.175 & 2.094 \\
H1 +2mm X \& Y & 0.060 & 1.180 & 2.104 \\
H1 -2mm X \& Y & 1.796 & 1.177 & 2.087 \\
H2 +2mm in X \& Y & -0.294 & 1.173 & 2.098 \\
H2 -2mm in X \& Y & 0.416 & 1.177 & 2.092 \\
Exp B field & -0.002 & 1.174 & 2.095 \\
BmSptSz .9mm & -0.261 & 1.173 & 2.095 \\
\hline \hline fluka2011.2c.3 & 0.870 & 1.175 & 2.097 \\
BMSptSz 1.5mm & 2.170 & 1.180 & 2.098 \\
TarPosZ +7mm & -0.262 & 1.175 & 2.096 \\
TarPosZ -7mm & 0.318 & 1.176 & 2.097 \\
NewHorn ImmWL & 3.457 & 1.192 & 2.081 \\
G4NuMI(FTFPv6) & -2.306 & 1.170 & 2.081 \\
\hline
\end{tabular}

Table B.7: The variation $\delta(\%) \nu_{e}$ and Energy (Mean and RMS) at $\mathrm{NO} \nu \mathrm{A}$ FD in the $0-10 \mathrm{GeV}$ range.

\begin{tabular}{cccc}
\hline Std(fluka2011.2b.6) & 0.000 & 0.366 & 1.959 \\
$+1 \mathrm{kA}$ & 0.168 & 0.367 & 1.960 \\
$-1 \mathrm{kA}$ & -0.093 & 0.366 & 1.958 \\
BposX+.5mm & 0.683 & 0.365 & 1.954 \\
BposX-.5mm & -0.235 & 0.368 & 1.964 \\
BposY+.5mm & -0.175 & 0.367 & 1.960 \\
BposY-.5mm & 0.450 & 0.366 & 1.958 \\
H1 +2mm X \& Y & 0.393 & 0.369 & 1.962 \\
H1 -2mm X \& Y & 1.786 & 0.369 & 1.954 \\
H2 +2mm in X \& Y & -0.299 & 0.367 & 1.963 \\
H2 -2mm in X \& Y & 0.472 & 0.366 & 1.955 \\
Exp B field & 0.016 & 0.366 & 1.959 \\
BmSptSz .9mm & -0.285 & 0.366 & 1.959 \\
\hline \hline fluka2011.2c.3 & 0.847 & 0.366 & 1.959 \\
BMSptSz 1.5mm & 2.360 & 0.367 & 1.959 \\
TarPosZ +7mm & -0.266 & 0.366 & 1.959 \\
TarPosZ -7mm & 0.339 & 0.367 & 1.959 \\
NewHorn ImmWL & 4.825 & 0.370 & 1.956 \\
G4NuMI(FTFPv6) & -1.368 & 0.363 & 1.953 \\
\hline
\end{tabular}

Table B.8: The variation $\delta(\%) \nu_{e}$ and Energy (Mean and RMS) at $\mathrm{NO} \nu \mathrm{A} F \mathrm{~F}$ in the $1-3 \mathrm{GeV}$ range. 


\begin{tabular}{cccccc}
\hline Shift/Parent & $\mu^{+}$ & $K^{+}$ & $K_{L}$ & Rest & Total \\
\hline Std(fluka2011.2b.6) & $7.413 \mathrm{E}-11$ & $5.391 \mathrm{E}-11$ & $1.695 \mathrm{E}-11$ & $1.450 \mathrm{E}-12$ & $1.464 \mathrm{E}-10$ \\
$+1 \mathrm{kA}$ & $7.350 \mathrm{E}-11$ & $5.412 \mathrm{E}-11$ & $1.695 \mathrm{E}-11$ & $1.394 \mathrm{E}-12$ & $1.460 \mathrm{E}-10$ \\
$-1 \mathrm{kA}$ & $7.453 \mathrm{E}-11$ & $5.372 \mathrm{E}-11$ & $1.694 \mathrm{E}-11$ & $1.391 \mathrm{E}-12$ & $1.466 \mathrm{E}-10$ \\
BposX+.5mm & $7.365 \mathrm{E}-11$ & $5.377 \mathrm{E}-11$ & $1.704 \mathrm{E}-11$ & $1.397 \mathrm{E}-12$ & $1.459 \mathrm{E}-10$ \\
BposX-.5mm & $7.386 \mathrm{E}-11$ & $5.388 \mathrm{E}-11$ & $1.703 \mathrm{E}-11$ & $1.386 \mathrm{E}-12$ & $1.462 \mathrm{E}-10$ \\
BposY+.5mm & $7.379 \mathrm{E}-11$ & $5.411 \mathrm{E}-11$ & $1.706 \mathrm{E}-11$ & $1.425 \mathrm{E}-12$ & $1.464 \mathrm{E}-10$ \\
BposY-.5mm & $7.363 \mathrm{E}-11$ & $5.366 \mathrm{E}-11$ & $1.687 \mathrm{E}-11$ & $1.406 \mathrm{E}-12$ & $1.456 \mathrm{E}-10$ \\
H1 +2mm X \& Y & $7.337 \mathrm{E}-11$ & $5.376 \mathrm{E}-11$ & $1.698 \mathrm{E}-11$ & $1.447 \mathrm{E}-12$ & $1.456 \mathrm{E}-10$ \\
H1 -2mm X \& Y & $7.305 \mathrm{E}-11$ & $5.386 \mathrm{E}-11$ & $1.694 \mathrm{E}-11$ & $1.406 \mathrm{E}-12$ & $1.453 \mathrm{E}-10$ \\
H2 +2mm in X \& Y & $7.343 \mathrm{E}-11$ & $5.386 \mathrm{E}-11$ & $1.700 \mathrm{E}-11$ & $1.402 \mathrm{E}-12$ & $1.457 \mathrm{E}-10$ \\
H2 -2mm in X \& Y & $7.302 \mathrm{E}-11$ & $5.390 \mathrm{E}-11$ & $1.695 \mathrm{E}-11$ & $1.407 \mathrm{E}-12$ & $1.453 \mathrm{E}-10$ \\
Exp B field & $7.399 \mathrm{E}-11$ & $5.388 \mathrm{E}-11$ & $1.698 \mathrm{E}-11$ & $1.399 \mathrm{E}-12$ & $1.463 \mathrm{E}-10$ \\
BmSptSz .9mm & $7.406 \mathrm{E}-11$ & $5.384 \mathrm{E}-11$ & $1.683 \mathrm{E}-11$ & $1.434 \mathrm{E}-12$ & $1.462 \mathrm{E}-10$ \\
\hline \hline fluka2011.2c.3 & $7.219 \mathrm{E}-11$ & $5.366 \mathrm{E}-11$ & $1.703 \mathrm{E}-11$ & $1.434 \mathrm{E}-12$ & $1.443 \mathrm{E}-10$ \\
BMSptSz 1.5mm & $7.011 \mathrm{E}-11$ & $5.320 \mathrm{E}-11$ & $1.734 \mathrm{E}-11$ & $1.378 \mathrm{E}-12$ & $1.420 \mathrm{E}-10$ \\
TarPosZ +7mm & $7.199 \mathrm{E}-11$ & $5.364 \mathrm{E}-11$ & $1.701 \mathrm{E}-11$ & $1.431 \mathrm{E}-12$ & $1.441 \mathrm{E}-10$ \\
TarPosZ -7mm & $7.177 \mathrm{E}-11$ & $5.367 \mathrm{E}-11$ & $1.706 \mathrm{E}-11$ & $1.421 \mathrm{E}-12$ & $1.439 \mathrm{E}-10$ \\
NewHorn ImmWL & $6.901 \mathrm{E}-11$ & $5.239 \mathrm{E}-11$ & $1.669 \mathrm{E}-11$ & $1.325 \mathrm{E}-12$ & $1.394 \mathrm{E}-10$ \\
G4NuMI(FTFPv6) & $7.793 \mathrm{E}-11$ & $5.620 \mathrm{E}-11$ & $1.716 \mathrm{E}-11$ & $0.000 \mathrm{E}+00$ & $1.513 \mathrm{E}-10$ \\
\hline
\end{tabular}

Table B.9: Number of $\nu_{e}$ at NOvA ND separated by parent types in the $0-10 \mathrm{GeV}$ range.

\begin{tabular}{cccccc}
\hline Shift/Parent & $\mu^{+}$ & $K^{+}$ & $K_{L}$ & Rest & Total \\
\hline Std(fluka2011.2b.6) & $4.698 \mathrm{E}-11$ & $9.498 \mathrm{E}-12$ & $4.864 \mathrm{E}-12$ & $2.090 \mathrm{E}-13$ & $6.155 \mathrm{E}-11$ \\
$+1 \mathrm{kA}$ & $4.643 \mathrm{E}-11$ & $9.517 \mathrm{E}-12$ & $4.856 \mathrm{E}-12$ & $2.060 \mathrm{E}-13$ & $6.101 \mathrm{E}-11$ \\
$-1 \mathrm{kA}$ & $4.713 \mathrm{E}-11$ & $9.489 \mathrm{E}-12$ & $4.848 \mathrm{E}-12$ & $2.100 \mathrm{E}-13$ & $6.168 \mathrm{E}-11$ \\
BposX+.5mm & $4.660 \mathrm{E}-11$ & $9.489 \mathrm{E}-12$ & $4.850 \mathrm{E}-12$ & $2.120 \mathrm{E}-13$ & $6.115 \mathrm{E}-11$ \\
BposX-.5mm & $4.673 \mathrm{E}-11$ & $9.495 \mathrm{E}-12$ & $4.883 \mathrm{E}-12$ & $2.260 \mathrm{E}-13$ & $6.133 \mathrm{E}-11$ \\
BposY+.5mm & $4.647 \mathrm{E}-11$ & $9.527 \mathrm{E}-12$ & $4.863 \mathrm{E}-12$ & $2.020 \mathrm{E}-13$ & $6.106 \mathrm{E}-11$ \\
BposY-.5mm & $4.654 \mathrm{E}-11$ & $9.478 \mathrm{E}-12$ & $4.827 \mathrm{E}-12$ & $1.900 \mathrm{E}-13$ & $6.103 \mathrm{E}-11$ \\
H1 +2mm X \& Y & $4.659 \mathrm{E}-11$ & $9.441 \mathrm{E}-12$ & $4.851 \mathrm{E}-12$ & $1.960 \mathrm{E}-13$ & $6.107 \mathrm{E}-11$ \\
H1 -2mm X \& Y & $4.648 \mathrm{E}-11$ & $9.494 \mathrm{E}-12$ & $4.867 \mathrm{E}-12$ & $2.000 \mathrm{E}-13$ & $6.104 \mathrm{E}-11$ \\
H2 +2mm in X \& Y & $4.634 \mathrm{E}-11$ & $9.501 \mathrm{E}-12$ & $4.864 \mathrm{E}-12$ & $2.140 \mathrm{E}-13$ & $6.091 \mathrm{E}-11$ \\
H2 -2mm in X \& Y & $4.631 \mathrm{E}-11$ & $9.497 \mathrm{E}-12$ & $4.859 \mathrm{E}-12$ & $2.130 \mathrm{E}-13$ & $6.088 \mathrm{E}-11$ \\
Exp B field & $4.707 \mathrm{E}-11$ & $9.495 \mathrm{E}-12$ & $4.847 \mathrm{E}-12$ & $2.110 \mathrm{E}-13$ & $6.162 \mathrm{E}-11$ \\
BmSptSz .9mm & $4.709 \mathrm{E}-11$ & $9.486 \mathrm{E}-12$ & $4.819 \mathrm{E}-12$ & $1.890 \mathrm{E}-13$ & $6.158 \mathrm{E}-11$ \\
\hline \hline fluka2011.2c.3 & $4.696 \mathrm{E}-11$ & $9.452 \mathrm{E}-12$ & $4.859 \mathrm{E}-12$ & $2.110 \mathrm{E}-13$ & $6.148 \mathrm{E}-11$ \\
BMSptSz 1.5mm & $4.523 \mathrm{E}-11$ & $9.390 \mathrm{E}-12$ & $4.987 \mathrm{E}-12$ & $2.180 \mathrm{E}-13$ & $5.982 \mathrm{E}-11$ \\
TarPosZ +7mm & $4.664 \mathrm{E}-11$ & $9.468 \mathrm{E}-12$ & $4.864 \mathrm{E}-12$ & $2.160 \mathrm{E}-13$ & $6.119 \mathrm{E}-11$ \\
TarPosZ -7mm & $4.649 \mathrm{E}-11$ & $9.453 \mathrm{E}-12$ & $4.875 \mathrm{E}-12$ & $2.190 \mathrm{E}-13$ & $6.104 \mathrm{E}-11$ \\
NewHorn ImmWL & $4.482 \mathrm{E}-11$ & $9.410 \mathrm{E}-12$ & $4.741 \mathrm{E}-12$ & $2.020 \mathrm{E}-13$ & $5.917 \mathrm{E}-11$ \\
G4NuMI(FTFPv6) & $4.843 \mathrm{E}-11$ & $9.981 \mathrm{E}-12$ & $4.936 \mathrm{E}-12$ & $0.000 \mathrm{E}+00$ & $6.335 \mathrm{E}-11$ \\
\hline
\end{tabular}

Table B.10: Number of $\nu_{e}$ at NOvA ND separated by parent types in the 1-3 GeV range. 


\begin{tabular}{cccc}
\hline Shift/Parent & $\delta(\%)$ & RMS $(\mathrm{GeV})$ & Mean \\
\hline Std(fluka2011.2b.6) & 0.000 & 2.514 & 3.338 \\
+1kA & 0.323 & 2.516 & 3.351 \\
-1kA & -0.100 & 2.507 & 3.333 \\
BposX+.5mm & 0.396 & 2.517 & 3.339 \\
BposX-.5mm & 0.194 & 2.515 & 3.339 \\
BposY+.5mm & 0.032 & 2.517 & 3.349 \\
BposY-.5mm & 0.593 & 2.512 & 3.338 \\
H1 +2mm X \& Y & 0.600 & 2.519 & 3.347 \\
H1 -2mm X \& Y & 0.809 & 2.515 & 3.349 \\
H2 +2mm in X \& Y & 0.507 & 2.516 & 3.349 \\
H2 -2mm in X \& Y & 0.791 & 2.517 & 3.348 \\
Exp B field & 0.126 & 2.513 & 3.339 \\
BmSptSz .9mm & 0.184 & 2.512 & 3.335 \\
\hline \hline fluka2011.2c.3 & 1.452 & 2.500 & 3.396 \\
BMSptSz 1.5mm & 1.586 & 2.506 & 3.404 \\
TarPosZ +7mm & -0.050 & 2.499 & 3.398 \\
TarPosZ -7mm & 0.045 & 2.500 & 3.401 \\
NewHorn ImmWL & 3.174 & 2.503 & 3.394 \\
G4NuMI(FTFPv6) & -5.064 & 2.539 & 3.356 \\
\hline
\end{tabular}

Table B.11: The variation $\delta(\%) \nu_{e}$ and Energy (Mean and RMS) at $\mathrm{NO} \nu \mathrm{A} F \mathrm{~F}$ in the $0-10 \mathrm{GeV}$ range.

\begin{tabular}{cccc}
\hline Shift/Parent & $\delta(\%)$ & RMS $(\mathrm{GeV})$ & Mean \\
\hline Std(fluka2011.2b.6) & 0.000 & 0.553 & 1.899 \\
$+1 \mathrm{kA}$ & 0.876 & 0.552 & 1.902 \\
$-1 \mathrm{kA}$ & -0.206 & 0.552 & 1.899 \\
BposX+.5mm & 0.646 & 0.553 & 1.896 \\
BposX-.5mm & 0.353 & 0.554 & 1.901 \\
BposY+.5mm & 0.793 & 0.554 & 1.900 \\
BposY-.5mm & 0.845 & 0.552 & 1.900 \\
H1 +2mm X \& Y & 0.774 & 0.553 & 1.897 \\
H1 -2mm X \& Y & 0.828 & 0.554 & 1.900 \\
H2 +2mm in X \& Y & 1.032 & 0.553 & 1.900 \\
H2 -2mm in X \& Y & 1.085 & 0.553 & 1.900 \\
Exp B field & -0.112 & 0.552 & 1.896 \\
BmSptSz .9mm & -0.053 & 0.552 & 1.899 \\
\hline \hline fluka2011.2c.3 & 0.116 & 0.551 & 1.912 \\
BMSptSz 1.5mm & 2.690 & 0.553 & 1.913 \\
TarPosZ +7mm & 0.163 & 0.552 & 1.913 \\
TarPosZ -7mm & 0.409 & 0.551 & 1.913 \\
NewHorn ImmWL & 3.451 & 0.553 & 1.915 \\
G4NuMI(FTFPv6) & -3.361 & 0.552 & 1.896 \\
\hline
\end{tabular}

Table B.12: The variation $\delta(\%) \nu_{e}$ and Energy (Mean and RMS) at $\mathrm{NO} \nu \mathrm{A}$ FD in the $1-3 \mathrm{GeV}$ range.

\begin{tabular}{cccccc}
\hline Shift/Parent & $\mu^{+}$ & $K^{+}$ & $K_{L}$ & Rest & Total \\
\hline Std(fluka2011.2b.6) & $7.879 \mathrm{E}-17$ & $6.313 \mathrm{E}-17$ & $1.899 \mathrm{E}-17$ & $1.630 \mathrm{E}-18$ & $1.625 \mathrm{E}-16$ \\
$+1 k A$ & $7.831 \mathrm{E}-17$ & $6.339 \mathrm{E}-17$ & $1.898 \mathrm{E}-17$ & $1.570 \mathrm{E}-18$ & $1.623 \mathrm{E}-16$ \\
$-1 \mathrm{kA}$ & $7.904 \mathrm{E}-17$ & $6.297 \mathrm{E}-17$ & $1.895 \mathrm{E}-17$ & $1.570 \mathrm{E}-18$ & $1.625 \mathrm{E}-16$ \\
BposX+.5mm & $7.849 \mathrm{E}-17$ & $6.297 \mathrm{E}-17$ & $1.909 \mathrm{E}-17$ & $1.590 \mathrm{E}-18$ & $1.622 \mathrm{E}-16$ \\
BposX-.5mm & $7.807 \mathrm{E}-17$ & $6.305 \mathrm{E}-17$ & $1.897 \mathrm{E}-17$ & $1.570 \mathrm{E}-18$ & $1.617 \mathrm{E}-16$ \\
BposY+.5mm & $7.824 \mathrm{E}-17$ & $6.331 \mathrm{E}-17$ & $1.908 \mathrm{E}-17$ & $1.600 \mathrm{E}-18$ & $1.622 \mathrm{E}-16$ \\
BposY-.5mm & $7.846 \mathrm{E}-17$ & $6.285 \mathrm{E}-17$ & $1.892 \mathrm{E}-17$ & $1.580 \mathrm{E}-18$ & $1.618 \mathrm{E}-16$ \\
H1 +2mm X \& Y & $7.768 \mathrm{E}-17$ & $6.298 \mathrm{E}-17$ & $1.900 \mathrm{E}-17$ & $1.640 \mathrm{E}-18$ & $1.613 \mathrm{E}-16$ \\
H1 -2mm X \& Y & $7.755 \mathrm{E}-17$ & $6.299 \mathrm{E}-17$ & $1.895 \mathrm{E}-17$ & $1.610 \mathrm{E}-18$ & $1.611 \mathrm{E}-16$ \\
H2 +2mm in X \& Y & $7.812 \mathrm{E}-17$ & $6.310 \mathrm{E}-17$ & $1.905 \mathrm{E}-17$ & $1.580 \mathrm{E}-18$ & $1.618 \mathrm{E}-16$ \\
H2 -2mm in X \& Y & $7.779 \mathrm{E}-17$ & $6.320 \mathrm{E}-17$ & $1.897 \mathrm{E}-17$ & $1.590 \mathrm{E}-18$ & $1.616 \mathrm{E}-16$ \\
Exp B field & $7.841 \mathrm{E}-17$ & $6.315 \mathrm{E}-17$ & $1.899 \mathrm{E}-17$ & $1.590 \mathrm{E}-18$ & $1.621 \mathrm{E}-16$ \\
BmSptSz .9mm & $7.859 \mathrm{E}-17$ & $6.315 \mathrm{E}-17$ & $1.891 \mathrm{E}-17$ & $1.610 \mathrm{E}-18$ & $1.623 \mathrm{E}-16$ \\
\hline \hline fluka2011.2c.3 & $7.514 \mathrm{E}-17$ & $6.294 \mathrm{E}-17$ & $1.906 \mathrm{E}-17$ & $1.620 \mathrm{E}-18$ & $1.588 \mathrm{E}-16$ \\
BMSptSz 1.5mm & $7.306 \mathrm{E}-17$ & $6.176 \mathrm{E}-17$ & $1.901 \mathrm{E}-17$ & $1.550 \mathrm{E}-18$ & $1.554 \mathrm{E}-16$ \\
TarPosZ +7mm & $7.482 \mathrm{E}-17$ & $6.289 \mathrm{E}-17$ & $1.905 \mathrm{E}-17$ & $1.600 \mathrm{E}-18$ & $1.584 \mathrm{E}-16$ \\
TarPosZ -7mm & $7.478 \mathrm{E}-17$ & $6.289 \mathrm{E}-17$ & $1.907 \mathrm{E}-17$ & $1.610 \mathrm{E}-18$ & $1.583 \mathrm{E}-16$ \\
NewHorn ImmWL & $7.185 \mathrm{E}-17$ & $6.149 \mathrm{E}-17$ & $1.864 \mathrm{E}-17$ & $1.510 \mathrm{E}-18$ & $1.535 \mathrm{E}-16$ \\
G4NuMI(FTFPv6) & $8.297 \mathrm{E}-17$ & $6.619 \mathrm{E}-17$ & $1.944 \mathrm{E}-17$ & $0.000 \mathrm{E}+00$ & $1.686 \mathrm{E}-16$ \\
\hline
\end{tabular}

Table B.13: Number of $\nu_{e}$ at NOvA FD separated by parent types in the $0-10 \mathrm{GeV}$ range. 


\begin{tabular}{cccccc}
\hline Shift/Parent & $\mu^{+}$ & $K^{+}$ & $K_{L}$ & Rest & Total \\
\hline Std(fluka2011.2b.6) & $5.370 \mathrm{E}-17$ & $1.122 \mathrm{E}-17$ & $5.180 \mathrm{E}-18$ & $1.200 \mathrm{E}-19$ & $7.021 \mathrm{E}-17$ \\
$+1 \mathrm{kA}$ & $5.355 \mathrm{E}-17$ & $1.124 \mathrm{E}-17$ & $5.170 \mathrm{E}-18$ & $1.100 \mathrm{E}-19$ & $7.006 \mathrm{E}-17$ \\
-1kA & $5.379 \mathrm{E}-17$ & $1.121 \mathrm{E}-17$ & $5.170 \mathrm{E}-18$ & $1.200 \mathrm{E}-19$ & $7.029 \mathrm{E}-17$ \\
BposX+.5mm & $5.359 \mathrm{E}-17$ & $1.119 \mathrm{E}-17$ & $5.160 \mathrm{E}-18$ & $1.200 \mathrm{E}-19$ & $7.005 \mathrm{E}-17$ \\
BposX-.5mm & $5.327 \mathrm{E}-17$ & $1.119 \mathrm{E}-17$ & $5.180 \mathrm{E}-18$ & $1.300 \mathrm{E}-19$ & $6.977 \mathrm{E}-17$ \\
BposY+.5mm & $5.342 \mathrm{E}-17$ & $1.119 \mathrm{E}-17$ & $5.170 \mathrm{E}-18$ & $1.300 \mathrm{E}-19$ & $6.991 \mathrm{E}-17$ \\
BposY-.5mm & $5.345 \mathrm{E}-17$ & $1.121 \mathrm{E}-17$ & $5.160 \mathrm{E}-18$ & $1.200 \mathrm{E}-19$ & $6.993 \mathrm{E}-17$ \\
H1 +2mm X \& Y & $5.330 \mathrm{E}-17$ & $1.113 \mathrm{E}-17$ & $5.170 \mathrm{E}-18$ & $1.200 \mathrm{E}-19$ & $6.972 \mathrm{E}-17$ \\
H1 -2mm X \& Y & $5.313 \mathrm{E}-17$ & $1.119 \mathrm{E}-17$ & $5.180 \mathrm{E}-18$ & $1.200 \mathrm{E}-19$ & $6.962 \mathrm{E}-17$ \\
H2 +2mm in X \& Y & $5.317 \mathrm{E}-17$ & $1.121 \mathrm{E}-17$ & $5.180 \mathrm{E}-18$ & $1.200 \mathrm{E}-19$ & $6.967 \mathrm{E}-17$ \\
H2 -2mm in X \& Y & $5.326 \mathrm{E}-17$ & $1.121 \mathrm{E}-17$ & $5.180 \mathrm{E}-18$ & $1.300 \mathrm{E}-19$ & $6.978 \mathrm{E}-17$ \\
Exp B field & $5.365 \mathrm{E}-17$ & $1.121 \mathrm{E}-17$ & $5.170 \mathrm{E}-18$ & $1.200 \mathrm{E}-19$ & $7.015 \mathrm{E}-17$ \\
BmSptSz .9mm & $5.385 \mathrm{E}-17$ & $1.125 \mathrm{E}-17$ & $5.150 \mathrm{E}-18$ & $1.100 \mathrm{E}-19$ & $7.036 \mathrm{E}-17$ \\
\hline \hline fluka2011.2c.3 & $5.106 \mathrm{E}-17$ & $1.117 \mathrm{E}-17$ & $5.180 \mathrm{E}-18$ & $1.200 \mathrm{E}-19$ & $6.753 \mathrm{E}-17$ \\
BMSptSz 1.5mm & $4.933 \mathrm{E}-17$ & $1.090 \mathrm{E}-17$ & $5.140 \mathrm{E}-18$ & $1.300 \mathrm{E}-19$ & $6.551 \mathrm{E}-17$ \\
TarPosZ +7mm & $5.064 \mathrm{E}-17$ & $1.118 \mathrm{E}-17$ & $5.180 \mathrm{E}-18$ & $1.300 \mathrm{E}-19$ & $6.713 \mathrm{E}-17$ \\
TarPosZ -7mm & $5.054 \mathrm{E}-17$ & $1.116 \mathrm{E}-17$ & $5.180 \mathrm{E}-18$ & $1.300 \mathrm{E}-19$ & $6.702 \mathrm{E}-17$ \\
NewHorn ImmWL & $4.832 \mathrm{E}-17$ & $1.117 \mathrm{E}-17$ & $5.010 \mathrm{E}-18$ & $1.300 \mathrm{E}-19$ & $6.462 \mathrm{E}-17$ \\
G4NuMI(FTFPv6) & $5.624 \mathrm{E}-17$ & $1.173 \mathrm{E}-17$ & $5.310 \mathrm{E}-18$ & $0.000 \mathrm{E}+00$ & $7.328 \mathrm{E}-17$ \\
\hline
\end{tabular}

Table B.14: Number of $\nu_{e}$ at NOvA FD separated by parent types in the 1-3 GeV range.

\begin{tabular}{cccc}
\hline Shift/Parent & $\delta(\%)$ & RMS(GeV) & Mean \\
\hline Std(fluka2011.2b.6) & 0.000 & 2.416 & 3.658 \\
+1kA & 0.172 & 2.417 & 3.668 \\
-1kA & 0.008 & 2.410 & 3.654 \\
BposX+.5mm & 0.238 & 2.419 & 3.660 \\
BposX-.5mm & 0.535 & 2.419 & 3.661 \\
BposY+.5mm & 0.187 & 2.420 & 3.671 \\
BposY-.5mm & 0.441 & 2.414 & 3.658 \\
H1 +2mm X \& Y & 0.761 & 2.425 & 3.672 \\
H1 -2mm X \& Y & 0.882 & 2.419 & 3.666 \\
H2 +2mm in X \& Y & 0.425 & 2.419 & 3.668 \\
H2 -2mm in X \& Y & 0.603 & 2.422 & 3.667 \\
Exp B field & 0.242 & 2.416 & 3.660 \\
BmSptSz .9mm & 0.164 & 2.415 & 3.659 \\
\hline \hline fluka2011.2c.3 & 2.323 & 2.407 & 3.740 \\
BMSptSz 1.5mm & 2.126 & 2.410 & 3.752 \\
TarPosZ +7mm & 0.035 & 2.405 & 3.741 \\
TarPosZ -7mm & 0.051 & 2.405 & 3.743 \\
NewHorn ImmWL & 3.117 & 2.414 & 3.732 \\
G4NuMI(FTFPv) & -6.423 & 2.447 & 3.668 \\
\hline
\end{tabular}

Table B.15: The variation $\delta(\%) \nu_{e}$ and Energy (Mean and RMS) at $\mathrm{NO} \nu \mathrm{A} F \mathrm{~F}$ in the $0-10 \mathrm{GeV}$ range.

\begin{tabular}{cccc}
\hline Shift/Parent & $\delta(\%)$ & RMS $(\mathrm{GeV})$ & Mean \\
\hline Std(fluka2011.2b.6) & 0.000 & 0.547 & 2.032 \\
$+1 \mathrm{kA}$ & 0.212 & 0.547 & 2.037 \\
-1kA & -0.104 & 0.546 & 2.034 \\
BposX+.5mm & 0.229 & 0.547 & 2.031 \\
BposX-.5mm & 0.632 & 0.547 & 2.032 \\
BposY+.5mm & 0.430 & 0.549 & 2.036 \\
BposY-.5mm & 0.411 & 0.546 & 2.034 \\
H1 +2mm X \& Y & 0.704 & 0.547 & 2.035 \\
H1 -2mm X \& Y & 0.851 & 0.548 & 2.033 \\
H2 +2mm in X \& Y & 0.779 & 0.547 & 2.034 \\
H2 -2mm in X \& Y & 0.617 & 0.547 & 2.035 \\
Exp B field & 0.094 & 0.547 & 2.033 \\
BmSptSz .9mm & -0.209 & 0.547 & 2.036 \\
\hline \hline fluka2011.2c.3 & 3.816 & 0.546 & 2.056 \\
BMSptSz 1.5mm & 2.998 & 0.546 & 2.055 \\
TarPosZ +7mm & 0.283 & 0.544 & 2.055 \\
TarPosZ -7mm & 0.457 & 0.545 & 2.056 \\
NewHorn ImmWL & 4.011 & 0.546 & 2.054 \\
G4NuMI(FTFPv6) & -8.843 & 0.548 & 2.031 \\
\hline
\end{tabular}

Table B.16: The variation $\delta(\%) \nu_{e}$ and Energy (Mean and RMS) at $\mathrm{NO} \nu \mathrm{A}$ FD in the $1-3 \mathrm{GeV}$ range. 


\section{Appendix $\mathrm{C}$}

\section{Supplementary Material for Hadron Production Uncertainty}

\section{C.1 Cross Sections from NA49}

The data used from both $\pi$ and $K$ invariant cross section measurements from NA49 for our studies. To derive the ratio between data and FLUKA prediction, extract the meson production yield using FLUKA based on simplified Carbon target configuration and $158 \mathrm{GeV}$ proton [78. The invariant cross section distributions from NA49 data and FLUKA prediction in related $x_{F}$ bins as a function of $p_{T}$ are shown in Figs C.1 - C.6.

\section{C.2 Extended $K$ Coverage Using $K / \pi$ Ratio}

The $K / \pi$ ratio from MIPP [76] is shown in Fig. C.7, which are measured for the $20<$ $p_{Z}<90 \mathrm{GeV}$ region, and help cover the $x_{F}>0.225\left(p_{Z}>27 \mathrm{GeV}\right)$ region which are not covered by the NA49 data results of K production. The cross section of $\mathrm{K}$ production in this extended phase space is calculated by multiplying these $K / \pi$ ratio by the $\pi$ cross section of NA49 data results. For simplicity of error treatment, use the larger one of the 

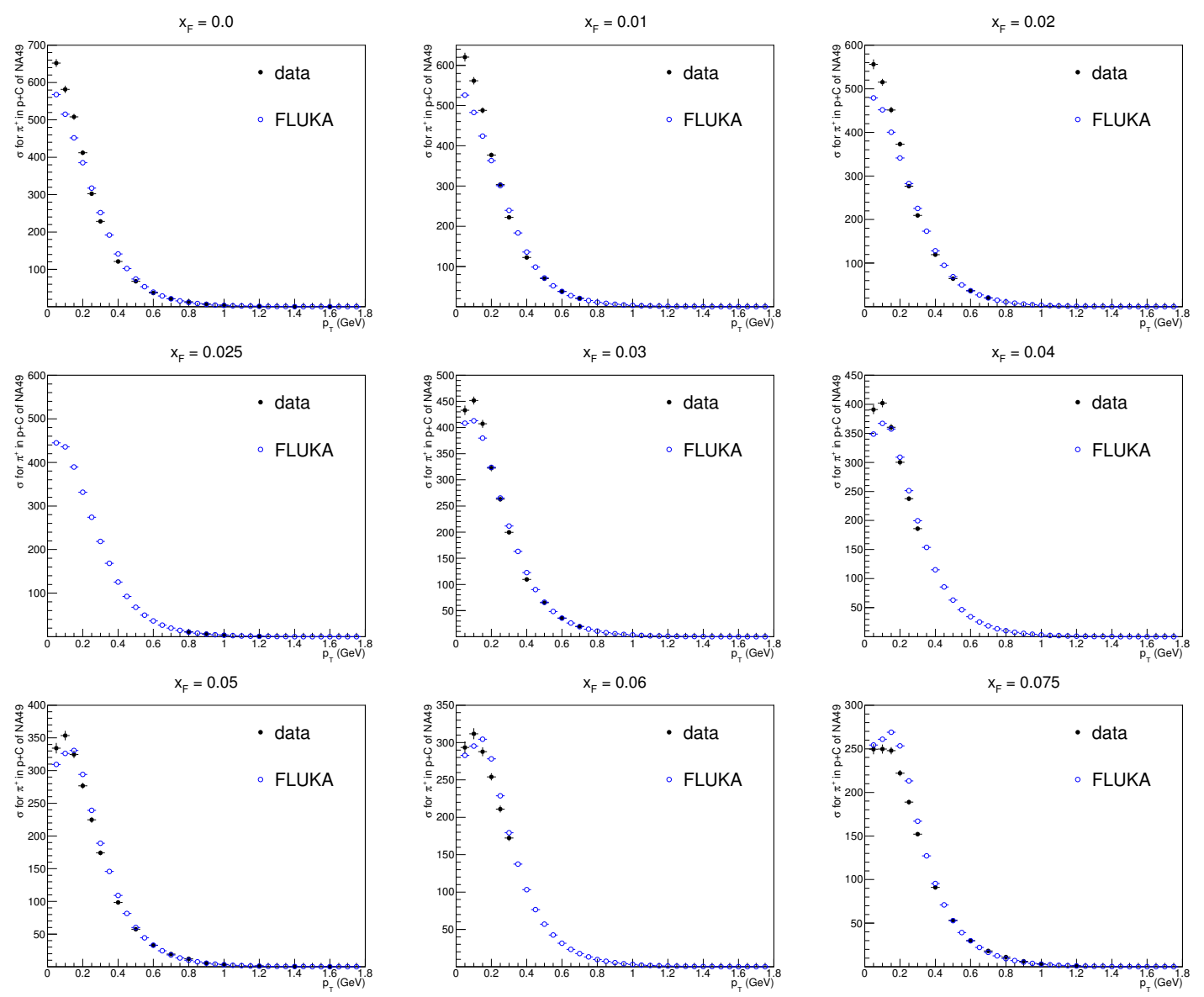

Figure C.1: Invariant cross section as a function of $p_{T}$ for different $x_{F}$ of $\pi^{+}$for NA49 from data and FLUKA simulation.

asymmetry uncertainty as the final uncertainty. 

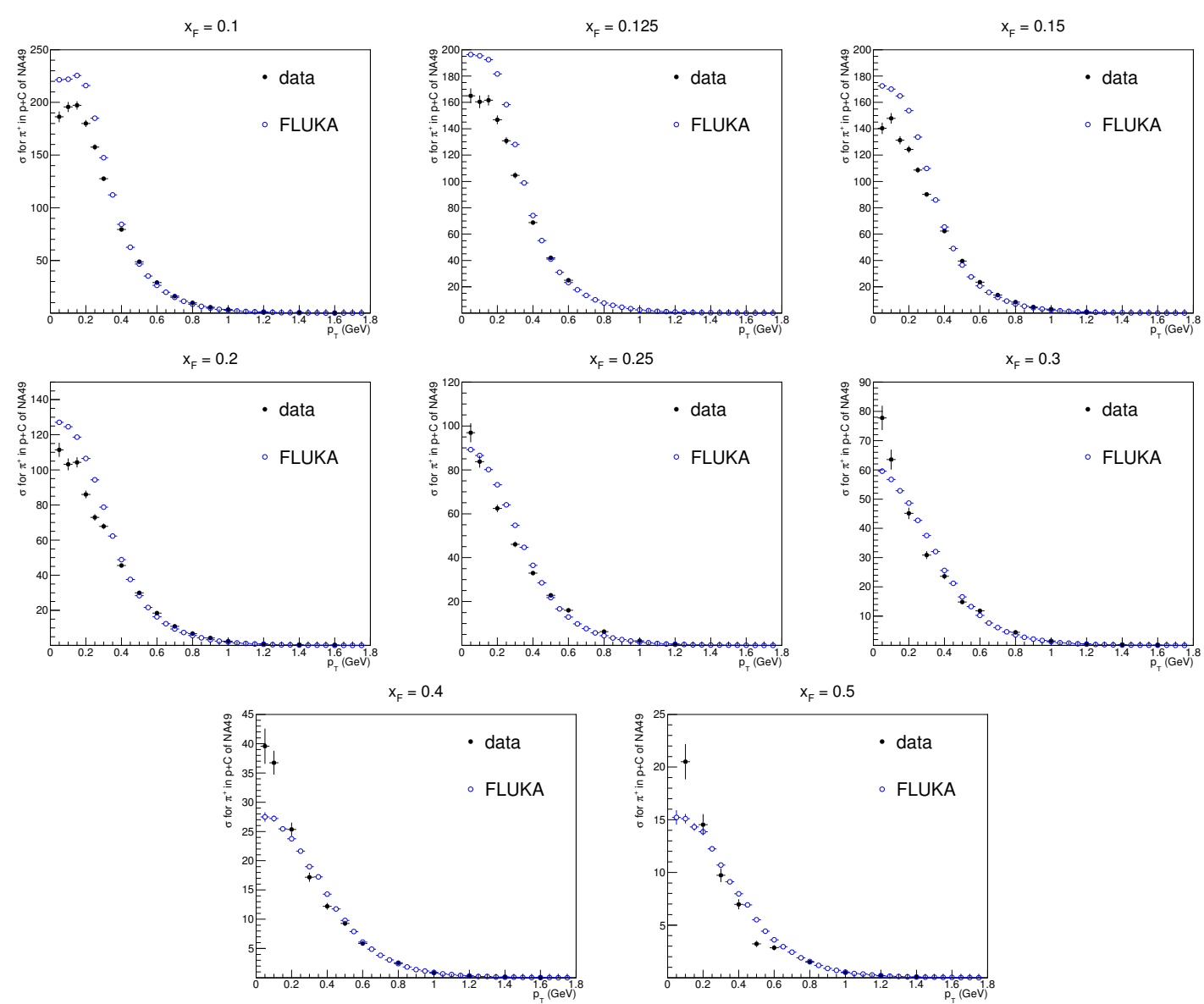

Figure C.2: Invariant cross section as a function of $p_{T}$ for different $x_{F}$ of $\pi^{+}$for NA49 from data and FLUKA simulation.

\section{C.3 $\pi^{ \pm}$Yields from MIPP}

The data results from MIPP (see Fig. C.8) has large coverage for the dominant phase space of NOvA. Simulated low energy NuMI beamline target files using FLUKA, and derive the ratio for the $\pi^{ \pm}$yields between MIPP data and FLUKA, then use those weights to derive flux uncertainty. 

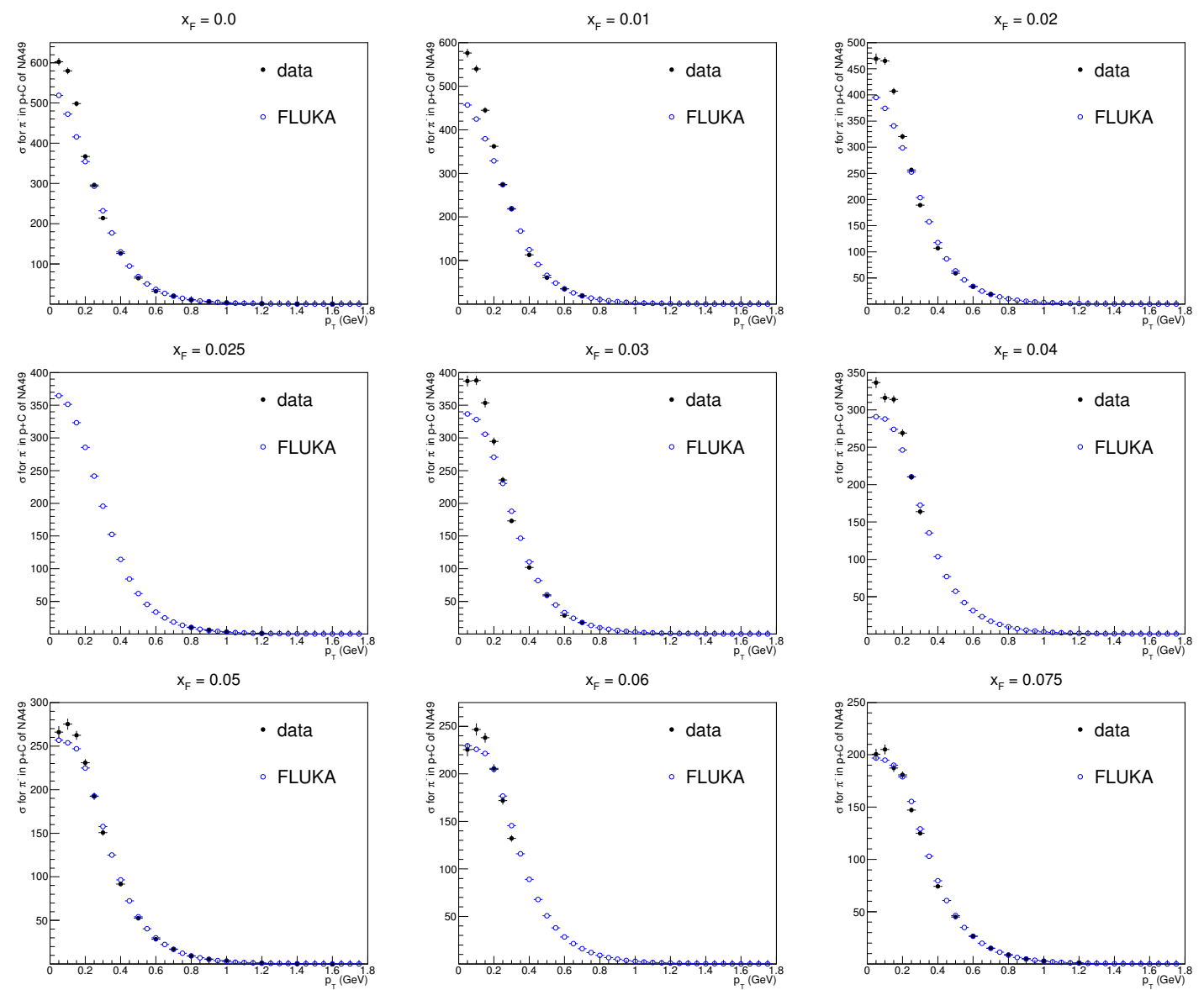

Figure C.3: Invariant cross section as a function of $p_{T}$ for different $x_{F}$ of $\pi^{-}$for NA49 from data and FLUKA simulation.

\section{C.4 Weights for $\pi$ Production}

The pion yield and invariant cross sections for the residual $\pi^{+}$between G4NuMI and

FLUKA using low energy target configuration. and $\pi^{-}$are shown in Figs. C.9 - C.14 

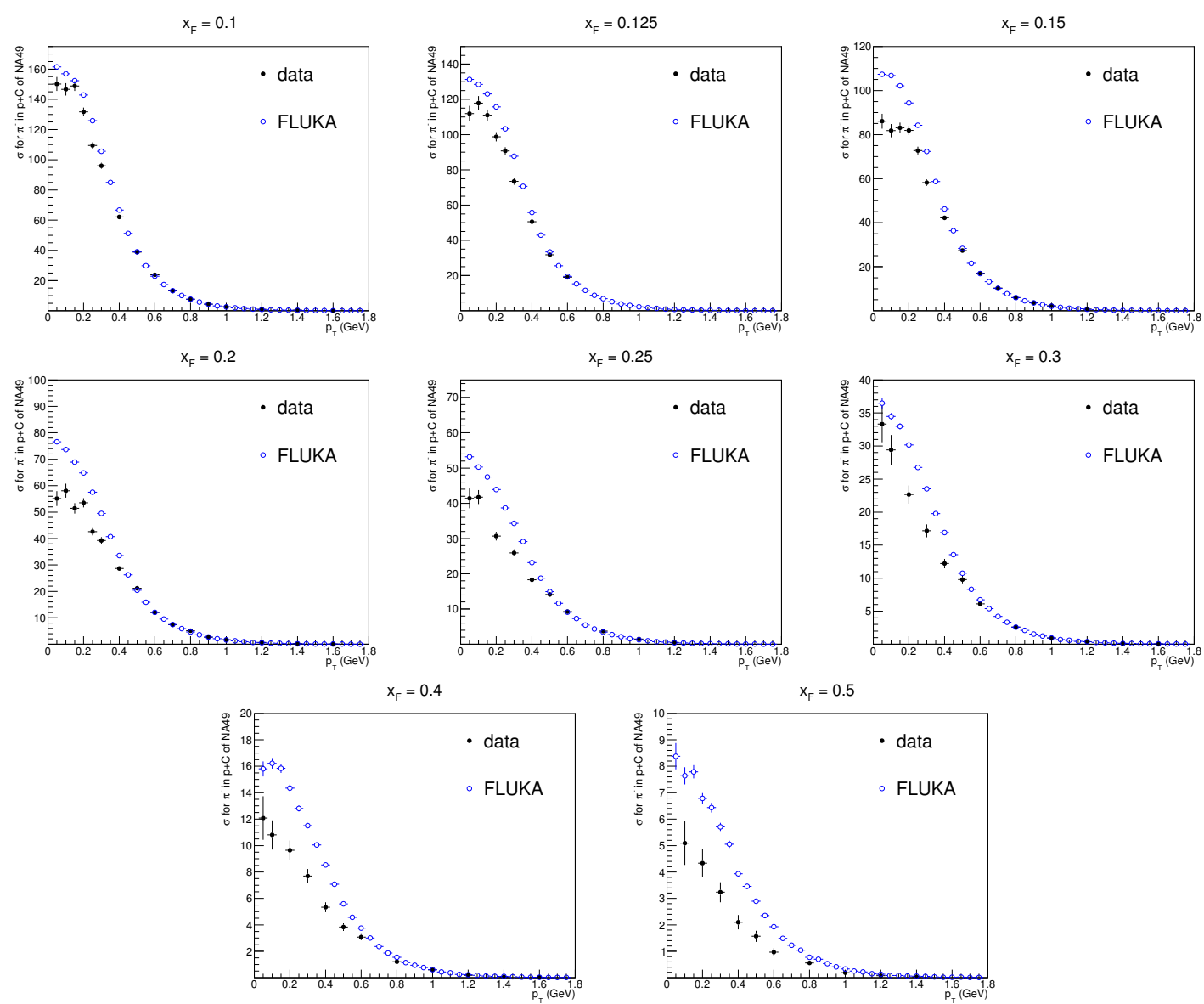

Figure C.4: Invariant cross section as a function of $p_{T}$ for different $x_{F}$ of $\pi^{-}$for NA49 from data and FLUKA simulation.

\section{C.5 Weights for $K^{-}$Production}

The invariant cross section distributions for data and FLUKA prediction are shown in Figs. C.15 and C.16. 

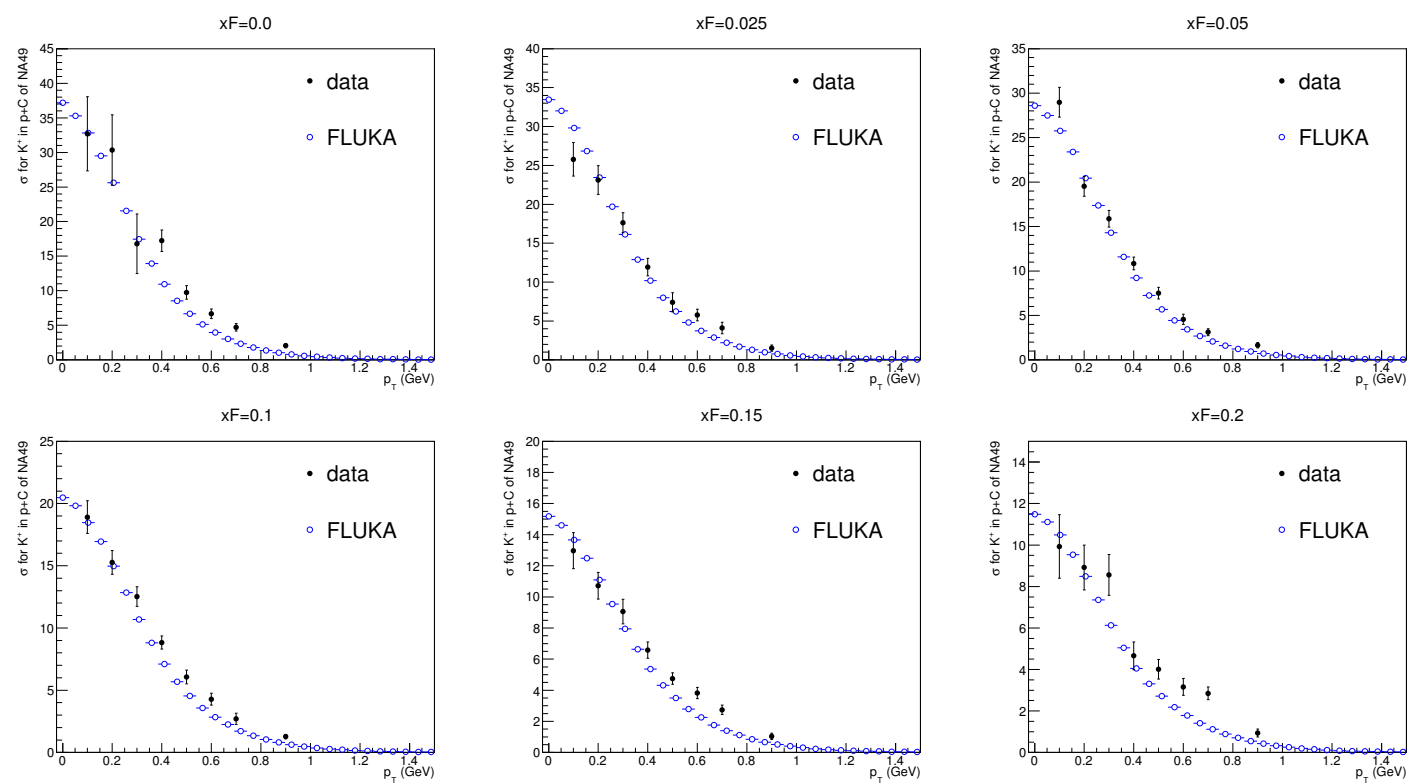

Figure C.5: Invariant cross section as a function of $p_{T}$ for different $x_{F}$ of $K^{+}$for NA49 from data and FLUKA simulation.

\section{C.6 Results Using NA49 Pion data}

We also performed a closure test by using NA49 pion invariant cross section results instead of MIPP pion yield. For this test, only apply these weights from NA49 pion results to the secondary pions. For the remaining non-secondary pions, we assign a $20 \%$ systematic uncertainty based on the difference between G4NuMI and FLUKA predictions.

The flux weight as a function of $\nu_{e}$ energy is shown in Figure. C.6. The weight is $\approx 0.97$ in $1-3 \mathrm{GeV}$ energy region. The uncertainty for the flux weight is shown in Figure. C.6, which is $\approx 10 \%$ in $1-3 \mathrm{GeV}$ energy region.

The pion invariant cross section from NA49 are used to cover the secondary $\pi$ ancestor in following phase space:

- $x_{F}<0.005$ with $p_{T}<2 \mathrm{GeV}$;

- $0.005<x_{F}<0.015$ with $p_{T}<0.75 \mathrm{GeV}$;

- $0.015<x_{F}<0.025$ with $p_{T}<0.75 \mathrm{GeV}$; 

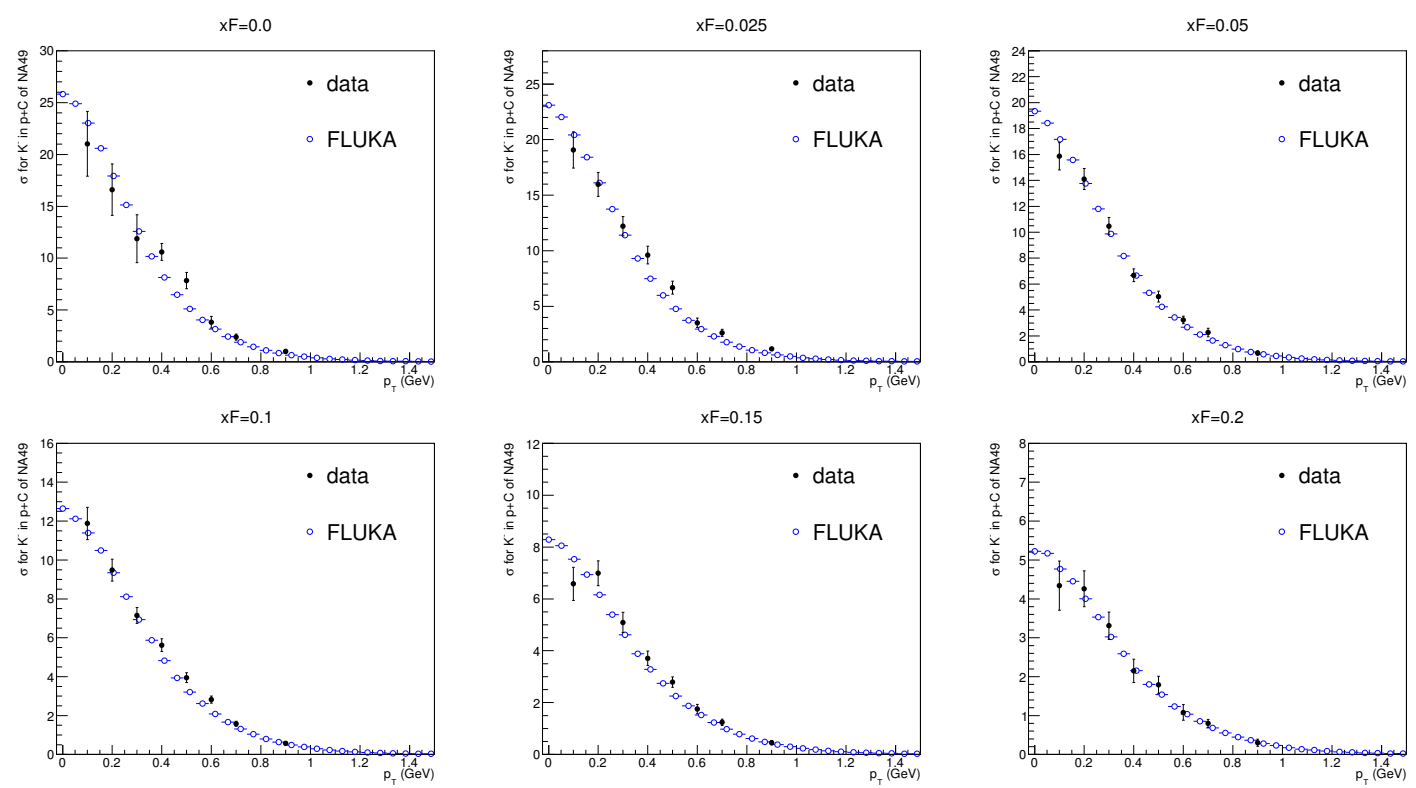

Figure C.6: Invariant cross section as a function of $p_{T}$ for different $x_{F}$ of $K^{-}$for NA49 from data and FLUKA simulation.

- $0.025<x_{F}<0.035$ with $p_{T}<0.75 \mathrm{GeV}$;

- $0.035<x_{F}<0.045$ with $p_{T}<0.325 \mathrm{GeV}$;

- $0.045<x_{F}<0.055$ with $p_{T}<2 \mathrm{GeV}$;

- $0.055<x_{F}<0.065$ with $p_{T}<0.325 \mathrm{GeV}$;

- $0.065<x_{F}<0.085$ with $p_{T}<1.3 \mathrm{GeV}$;

- $0.085<x_{F}<0.1125$ with $p_{T}<2 \mathrm{GeV}$;

- $0.1125<x_{F}<0.1375$ with $p_{T}<0.65 \mathrm{GeV}$;

- $0.1375<x_{F}<0.1625$ with $p_{T}<1.3 \mathrm{GeV}$;

- $0.175<x_{F}<0.225$ with $p_{T}<2 \mathrm{GeV}$;

- $0.225<x_{F}<0.275$ with $p_{T}<1.3 \mathrm{GeV}$;

- $0.275<x_{F}<0.325$ with $p_{T}<1.7 \mathrm{GeV}$;

- $0.325<x_{F}<0.45$ with $p_{T}<1.7 \mathrm{GeV}$; 

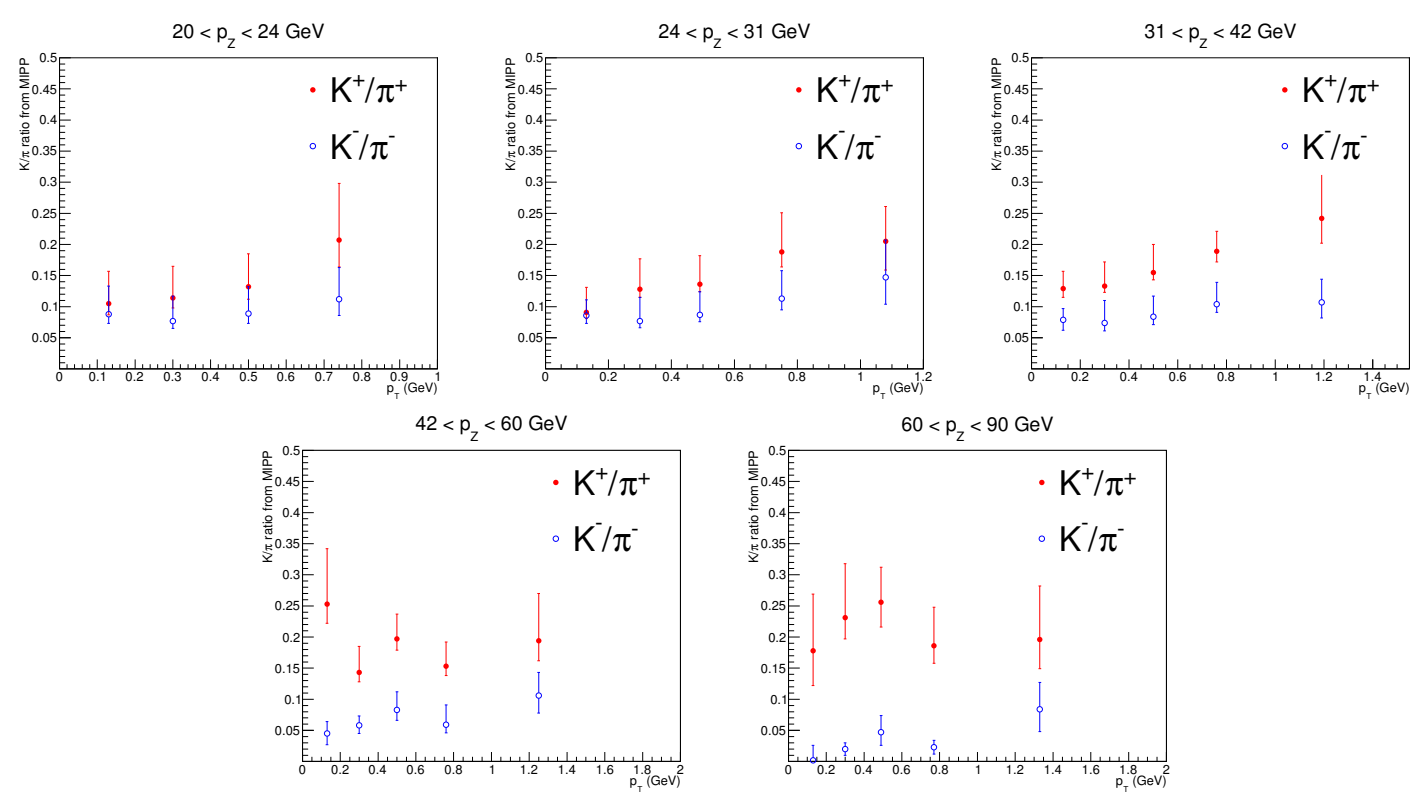

Figure C.7: $K / \pi$ ratio from MIPP.
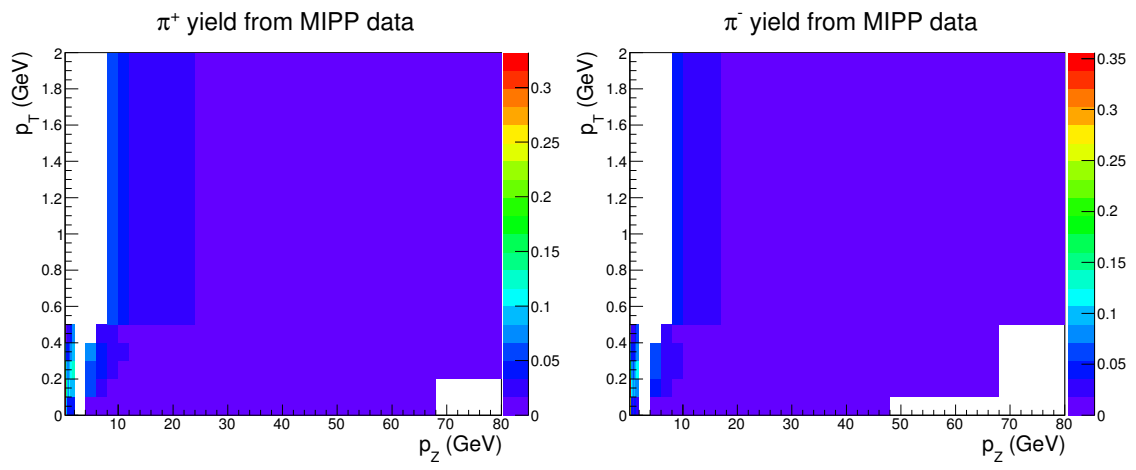

Figure C.8: $\pi^{ \pm}$yields from MIPP data.

- $0.45<x_{F}<0.55$ with $0.05<p_{T}<1.3 \mathrm{GeV}$;

The pion invariant cross section distributions for data and FLUKA in above phase space are shown in Figs. C.18 - C.21. The event weights as a function of $p_{T}$ for each $x_{F}$ bin are shown in the bottom part of these distributions. 

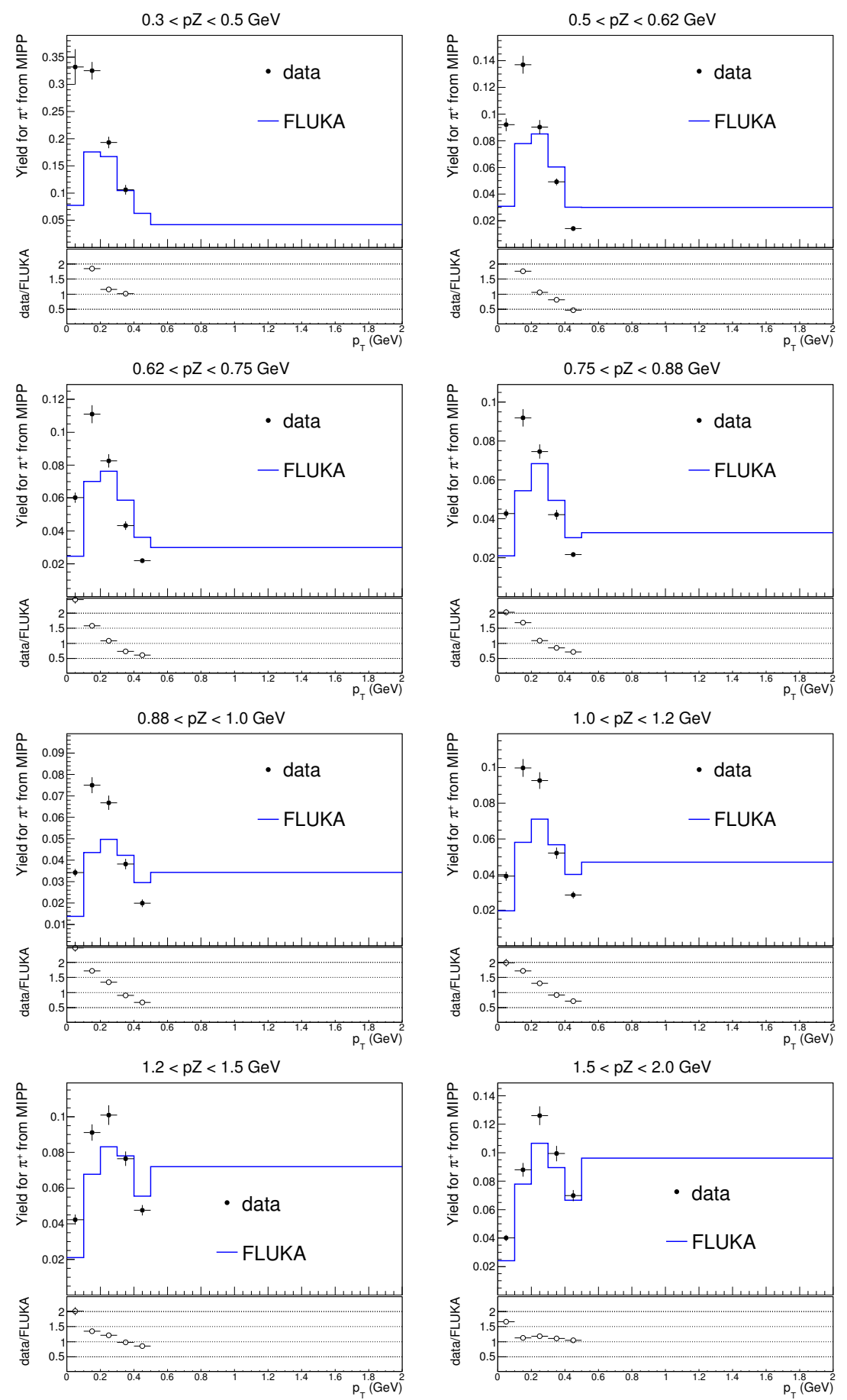

Figure C.9: Invariant cross section and corresponding ratio between data and FLUKA prediction for $\pi^{+}$production of MIPP. 

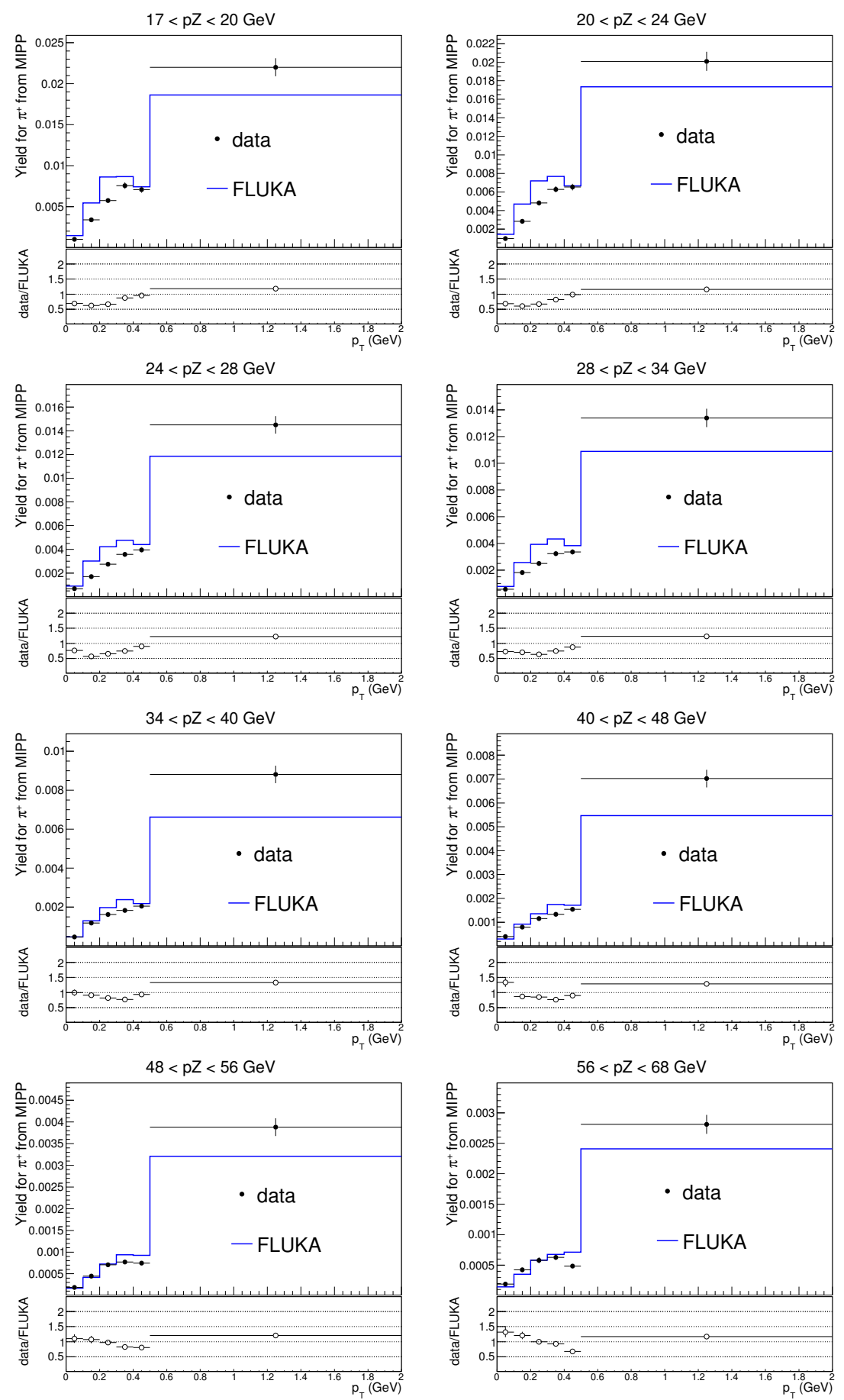

Figure C.10: Invariant cross section and corresponding ratio between data and FLUKA prediction for $\pi^{+}$production of MIPP. 

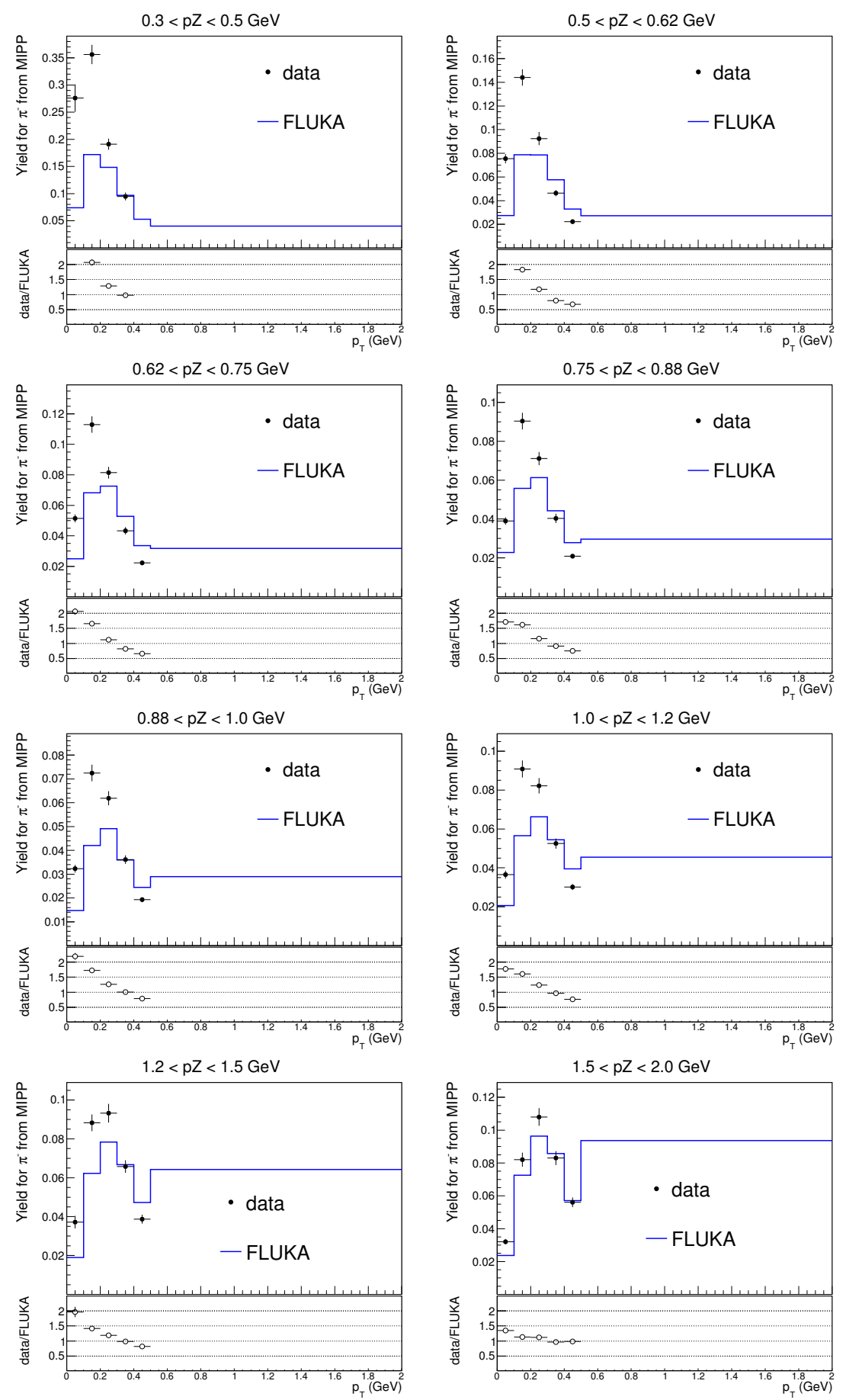

Figure C.11: Invariant cross section and corresponding ratio between data and FLUKA prediction for $\pi^{-}$production of MIPP. 

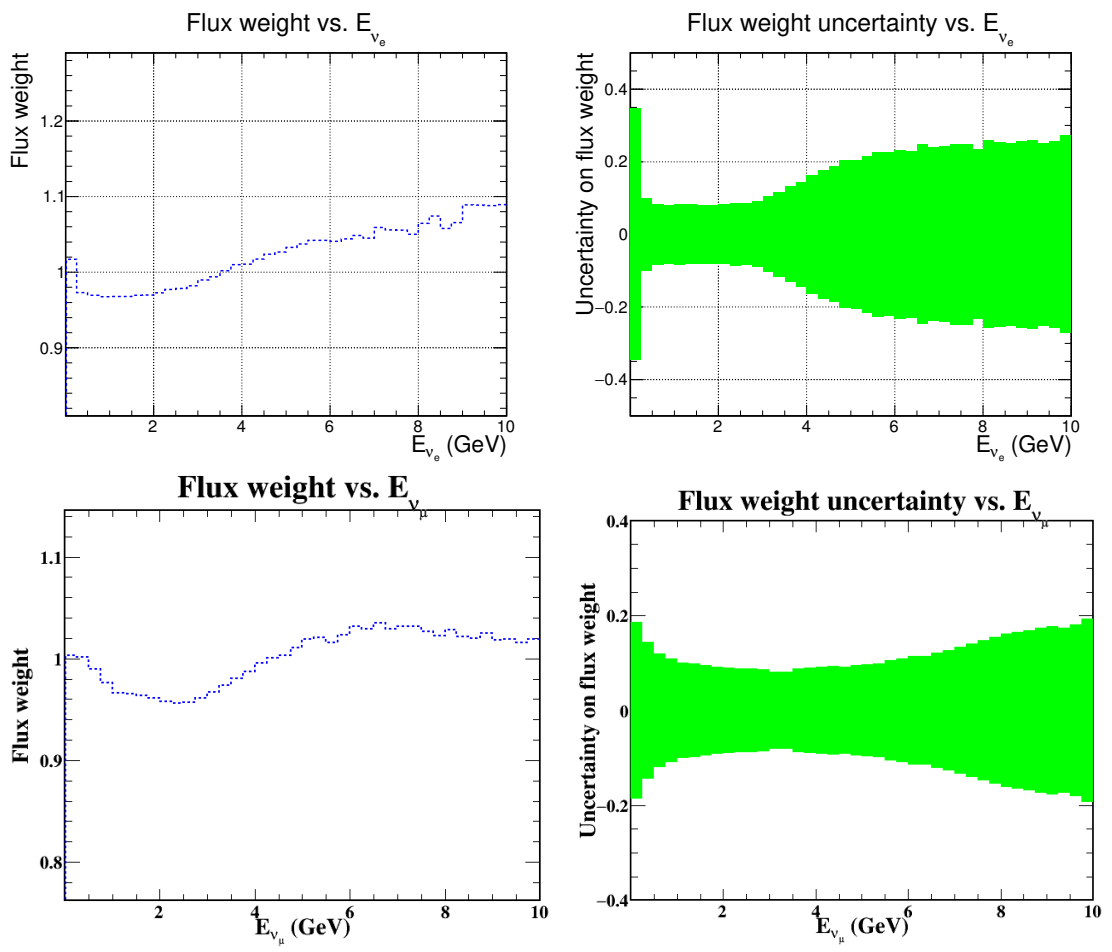

Figure C.17: Flux weight as a function of $\nu_{e}$ and $\nu_{\mu}$ energy, where the event weights from NA49 pion results are used. Uncertainty for the flux weight as a function of $\nu_{e}$ and $\nu_{\mu}$ energy, where the event weights from NA49 pion results are used. 

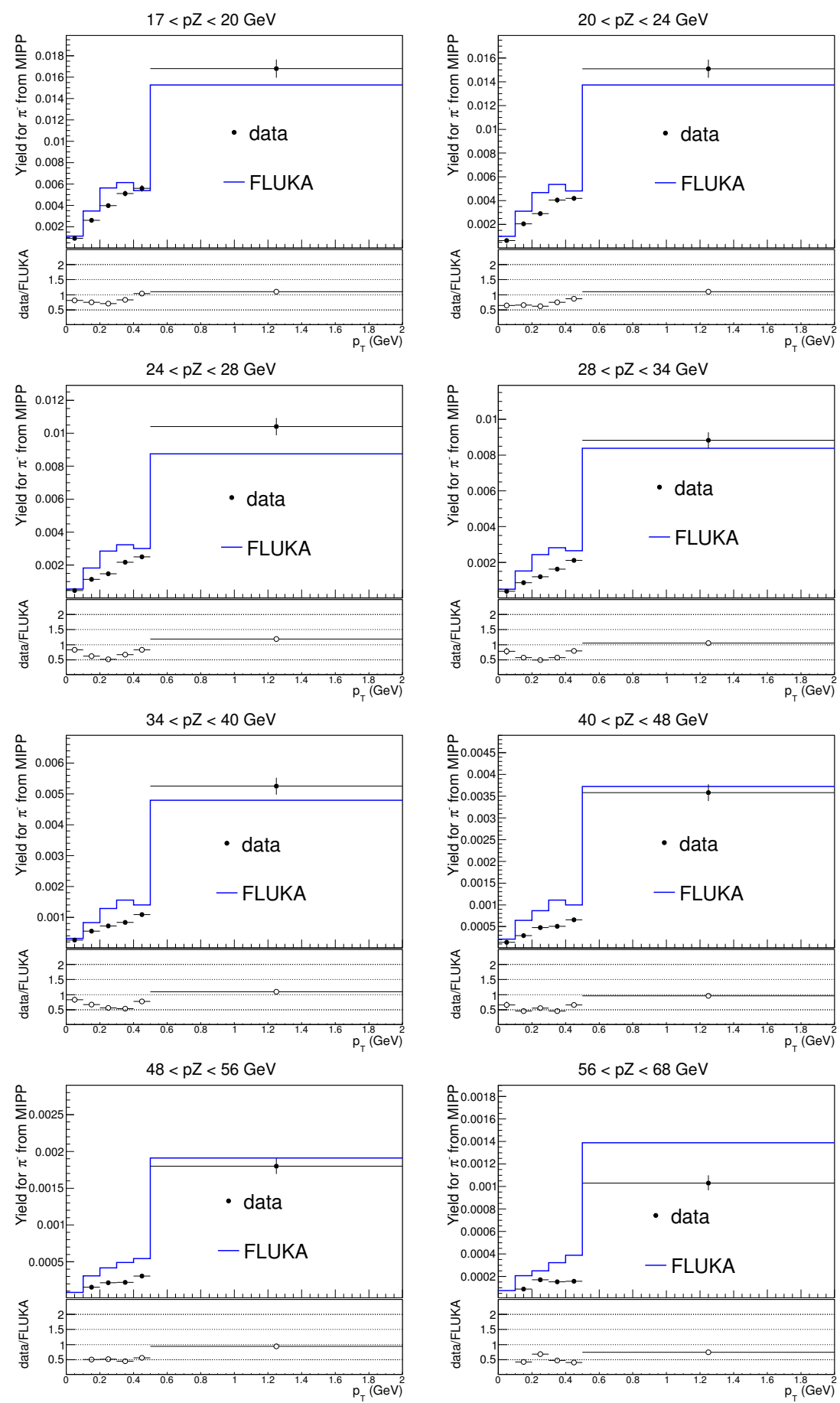

Figure C.13: Invariant cross section and corresponding ratio between data and FLUKA prediction for $\pi^{-}$production of MIPP. 

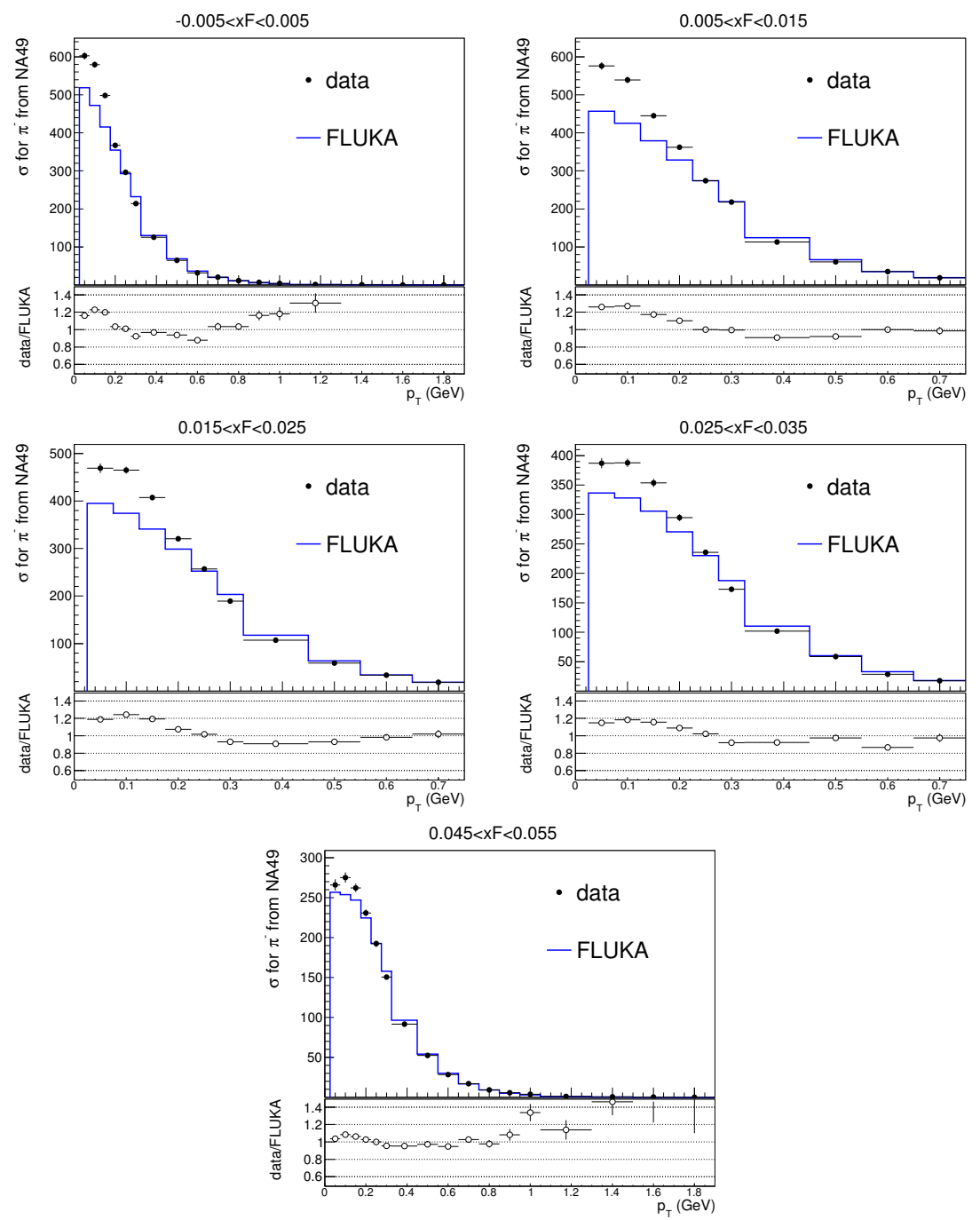

Figure C.14: Invariant cross section and corresponding ratio between data and FLUKA prediction for $\pi^{-}$production of NA49. 

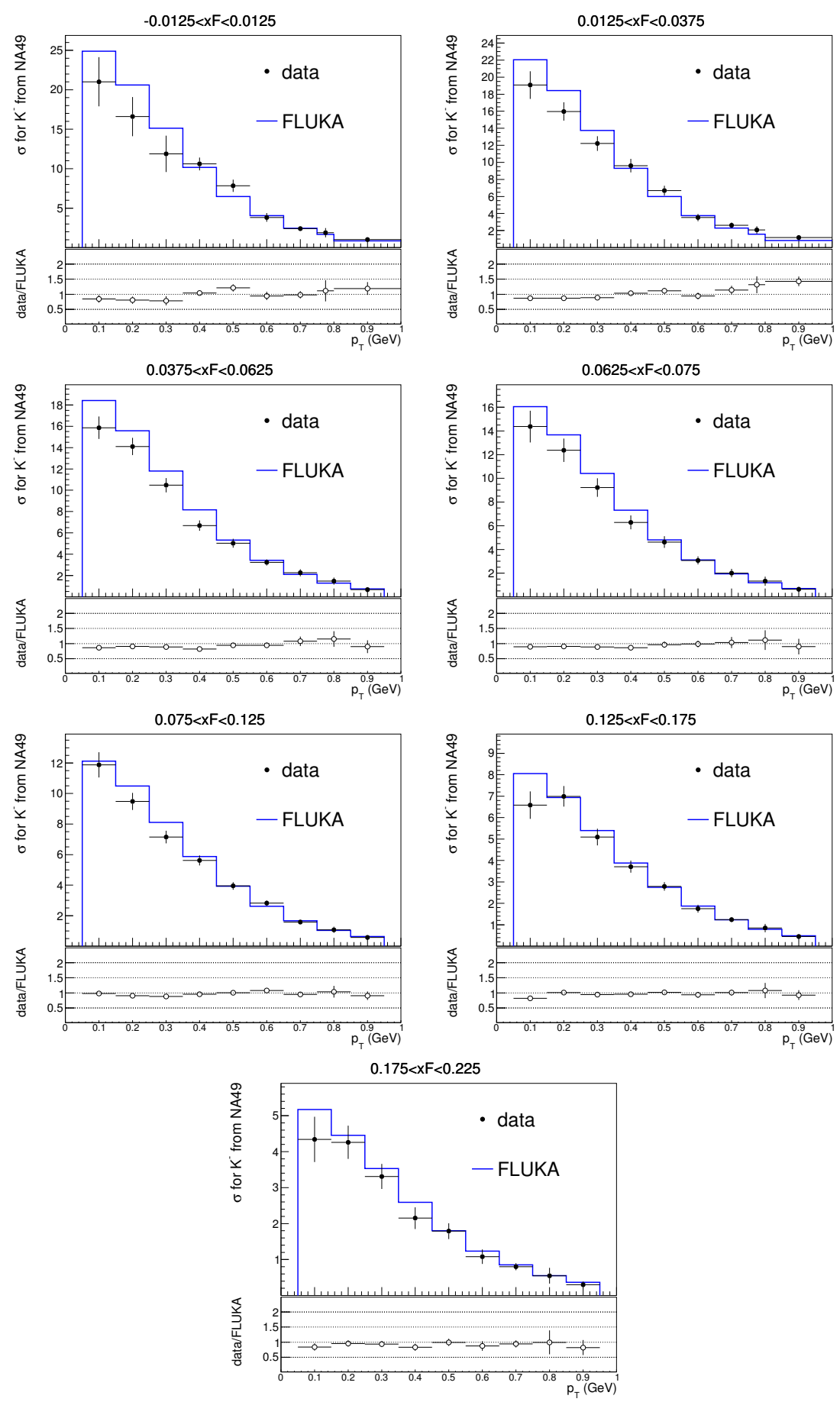

Figure C.15: Invariant cross section and corresponding ratio between data and FLUKA prediction for $K^{-}$production. 

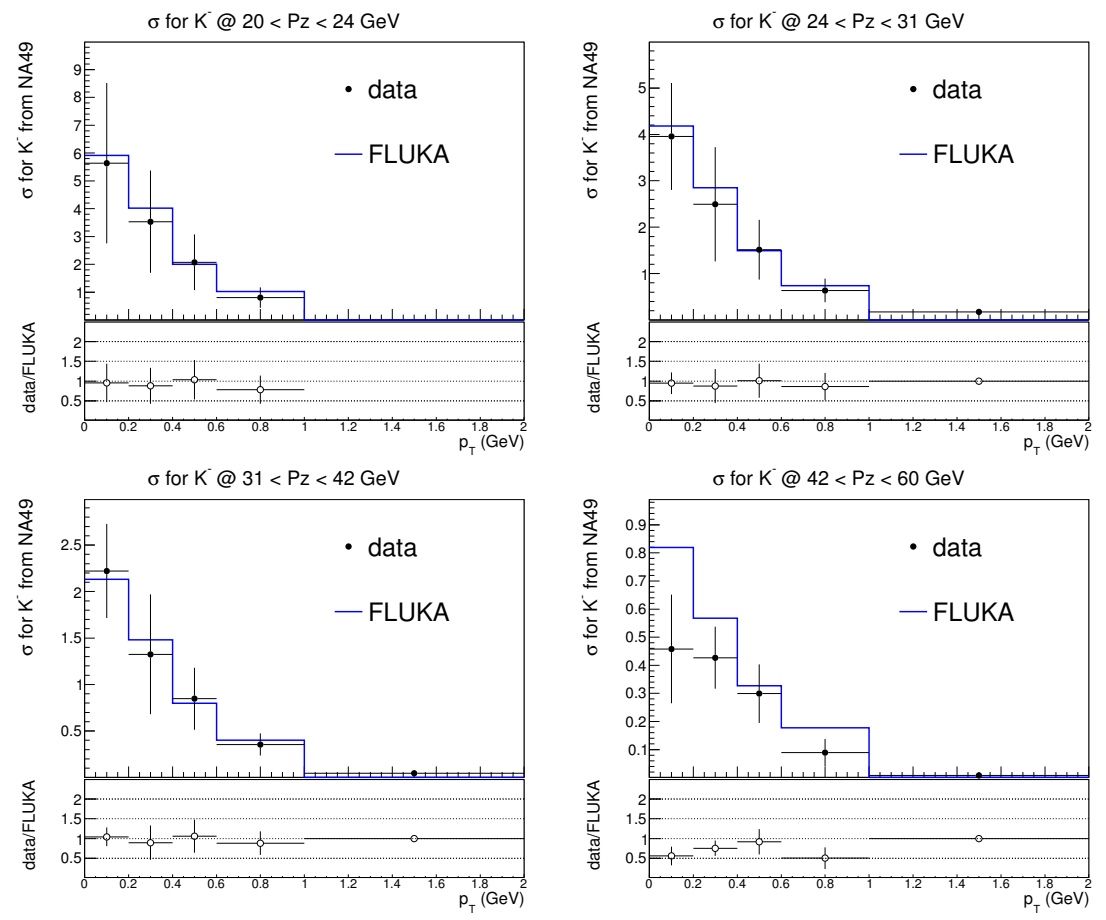

Figure C.16: Invariant cross section and corresponding ratio between data and FLUKA prediction for $K^{-}$production in the extended phase space using the $K^{-} / \pi^{-}$ratio and $\pi^{-}$production cross section. 

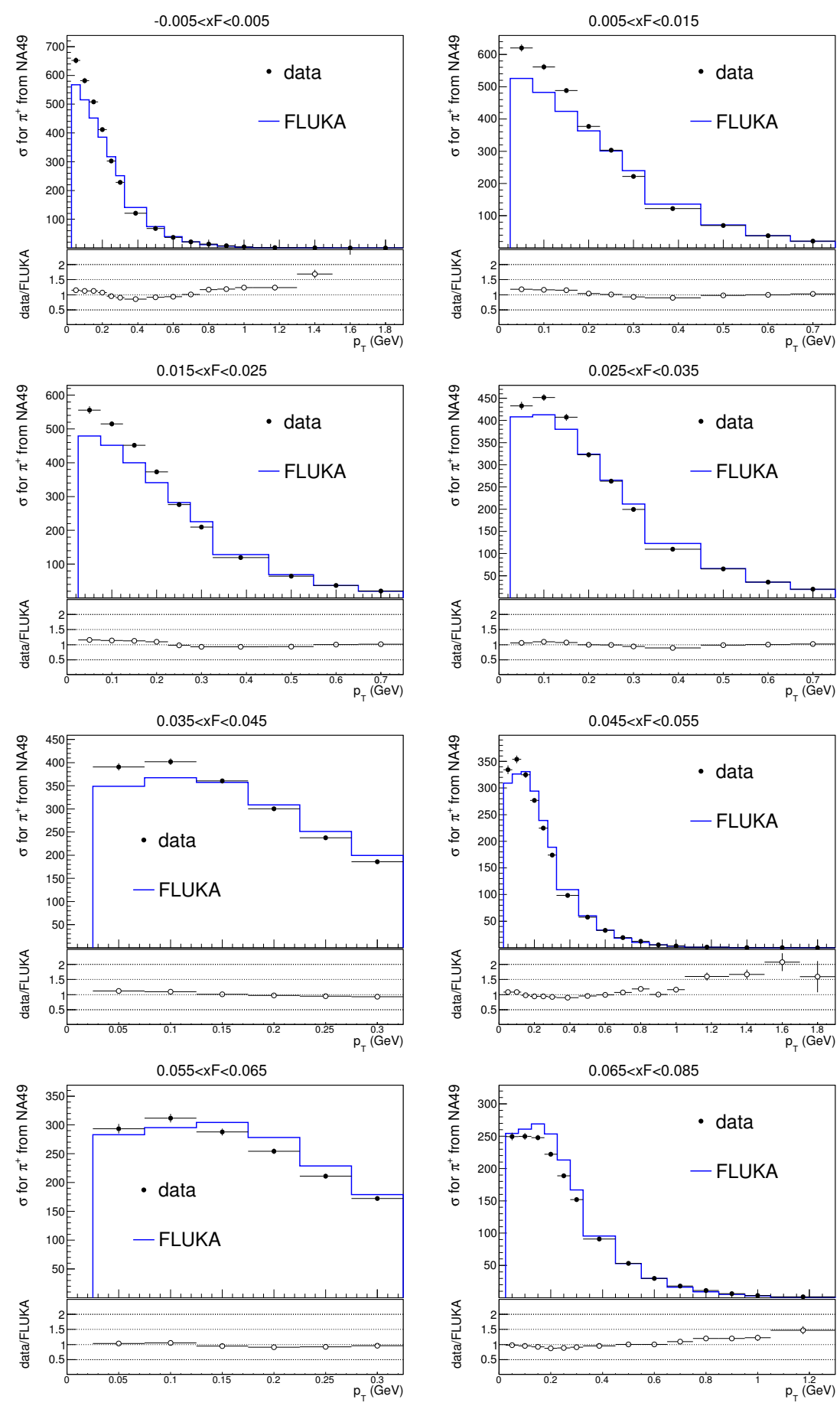

Figure C.18: Invariant cross section and corresponding ratio between data and FLUKA prediction for $\pi^{+}$production of NA49. 

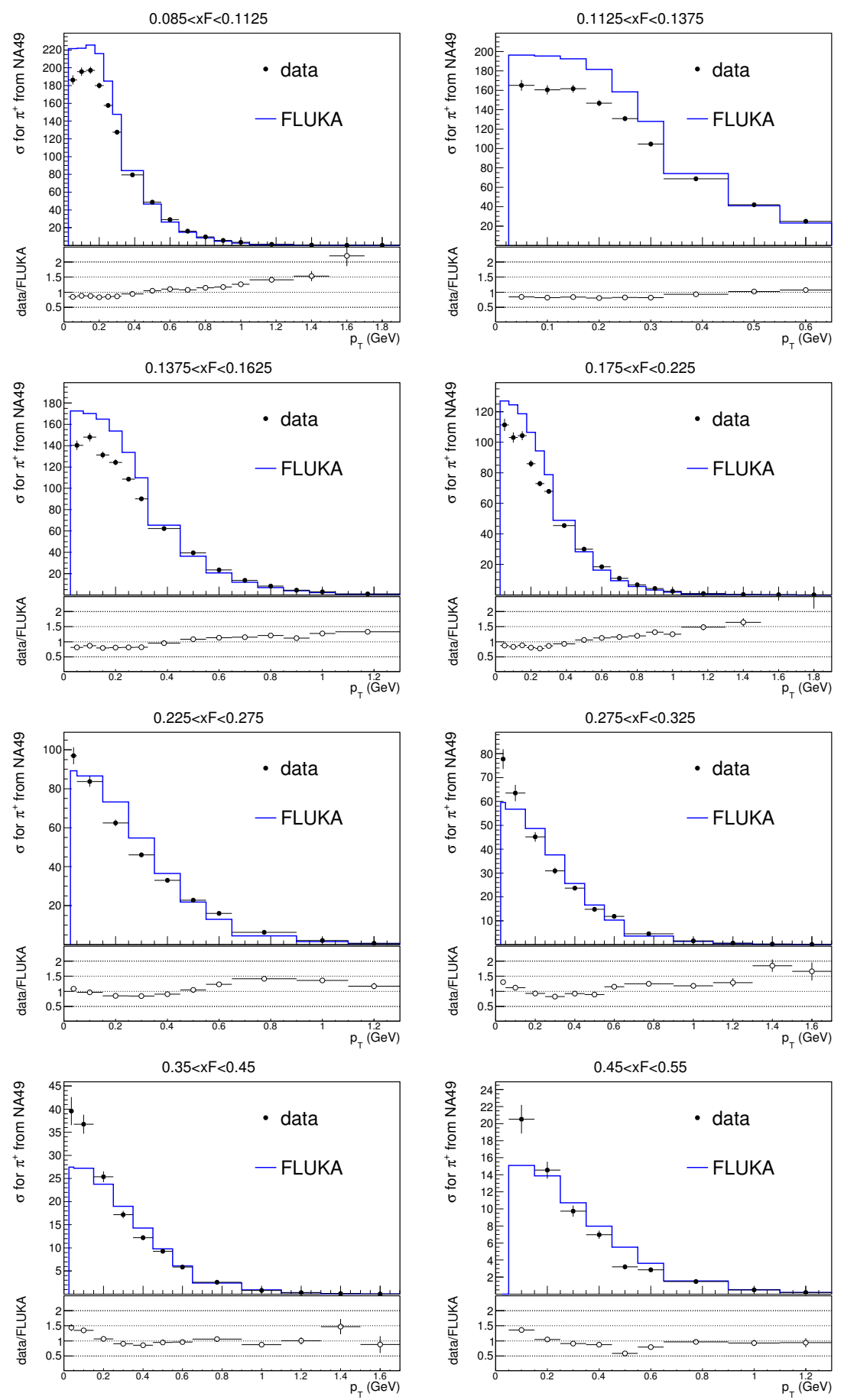

Figure C.19: Invariant cross section and corresponding ratio between data and FLUKA prediction for $\pi^{+}$production of NA49. 

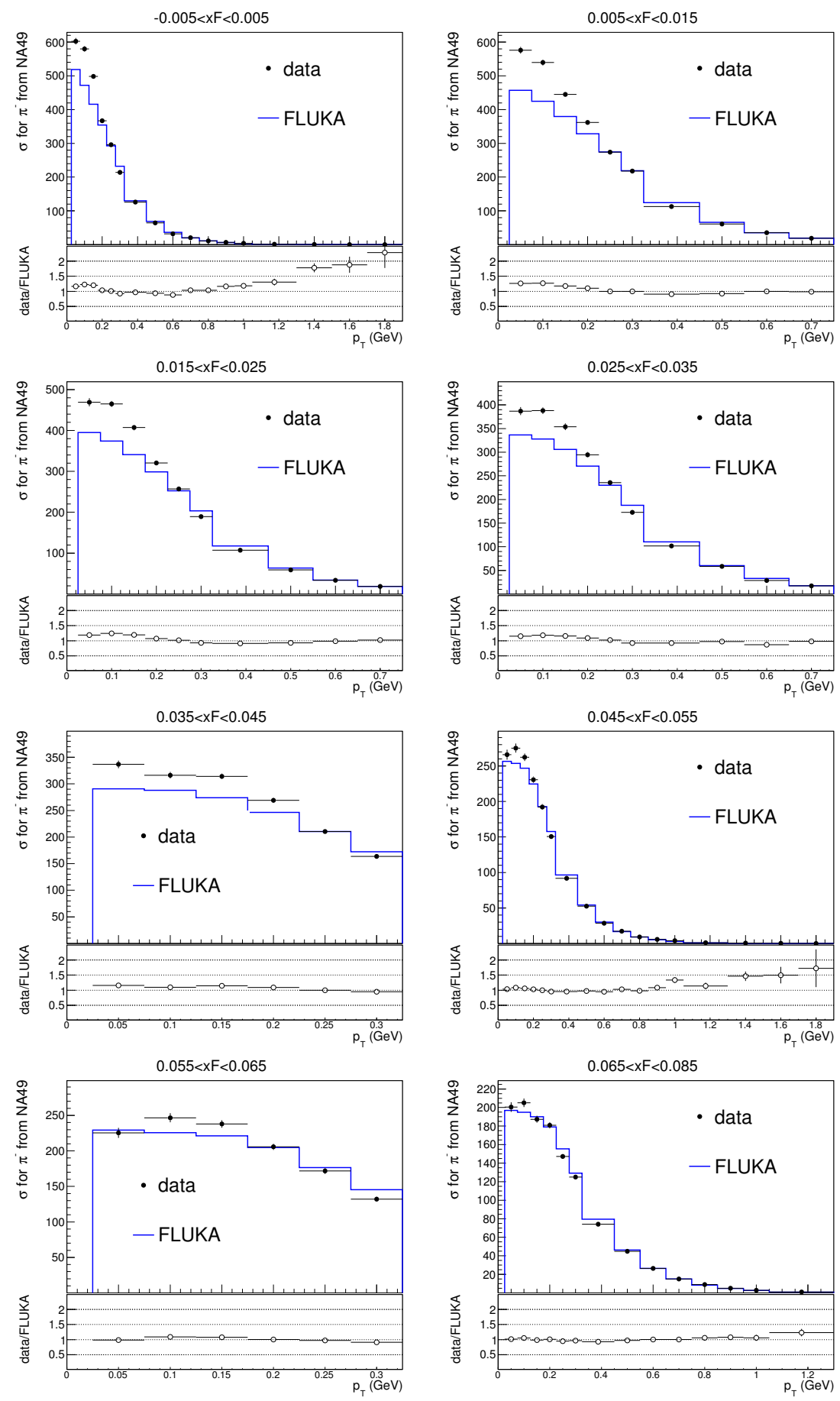

Figure C.20: Invariant cross section and corresponding ratio between data and FLUKA prediction for $\pi^{-}$production of NA49. 

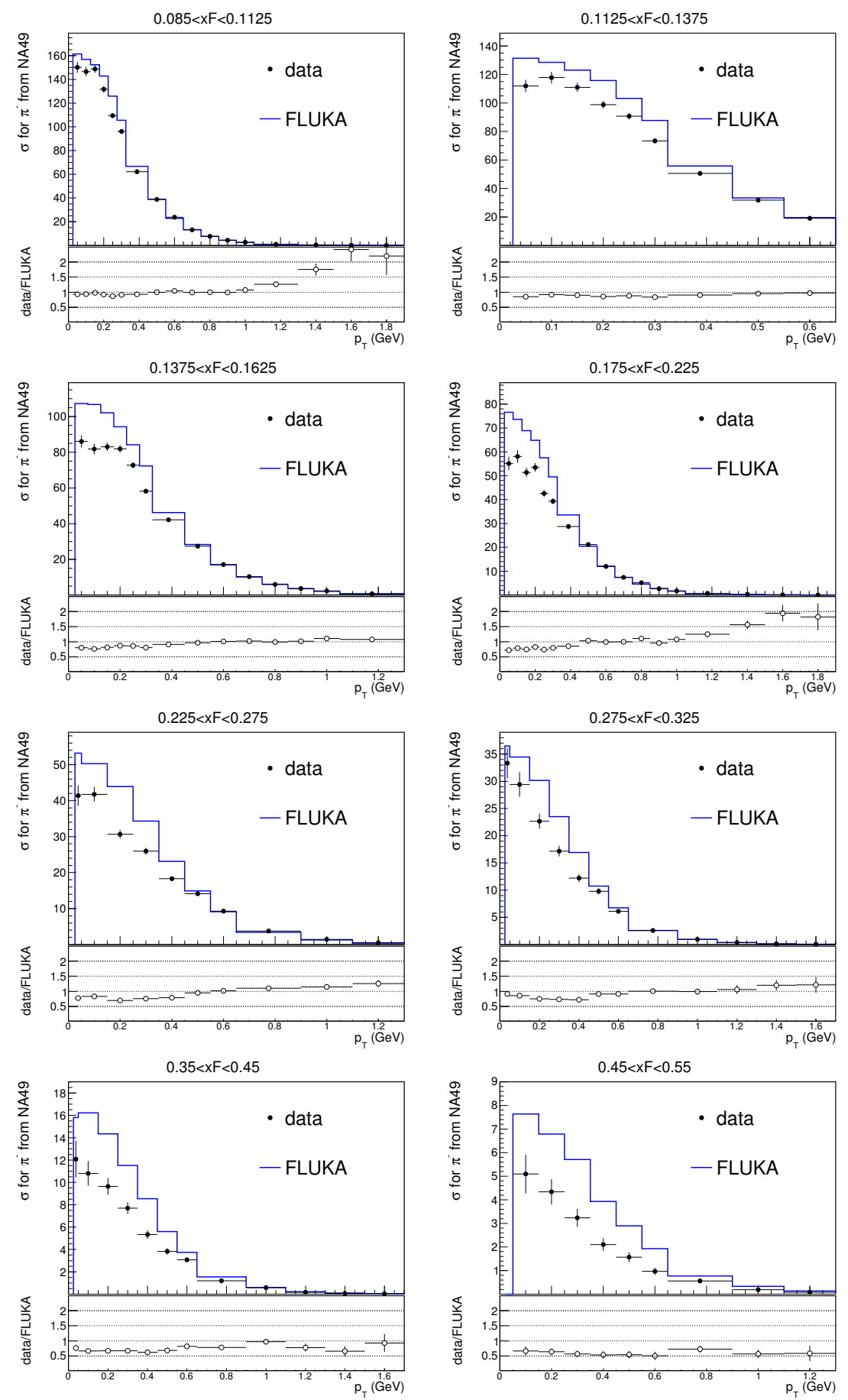

Figure C.21: Invariant cross section and corresponding ratio between data and FLUKA prediction for $\pi^{-}$production of NA49. 


\section{Appendix D}

\section{Supplementary Material for Chapter 6 Part 1}
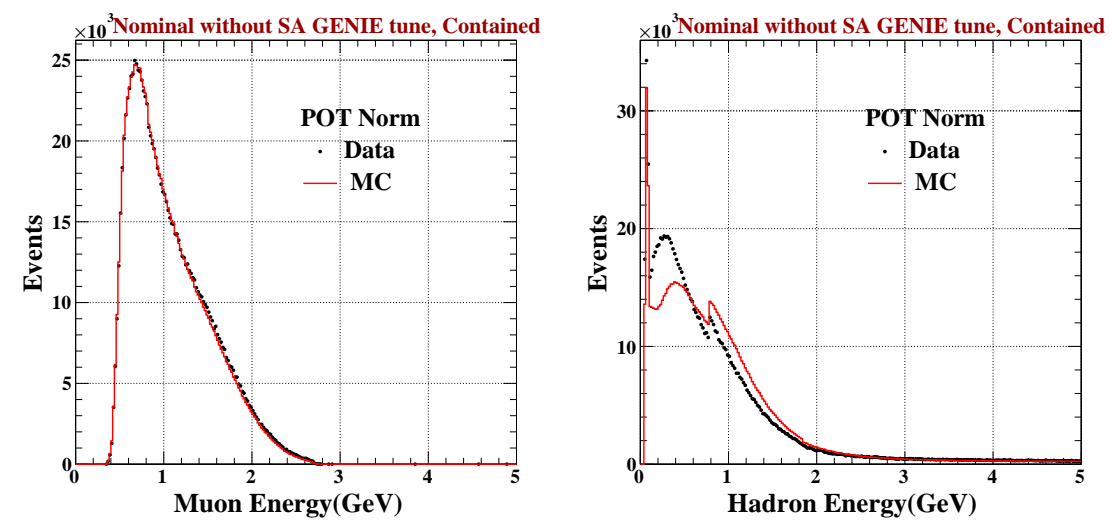

Figure D.1: Left: $\mathrm{E}_{\mu}$ distribution of Data-MC for contained sample without GENIE Tune. Right: $\mathrm{E}_{\text {had }}$ distribution of Data-MC for contained sample without GENIE Tune. 

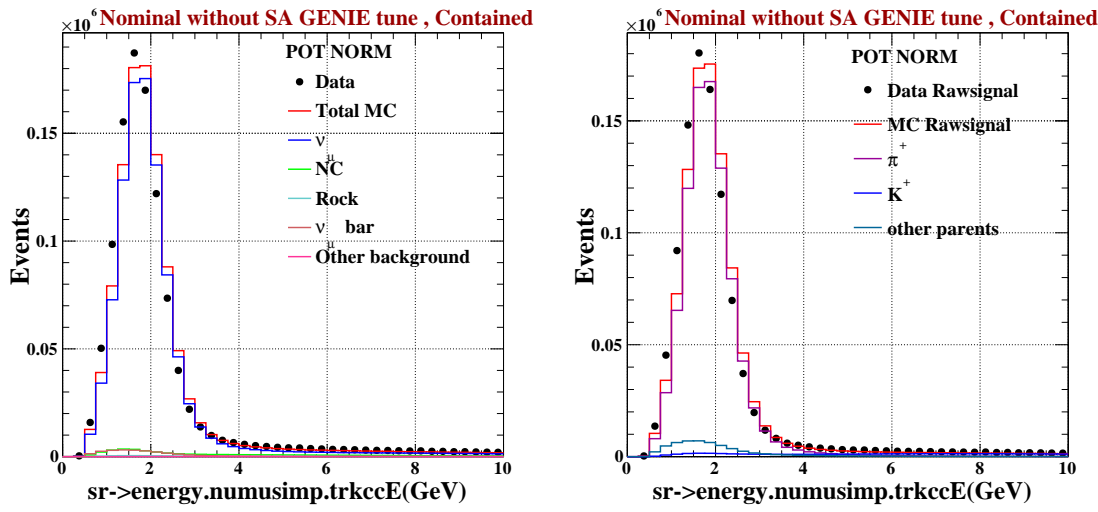

Figure D.2: Reconstructed Neutrino Energy distribution for contained sample without GENIE Tune, Left: Data events and MC with separate background. Right: Raw data signal and MC.

\begin{tabular}{|c|c|c|c|c|}
\hline Variable & $.75-3$ & $3-4.5$ & $4.5-10$ & $10-20$ \\
\hline Data & 918740 & 48603 & 67335 & 29926 \\
\hline $\mathrm{MC}$ & 919577.79 & 49264.03 & 57745.48 & 28744.44 \\
\hline Raw Data Signal (Data-MC $\mathrm{MC}_{b k}$ ) & 873682.81 & 39337.75 & 49951.03 & 23451.64 \\
\hline Signal MC & 874520.6 & 39998.78 & 40361.51 & 22270.08 \\
\hline MC Background & 45057.19 & 9265.25 & 17383.97 & 6474.36 \\
\hline From Pion MC & 819056.4 & 29020.22 & 8786.21 & 955.18 \\
\hline From Kaon MC & 11258.44 & 6348.91 & 27218.65 & 20100.1 \\
\hline From other parents & 44205.76 & 4629.65 & 4356.65 & 1214.81 \\
\hline Raw & Signal & w.r.t & parent & type \\
\hline RawDtSigPi & 818218.61 & 28359.19 & 18375.73 & 2155.55 \\
\hline RawDataSigK & 61958.04 & 6548.23 & 36808.17 & 21281.65 \\
\hline Signal MCpi & 819056.40 & 29020.22 & 8786.21 & 955.18 \\
\hline Signal MCK & 11258.44 & 6348.91 & 27218.65 & 20100.10 \\
\hline$N_{\pi}$ & 1.00 & 0.98 & 2.09 & 2.26 \\
\hline$N_{K}$ & 5.50 & 1.03 & 1.35 & 1.06 \\
\hline
\end{tabular}

Table D.1: $\nu_{\mu}-$ CC event counts for Contained without GENIE Tune. 

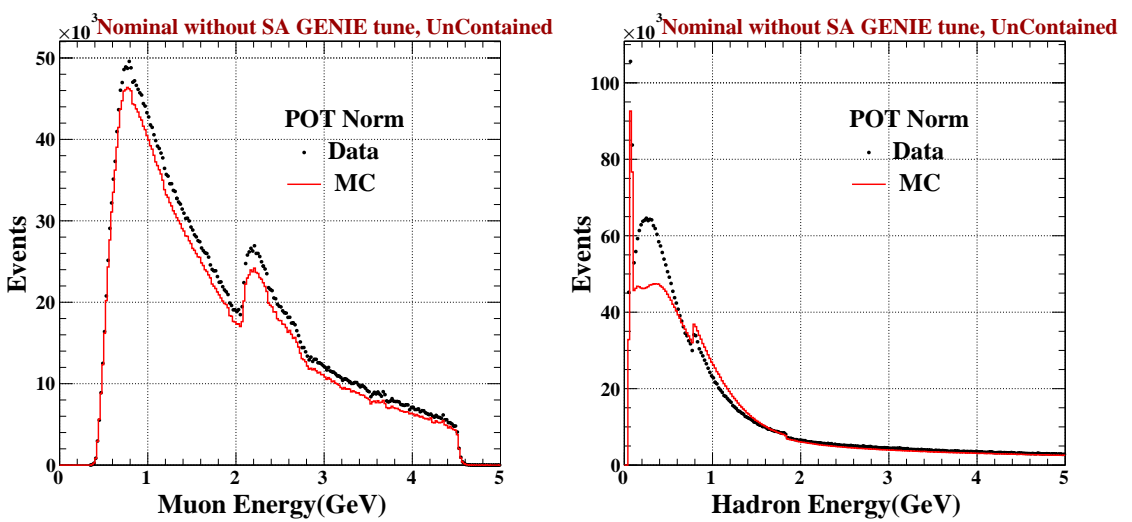

Figure D.3: Left: $\mathrm{E}_{\mu}$ distribution of Data-MC for uncontained sample with out GENIE Tune. Right: $\mathrm{E}_{\text {had }}$ distribution of Data-MC for uncontained sample without GENIE Tune.
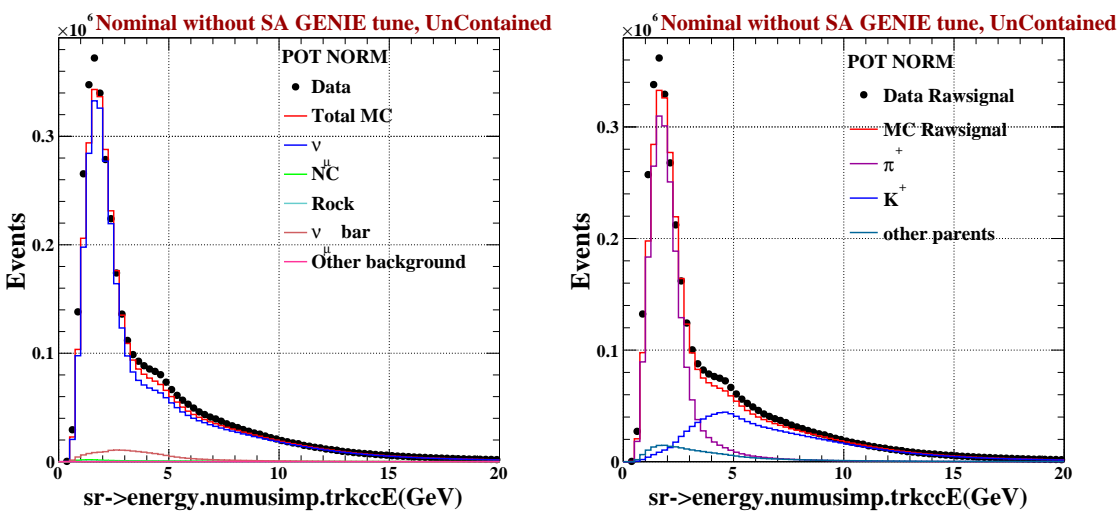

Figure D.4: Reconstructed Neutrino Energy(TrkCCE) distribution for uncontained sample with out GENIE Tune, Left: Data events and MC with separate background. Right: Raw data signal and MC.
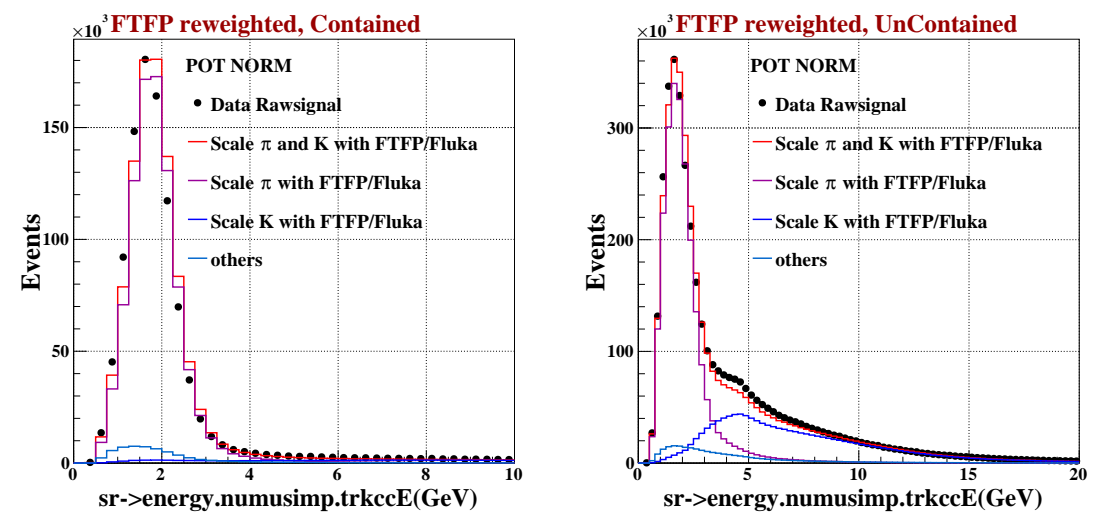

Figure D.5: Reconstructed Neutrino Energy(TrkCCE) distribution rewieghted with FTFP/Fluka weight, Left: Contained and Right: UnContained. 


\begin{tabular}{|c||c|c|c|c|}
\hline Variable & $.75-3$ & $3-4.5$ & $4.5-10$ & $10-20$ \\
\hline Data & 2276153 & 560455 & 923601 & 297517 \\
MC & 2113862.11 & 520164.02 & 832456.11 & 283739.93 \\
Raw Data Signal (Data-MC ${ }_{b k g}$ ) & 2184904.01 & 499936.49 & 857261.99 & 281144.04 \\
Signal MC & 2022613.11 & 459645.51 & 766117.1 & 267366.97 \\
MC Background & 91248.99 & 60518.51 & 66339.01 & 16372.96 \\
From Pion MC & 1801360.4 & 174777.32 & 82924.49 & 8752.99 \\
From Kaon MC & 111283.32 & 232216.85 & 623651.9 & 243009.74 \\
From otherparent & 109969.39 & 52651.35 & 59540.7 & 15604.24 \\
\hline Raw & Signal & w.r.t & parent & type \\
\hline RawDtSigPi & 1963651.29 & 215068.30 & 174069.38 & 22530.06 \\
RawDataSigK & 273574.22 & 272507.83 & 714796.79 & 256786.82 \\
Signal MCpi & 1801360.40 & 174777.32 & 82924.49 & 8752.99 \\
Signal MCK & 111283.32 & 232216.85 & 623651.90 & 243009.74 \\
$N_{\pi}$ & 1.09 & 1.23 & 2.10 & 2.57 \\
$N_{K}$ & 2.46 & 1.17 & 1.15 & 1.06 \\
\hline
\end{tabular}

Table D.2: $\nu_{\mu}$ - CC event counts for Uncontained without GENIE Tune.

\begin{tabular}{|c|}
\hline$\pi^{+}$norm for .75-3 GeV in contained sample $\tilde{1.0}$ Table 6.4 \\
\hline after normalization of $\pi^{+}($eq.1) for Uncontained sample \\
$N_{K}$ is 1.15(4-10GeV)- $N_{\pi} 1 .(0.75-3 \mathrm{GeV})$ \\
$<-$ after iteration \\
\hline$\pi^{+}--2.04 \%$ \\
$K^{+}--1.71 \%$ \\
\hline
\end{tabular}

Table D.3: $K^{+}$Norm without GENIE Tune

\begin{tabular}{|c|}
\hline$\pi^{+}$norm for .75-3 GeV in contained sample 0.97 Table 6.4 \\
\hline After normalization of $\pi^{+}($eq.1) for Uncontained sample \\
$N_{K}$ is $1.13(4.5-10 \mathrm{GeV})-N_{\pi} 0.96(0.75-3 \mathrm{GeV})$ \\
$N_{K}$ is $1.14(4.5-10 \mathrm{GeV})-N_{\pi} 0.96(0.75-3 \mathrm{GeV})$ \\
$<-$ after iteration \\
$\Delta\left(\pi^{+}\right) 2.04 \%$ \\
$\Delta\left(K^{+}\right) 2.6 \%$ \\
\hline
\end{tabular}

Table D.4: $K^{+}$Norm rewieghted with FTFP/Fluka weight. 

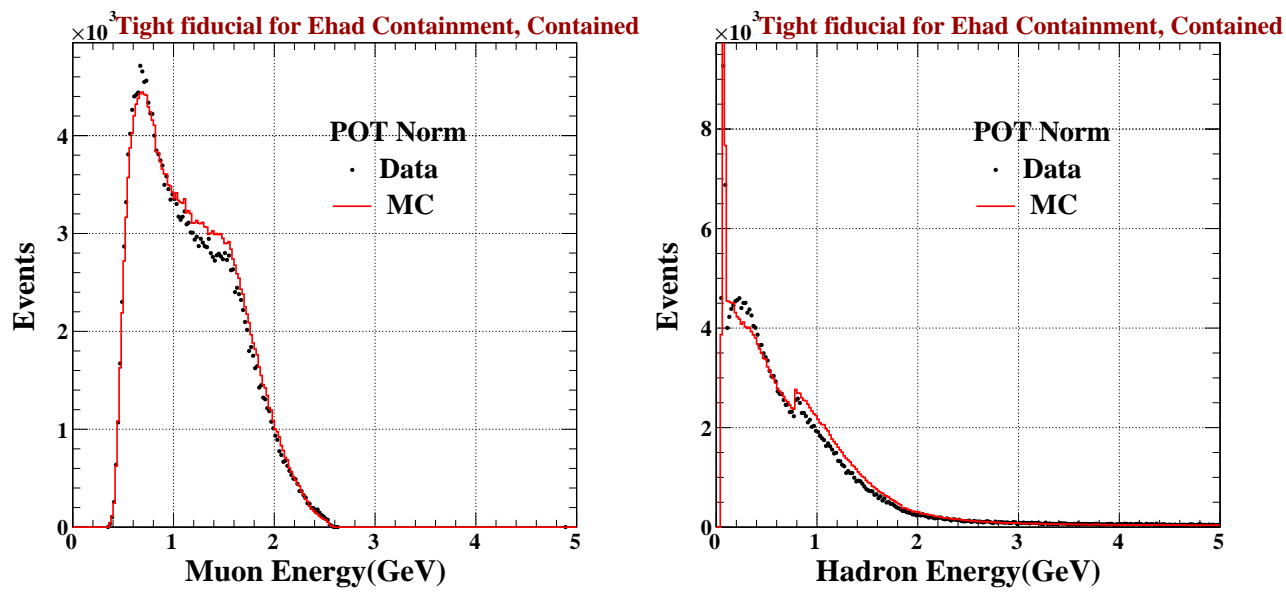

Figure D.6: Left: $\mathrm{E}_{\mu}$ distribution of Data-MC for contained sample. Right: $\mathrm{E}_{\text {had }}$ distribution of Data-MC for contained sample for tight fiducial cut.
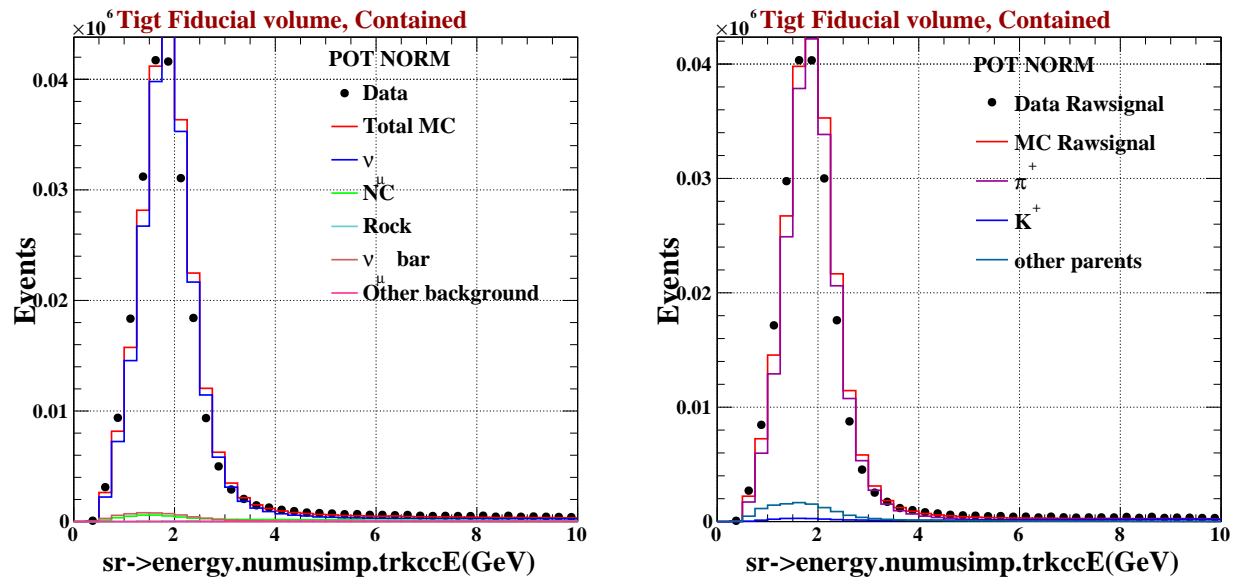

Figure D.7: Reconstructed Neutrino Energy(TrkCCE) distribution for contained sample tight fiducial cut, Left: Data events and MC with separate background. Right: Raw data signal and MC. 


\begin{tabular}{|c||c|c|c|c|}
\hline Variable & $.75-3$ & $3-4.5$ & $4.5-10$ & $10-20$ \\
\hline Data & 206102 & 9705 & 12261 & 6557 \\
MC & 215691.24 & 10092.74 & 9990.3 & 5960.94 \\
Raw Data Signal (Data-MC ${ }_{b k g}$ ) & 196944.6 & 7964.48 & 8846.73 & 5235.66 \\
Signal MC & 206533.83 & 8352.22 & 6576.03 & 4639.61 \\
MC Background & 9157.4 & 1740.52 & 3414.27 & 1321.34 \\
From Pion MC & 194393.99 & 6719.86 & 1824.87 & 205.86 \\
From Kaon MC & 1810.81 & 676.23 & 3916.02 & 4182.58 \\
From otherparent & 10329.03 & 956.14 & 835.13 & 251.17 \\
\hline Raw & Signal & w.r.t & parent & type \\
\hline RawDtSigPi & 184804.76 & 6332.12 & 4095.58 & 834.00 \\
RawDataSigK & 8271.15 & 756.25 & 6186.73 & 4778.63 \\
Signal MCpi & 194393.99 & 6719.86 & 1824.87 & 205.86 \\
Signal MCK & 1810.81 & 676.23 & 3916.02 & 4182.58 \\
$N_{\pi}$ & 0.95 & 0.94 & 2.24 & 4.05 \\
$N_{K}$ & 4.57 & 1.12 & 1.58 & 1.14 \\
\hline
\end{tabular}

Table D.5: $\quad \nu_{\mu}-\mathrm{CC}$ event counts for Contained for $\mathrm{E}_{\text {had }}$ Containment, tight fiducial volume.
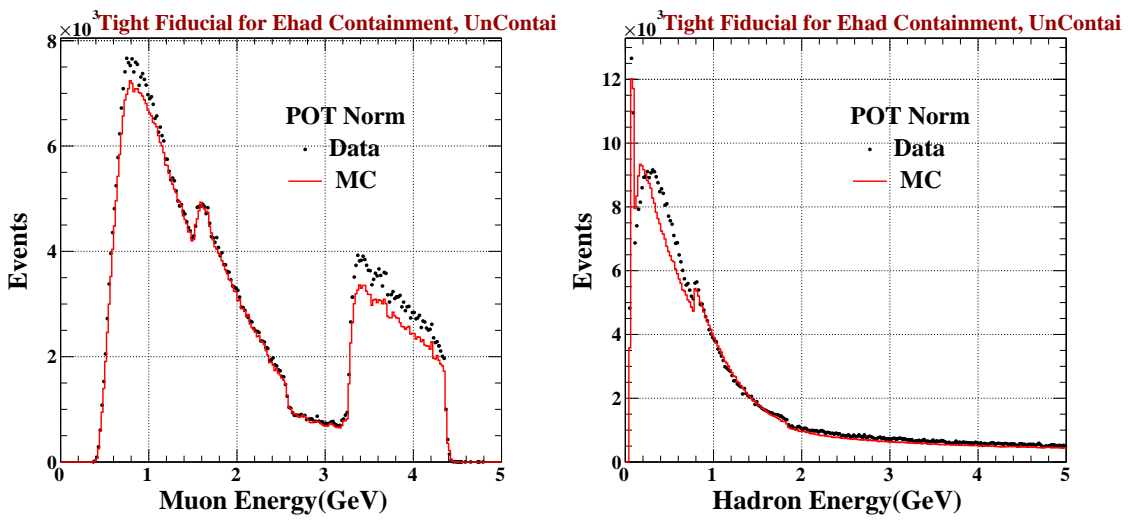

Figure D.8: Left: $\mathrm{E}_{\mu}$ distribution of Data-MC for uncontained sample. Right: $\mathrm{E}_{\text {had }}$ distribution of Data-MC for uncontained sample for tight fiducial cut. 

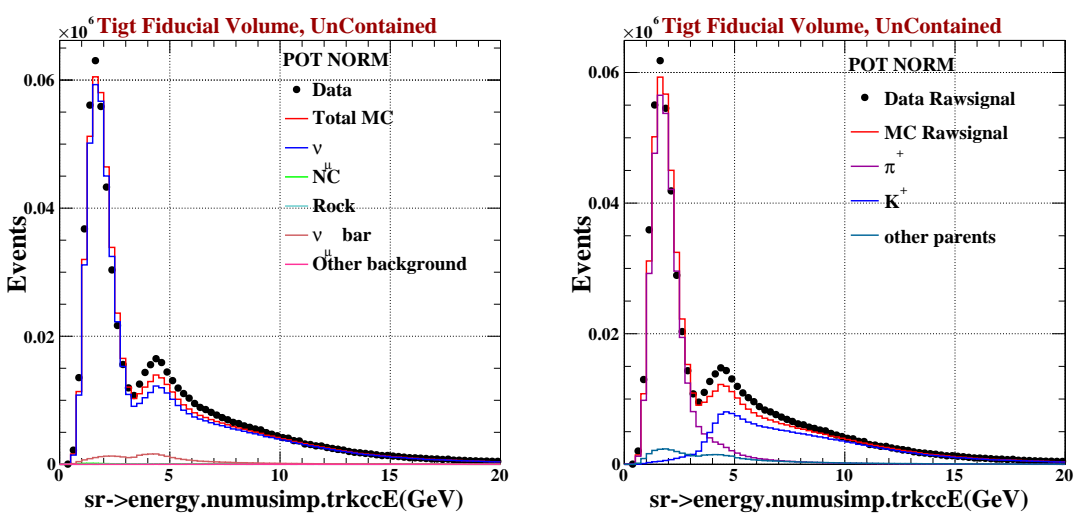

Figure D.9: Reconstructed Neutrino Energy(TrkCCE) distribution for uncontained sample for tight fiducial cut, Left: Data events and MC with separate background. Right: Raw data signal and MC.

\begin{tabular}{|c||c|c|c|c|}
\hline Variable & $.75-3$ & $3-4.5$ & $4.5-10$ & $10-20$ \\
\hline Data & 336214 & 81621 & 183451 & 66448 \\
MC & 333593.22 & 72295.68 & 159960.52 & 63312.51 \\
Raw Data Signal (Data-MC ${ }_{b k g}$ ) & 325734.29 & 72651.07 & 170941.25 & 63439.3 \\
Signal MC & 323113.5 & 63325.74 & 147450.76 & 60303.8 \\
MC Background & 10479.71 & 8969.93 & 12509.75 & 3008.7 \\
From Pion MC & 300454.46 & 29062.41 & 16910.61 & 1990.88 \\
From Kaon MC & 5935.44 & 25839.44 & 118423.69 & 54844.15 \\
From otherparent & 16723.61 & 8423.89 & 12116.47 & 3468.78 \\
\hline Raw & Signal & w.r.t & parent & type \\
\hline RawDtSigPi & 303075.24 & 38387.73 & 40401.09 & 5126.37 \\
RawDataSigK & 15986.63 & 35164.77 & 141914.17 & 57979.64 \\
Signal MCpi & 300454.46 & 29062.41 & 16910.61 & 1990.88 \\
Signal MCK & 5935.44 & 25839.44 & 118423.69 & 54844.15 \\
$N_{\pi}$ & 1.01 & 1.32 & 2.39 & 2.57 \\
$N_{K}$ & 2.69 & 1.36 & 1.20 & 1.06 \\
\hline
\end{tabular}

Table D.6: $\nu_{\mu}-\mathrm{CC}$ event counts for Uncontained for $\mathrm{E}_{\text {had }}$ Containment, tight fiducial volume.

\begin{tabular}{|c|}
\hline$\pi^{+}$norm for .75-3 $\mathrm{GeV}$ in contained sample 0.95 Table 6.4 \\
\hline After normalization of $\pi^{+}$(eq.1) for Uncontained sample \\
$N_{K}$ is $1.21(4.5-10 \mathrm{GeV})-N_{\pi} 0.95(0.75-3 \mathrm{GeV})$ \\
$N_{K}$ is $1.21(4.5-10 \mathrm{GeV})-N_{\pi} 0.95(0.75-3 \mathrm{GeV})$ \\
$<-$ after iteration \\
\hline$\pi^{+}---3.1 \%$ \\
$K^{+}--3.4 \%$ \\
\hline
\end{tabular}

Table D.7: $K^{+}$Norm for $\mathrm{E}_{\text {had }}$ Containment, tight fiducial volume. 


\section{Appendix E}

\section{Supplementary Material for}

\section{Chapter 6 Part 2}
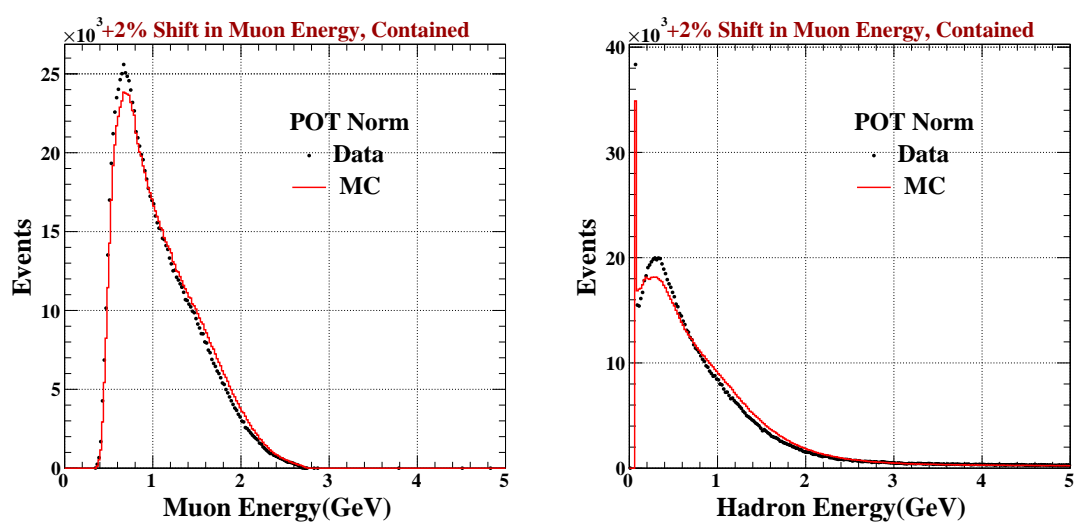

Figure E.1: Left: $\mathrm{E}_{\mu}$ distribution of Data-MC for contained sample. Right: $\mathrm{E}_{\text {had }}$ distribution of Data-MC for contained sample for $+2 \%$ shift in muon energy. 

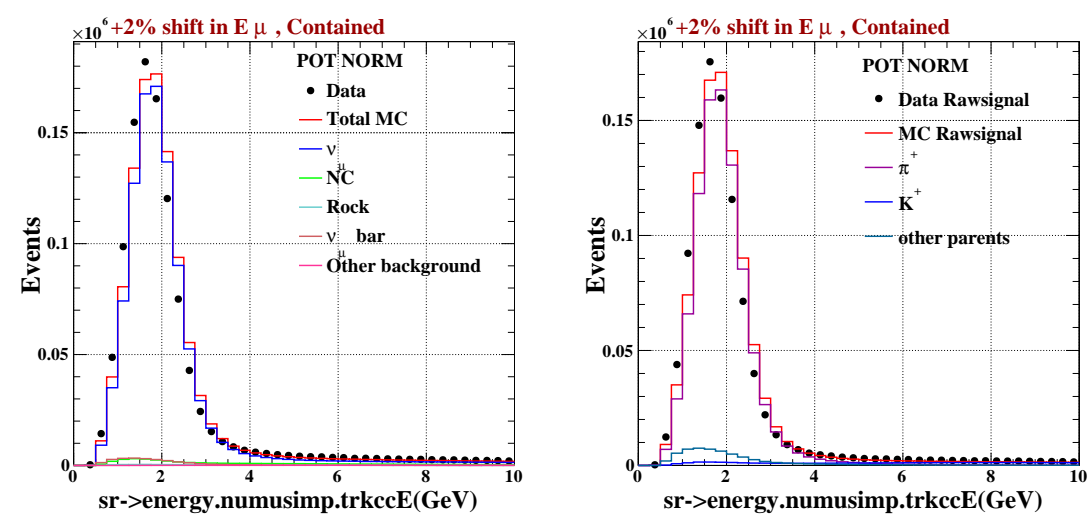

Figure E.2: Reconstructed Neutrino Energy (TrkCCE) distribution for contained sample $+2 \%$ shift in muon energy, Left: Data events and MC with separate background. Right: Raw data signal and MC.

\begin{tabular}{|c|c|c|c|c|}
\hline Variable & $.75-3$ & $3-4.5$ & $4.5-10$ & $10-20$ \\
\hline Data & 911928 & 52693 & 68291 & 32678 \\
\hline $\mathrm{MC}$ & 927253.39 & 56911.26 & 58750.73 & 31256.28 \\
\hline Raw Data Signal (Data-MC $\mathrm{MC}_{b k}$ ) & 868313.4 & 43429.8 & 50524.86 & 25552.4 \\
\hline Signal MC & 883638.8 & 47648.06 & 40984.59 & 24130.68 \\
\hline MC Background & 43614.6 & 9263.2 & 17766.14 & 7125.6 \\
\hline From Pion MC & 826717.92 & 36921.2 & 10172.54 & 1110.85 \\
\hline From Kaon MC & 10797.42 & 5788.72 & 26169.86 & 21689.74 \\
\hline From otherparent & 46123.45 & 4938.15 & 4642.18 & 1330.09 \\
\hline Raw & Signal & w.r.t & parent & type \\
\hline RawDtSigPi & 811392.53 & 32702.94 & 19712.82 & 2607.34 \\
\hline RawDataSigK & 60485.76 & 4461.87 & 35710.13 & 23111.46 \\
\hline Signal MCpi & 826717.92 & 36921.20 & 10172.54 & 1110.85 \\
\hline Signal MCK & 10797.42 & 5788.72 & 26169.86 & 21689.74 \\
\hline$N_{\pi}$ & 0.98 & 0.89 & 1.94 & 2.35 \\
\hline$N_{K}$ & 5.60 & 0.77 & 1.36 & 1.07 \\
\hline
\end{tabular}

Table E.1: $\nu_{\mu}-\mathrm{CC}$ event counts for Contained for $\mathrm{E}_{\mu}$ Shift $+2 \%$ in MC. 

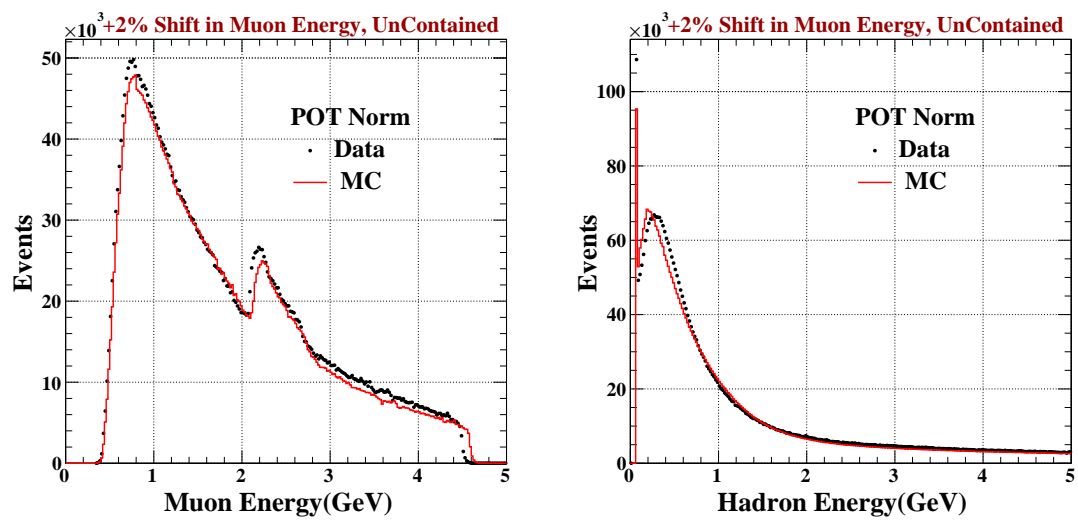

Figure E.3: Left: $\mathrm{E}_{\mu}$ distribution of Data-MC for contained sample. Right: $\mathrm{E}_{\text {had }}$ distribution of Data-MC for uncontained sample for $+2 \%$ shift in muon energy.
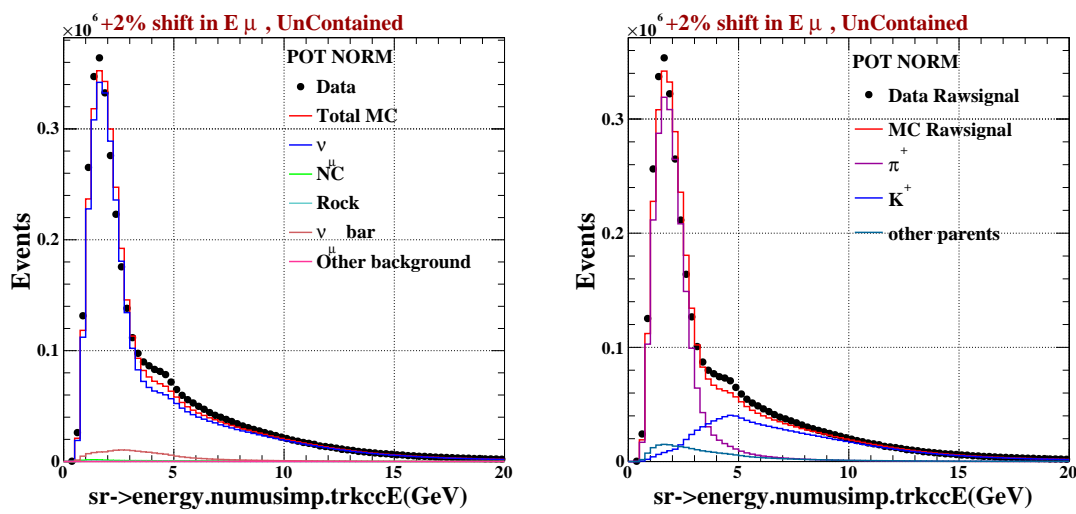

Figure E.4: Reconstructed Neutrino Energy(TrkCCE) distribution for uncontained sample $+2 \%$ shift in muon energy, Left: Data events and MC with separate background. Right: Raw data signal and MC.
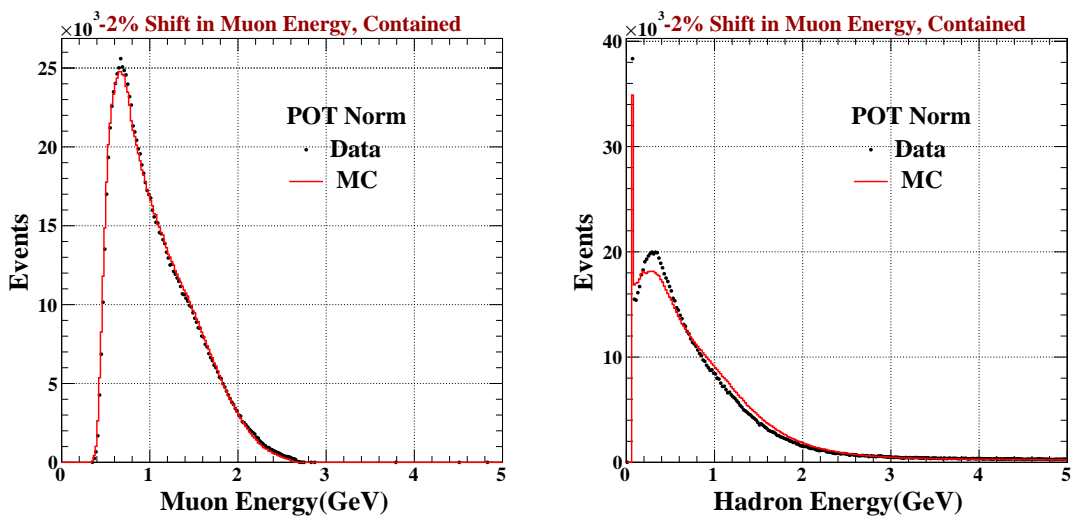

Figure E.5: Left: $\mathrm{E}_{\mu}$ distribution of Data-MC for contained sample. Right: $\mathrm{E}_{\text {had }}$ distribution of Data-MC for contained sample for $-2 \%$ shift in muon energy. 


\begin{tabular}{|c||c|c|c|c|}
\hline Variable & $.75-3$ & $3-4.5$ & $4.5-10$ & $10-20$ \\
\hline Data & 2253246 & 549947 & 930049 & 322079 \\
MC & 2253888.47 & 507224.74 & 835245.79 & 311011.96 \\
Raw Data Signal (Data-MC ${ }_{b k g}$ ) & 2161590.18 & 492231.24 & 861629.38 & 304247.71 \\
Signal MC & 2162232.65 & 449508.98 & 766826.17 & 293180.67 \\
MC Background & 91655.82 & 57715.76 & 68419.62 & 17831.29 \\
From Pion MC & 1950259.71 & 195169.57 & 90356.29 & 10105.95 \\
From Kaon MC & 98105.77 & 203267.34 & 614808.83 & 266086.24 \\
From otherparent & 113867.18 & 51072.07 & 61661.05 & 16988.47 \\
\hline Raw & Signal & w.r.t & parent & type \\
\hline RawDtSigPi & 1949617.24 & 237891.83 & 185159.51 & 21172.99 \\
RawDataSigK & 121228.95 & 245989.60 & 709612.04 & 277153.28 \\
Signal MCpi & 1950259.71 & 195169.57 & 90356.29 & 10105.95 \\
Signal MCK & 98105.77 & 203267.34 & 614808.83 & 266086.24 \\
$N_{\pi}$ & 1.00 & 1.22 & 2.05 & 2.10 \\
$N_{K}$ & 1.24 & 1.21 & 1.15 & 1.04 \\
\hline
\end{tabular}

Table E.2: $\nu_{\mu}-\mathrm{CC}$ event counts for Uncontained for $\mathrm{E}_{\mu}$ Shift $+2 \%$ in MC.

\begin{tabular}{|c|}
\hline$\pi^{+}$norm for .75-3 GeV in contained sample0̃.981 Table 6.4 \\
\hline After normalization of $\pi^{+}$(eq.1) for Uncontained sample \\
$N_{K}$ is $1.16(4.5-10 \mathrm{GeV})-N_{\pi} 0.98(0.75-3 \mathrm{GeV})$ \\
$N_{K}$ is $1.16(4.5-10 \mathrm{GeV})-N_{\pi} 0.98(0.75-3 \mathrm{GeV})$ \\
$<-$ after iteration \\
\hline$\pi^{+}--0.1 \%$ \\
$K^{+}--0.9 \%$ \\
\hline
\end{tabular}

Table E.3: $K^{+}$Norm for for $\mathrm{E}_{\mu}$ Shift $+2 \%$ in MC.
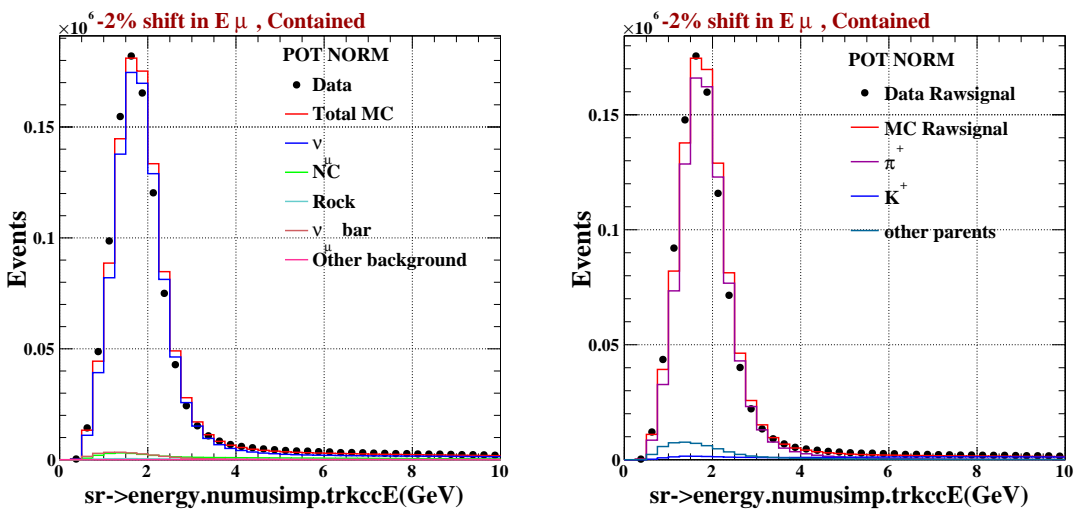

Figure E.6: Reconstructed Neutrino Energy(TrkCCE) distribution for contained sample $-2 \%$ shift in muon energy, Left: Data events and MC with separate background. Right: Raw data signal and MC. 


\begin{tabular}{|c||c|c|c|c|}
\hline Variable & $.75-3$ & $3-4.5$ & $4.5-10$ & $10-20$ \\
\hline Data & 911928 & 52693 & 68291 & 32678 \\
MC & 929213.01 & 53395.19 & 58259.48 & 30982.46 \\
Raw Data Signal (Data-MC ${ }_{b k g}$ ) & 868338.76 & 43571.36 & 50640.37 & 25614.49 \\
Signal MC & 885623.77 & 44273.55 & 40608.85 & 23918.96 \\
MC Background & 43589.24 & 9121.64 & 17650.63 & 7063.51 \\
From Pion MC & 828648.62 & 33739.68 & 9852.61 & 1093.25 \\
From Kaon MC & 10946.13 & 5777.89 & 26189.26 & 21511.1 \\
From otherparent & 46029.02 & 4755.98 & 4566.98 & 1314.61 \\
\hline Raw & Signal & w.r.t & parent & type \\
\hline RawDtSigPi & 811363.61 & 33037.49 & 19884.12 & 2834.12 \\
RawDataSigK & 30405.07 & 5881.54 & 36220.78 & 23206.64 \\
Signal MCpi & 828648.62 & 33739.68 & 9852.61 & 1093.25 \\
Signal MCK & 10946.13 & 5777.89 & 26189.26 & 21511.10 \\
$N_{\pi}$ & 0.98 & 0.98 & 2.02 & 2.59 \\
$N_{K}$ & 2.78 & 1.02 & 1.38 & 1.08 \\
\hline
\end{tabular}

Table E.4: $\quad \nu_{\mu}-\mathrm{CC}$ event counts for Contained for $\mathrm{E}_{\mu}$ Shift $-2 \%$ in MC
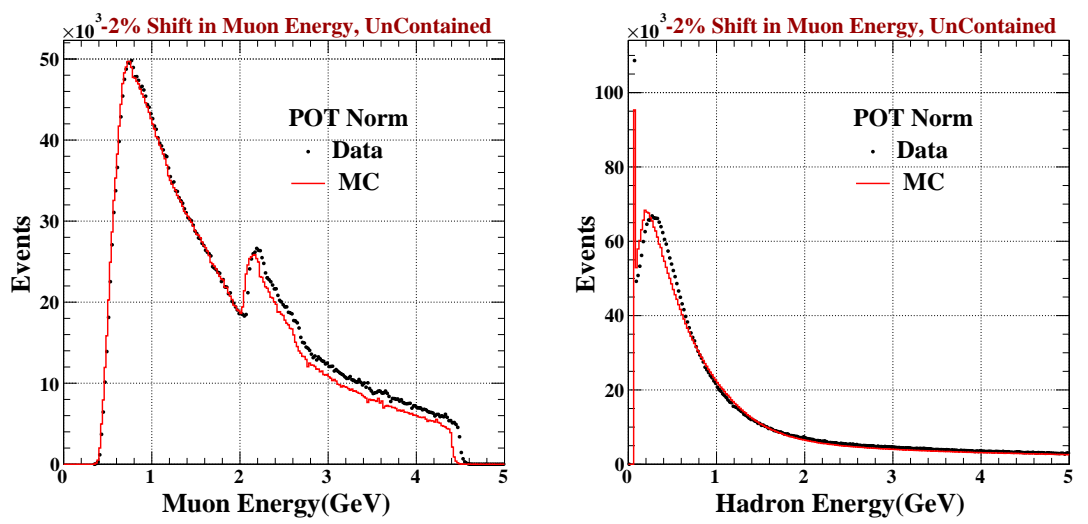

Figure E.7: Left: $\mathrm{E}_{\mu}$ distribution of Data-MC for contained sample. Right: $\mathrm{E}_{\text {had }}$ distribution of Data-MC for contained sample for $-2 \%$ shift in muon energy. 

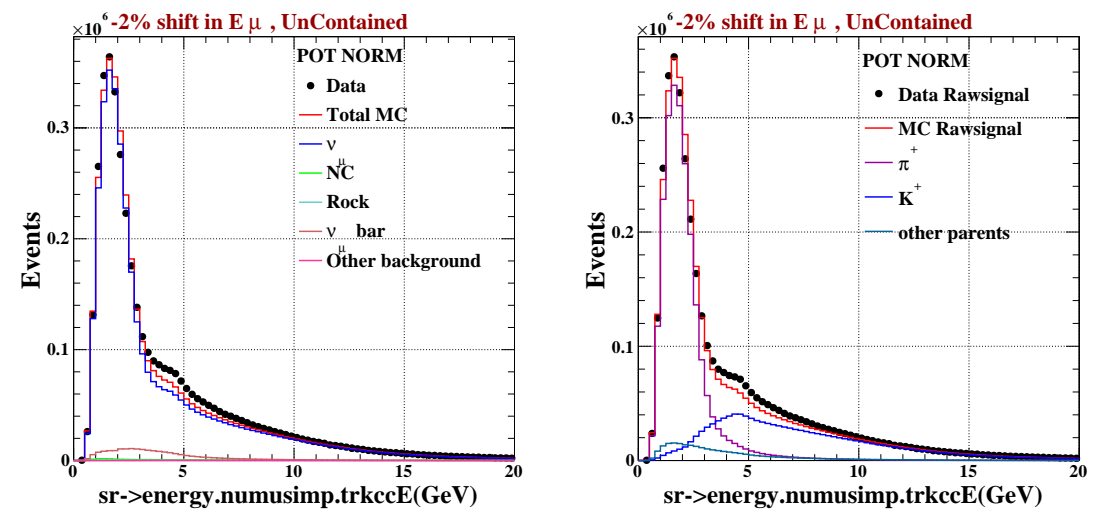

Figure E.8: Reconstructed Neutrino Energy(TrkCCE) distribution for uncontained sample $-2 \%$ shift in muon energy, Left: Data events and MC with separate background. Right: Raw data signal and MC.

\begin{tabular}{|c||c|c|c|c|}
\hline \multicolumn{1}{|c||}{ Variable } & $.75-3$ & $3-4.5$ & $4.5-10$ & $10-20$ \\
\hline Data & 2253246 & 549947 & 930049 & 322079 \\
MC & 2287902.77 & 497483.4 & 813087.69 & 303769.03 \\
Raw Data Signal (Data-MC ${ }_{b k g}$ ) & 2158288.7 & 492407.38 & 864748.41 & 304649.51 \\
Signal MC & 2192945.47 & 439943.78 & 747787.09 & 286339.53 \\
of Bkgrnd & 94957.3 & 57539.62 & 65300.59 & 17429.49 \\
from Pion & 1969237.08 & 176933.75 & 84993.06 & 9774.07 \\
from Kaon & 106829.11 & 212611.45 & 603474.07 & 259957.74 \\
from otherparent & 116879.28 & 50398.58 & 59319.96 & 16607.73 \\
\hline Raw & Signal & w.r.t & parent & type \\
\hline RawDtSigPi & 1934580.31 & 229397.35 & 201954.37 & 28084.04 \\
RawDataSigK & 86200.58 & 265075.05 & 720435.39 & 278267.71 \\
Signal MCpi & 1969237.08 & 176933.75 & 84993.06 & 9774.07 \\
Signal MCK & 106829.11 & 212611.45 & 603474.07 & 259957.74 \\
$N_{\pi}$ & 0.98 & 1.30 & 2.38 & 2.87 \\
$N_{K}$ & 0.81 & 1.25 & 1.19 & 1.07 \\
\hline
\end{tabular}

Table E.5: $\quad \nu_{\mu}$ - CC event counts for Uncontained for $\mathrm{E}_{\mu}$ Shift $-2 \%$ in MC.

\begin{tabular}{|c|}
\hline$\pi^{+}$norm for .75-3 $\mathrm{GeV}$ in contained sample0̃.98 Table 6.4 \\
\hline After normalization of $\pi^{+}$(eq.1) for uncontained sample \\
$N_{K}$ is $1.20(4.5-10 \mathrm{GeV})-N_{\pi} 0.977(0.75-3 \mathrm{GeV})$ \\
$N_{K}$ is $1.20(4.5-10 \mathrm{GeV})-N_{\pi} 0.977(0.75-3 \mathrm{GeV})$ \\
$<-$ after iteration \\
\hline$\pi^{+}---0.3 \%$ \\
$K^{+}--2.6 \%$ \\
\hline
\end{tabular}

Table E.6: $K^{+}$Norm for for $\mathrm{E}_{\mu}$ Shift $-2 \%$ in MC. 


\section{Appendix F}

\section{Supplementary Material for}

\section{Chapter 6 Part 3}
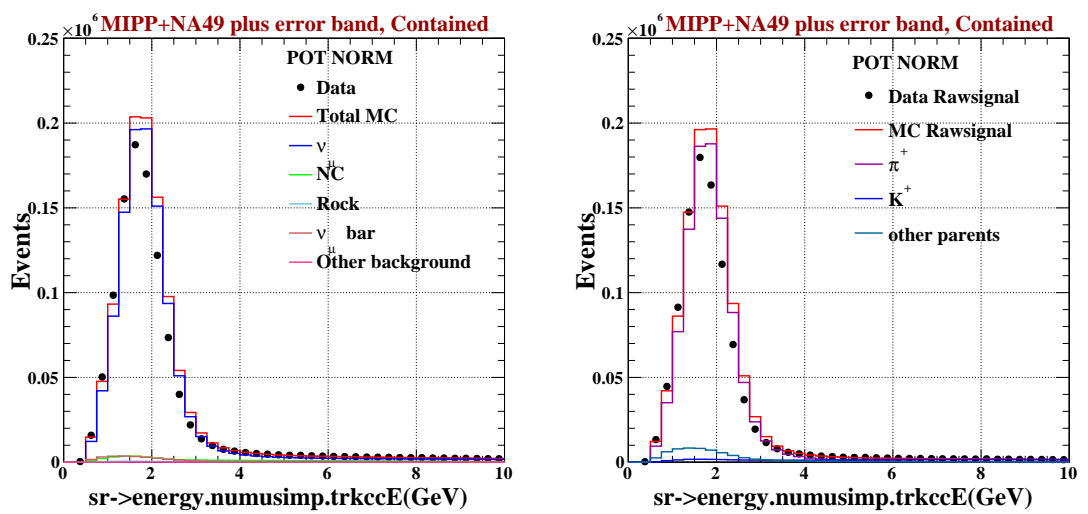

Figure F.1: Reconstructed Neutrino Energy(TrkCCE) distribution for contained sample MIPP+NA49 error band plus only, Left: Data events and MC with separate background. Right: Raw data signal and MC. 


\begin{tabular}{|c|c|c|c|c|}
\hline Variable & $.75-3$ & $3-4.5$ & $4.5-10$ & $10-20$ \\
\hline Data & 918740 & 48603 & 67335 & 29926 \\
\hline $\mathrm{MC}$ & 1040248.88 & 54823.6 & 67331.5 & 33874.35 \\
\hline Raw Data Signal (Data-MC $\mathrm{M}_{b k g}$ ) & 869283.01 & 38119.36 & 46936.31 & 22517.63 \\
\hline Signal MC & 990791.9 & 44339.96 & 46932.82 & 26465.98 \\
\hline MC Background & 49456.99 & 10483.64 & 20398.69 & 7408.37 \\
\hline From Pion MC & 926467.01 & 32127.61 & 9937.44 & 1150.01 \\
\hline From Kaon MC & 12775.84 & 7138.06 & 32026.88 & 23883.15 \\
\hline From otherparent & 51549.05 & 5074.28 & 4968.49 & 1432.83 \\
\hline Raw & Signal & w.r.t & parent & type \\
\hline RawDtSigPi & 804958.12 & 25907.01 & 9959.95 & 137.20 \\
\hline RawDataSigK & 11857.49 & 3557.24 & 32030.38 & 19934.80 \\
\hline Signal MCpi & 926467.01 & 32127.61 & 9937.44 & 1150.01 \\
\hline Signal MCK & 12775.84 & 7138.06 & 32026.88 & 23883.15 \\
\hline$N_{\pi}$ & 0.87 & 0.81 & 1.00 & 0.12 \\
\hline$N_{K}$ & 0.93 & 0.50 & 1.00 & 0.83 \\
\hline
\end{tabular}

Table F.1: $\nu_{\mu}-$ CC event counts for Contained for MIPP+NA49 error band plus.

\begin{tabular}{|c|}
\hline$\pi^{+}$norm for .75-3 $\mathrm{GeV}$ in contained sample 0.87 Table 6.4 \\
\hline After normalization of $\pi^{+}$(eq.1) for Uncontained sample \\
$N_{K}$ is $1.18(4.5-10 \mathrm{GeV})-N_{\pi} 0.87(0.75-3 \mathrm{GeV})$ \\
$N_{K}$ is $1.18(4.5-10 \mathrm{GeV})-N_{\pi} 0.87(0.75-3 \mathrm{GeV})$ \\
$<$-after iteration \\
\hline$\pi^{+}--11.7 \%$ \\
$K^{+}--0.9 \%$ \\
\hline
\end{tabular}

Table F.2: $K^{+}$Norm for MIPP+NA49 error band plus only to contained.
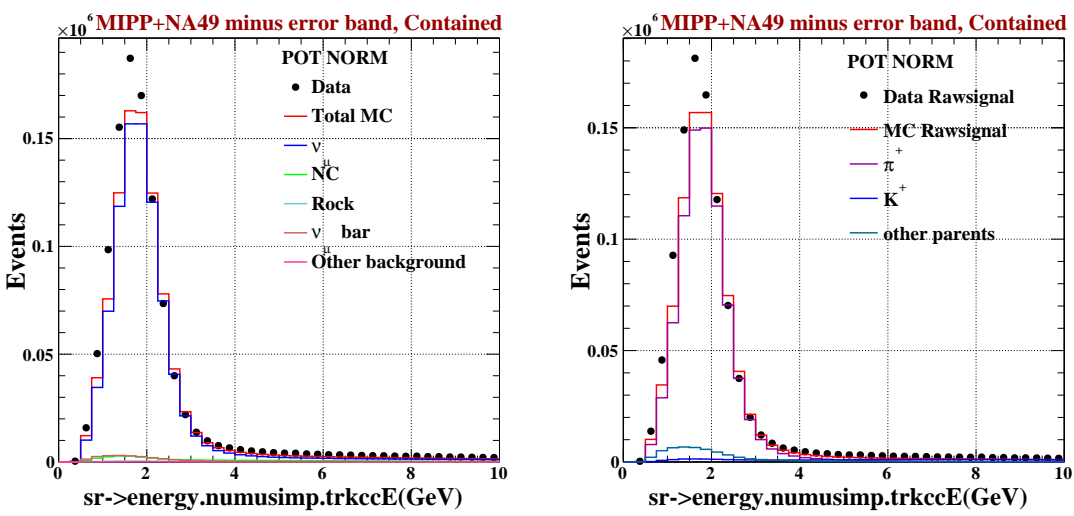

Figure F.2: Reconstructed Neutrino Energy(TrkCCE) distribution for contained sample MIPP+NA49 Error Band Minus Only, Left: Data events and MC with separate background. Right: Raw data signal and MC. 


\begin{tabular}{|c||c|c|c|c|}
\hline Variable & $.75-3$ & $3-4.5$ & $4.5-10$ & $10-20$ \\
\hline Data & 918740 & 48603 & 67335 & 29926 \\
MC & 833691.9 & 42962.93 & 47943.41 & 23579.54 \\
Raw Data Signal (Data-MC $\mathrm{Mk}_{b g}$ ) & 879028.83 & 40754.52 & 53076.25 & 24408.25 \\
Signal MC & 793980.73 & 35114.46 & 33684.65 & 18061.79 \\
MC Background & 39711.17 & 7848.48 & 14258.75 & 5517.75 \\
From Pion MC & 742568.42 & 25773.26 & 7675.48 & 760.27 \\
From Kaon MC & 9889.8 & 5277.06 & 22275.61 & 16305.47 \\
From otherparent & 41522.51 & 4064.13 & 3733.57 & 996.06 \\
\hline Raw & Signal & w.r.t & parent & type \\
\hline RawDtSigPi & 827616.52 & 31413.33 & 27067.07 & 7106.73 \\
RawDataSigK & 102488.04 & 10917.13 & 41667.20 & 22651.93 \\
Signal MCpi & 742568.42 & 25773.26 & 7675.48 & 760.27 \\
Signal MCK & 9889.80 & 5277.06 & 22275.61 & 16305.47 \\
$N_{\pi}$ & 1.11 & 1.22 & 3.53 & 9.35 \\
$N_{K}$ & 10.36 & 2.07 & 1.87 & 1.39 \\
\hline
\end{tabular}

Table F.3: $\quad \nu_{\mu}-\mathrm{CC}$ event counts for Contained for MIPP+NA49 error band minus.

\begin{tabular}{|c|}
\hline$\pi^{+}$norm for .75-3 $\mathrm{GeV}$ in contained sample1.12 Table 6.4 \\
\hline After normalization of $\pi^{+}$(eq.1) for Uncontained sample \\
$N_{K}$ is $1.15(4.5-10 \mathrm{GeV})-N_{\pi} 1.11(0.75-3 \mathrm{GeV})$ \\
$N_{K}$ is $1.15(4.5-10 \mathrm{GeV})-N_{\pi} 1.11(0.75-3 \mathrm{GeV})$ \\
$<$-after iteration \\
\hline$\pi^{+}---13 \%$ \\
$K^{+}---1.7 \%$ \\
\hline
\end{tabular}

Table F.4: $K^{+}$Norm for MIPP+NA49 error band minus only to contained.
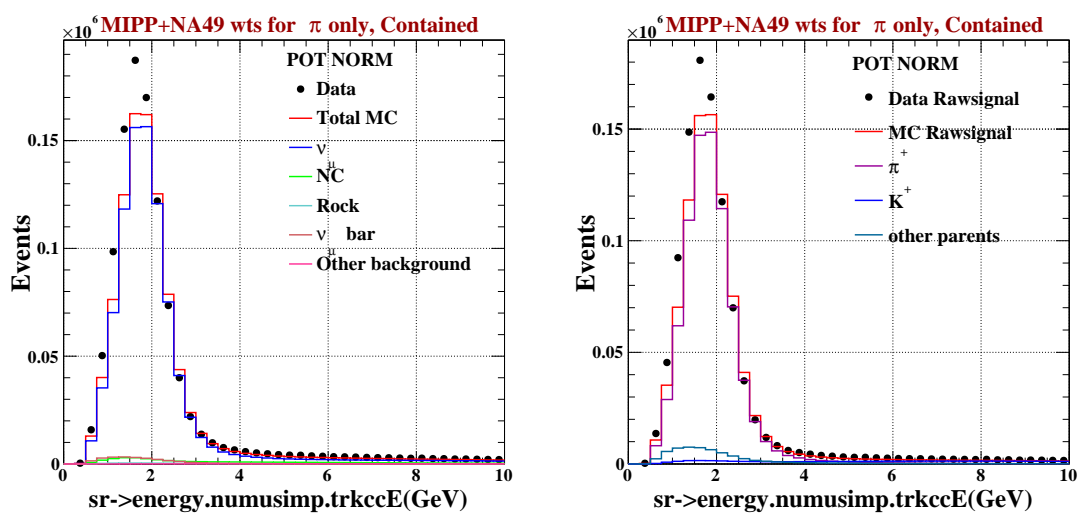

Figure F.3: Reconstructed Neutrino Energy(TrkCCE) distribution for contained sample MIPP+NA49 weight for pion only, Left: Data events and MC with separate background. Right: Raw data signal and MC. 


\begin{tabular}{|c||c|c|c|c|}
\hline Variable & $.75-3$ & $3-4.5$ & $4.5-10$ & $10-20$ \\
\hline Data & 918740 & 48603 & 67335 & 29926 \\
MC & 837432.07 & 45839.65 & 57512.36 & 28850.28 \\
Raw Data Signal (Data-MC Mkg $_{1}$ ) & 876150.64 & 39519.17 & 49976.74 & 23453.13 \\
Signal MC & 794842.71 & 36755.82 & 40154.1 & 22377.41 \\
MC Background & 42589.36 & 9083.83 & 17358.26 & 6472.87 \\
From Pion MC & 736974.11 & 25979.05 & 8651.82 & 1068.66 \\
From Kaon MC & 11332.82 & 6207.56 & 27151.25 & 20094.31 \\
From otherparent & 46535.78 & 4569.21 & 4351.03 & 1214.44 \\
\hline Raw & Signal & w.r.t & parent & type \\
\hline RawDtSigPi & 818282.04 & 28742.40 & 18474.46 & 2163.48 \\
RawDataSigK & 101990.54 & 8970.91 & 36973.89 & 21170.03 \\
Signal MCpi & 736974.11 & 25979.05 & 8651.82 & 1068.66 \\
Signal MCK & 11332.82 & 6207.56 & 27151.25 & 20094.31 \\
$N_{\pi}$ & 1.11 & 1.11 & 2.14 & 2.02 \\
$N_{K}$ & 9.00 & 1.45 & 1.36 & 1.05 \\
\hline
\end{tabular}

Table F.5: $\quad \nu_{\mu}-\mathrm{CC}$ event counts for Contained for MIPP+NA49 weight for pion only.
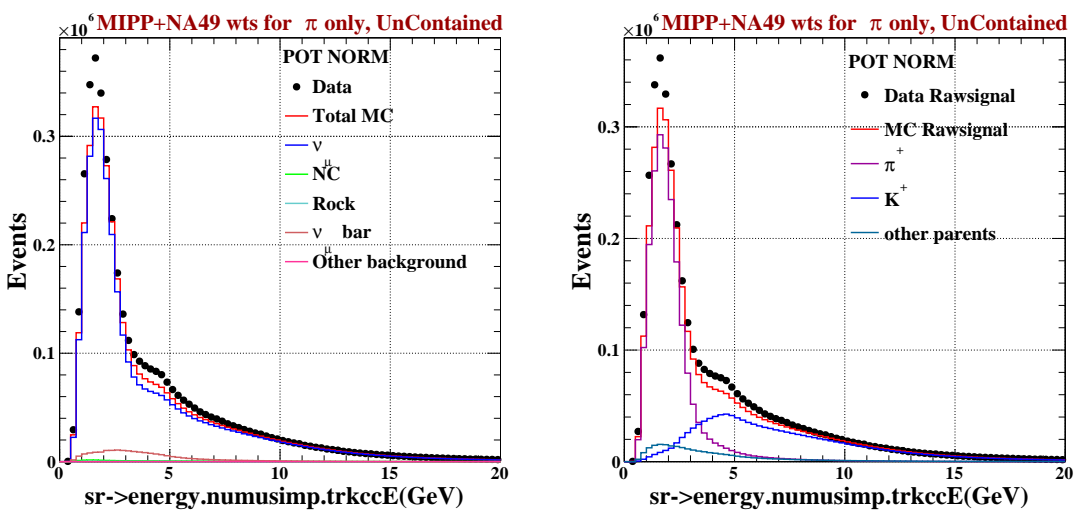

Figure F.4: Reconstructed Neutrino Energy(TrkCCE) distribution for uncontained sample MIPP+NA49 weight for pion only, Left: Data events and MC with separate background. Right: Raw data signal and MC.

\begin{tabular}{|c||c|c|c|c|}
\hline Variable & $.75-3$ & $3-4.5$ & $4.5-10$ & $10-20$ \\
\hline Data & 2276153 & 560455 & 923601 & 297517 \\
MC & 2065675.01 & 493716.96 & 822907.31 & 284600.5 \\
Raw Data Signal (Data-MC $M_{b k g}$ ) & 2182842.76 & 502529.95 & 858753.28 & 281174.59 \\
Signal MC & 1972364.77 & 435791.92 & 758059.59 & 268258.09 \\
MC Background & 93310.24 & 57925.05 & 64847.72 & 16342.41 \\
From Pion MC & 1746671.78 & 163459.2 & 84237.62 & 9723.72 \\
From Kaon MC & 107942.85 & 220995.32 & 615038.79 & 242935.13 \\
From otherparent & 117750.14 & 51337.39 & 58783.18 & 15599.24 \\
\hline Raw & Signal & w.r.t & parent & type \\
\hline RawDtSigPi & 1957149.77 & 230197.24 & 184931.30 & 22640.23 \\
RawDataSigK & 318420.84 & 287733.36 & 715732.47 & 255851.63 \\
Signal MCpi & 1746671.78 & 163459.20 & 84237.62 & 9723.72 \\
Signal MCK & 107942.85 & 220995.32 & 615038.79 & 242935.13 \\
$N_{\pi}$ & 1.12 & 1.41 & 2.20 & 2.33 \\
$N_{K}$ & 2.95 & 1.30 & 1.16 & 1.05 \\
\hline
\end{tabular}

Table F.6: $\nu_{\mu}$ - CC event counts for Uncontained for MIPP+NA49 weight for pion only. 


\begin{tabular}{|c|}
\hline$\pi^{+}$norm for .75-3 GeV in contained sample $\tilde{1} .11$ Table 6.4 \\
\hline After normalization of $\pi^{+}($eq.1) for un-contained sample \\
$N_{K}$ is $1.15(4.5-10 \mathrm{GeV})-N_{\pi} 1.11(0.75-3 \mathrm{GeV})$ \\
$N_{K}$ is $1.15(4.5-10 \mathrm{GeV})-N_{\pi} 1.11(0.75-3 \mathrm{GeV})$ \\
$<-$ after iteration \\
$\pi^{+}--11.7 \%$ \\
$K^{+}--1.7 \%$ \\
\hline
\end{tabular}

Table F.7: $K^{+}$Norm for MIPP+NA49 weight for pion only.
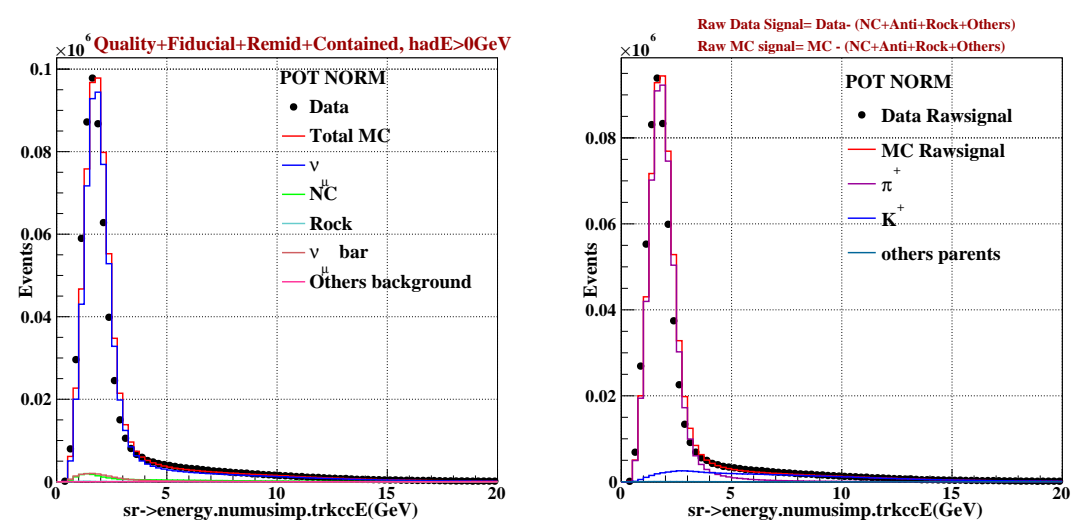

Figure F.5: An example: fReweightMaCCRES, Contained.
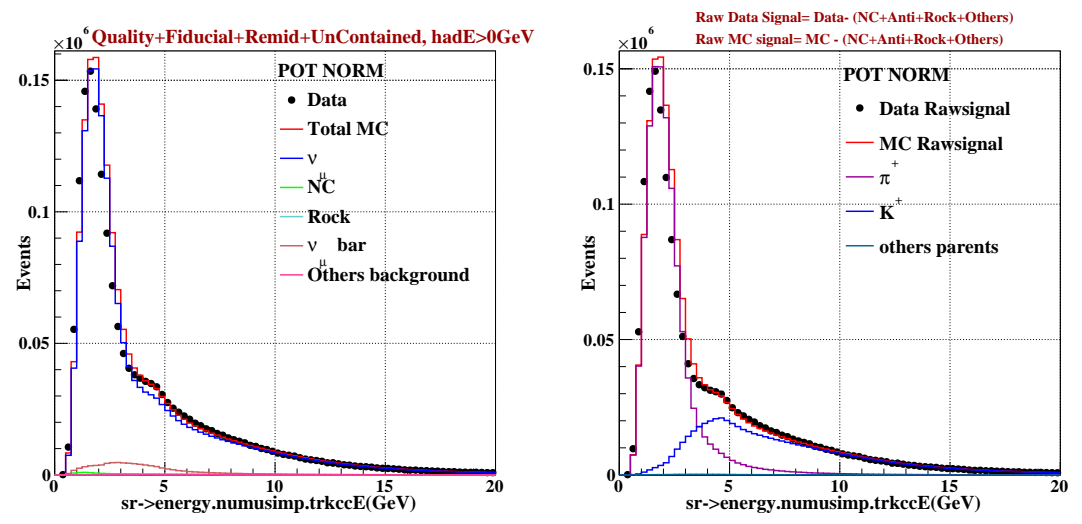

Figure F.6: An example: fReweightMaCCRES, UnContained. 


\begin{tabular}{|c|c|c|}
\hline GENIE Parameter & $\frac{(\text { Nom-Shift })}{\text { Nom }} \%$ for $+1 \sigma$ & $\frac{(\text { Nom-Shift })}{\text { Nom }} \%$ for $-1 \sigma$ \\
\hline 6:fReweightMaCCQE & 0.397 & -0.186 \\
\hline 7:fReweightVecCCQEshape & -0.004 & 0.004 \\
\hline 11:fReweightMaCCRES & 1.925 & -1.466 \\
\hline 12:fReweightMvCCRES & 0.925 & -0.715 \\
\hline 16:fReweightMaNCRES & 0.003 & -0.001 \\
\hline 17:fReweightMvNCRES & 0.001 & 0 \\
\hline 20:fReweightRvpCC1pi & 0.302 & -0.304 \\
\hline 21:fReweightRvpCC2pi & 0.714 & -0.722 \\
\hline 22:fReweightRvpNC1pi & 0.001 & -0.001 \\
\hline 23:fReweightRvpNC2pi & 0.002 & -0.002 \\
\hline 24:fReweightRvnCC1pi & 1.388 & -1.421 \\
\hline 25:fReweightRvnCC2pi & 0.891 & -0.904 \\
\hline 26:fReweightRvnNC1pi & 0.001 & -0.001 \\
\hline 27:fReweightRvnNC2pi & 0.001 & -0.001 \\
\hline 28:fReweightRvbarpCC1pi & 0.143 & -0.143 \\
\hline 29:fReweightRvbarpCC2pi & 0.105 & -0.105 \\
\hline 32:fReweightRvbarnCC1pi & 0.027 & -0.027 \\
\hline 33:fReweightRvbarnCC2pi & 0.062 & -0.062 \\
\hline 36:fReweightAhtBY & 0.303 & -1.434 \\
\hline 37:fReweightBhtBY & -1.889 & 0.78 \\
\hline 38:fReweightCV1uBY & -1.294 & 0.232 \\
\hline 39:fReweightCV2uBY & 0.132 & -1.251 \\
\hline 50:fReweightMFP'pi & 2.453 & -2.302 \\
\hline 51:fReweightMFP'N & 1.039 & -1.207 \\
\hline 52:fReweightFrCEx pi & 0.015 & -0.015 \\
\hline 53:fReweightFrElas pi & 0.012 & -0.012 \\
\hline 54:fReweightFrInel'pi & -0.032 & 0.024 \\
\hline 55:fReweightFrAbs pi & -0.022 & 0.01 \\
\hline 57:fReweightFrCEx ${ }^{*} \mathrm{~N}$ & 0.006 & -0.006 \\
\hline 58:fReweightFrElas ${ }^{\circ} \mathrm{N}$ & 0.016 & -0.016 \\
\hline 59:fReweightFrInel ${ }^{\circ} \mathrm{N}$ & -0.009 & 0.009 \\
\hline 60:fReweightFrAbs ${ }^{\circ} \mathrm{N}$ & -0.021 & 0.021 \\
\hline 61:fReweightFrPiProd ${ }^{\circ} \mathrm{N}$ & 0.011 & -0.011 \\
\hline 62:fReweightCCQE PauliSupViaKF & -0.06 & 0.055 \\
\hline 65:fReweightBR1eta & -0.022 & 0.022 \\
\hline 66:fReweightTheta_Delta2Npi & 0.003 & 0.002 \\
\hline
\end{tabular}

Table F.8: Effect of GENIE parameters $\pm \sigma$, UnContained Sample $\mathrm{K}^{+}$Norm. 


\begin{tabular}{|c|c|c|}
\hline GENIE Parameter & $\frac{(\text { Nom-Shift })}{\text { Nom }} \%$ for $+1 \sigma$ & $\frac{(\text { Nom-Shift })}{\text { Nom }} \%$ for $-1 \sigma$ \\
\hline 6:fReweightMaCCQE & 2.682 & -1.714 \\
\hline 7:fReweightVecCCQEshape & 0.121 & -0.121 \\
\hline 11:fReweightMaCCRES & 4.924 & -5.666 \\
\hline 12:fReweightMvCCRES & 2.824 & -2.727 \\
\hline 16:fReweightMaNCRES & 0.168 & -0.127 \\
\hline 17:fReweightMvNCRES & 0.039 & -0.032 \\
\hline 20:fReweightRvpCC1pi & 0.641 & -0.649 \\
\hline 21:fReweightRvpCC2pi & 1.41 & -1.45 \\
\hline 22:fReweightRvpNC1pi & 0.041 & -0.041 \\
\hline 23:fReweightRvpNC2pi & 0.076 & -0.076 \\
\hline 24:fReweightRvnCC1pi & 2.862 & -3.029 \\
\hline 25:fReweightRvnCC2pi & 0.97 & -0.988 \\
\hline 26:fReweightRvnNC1pi & 0.104 & -0.104 \\
\hline 27:fReweightRvnNC2pi & 0.048 & -0.048 \\
\hline 28:fReweightRvbarpCC1pi & 0.067 & -0.067 \\
\hline 29:fReweightRvbarpCC2pi & 0.023 & -0.023 \\
\hline 31:fReweightRvbarpNC2pi & 0.002 & -0.002 \\
\hline 32:fReweightRvbarnCC1pi & 0.013 & -0.013 \\
\hline 36:fReweightAhtBY & 0.03 & -0.116 \\
\hline 37:fReweightBhtBY & -0.154 & 0.075 \\
\hline 38:fReweightCV1uBY & -0.095 & 0.016 \\
\hline 39:fReweightCV2uBY & 0.009 & -0.093 \\
\hline 50:fReweightMFP pi & 1.149 & -1.048 \\
\hline 51:fReweightMFP'N & 0.854 & -0.94 \\
\hline 52:fReweightFrCEx pi & -0.017 & 0.017 \\
\hline 53:fReweightFrElas pi & 0.017 & -0.017 \\
\hline 54:fReweightFrInel pi & 0.099 & -0.092 \\
\hline 55:fReweightFrAbs pi & -0.073 & 0.071 \\
\hline 56:fReweightFrPiProd pi & -0.006 & 0.006 \\
\hline 57:fReweightFrCEx ${ }^{\circ} \mathrm{N}$ & -0.003 & 0.003 \\
\hline 58:fReweightFrElas ${ }^{*} \mathrm{~N}$ & 0.05 & -0.05 \\
\hline 59:fReweightFrInel ${ }^{\circ} \mathrm{N}$ & 0.013 & -0.013 \\
\hline 60:fReweightFrAbs ${ }^{*} \mathrm{~N}$ & -0.04 & 0.04 \\
\hline 61:fReweightFrPiProd ${ }^{\circ} \mathrm{N}$ & -0.012 & 0.012 \\
\hline 62:fReweightCCQEPauliSupViaKF & -0.829 & 0.771 \\
\hline 65:fReweightBR1eta & -0.281 & 0.28 \\
\hline
\end{tabular}

Table F.9: Effect of GENIE parameters $\pm \sigma$, Contained Sample $\pi^{+} 1-3(\mathrm{GeV})$ Norm. 


\section{Appendix G}

\section{Supplementary Material for \\ Chapter 7}

\section{G.1 Data/MC Comparison}

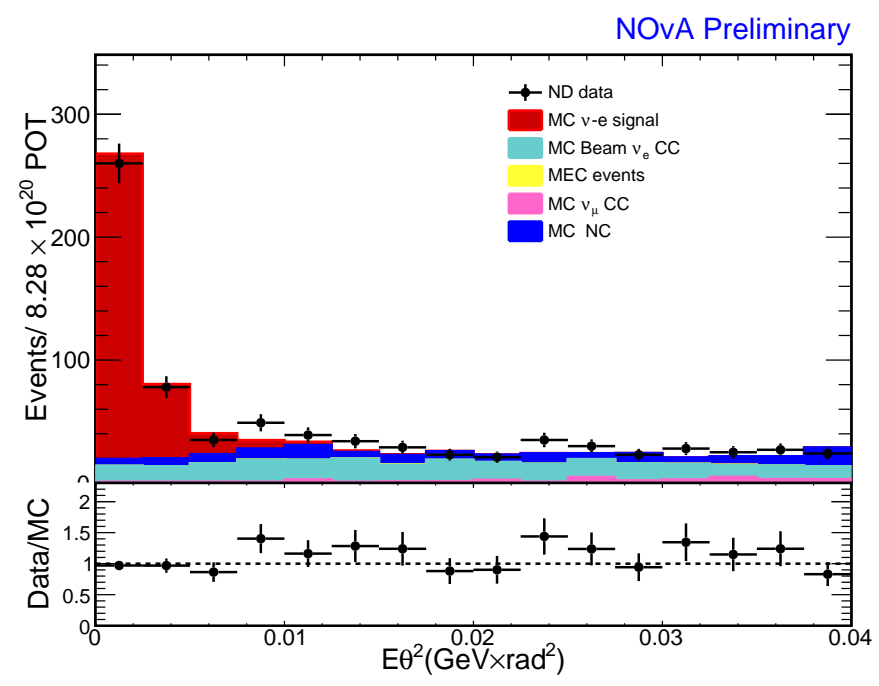

Figure G.1: Data/MC of E $\theta^{2}$ before background MC corrections, with separate $\nu_{\mu}$ and $\mathrm{NC}$ background distributions. 


\begin{tabular}{|c|c|c|}
\hline Genie Parameter & $+1 \sigma(\%$ to $\mathrm{bkg})$ & $-1 \sigma(\%$ to bkg $)$ \\
\hline 1:fReweightMaNCEL & -2.14 & 3.51 \\
\hline 2: fReweightEtaNCEL & -0.15 & 0.16 \\
\hline 6:fReweightMaCCQE & -2.41 & 2.86 \\
\hline 7:fReweightVecCCQEshape & -1.69 & 1.68 \\
\hline 11:fReweightMaCCRES & -0.66 & 0.89 \\
\hline 12:fReweightMvCCRES & -0.42 & 0.54 \\
\hline 14:fReweightMaNCRESshape & 1.26 & -1.38 \\
\hline 15:fReweightMvNCRESshape & 0.1 & -0.11 \\
\hline 16:fReweightMaNCRES & 2.87 & -6.06 \\
\hline 17:fReweightMvNCRES & 0.26 & -0.37 \\
\hline 21:fReweightRvpCC2pi & -0.3 & 0.3 \\
\hline 22:fReweightRvpNC1pi & -0.3 & 0.3 \\
\hline 23:fReweightRvpNC2pi & -0.3 & 0.3 \\
\hline 24:fReweightRvnCC1pi & -0.96 & 0.94 \\
\hline 36:fReweightAhtBY & -0.01 & 0.02 \\
\hline 37:fReweightBhtBY & -0.01 & 0.04 \\
\hline 38:fReweightCV1uBY & -0.49 & 0.43 \\
\hline 39:fReweightCV2uBY & 0.43 & -0.43 \\
\hline 50:fReweightMFP_pi & 1.09 & -0.9 \\
\hline 51:fReweightMFP_N & 1.93 & -1.31 \\
\hline 52:fReweightFrCEx_pi & -0.21 & 0.21 \\
\hline 53:fReweightFrElas_pi & 0.01 & -0.01 \\
\hline 54:fReweightFrInel_pi & -0.46 & 0.32 \\
\hline 55:fReweightFrAbs_pi & 0.48 & -0.57 \\
\hline 56:fReweightFrPiProd_pi & 0.04 & -0.04 \\
\hline 57:fReweightFrCEx_N & 1.28 & -1.28 \\
\hline 60:fReweightFrAbs_N & -0.29 & 0.3 \\
\hline 61:fReweightFrPiProd_N & 0.23 & -0.23 \\
\hline 62:fReweightCCQEPauliSupViaKF & -0.62 & -2.99 \\
\hline 64:fReweightBR1gamma & 0.01 & -0.01 \\
\hline 65:fReweightBR1eta & 0.3 & -0.3 \\
\hline 66:fReweightTheta_Delta2Npi & -0.21 & -0.31 \\
\hline $\operatorname{coh}$ & -0.37 & 0.37 \\
\hline
\end{tabular}

Table G.1: Summary of the effect of GENIE knobs variation on this analysis, for GENIE knobs.

\section{G.2 One Loop Electroweak Radiative Corrections to}

\section{Neutrino-Electron Scattering}

The cross-section for the three-level neutrino-electron scattering is given in the Equation 7.2, which is the cross-section model for Neutrino-Electron Scattering in our simulation using GENIE event generator. As we mentioned in section 7.2 , that we need to 
modify the Equation 7.2 to use modern values of the electroweak couplings. This is done by changing the chiral couplings, $\mathrm{C}_{L L}$ and $\mathrm{C}_{L R}$, to one-loop values predicted using global fits to electroweak data [99]. Table G.2 compares the values for these couplings in GENIE to the values we used in this analysis. Also for one-loop electroweak radiative corrections the Equation 7.2 has been modified the expressions for the $\nu_{\mu} e, \overline{\nu_{\mu}} e, \nu_{e} e$ and $\overline{\nu_{e}} e$ as shown below:

\begin{tabular}{|c|c|c|c|}
\hline & $C_{L L}^{\nu_{e} e}$ & $C_{L L}^{\nu \mu} e$ & $C_{L R}^{\nu e}$ \\
\hline GENIE 2.10.4 & 0.7277 & -0.2723 & 0.2277 \\
One loop & 0.7276 & -0.2730 & 0.2334 \\
\hline
\end{tabular}

Table G.2: Electroweak couplings in GENIE and in our oneloop calculation of $\nu \mathrm{e}^{-}$elastic scattering

$$
\begin{aligned}
\frac{d \sigma\left(\nu_{l} e^{-} \rightarrow \nu_{l} e^{-}\right)}{d y}= & \frac{G_{F}^{2} s}{\pi}\left[\left(C_{L L}^{\nu_{l} e}\right)^{2}\left(1+\frac{\alpha_{E M}}{\pi} X_{1}\right)+\left(C_{L R}^{\nu e}\right)^{2}(1-y)^{2}\left(1+\frac{\alpha_{E M}}{\pi} X_{2}\right)\right] \\
\frac{d \sigma\left(\overline{\nu_{l}} e^{-} \rightarrow \overline{\nu_{l}} e^{-}\right)}{d y}= & \frac{G_{F}^{2} s}{\pi}\left[\left(C_{L R}^{\nu e}\right)^{2}\left(1+\frac{\alpha_{E M}}{\pi} X_{1}\right)+\left(C_{L L}^{\nu_{l} e}\right)^{2}(1-y)^{2}\left(1+\frac{\alpha_{E M}}{\pi} X_{2}\right)\right. \\
& \left.-\frac{C_{L L}^{\nu_{l} e} C_{L R}^{\nu e} m y}{E_{\nu}}\left(1+\frac{\alpha_{E M}}{\pi} X_{3}\right)\right]
\end{aligned}
$$

where $\mathrm{E}_{\nu}$ is the neutrino energy, $\mathrm{s}$ is the Mandelstam invariant representing the square of the total energy in the center-of-mass frame, $\mathrm{m}$ is the electron mass and $\mathrm{y}=\frac{T_{e}}{E_{\nu}}$. The $\mathrm{X}_{i}$ correction terms are:

$$
\begin{aligned}
X_{1}= & \frac{1}{12}(6 y+12 \log (1-y)-6 \log (y)-5) \log \left(\frac{2 E_{\nu}}{m}\right)-\frac{L i_{2}(y)}{2}+\frac{y^{2}}{24}-\frac{11 y}{12} \\
& -\frac{1}{2} \log ^{2}\left(\frac{1}{y}-1\right)+y \log (y)-\frac{1}{12}(6 y+23) \log (1-y)+\frac{\pi^{2}}{12}-\frac{47}{36}
\end{aligned}
$$




$$
\begin{gathered}
X_{2}=\frac{\left.\left(-4 y^{2}+\left(-6 y^{2}+6 y-3\right) \log (y)+11 y+6(1-y)^{2} \log (1-y)-7\right) \log \left(\frac{2 E_{\nu}}{m}\right)\right)}{6(1-y)^{2}} \\
+\frac{\left(-y^{2}+y-\frac{1}{2}\right)\left(\operatorname{Li} 2(y)+\log ^{2}(y)-\frac{\pi^{2}}{6}\right.}{(1-y)^{2}}+\frac{\left(4 y^{2}+2 y-3\right) \log (y)}{4(1-y)^{2}}-\frac{31-49 y}{72(1-y)} \\
+\frac{(10 y-7) \log (1-y)}{6(1-y)}+\log (1-y)\left(\log (y)-\frac{1}{2} \log (1-y)\right) \\
\quad=\log \left(-\frac{m}{\sqrt{y E_{\nu}\left(2 m+y E_{\nu}\right)}+m+y E_{\nu}}+1-y\right) \\
\quad \times\left(\frac{\left(m+y E_{\nu}\right) \log \left(\frac{\sqrt{y E_{\nu}\left(2 m+y E_{\nu}\right)}+m+y E_{\nu}}{m}\right)}{\sqrt{y E_{\nu}\left(2 m+y E_{\nu}\right)}}-1\right)
\end{gathered}
$$

where $\operatorname{Li}_{2}(z)$ represents Spence's function, $\int_{0}^{z} \frac{-\log (1-u)}{u} d u$. After integration over y and using Mathematica software approximations (just an example for $\nu_{\mu}$ ):

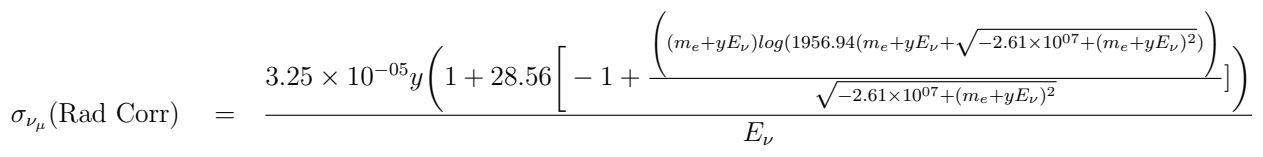

$$
\begin{aligned}
& +7.45 \times 10^{-02}\left(1+2.32 \times 10^{-03}\left[-1.306+\frac{\pi^{2}}{12}-\frac{11 y}{12}+\frac{y^{2}}{24}-\left(1.917+\frac{y}{2}\right) \log (1-y)-\frac{\log \left(\frac{1-y}{y}\right)^{2}}{2}\right.\right. \\
& \left.\left.+\log \left(3913.9 E_{\nu}\right)\left(-0.417+\frac{y}{2}+\log (1-y)-\frac{\log (y)}{2}\right)+y \log (y)-\frac{\operatorname{Poly} \log (2, y)}{2}\right]\right) \\
& +0.055(1-y)^{2}\left(1+2.32 \times 10^{-03}\left[-\frac{31-49 y}{72(1-y)}+\frac{(-35+5 y) \log (1-y)}{3(1-y)}\right.\right. \\
& +\frac{\left(-0.75+\frac{y}{2}+y^{2}\right) \log (y)}{(1-y)^{2}}+\log (1-y)\left(-\frac{\log (1-y)}{2}+\log (y)\right) \\
& +\log \left(3913.89 E_{\nu}\right)\left(-0.67-\frac{1}{2(1-y)}+\log (1-y)-\frac{\log (y)}{2(1-y)^{2}}+\frac{y \log (y)}{1-y}\right) \\
& \left.\left.+\frac{(-0.5+(1-y) y)\left(-\frac{\pi^{2}}{6}+(\log (y))^{2}+\operatorname{Poly} \log (2, y)\right)}{(1-y)^{2}}\right]\right) \\
& \sigma_{\nu_{\mu}}(\mathrm{nom})=7.42 \times 10^{-02}+0.052(1-y)^{2}-\frac{3.17 \times 10^{-05} y}{E_{\nu}}
\end{aligned}
$$




$$
\text { Weight }_{\nu_{\mu}}=\frac{\sigma_{\nu_{\mu}}(\text { Rad Corr })}{\sigma_{\nu_{\mu}}(\text { nom })}
$$

Similarly, we calculated the weight for $\overline{\nu_{\mu}} e, \nu_{e} e$ and $\overline{\nu_{e}} e$. The distributions of cross-sections corrected, nominal and their ratio (weights) for respective neutrino flavor w.r.t to y and $\mathrm{E}_{\nu}$ are shown in Figures G.4 and G.2, G.3.
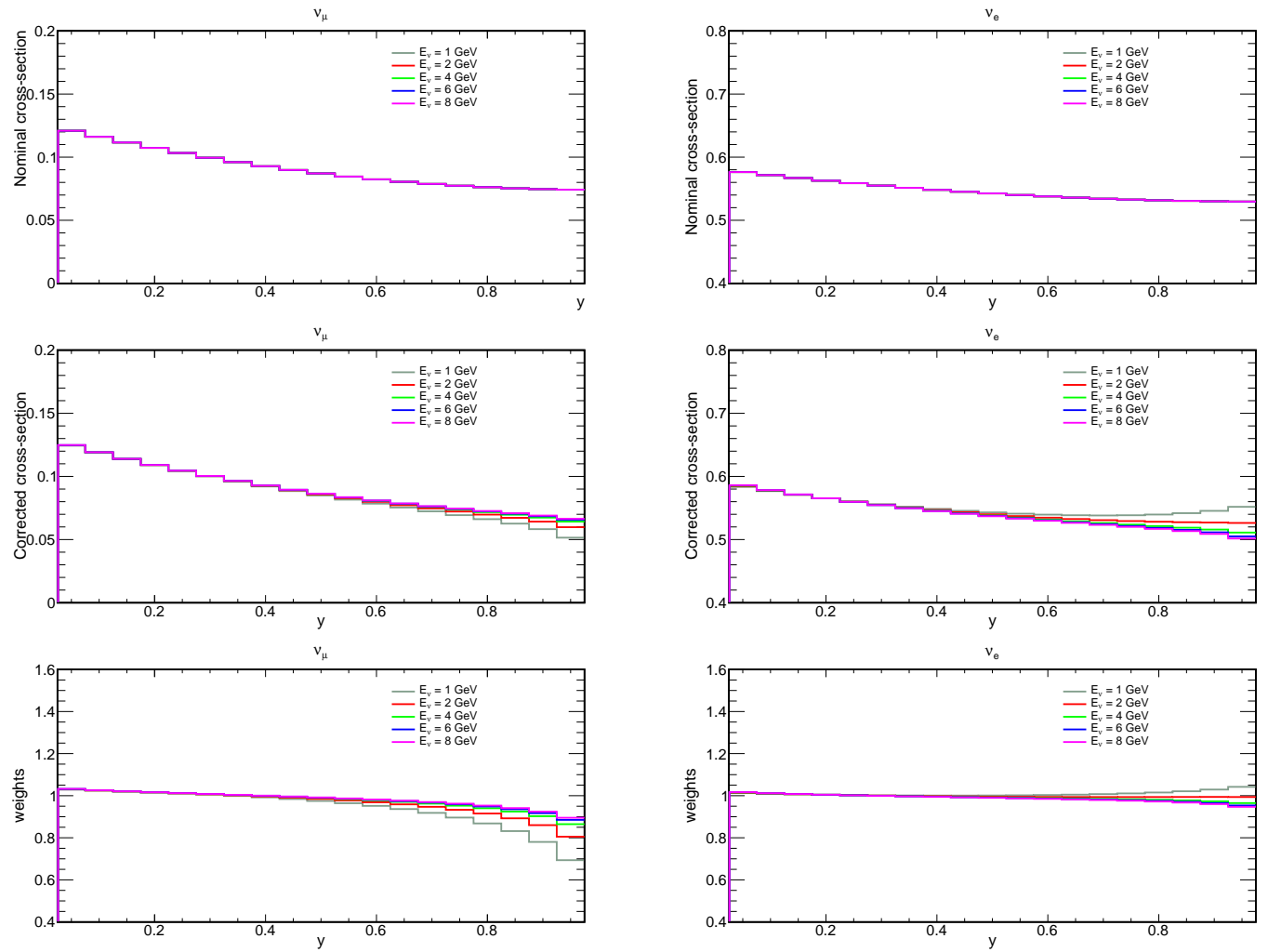

Figure G.2: Distribution of $\sigma_{\nu_{\mu}}$ (left) and $\sigma_{\nu_{e}}$ (right) for corrected, nominal and ratio as function of $\mathrm{y}$ at fixed $\mathrm{E}_{\nu}$.

\section{G.3 Particle Likelihoods for $\nu$ - e Elastic Scattering}

\section{Identification}

We use a based particle likelihood method to identify $\nu$-e elastic scattering events. This

method is similar to the one used in the electron-neutrino oscillation analysis [115]. For 

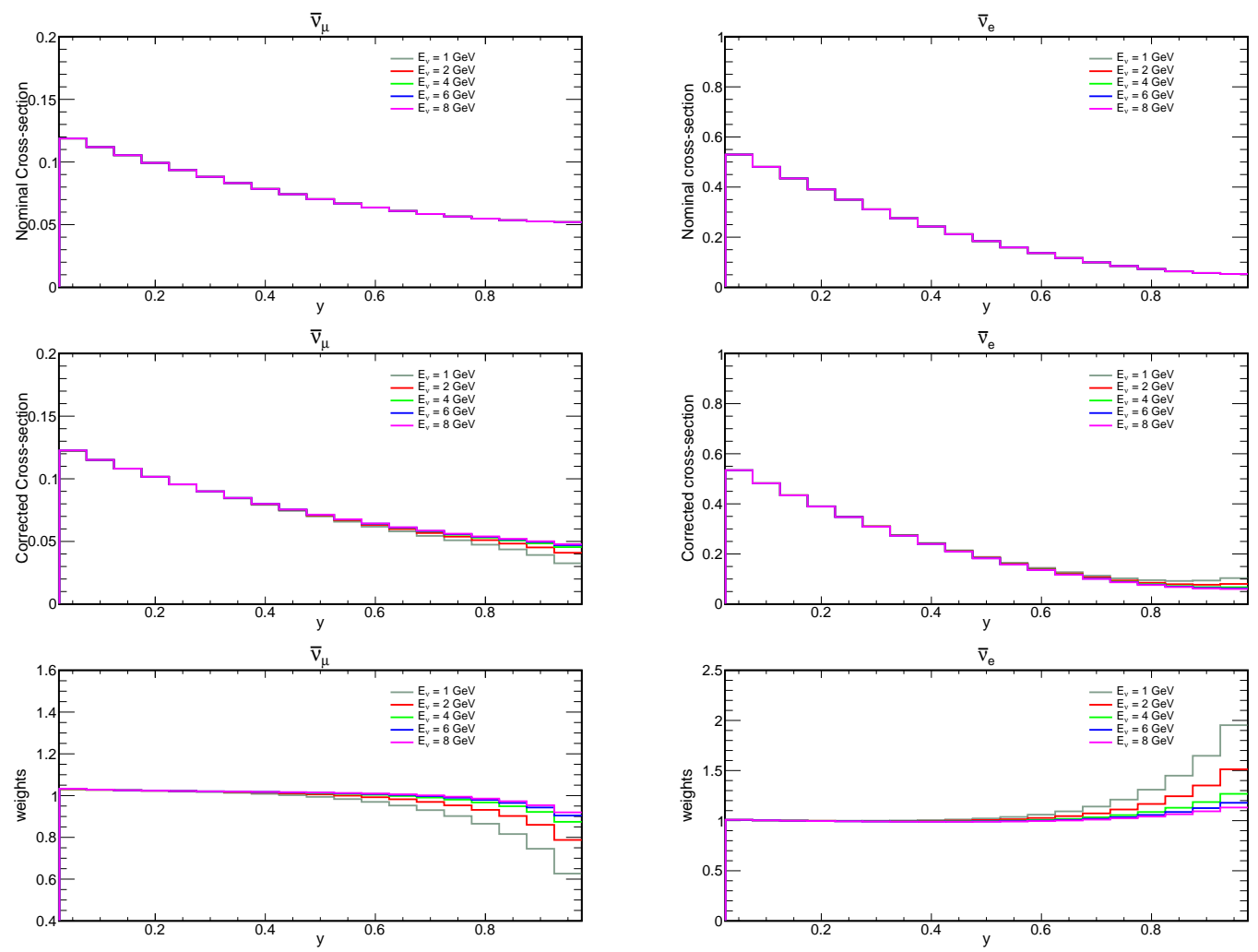

Figure G.3: Distribution of $\sigma \bar{\nu}_{\mu}$ (left) and $\sigma \bar{\nu}_{e}$ (right) for corrected, nominal and ratio as function of $\mathrm{y}$ at fixed $\mathrm{E}_{\nu}$.
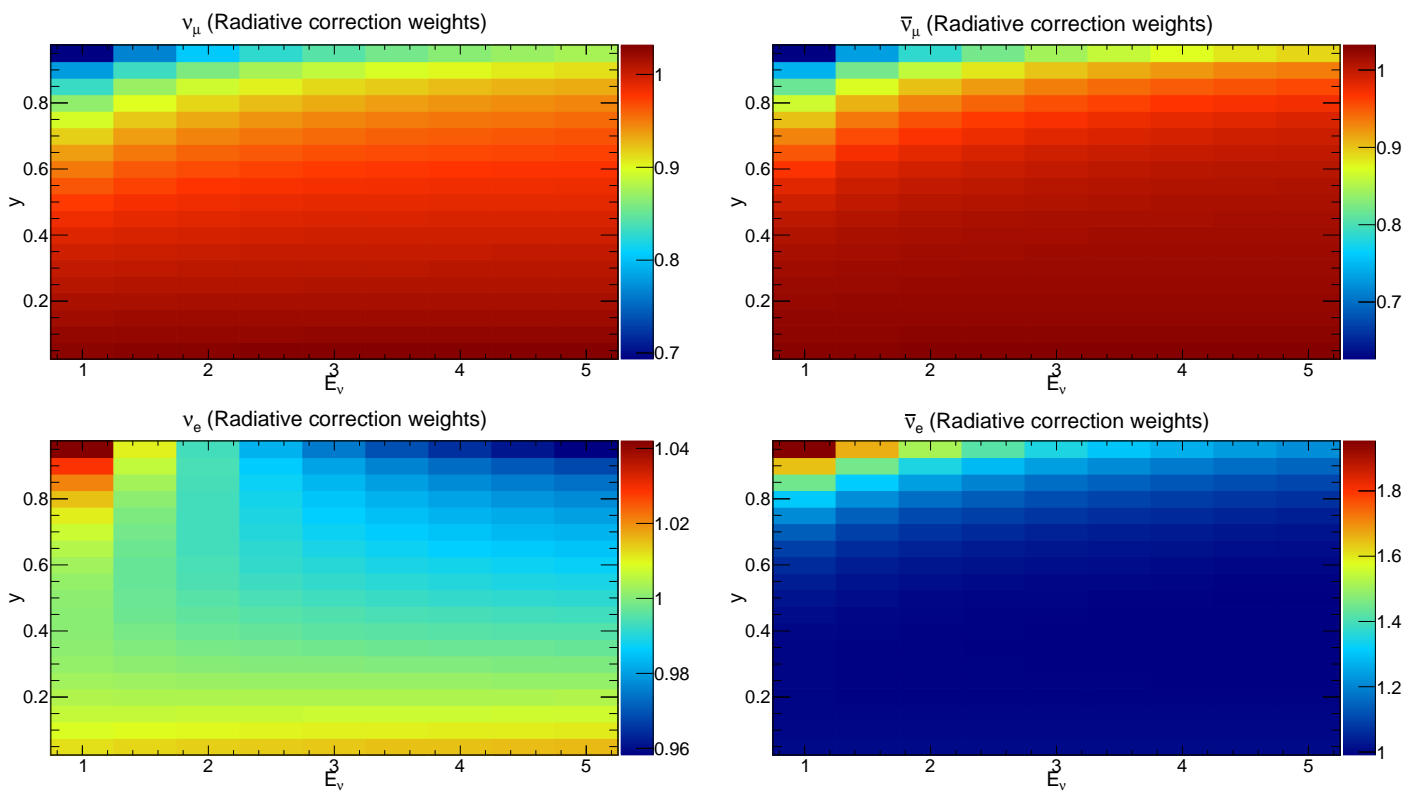

Figure G.4: Distribution of $\frac{\sigma_{\nu_{\mu}}(\text { RadCorr })}{\sigma_{\nu_{\mu}}(\text { nom })}$ in $\mathrm{y}$ and $\mathrm{E}_{\nu}$ space. 
an unidentified particle, the measured is compared with the expected under each particle hypothesis in each plane and each transverse cell chain (defined later) to construct the probability and likelihood for particle identification. In this way we can describe the 3-D development of a shower in detail.

Using the cell energy, we calculate in the longitudinal and transverse directions. The longitudinal is calculated plane-by-plane. It is defined as the total shower energy in a plane divided by the path length in that plane (the thickness of a plane divided by the cosine of the incident angle of the shower). The transverse is calculated using the following method. (1) A line connecting the start and end point of the shower is defined as the longitudinal central line of the shower. (2) In each plane of the shower, the cell where the longitudinal central line penetrates through is defined as the core cell of the shower in that plane and is assigned a transverse cell index of zero. (3) In a given plane, the next cells out from the core cell in the positive or negative transverse direction are both assigned a transverse cell index of 1, and so on for transverse cell indices up to 20. (4) Cells along the entire shower with the same transverse cell index are clustered as a transverse cell chain (5) For a given transverse cell index, the cell energies in the corresponding transverse cell chain along the entire shower are summed and divided by the total path length of the chain to give the total corresponding to that transverse cell index. (6) The average for each transverse cell index is calculated.

By matching the reconstructed shower direction to truth, we select Monte Carlo (MC) $e, \gamma, \mu, \pi^{0}, p, n$, and $\pi^{ \pm}$showers from neutrino MC events to extract the expected distribution histograms for each plane and each transverse cell chain. To consider energy dependence, we evenly divide the shower energy range 0-20 GeV (0-5 GeV) into 40 (10) bins for electrons (other particles), then obtain expected histograms in these energy bins.

For a given shower in the test sample, the measured value in each plane (transverse cell chain) is compared to the expected histogram of each type of particle to calculate the probability to be each particle's hypothesis in that plane (transverse cell chain). In 
the expected histogram, we find the index $i$ of the bin which the measured value falls into. The probability that the measured value falls into the $i$-th bin is then calculated as: $P_{i}=\left(N \times n_{\text {bin }}\right) / N_{\text {tot }}$, where $n_{\text {bin }}$ is the number of bins in the expected histogram, and $N_{t o t}$ is the total number of entries in the histogram. The scale factor $n_{b i n}$ is applied in order to avoid issues caused by the limit of the machine precision when we $\operatorname{sum} \ln \left(P_{i}\right)$ over planes or transverse cell chains to calculate the overall particle likelihood.

The likelihood of each particle's hypothesis in the $i$-th plane (transverse cell chain) is defined as: $L L_{i}=\ln \left(P_{i}\right)$. The overall longitudinal and transverse log likelihoods are defined as: $L L_{L}=\Sigma L L_{i} / N_{p}$ and $L L_{T}=\Sigma L L_{i} / N_{t}$, where $N_{p}$ is the number of planes and $N_{t}$ is the number of transverse cells in the shower. Differences between longitudinal and transverse log likelihoods for the electron and other particle hypotheses can be used to identify electrons. Differences between longitudinal and transverse log likelihoods for electron and other particle hypotheses can be used to identify electrons.

To identify $\nu$-e elastic scattering events, 12 variables are used to form the input to an artificial feed-forward neutral network (ANN). These inputs consist of 12 differences between $\mathrm{dE} / \mathrm{dx}$ based longitudinal and transverse log-likelihoods for electron and the six other-particle hypotheses $\left(\gamma, \mu, \pi^{0}, p, n\right.$ and charged $\left.\pi\right)$ applied to the most energetic shower $\left[L L_{L}(e)-L L_{L}(\gamma), L L_{T}(e)-L L_{T}(\gamma),\right]$. The likelihoods for $\gamma, \mu, \pi^{0}, p, n, \pi^{+}$ are the same as the LID algorithm [115], while longitudinal and transverse likelihoods for electrons are created with a single electron MC sample.
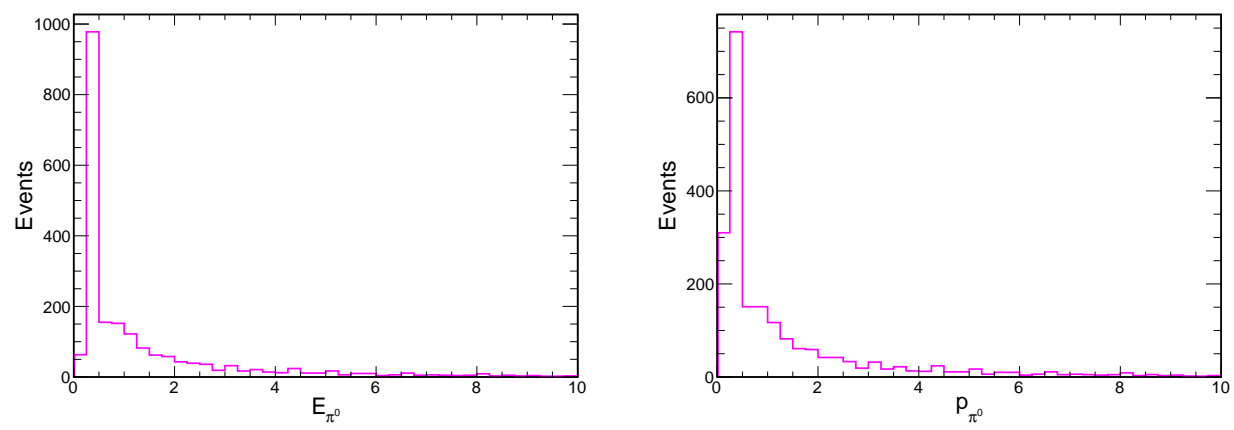

Figure G.5: DFR true energy and momentum distributions. 

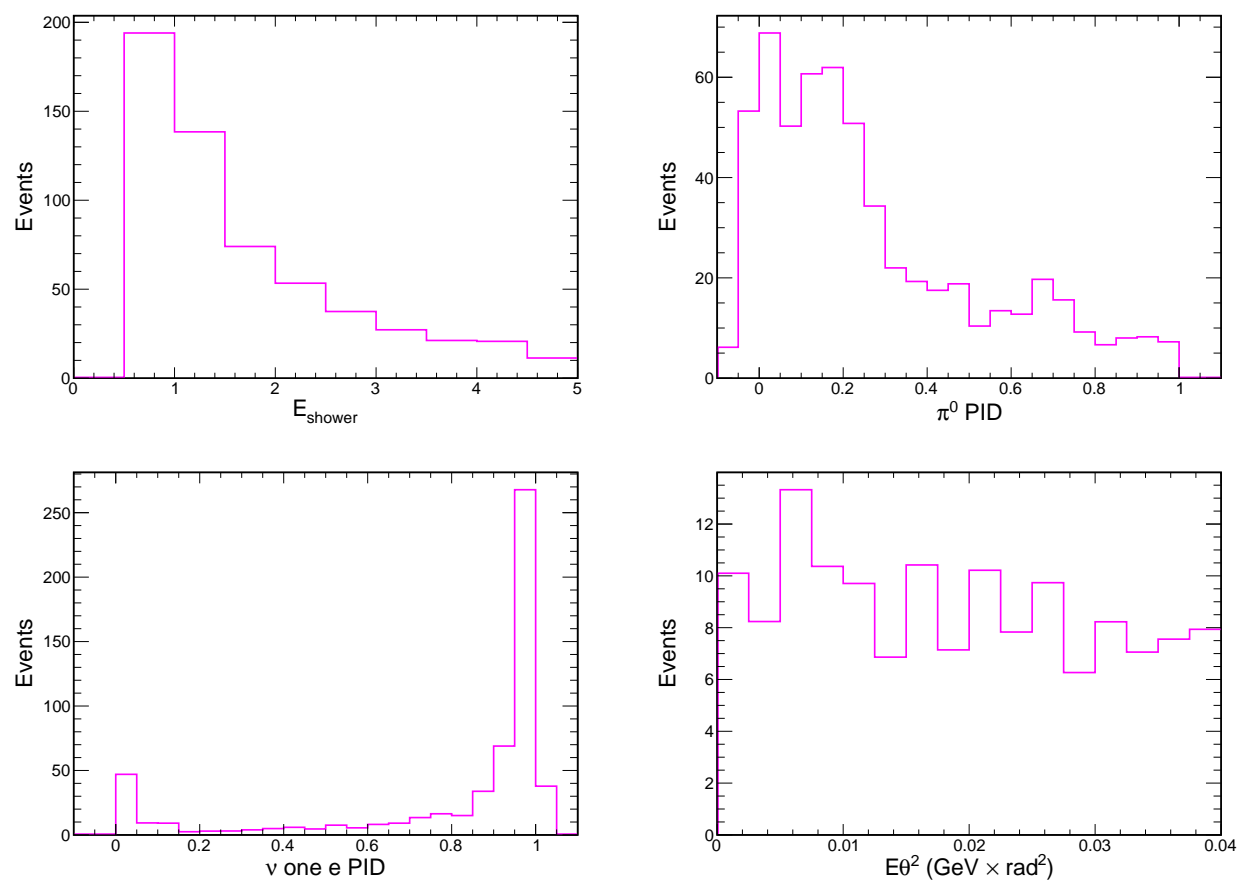

Figure G.6: DFR reconstructed shower energy, $\mathrm{E} \theta^{2}$, annLPID and $e / \pi^{0} \mathrm{PID}$ distributions before the cut on PIDs and kinematics $\left(\mathrm{E} \theta^{2}\right)$ distribution i.e distributions with preselection+fiducial $+\mathrm{E}<5 \mathrm{GeV}$.
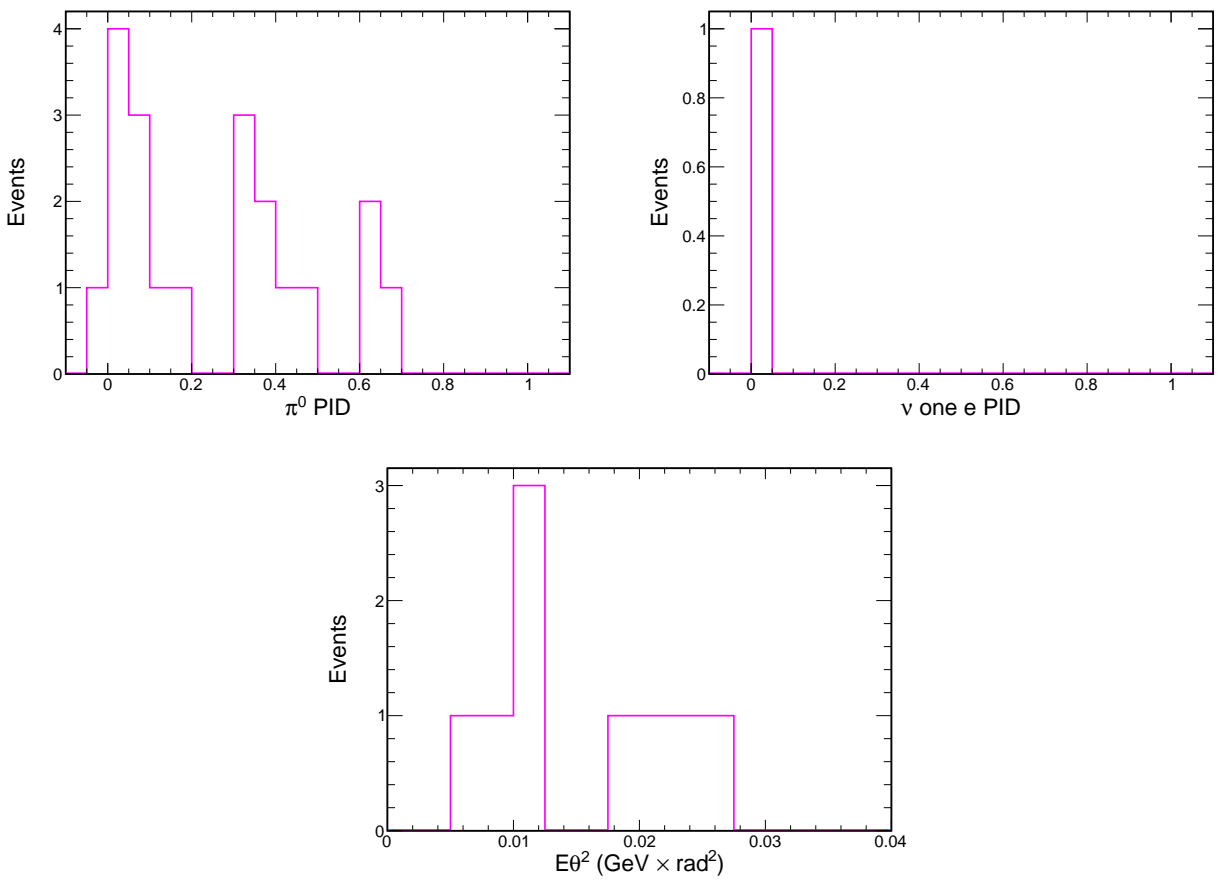

Figure G.7: DFR reconstructed shower energy, $\operatorname{Cos}(\theta), \mathrm{E} \theta^{2}$, annLPID and $e / \pi^{0} \mathrm{PID}$ distributions after the cut on PIDs and knematics $\left(\mathrm{E} \theta^{2}\right)$ distributions. 


\section{G.4 NC Diffractive (DFR) $\pi^{0}$}

Using third analysis production R17-03-09-prod3genie.c (GENIE 2.12.2) and reconstruction R17-03-01-prod3reco.d release, we produced set of caf files having 98k DFR $\pi^{0}$ 's events in ND with our neutrino-electron elastic scattering PID and $e / \pi^{0}$ PID. Normalized the DFR events to $34 \%$ of $\mathrm{COH} \pi^{0}=1.6$ at the Generated-level [11] as shown in Figure G.5, G.6 and after our selection kinematics distributions for DFR are shown in Figure G.7. 


\section{List of Publications}

1. Search for active-sterile neutrino mixing using neutral-current interactions in NOvA, NOvA Collaboration (P. Adamson (Fermilab) et al.) , Published in Phys. Rev. D. 96(2017), 072006.

2. PRL: Constraints on Oscillation Parameters from $\nu_{e}$ Appearance and $\nu_{\mu}$ Disappearance in $\boldsymbol{N O \boldsymbol { v } A}$, NOvA Collaboration (P. Adamson (Fermilab) et al.), Published in Phys.Rev.Lett. 118 (2017) no.23, 231801.

3. PRL: Measurement of the neutrino mixing angle $\theta_{23}$ in NOvA, NOvA Collaboration (P. Adamson (Fermilab) et al.), Published in Phys.Rev.Lett. 118 (2017) no.15, 151802.

4. Proceeding: Constraints on the Neutrino Flux in NOvA using the Near Detector Data, Kulddep Kaur Maan for NOvA Collaboration, 38th International Conference on High Energy Physics (ICHEP 2016), Published in PoS ICHEP2016 (2016) 931.

5. Proceeding: Constraint of the Charged Kaon Yield using $\nu_{\mu}$ Data in the NOvA Near Detector \& Prediction of the $\nu_{e}$ Flux, Kulddep Kaur Maan et al. for NOvA Collaboration, 18th International Workshop on Neutrino Factories and Future Neutrino Facilities Search (NuFact16), Proceedings to be published.

6. PRL: First measurement of electron neutrino appearance in NOvA, NOvA Collaboration (P. Adamson (Fermilab) et al.). Jan 19, 2016. 7 pp. Published in Phys.Rev.Lett. 116 (2016) no.15, 151806. 
7. PRD: First measurement of muon-neutrino disappearance in NOvA, NOvA Collaboration (P. Adamson (Fermilab) et al.). Jan 19, 2016. 8 pp. Published in Phys.Rev. D93 (2016) no.5, 051104.

8. Proceeding: Neutrino Flux Studies at $\boldsymbol{N O \boldsymbol { v A }}$, For the NOvA Collaboration, Published in DPF2015-262, arXiv:1511.00287.

9. Proceeding: Neutrino Flux at NOvA: Empirical Constraint and Sytematics Errors, 21st DAE-BRNS (2014) High Energy Physics Symposium, Published in Springer Proc.Phys. 174 (2016) 311-315.

10. Proceeding: Component study of the NuMI neutrino beam for NOvA experiment at Fermilab, Published in DAE Symp.Nucl.Phys. 58 (2013) 822-825 


\section{Bibliography}

[1] W. Pauli, "Letter to the physical society of Tubingen," 1930.

[2] Wolfgang, "Dear radioactive ladies and gentlemen," Phys. Today, vol. 31N9, p. $27,1978$.

[3] E. Fermi, "An attempt of a theory of beta radiation. 1." Z. Phys., vol. 88, pp. 161-177, 1934.

[4] J. Beringer and et al., "Review of particle physics," Phys. Rev. D, vol. 86, p. 010001, Jul 2012. [Online]. Available: https://link.aps.org/doi/10.1103/ PhysRevD.86.010001

[5] C. L. Cowan, Jr, F. Reines, F. B. Harrison, H. W. Kruse, and A. D. McGuire, "Detection of the free neutrino: a confirmation," Science (New York, N.Y.), vol. 124, no. 3212, pp. 103-4, Jul 1956. [Online]. Available: https://doi.org/10.1126/science.124.3212.103

[6] F. Reines and C. L. Cowan, "Detection of the free neutrino," Phys. Rev., vol. 92, pp. 830-831, Nov 1953. [Online]. Available: https: //link.aps.org/doi/10.1103/PhysRev.92.830

[7] T. D. Lee and C. N. Yang, "Question of parity conservation in weak interactions," Phys. Rev., vol. 104, pp. 254-258, Oct 1956. [Online]. Available: https://link.aps.org/doi/10.1103/PhysRev.104.254

[8] C. S. Wu, E. Ambler, R. W. Hayward, D. D. Hoppes, and R. P. Hudson, "Experimental test of parity conservation in beta decay," Phys. Rev., vol. 105, pp. 1413-1415, Feb 1957. [Online]. Available: https://link.aps.org/doi/10.1103/PhysRev.105.1413 
[9] T. D. Lee and C. N. Yang, "Parity nonconservation and a two-component theory of the neutrino," Phys. Rev., vol. 105, pp. 1671-1675, Mar 1957. [Online]. Available: https://link.aps.org/doi/10.1103/PhysRev.105.1671

[10] G. Danby, J.-M. Gaillard, K. Goulianos, L. M. Lederman, N. Mistry, M. Schwartz, and J. Steinberger, "Observation of high-energy neutrino reactions and the existence of two kinds of neutrinos," Phys. Rev. Lett., vol. 9, pp. 36-44, Jul 1962. [Online]. Available: https://link.aps.org/doi/10. 1103/PhysRevLett.9.36

[11] S. L. Glashow, "Partial-symmetries of weak interactions," Nuclear Physics, vol. 22, no. 4, pp. 579-588, 1961. [Online]. Available: http: //www.sciencedirect.com/science/article/pii/0029558261904692

[12] S. Weinberg, "A model of leptons," Phys. Rev. Lett., vol. 19, pp. 1264-1266, Nov 1967. [Online]. Available: https://link.aps.org/doi/10.1103/ PhysRevLett.19.1264

[13] J. R. Patterson, "Lepton universality," 1995. [Online]. Available: http: //www.slac.stanford.edu/pubs/beamline/pdf/95i.pdf

[14] A. Salam and J. Ward, "Electromagnetic and weak interactions," Physics Letters, vol. 13, no. 2, pp. 168 - 171, 1964. [Online]. Available: http://www.sciencedirect.com/science/article/pii/0031916364907115

[15] Hasert, F. J. et al., "Observation of Neutrino Like Interactions Without Muon Or Electron in the Gargamelle Neutrino Experiment," Phys. Lett., vol. B46, pp. 138-140, 1973, [,5.15(1973)].

[16] B. C. Barish, "Caltech - fermilab neutral current search," FERMILAB-CONF76-116-E, 1974. 
[17] K. Kodama et al., "Observation of tau neutrino interactions," Phys. Lett., vol. B504, pp. 218-224, 2001. [Online]. Available: 10.1016/S0370-2693(01)00307-0

[18] Bahcall, J. N, Davis, and R. Jr, "Solar neutrinos: a scientific puzzle," Science, vol. 191, no. 4224, pp. 264-7, Jan 1976. [Online]. Available: https://doi.org/10.1126/science.191.4224.264

[19] Hirata, Kajita, and et al., "Observation of $8 \mathrm{~b}$ solar neutrinos in the kamiokande-ii detector," Phys Rev Lett, vol. 63, no. 1, pp. 16-19, Jul 1989. [Online]. Available: https://doi.org/10.1103/PhysRevLett.63.16

[20] Fukuda and et al., "Evidence for oscillation of atmospheric neutrinos," Phys. Rev. Lett., vol. 81, pp. 1562-1567, Aug 1998. [Online]. Available: https://link.aps.org/doi/10.1103/PhysRevLett.81.1562

[21] B. Aharmim and et al., "Determination of the $\nu_{e}$ and total ${ }^{8} \mathrm{~B}$ solar neutrino fluxes using the sudbury neutrino observatory phase i data set," Phys. Rev. C, vol. 75, p. 045502, Apr 2007. [Online]. Available: https://link.aps.org/doi/10.1103/PhysRevC.75.045502

[22] C. Giunti and C. W. Kim, "Fundamentals of neutrino physics and astrophysics," Oxford, UK: Univ. Pr. (2007) 710 p, pp. p139-144.

[23] H. J. Lipkin, Quantum theory of neutrino oscillations for pedestrians: simple answers to confusing questions. Physics Letters B, 2006. [Online]. Available: http://arxiv.org/abs/hep-ph/0505141

[24] J. L. Hewett et al., "Fundamental Physics at the Intensity Frontier," 2012. [Online]. Available: http://inspirehep.net/record/1114323/files/arXiv: 1205.2671.pdf

[25] Y. Ashie and et al., "Measurement of atmospheric neutrino oscillation parameters by super-kamiokande i." 
[26] P. Adamson and et al., "Measurement of neutrino and antineutrino oscillations using beam and atmospheric data in minos," Phys. Rev. Lett., vol. 110, p. 251801, Jun 2013. [Online]. Available: https: //link.aps.org/doi/10.1103/PhysRevLett.110.251801

[27] Eguch and et al., "First results from kamland: Evidence for reactor antineutrino disappearance," Phys. Rev. Lett., vol. 90, p. 021802, Jan 2003. [Online]. Available: https://link.aps.org/doi/10.1103/PhysRevLett.90.021802

[28] G. Zeller, "Review of Particle Physics," 2017. [Online]. Available: http://pdg.lbl.gov/2015/reviews/rpp2015-rev-nu-cross-sections.pdf

[29] (2015, Mar) Fermilab accelerator complex. [Online]. Available: https://science.energy.gov/hep/facilities/user-facilities/ fermilab-accelerator-complex $/ ? \mathrm{p}=1$

[30] P. Adamson and et al., "Improved search for muon-neutrino to electronneutrino oscillations in minos," Phys. Rev. Lett., vol. 107, p. 181802, Oct 2011. [Online]. Available: https://link.aps.org/doi/10.1103/PhysRevLett. 107.181802

[31] L. Aliaga and et al., "Design, calibration, and performance of the minerva detector," Nuclear Instruments and Methods in Physics Research Section A: Accelerators, Spectrometers, Detectors and Associated Equipment, vol. 743, pp. 130 - 159, 2014. [Online]. Available: http://www.sciencedirect.com/ science/article/pii/S0168900214000035

[32] J. H. M. Martens and K. Anderson, "Target and horn configuration for the nova experiment," Tech. Rep., 2009.

[33] Ferrari and et al, "FLUKA: A multi-particle transport code (Program version 2005)," CERN-2005-010, SLAC-R-773, INFN-TC-05-11, 2005. 
[34] T. Bhlen, F. Cerutti, M. Chin, A. Fass, A. Ferrari, P. Ortega, A. Mairani, P. Sala, G. Smirnov, and V. Vlachoudis, "The fluka code: Developments and challenges for high energy and medical applications," Nuclear Data Sheets, vol. 120, pp. 211 - 214, 2014. [Online]. Available: http://www.sciencedirect.com/science/article/pii/S0090375214005018

[35] S. Agostinelli and et al., "Geant4-a simulation toolkit," Nuclear Instruments and Methods in Physics Research, 2003.

[36] P. R. Sala and S. Vanini., "Flugg: Fluka + geant4 geometry for simulation in hep," 2003. [Online]. Available: url:http://www.fluka.org/content/tools/ flugg/

[37] A. Himmel., "The numi beam simulation with flugg," NuMI/MINOS Collaboration internal document 6316, 2010.

[38] F. Collaboration, "A quick look at fluka's physics, structure and capabilities," 2015. [Online]. Available: http://www.fluka.org/fluka.php?id=man_onl\& $\mathrm{sub}=3$

[39] N. Collaboration, "The numi technical design handbook." [Online]. Available: http://www-numi.fnal.gov/numwork/tdh/tdh_index.html

[40] S. Mufson and et al., "Liquid scintillator production for the nova experiment." NOvA Collaboration internal document 13014., 2010.

[41] E. Arrieta and et al, "Michigan state university fiber loop transmission qa device," NOvA Collaboration Internal Document 7542, 2012.

[42] L. Vinton, "Calorimetric energy scale calibration of the nova detectors," NOvA Collaboration Internal document 13579.

[43] C. Backhouse and A. Radovic, "The attenuation and threshold correction of the nova detectors," NOvA Collaboration Internal document 13579. 
[44] S. Eidelman and et al., "Particle data group," Phys. Lett. B, vol. 592, 2004.

[45] M. Baird, E. Niner, and K. Sachdev, "Paper on nova event reconstruction for the 2015 chep conference," NOvA Collaboration, Tech. Rep., 2015.

[46] L. Fernandes and M. Oliveira, "Real-time line detection through an improved hough transform voting scheme," vol. Pattern Recognition 41, 2008, pp. 299 -314 .

[47] M. Ohlsson and C. Peterson, "Track finding with deformable templates - the elastic arms approach." Computer Physics Communications 71, 1992, pp. $77-98$.

[48] N. J. Raddatz, "Measurement of muon neutrino disappearance with nonfiducial interactions in the nova experiment," NOvA Collaboration Internal document 15264, 2016.

[49] N. Raddatz, "A technical note describing the numu pid algorithm remid," NOvA Collaboration Internal document 11206, 2014.

[50] S. M. Lein, " $\nu_{\mu}$ charged current energy estimators," NOvA Collaboration Internal document 15211, 2016.

[51] E. N. Jianming Bian and K. Sachdev, "Lid and e/ $\pi^{0}$ identifier," NOvA Collaboration Internal document 15344, 2016.

[52] A. Kaidalov and K. Ter-Martirosyan, Phys. Rev. Lett. B, vol. 117, 1982.

[53] M. Guthrie and et al., Nucl. Instr. Meth, 1968.

[54] H. Bertini and et al., Nucl. Phys. A, vol. 169, 1971.

[55] G. Collaboration, "Reference physics lists," 2013. [Online]. Available: http://geant4.cern.ch/support/proc 
[56] R. Hatcher, "Technote on beam mc simulation," NOvA Collaboration Internal document 6941, 2015.

[57] G. Kafka, "Technote on flux reader," NOvA Collaboration Internal document 12089, 2015.

[58] A. P., L. Aliaga, and et al., "First measurement of electron neutrino appearance in nova," Phys. Rev. Lett., vol. 116, p. 151806, Apr 2016. [Online]. Available: https://link.aps.org/doi/10.1103/PhysRevLett.116.151806

[59] P. Adamson and et al., "First measurement of muon-neutrino disappearance in nova," Phys. Rev. D, vol. 93, p. 051104, Mar 2016. [Online]. Available: https://link.aps.org/doi/10.1103/PhysRevD.93.051104

[60] P. Adamson, D. Ambrose, N. Anfimov, and et al., "Search for active-sterile neutrino mixing using neutral-current interactions in nova," Phys. Rev. D, vol. 96, p. 072006, Oct 2017. [Online]. Available: https://link.aps.org/doi/10.1103/PhysRevD.96.072006

[61] P. Adamson, L. Aliaga, D. Ambrose, N. Anfimov, and et al., "Measurement of the neutrino mixing angle $\theta_{23}$ in nova," Phys. Rev. Lett., vol. 118, p. 151802, Apr 2017. [Online]. Available: https: //link.aps.org/doi/10.1103/PhysRevLett.118.151802

[62] P. Adamson, L. Aliaga, D. Ambrose, and et al., "Constraints on oscillation parameters from $\nu_{e}$ appearance and $\nu_{\mu}$ disappearance in nova," Phys. Rev. Lett., vol. 118, p. 231801, Jun 2017. [Online]. Available: https://link.aps.org/doi/10.1103/PhysRevLett.118.231801

[63] P. Adamson and et al., "New constraints on oscillation parameters from nue appearance and numu disappearance in the nova experiment," 2018. 
[64] Kuldeep K. Maan and H. Duyang and Sanjib R. Mishra, "Flux systematics for noa," NOvA Collaboration Internal document 12175, 23 Oct, 2014.

[65] Kuldeep K. Maan and et al., "Flux systematics for nova," NOvA Collaboration Internal document 12916, 3 March, 2015.

[66] L. Cremonesi, "Supporting technical note describing the addition of the water layer and detailed horn geometry used for the evaluation of the second analysis systematic," NOvA Collaboration Internal document 15296, April 2016.

[67] "Systematic uncertainties in the numi beam flux," MINOS Collaboration Internal document 1283-v4, vol. 4, May 02, 2007.

[68] S. R. Phan-Budd and L. Goodenough, "Technical note on the nova beam monitoring for 2015 summer analysis," NOvA Collaboration Internal document 13572, June 23, 2015.

[69] T. Carroll, "Numi-x: Effects of numi beam shifts and beam divergence," $M I-$ NOS Collaboration Internal document 1283-v4), October 31, 2014.

[70] J. Hylen, "Numi status," NOvA Collaboration Internal document 14706, 2016.

[71] F. b. Phil Schlabach, "Numi-x collaboration internal document 8791," NB1 $2014,2014$.

[72] A. F. t. T. T. Bohlen et al., Nucl. Data Sheets 120, 211; Reports No. CERN2005-10, No. INFN/TC_05/11, No. SLAC-R-773, 2014, 2005.

[73] X. Bu, https://arxiv.org/abs/1601.01213, 2016.

[74] N. E. Collaboration, Eur. Phys. J., vol. C49, 2007.

[75] G. Tinti, "Sterile neutrino oscillations in minos and hadron production in pc collisions," 2010. 
[76] A. Lebedev, "Ratio of pion kaon production in proton carbon interactions," 2007.

[77] J. M. Paley and et al., "Measurement of charged pion production yields off the numi target," Phys. Rev. D, vol. 90, p. 032001, Aug 2014. [Online]. Available: https://link.aps.org/doi/10.1103/PhysRevD.90.032001

[78] R. S. Pedro Mediano, "Nova collaboration internal document 11169," 2014.

[79] Q. W. et al. [NOMAD Collaboration], "A precise measurement of the muon neutrino-nucleon inclusive charged current cross-section off an isoscalar target in the energy range (2.5-40) gev by nomad," Phys. Lett. B 660, 19, 2008.

[80] C. Backhouse, "The cafana framework technote," NOvA Collaboration Internal document 9222.

[81] M. Tamsett, "Cosmic track code review," NOvA Collaboration, Tech. Rep., 2013.

[82] X. Bu, NOvA Collaboration Internal document 13325, 2015.

[83] R. Hatcher, "Technote on beam mc simulation," NOvA Collaboration Internal Document 6941, 2015.

[84] J. Wolcott, H. Gallagher, T. Olson, and T. Mann, "Genie central value tune and uncertainties for second analysis," 2016.

[85] K. K. Maan, " $K^{+}$normalization \& the $\nu_{e}$ flux using nd $\nu_{\mu}$ cc data update," NOvA Collaboration Internal document 14999, 2016.

[86] P. S. Steve Brice, Xuebing Bu and K. K. Maan, "Event-weighted hadron production uncertainty measurement for $\nu_{e}$ flux," NOvA Collaboration Internal document 14129, 2015.

[87] J. Hartnell and et al., NOvA Collaboration Internal document 5232, 2016. 
[88] B. Behera, "Technote on geant systematic study in near detector," NOvA Collaboration Internal document 15776, 2016.

[89] F. H. e. a. Gargamelle collab., Phys. Lett. B 46 (1973) 121, Phys. Lett. $B 46$ (1973) 138

[90] J. D. et al. (CHARM), Z.Phys. C41, p. 567, 1989.

[91] L. Ahrens and et al., Phys.Rev. D41, 3297, 1990.

[92] C. A. et al. [LBNE Collaboration], "The long-baseline neutrino experiment: Exploring fundamental symmetries of the universe."

[93] M. C. et al., Report No. CERN-ATL-SOFT-99-004, 1999.

[94] S. A. et al., Nucl. Instrum. Methods Phys. Res., Sect. A 506, 250, 2003.

[95] C. A. et al., Nucl. Instrum. Methods Phys. Res., Sect. A 614, 87, 2010.

[96] A. Aurisano, C. Backhouse, R. Hatcher, N. Mayer, J. Musser, R. Patterson, R. Schroeter, and A. Sousa, "The nova simulation chain," Journal of Physics. Conference Series, vol. 664, no. 7, 122015.

[97] J. N. Bahcall and et al., Phys.Rev. D51, 6146, 1995. [Online]. Available: arXiv:astro-ph/9502003[astro-ph]

[98] J. Erler and S. Su, Prog. Part. Nucl. Phys. 71, 119 (2013). [Online]. Available: :10.1016/j.ppnp.2013.03.004[arXiv:1303.5522[hep-ph]].

[99] J. Park and et al., "Measurement of neutrino flux from neutrino-electron elastic scattering," https://arxiv.org/pdf/1512.07699.pdf, 2016.

[100] D. d. O. Benhar and I. Sick, "Inclusive quasi-elastic electron-nucleus scattering," Rev. Mod. Phys. 80, 189 (2008) doi:10.1103/RevModPhys.80.189 [nuclex/0603029]. 
[101] M. D. Baird, "An analysis of muon neutrino disappearance from the numi beam using an optimal track fitter," FERMILAB-THESIS-2015-24.

[102] M. E. et al., "Proc. of 2nd international conference on knowledge discovery and knowledge engineering and knowledge management," pp. pp226-231, 1996.

[103] E. D. Niner, "Observation of electron neutrino appearance in the numi beam with the nova experiment," FERMILAB-THESIS-2015-16.

[104] J. Bian and N. Collaboration, "First results of $\nu_{e}$ appearance analysis and electron neutrino identification at nova," arXiv:1510.05708 [hep-ex].

[105] J. Wolcott and et al., NOvA Collaboration Internal document 15214, 2016.

[106] K. K. Maan and et al., NOvA Collaboration Internal Document 15545, 2016.

[107] N. Collaboration, "Reconstructed variables in nova," 2014. [Online]. Available: https://cdcvs.fnal.gov/redmine/projects/novaart/wiki/CAF_Tree_ Structure_and_Variable_Listing

[108] K. Sachdev, "A data-driven method of background prediction at nova." [Online]. Available: arXiv:1310.0119[hep-ex]

[109] H. Duyang and N. Collaboration], "Cosmic ray induced em showers in the no $\nu$ a detectors." [Online]. Available: arXiv:1511.00351[physics.ins-det]

[110] L. Vinton, NOvA Collaboration Internal document 15216, 2016.

[111] H. Duyang, NOvA Collaboration Internal document 14600, 2016.

[112] L. Cremonesi and et al., "A technote describing the derivation and size of numi flux uncertainties used in the second nova analyses," NOvA Collaboration Internal document 15296. 
[113] A. Analyses, "Fiducial mass systematic," NOvA Collaboration Internal document $1323 \%$.

[114] D. Pershey, NOvA Collaboration Internal document 15388, 2016.

[115] K. Sachdev, J. Bian, and E. Niner, NOvA Collaboration Internal Document $13590,2015$. 